\title{
Optical Radiation Measurements \\ Based on Detector Standards
}

George P. Eppeldauer, Editor

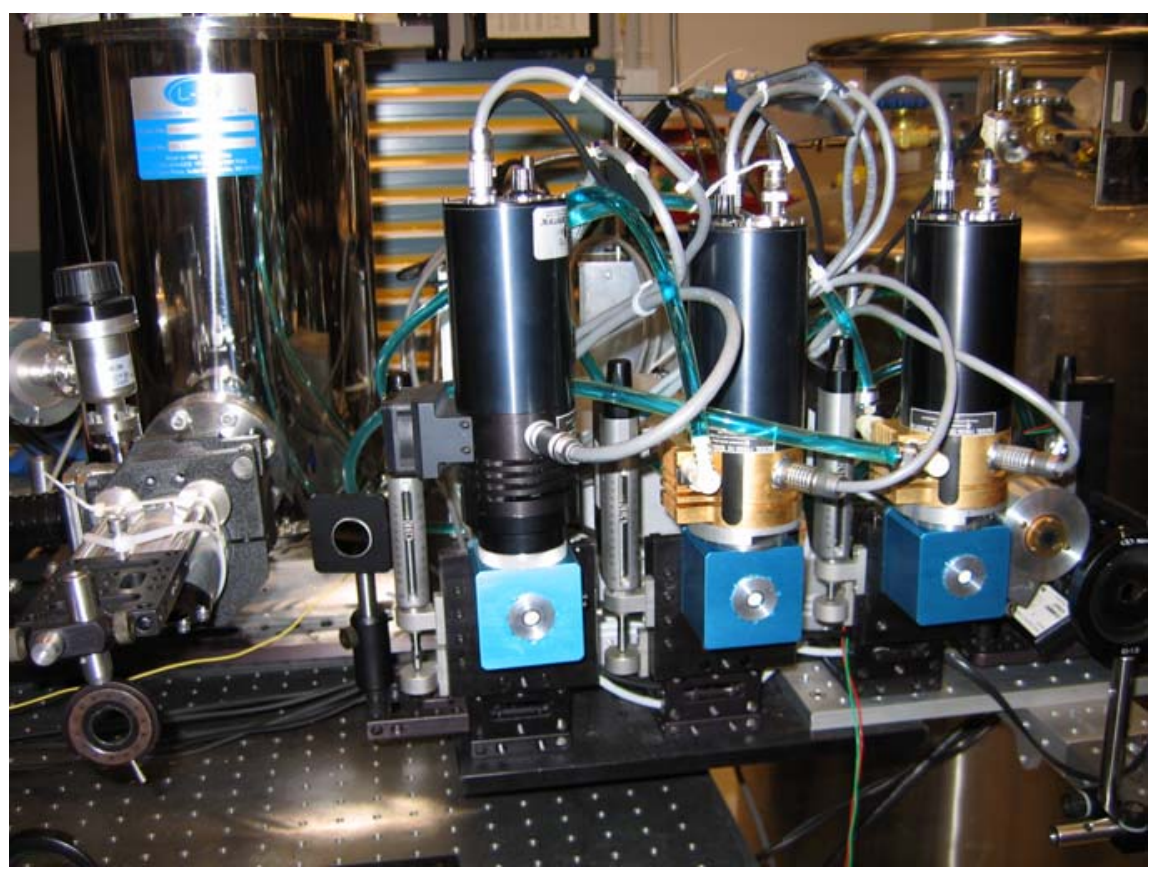




\section{Optical Radiation Measurements Based on Detector Standards}

George P. Eppeldauer, Editor

Optical Technology Division

Physics Laboratory

National Institute of Standards and Technology

Gaithersburg, MD 20899-8441

March 2009

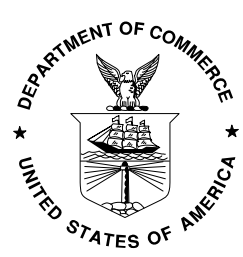

U.S. Department of Commerce

Otto J. Wolff, Acting Secretary

National Institute of Standards and Technology

Patrick Gallagher, Deputy Director 
Certain commercial entities, equipment, or materials may be identified in this document in order to describe an experimental procedure or concept adequately. Such identification is not intended to imply recommendation or endorsement by the National Institute of Standards and Technology, nor is it intended to imply that the entities, materials, or equipment are necessarily the best available for the purpose.

National Institute of Standards and Technology Technical Note 1621

Natl. Inst. Stand. Technol. Tech. Note 1621, 371 pages (March 2009) CODEN: NTNOEF

\section{Front Cover:}

Spectral responsivity calibrations of three transfer-standard extended-InGaAs radiometers against the primary-standard cryogenic radiometer at the SIRCUS facility in the $1 \mu \mathrm{m}$ to $2.5 \mu \mathrm{m}$ wavelength range. 


\section{Preface}

Improved detector technology in the past two decades opened a new era in the field of optical radiation measurements. Lower calibration and measurement uncertainties can be achieved with modern detector/radiometer standards than traditionally used source standards (blackbodies and lamps). The achievable lower uncertainties provided the motivation to decrease the gap between the $0.02 \%(k=2)$ relative expanded uncertainty of cryogenic radiometer measurements and the two to three orders of magnitude higher uncertainties of field-level optical radiation measurements.

The NIST Technical Note (TN) 1438 published in 2001 summarized the research and development work carried out in the Optical Sensor Group of the Optical Technology Division (OTD) since 1989 to develop high optical and electronic performance radiometers with selected optical detectors for the $200 \mathrm{~nm}$ to $20 \mu \mathrm{m}$ wavelength range. In this present Technical Note, which is the continuation of the TN 1438, 36 research papers, published by the OTD from 1996 to present, have been selected to show how the high-performance optical radiometers were converted into standards and used to realize new spectral-responsivity-based scales. In order to utilize the increased performance of the radiometers, new calibration-facilities have been developed to realize, maintain, and disseminate the detector/radiometer standards-based scales. As a result of the work described in these two TNs most of the calibrations at the OTD are performed with the new-generation detector/radiometer standards.

The spectral power responsivity calibrations that started at the OTD about 30 years ago have been extended from the silicon wavelength range for the $210 \mathrm{~nm}$ to $20 \mu \mathrm{m}$ range. New reference responsivity scales have been developed on the newly developed Spectral Irradiance and Radiance Responsivity Calibrations with Uniform Sources Facility (SIRCUS), where instead of traditional monochromators, tunable laser-driven integrating sphere sources are used to decrease detector and radiometer measurement uncertainties. The spectral power responsivity calibrations have also been extended to spectral irradiance and radiance responsivity calibrations.

The irradiance mode reference scale was needed for the SIRCUS facility to improve the NIST realized SI unit, the candela, and to realize the first spectral-responsivity-based tristimulus color scale. New filter-radiometers including photometers, and tristimulus colorimeters have been developed for the scale extensions with electronic and radiometric characteristics that will not limit the scale realization uncertainties. Silicon-trap and sphere-input InGaAs detectors have been developed as highest level transfer standards that can perform $0.05 \%(k=2)$ responsivity uncertainties between $400 \mathrm{~nm}$ and $1650 \mathrm{~nm}$. These SIRCUS reference standards are directly traceable to the primary-standard optical power measuring electrical-substitution cryogenic radiometers.

The radiance responsivity scale was needed to calibrate new-generation optical-radiation thermometers to realize the detector-responsivity-based radiance temperature scale and the SI unit, the kelvin. The most accurate temperature determination of blackbody sources is based on the spectral irradiance and radiance responsivity determinations of the filter (band-pass) radiometers that measure the radiation from blackbody sources. For temperature scale disseminations, improved transfer- and working-standard radiometers have been developed with selected Si, InGaAs, and extended-InGaAs detectors to perform direct thermodynamic temperature measurements in a wide temperature range. As an alternative to the source-based International Temperature Scale (ITS-90), low-uncertainty detector-based radiation temperature measurements can be directly performed with the new Si and InGaAs radiation thermometers between $2700 \mathrm{~K}$ and $430 \mathrm{~K}$. 
For routine spectral power responsivity calibrations in an extended wavelength range, low noiseequivalent-power (NEP) pyroelectric radiometers have been developed that can be operated at the low-power-output of traditional monochromators. These radiometers, traceable to the above reference detectors, extended the routine spectral power responsivity calibrations to a wavelength range from $250 \mathrm{~nm}$ to $19 \mu \mathrm{m}$.

The low measurement uncertainties of the radiometric reference scale realizations required detector output signal measurements with uncertainties smaller than the uncertainty of the radiometric scale realization itself. In order to perform $0.01 \%(k=2)$ electrical output signal measurement uncertainties for the detectors, traceability of the photocurrent measurements has been established to NIST electrical standards. The calibrated photocurrent meters and the different input optics to measure the different radiometric, photometric, and color quantities are integral parts of the new generation radiometers.

Using the calibrated radiometers, the reference responsivity scales have been transferred to other NIST calibration facilities to decrease calibration and measurement uncertainties. Now, the spectral irradiance and radiance scales (disseminated by lamps) are derived from blackbody sources that are calibrated against the filter radiometer standards. Also, the radiometer standards are used to validate other existing source-based scales such as the irradiance from a synchrotron radiation source. The reference radiometers with the new calibration facilities have also been used for the absolute radiometric calibration of digital imaging systems. As a result of the photometric scale improvement and the spectral-responsivity-based tristimulus color scale development, both regular and special light sources (discharge lamps, color displays/monitors, solid-state light sources, etc.) can be measured with low uncertainty resulting in significant energy saving and quality improvement.

With the calibrated radiometers, the NIST detector-standards-based radiometric scales are also disseminated to industry, military, and academia. The new calibrations with the lower measurement uncertainties support the economic growth and competitiveness of the U.S. electrooptical, lighting, paint, textile, and other industries. They also support the National Aeronautics and Space Administration's (NASA) remote sensing activities. With the improved uncertainties of radiometric calibrations and measurements, it became possible to monitor the smaller than $0.1 \%$ long-term changes of the Earth. The detector-standards-based radiance scale is used to validate the calibration sources for the Earth Observing System of the U.S. Global Change Research Program. The OTD developed detector-standards-based infrared calibration systems have also significantly increased the competitiveness of the U.S. military-defense and security.

George P Eppeldauer, Ph.D.

Electronics Engineer, Development and Calibration of Optical Radiometers

Optical Sensor Group, Optical Technology Division

National Institute of Standards and Technology

100 Bureau Drive, Gaithersburg, MD 20899-8441 


\section{Table of Contents}

Preface iii

1. "Facility for spectral irradiance and radiance responsivity calibrations using uniform sources,” Applied Optics, Vol. 45, No. 32, p. 8218-8237, 2006, S. W. Brown, G. P. Eppeldauer, and K. R. Lykke. Reproduced with permission of the Optical Society of America

2. "Radiometer standard for absolute responsivity calibrations from $950 \mathrm{~nm}$ to $1650 \mathrm{~nm}$ with $0.05 \%(k=2)$ uncertainty,” NEWRAD-2008 presentation, to be published in Metrologia, G. P. Eppeldauer, H. W. Yoon, Y. Zong, T. C. Larason, A. Smith, and M. Racz.

3. “Optical characterization of diffuser-input standard irradiance meters," Optika'98, 5th Congress on Modern Optics, SPIE Proc. Vol. 3573, 220-224, 1998. G. Eppeldauer, M. Racz, and T. Larason .34

4. “Near infrared radiometer standards,” SPIE Proceedings, Vol. 2815, 42-54, 1996. G. Eppeldauer

5. "Calibration of a pyroelectric detector at $10.6 \mu \mathrm{m}$ with the National Institute of Standards and Technology high-accuracy cryogenic radiometer,” Applied Optics, Vol. 36, No.16, 3614-3621, 1997. T. R. Gentile, J. M. Houston, G. Eppeldauer, A. L. Migdall, and C. L. Cromer. Reproduced with permission of the Optical Society of America.....

6. "Realization of an infrared spectral response scale on a cryogenic bolometer," Metrologia, Vol. 35, 307-315, 1998. A. Migdall and G. Eppeldauer.

7. "Spectral power and irradiance responsivity calibration of InSb working-standard radiometers,” Applied Optics, Vol. 39, No. 31, 5739-5744, 2000. G. Eppeldauer and M. Racz. Reproduced with permission of the Optical Society of America

8. “Spectral responsivity determination of a transfer-standard pyroelectric radiometer," SPIE Proceedings, Vol. 4818, 118-126, 2002. G. P. Eppeldauer, M. Racz, and L. M. Hanssen

9. "Spectral irradiance responsivity measurements between $1 \mu \mathrm{m}$ and $5 \mu \mathrm{m}$," SPIE Proceedings, Vol. 5543, 248-257, 2004. G. P. Eppeldauer, J. P. Rice, J. Zhang, and K. R. Lykke

10. "Development and calibration of pyroelectric radiometer standards at NIST," SPIE Proc. Vol. 6201, pp. 620119-1 to 620119-12, 2006, G. P. Eppeldauer, J. Zeng, and L. M. Hanssen 
11. "Extension of the NIST infrared spectral responsivity scale to routine monochromatorbased calibrations using improved-NEP pyroelectric detectors,” NEWRAD-2008 presentation, to be published in the Metrologia, G. P. Eppeldauer, J. Zeng, H. W. Yoon, B. Wilthan, T. C. Larason, and L. M. Hanssen

12. "Responsivity calibration methods for 365-nm irradiance meters," also in IEEE Transactions on Instrumentation and Measurement, Vol. 50, No. 2, p. 474-477, April, 2001, T. C. Larason, S. W. Brown, , G. P. Eppeldauer, and K. R. Lykke

13. "Realization of a spectral radiance responsivity scale with a laser-based source and Si radiance meters,” Metrologia, Vol. 37, p. 531-534, 2000, G. P. Eppeldauer, S. W. Brown, T. C. Larason, M. Racz, and K. R. Lykke

14. "Uncertainties of Spectral Responsivity Measurements," Proc. of the 2nd CIE Expert Symposium on Measurement Uncertainty, p. 133-138, CIE Central Bureau, 27 Kegelgasse, A-1030, Vienna, Austria, 2006, G. P. Eppeldauer, G. Sauter, and J. L. Gardner

15. "Realization of the National Institute of Standards and Technology detector-based spectral irradiance scale,” Applied Optics, Vol. 41, No. 28, 5879-5890, 2002. H. W. Yoon, C. E. Gibson, and P. Y. Barnes. Reproduced with permission of the Optical Society of America......140

16. “The NIST detector-based luminous intensity scale,” J. Res. Natl. Inst. Stand. Technol. Vol. 101, 109-132, 1996. C. L. Cromer, G. Eppeldauer, J. E. Hardis, T. C. Larason, Y. Ohno, and A. C. Parr.

17. "Spectral response based calibration method of tristimulus colorimeters," J. Res. Natl. Inst. Stand. Technol. Vol. 103, 615-619, 1998. G. Eppeldauer

18. "Design and characterization of a photometer-colorimeter standard," Applied Optics, Vol. 43, No. 13, 2621-2631, 2004. G. P. Eppeldauer and M. Racz. Reproduced with permission of the Optical Society of America

19. "New photometer standards for low uncertainty illuminance scale realization," The 26th Session of the CIE, July 4-11, 2007, Beijing, China, Proceedings Volume 1, pp. D2-99 to D2-102, G. P. Eppeldauer, C. C. Miller, and Y. Ohno

20. "Improved accuracy photometric and tristimulus-color scales based on spectral irradiance responsivity,” 25th Session of the CIE, Conference Proceedings, Vol. 1, D2-30 to D2-33, 2003. G. P. Eppeldauer, S. W. Brown, C. C. Miller, and K. R. Lykke

21. "Realization and application of a detector-based tristimulus color scale at the National Institute of Standards and Technology, USA,” AIC Colour 05 - 10th Congress of the International Colour Association, Proc. Part I (Editors, J. L. Nieves and J. H-Andres), p.693-696, 2005, G. P. Eppeldauer, S. W. Brown, K. R. Lykke, and Y. Ohno. 201 
22. "Detector-based calibration procedures for tristimulus colorimeter standards," CIE Expert Symposium on Advances in Photometry and Colorimetry, Turin, Italy, 2008, G. P. Eppeldauer, C. C. Miller, and Y. Ohno ..... 205

23. "Directional error correction of radiometric and photometric standard detectors", Metrologia, Vol. 37, p. 489-492, 2000, M. Racz and G. Eppeldauer

24. "The realization and the dissemination of the detector-based kelvin," in Proc. of the 9th International Symposium on Temperature and Thermal Measurements in Industry and Science TEMPMEKO 2004, Dubrovnik Croatia, 22-25 June 2004, D. Zvizdic, L. G. Bermanec, T. Stasic, T. Veliki eds., 59-70, 2004, H. W. Yoon, C.E. Gibson, D. W. Allen, R. D. Saunders, M. Litorja, S. W. Brown, G. P. Eppeldauer, K. R. Lykke. 216

25. "Thermodynamic-temperature determinations of the Ag and Au freezing temperatures using a detector-based radiation thermometer," Applied Optics, Vol. 46, No. 15, p. 28702880, 2007, H. W. Yoon, D. W. Allen, C.E. Gibson, M. Litorja, R. D. Saunders, S. W. Brown, G. P. Eppeldauer, K. R. Lykke. Reproduced with permission of the Optical Society of America .228

26. "Uncertainty analysis and maintenance of the NIST detector-based temperature scale, Acta Metrologica Sinica,” Vol. 29, No 4A, October, 2008, H. W. Yoon, C .E. Gibson, G. P. Eppeldauer, A. W. Smith, S. W. Brown, and K. R. Lykke .239

27. "SSE- and Noise-Optimized InGaAs Radiation Thermometer," Int. J. Thermophysics, Vol. 28, No. 6, p. 2076-2086, Springer, Online: December 2007,” H. W. Yoon, C. E. Gibson, V. Khromchenko, and G. P. Eppeldauer....

28. "Linearity of InGaAs photodiodes," Poster presentation, 8th NEWRAD Conference, NIST, Gaithersburg, MD, May 20-24, 2002. Metrologia Vol. 40, S154-S158, 2003, H. W. Yoon, J. J. Butler, T. C. Larason, G. P. Eppeldauer. Reproduced with permission of the BIPM. .263

29. "Short-wave infrared radiometers design and characterization,” SPIE Defense \& Security Symposium, Infrared Technology and Applications XXXIII, 9-13 April, 2007, Orlando, FL, Proceedings Volume 6542, Part 1, pp. 654200-1 to 65420O-10, G. P. Eppeldauer and H. W. Yoon

30. "Measurement of thermal radiation using regular glass optics and short-wave infrared detectors,” Optics Express, Vol. 16, No. 2, pp. 937-949, January 21, 2008, H. W. Yoon and G. P. Eppeldauer

31. "AC-mode Short-Wavelength IR Radiation Thermometers for Measurement of Ambient Temperatures,” Int. J. Thermophysics, Vol. 29, p. 1041-1051, Springer, 2008, G. P. Eppeldauer and H. W. Yoon .... 
32. "System-level calibration of a transfer radiometer used to validate EOS radiance scales," Int. J. Remote Sensing, Vol. 24, No. 2, 339-356, 2003. B. C. Johnson, S. W. Brown, G. P. Eppeldauer, and K. R. Lykke. Reprinted by permission of the publisher, Taylor \& Francis Ltd.

33. "Spectral Irradiance and Radiance Responsivity Calibrations using Uniform Sources (SIRCUS) facility at NIST,” SPIE Symposium, Denver, CO, August 2-6, 2004, Proc. Vol. 5542, p. 363-374, S. W. Brown, G. P. Eppeldauer, J. P. Rice, J. Zhang, and K. R. Lykke 321

34. “Absolute radiometric calibration of digital imaging systems," SPIE Proceedings, Vol. 4306, 13-21, 2001. S. W. Brown, T. C. Larason, C. Habazuit, G. P. Eppeldauer, Y. Ohno, and K. R. Lykke

35. "Uniform calibration of night vision goggles and test sets," SPIE Europe Security and Defence Symposium, Electro-Optical and Infrared Systems: Technology and Applications IV, Vol. 6737, p. 67370M-1 to 67370M-16, 2007, G. P. Eppeldauer

36. “Traceability of NIST photocurrent measurements to electrical standards,” NEWRAD2008 presentation, to be published in the J. Res. Natl. Inst. Stand. Technol., G. P.

Eppeldauer 


\title{
Facility for spectral irradiance and radiance responsivity calibrations using uniform sources
}

\author{
Steven W. Brown, George P. Eppeldauer, and Keith R. Lykke
}

\begin{abstract}
Detectors have historically been calibrated for spectral power responsivity at the National Institute of Standards and Technology by using a lamp-monochromator system to tune the wavelength of the excitation source. Silicon detectors can be calibrated in the visible spectral region with combined standard uncertainties at the $0.1 \%$ level. However, uncertainties increase dramatically when measuring an instrument's spectral irradiance or radiance responsivity. We describe what we believe to be a new laser-based facility for spectral irradiance and radiance responsivity calibrations using uniform sources (SIRCUS) that was developed to calibrate instruments directly in irradiance or radiance mode with uncertainties approaching or exceeding those available for spectral power responsivity calibrations. In SIRCUS, the emission from high-power, tunable lasers is introduced into an integrating sphere using optical fibers, producing uniform, quasi-Lambertian, high-radiant-flux sources. Reference standard irradiance detectors, calibrated directly against national primary standards for spectral power responsivity and aperture area measurement, are used to determine the irradiance at a reference plane. Knowing the measurement geometry, the source radiance can be readily determined as well. The radiometric properties of the SIRCUS source coupled with state-of-the-art transfer standard radiometers whose responsivities are directly traceable to primary national radiometric scales result in typical combined standard uncertainties in irradiance and radiance responsivity calibrations of less than $0.1 \%$. The details of the facility and its effect on primary national radiometric scales are discussed.
\end{abstract}

OCIS codes: $\quad 120.0120,120.5630,120.3930$.

\section{Introduction}

Detectors are calibrated for spectral power responsivity over the spectral range from 350 to $1000 \mathrm{~nm}$ at the National Institute of Standards and Technology (NIST) on the spectral comparator facility ${ }^{1}$ (SCF) by using a lamp-monochromator system to tune the wavelength of the excitation source and silicon working standard detectors with responsivities directly traceable to the NIST high-accuracy cryogenic radiometer (HACR), the primary U.S. national radiometric standard for optical power measurement. ${ }^{2,3}$ Silicon detectors can be calibrated for optical power responsivity in the visible spectral region on the SCF with combined expanded uncertainties $(k=2)$ at the $0.2 \%$ level. However, quantities of interest are often the amount of light falling on a surface (irradiance) or the amount of light emitted from a source (radiance). In many cases, spectral information is required as

The authors are with the National Institute of Standards and Technology, Gaithersburg, Maryland 20899. S. Brown's e-mail address is steven.brown@nist.gov.

Received 14 February 2006; accepted 27 April 2006; posted 7 July 2006 (Doc. ID 68064). well. Consequently, the calibration of filter-based irradiance and radiance meters is frequently needed.

Because of the low flux in the lamp-monochromator system, instruments cannot be directly calibrated for irradiance or radiance responsivity on the $\mathrm{SCF}$, and more complicated approaches must be taken that often increase the expanded uncertainty $(k=2)$ in the measurements to the $0.5 \%$ level or greater. ${ }^{4}$ In addition, the low flux associated with lamp-monochromator excitation sources $(\sim 1 \mu \mathrm{W})$ limits the effective dynamic range of the system. The out-of-band response of filter radiometers can be measured only to approximately $0.001 \%$ of the peak response while a dynamic range greater than $10^{6}$ can be required for high-accuracy applications. In more advanced applications, spatially nonuniform (e.g., infrared or ultraviolet) irradiance meters cannot be calibrated accurately by using the traditional power measurement mode on the SCF and detector-array-based imaging systems require tests in uniform, monochromatic fields.

With the development of tunable dye lasers in 1970,5,6 high-power, monochromatic, tunable sources became available for scientific use. Replacing the lamp-monochromator source with a tunable laser 
source has a number of advantages for radiometric applications, in particular the high-power, very narrow spectral bandwidth, and the extremely low wavelength uncertainty of the laser-based source. Schaefer and Eckerle ${ }^{7}$ developed the first laser-based radiometric facility at NIST in 1984. In 1986, independent irradiance scales maintained by NIST based on blackbody or synchrotron radiation were compared with scales established on the laser calibration facility based on silicon photodiode physics traceable to cryogenic radiometry. ${ }^{8}$ In 1990 , absolute spectral radiometric measurements of the melting and freezing points of a gold blackbody were made at NIST using the laser-based facility. ${ }^{9}$ The radiometric temperature was in agreement with the gold-point value established in the International Temperature Scale of 1990 (ITS-90). In the same time frame, a laser-based radiometric calibration facility was established at the National Physical Laboratory (NPL) in the United Kingdom, and the radiometric freezing and melting temperatures of gold, silver, and aluminum blackbodies were determined with a radiometric uncertainty of $0.04 \% .{ }^{10}$ The results were compared with thermodynamic temperatures established by using constantvolume gas thermometry. Subsequently, several primary national radiometric standards laboratories have developed or are developing laser-based calibration facilities, including the NPL in the UK, ${ }^{11}$ the Helsinki University of Technology (HUT) in Finland, ${ }^{12}$ and the Physikalisch-Technische Bundesanstalt (PTB) in Germany, ${ }^{13}$ as well as at NIST. In this work, a new laser-based facility developed at NIST for spectral irradiance and radiance responsivity calibrations using uniform sources (SIRCUS) is described.

This facility expands on previous work through the integration of $\mathrm{cw}$, continuously tunable lasers that cover wide ranges of the spectrum as well as with the development of high-performance transfer standard detectors. The expanded continuous spectral coverage has been made possible by recent and continued advances in laser technology. By exploiting the developments in laser and detector technologies in SIRCUS and calibrating filter radiometers that are used in radiance temperature (pyrometers) and photometry (photometers), the uncertainty of the two Systeme Internationale (SI) units maintained by the NIST Optical Technology Division, the kelvin and the candela, can be reduced.

In Section 2 we describe the principal components of the facility and give a representative uncertainty budget for an instrument calibration. In Section 3 we compare the SCF with SIRCUS. In Section 4 we discuss the effect on NIST scales and facilities. In Section 5, we give examples of calibrations that illustrate the flexibility of the new facility.

\section{Description of Spectral Irradiance and Radiance Responsivity Calibrations Using Uniform Sources}

In the SIRCUS facility, emission from high-power, tunable lasers is introduced into an integrating sphere producing uniform, quasi-Lambertian, highly radiant flux sources. Reference standard irradiance detectors, calibrated directly against national primary standards for spectral power responsivity, are used to determine the irradiance at a reference plane. Knowing the measurement geometry, the source radiance can be readily determined as well. Instruments are calibrated directly in irradiance or radiance mode with uncertainties approaching or exceeding those available for spectral power responsivity calibrations.

There are three components to the SIRCUS facility: an ultraviolet, visible, and near-infrared (UV-VisNIR) SIRCUS; an infrared (IR) SIRCUS; and a highaccuracy electrical substitution cryogenic radiometer system called the primary optical watt radiometer (POWR). ${ }^{14}$ POWR is a primary standard facility for the measurement of optical power. The UV-Vis-NIR facility currently operates over the spectral region from 210 to $960 \mathrm{~nm}$; extensions to the short-wave IR, out to $2.5 \mu \mathrm{m}$, are planned. The IR SIRCUS facility has a spectral coverage from 700 to $5.3 \mu \mathrm{m}$ using tunable optical parametric oscillator (OPO) systems and coverage to $11 \mu \mathrm{m}$ using discrete laser sources. Lasers from both SIRCUS facilities can be introduced into the POWR radiometer for detector responsivity measurements. Typically, reference instruments used on SIRCUS are calibrated against POWR. Occasionally, a second, working-standard absolute cryogenic radiometer (ACR) is used. ${ }^{15}$ This system is smaller and easier to operate than POWR. It is located on a portable table and can be readily moved between facilities. Intercomparisons using transfer standard detectors establish the equivalence between the two cryogenic radiometers.

The spectral coverage available with the tunable lasers ultimately defines the spectral range of the SIRCUS facility while the quality of the reference standard detectors and their radiometric uncertainties ultimately determine the uncertainty achievable on SIRCUS. In this work we focus on the UV-VisNIR facility and limit the discussion to the spectral range from 210 to $960 \mathrm{~nm}$ where silicon reference detectors are available. A general description of the SIRCUS facility is given in Subsection 2.A. Sources and reference detectors are discussed in Subsections 2.B and 2.C. Subsection 2.D outlines the calibration method. The uncertainties achievable for radiance and irradiance responsivity calibrations are discussed in Subsection 2.E.

It should be noted that our identification of commercial equipment is for information purposes only. It does not imply recommendation or endorsement by the National Institute of Standards and Technology, nor does it imply that the equipment identified is necessarily the best available for the purpose.

\section{A. Setup}

The SIRCUS facility is shown schematically in Fig. 1. The output of a high-power, tunable laser is first directed through an intensity stabilizer that controls the relative optical power in the beam to within $0.1 \%$ of the set point. A portion of the laser beam is sent into a traveling Michelson interferometer that mea- 


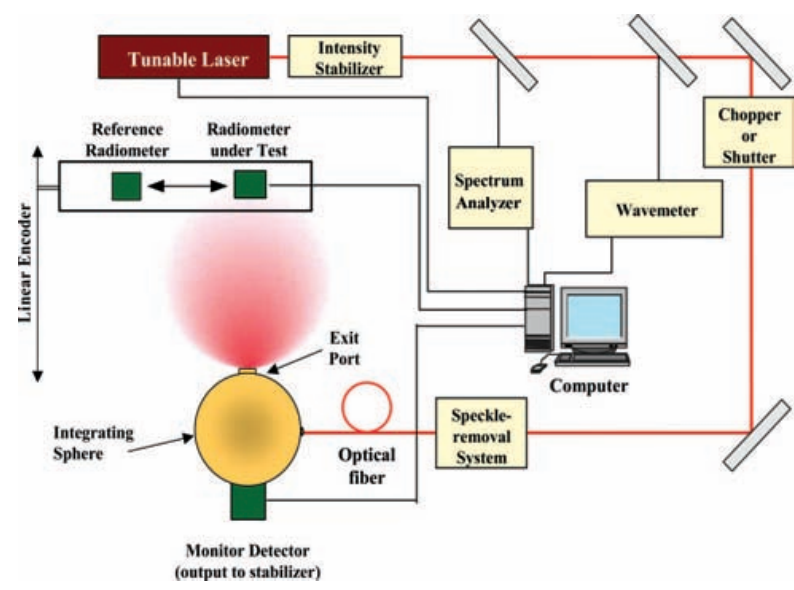

Fig. 1. (Color online) Schematic diagram of the SIRCUS facility.

sures the wavelength of the radiation to within $0.001 \mathrm{~nm}$. A beam splitter sends another portion of the laser beam into a Fabry-Perot interferometer to measure the bandwidth and mode stability of the laser. The laser radiation is typically introduced into an integrating sphere, often using an optical fiber. Different size spheres are used depending on the application. Occasionally, a collimator coupled to the sphere is used as a calibration source. Speckle in the image from the source, originating from interference due to the coherent nature of the laser radiation, is effectively removed either by rastering the beam inside the sphere with a galvanometer-driven mirror ${ }^{9}$ or by placing a short length of optical fiber in an ultrasonic bath. ${ }^{11,16}$ Note that the speckle is still present but is altered on a much shorter time constant than the observing radiometers, effectively averaging out the distribution. A monitor photodiode is located on the sphere to correct for any radiant flux changes in the sphere output between measurements with the reference instrument and the device under test. The sources and detectors are located inside a light-tight box (1.4 m wide, $1.2 \mathrm{~m}$ tall, $3.2 \mathrm{~m}$ long) that has been covered on the inside with Ultrapol, a material with excellent light-absorbing properties: The measured reflectance is of the order of $0.1 \%-$ $0.3 \%$ from $300 \mathrm{~nm}$ to $2.5 \mu \mathrm{m}$. Two baffles are typically installed between the source and the detectors to minimize the effects of stray radiation on the measurement.

The calibrations are performed by direct substitution against primary standard reference irradiance meters (see Subsection 2.C). For both irradiance and radiance responsivity calibrations, the distance between the source aperture and the defining aperture on the reference standard detector is required. An electronic ruler with a resolution of $5 \mu \mathrm{m}$ measures the distance between the source and the detectors.

\section{B. Sources}

In this subsection we go into detail on the laser systems as well as the different calibration sources, namely, the integrating spheres and collimators used on SIRCUS.

\section{Lasers}

A number of different lasers are used to cover the spectral range from about 210 to $960 \mathrm{~nm}$ (see Fig. 2). Continuous tunability is provided by dye lasers and Ti:sapphire lasers that are pumped by either an argon-ion laser $(25 \mathrm{~W}$, all lines visible; $7 \mathrm{~W}$, all lines UV) or a frequency-doubled Nd:vanadate laser $(10 \mathrm{~W}, 532 \mathrm{~nm})$. A number of discrete wavelengths are provided by the pump lasers themselves.

Dye laser use has declined in recent years because of the development of the solid-state Ti:sapphire laser, but the dye laser is still the easiest method for producing high-power, tunable, cw light in the visible. ${ }^{17,18}$ Dye lasers at SIRCUS cover the spectral region from 415 to $700 \mathrm{~nm}$; the dyes used include Stilbene 3 (415-475 nm), Coumarin 480 (470-520 nm), Coumarin 521 (505-565 nm), ${ }^{19}$ Rhodamine 6G (R6G; 550$620 \mathrm{~nm})$, and DCM (610-700 nm). A separate laser is used for each dye so that the full tuning range can be rapidly covered, and no dye change or optical reconfiguration is required. The longer-wavelength dye lasers, with DCM and R6G dyes, are ring lasers. They are located on a table separate from the other three dye lasers and are pumped by the $532 \mathrm{~nm}$ output from a frequency-doubled Nd:vanadate laser. The Coumarin 521, Coumarin 480, and Stilbene 3 lasers are pumped with the output of an argon-ion laser. Separate optical fibers, located on each laser table, couple the radiation from the lasers into the integrating spheres.

Ti:sapphire lasers cover the spectral range from 680 to $1050 \mathrm{~nm} .{ }^{20}$ A frequency doubling of Ti:sapphire lasers enables us to tune from 350 to $500 \mathrm{~nm}$. Two different types of frequency doubling are available: The first approach uses an external power buildup cavity that requires a frequency-stabilized, singlemode pump laser. ${ }^{21}$ The second is a home-built intracavity-doubled Ti:sapphire laser that requires no frequency stabilization. This system uses a simple

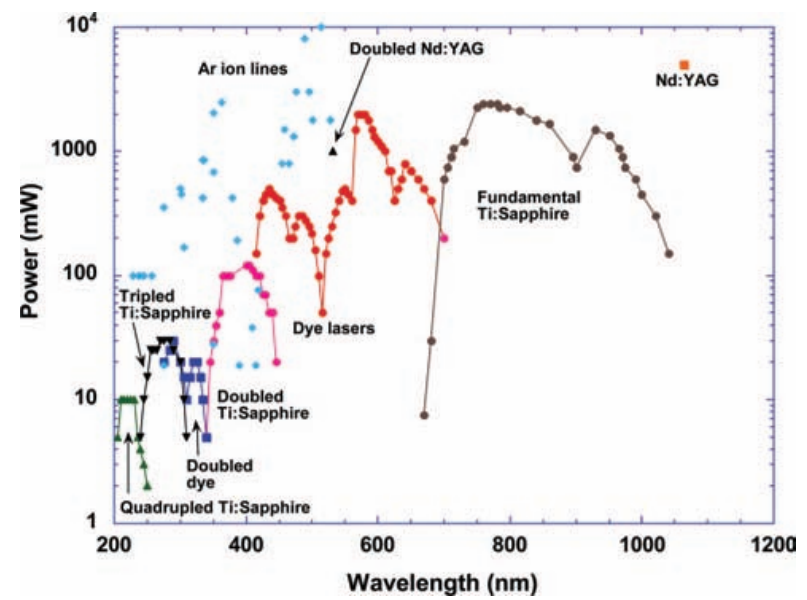

Fig. 2. (Color online) Lasers, output power, and spectral coverage of UV-Vis-IR SIRCUS. 
$z$-cavity Ti:sapphire laser. When doubling is required, the output coupler is removed and two mirrors are used in its place: a fold mirror (with a $5 \mathrm{~cm}$ focal length) and a flat mirror. The doubling crystal, typically lithium barium oxide (LBO), is located near the focus of the curved mirror. ${ }^{22}$ One of the attractive features of the intracavity-doubling design is that the system lases without the doubling crystal in placeensuring the proper cavity alignment prior to installation of the doubling crystal. It is also less sensitive to acoustic noise and small frequency fluctuations in the laser than the external-cavity system.

Over $300 \mathrm{~mW}$ of optical power is available from the dye lasers and the Ti:sapphire laser (with optical powers of $1 \mathrm{~W}$ or greater over much of the spectral range). The power available from the $\mathrm{cw}$, frequencydoubled radiation is typically in the range from 100 to $200 \mathrm{~mW}$. A variety of standing-wave and ring lasers are currently used on SIRCUS. With the exception of the external buildup cavity approach to frequencydoubled light, simple, inexpensive standing-wave dye lasers and Ti:sapphire lasers equipped with an intracavity, uncoated etalon to ensure narrowband operation are sufficient for all foreseeable radiometric applications.

Quasi-cw, mode-locked laser systems have been shown in the past to be equivalent to $\mathrm{cw}$ systems for radiometric calibrations of silicon radiometers, with no additional uncertainties in the calibration arising from the quasi-cw nature of the radiation. ${ }^{23,24} \mathrm{~A}$ commercial mode-locked system was recently installed in the facility. The system includes an OPO system for generation of visible and infrared light, and a frequency doubler, tripler, and quadrupler system for generation of visible and UV radiation. These nonlinear optical systems take advantage of the pulsed nature of the radiation: It is significantly easier to generate sum and difference frequency radiation using a mode-locked laser rather than a true cw laser because of the high peak power of the mode-locked source. This system generates almost continuously tunable radiation from 210 to $3 \mu \mathrm{m}$. One useful feature of the quasi-cw system is that the bandwidth of the radiation is considerably broader than radiation from the cw systems. This may help to alleviate the measurement problems associated with interference fringes (discussed in Section 6).

Computer-controlled actuators rotate the angle of the birefringent tuner in the dye and Ti:sapphire lasers. The minimum step size is determined by the free spectral range of the etalon in the lasers. For a $1 \mathrm{~mm}$ thick etalon, the thickness of the etalons used on SIRCUS, the minimum wavelength step using the birefringent tuner is $0.16 \mathrm{~nm}$ at $600 \mathrm{~nm}$. For finer spectral resolution, the etalon angle is changed as well. Controlling both the birefringent tuner and the etalon angle, wavelength steps of $0.01 \mathrm{~nm}$ or less can be readily achieved.

\section{Integrating Spheres}

Different integrating spheres are used, depending on the radiometric calibration. Small-diameter integrat- ing spheres-with diameters ranging from 2.54 to $5.08 \mathrm{~cm}$ - equipped with precision apertures with diameters ranging from 3 to $8 \mathrm{~mm}$ are typically used for irradiance responsivity calibrations. Note that the areas of the exit apertures are normally measured at the NIST facility for aperture area measurement. ${ }^{25}$ Larger-diameter spheres- $30 \mathrm{~cm}$ in diameter-with 5 to $10 \mathrm{~cm}$ diameter exit ports are used for radiance measurements. The spheres are made of a sintered polytetrafluoroethylene-based (PTFE-based) coating that has high diffuse reflectance from approximately $250 \mathrm{~nm}$ to $2.5 \mu \mathrm{m} .{ }^{26}$ Typical irradiance levels at $1 \mathrm{~m}$ using a $2.54 \mathrm{~cm}$ diameter integrating sphere with a $5 \mathrm{~mm}$ diameter aperture range from approximately 1 to $10 \mu \mathrm{W} / \mathrm{cm}^{2}$. Radiance levels between 1 and $5 \mathrm{~mW} / \mathrm{cm}^{2} / \mathrm{sr}$ are standard for a $30 \mathrm{~cm}$ diameter sphere with a $7.5 \mathrm{~cm}$ diameter output port. A monitor detector is mounted on the sphere wall to monitor and correct for fluctuations in the source radiant flux during a calibration. The irradiance is uniform to within $0.1 \%$ over several centimeters at a $1 \mathrm{~m}$ separation from the integrating sphere. Similarly, the radiance from the integrating sphere is uniform to within $0.1 \%$ over the central $90 \%$ of the exit aperture.

\section{Collimator Sources}

In earlier work at the NPL, collimated sources made from spheres with lenses were used in the calibration of a number of filter radiometers and transmission of lenses. ${ }^{11}$ In SIRCUS, off-axis parabolic mirrors are used to prepare the quasi-collimated source. Mirrors have the advantage over lenses of achromaticity from the UV to the IR. In addition to the collimators in SIRCUS, both on- and off-axis collimator sources have been developed in collaboration with NASA ${ }^{27}$ and the U.S. Geological Survey ${ }^{28}$ (USGS) for the characterization and calibration of large-aperturearea telescopes. Nonuniformities of $1 \%$ or less are achieved over a $5 \mathrm{~cm}$ diameter area with an irradiance up to 2 orders of magnitude greater than with the integrating sphere alone.

\section{Detectors}

The uncertainty in the spectral irradiance responsivity of reference standard detectors is the largest component in the SIRCUS calibration uncertainty budget. Ultimately, the quality and characteristics of the reference standard detectors determine the uncertainty achievable on SIRCUS. Silicon tunnel-trap detectors, configured to operate in both radiant power and irradiance measurement modes, are the highest-level reference standards used in the SIRCUS facility. They cover the spectral range from 300 to $960 \mathrm{~nm}$. Reflectance traps with specially designed Si detectors that are stable under UV exposure are used for calibrations in the 210 to $350 \mathrm{~nm}$ spectral region.

1. Reference Standard Silicon Tunnel-Trap Detector Trap detectors consist of multiple silicon photodiodes oriented such that the incident radiation reflected from one detector impinges on a second detector. ${ }^{29}$ Hamamatsu model S1337 (Ref. 30) photodiodes are 


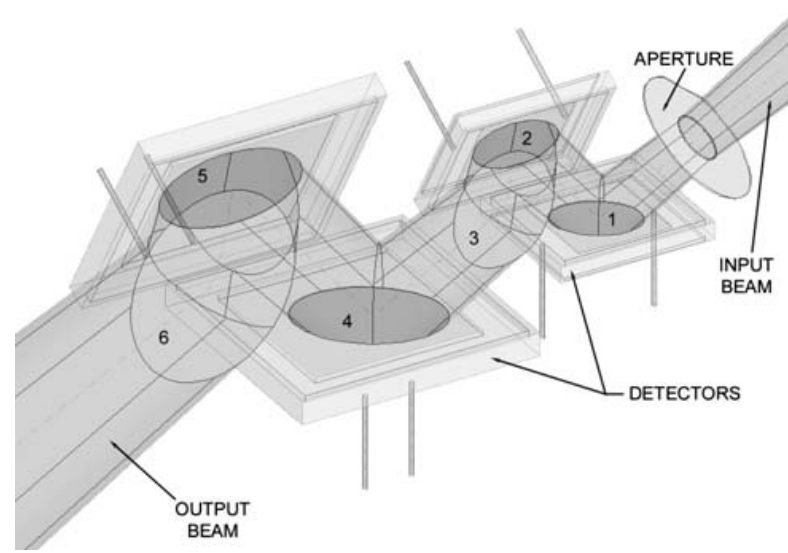

Fig. 3. Beam propagation through the six-element transmission tunnel-trap detector. Detectors 3 and 6 have been removed from the diagram.

typically used in trap detectors to utilize the physical model of internal quantum efficiency (IQE) for spectral responsivity extrapolation and interpolation. ${ }^{3}$ The $\mathrm{IQE}$ is the ratio of the number of collected electrons to the number of photons absorbed by the detector after the front surface reflection loss; the S1337 photodiodes are chosen because of their extremely high IQE (close to 1). A detector's external quantum efficiency (EQE) is equal to the IQE times the term 1 - reflectance. In trap detectors, the reflection loss is minimized by having incident reflected radiation impinge on the next photodiode in succession, leading to the absorption of almost all the incident radiation. Measuring the sum of the photocurrents from the photodiodes that make up the trap detector, the $\mathrm{EQE}$ can be very close to unity. This type of detector is frequently called a quantum-flat detector.

The schematic of the transmission tunnel-trap detectors used on SIRCUS, with the input aperture included, is shown in Fig. $3 .{ }^{31}$ By choosing different size photodiodes, the aperture area and the field of view (FOV) could be maximized. Two $10 \mathrm{~mm} \times$ $10 \mathrm{~mm}$ and four $18 \mathrm{~mm} \times 18 \mathrm{~mm}$ silicon photodiodes, equivalent to the Hamamatsu S1337 model photodiodes, were packaged in a triangular-shaped, light-trapping arrangement. The photodiodes were selected for large and equal shunt resistance, an important consideration for the signal current-tovoltage conversion. ${ }^{31}$ The beam propagates in the tunnel-trap detector from the first photodiode (behind the aperture) to the last (sixth) photodiode. Photodiodes 3 and 6 are not shown for better illustration. The small amount of radiation that exits the back of the trap detector can be utilized for alignment or can be measured for detector transmittance measurements in spectral responsivity determination. A conical light trap attached to the trap detector output absorbs the transmitted radiation and minimizes the effect of ambient stray light for low-level optical radiation measurements. The photodiodes in the trap detectors are not temperature controlled. The trap detectors have been characterized for both electri- cal and optical performance. In Subsection 2.D, the trap detector's response linearity, spatial uniformity, and angular responsivity are discussed along with the spectral power responsivity calibration against a high-accuracy cryogenic radiometer.

\section{Monitor Detectors}

Silicon detectors mounted on the integrating sphere sources monitor the source radiance and correct for any small changes that occur during a calibration caused by fluctuations in the laser power input into the integrating spheres. To avoid interference fringes in the monitor signal (caused by residual coherence in the output radiation of a sphere source), the window of the silicon monitor detector is removed, or a diffuser is placed between the silicon detector and the integrating sphere. Both the reference standard trap detectors and the monitor detectors operate in dc measurement mode. ${ }^{32}$

\section{Method of Calibration}

A calibration relates the measured quantity from a radiometer, usually current or voltage, to the radiometric quantity being measured, e.g., radiance or irradiance, through the instrument's responsivity. Determination of an instrument's responsivity and an evaluation of the associated uncertainties are required in a calibration. The substitution method is used for calibrations in SIRCUS. NIST reference standard irradiance meters determine the irradiance at a reference plane. From the irradiance at the reference plane, which is usually the plane of the detector aperture, the radiance of a source can be determined by knowing the distance between the detector and source apertures, as well as the area of the source aperture. The instrument being calibrated, or device under test (DUT), is then placed in front of the source and its signal is recorded. The laser is blocked and the dark signal is recorded prior to each measurement. The responsivity (at the excitation wavelength) is the instrument's net output signal (with the dark signal subtracted from the light signal) divided by the radiometric quantity to be measured.

The characterization of an instrument and an understanding and evaluation of all meaningful sources of uncertainty are crucial for a proper calibration. A calibration without an associated uncertainty table is of limited use. The evaluation and expression of uncertainty is generally difficult and time consuming; it is not unusual to have incomplete or inaccurate information in an uncertainty table. Determining how best to express a particular uncertainty component can be confusing. There are a variety of useful references that provide definitions and recommendations for describing and establishing the uncertainties encountered when calibrating a radiometer. ${ }^{33}$

A measurement equation is a mathematical expression describing the relationship between the measured source-related radiometric quantity and the instrument responsivity. The simplified measurement equation for an irradiance responsivity calibration is 


$$
R_{\text {DUT }}=\frac{i_{\text {DUT }}}{i_{\text {trap }}} R_{\text {trap }}=\frac{i_{\text {DUT }}}{i_{\text {trap }}} s_{\text {trap }} A,
$$

where $R_{\text {DUT }}$ is the irradiance responsivity of the device under test, $R_{\text {trap }}$ is the irradiance responsivity of the trap, $s_{\text {trap }}$ is the power spectral responsivity of the trap, $A$ is the area of the aperture on the trap, and $i$ is the photocurrent. The photocurrent is converted to voltage in a transimpedence amplifier and then measured with a multimeter.

Equation (1) assumes that the reference planes of the DUT and trap are in the same plane, i.e., that the defining apertures are located the same distance from the point source. In many cases, they are located in different planes. In Subsection 2.D.4, it is shown that, for the aperture sizes and operating distances used on SIRCUS, the sources can be treated as point sources. For a point-source geometry,

$$
I(\lambda)=E_{1}(\lambda) d_{1}^{2}=E_{2}(\lambda) d_{2}{ }^{2},
$$

where $I(\lambda)$ is the radiant intensity of the source; $E_{1}$ is the irradiance at reference plane 1 , located a distance $d_{1}$ from the source; and $E_{2}$ is the irradiance at reference plane 2 , located a distance $d_{2}$ from the source. In many cases, the reference plane of the DUT is not known and must be determined radiometrically.

There are two types of uncertainty components, designated Type A and Type B. Type A uncertainties are evaluated using statistical methods, and Type B uncertainties are evaluated using models or other external information. The term standard uncertainty refers to an estimated standard deviation. Assuming each uncertainty component is independent from the others (the components are uncorrelated), the combined standard uncertainty is the root sum square of the individual uncertainty components. Often, the different variables are not completely independent from one another, and correlations between these variables need to be taken into account. 34,35

The expanded uncertainty is the product of the combined standard uncertainty and a coverage factor $k$, where the value of $k$ is chosen based on the desired level of confidence. Typically the expanded uncertainty is reported with $k=2$, corresponding to a confidence level of $95 \%$. A confidence level of $95 \%$ means that there is a 1 in 20 chance that a measurement will fall outside the interval. A coverage factor $k=3$ corresponds to a confidence level of $99 \%$, meaning there is a $1 \%$ chance that a measurement will fall outside the stated interval. In reporting the uncertainty for a measurement, the components of standard uncertainty are listed and their designation stated (A or B). The uncertainty table should also identify and distinguish random components from systematic components that are common to all wavelengths. This information is important when combining the spectral measurements, for example in measuring the band-integrated response of filtered detectors.

The dominant uncertainties of the spectral irradiance responsivity scale originate from systematic errors in the absolute scale transfer from the cryogenic radiometer. Temperature variations within the facil-

\begin{tabular}{|c|c|c|}
\hline \multirow[b]{2}{*}{ Uncertainty Component } & \multicolumn{2}{|c|}{$\begin{array}{c}\text { Relative Standard } \\
\text { Uncertainty } \\
{[\%]}\end{array}$} \\
\hline & Type A & Type B \\
\hline \multicolumn{3}{|l|}{ Reference detector responsivity } \\
\hline $\begin{array}{l}\text { Radiant power responsivity } \\
\quad(400 \text { to } 920 \mathrm{~nm})\end{array}$ & 0.025 & \\
\hline Aperture area & 0.004 & \\
\hline Response uniformity & 0.005 & \\
\hline Cosine dependence & 0.01 & \\
\hline Polarization & & 0.00 \\
\hline Linearity & 0.00 & \\
\hline Temperature & & 0.003 \\
\hline \multicolumn{3}{|l|}{ Source characteristics } \\
\hline Radiant flux & 0.005 & \\
\hline Wavelength $(<0.01 \mathrm{~nm})$ & & 0.005 \\
\hline Irradiance uniformity & 0.005 & \\
\hline $\begin{array}{l}\text { Determination of the } \\
\text { reference plane }\end{array}$ & 0.01 & \\
\hline$I-V$ Gain & 0.01 & \\
\hline Voltmeter reading & & 0.005 \\
\hline Irradiance & \multicolumn{2}{|c|}{0.032} \\
\hline $\begin{array}{l}\text { Transfer to device under test } \\
\text { (estimated) }\end{array}$ & \multicolumn{2}{|c|}{0.030} \\
\hline $\begin{array}{l}\text { Combined standard } \\
\quad \text { uncertainty }(k=1)(\%)\end{array}$ & \multicolumn{2}{|c|}{0.044} \\
\hline
\end{tabular}

Table 1. SIRCUS Uncertainty for an Irradiance Responsivity Calibration

ity, while small, may also contribute to the overall uncertainty in certain spectral regions. The radiometric stability of the source (and monitor detector) and the irradiance uniformity at a reference plane contribute to the overall uncertainty in the measurement. Additional uncertainties from the radiometric characteristics of the DUT need to be quantified to establish the uncertainty in the calibration (and subsequent measurements of a source). Typically, the instrument's response linearity, temperature dependence, polarization dependence, and out-of-FOV blocking are measured. The current-to-voltage converter and the multimeter used to measure the signal need to be characterized and their contribution to the overall uncertainty established. Finally, both short-term stability (repeatability) and long-term stability (to monitor degradation in time) should be considered.

In the following subsections, we discuss the uncertainty components in a typical irradiance responsivity calibration. The components are combined into an uncertainty budget in Table 1 . These are representative numbers only. The uncertainty in an irradiance responsivity calibration may differ slightly, depending on the details of the calibration and the DUT.

\section{Detector-Based Spectral Irradiance Responsivity} Scale

The reference standard tunnel-trap detectors hold the spectral irradiance responsivity scale on SIRCUS. As no light is reflected back from the trap detector to the aperture, the aperture area and the trap detector spectral radiant power responsivity can be determined sep- 


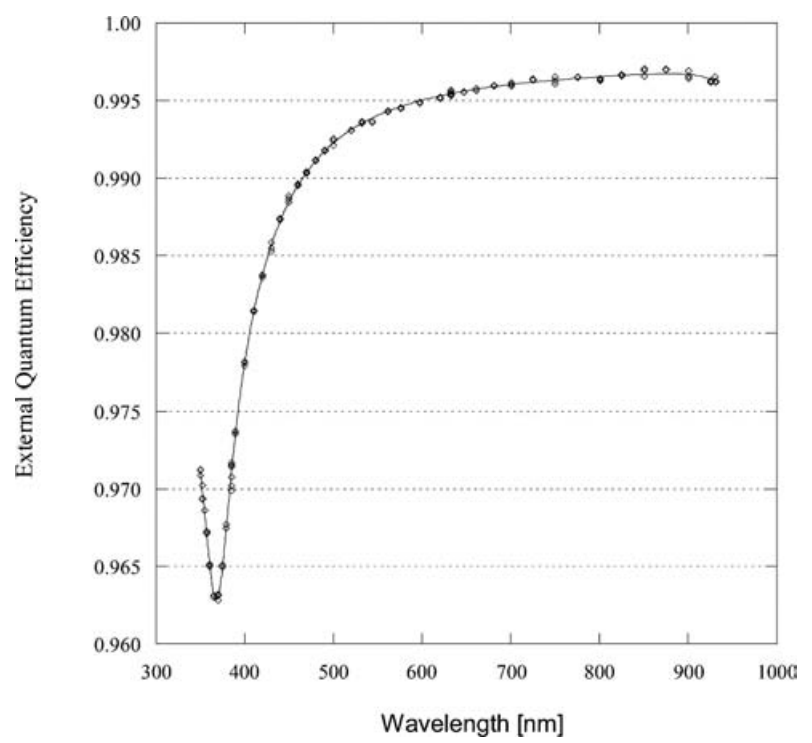

Fig. 4. Silicon tunnel-trap detector EQE versus wavelength.

arately. The spectral irradiance responsivity of the trap detector is calculated as the product of the aperture area and the spectral radiant power responsivity of the trap detector. The spectral radiant power responsivity of the tunnel trap was measured by direct substitution against the working standard ACR (Section 2). The spectral power responsivity (in $\mathrm{A} / \mathrm{W}$ ) is proportional to the EQE and the wavelength. The proportionality factor is $e / h c$, where $e$ is the elementary electron charge, $c$ is the speed of light in vacuum, and $h$ is the Planck constant. The measured EQE of one of the working standard trap detectors (T-01) versus the wavelength (82 laser wavelengths) is shown in Fig. 4. The EQE is equal to 0.996 to within $0.1 \%$ between 600 and $920 \mathrm{~nm}$. The relative combined standard uncertainties in the detector responsivity are $0.05 \%$ between 400 and $960 \mathrm{~nm}$ and $0.1 \%$ between 350 and $400 \mathrm{~nm}$. A fit to the data points, illustrated in Fig. 4, shows that the reproducibility error of the responsivity determinations is very small, especially between 500 and $900 \mathrm{~nm}$. That is, the uncertainty in the fit to the $\mathrm{EQE}$ is smaller than the uncertainty determination at each wavelength. Additionally, there are correlations among these absolute points. ${ }^{35}$ Neither have been considered or included in the uncertainty budget listed in Table 1.

The trap detector was then equipped with a precision circular aperture whose area was measured on an interferometrically controlled $x-y$ translation stage with a video microscope by using edge detection. ${ }^{25}$ The relative standard uncertainty of the area determination for the $5 \mathrm{~mm}$ diameter aperture of the trap detector was $0.004 \% .25$

The spectral radiant power responsivity of the reference standard trap detectors was measured in underfilled (total beam power measurement) mode. In the irradiance mode, the incident flux overfills the aperture. The radiant flux falls on different regions of the trap detector for the two measurement modes. While

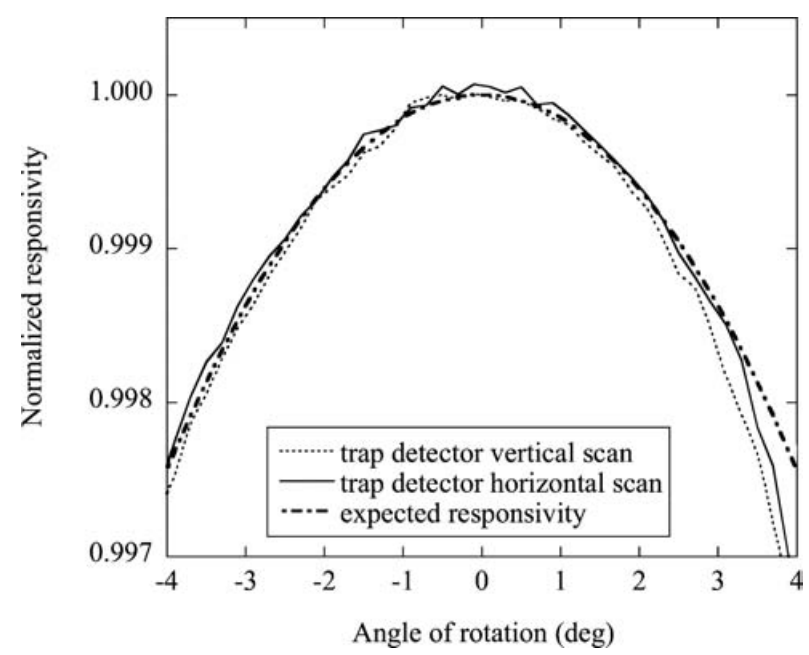

Fig. 5. Angular responsivity of the tunnel-trap detector.

typically small for silicon trap detectors, the spatial nonuniformity in responsivity of the trap detector can produce the ultimate limiting uncertainty component for the reference irradiance responsivity scale when the primary standard, in our case the ACR, measures radiant power. Dust is the primary cause of nonuniformity in the responsivity because the reference trap detectors are nonwindowed devices. To propagate the low uncertainties in power responsivity to irradiance responsivity, the detector's spatial response uniformity, as well as the area of the defining aperture, must be measured. The response of a reference trap detector was recently found to be uniform to within $0.01 \%$ over the entire area of the entrance window (with the aperture removed).

When measuring a point source, the expected responsivity in irradiance mode is a cosine dependence on the angle of rotation of the detector from the plane normal to the incident flux. Deviations from the expected response can give rise to errors when measuring extended sources. Figure 5 shows the normalized angular responsivity of the tunneltrap detector when it is equipped with a circular aperture of $5 \mathrm{~mm}$ in diameter. The responsivity deviation from the cosine function is less than $0.02 \%$ within a $6^{\circ} \mathrm{FOV}$. For an $8^{\circ} \mathrm{FOV}$, the deviation is approximately $0.05 \%$. This feature makes it possible to use these trap detectors as transfer standards for monochromator-based facilities where the beam convergence angle is smaller than the unvignetted FOV of the tunnel-trap detector.

\section{Radiometric Characteristics of the Trap Detector} The radiation from an integrating sphere is typically unpolarized. ${ }^{36}$ If polarized light is required for a calibration, a collimator can be used with a polarizer in the beam.

Linearity is easily checked by changing the laser power with the intensity stabilizer at the input to the fiber. The ratio of the signal of the DUT or reference trap to the monitor signal as a function of power should 


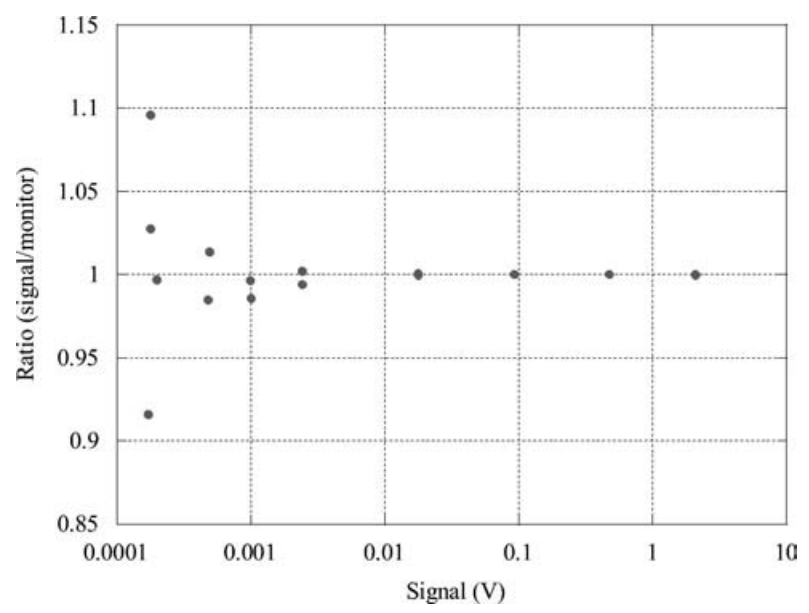

Fig. 6. Detector response linearity. The signal-to-monitor ratio as a function of the signal (related to incident optical power).

be a straight horizontal line. Figure 6 is a representative plot of a silicon detector's response linearity over four decades. Some silicon detectors have been measured to be linear over 14 decades. 37,38

The temperature in the SIRCUS facility was maintained at a nominal temperature of $22{ }^{\circ} \mathrm{C}$. Typical variations were of the order of $0.5^{\circ} \mathrm{C}$ or less, though in extreme cases, variations of the order of $\pm 2{ }^{\circ} \mathrm{C}$ were observed. The temperature, pressure, and humidity inside the light-tight enclosure can be monitored and corrections can be made if necessary. The temperature coefficient of Hamamatsu S1337 photodiodes (the type of silicon photodiode used in the trap detectors) has been measured. ${ }^{1}$ It was found that there was negligible dependence below $950 \mathrm{~nm}$ $(<0.01 \% / \mathrm{K})$ but that the dependence rose quickly to $\sim 0.2 \% / \mathrm{K}$ by $1000 \mathrm{~nm} .{ }^{39} \mathrm{~A}$ similar dependence should be seen in trap detectors using these diodes.

\section{Source Characteristics}

The radiant power and wavelength stability are two of the primary uncertainty components. A laser intensity stabilizer keeps the laser power level constant to within $0.1 \%$. The lasers used as excitation sources are equipped with etalons to reduce the wavelength uncertainty from mode hopping. The wavelength uncertainty is of the order of $10^{-3} \mathrm{~nm}$ with a typical bandwidth of the order of $10^{-3} \mathrm{~nm}$. The wavelength uncertainty is dominated by the dependence of the index of refraction of air on temperature, humidity, and pressure. The wavemeter corrects for the temperature and pressure dependence and can be programmed to correct for the humidity.

The sequence for measurements of a DUT is to first measure the trap and monitor signal, then move the stage so that the DUT versus the monitor signal is measured, then move back to the trap measurement. If any change is observed in the trap-to-monitor ratio greater than $\sim 0.01 \%$, additional measurements are made and the data are averaged. Typically, from 9 to 16 samples are averaged for each data point. Occa- (a)

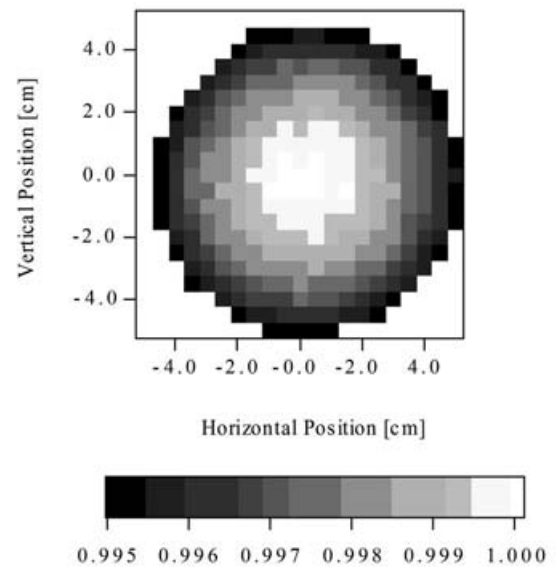

(b)

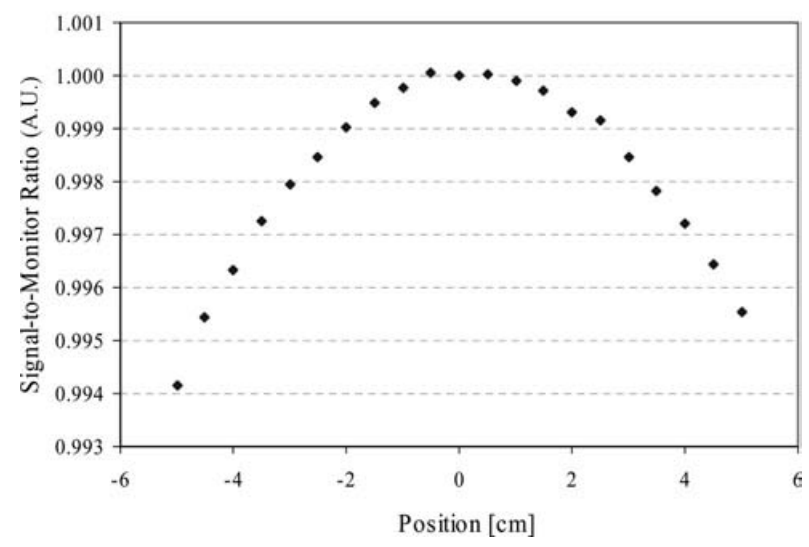

Fig. 7. Irradiance uniformity at a reference plane $1 \mathrm{~m}$ from the irradiance source. (a) Two-dimensional map of the irradiance distribution; (b) horizontal slice through the vertically centered irradiance.

sionally additional samples are taken, for example, when measuring the out-of-band response of a filter radiometer under test.

The irradiance at a reference plane should be spatially uniform to limit the magnitude of corrections for different size entrance pupils. In Fig. 7(a) we show the irradiance distribution at a reference plane approximately $1 \mathrm{~m}$ from the exit port of a $25.4 \mathrm{~mm}$ integrating sphere equipped with a $5 \mathrm{~mm}$ diameter aperture. In Fig. 7(b) we show a horizontal slice across the center of the distribution. As shown in Fig. 7, the irradiance is uniform to within $0.1 \%$ over the central $\pm 2 \mathrm{~cm}$ in both the horizontal and vertical directions. The entrance apertures of most irradiance meters calibrated on SIRCUS have diameters significantly smaller than $4 \mathrm{~cm}$, and no correction for irradiance nonuniformity at the reference plane is applied.

\section{Distance}

The distance between the source and trap detector apertures (reference planes) is measured optically and mechanically. For a point-source geometry, the irradiance should follow an inverse square law, i.e., the ir- 


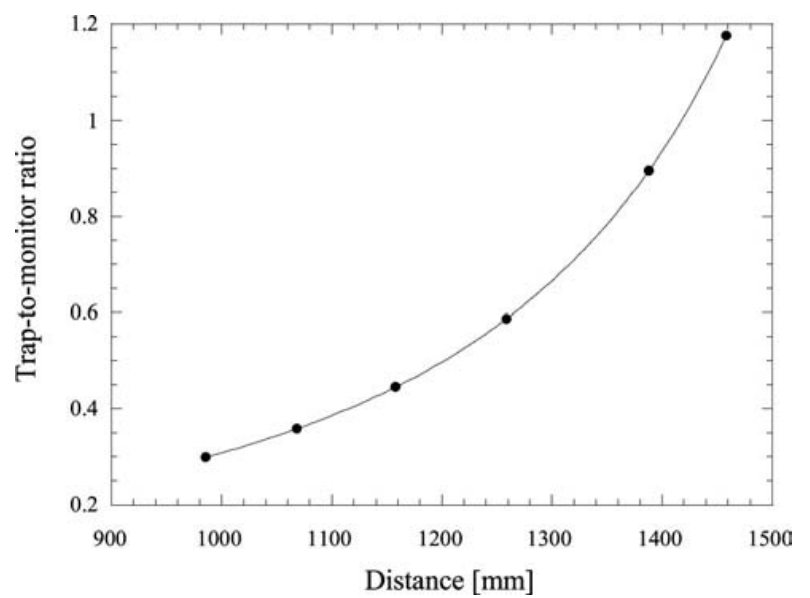

Fig. 8. Radiometric determination of the distance between two aperture planes. Dots are measured trap-to-monitor ratios; solid curve is a fit to the data.

radiance should be a function of $1 / d^{2}$, where $d$ is the distance between the two reference planes. The relative distance between the source and the detector is measured with an electronic ruler that has a quoted resolution of $5 \mu \mathrm{m} .{ }^{40}$ The three-axis detector translation stage is moved in the horizontal direction, and the detector and monitor signals are acquired as a function of relative distance. The ratio of the trap signal to the monitor signal is then plotted as a function of distance and fit with a nonlinear least-squares algorithm. ${ }^{41}$ Figure 8 illustrates an optical distance measurement, with the dots measuring trap-tomonitor ratios and the solid curve being the fit to the data. The data were fit to the expression

$$
y=m_{1} /\left(m_{0}-m_{2}\right)^{2},
$$

where the radiant intensity is given by $m_{1}$, the zero offset of the fixed source is $m_{2}$, and $m_{0}$ is the position of the stage measured by the electronic ruler. The uncertainty in the fit to $m_{2}$ is $28.7 \mu \mathrm{m}$. Since we know the zero offset from this fit, we also know the absolute separation at any value of the electronic ruler with an uncertainty of $28.7 \mu \mathrm{m}$. To validate the radiometric measurements, we also measured the aperture separation by using a calibrated ruler. The two separate techniques used to measure the distance between the source and the detector reference planes agreed usually within $50 \mu \mathrm{m}$. Given a $50 \mu \mathrm{m}$ uncertainty in the trap reference plane, the uncertainty in the irradiance at a given reference plane for distances of $1 \mathrm{~m}$ or greater is less than $0.01 \%$.

For radiometers with a known reference plane, we use a micrometer to make sure that the DUTs and the reference trap aperture lie in the same plane. By using a micrometer, the reference planes can be aligned to within approximately $10 \mu \mathrm{m}$. Consequently, this approach significantly reduces the uncertainty in the irradiance at the DUT reference plane. If the DUT reference plane is not known, it is optically determined by using Eq. (3). For a non-point-source geometry, the full equation including the spatial extent of the source and detector is required ${ }^{42}$; the uncertainties remain the same.

\section{Current-to-Voltage Conversion (I-V Amplification)}

The uncertainty of the current-to-voltage $(I-V)$ conversion $^{43}$ was $0.01 \%$ for gain selections between $10^{4} \mathrm{~V} / \mathrm{A}$ and $10^{7} \mathrm{~V} / \mathrm{A}$. At these gains, the uncertainty of the feedback resistors in the $I-V$ converter was $0.01 \%(k=1)$ for the decade nominal resistance values. The uncertainty increased to $0.014 \%$ at the $10^{8} \mathrm{~V} / \mathrm{A}$ gain where two $50 \mathrm{M} \Omega$ resistors (each with $0.01 \%$ resistance uncertainty) were connected serially. Because the resistance uncertainty of $10^{9} \Omega$ resistors is $0.5 \%$ or higher, a gain of $10^{9} \mathrm{~V} / \mathrm{A}$ was radiometrically calibrated against a gain of $10^{8} \mathrm{~V} / \mathrm{A}$ by measuring the same stabilized optical power. The gain uncertainty for this highest gain selection was $0.02 \%$. The low uncertainties in gain can be achieved only if the dark reading is substracted from the signal plus the dark reading of the trap detector radiometer (which includes the $I-V$ converter) using a shutter positioned upstream in the laser beam. The temperature coefficients of the resistances were $0.001 \% /{ }^{\circ} \mathrm{C}$ for gain selections between $10^{4} \mathrm{~V} / \mathrm{A}$ and $10^{7} \mathrm{~V} / \mathrm{A}$. The maximum temperature change in the lighttight box is $2{ }^{\circ} \mathrm{C}$. Given an equal probability distribution for the temperature, the uncertainty in the resistance arising from the temperature coefficient is $(0.001 \%) \times 2 / 3=7 \times 10^{-4} \% .^{33}$

\section{Transfer to Test Instrument}

To achieve the lowest possible uncertainties on SIRCUS, the instrument should be designed with the calibration in mind. The uncertainty in the transfer to a test instrument is listed in Table 1 as $0.03 \%$. This is for a well-designed, well-characterized, stable radiometer. Nonideal instrument characteristics such as stability, temperature dependence, long-term drift, and out-of-field response, all contribute to increased overall uncertainty in a calibration. Interference fringes from multiple reflections of incident radiation at optical surfaces have been observed in the calibration of instruments with windows and other optical elements if they are not wedged or antireflection coated. For example, the absolute spectral responsivity of a pyrometer (discussed in Subsection 4.A) calibrated on SIRCUS is shown in Fig. 9, in both a logarithmic [Fig. 9(a)] and a linear [Fig. 9(b)] scale. Interference fringes (the sinusoidal oscillations in the responsivity) are emphasized in the expanded view [Fig. 9(c)]. The presence of interference fringes increases the difficulty in the calibration if they must be measured during the calibration or increases the uncertainty in the calibration.

As an aside, we have found that interference fringes are ubiquitous, caused mainly by the window on the photodiode or parallel-surface windows in the device, or filters. ${ }^{12}$ A complete mapping of the fringes is possible on SIRCUS because of the narrow spectral bandwidth and wavelength stability of the lasers and because the laser wavelength can be tuned automatically. In certain calibration approaches-for example, 


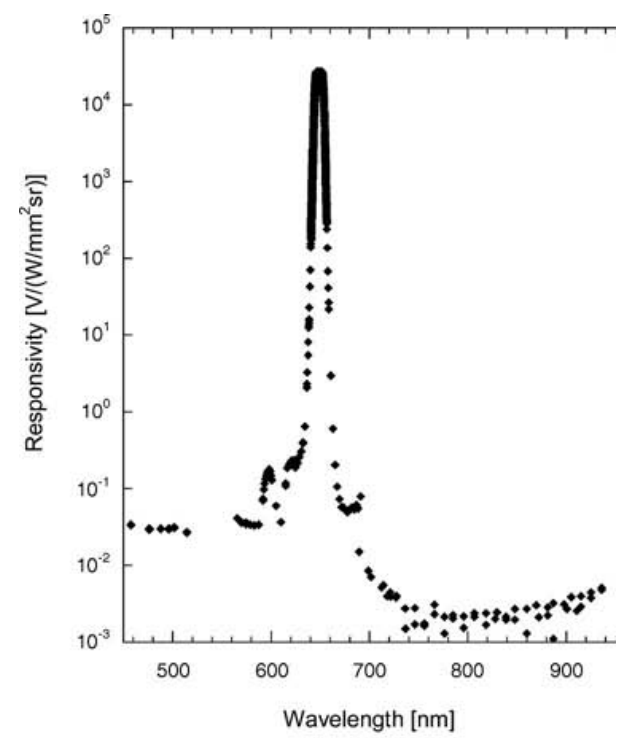

(a)

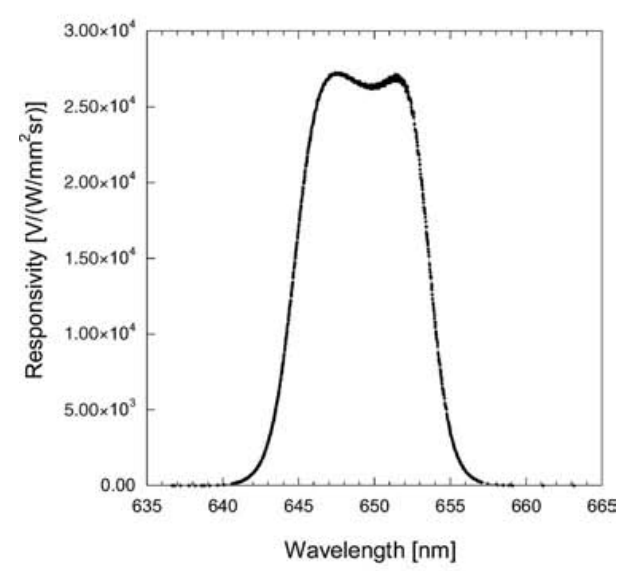

(b)

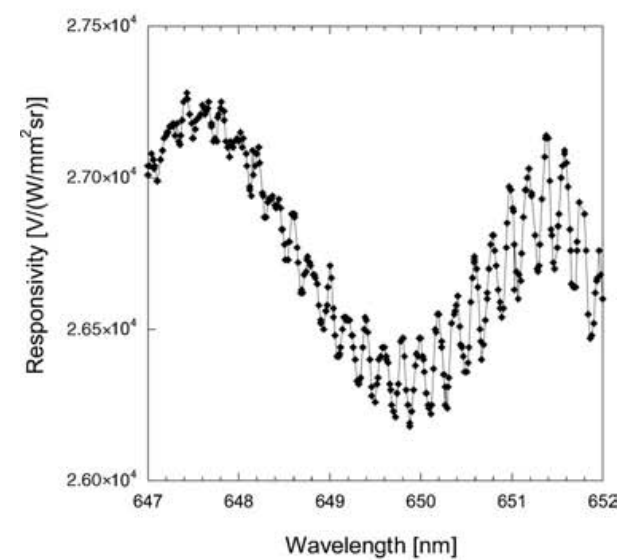

(c)

Fig. 9. Absolute spectral irradiance responsivity of a filter radiometer, namely, the AP1 pyrometer, shown on (a) a logarithmic and (b) a linear scale. (c) Expanded view of the responsivity showing large oscillations arising from multiple reflections of the incident flux between surfaces within the instrument. using lamp-monochromator systems - the fringes are not observed. Interference fringes occur when the coherence length of the source is as long as or longer than the size of the interfering structure. For example, interference fringes will most likely be present from multilayer coatings even for an incoherent source such as a lamp. If they are not observed, it is likely because the fine structure in the spectral responsivity caused by interference fringes is being averaged over the finite source bandwidth. However, the averaging may not be complete, and artifacts caused by the incomplete cancellation of the fringes can lead to erroneous results. Also, errors can arise in the determination of an instrument's effective aperture area using the scanning method if fringes are present. ${ }^{12}$ In this case, the instrument should be calibrated directly in the irradiance mode to obtain the lowest achievable uncertainties.

\section{Radiance Responsivity Calibrations}

For radiance responsivity calibrations, the uncertainty budget differs only slightly from the uncertainty budget derived for irradiance responsivity calibrations. In a radiance responsivity calibration, the average radiance over the entire integrating sphere exit port area is measured by the reference trap detector, while instruments being calibrated typically image variously sized central regions of the exit port. Errors in calibration can occur if the sphere radiance is nonuniform, giving different average radiance depending on the area of the exit port being imaged. While the sphere's nonuniformity can be mapped and corrections applied, for the lowest uncertainty measurements, it is desirable to keep the sphere's nonuniformity as small as possible. Many researchers have calculated baffle arrangements for spheres to improve the radiance uniformity within the exit port for different input source configurations. We have found empirically that if the input laser beam impinges on the sphere wall close to the exit port, no baffles inside the sphere are needed.

The integrating spheres used for radiance responsivity calibrations on SIRCUS have been designed to allow the incident laser light to hit the sphere wall near the exit port. The spatial uniformity of one of the spheres, a $30 \mathrm{~cm}$ diameter Spectralon-coated integrating sphere with a $7.5 \mathrm{~cm}$ diameter aperture, was mapped using a radiometer with a narrow $\left(\sim 1^{\circ}\right)$ FOV. Fixed at a distance of $0.5 \mathrm{~m}$ from the sphere, the target spot diameter of the radiometer at the sphere exit port was approximately $12.5 \mathrm{~mm}$. The radiometer was translated in $6 \mathrm{~mm}$ increments and the radiance recorded at each position. The sphere radiance was spatially uniform to within $\pm 0.1 \%$ over the entire sphere exit port (Fig. 10). Radiance is a conserved quantity. As long as the DUT is imaging an area within the exit port, the distance from the DUT to the sphere exit port is not critical for radiance responsivity calibrations. 


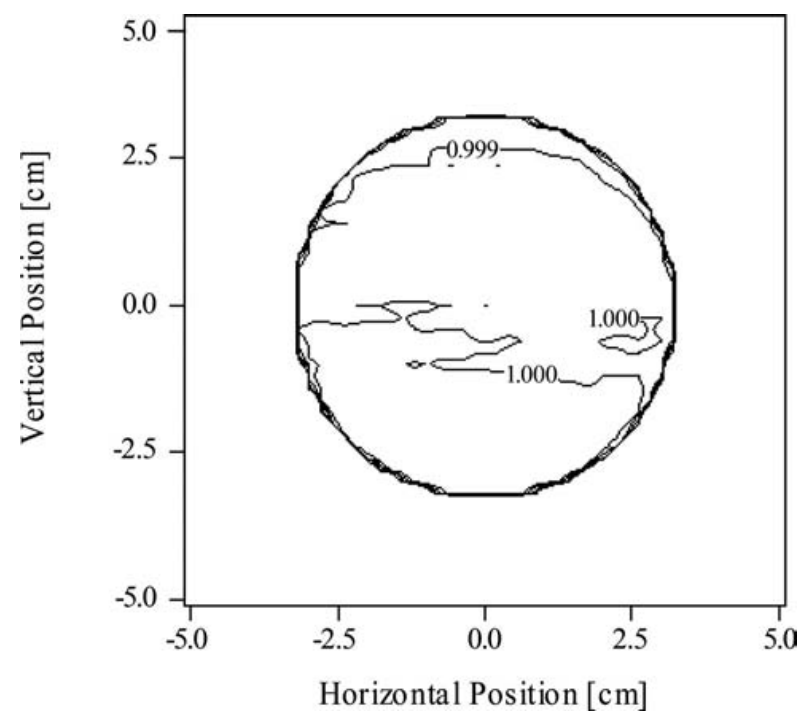

Fig. 10. Contour plot of the sphere radiance uniformity within the exit port; $0.1 \%$ steps.

\section{E. Calibration Procedure}

Before an instrument can be calibrated, it must be aligned with respect to the integrating sphere, as must the reference standard detector. To align the system, a laser is used to define an optical axis. Both the integrating sphere and the detectors are then aligned to the optical axis. The optical axis is typically set to be parallel to the direction of motion of a linear translation stage that moves perpendicular to the sphere exit port (the $z$ axis defined in Fig. 11). The $z$ axis translation stage sits on a pair of rails and is manually translated. A linear encoder records the stage position along the rails.

As illustrated in Fig. 11, to define the optical axis, a laser beam is incident on a pellicle beam splitter that is mounted on the $z$-axis rail system. A mirror is positioned behind the pellicle to retroreflect the light directly back into the laser. The pellicle is in a mirror mount and is adjusted to reflect light that is then aligned to be parallel to the translation stage. This is done by first mounting an iris near the pellicle beam splitter on the translation stage and

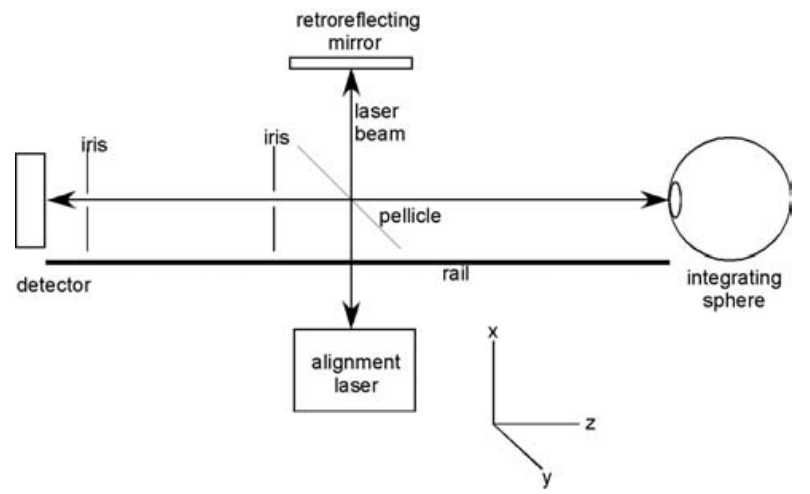

Fig. 11. Schematic of the alignment method for SIRCUS. aligning it so that the laser light is centered on the iris aperture. Then the iris is moved by using the translation stage to lie some distance away from the pellicle. The angle of the pellicle beam splitter is adjusted so that the light reflected from it goes through the iris aperture. These two steps are repeated until the beam is centered on the iris at the two translation stage positions. At this point, the optical beam is parallel to the translation stage, and the optical axis is defined.

To align the detectors, the motorized $x-y$ translation stage is positioned such that the laser is centered on each detector (one at a time), and the reflection from a flat reference plane on the detector is parallel with the incident beam. For each detector, a computer registers and stores the alignment position. The retroreflected beam (from the mirror behind the pellicle) is automatically aligned parallel to the rail. The sphere exit port is then centered on this beam (and the angle adjusted to coalign the reflected laser beam with the incident beam).

The alignment between the detectors and the integrating sphere is radiometrically validated by translating the detectors in the $x$ and $y$ directions and recording the signals as a function of stage position. The stage position giving the maximum signal from each instrument is compared with the stage position derived from the optical alignment procedure. The two positions typically agree to within $1 \mathrm{~mm}$, well within the desired alignment uncertainty.

\section{Calibration Protocol}

The entire data collection sequence is automated. Initially, an electronic shutter that blocks the laser radiation before it enters the optical fiber is closed, and a background signal is acquired for both the reference standard trap detector and the sphere monitor. Then the shutter opens and the signals from the trap and the monitor on the sphere are recorded. The signals are initially amplified using a current-to-voltage amplifier ${ }^{44}$ and then fed into a digital multimeter. ${ }^{45}$ The signal from the multimeter is averaged for several seconds. The mean reference-to-monitor ratio and the standard deviation of the ratio are recorded. After this, the stage moves to the DUT position, and the data acquisition sequence is repeated. This gives the DUT mean signal-to-monitor ratio and the standard deviation of the ratio. The standard deviations of the ratios are monitored; trap detector measurement standard deviations larger than $0.01 \%$ are an indication of laser power instabilities.

Under routine calibration conditions, the intensitystabilized laser wavelength is read by the wavemeter and transferred to the computer during each measurement. Along with mean ratios, the mean and standard deviations of the wavelength are recorded. This allows ready identification of laser wavelength instabilities during a scan. Occasionally, for faster data acquisition, the wavelength is recorded only at the beginning of the acquisition sequence. Following the data acquisition 
sequence, the wavelength is changed and the sequence is repeated.

\section{Comparison with the National Institute of Standards and Technology Lamp-Monochromator-Based Calibration Facility}

The high optical power available with laser-based calibration systems enables us to calibrate instruments directly for either radiance or irradiance responsivity. The low wavelength uncertainty of the laser is instrumental in reducing the calibration uncertainty for filtered instruments. The source radiance uniformity also permits rapid characterization and calibration of digital imaging systems. Some of the advantages of the laser-based calibration approach over a conventional lamp-monochromator-based approach are illustrated by the calibration of a photoelectric pyrometer $(\mathrm{PEP})$, an instrument used to radiometrically determine the temperature of a blackbody. ${ }^{46}$ The instrument is equipped with a narrow bandpass filter $(\sim 1 \mathrm{~nm})$ for spectral selectivity.

For accurate radiance temperature determinations, the instrument's spectral out-of-band responsivity must be measured as well as its in-band responsivity. Figure 12 shows the relative spectral responsivity of

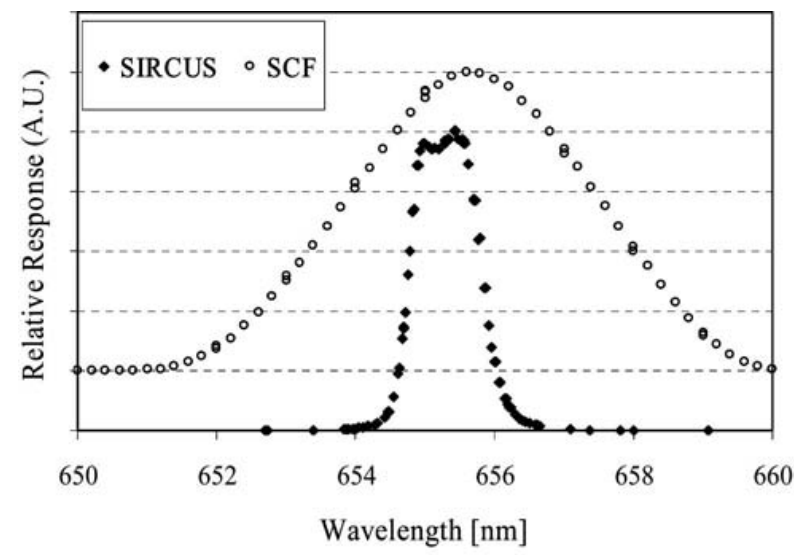

(a)

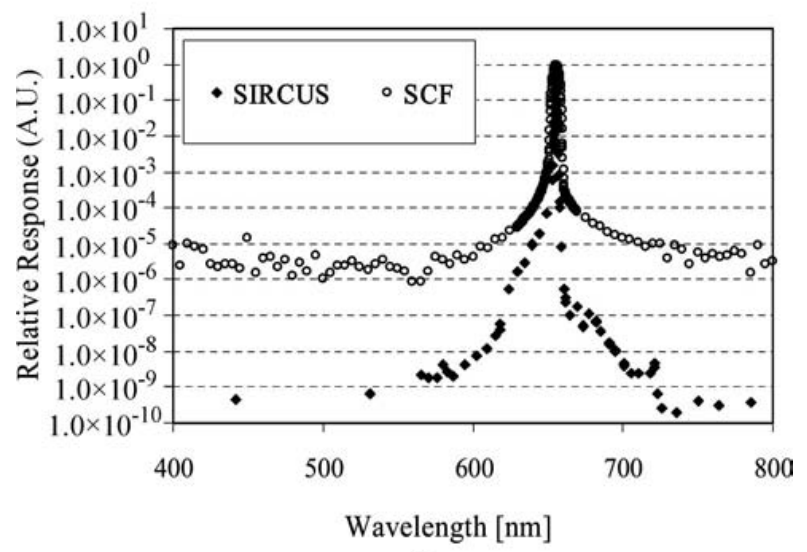

(b)

Fig. 12. Relative spectral responsivity of the PEP measured on SIRCUS and on the SCF: (a) linear scale; (b) log scale. the PEP determined on SIRCUS compared with the relative spectral responsivity determined using the lamp-monochromator system in the SCF. As shown in Fig. 12(a), the spectral responsivity measured with the lamp-monochromator system is dominated by the spectral bandwidth of the source and deconvolution of the spectrum by using the source slit scatter function is required. In contrast, the fine detail in the spectral responsivity is easily measured on SIRCUS because of the monochromatic nature of the source. Note that there are several overlying data points at each wavelength along both the rising and falling edges, demonstrating the extreme wavelength stability of the SIRCUS facility. Because of the low flux in the lampmonochromator system, the out-of-band responsivity is limited to approximately $10^{-6}$ [Fig. 12(b)]. In contrast, the out-of-band responsivity can be measured to the $10^{-9}$ level in the SIRCUS facility.

In SIRCUS, instruments are calibrated in their operational state: at the system level, with entrance pupils overfilled. This approach avoids unforeseen errors that can occur using other calibration approaches. For example, consider the measurements of the relative spectral responsivity of a single channel filter radiometer known as a standard lamp monitor (SLM). ${ }^{47}$ The SLM can be operated in irradiance or radiance mode, depending on the foreoptics. The irradiance mode configuration has a Teflon diffuser, a window, an interference filter, and a silicon photodiode. The instrument's relative spectral responsivities (RSRs) are used to band integrate the response to an illumination source. The RSR of a SLM was measured on the SCF. During these measurements, the flux from the monochromator exit slit was imaged onto the center of the diffuser. In this case, the irradiance collector was underfilled by the incident radiant flux. The SLM was also calibrated in irradiance mode for absolute spectral responsivity on SIRCUS. In this case, the irradiance collector was overfilled by the flux from the laser-illuminated integrating sphere. A comparison of the two results by using peak-normalized data showed that the relative spectral responses did not agree (Fig. 13). There was no dependence on the $f / \#$ of the incoming flux. However, spatial maps of the relative response at multiple

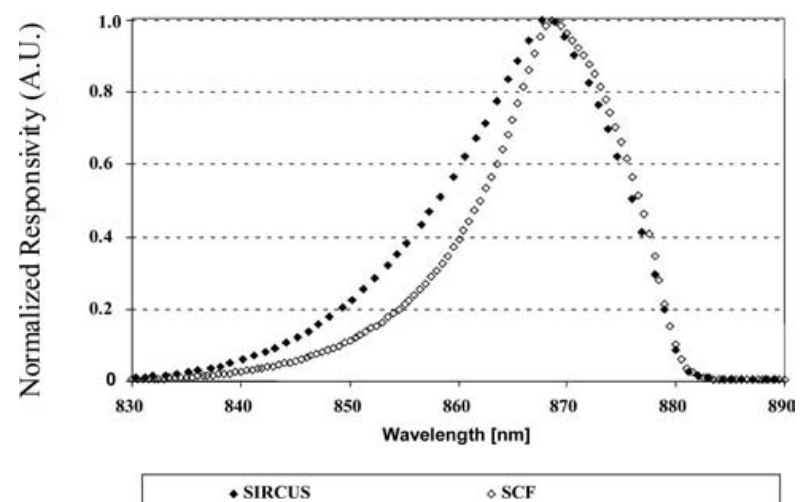

Fig. 13. Relative spectral responsivity of the SLM measured in underfilled mode (SCF) and in overfilled mode (SIRCUS). 
fixed wavelengths within the in-band region showed that the irradiance responsivity was not spatially uniform (due to the diffuser), leading to the observed differences. These measured differences can cause errors in the band-averaged measurements of spectral irradiance when the spectrum of the source being measured differs from that of the calibration source.

\section{Application to Primary Radiometric Quantities and Units}

The NIST Optical Technology Division is responsible for maintaining two fundamental SI units, the unit for temperature, the kelvin, above $1234.96 \mathrm{~K}$, and the unit for luminous intensity, the candela. In the following subsections we describe the effect of SIRCUS calibrations on reference standard pyrometers used to measure the radiance temperature of blackbody sources and photometers used to measure the illuminance of standard lamps. Using SIRCUS to develop a detector-based validation of the spatial distribution of the radiant flux from a standard synchrotron source predicted by the Schwinger equation is also discussed.

\section{A. Radiance Temperature}

For temperatures above the freezing temperature of silver, the ITS-90 (Ref. 48) is defined in terms of spectral radiance ratios to one of the silver-, gold-, or aluminum-freezing temperature blackbodies using the Planck radiance law. ${ }^{49}$ In the ITS-90, the assigned temperatures for the aluminum, silver, and gold freezing points result from thermometry using ratio pyrometry from the mean of two different and conflicting constant-volume gas thermometry measurements at lower temperatures. There are thermodynamic temperature uncertainties of the freezing points of the primary metal blackbodies that arise primarily from the uncertainties in the lower temperature gas thermometry.

Because of the use of spectral radiance ratios, the temperature uncertainties of ITS-90 assigned blackbody, $u\left(T_{\mathrm{BB}}\right)$, increase as the square of the temperature ratios according to

$$
u\left(T_{\mathrm{BB}}\right)=\frac{u\left(T_{\mathrm{FP}}\right)}{T_{\mathrm{FP}}{ }^{2}} T_{\mathrm{BB}}{ }^{2},
$$

where $T_{\mathrm{FP}}$ and $u\left(T_{\mathrm{FP}}\right)$ are the temperature and the uncertainty of the fixed-point blackbody and $T_{\mathrm{BB}}$ is the temperature of the higher temperature blackbody. The increases in the temperature uncertainties can be reduced by using absolute radiometry with pyrometers traceable to cryogenic radiometers, and the resulting temperature uncertainties can be smaller than those measured using the ITS-90 techniques. ${ }^{10,50}$

To decrease the uncertainties in the radiance temperature scale at NIST and, ultimately, in the thermodynamic temperature assignment of the metal freezing-point temperatures, a pyrometer-denoted the Absolute Pyrometer-1 (AP1) — was built to radiometrically measure blackbody temperatures. ${ }^{50,51}$ Spec- tral selection is achieved by using a narrowband interference filter with a center wavelength at $650 \mathrm{~nm}$ and a bandpass of $10 \mathrm{~nm}$. The filter is temperature stabilized to near room temperature, and the hermetically sealed silicon photodiode has a two-stage thermoelectric cooler for operation at $-15^{\circ} \mathrm{C}$. It has a noise-equivalent power (NEP) of $3 \mathrm{fW}$ at an electrical bandwidth of $0.3 \mathrm{~Hz}$. The radiometric characteristics, e.g., stability and linearity, for example, of the AP1 have been established. 51

The AP1 was calibrated for absolute spectral radiance responsivity on SIRCUS. As shown in Fig. 9, it has a peak responsivity between 647 and $652 \mathrm{~nm}$, a FWHM bandwidth of approximately $10 \mathrm{~nm}$, and out-of-band blocking better than $10^{-7}$. Interference fringes with an amplitude of $0.5 \%$ were observed in the responsivity. The absolute spectral responsivity was therefore measured with $0.03 \mathrm{~nm}$ resolution. The AP1 subsequently measured the melt and freeze cycles of silver and gold fixed-point blackbodies. The signal from the AP1 is converted to temperature using the equation:

$$
i_{u}=\int s_{L}(\lambda) \varepsilon_{u} L_{u}(\lambda, T) \mathrm{d} \lambda
$$

where $s_{L}(\lambda)$ is the absolute spectral responsivity of the $\mathrm{AP} 1, \varepsilon_{u}$ is the emissivity of the blackbody, and $L_{u}(\lambda, T)$ is the radiance of the blackbody derived from Planck's equation. ${ }^{50}$

The noise-equivalent temperature at the gold (and silver) freezing temperature is $\approx 2 \mathrm{mK}$, and the noise will not be the dominant component of the total temperature uncertainties. The AP1 was calibrated twice on SIRCUS, once in 2003 and again in 2005. Using the SIRCUS 2003 calibration, the expanded uncertainty $(k=2)$ in the radiometric measurement of the gold (or silver) freezing-point blackbody was approximately $0.15 \%$. The radiometric uncertainties can be related to the uncertainties of the temperature determinations from the derivative of the Wien approximation, which shows the relationship between the uncertainty in radiance, $L$, to the uncertainty in blackbody temperature, $T$ :

$$
\frac{\Delta L}{L}=\frac{c_{2}}{\lambda} \frac{\Delta T}{T^{2}} .
$$

In Eq. (6), $c_{2}$ is the second radiation constant, and $\lambda$ is the wavelength. Using Eq. (6), an uncertainty of $0.15 \%$ in radiance responsivity at $650 \mathrm{~nm}$ will lead to an uncertainty of $121 \mathrm{mK}$ in the measurement of the melting and freezing temperature of the gold-point blackbody, which is slightly larger than the ITS-90 uncertainty, as shown in Table 2.52 The AP1 was recently recalibrated on SIRCUS by using the 2004 trap-detector responsivity scale (Table 1). By using the new calibration, the expanded uncertainty in the radiometric measurement of the gold point is reduced to approximately $0.09 \%$. With the reduced uncer- 
Table 2. Comparison of the Results of Radiometric Determinations of the Gold- and Silver-Point Blackbody Temperatures Using the AP1 Pyrometer with the ITS-90 Values

\begin{tabular}{lccccc}
\hline & \multicolumn{3}{c}{$u\left(T_{\mathrm{AP} 1}\right)$} & \multicolumn{3}{c}{$u\left(T_{90}\right)$} \\
& $\begin{array}{c}T_{\mathrm{AP} 1} \\
(\mathrm{~K})\end{array}$ & $(k=2)$ & $\begin{array}{c}T_{90} \\
(\mathrm{~K})\end{array}$ & $\begin{array}{c}(\mathrm{K}) \\
(k=2)\end{array}$ & $\begin{array}{c}T_{\mathrm{AP} 1}-T_{90} \\
(\mathrm{~K})\end{array}$ \\
\hline $\begin{array}{c}\text { Au-point } \\
\text { blackbody }\end{array}$ & 1337.344 & 0.121 & 1337.33 & 0.100 & 0.014 \\
$\begin{array}{c}\text { Ag-point } \\
\text { blackbody }\end{array}$ & 1234.956 & 0.106 & 1234.93 & 0.080 & 0.026 \\
\hline
\end{tabular}

tainty from SIRCUS, the corresponding temperature uncertainty derived from the uncertainty in the radiometric measurement of the gold-point blackbody can be reduced to approximately $72 \mathrm{mK}$, significantly lower than the ITS-90 uncertainty.

These results are, in effect, the first step toward implementation of a detector-based thermodynamic temperature scale. SIRCUS can be used to calibrate optical pyrometers directly, eliminating the dependence on a blackbody and the ITS-90. As discussed by Yoon et al., ${ }^{50,53}$ moving from a source-based to a detector-based spectral irradiance (and radiance) scale can greatly reduce the radiometric uncertainty in the measurement of higher temperature sources.

\section{B. Photometry}

The redefinition of the candela in 1979 coupled photometric and radiometric units ${ }^{54}$ and made it possible to realize and maintain photometric units by using detectors as well as sources. Following the redefinition, many national laboratories, including NIST, derived and maintained the candela (and derived photometric units) using calibrated standard detectors traceable to cryogenic radiometry rather than standard lamps traceable to primary standard blackbodies and international temperature scales. ${ }^{55,56}$ Since 1992 , the candela has been maintained at NIST by using a group of eight standard photometers. ${ }^{57}$ The overall relative expanded uncertainty $(k=2)$ for the NIST illuminance unit realization is currently $0.39 \% .57$

There are several components in the uncertainty budget related to the determination of the photometer relative spectral responsivity using the lampmonochromator system in the SCF.1,57 The uncertainties in these components can be significantly reduced or eliminated by realizing the illuminance scale on SIRCUS. The spectral responsivity scale will be reduced from a standard uncertainty of $0.11 \%$ currently measured on the SCF to $0.075 \%$ because of the reduction of transfer steps from the high-accuracy cryogenic radiometer. The luminous efficiency function defined by the Commission Internationale de l'Eclairage (CIE), $V(\lambda)$, is a strong function of wavelength. The SIRCUS wavelength scale, with uncertainties reduced to less than $0.01 \mathrm{~nm}$ from the SCF's wavelength uncertainty of $0.1 \mathrm{~nm}$, reduces the relative standard uncertainty for this component from $0.04 \%$ to $<0.004 \%$. A significant set of uncertainty components results from the geometry of the SCF output beam. The SCF uses $f / 9$ optics to focus the light to a $1 \mathrm{~mm}$ spot that is used to calibrate the photometers, whereas the photometers are normally used in a far-field condition. SIRCUS can calibrate photometers in a geometrical configuration identical to the application geometry, eliminating errors caused by different interreflection patterns inside the photometer between the calibration and application.

In addition, since SIRCUS calibrates the detector in irradiance mode instead of power mode, the uncertainty of the aperture size and the uncertainty due to the nonuniform spatial response of the photometer are eliminated. Combined the uncertainty contribution from these components can be reduced from $0.12 \%$ (Ref. 57) to less than $0.01 \%$. The overall relative expanded uncertainty $(k=2)$ for the NIST illuminance unit realization is expected to be reduced to $0.24 \%$, a $40 \%$ reduction from the uncertainty in the current scale, when the new illuminance scale is realized. The new illuminance scale will lead to an expected overall relative expanded uncertainty of $0.27 \%(k=2)$ for the NIST candela.

The current photometric working standard photometers were not calibrated on SIRCUS because large interference fringes (of the order of 10\%) were observed in the spectral responsivity of those instruments. A trap-detector-based tristimulus colorimeter was developed to realize a detector-based color scale (see Subsection 5.A.). As a first step toward the new SIRCUS-based photometric scale, the irradiance responsivity of the photometric $(Y)$ channel of the colorimeter was determined. An aperture mounted on the front surface of the colorimeter defines the reference plane for irradiance and illuminance measurements. Because of the thick color filter combinations ${ }^{58}$ and the removed photodiode windows, all four channels of this colorimeter showed fringe-free operation on SIRCUS. Use of feedback resistors in the photocurrent meters with $0.001 \% /{ }^{\circ} \mathrm{C}$ temperature coefficient of resistance, $0.01 \%$ uncertainty of their decade nominal values, and realizing loop gains larger than $10^{4} \mathrm{in} \mathrm{dc}$ measurements for all signal-gain selections, the uncertainty of the photocurrent-to-voltage conversion was $0.01 \% .31$

From the SIRCUS-measured spectral irradiance responsivity of the photometric channel (between 360 and $950 \mathrm{~nm}$ ), the illuminance responsivity was calculated. ${ }^{59}$ Thereafter standard lamps were measured in the photometry calibration facility to compare the illuminance responsivities of the same channel determined from both the existing (SCF-based) and the new (SIRCUS-based) scales. The colorimeter was calibrated on SIRCUS twice, with the calibrations separated in time by 15 months. The illuminance responsivity of the photometric channel from the two SIRCUS calibrations agreed within $0.1 \%$. The average illuminance responsivity from the two SIRCUS calibrations was $4.911 \mathrm{nA} / \mathrm{lx}, 0.35 \%$ higher than the SCF-based illuminance responsivity.

Our investigations showed that the SCF-based illuminance responsivity (used in the present photo- 
metric scale) is low by approximately $3 \%$ because of a beam-clipping problem caused by the small apertures in the working standard photometers. Comparisons of the spectral responsivity determined on SIRCUS and the SCF show spectral shifts as large as $0.5 \mathrm{~nm}$ and responsivity differences as large as $1 \%$.

\section{Synchrotron Radiation}

Schwinger described the nature of radiation that is emitted from the acceleration of relativistic particles. ${ }^{60}$ In a synchrotron accelerator, electrons are confined to a circular path and continuously emit radiation tangential to their direction. Magnets are used to control the energy and direction of electrons in the synchrotron. Like blackbody radiation, synchrotron radiation is absolutely calculable from the knowledge of a very few parameters. Blackbody radiation follows from Planck's law: Knowledge of the temperature and emittance allows for calculation of all radiation emanating from the blackbody. Synchro-

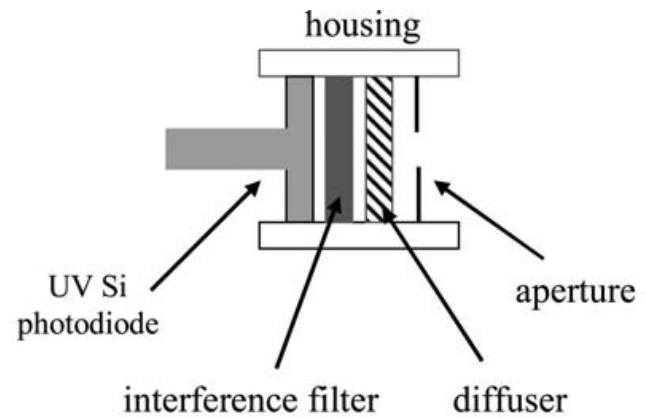

(a)

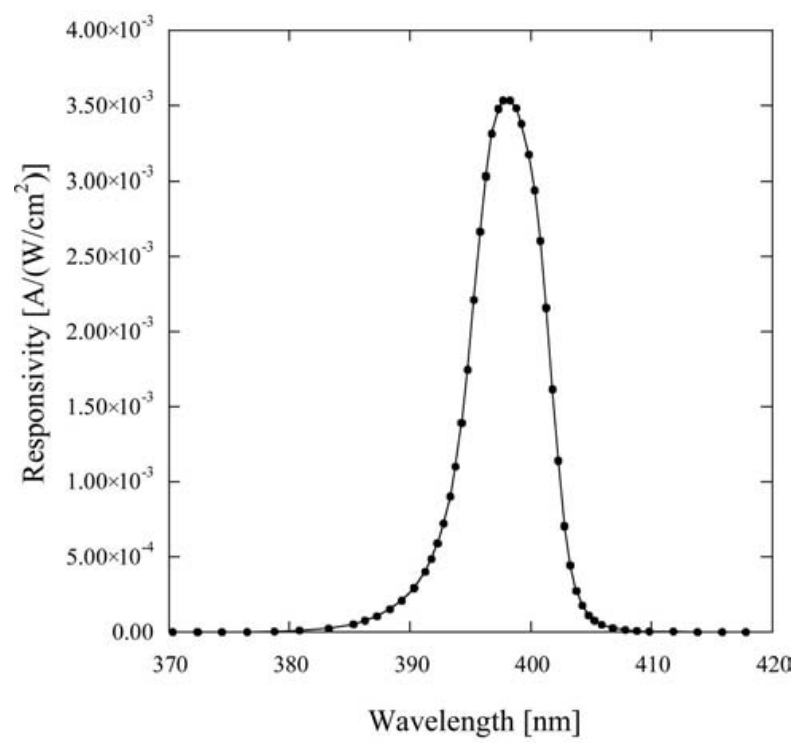

(b)

Fig. 14. (a) Schematic diagram of the filter radiometers that were calibrated for irradiance responsivity on SIRCUS and used to measure the angular distribution of radiation and intensity at SURF III. (b) Absolute spectral irradiance responsivity of the $400 \mathrm{~nm}$ filter radiometer.

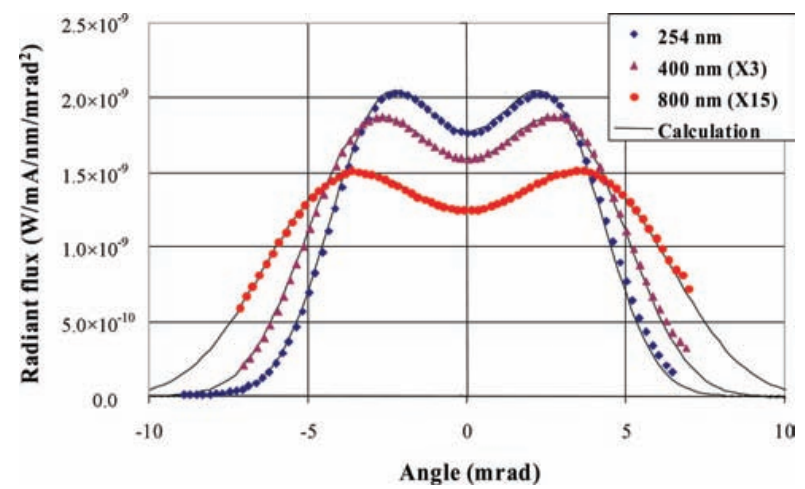

Fig. 15. (Color online) Angular distribution pattern from SURF measured with three filter radiometers. The solid curve is the calculated distribution pattern.

tron radiation is defined by the Schwinger equation: The electron beam current, magnetic field, and the orbital radius completely define the radiation field.

The NIST Synchrotron Ultraviolet Radiation Facility (SURF III) is a particularly simple synchrotron storage ring. The electrons are confined to a circular orbit in a vacuum chamber by a dipole magnet with an extremely uniform magnetic field $\left(<10^{-4}\right.$ nonuniformity in the magnetic field). The angular distribution and intensity of radiation is dependent on the electron beam energy and the wavelength of the emitted radiation. To validate the expected spatial distribution, three filter radiometers were constructed with band-center wavelengths at 254, 400, and $800 \mathrm{~nm}$, respectively. A simplified schematic of the filter radiometers is shown in Fig. 14(a). These filter radiometers contain a precision aperture, a diffuser, an interference filter, and a photodiode, and they are not polarization sensitive. The filter radiometers were calibrated for irradiance responsivity on SIRCUS; the absolute spectral responsivity of the $400 \mathrm{~nm}$ filter radiometer is shown in Fig. 14(b). They subsequently measured the angular distribution and intensity of the emitted radiation at a known beam current and magnetic field at SURF III. ${ }^{61}$ The filter radiometer results are shown in Fig. 15. By numerically integrating the response of the radiometers and the calculated angular distribution of the intensity of emitted radiation, the spatial distribution of the radiant flux from SURF III can be calculated for different wavelengths. The quantitative results for the three filter radiometer wavelengths are given by the solid curves in Fig. 15. There are no adjustable parameters in the calculations. The results at $400 \mathrm{~nm}$ have been multiplied by a factor of 3 and the results at 800 by a factor of 15 to show all the results on the same figure. The calculated, or predicted signals as a function of angle agree with the measurements to better than $0.5 \%$.

\section{Applications}

The unique characteristics of SIRCUS give us the versatility to address a wide range of optical metrology issues in addition to applications related to the 
measurement of fundamental sources or to the realization of fundamental SI units. We briefly discuss other applications in the following subsections.

\section{A. Detector-Based Color Scale}

A tristimulus colorimeter standard was developed by using temperature-stabilized filters in front of a silicon tunnel-trap detector to realize a detector-based color scale. ${ }^{58}$ The tristimulus colorimeter is composed of four channels consisting of filter combinations with the trap detector designed to mimic the CIE color-matching functions ${ }^{62}$ with small spectral mismatch. The spectral mismatch of the realized channels is characterized by the CIE $f_{1}^{\prime}$ values. ${ }^{63}$ The filter combinations are mounted in a temperature-controlled (heated) filter wheel that can be moved between the trap detector and the front aperture. The aperture plane is the reference plane of the illuminance-mode photometercolorimeter, and it is in the plane of the front cover. A precision current-to-voltage converter is attached to the top of the instrument. Electronic and radiometric characterizations, including current-to-voltage conversion uncertainty and spatial, angular, and spectral responsivity, have been done.

The tristimulus colorimeter was calibrated on SIRCUS as a first step in the establishment of a detector-based color scale. ${ }^{64}$ When utilizing the uniform irradiance at the detector reference plane from the small sphere source of the SIRCUS, the nonuniformity of spatial responsivity of the filters and the detector in the tristimulus colorimeter will not contribute to measurement uncertainty. Also, interreflections between optical components will be the same for the calibration and subsequent applications, and their contribution to radiometric measurement error will cancel. The combined expanded uncertainties $(k=2)$ in the absolute spectral irradiance responsivities of the individual channels, neglecting correlations, are estimated to be $0.15 \%$ or less. From the spectral irradiance responsivity curves, the broadband calibration factors of the colorimeter channels can be calculated, and the tristimulus values of a test light source can be measured. For a Planckian radiator (a light source with a well-known spectral distribution), a $0.15 \%$ uncertainty in the spectral irradiance responsivity of any one channel of a tristimulus colorimeter will result in a chromaticity coordinate uncertainty of 0.0004 for both $x$ and $y$. These uncertainties correspond to an uncertainty of $4 \mathrm{~K}$ in color temperature, a factor of 2 less than the uncertainties of current primary color temperature lamp standards.

\section{B. Stray Light Characterization of Spectrographs}

Spectrographs are spectroradiometers with multielement array detectors that can acquire an entire spectral image over some finite spectral region simultaneously. Spectrographs commonly consist of an entrance port, a dispersing element (such as a grating) to spatially resolve the spectral components of the incident radiation, and mirrors to image the entrance port (often a slit) onto a reference plane where the array detector is located. Because of the dispersing element, the spatial image of the entrance port falls on different regions of the detector array, depending on its wavelength; broadband sources form an image across the entire array. The spectral coverage of a spectrograph is determined by the size of its detector array, the dispersion properties of its grating, and its optical layout. Compared with conventional, scanned grating systems, source spectral distributions can be acquired in a matter of seconds, as opposed to minutes. The ability to rapidly acquire a spectrum has led to the use of array-based systems in a variety of radiometric, photometric, and colorimetric applications where acquisition speed is an issue, for instance, on a production line, or in cases where the source being measured is not stable over extended periods of time.

The measurement uncertainties of spectrographs are often larger than conventional scanning spectrometers. Spectrographs are single grating instruments and there are intrinsic limitations in the background signal originating from radiation scattered from imperfections in the grating and other optical elements. This unwanted background radiation, called stray light, while small- of the order of $10^{-4}$ or less of the incident spectral radiance in a single grating spectrograph-can give rise to unforeseen errors, often much larger than anticipated, when the spectral distribution of a source being measured differs significantly from the spectral distribution of the calibration source. In comparison, a conventional scanning spectrometer is often constructed as a doublemonochromator instrument (double grating or prism grating) that has a stray light level lower than $10^{-6}$.

In many applications, the spectral distributions of the test source and the calibration source differ significantly. Spectrometers are typically calibrated against standard incandescent lamps with correlated color temperatures (CCTs) ranging from 2800 to $3100 \mathrm{~K}$. Most of the emission from these sources lies in the red and NIR regions. Measurement errors, often significant, arising from stray light are inevitable when instruments calibrated against standard lamps subsequently measure light sources that have dissimilar spectral distributions, such as LEDs, displays, discharge lamps, and fluorescent lamps. In these situations, stray light is often the dominant source of spectrograph measurement error.

Algorithms have been developed to correct the output of a spectrograph for stray light based on the spectral imaging properties of the instrument. The tunable lasers available on SIRCUS are used to characterize a spectrograph's response for stray light. Figure 16 is a semilogarithm plot of spectra acquired from a spectrograph with excitation wavelengths ranging from 350 to $800 \mathrm{~nm}$. Each spectrum is a single image normalized by the peak value. The strong central peak in each image (or spectrum) in Fig. 16 corresponds to the image of the entrance slit formed on the detector array. The broad shoulder and finite baseline are signals from radiation that is not 


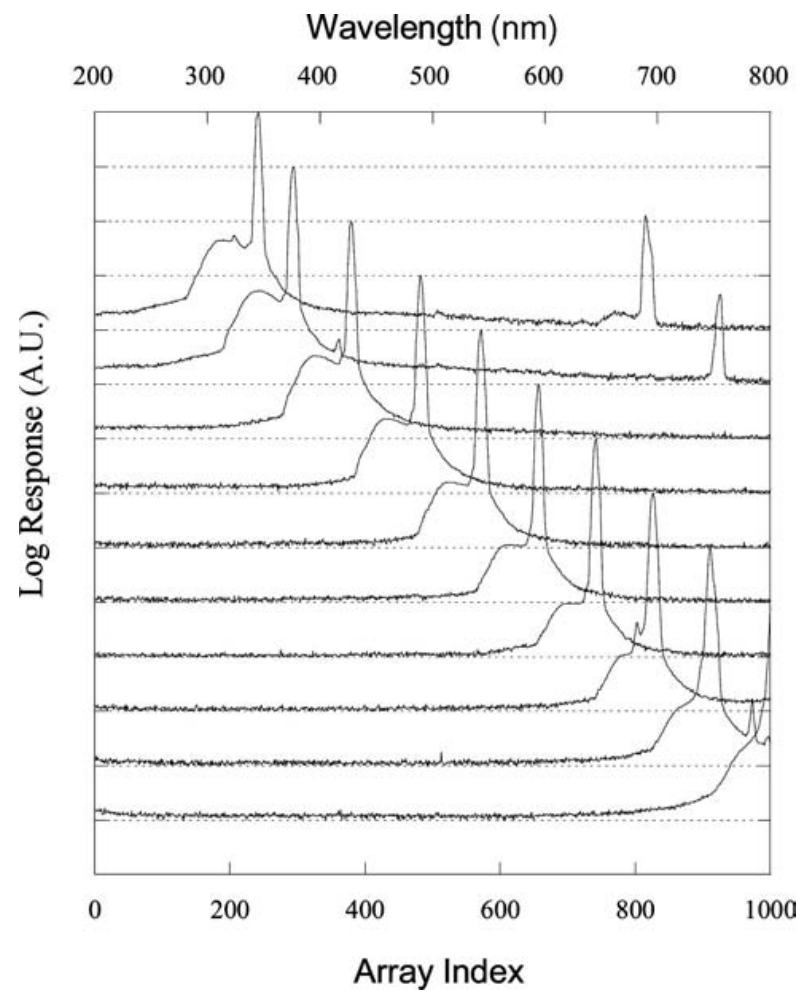

Fig. 16. Spectrograph images as the incident wavelength is changed and the central image moves across the array.

properly imaged onto the detector, i.e., from stray light within the spectrograph. By measuring the system response at a number of wavelengths, the evolution of the image as the excitation wavelength moves across the array can be determined. Based on these measurements, a stray light correction algorithm can be implemented that reduces the errors and associated uncertainties arising from stray light an order of magnitude or more. ${ }^{47,65,66}$ For an accurate characterization of spectrometers for this correction, the input optics must be illuminated by uniform monochromatic radiation at many wavelengths in irradiance or radiance mode. The SIRCUS facility is effectively utilized to achieve the necessary measurements.

\section{Solar Irradiance}

Sun photometers are filter radiometers used to determine atmospheric optical properties at different wavelengths. Primary standard reference sun photometers are calibrated at high altitudes by using the LangleyBouguer technique. In this calibration approach, a series of measurements at different solar zenith angles are made over the course of a day, giving a range of measured signals as a function of zenith angle (or air mass). The measurements are then extrapolated back to zero air mass, giving an instrument signal corresponding to the expected signal in the absence of any intervening atmosphere. ${ }^{67}$ Reductions in the uncertainties in irradiance responsivity calibrations of primary standard reference Sun photometers using laboratory standards would allow for meaningful com- parisons with the results from the Langley-Bouguer method, resulting in independent values for the exoatmospheric solar irradiance at the set of measurement wavelengths.

Global networks of Sun photometers are used to provide detailed mapping of atmosphere optical properties, for example, aerosol concentrations and distributions. Sun photometers that are part of the Aerosol Robotic Network ${ }^{68}$ (AERONET) are calibrated for irradiance responsivity against reference primary standard Sun photometers using the cross-calibration technique at the NASA Goddard Space Flight Center (GSFC). ${ }^{67}$ The cross-calibration technique consists of near-simultaneous solar observations with the uncalibrated instrument and a calibrated reference Sun photometer. Reducing the uncertainties in network instruments by using laboratory standards would validate the uncertainties in the calibration transfer and could improve the characterization of the atmosphere.

Because Sun photometers measure solar irradiance, their responsivity is typically low, and they are difficult to calibrate using lamp-monochromator systems. To demonstrate the feasibility of calibrating Sun photometers on SIRCUS, two multichannel Sun photometers were calibrated and the results compared with standard cross calibrations. ${ }^{69}$ To compare the cross-calibration results to the SIRCUS calibrations, the SIRCUS-derived irradiance responsivities $s(\lambda)$ were used to predict a top-of-the-atmosphere (TOA) signal $V_{o}(\mathrm{SIRCUS})$ :

$$
V_{o}(\operatorname{SIRCUS})=\int s(\lambda) E(\lambda) \mathrm{d} \lambda
$$

where $s(\lambda)$ is the spectral responsivity of one of the radiometer channels and $E(\lambda)$ is an exoatmospheric solar irradiance spectrum. In this work we used the exoatmospheric solar irradiance spectrum developed by Thuillier et al. ${ }^{70}$ To perform the integration, the $s(\lambda)$ and the $E(\lambda)$ were interpolated to a uniform wavelength interval of $0.25 \mathrm{~nm}$ and integrated. The $750 \mathrm{~nm}$ channel results agreed with the cross calibration to within $0.15 \%$, the $490 \mathrm{~nm}$ channel to within $0.85 \%$, and the $440 \mathrm{~nm}$ channel to within $5.76 \%$.

The dominant source of uncertainty in the SIRCUSbased predicted TOA signal was the uncertainty in the solar irradiance spectrum. Assuming an $\sim 1.5 \%$ combined standard uncertainty in the absolute value and given a step size of $1 \mathrm{~nm}$ in the region of interest, we were able to validate the cross calibration with an expanded uncertainty $(k=2)$ of $\sim 4 \%$. The agreement for the 490 and $750 \mathrm{~nm}$ channels were within the combined uncertainties, but the $440 \mathrm{~nm}$ results were not and warrant further investigation.

The measurements demonstrated that SIRCUS irradiance levels were high enough to calibrate Sun photometers. Current uncertainties in the exoatmospheric solar irradiance depend on the wavelength, but in atmospheric transmittance windows in the vis- 
ible spectral region, they are estimated to be approximately $0.5 \%$ to $1 \%$. Calibration of primary standard Sun photometers on SIRCUS with $0.1 \%$ uncertainties or less would enable us to differentiate between exoatmospheric solar irradiance spectra and could lead to a reduction in the uncertainties in the exoatmospheric solar irradiance spectrum. This has implications for global climate change studies because the exoatmospheric solar irradiance is one of the primary forcing functions driving the global climate.

Additional applications include the development of wavelength standard reference materials with reduced uncertainty ${ }^{71}$ and the characterization and calibration of instruments with large entrance pupils, such as telescopes and remote-sensing sensors. ${ }^{72}$

\section{Summary}

We have described a laser-based calibration facility for irradiance- and radiance-measuring instruments. In this facility, high-power tunable lasers were introduced into integrating spheres by using optical fibers, producing uniform, quasi-Lambertian, highly radiant flux sources. Reference standard irradiance detectors, calibrated directly against national primary standards for spectral power responsivity, were used to determine the irradiance at a reference plane. Instruments were calibrated directly in irradiance or radiance mode with uncertainties approaching or lower than those available for spectral power responsivity calibrations in traditional lamp-monochromator facilities. The implications for fundamental radiometric measurements, including radiance temperature, photometric, and synchrotron source measurements, were described. Finally, advanced applications to realize the first detector-based color scale, to correct stray light in spectrographs, and to measure solar irradiance by using this facility were briefly outlined.

Work continues on reducing the uncertainties associated with measurements on SIRCUS as well as the spectral coverage and laser powers available. In Table 1 we calculated the uncertainty in the irradiance responsivity at a single wavelength. In practice, in determining the uncertainty in measuring the irradiance of a source by using a filter radiometer as an example, the responsivity was integrated over a wavelength range. The irradiance responsivity was determined for a large number of wavelengths, typically 100 or more, over the in-band range. Calculating the bandintegrated responsivity, the random components in the uncertainty budget were reduced by the square root of the number of measurements, and only systematic components in the uncertainty budget contributed to the uncertainty in the measurement. We have neglected the reduction in the uncertainty from integrating the responsivity over a finite number of elements.

The spectral coverage of the SIRCUS facility for spectral responsivity calibrations is determined by the high-reflectance range of the coating of the integrating sphere sources, the wavelength coverage of the standard reference and monitor detectors, and the spectral coverage of the tunable laser systems. Spectralon and similar PTFE-based coatings have high reflectance from 250 to $2500 \mathrm{~nm}$. The spectral coverage of the UV-Vis-NIR SIRCUS facility has been extended into the UV and the short-wave infrared (SWIR) regions with the integration of a quasicontinuous-wave, mode-locked OPO-sum-frequency system. This system gave us nearly continuous tunability from 210 to $3.0 \mu \mathrm{m}$.

Research is being conducted on reference detectors to extend the radiant power responsivity scale from 350 down to $200 \mathrm{~nm}$ and from 1 out to $2.5 \mu \mathrm{m}$ and beyond. Reference UV reflectance-type trap detectors using nitride-passivated UVG-100 diodes $^{73}$ as well as PIN-UV100 diodes ${ }^{73}$ have been developed to extend the wavelength coverage from 350 down to $200 \mathrm{~nm}$. Recently, a UV trap detector was calibrated for radiant power responsivity over the spectral range from 210 to $400 \mathrm{~nm}$ with an expanded uncertainty $(k=2)$ of $0.05 \%$ or less. Finally, a variety of irradiance-measuring working standard radiometers have been developed for the NIR range, including single-element Ge, InGaAs, and extended InGaAs detectors. ${ }^{74,75}$ With their calibration against an ACR, the low uncertainty in the SIRCUS irradiance scale will be extended into the IR spectral range.

The authors thank the U.S. Air Force Metrology for funding this work under contract 98-435. They also acknowledge the early and continued support of John Grangaard, USAF (ret.) and the significant contributions to the work by B. Carol Johnson, Howard Yoon, Yuqin Zong, Thomas C. Larason, C. Cameron Miller, and Ping-Shine Shaw from the NIST Optical Technology Division.

\section{References}

1. T. C. Larason, S. S. Bruce, and A. C. Parr, Spectroradiometric Detector Measurements (U.S. Government Printing Office, 1998).

2. T. R. Gentile, J. M. Houston, J. E. Hardis, C. L. Cromer, and A. C. Parr, "National Institute of Standards and Technology high-accuracy cryogenic radiometer," Appl. Opt. 35, 10561068 (1996).

3. T. R. Gentile, J. M. Houston, and C. L. Cromer, "Realization of a scale of absolute spectral response using the NIST highaccuracy cryogenic radiometer," Appl. Opt. 35, 4392-4403 (1996).

4. G. Eppeldauer, M. Racz, and T. Larason, "Optical characterization of diffuser-input standard irradiance meters," in Proc. SPIE 3573, 220-224 (1998).

5. O. G. Peterson, S. A. Tuccio, and B. B. Snavely, "cw operation of an organic dye solution laser," Appl. Phys. Lett. 17, 245-247 (1970).

6. J. M. Yarborough, "cw dye laser emission spanning the visible spectrum," Appl. Phys. Lett. 24, 629-630 (1974).

7. A. R. Schaefer and K. L. Eckerle, "Spectrophotometric tests using a dye-laser-based radiometric characterization facility," Appl. Opt. 23, 250-256 (1984).

8. A. R. Schaefer, R. D. Saunders, and L. R. Hughey, "Intercomparison between independent irradiance scales based on silicon photodiodes physics, gold-point blackbody radiation, and synchrotron radiation," Opt. Eng. 25, 892-896 (1986).

9. K. D. Mielenz, R. D. Saunders, and J. B. Shumaker, "Spectroradiometric determination of the freezing temperature of gold," J. Res. Natl. Inst. Stand. Technol. 95, 49-67 (1990).

10. N. P. Fox, J. E. Martin, and D. H. Nettleton, “Absolute spectral 
radiometric determination of the thermodynamic temperatures of the melting/freezing points of gold, silver, and aluminium," Metrologia 28, 357-374 (1991).

11. V. E. Anderson, N. P. Fox, and D. H. Nettleton, "Highly stable, monochromatic and tunable optical radiation source and its application to high accuracy spectrophotometry," Appl. Opt. 31, 536-545 (1992)

12. M. Noorma, P. Toivanen, F. Manoocheri, and E. Ikonen, "Characterization of filter radiometers with a wavelength-tunable laser source," Metrologia 40, S220-S223 (2003).

13. A. Sperling, Physikalisch-Technische Bundesanstalt, Braunschweig, Germany (personal communication, 2005).

14. J. M. Houston and J. P. Rice, "NIST reference cryogenic radiometer designed for versatile performance," Metrologia 43, S31-S35 (2006).

15. L-1 Standards and Technology, Ijamsville, Md.

16. D. J. Pugh and K. Jackson, "Automatic gauge block measurement using multiple wavelength interferometry," in Proc. SPIE 656, 244-250 (1986).

17. T. F. Johnston, R. H. Brady, and W. Proffitt, "Powerful singlefrequency ring dye laser spanning the visible spectrum," Appl. Opt. 21, 2307-2316 (1982).

18. T. F. Johnston, "Lasers, dye," in Encyclopedia of Physical Science and Technology, R. A. Meyers, ed. (Academic, 2002), pp. 315-359.

19. L. E. Jusinski and C. A. Taatjes, "Efficient and stable operation of an Ar+-pumped continuous-wave ring laser from 505-560 nm using a coumarin dye," Rev. Sci. Instrum 72, 2837-2838 (2001).

20. P. F. Moulton, "Spectroscopic and laser characteristics of Ti: $\mathrm{Al}_{2} \mathrm{O}_{3}$," J. Opt. Soc. Am. B 3, 125-133 (1986).

21. Spectra-Physics, Inc., Wavetrain frequency doubler with a Coherent, Inc. Model 899 Ti:sapphire laser.

22. W.-L. Zhou, Y. Mori, T. Sasaki, S. M. Nakai, K. Nakano, S. Niikura, and B. Craig, "Intracavity frequency doubling of a continuous wave Ti:sapphire laser with over $70 \%$ conversion efficiency," Appl. Phys. Lett. 66, 2463-2465 (1995).

23. T. R. Gentile and C. L. Cromer, "Mode-locked lasers for highaccuracy radiometry," Metrologia 32, 585-587 (1996).

24. W. S. Hartree, P. R. Haycocks, and N. P. Fox, "The use of a mode-locked laser for ultraviolet radiometry," Metrologia 35, 339-343 (1998).

25. J. Fowler and M. Litorja, "Geometric area measurements of circular apertures for radiometry at NIST," Metrologia 40, S9-S12 (2003).

26. Spectralon, Labsphere, Inc., No. Sutton, N.H.

27. E. A. Early, B. C. Bush, S. W. Brown, D. W. Allen, and B. C. Johnson, "Radiometric calibration of the Scripps Earth Polychromatic Imaging Camera," in Proc. SPIE 4483, 77-84 (2001).

28. T. Stone, U.S. Geological Survey, Flagstaff, Ariz., (personal communication, 2005).

29. E. F. Zalewski and C. R. Duda, "Silicon photodiode device with 100\% external quantum efficiency," Appl. Opt. 22, 2867-2873 (1983).

30. Hamamatsu, "Photodiodes," Catalog 1990-91, 16.

31. G. P. Eppeldauer and D. C. Lynch, "Opto-mechanical and electronic design of a tunnel-trap Si- radiometer," J. Res. Natl. Inst. Stand. Technol. 105, 813-828 (2000).

32. G. Eppeldauer, "Noise-optimized silicon radiometers," J. Res. Natl. Inst. Stand. Technol. 105, 209-219 (2000).

33. Guide to the Expression of Uncertainty in Measurement (International Organization for Standardization, Geneva, 1993).

34. J. L. Gardner, "Correlated color temperature-uncertainty and estimation," Metrologia 37, 381-384 (2000).

35. J. L. Gardner, "Correlations in primary spectral standards," Metrologia 40, S167-S176 (2003).

36. K. R. Lykke, P.-S. Shaw, L. M. Hanssen, and G. P. Eppeldauer,
"Development of a monochromatic, uniform source facility for calibration of radiance and irradiance detectors from $0.2 \mathrm{mi}-$ crometer to 12 micrometer," Metrologia 35, 479-484 (1998).

37. G. Eppeldauer and J. E. Hardis, "Fourteen-decade photocurrent measurements with large-area silicon photodiodes at room temperature," Appl. Opt. 30, 3091-3099 (1991).

38. G. Eppeldauer, M. Racz, and L. M. Hanssen, "Spectral responsivity determination of a transfer-standard pyroelectric radiometer," in Proc. SPIE 4818, 118-126 (2002).

39. G. P. Eppeldauer, S. W. Brown, T. C. Larason, M. Racz, and K. R. Lykke, "Realization of a spectral radiance responsivity scale with a laser-based source and $\mathrm{Si}$ radiance meters," Metrologia 37, 531-534 (2000).

40. Burgthaler Electronik GmbH.

41. KaleidaGraph, Synergy Software, Reading, Pa.

42. J. H. Walker, R. D. Saunders, J. K. Jackson, and D. A. McSparron, Spectral Irradiance Calibrations (U.S. Government Printing Office, 1987).

43. G. Eppeldauer, Optical Radiation Measurement with Selected Detectors and Matched Electronic Circuits between $200 \mathrm{~nm}$ and $20 \mu \mathrm{m}$ (U.S. Government Printing Office, 2001).

44. Stanford Research Systems model SR570 or home built.

45. Hewlett Packard Model HP3458A.

46. C. E. Gibson, B. K. Tsai, and A. C. Parr, Radiance Temperature Calibrations (U.S. Government Printing Office, 1998).

47. S. W. Brown, D. K. Clark, B. C. Johnson, H. W. Yoon, K. R. Lykke, S. J. Flora, M. E. Feinholz, M. A. Yarbrough, R. A. Barnes, Y. S. Kim, T. Stone, and J. Mueller, eds., Advances in Radiometry for Ocean Color (NASA, 2004).

48. H. Preston-Thomas, "The international temperature scale of 1990 (ITS-90)," Metrologia 27, 3-10 (1990).

49. D. Halliday and R. Resnick, Fundamentals of Physics (Wiley, 1981).

50. H. W. Yoon, D. W. Allen, C. E. Gibson, R. D. Saunders, B. C. Johnson, S. W. Brown, and K. R. Lykke, "Temperature scales using radiation thermometers calibrated from absolute irradiance and radiance responsivity," in NCSL International Workshop and Symposium (Orlando, Fla., 2003).

51. D. W. Allen, R. D. Saunders, B. C. Johnson, C. E. Gibson, and H. W. Yoon, "The development and characterization of an absolute pyrometer calibrated for radiance responsivity," in International Temperature Symposium (Chicago, Ill., 2003).

52. H. W. Yoon, C. E. Gibson, D. W. Allen, R. D. Saunders, M. Litorja, S. W. Brown, G. P. Eppeldauer, and K. R. Lykke, "The realization and the dissemination of the detector-based kelvin," in Proceedings of Tempmeko 04 (Dubrovnik, Croatia, 2004).

53. H. W. Yoon, C. E. Gibson, and P. Y. Barnes, "Realization of the National Institute of Standards and Technology detectorbased spectral irradiance scale," Appl. Opt. 41, 5879-5890 (2002).

54. Principles Governing Photometry (Bureau International des Poids et Mesures, 1983).

55. R. L. Booker and D. A. McSparron, Photometric Calibrations (U.S. Government Printing Office, 1987).

56. C. L. Cromer, G. Eppeldauer, J. E. Hardis, T. Larason, and A. C. Parr, "National Institute of Standards and Technology detector-based photometric scales," Appl. Opt. 32, 2936-2948 (1993).

57. Y. Ohno, NIST Measurement Services: Photometric Calibrations (U.S. Government Printing Office, 1997).

58. G. P. Eppeldauer and M. Racz, "Design and characterization of a photometer-colorimeter standard," Appl. Opt. 43, 2621-2631 (2004).

59. G. P. Eppeldauer, S. W. Brown, C. C. Miller, and K. R. Lykke, "Improved accuracy photometric and tristimulus-color scales based on spectral irradiance responsivity," Proceedings of the 
25th Session of the Commission Internationale de l'Eclairage, Vol. 1, pp. D2-30-D32-33 (2003).

60. J. Schwinger, "On the classical radiation of accelerated electrons," Phys. Rev. 75, 1912-1925 (1949).

61. P.-S. Shaw, U. Arp, H. W. Yoon, R. D. Saunders, A. C. Parr, and K. R. Lykke, "A SURF beamline for synchrotron sourcebased radiometry," Metrologia 40, S124-S127 (2003).

62. R. McCluney, Introduction to Radiometry and Photometry (Artech House, 1994).

63. J. D. Schanda, "Colorimetry," in Handbook of Applied Photometry, C. DeCusatis, ed. (Springer, 1998), pp. 327-412.

64. G. P. Eppeldauer, "Spectral response based calibration method of tristimulus colorimeters," J. Res. Natl. Inst. Stand. Technol. 103, 615-619 (1998).

65. S. W. Brown, B. C. Johnson, M. E. Feinholz, M. A. Yarborough, S. J. Flora, K. R. Lykke, and D. K. Clark, "Stray-light correction algorithm for spectrographs," Metrologia 40, S81-S84 (2003).

66. Y. Zong, S. W. Brown, K. R. Lykke, and Y. Ohno, "A simple spectral stray light correction method for array spectroradiometers," Appl. Opt. 45, 1111-1119 (2006).

67. C. Pietras, M. Miller, R. Frouin, E. J. Welton, and I. Slutsker, "Calibration of sun photometers and sky radiance sensors," in In Situ Aerosol Optical Thickness Collected by the SIMBIOS Program (1997-2000): Protocols, Data QC, and Analysis,
R. B. G. S. Fargion and C. McClain, eds. (NASA Goddard Space Flight Center, 2001).

68. B. N. Holben, T. F. Eck, I. Slutsker, D. Tanre, J. P. Buis, A. Setzer, E. Vermote, J. A. Reagan, Y. J. Kaufman, T. Nakajima, F. Leaven, I. Jankowiak, and A. Smirnov, "Aeronet-a federated instrument network and data archive for aerosol characterization," Remote Sens. Environ. 66, 1-16 (1998).

69. N. Souaidia, C. Pietras, G. Fargion, R. A. Barnes, R. Frouin, K. R. Lykke, B. C. Johnson, and S. W. Brown, "Comparison of laser-based and conventional calibrations of sun photometers," in Proc. SPIE 4481, 61-72 (2003).

70. G. Thuillier, M. Herse, T. Foujots, W. Peetermans, W. Gillotay, P. C. Simon, and H. Mande, "The solar spectral irradiance from 200 to $2400 \mathrm{~nm}$ as measured by the SOLSPEC spectrometer from the ATLAS and EUREKA missions," Sol. Phys. 214, 1-22 (2003).

71. E. A. Early, NIST, Gaithersburg, Md. (personal communication, 2005)

72. T. Stone, U.S. Geological Survey, Flagstaff, Ariz. (personal communication, 2005).

73. R. Korde, International Radiation Detectors, Inc., Torrance, Calif. (personal communication, 2005).

74. G. Eppeldauer, "Near infrared radiometer standards," in Proc. SPIE 2815, 42-54 (1996).

75. G. Eppeldauer, "Electronic characteristics of Ge and InGaAs radiometers," in Proc. SPIE 3061-97, 833-838 (1997). 


\title{
Radiometer standard for absolute responsivity calibrations from $950 \mathrm{~nm}$ to $1650 \mathrm{~nm}$ with $0.05 \%(k=2)$ uncertainty
}

\author{
G. P. Eppeldauer, H. W. Yoon, Y. Zong, T. C. Larason, A. Smith, and M. Racz \\ National Institute of Standards and Technology \\ Gaithersburg, Maryland USA
}

\begin{abstract}
A near-IR radiometer standard with similar performance to silicon trap-detectors has been developed to calibrate detectors and radiometers for absolute spectral power, irradiance, and radiance responsivities between $950 \mathrm{~nm}$ and $1650 \mathrm{~nm}$. The new radiometer standard is utilized at the Spectral Irradiance and Radiance Responsivity Calibrations using Uniform Sources (SIRCUS) which is the reference calibration facility of NIST for absolute responsivity. The radiometer is a sphere-detector with a unique geometrical arrangement and it can convert the radiant-power responsivity scale of the primarystandard cryogenic-radiometer into a reference irradiance responsivity scale. The $0.05 \%$ $(k=2)$ scale conversion uncertainty is dominated by the two largest uncertainty components of the radiometer: The spatial non-uniformity of responsivity of less than $0.05 \%$ in power mode and the $0.03 \%$ angular responsivity deviation from the cosine function in a five-degree angular range in irradiance mode. These small uncertainty components are the results of a tilted input aperture (relative to the sphere-axis) and four symmetrically positioned InGaAs detectors around the incident beam-spot in the sphere. With the new radiometer standard, it is expected that a thermodynamic temperature uncertainty of $10 \mathrm{mK}(k=2)$ can be achieved at $157{ }^{\circ} \mathrm{C}$ the freeze temperature of the In fixed point blackbody.
\end{abstract}

\section{Introduction}

The primary basis of modern radiometry is the measurement of optical power with a cryogenic electrical-substitution radiometer. The spectroradiometric quantities such as spectral irradiance and spectral radiance can be derived from the primary power measurements. However, the derivation of the spectroradiometric quantities from optical power requires the use of spatially-uniform and Lambertian detectors. These detectors serve as power-to-irradiance converters. In the visible and near-infrared wavelength regions, up to $960 \mathrm{~nm}$, silicon trap detectors, introduced in 1983 [1], are the lowest uncertainty transfer standards. Using these silicon reference detectors, $0.06 \%(k=2)$ uncertainty can be achieved in irradiance measurement mode [2]. In the near-infrared (NIR) region, between $950 \mathrm{~nm}$ and $1650 \mathrm{~nm}$, where the use of Si detectors is not possible, trap detectors using either Ge or InGaAs photodiodes have been constructed [3, 4]. Since the $\mathrm{Ge}$ or InGaAs photodiodes have much smaller shunt resistances than $\mathrm{Si}$, the combinations of large area, NIR diodes in parallel electrical connections have resulted in low shunt resistance devices with much worse radiometric and electronic performances than Si trap detectors [5]. Also, the spatial non-uniformity of the internal-quantumefficiency limited their power measurement uncertainties to about $0.3 \%(k=2)$. 
Another approach to obtain detectors with high spatial uniformity and Lambertian angular responsivity is to utilize an integrating sphere as the input to detectors which are directly attached to the sphere. Previous designs using InGaAs photodiodes have utilized either a single photodiode placed perpendicularly from the entrance aperture or multiple diodes facing the sphere center [6, 7].

Among other applications, the need for low-uncertainty radiance responsivity calibrations is driven by the possible thermodynamic temperature realizations in the near-infrared wavelength region where a $0.05 \%(k=2)$ absolute radiance responsivity uncertainty is needed to achieve a $10 \mathrm{mK}(k=2)$ uncertainty in the measurement of the In-point freezing blackbody at $157^{\circ} \mathrm{C}$. In the last 25 years, these low uncertainty requirements could not be achieved with regular near-IR detectors; it was possible only with silicon trap detectors up to $960 \mathrm{~nm}$. In order to achieve this low uncertainty in the near-IR, a new type of power-to-irradiance converting radiometer standard is needed that will not limit the uncertainty of the spectral responsivity propagation from the primary standard cryogenic radiometer. This new radiometer will be the reference detector of the Spectral Irradiance and Radiance Responsivity Calibrations using Uniform Sources (SIRCUS) facility for the near-IR range where up until now these low uncertainty spectral responsivity calibrations could not be performed.

We describe the design and characterization of a novel near-IR radiometer transferstandard that makes it possible to perform spectral power and irradiance responsivity calibrations with uncertainties lower than $0.05 \%(k=2)$.

\section{Preliminary sphere-detector experiments}

For spatial and angular responsivity tests, a Ge radiometer was attached to the exit port of a $5 \mathrm{~cm}$ diameter Spectralon ${ }^{\#}$ coated integrating sphere with $90^{\circ}$ angle between the entrance and exit ports. The spatial uniformity of this simple (no baffles) and asymmetric Ge sphere-detector with an $8 \mathrm{~mm}$ diameter input-aperture is shown in Fig. 1. The aperture plane was (traditionally) normal to the sphere-axis. The obtained maximum nonuniformity is $0.6 \%$ at $1500 \mathrm{~nm}$ using a $1.1 \mathrm{~mm}$ diameter scanning beam with $0.5 \mathrm{~mm}$ increments. The poor uniformity is caused by the asymmetric arrangement of the sphere input and output ports.

The angular responsivity test was performed with the same sphere-detector arrangement, but the sphere-aperture diameter was decreased to $3.5 \mathrm{~mm}$ to obtain improved sphere performance and better Lambertian angular response. First, as in traditional spheredetector designs, the aperture plane was normal $\left(0^{\circ}\right.$ tilt $)$ to the sphere optical axis. The incident beam was produced by an FEL lamp, located $3 \mathrm{~m}$ away from the detector. As shown in Fig. 2, at $0^{\circ}$ tilt (see legend), the angular response curve is flattened around the $0^{\circ}$ incidence angle because part of the reflected incident beam escaped through the entrance aperture of the sphere. The curve normalization was performed at the $0^{\circ}$ incidence angle. As a result of this problem, the angular responsivity is different from the cosine function, the ideal angular responsivity of detector irradiance measurements. The 
difference from the cosine is about $0.2 \%$ at $+4^{\circ}$ and $-4^{\circ}$ incidence angles. The structures within the response curve are dominated by lamp instabilities.

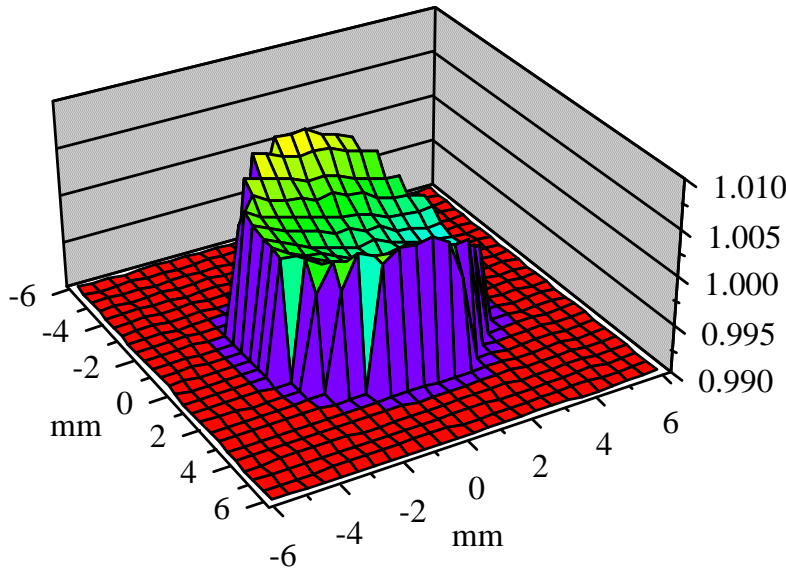

Relative Responsivity

Fig. 1. Spatial uniformity of responsivity of a traditional Ge sphere detector at $1500 \mathrm{~nm}$.

In the following steps, the angular responses of the Ge sphere-detector were measured at different aperture tilt-angles (between the normal of the aperture-plane and the sphereaxis). The tilt-angles were increased to check if the angular response relative to the above measurement (at $0^{\circ}$ tilt) can be improved. The incidence angle (x-axis) was changed as above, by rotating the sphere-detector around the center of the sphere-aperture. During these angular response scans, the sphere-detector was rotated in a plane perpendicular to the plane where the entrance and the exit ports are. The normal of the aperture plane was increased from $0^{\circ}$ (relative to the sphere axis) to $18^{\circ}$ in increments shown in Fig. 2. During the angular response tests, at all tilt angles, the incident beam (at the $0^{\circ}$ incidence angle) was always perpendicular to the plane of the input aperture. The measured response curves are shown in a $+/-10^{\circ}$ range of the incidence angle. At a large enough tilt-angle of the aperture plane (relative to the sphere axis), the angular response curve becomes similar to the cosine function. For aperture-plane tilt angles of $12^{\circ}$ and $18^{\circ}$, the first specular reflection from the sphere wall could not escape from the sphere (as it happened at $0^{\circ}$ tilt) therefore the normalized responses do not show a suppressed cosine curve anymore. The reason of the slight angular shift relative to the cosine function is a close to $1^{\circ}$ alignment error in the rotation of the sphere-detector.

The above spatial and angular responsivity results show that traditional sphere-detectors, with $90^{\circ}$ angle between the entrance and exit ports and where the aperture plane is perpendicular (not tilted) to the sphere axis, have significant spatial and angular responsivity errors, therefore, they are not suitable for absolute power and irradiance mode measurements with the required less than $0.1 \%(k=2)$ responsivity uncertainty. It also has been shown with the preliminary angular response tests (at different aperture tilt angles relative to the sphere axis) that the non-Lambertian angular response can be significantly improved if the input aperture plane is tilted at least $12^{\circ}$ relative to the sphere axis. This improvement was verified in the rotation plane perpendicular to the plane where the aperture and the detector are located. 


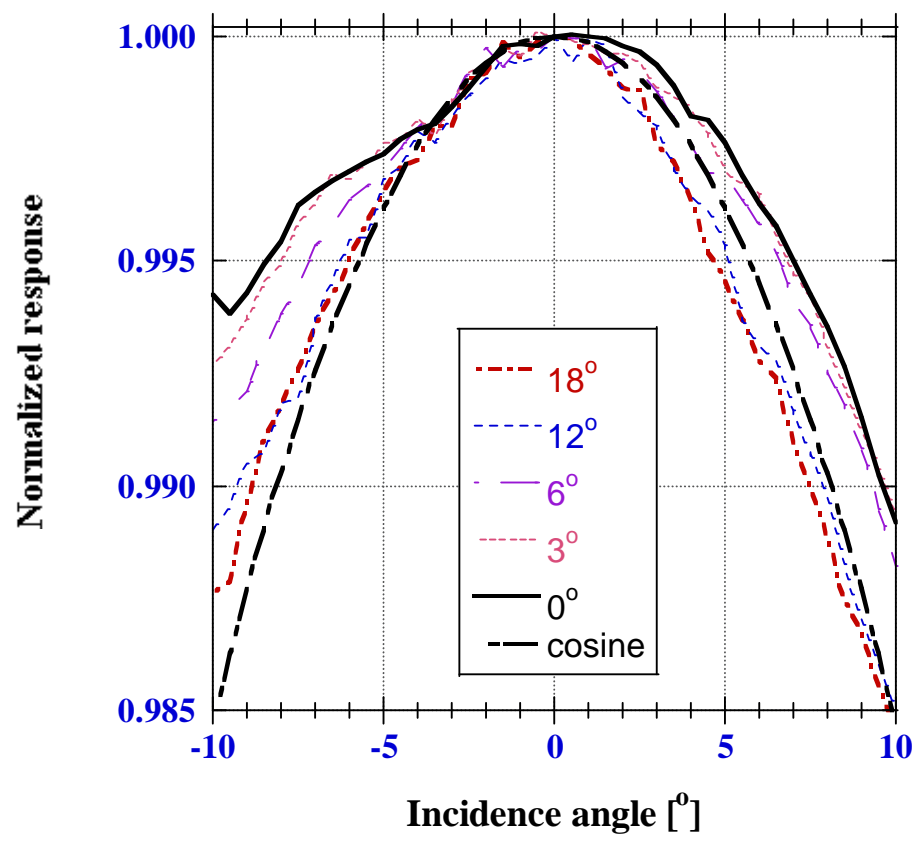

Fig. 2. Angular response of a Ge sphere-detector at different tilt angles of the input aperture plane relative to the sphere-axis.

The influence of the sphere-detector asymmetry $\left(90^{\circ}\right.$ between the sphere aperture and the detector) for the angular response was tested as well. This test was performed with the $18^{\circ}$ tilt (of the aperture plane relative to the sphere-axis) in the plane where the aperture and the detector are located. To make this test, the previous angular response measurements were repeated after a $90^{\circ}$ rotation of the sphere-detector (around its optical axis) and the previous measurement geometry (pivot point and fixed lamp distance) was used again. The obtained angular response curve significantly deviated from the cosine function. The measured asymmetric curve is shown in Fig. 3 (at $18^{\circ}$ tilt of the aperture plane relative to the sphere-axis). The asymmetric angular response is caused by the asymmetric arrangement of the sphere entrance and exit ports in the plane of the rotation. (The rotation changes the incidence angle.) The deviation from the cosine is about $0.4 \%$ at a $4^{\circ}$ incidence angle. This error in the angular responsivity can be significantly decreased if multiple detectors are symmetrically arranged relative to the incident-beamproduced spot on the sphere wall. A symmetrical detector arrangement can improve the spatial uniformity of responsivity as well.

The preliminary angular-response measurement results verified that using $18^{\circ}$ tilt in the plane of the input aperture (relative the sphere-axis), the escaping radiation can be trapped and the angular responsivity to the cosine can be restored. Probably, there is a very small enhanced reflection peak at $0^{\circ}$ in the BRDF of the Spectralon wall-coating since this material is not an ideal Lambertian reflector. This material-characteristic produced the flattened angular response. Also, the trapped specular-component of the first reflection could improve the spatial uniformity of responsivity which is important for 
low-uncertainty radiant power measurements. The results in Fig. 3 show that the traditional $90^{\circ}$ aperture-detector arrangement should be changed to a symmetrical detector arrangement relative to the incident-beam-produced spot on the back of the sphere-wall. A symmetrical detector arrangement can be implemented using multiple detectors instead of a single element detector.

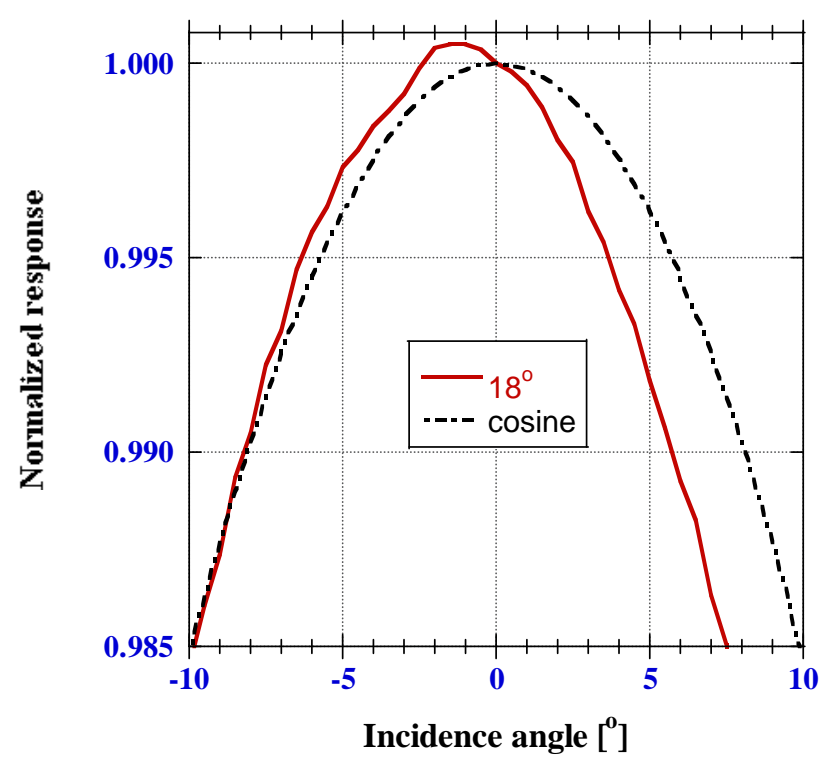

Fig. 3. Angular response of a traditional Ge sphere-detector where the $90^{\circ}$ separated entrance and exit ports are in the plane of the rotation.

\section{Design of the sphere-InGaAs radiometer}

In order to improve the spatial and angular uniformity of the responsivity and to obtain lower responsivity uncertainties in a calibration transfer, a near-IR transfer standard radiometer has been designed. The new radiometer has an integrating-sphere receiver with the optical axis of the entrance aperture tilted away from the sphere-axis. Four temperature-controlled InGaAs photodiodes, selected for high shunt resistance, are symmetrically positioned in the sphere wall around the incident beam spot such that they cannot see each other.

The geometrical arrangement for the input of the sphere-detector is shown in Fig. 4. A new position is needed for the aperture to obtain the maximum useful acceptance angle without beam clipping and to avoid any flux loss through the entrance aperture owing to the back reflection of the specular component of the incident beam from the sphere wall. The tilted aperure and the two unclipped beam-limits are illustrated. The incidence angle of the left limit-beam (relative to the sphere axis) is $6^{\circ}$, the other side limit-beam is determined by the sphere wall. The new entrance axis of the sphere is the aperture axis, which splits the angle between two limit-beams. The measurements and calculations for the available components showed that the optimum value of the angle between the sphere axis and the aperture axis is $17.5^{\circ}$. 


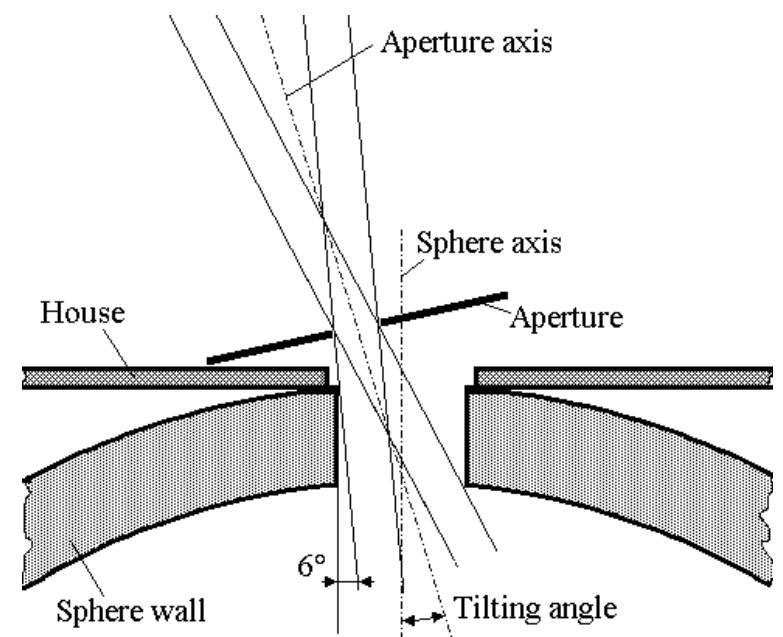

Fig. 4. Sphere-detector with a tilted aperture input.

The cross-section of the sphere-InGaAs detector housing is shown in Fig. 5. The aperture axis is tilted by $18^{\circ}$. The housing is temperature controlled with a thermo-electric (TE) cooler/heater attached to the back of the Al-housing. As shown on the picture of the sphere-detector in Fig. 6, a heat sink is attached to the back side of the TE cooler/heater. The four $1 \mathrm{~mm}$ diameter InGaAs detectors are selected for equal shunt resistance and they are parallel connected with each other. They are symmetrically positioned around the incident beam produced "hot" spot such that they cannot see each other. The stabilized $26{ }^{\circ} \mathrm{C}$ temperature of the sphere-housing propagates to the metal cans of the detectors.

\section{Sphere-InGaAs radiometer characterizations}

In Fig. 6, the sphere-InGaAs detector with a $5 \mathrm{~mm}$ diameter input aperture was characterized at the Spectral Comparator Facility (SCF) by scanning with a $1 \mathrm{~mm}$ diameter beam. The measured spatial uniformity at $1200 \mathrm{~nm}$ is shown in Fig. 7. The maximum-to-minimum responsivity change is less than $0.05 \%$. The spatial uniformity tests were repeated at $1000 \mathrm{~nm}$ and $1600 \mathrm{~nm}$. The pattern of the spatial non-uniformity of responsivity was different at each wavelength. The maximum-to-minimum responsivity change was also $0.05 \%$ at $1000 \mathrm{~nm}$ and it increased to $0.075 \%$ at $1600 \mathrm{~nm}$. With a larger beam spot positioned in the aperture-center, the beam positioning error in the power responsivity measurement can be decreased by about a factor of two.

The noise-equivalent-power (NEP) was determined from output noise and spectral power responsivity measurements between $950 \mathrm{~nm}$ and $1650 \mathrm{~nm}$. Figure 8 shows the NEP results from two spectral scans compared to the NEP of a Ge trap detector [8]. The 


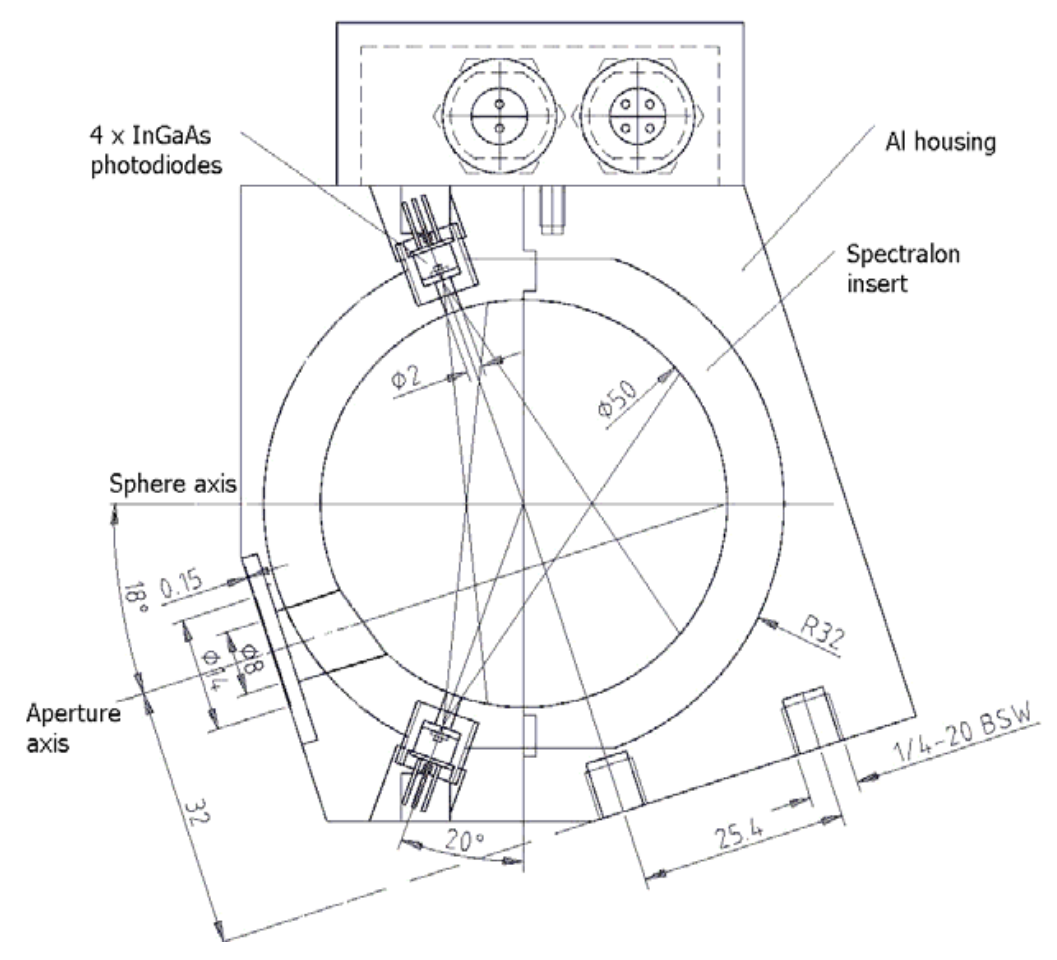

Fig. 5. Cross-section of the sphere-InGaAs detector.

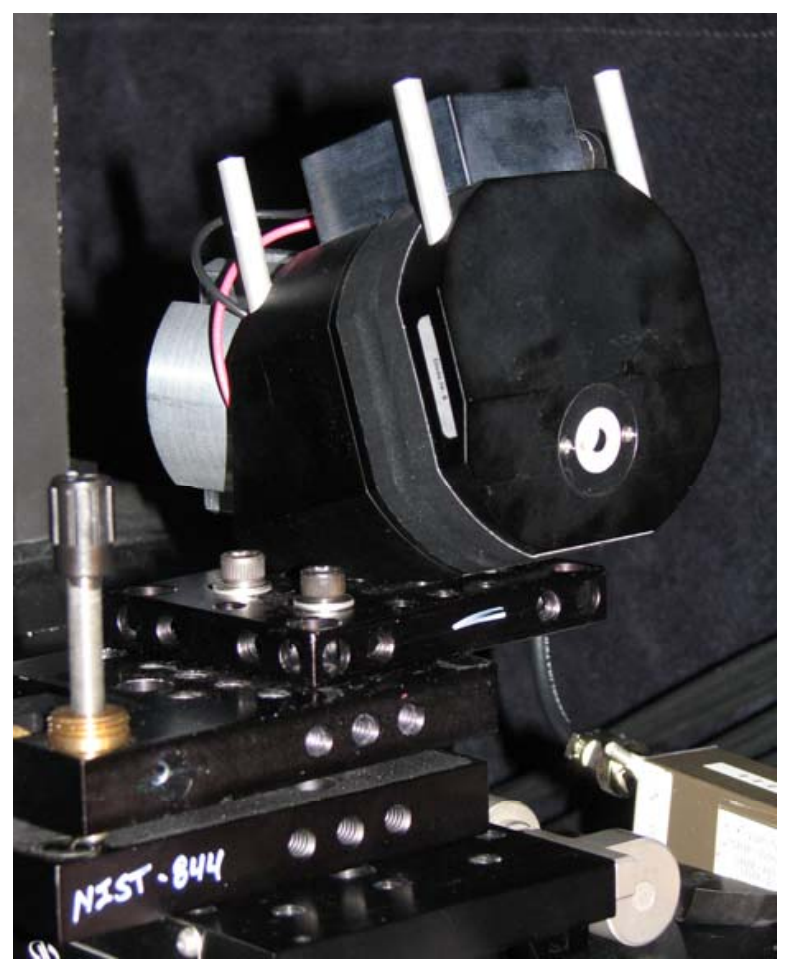

Fig. 6. Picture of the sphere-InGaAs detector. 


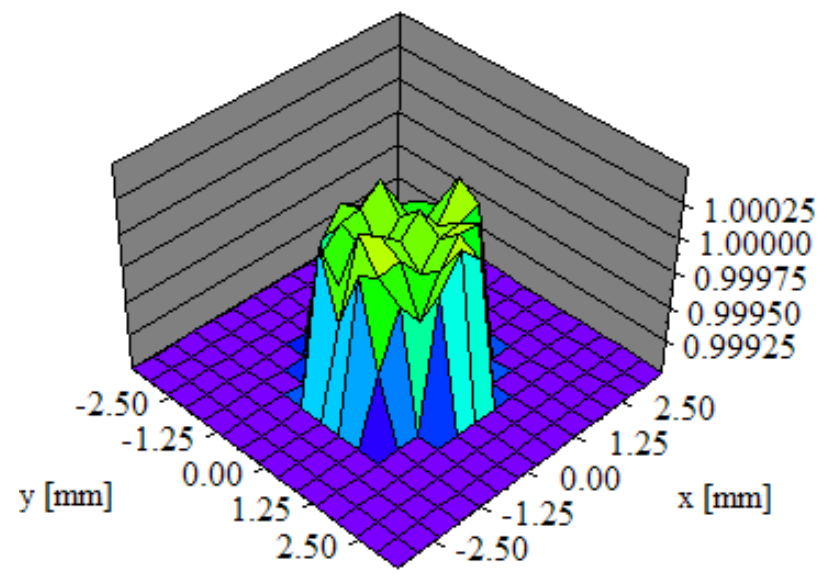

Relative Responsivity

Fig. 7. Spatial uniformity of the sphere-IGA detector with a $5 \mathrm{~mm}$ diameter input-aperture at $1200 \mathrm{~nm}$.

obtained data verify that the dominating noise of the sphere-InGaAs detector is white noise, specifically, resistor noise. The resistor noise, which originates from the parallel connection of the shunt resistance and the feedback resistance, is dominated by the resultant shunt resistance of the four parallel connected 1-mm detectors. The resultant shunt resistance was $33 \mathrm{M} \Omega$ for four non-selected photodiodes (set-1) and $50 \mathrm{M} \Omega$ for four photodiodes selected for equal shunt resistance of $200 \mathrm{M} \Omega$ (set-2). The expanded uncertainty of the shunt resistance measurements was $10 \%(k=2)$. The feedback resistance of the current-to-voltage converter during the spectral measurements was $100 \mathrm{M} \Omega$. The average NEP was $5 \mathrm{pW} / \mathrm{Hz}^{1 / 2}$ for run- 1 and $5.5 \mathrm{pW} / \mathrm{Hz}^{1 / 2}$ for run-2 using set-1 with the $33 \mathrm{M} \Omega$ shunt resistance. Since the resultant shunt resistance of the Ge trap detector is only $1 \mathrm{k} \Omega$, its dominating noise is $1 / \mathrm{f}$ noise. The $1 / \mathrm{f}$ noise determined NEP of the Ge trap detector was $30 \mathrm{pW} / \mathrm{Hz}^{1 / 2}$.

Figure 9 shows the repeatability of four spectral responsivity scans at the SCF. The responsivity differences shown on the Y-axis are in the $0.05 \%$ level between $950 \mathrm{~nm}$ and $1350 \mathrm{~nm}$ and also between $1450 \mathrm{~nm}$ and $1650 \mathrm{~nm}$. The larger differences at $1400 \mathrm{~nm}$ are caused by the atmospheric absorption.

The sphere-InGaAs detector angular responsivities were measured on a photometer bench. The illuminating source was a Wi41G lamp. This lamp had significantly smaller intensity fluctuations than the FEL lamp used in the preliminary experiments shown in Fig. 2. Otherwise, these measurements were performed similarly to the preliminary tests. Since the separation between the lamp and the sphere-detector was about $3 \mathrm{~m}$, the lamp operated like a "point source" for the sphere-InGaAs detector. Two baffles were located between the lamp and the sphere-detector to minimize stray light on the detector. The detector was mounted on a rotation stage and it was rotated around the aperture-center in the horizontal plane. To repeat the angular responsivity measurements in the vertical plane, the sphere-detector was $90^{\circ}$ rotated on the rotation stage around its optical axis. The measurement results are shown in Fig. 10. The ratios of the measured responses to 
the cosine function are shown versus the rotation (incidence) angle of the sphere-InGaAs detector. The curves are normalized. The deviations from the cosine function are $0.03 \%$ if the (full-angle) field-of-view of the sphere-InGaAs detector which is limited to $5^{\circ}$.

The long-term stability of the sphere-InGaAs detector is an important issue. Our 2-years long stability tests on several InGaAs makes and models verified that these photodiodes

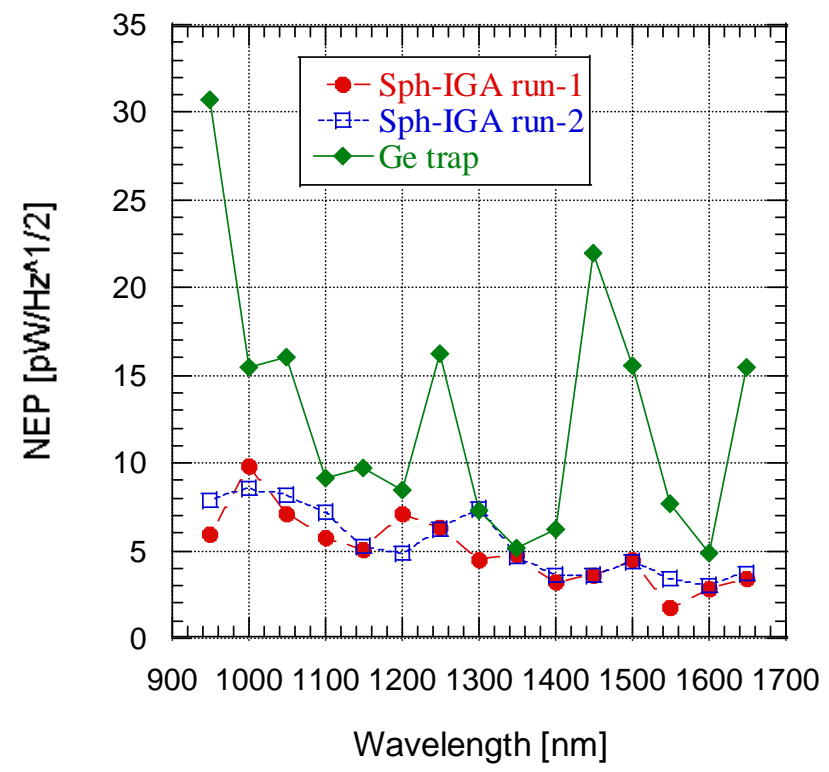

Fig. 8. NEP comparison of the sphere-IGA and the Ge-trap detectors.

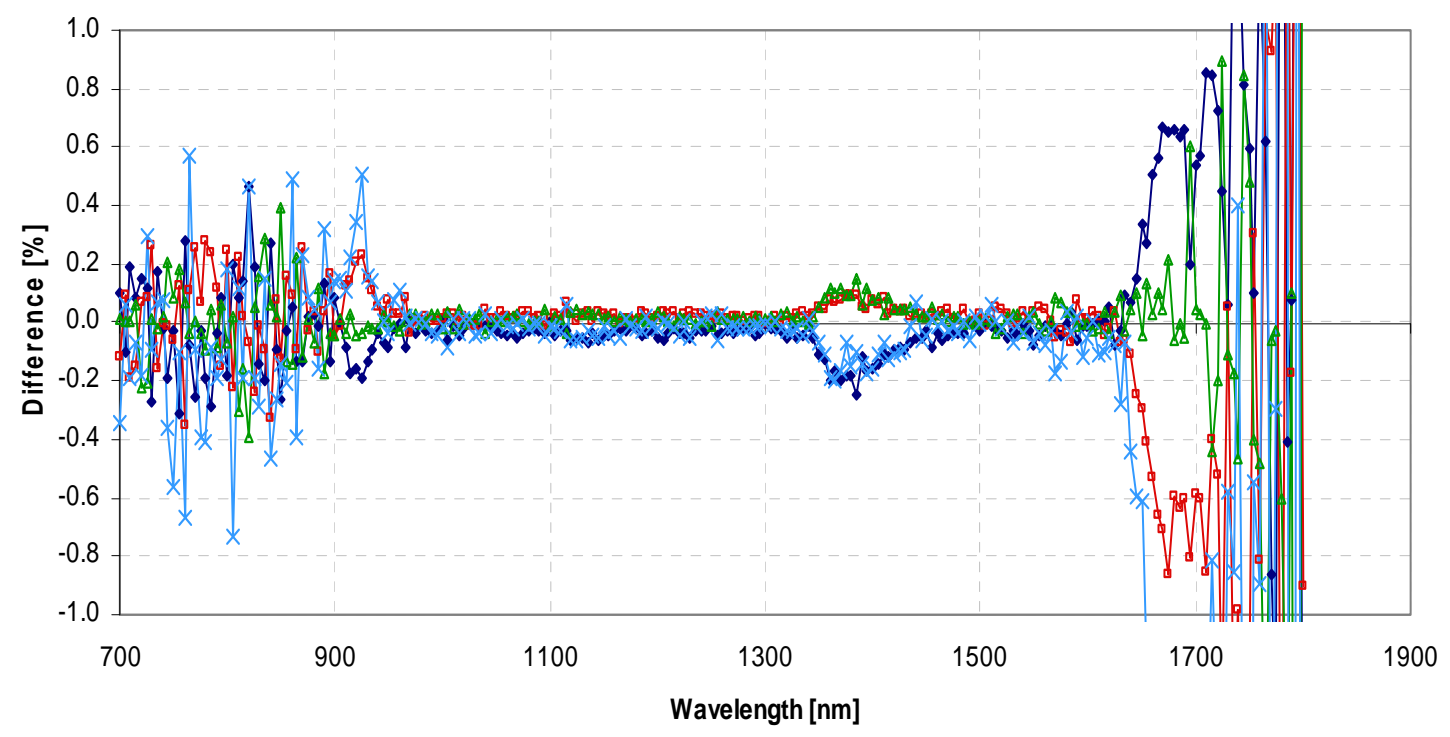

Fig. 9. Responsivity differences (repeatability) of four spectral responsivity scans. 
(even if they are not temperature stabilized) exhibit less than $0.2 \%$ instabilities between $1.0 \mu \mathrm{m}$ and $1.6 \mu \mathrm{m}$. Also, when measurements are not performed, a protecting cap is mounted to the entrance port of the sphere to keep the dust and dirt outside. The low-NEP (high sensitivity) makes it possible to decrease throughput changes with gray spherecoatings where the reflectance factors are significantly smaller than unity. The sphereInGaAs detector will be calibrated against the cryogenic radiometer yearly, to calibrate out long-term changes.

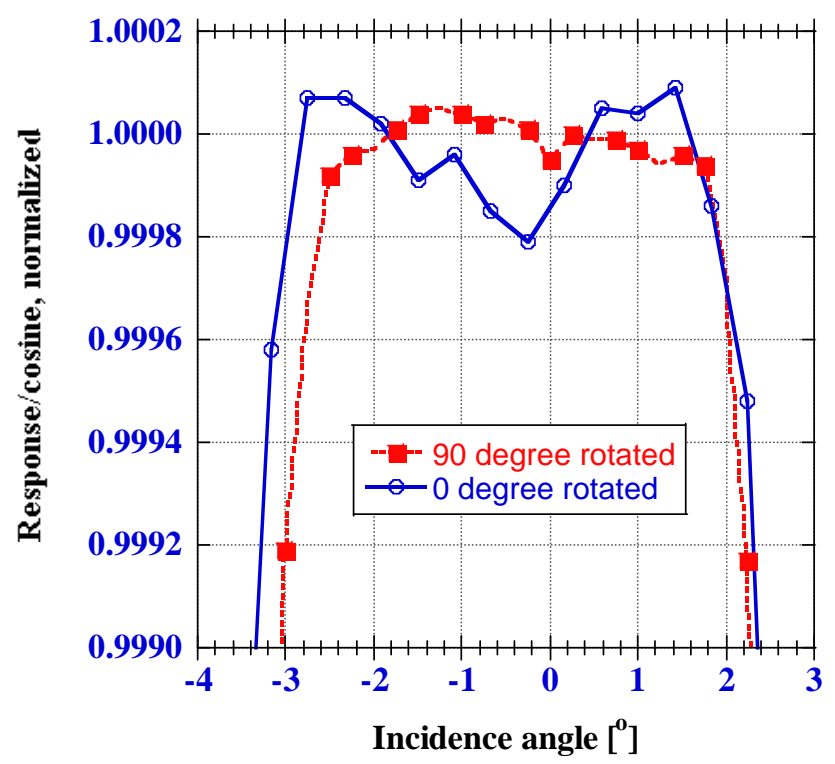

Fig. 10. Response/cosine versus incident angle to the normal of the $8 \mathrm{~mm}$ diameter input-aperture of the sphere-detector.

\section{Calibration procedure}

The SIRCUS scale derivation is illustrated in Fig. 11. The new sphere-InGaAs detector (shown with the earlier used $90^{\circ}$ aperture-detector arrangement) is calibrated against the cryogenic radiometer for absolute spectral power responsivity. Following the radiant power responsivity scale transfer, an irradiance mode tie point is derived from a silicon trap detector at about $950 \mathrm{~nm}$. An alternative solution is to use an area-calibrated aperture in front of the sphere-detector for power-to-irradiance mode conversion. The irradiance mode sphere-InGaAs detector is the reference detector for the SIRCUS in the NIR range to $1650 \mathrm{~nm}$.

Other (test) irradiance detectors can be calibrated against the sphere-InGaAs reference detector when they both measure the irradiance from an integrating sphere source of the SIRCUS. For absolute radiance responsivity calibrations, a large sphere-source is used at SIRCUS and its exit-port radiance is determined against the new sphere-InGaAs detector when the distance $d$ is measured between the source aperture and the detector aperture. Test radiance meters are calibrated against the detector-based radiance of the large 
sphere-source. In order to achieve the required $0.05 \%(k=2)$ absolute radiance responsivity uncertainty, the uncertainty components are kept small and the sphereInGaAs detector will not limit the overall uncertainty of the responsivity scale transfer.

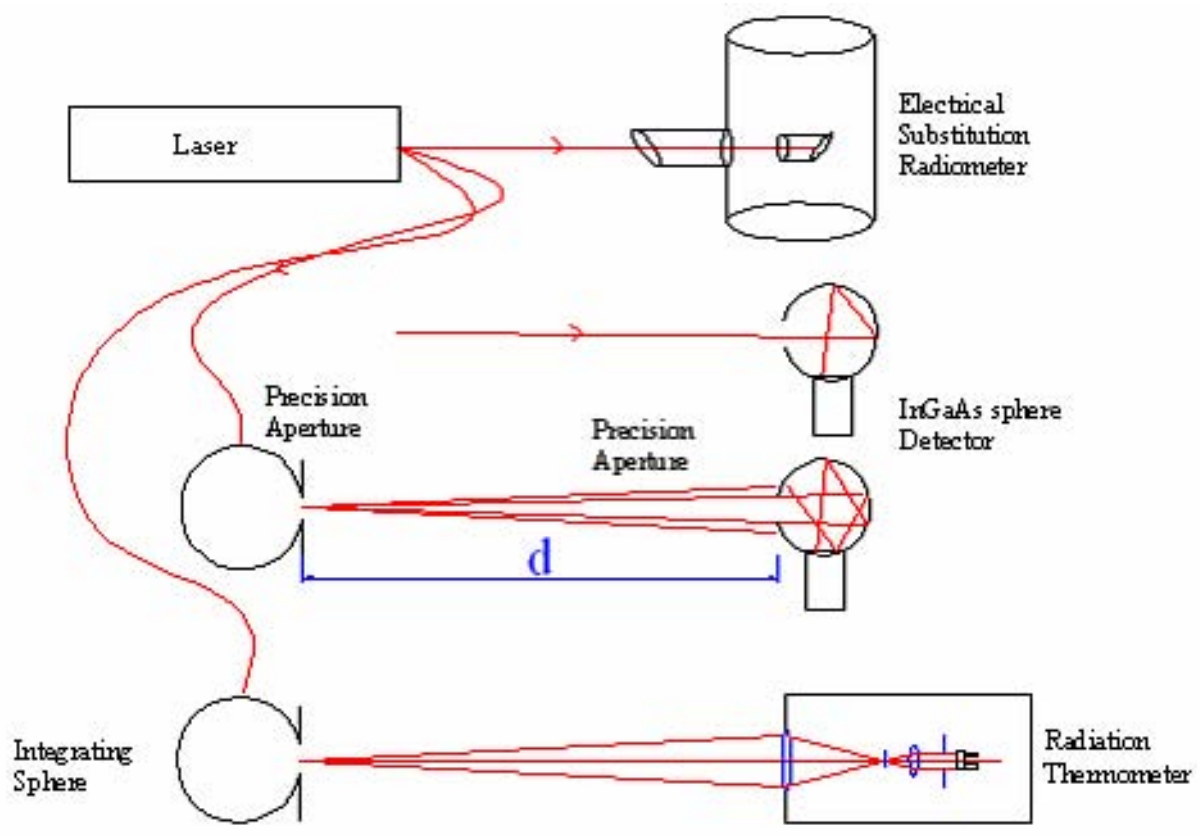

Fig. 11. The SIRCUS responsivity scale derivation using a sphere-InGaAs detector.

\section{Future applications}

In addition to the SIRCUS irradiance and radiance responsivity scale extension to the near-IR, the sphere-InGaAs detector can be used for fiber coupled spectral power responsivity scale transfer with sub-pW NEP and high coupling efficiency. The sphereInGaAs detector with the $0.05 \%(k=2)$ radiance responsivity uncertainty, also can be used to determine the thermodynamic temperature of a $157{ }^{\circ} \mathrm{C}$ Indium point (blackbody radiator) with $10 \mathrm{mK}(k=2)$ uncertainty. Also, because of its excellent spatial uniformity of responsivity, it can be applied for international spectral power responsivity comparisons with decreased uncertainties compared to presently used single-element or trap near-IR quantum detectors even if the incident beam sizes are different (as was the case in the CCPR K2.a). We are planning to increase the area of the InGaAs detectors to increase responsivity. Also, the detectors will be cooled to increase their shunt resistance and to obtain lower output noise. According to our estimation, the NEP of an improved sphere-InGaAs detector can be as low as $50 \mathrm{fW} / \mathrm{Hz}^{1 / 2}$. This is equal to the NEP of silicon tunnel-trap detectors built with shunt-resistance selected photodiodes. We also plan to extend the spectral range to $2.5 \mu \mathrm{m}$ using cooled extended-InGaAs detectors. 


\section{Conclusions}

This is the first time that a near-IR transfer standard detector with similar performance to silicon trap detectors has been developed. Recent responsivity scale realizations on the SIRCUS facility demonstrated that silicon trap detectors can propagate the low uncertainty radiant power scale of the cryogenic radiometer to $960 \mathrm{~nm}$ with a minimal increase in the uncertainty. Because of the increasing demand for less than $0.1 \%(k=2)$ responsivity calibrations at the SIRCUS facility between $950 \mathrm{~nm}$ and $1650 \mathrm{~nm}$, a transfer standard near-IR radiometer was needed that can propagate the primary responsivity scale similarly to the silicon trap detectors. A novel sphere-input radiometer (with tilted input aperture to the sphere axis and a symmetrical arrangement of four InGaAs detectors to the incident beam spot on the sphere-wall) has been developed with electronic and radiometric characteristics similar to silicon trap detectors. The two dominant responsivity uncertainty components (originating from the spatial non-uniformity and the angular responsivity deviation from the cosine) in a power to irradiance/radiance scale transfer could be decreased to less than $0.05 \%(k=2)$. The new device with its improved responsivity uncertainty is used as the highest level reference radiometer of the SIRCUS facility for near-IR irradiance and radiance mode spectral responsivity calibrations. The radiometer standard can be utilized to realize a detector-based radiation temperature scale down to $157{ }^{\circ} \mathrm{C}$ with a $10 \mathrm{mK}(k=2)$ uncertainty. There are also other application areas, such as fiber coupled spectral power responsivity measurements where the coupling efficiency can be high using this sphere-input standard. It also can be used as a traveling transfer detector to compare international near-IR responsivity scales in both power and irradiance measurement modes with significantly lowered responsivity uncertainties.

\# Certain commercial equipment, instruments, or materials are identified in this paper to foster understanding. Such identification does not imply recommendation or endorsement by the National Institute of Standards and Technology, nor does it imply that the material or equipment are necessarily the best available for the purpose.

\section{References}

1. E. F. Zalewski and C. R. Duda, "Silicon photodiode device with $100 \%$ external quantum efficiency,” Applied Optics, Vol. 22, No.18, p. 2867-2873, 1983.

2. S. W. Brown, G. P. Eppeldauer, and K. R. Lykke, "Facility for Spectral Irradiance and Radiance Responsivity Calibrations using Uniform Sources," Applied Optics, Vol. 45, No. 32, p. 8218-8237, 2006.

3. K. D. Stock, R. Heine, and H. Hofer, "Spectral characterization of Ge trap detectors and photodiodes used as transfer standards,” Metrologia, Vol. 40, p. S163-S166, 2003.

4. M. Lopez, H. Hofer and S. Kuck, "High accuracy measurement of the absolute spectral responsivity of Ge and InGaAs trap detectors by direct calibration against an electrically 
calibrated cryogenic radiometer in the near- infrared,” Metrologia, Vol. 43, p. 508-514, 2006.

5. A. Lamminpaa, M. Noorma, T. Hyyppa, F. Manoocheri, P. Karha, and E. Ikonen, "Characterization of germanium photodiodes and trap detector," Meas. Sci. Technol., Vol. 17, p. 908-912, 2006.

6. Ana Carrasco-Sanz, Felix Rodrıguez-Barrios, Pedro Corredera, Sonia Martın-Lopez, Miguel Gonzalez-Herraez and Marıa Luisa Hernanz, "An integrating sphere radiometer as a solution for high power calibrations in fiber optics," Metrologia, Vol. 43, p. S145S150, 2006.

7. L. P. Boivin "Properties of sphere radiometers suitable for high-accuracy cryogenicradiometer-based calibrations in the near-infrared," Metrologia, Vol. 37, p. 273-8, 2000.

8. J. H. Lehman and X. Li, “A transfer standard for optical fiber power metrology," Applied Optics, Vol. 38, p. 7164-7166, 1999. 


\title{
Optical characterization of diffuser-input standard irradiance meters
}

\author{
George Eppeldauer ${ }^{a}$, Miklós Rácz ${ }^{b}$, and Thomas Larason $^{a}$ \\ ${ }^{a}$ Optical Technology Division of the National Institute of Standards and Technology \\ Gaithersburg, MD 20899, USA \\ ${ }^{b}$ Guest Researcher at National Institute of Standards and Technology from the Hungarian \\ Academy of Sciences Research Institute for Technical Physics and Materials Science \\ P.O. Box 49, Budapest-114, Hungary 1525
}

\begin{abstract}
Standards quality irradiance meters have been developed at the National Institute of Standards and Technology (NIST) to realize a detector based spectral irradiance scale. The design criteria and the optical and radiometric characterization of diffuser-input irradiance meters are discussed. The input geometry optimization is described for a broad-band InGaAs irradiance meter when measuring a $2856 \mathrm{~K}$ incandescent light source. The directional responsivity of the fabricated InGaAs and Si irradiance meters matches the cosine function with a maximum deviation of $0.15 \%$ within a $12^{\circ}$ field-of-view (FOV). Irradiance from sources of different sizes (incident beams with different $f$ /numbers) can be measured over a wavelength range of $350 \mathrm{~nm}$ to $1800 \mathrm{~nm}$.
\end{abstract}

Keywords: aperture, cosine function, directional responsivity, detector, diffuser, irradiance, photodiode, radiometer, spectral responsivity, working standard.

\section{INTRODUCTION}

The detector spectral responsivity scale of NIST is based on an electrical substitution High Accuracy Cryogenic Radiometer (HACR) ${ }^{1}$. The HACR measures the radiant power of intensity stabilized laser lines with a relative standard uncertainty of $0.021 \%$. After a scale derivation procedure ${ }^{2}$, large-area, single-element $\mathrm{Si}^{3}$ and $\mathrm{Ge}^{4,5}$ photodiode working standards are calibrated for spectral power responsivity. The uncertainty of the spectral power responsivity is wavelength dependent. The relative standard uncertainty is about $0.1 \%$ in the visible, $1 \%$ in the $\mathrm{UV}$, and $2.5 \%$ in the near-infrared range when the responsivity is transferred to test detectors ${ }^{2}$.

In order to extend the detector based spectral power responsivity calibrations to irradiance responsivity, standards quality irradiance meters had to be developed. The beam geometry at calibration and the different applications varies from parallel (collimated) to $12^{\circ}$ full angle ( $6^{\circ}$ incidence angle). In order to achieve high measurement accuracy, the directional characteristics of the detectors have to follow the cosine law in this angular range. The directional characteristics were controlled up to a $10^{\circ}$ incidence angle to assure the proper cosine response of the detectors within the desired angular range. The design and optical characterization of these meters are discussed for an InGaAs irradiance meter. The described optimization procedure was utilized for a Si irradiance meter as well.

\section{SPECTRAL CHARACTERISTICS}

The spectral characteristics of the irradiance meters are determined by the photodiode and the flashed opal glass diffuser used in the meter. In Fig. 1 the spectral responsivities of the Si and InGaAs photodiodes and the irradiance meters can be seen. The magnitudes of the irradiance responsivities are similar for the two meters because the larger aperture $(8.0 \mathrm{~mm}$ diameter) of the Si meter produces higher irradiance responsivity than the smaller aperture (6.4 mm diameter) of the InGaAs meter. Sources with either monochromatic or known (e.g., Planckian) spectral power distributions can be measured with these broadband irradiance meters. 


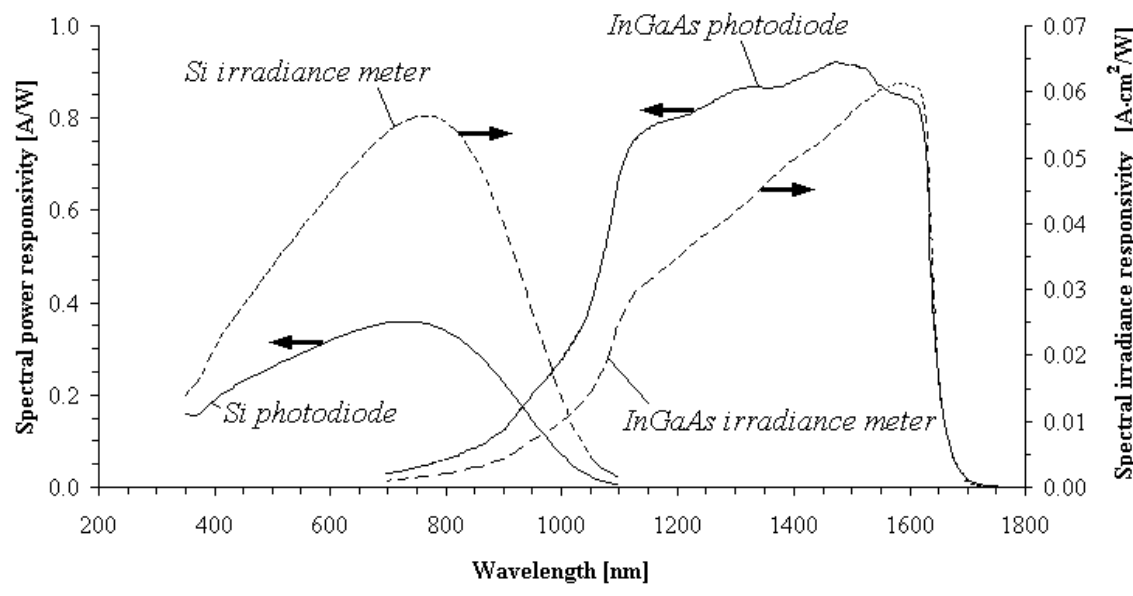

Fig. 1. Spectral responsivities of the Si and InGaAs photodiodes and the corresponding irradiance meters.

\section{DIRECTIONAL CHARACTERISTICS}

The directional responsivity measurements were performed on the NIST photometry bench. Several tungsten incandescent lamps were used as non-calibrated radiation sources. The color temperature of the lamps was set to $2856 \mathrm{~K}$ or lower. The irradiance meters to be measured were mounted on a rotational stage and the input apertures were positioned on the rotation axis. The distance between the lamps and the rotation axis was about $3.25 \mathrm{~m}$. The quality of the directional responsivity data was characterized with the Commission Internationale de L’Éclairage (CIE) directional error $f_{2}(\varepsilon, \phi)$ :

$$
f_{2}(\varepsilon, \phi)=\frac{E_{\text {reading }}(\varepsilon, \phi)}{E_{\text {reading }}\left(\varepsilon=0^{\circ}\right) \cdot \cos \varepsilon}-1
$$

where $E_{\text {reading }}(\varepsilon, \phi)$ and $E_{\text {reading }}\left(\varepsilon=0^{\circ}\right)$ are the readings for an irradiance, $E$, arriving at the angles of incidence $\varepsilon$ and $0^{\circ}$ respectively. $\phi$ is the azimuth angle. Because the irradiance meters are circularly symmetrical, they were only tested at two perpendicular $\phi$ values.

Drawings of the NIST developed diffuser-input irradiance meters can be seen in Fig. 2. The FOV limiters reject optical radiation outside the FOV. The input apertures determine the reference plane and the active area of the irradiance meters. The diffusers dominate the directional responsivity of the meters. The detectors are temperature controlled by thermoelectric coolers and are electrically shielded. The temperature of the InGaAs and Si photodiodes are controlled to $-30{ }^{\circ} \mathrm{C}$ and $+25^{\circ} \mathrm{C}$ respectively.
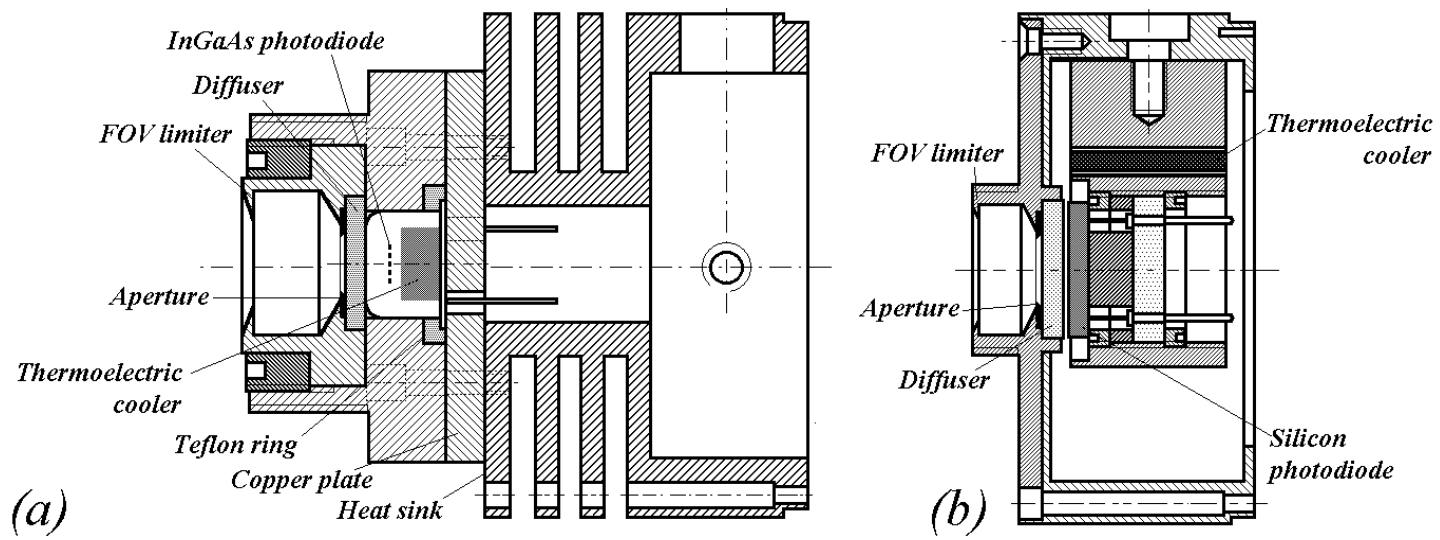

Fig. 2. Input optics cross-section of the InGaAs (a) and Si (b) irradiance meters. 
The geometry of the input optics of the irradiance meters was optimized experimentally. Several flashed opal glass diffusers were tested with a Cary 4a spectroradiometer in the $400 \mathrm{~nm}$ to $1700 \mathrm{~nm}$ wavelength range because their visual appearance was not the same. The diffusers were separated into four groups according to their diffuse reflectances of about $27 \%, 31 \%, 35 \%$, and $40 \%$ at $1400 \mathrm{~nm}$. As can be seen in Fig. 3, the diffuse reflectance of the samples decreases with increasing wavelength.

Four sizes of apertures were also tested. Their diameters were approximately $3.5 \mathrm{~mm}, 6.4 \mathrm{~mm}, 8.0 \mathrm{~mm}$, and $11.5 \mathrm{~mm}$. The optimal aperture-diffuser pair was chosen experimentally. Every possible combination was measured for angular response and the $\operatorname{CIE} f_{2}(\varepsilon)$ error functions were evaluated. Fig. 4 shows the $f_{2}(\varepsilon)$ functions for the $0^{\circ}$ to $10^{\circ}$ incidence angle range at a given $\phi$ value. The $f_{2}(\varepsilon)$ functions can be seen for combinations with the $6.4 \mathrm{~mm}$ aperture and the four different diffusers in Fig. 4a. Whereas Fig. 4b shows the $f_{2}(\varepsilon)$ functions for combinations built with a $40 \%$ reflectance diffuser and the four different apertures.

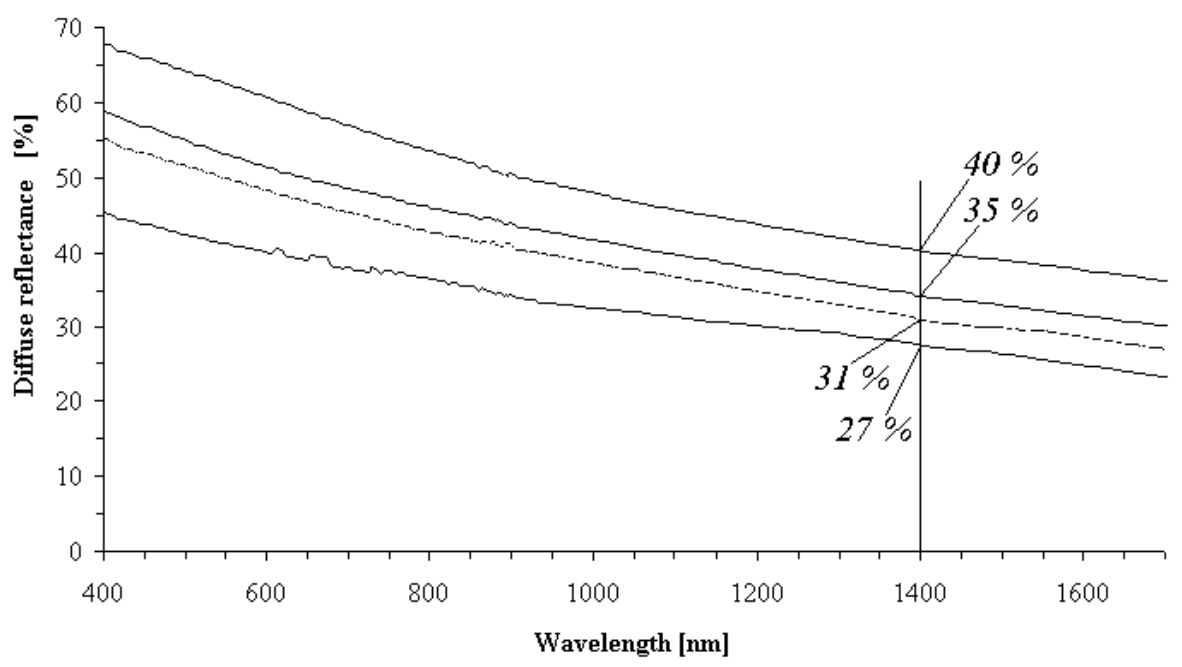

Fig. 3. Diffuse spectral reflectance of the flashed opal glass diffusers. The vertical line shows the wavelength of the diffuser selection.

Aperture $6.4 \mathrm{~mm}$

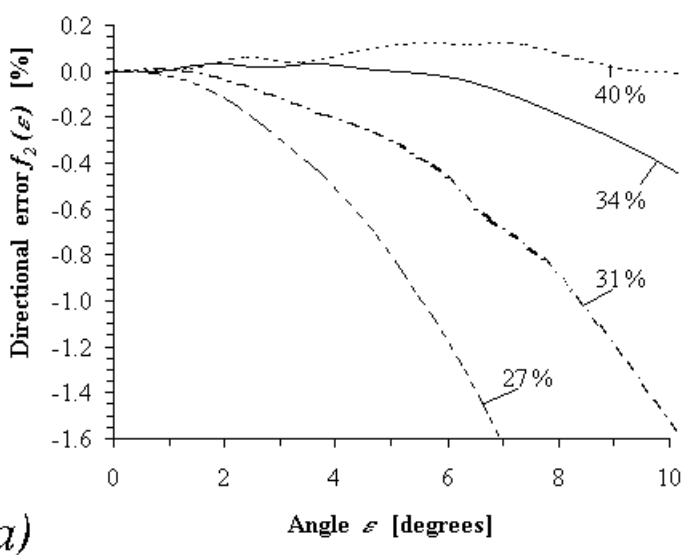

Diffise reflectance $40 \%$

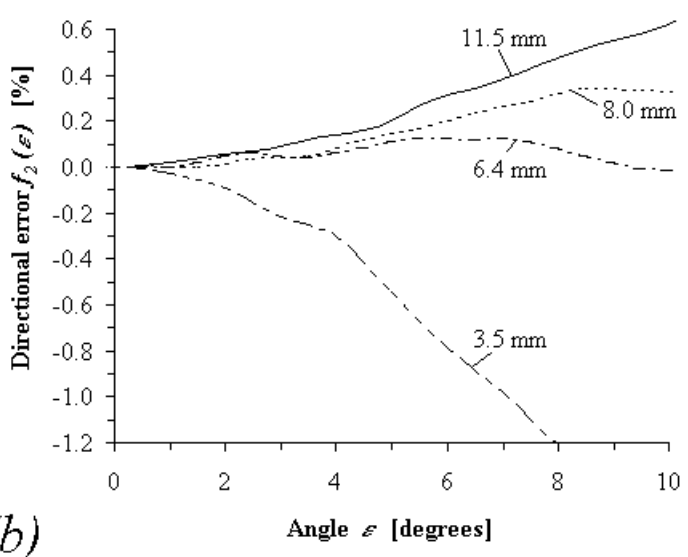

Fig. 4. Directional error of different aperture-diffuser pairs for a circular InGaAs photodiode with a diameter of $5.0 \mathrm{~mm}$. Figure 4a shows the measurement results for four different diffusers with the $6.4 \mathrm{~mm}$ aperture. Figure $4 \mathrm{~b}$ shows the results for four different apertures with a diffuser reflectance of $40 \%$. 
As can be seen, the angular responsivity depends upon the diameter of the aperture and the quality of the diffuser. Two pairs of aperture-diffuser were found to have adequate directional characteristics, the $6.4 \mathrm{~mm}$ aperture with a $40 \%$ diffuser and the $8 \mathrm{~mm}$ aperture with a $35 \%$ diffuser. The $6.4 \mathrm{~mm}$ aperture and $40 \%$ diffuser pair had better spatial responsivity characteristics and was selected for the InGaAs irradiance meter. The selected diffuser pair had an effective area $11.0 \%$ smaller than the geometrically measured area. For the other pair, the effective area was $18.4 \%$ smaller than the geometrically measured area because of the larger spatial nonuniformity.

It follows from Figs. 3 and 4a that the optimum cosine response depends on the wavelength. Consequently, the input geometry has to be optimized for the wavelength (or the spectral distribution) of the source to be measured.

\section{RESPONSIVITY CALIBRATION AND SPATIAL CHARACTERISTICS}

An $x-y$ scanning procedure was used to determine the integrated irradiance responsivity and the effective aperture area of irradiance and illuminance meters ${ }^{6}$. This irradiance calibration method uses only detector standards instead of conventional standard sources. The procedure where a small beam overscans the input aperture of the meter, also provides information on the spatial responsivity uniformity of the detector. The output signal of the detector was measured as a function of the position of the beam. The wavelength was selected for the sensitivity range of the photodiode, $780 \mathrm{~nm}$ for Si and $1500 \mathrm{~nm}$ for InGaAs. Fig. 5 shows the spatial responsivity of the InGaAs meter with the $6.4 \mathrm{~mm}$ aperture and the $40 \%$ diffuser. The diameter of the beam was $1.1 \mathrm{~mm}$. Each distance step of the scan was $0.5 \mathrm{~mm}$. The position of the beam relative to the center of the aperture is measured on the horizontal axes, while the output signal of the irradiance meter is drawn on the vertical axis. The irradiance meter output signal is normalized to the signal measured in the central position. In Fig. 5a the response uniformity of the active area is shown and in Fig. 5b the radiation scattered from the area surrounding the aperture can be seen.
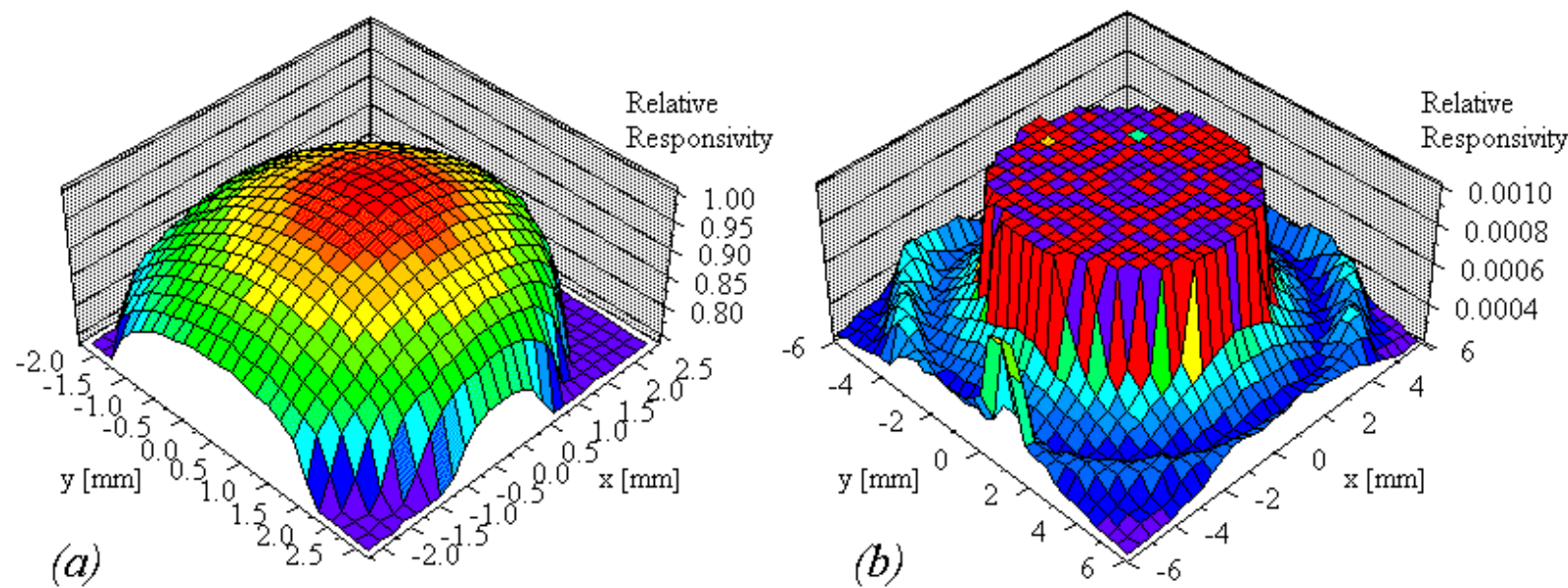

Fig. 5. Spatial responsivity distribution plots of the diffuser-input InGaAs irradiance meter with a $6.4 \mathrm{~mm}$ aperture and a $40 \%$ diffuser. Figure 5 a shows the uniformity of the sensitive area, while Fig. $5 \mathrm{~b}$ shows the stray radiation.

The effective area of an irradiance meter can be calculated as the ratio of the integrated irradiance responsivity to the radiant power responsivity in the center of the aperture. The effective area is utilized in flux transfers when the measured irradiance (in the meter's aperture plane) is transferred to another device, e.g., the exit port of a radiance source $^{7}$. In such cases, geometrical aperture area measurements are not needed. When a diffuser is used between the aperture and the photodiode to maintain the cosine response of an irradiance meter, a side effect is the decreased responsivity toward the edges of the photodiode. Because of this side effect, the effective area can be significantly different from the geometrically measured area of the aperture. The measured effective area of the InGaAs irradiance meter with the $6.4 \mathrm{~mm}$ aperture was $28.6 \mathrm{~mm}^{2} \pm 0.5 \%$, while the geometrical area is $32.08 \mathrm{~mm}^{2} \pm 0.075 \%$. The effective area of the Si meter was measured at two different wavelengths of $563 \mathrm{~nm}$ and $815 \mathrm{~nm}$. The area change was $0.29 \%$, well within the uncertainty of the radiometric effective area measurement. 


\section{UNCERTAINTIES}

The uncertainty components in addition to the uncertainty of the spectral power responsivity calibrations ${ }^{2}$ originate from several sources. The uncertainty of the integrated irradiance measurement method is estimated to be $0.5 \%$, the directional error of $0.15 \%$ for the optimized meters, and the integrated stray radiation of $0.1 \%$. The relative combined standard uncertainty of an irradiance measurement with the Si meter is $1.6 \%$ at $350 \mathrm{~nm}$. The standard uncertainty decreases to $0.94 \%$ at $400 \mathrm{~nm}$ and is almost constant at $0.54 \%$ in the visible to $925 \mathrm{~nm}$. From $925 \mathrm{~nm}$ to $1800 \mathrm{~nm}$ where the InGaAs irradiance meter is used, the estimated standard uncertainty is about $1.2 \%$ from $925 \mathrm{~nm}$ to $1345 \mathrm{~nm}$ and increases to about $2.6 \%$ between $1350 \mathrm{~nm}$ and $1800 \mathrm{~nm}$.

\section{CONCLUSION}

Diffuser-input irradiance meters have been developed with InGaAs and Si photodiodes as working standards. The wavelength range of the two irradiance meters covers the $350 \mathrm{~nm}$ to $1800 \mathrm{~nm}$ wavelength region. The irradiance meters were calibrated against the NIST detector spectral power responsivity scale. The flashed opal glass diffusers were measured and grouped according to their diffuse reflectance. A range of apertures was tested with the different diffusers. Diffuser and aperture pairs were selected for optimum cosine response and minimum spatial responsivity non-uniformity. The CIE directional error was $f_{2}(\varepsilon)=0.15 \%$ or less for the irradiance meters in the $0^{\circ}$ to $10^{\circ}$ incidence angle range. The total irradiance responsivity of the meters was determined from spatial responsivity integrals over the aperture area during a scanning procedure. The standard irradiance meters, that hold the spectral irradiance responsivity scale of the SCF facility, can measure spectral irradiance with an estimated combined standard uncertainty of $0.54 \%$ in the visible range.

\section{ACKNOWLEDGMENTS}

The authors thank Donald Lynch of Reyer Corporation for the irradiance meter fabrication, Yuqin Zong for helping in the directional response measurements, Sally Bruce for making the spectral responsivity and spatial uniformity measurements, Joel Fowler for the aperture area measurements, and Yvonne Barnes for helping in the diffuse reflectance measurements.

\section{REFERENCES}

1. Gentile, T. R., Houston, J. M., Hardis, J. E., Cromer, C. L., and Parr, A. C., "National Institute of Standards and Technology high accuracy cryogenic radiometer,” Appl. Opt. 35 (7), 1056-1068 (1996).

2. Larason, T. C., Bruce, S. S., Parr, A. C., "Spectroradiometric Detector Measurements: Part I-Ultraviolet Detectors and Part II-Visible to Near-Infrared Detectors," National Institute of Standards and Technology Special Publication 250-41 (1998).

3. Eppeldauer, G. and Hardis, J. E., "Fourteen-decade photocurrent measurements with large-area silicon photodiodes at room temperature," Appl. Opt. 30 (22), 3091-3099 (1991).

4. Eppeldauer, G., “Near Infrared Radiometer Standards,” SPIE 2815 Optical Radiation Measurements III. $42-54$ (1996).

5. Eppeldauer, G., "Electronic characteristics of Ge and InGaAs radiometers," SPIE 3061 Infrared Technology and Applications XXIII. 833-838 (1997).

6. Cromer, C. L., Eppeldauer, G., Hardis, J. E., Larason, T. C., Ohno, Y., Parr, A. C., “The NIST Detector-Based Luminous Intensity Scale,” J. Res. Natl. Inst. Stand. Technol., 101 (2), 109-132 (1996).

7. Johnson, B. C., Cromer, C. L., Saunders R. D., Eppeldauer, G., Fowler, J., Sapritsky, V. I. and G. Dézsi, “A Method of Realizing Spectral Irradiance Based on an Absolute Cryogenic Radiometer," Metrologia, 30, 309315 (1993). 


\title{
Near Infrared Radiometer Standards
}

\author{
George Eppeldauer \\ Optical Technology Division \\ National Institute of Standards and Technology \\ Gaithersburg, Maryland 20899
}

\begin{abstract}
$\underline{\text { ABSTRACT }}$
We are developing new detector-based radiometric scales in the near infrared to extend existing spectral radiant power calibrations ${ }^{1}$ to spectral irradiance and radiance calibrations. High accuracy irradiance and radiance meters have been developed to realize these new detector-based radiometric scales. Mechanical, optical, and radiometric design considerations of Ge and InGaAs radiometers are discussed. Calibration principles and design considerations of the calibration setup are also described. Several results of preliminary radiometric characterizations are reported.
\end{abstract}

\section{INTRODUCTION}

At present, standard quality high-accuracy radiometers for spectral irradiance and radiance measurements are not available in the near infrared (NIR) wavelength range. Most national laboratories offer detector calibrations only for spectral radiant power responsivity. Irradiance meters ${ }^{2}$ frequently show response changes if the geometry $(\mathrm{f} / \#)$ of the measured optical radiation changes. This usually happens in applications where the beam geometry reaching the irradiance meter is different than at calibration. In these situations the reflectance pattern inside the radiometer input optics can change. This change will result in irradiance response errors. Similarly, radiance meters frequently measure signal from outside of the source target area ${ }^{3}$. Here, again, the measurement results contain significant errors. In cases like this, the input optics of these radiance meters may not be properly designed, resulting in a poor out of field-of-view (FOV) blocking ${ }^{4}$.

Sensitivity of NIR radiometers is an important issue in many radiometric applications. Typically, the peak radiant power level at the exit slit of a monochromator is a few $\mu \mathrm{W}$ only. The low power end of the signal can be several orders of magnitude smaller. In most monochromator applications, photomultiplier detectors are used to measure low level NIR signals. Similarly, low temperature black-bodies have extremely small radiance in the visible range and somewhat larger in the NIR. Radiance temperature meters measure radiance (scaled in temperature) in a narrow wavelength interval. Frequently used photomultiplier tubes ${ }^{5}$ could be substituted with high sensitivity NIR detector-circuit packages in these radiance temperature meters by switching from the visible to the NIR range.

The quality of NIR detectors have improved a lot in the past decade. As a result of improved fabrication procedures, a $40 \%$ to $50 \%$ internal quantum efficiency increase was reported on Judson $\mathrm{J} 16 \mathrm{P}^{6}{ }^{6}$ Ge photodiodes at the beginning of the decade ${ }^{7}$. The peak internal quantum efficiency was between 0.8 and 0.9 . The reported shunt resistance of a $10 \mathrm{~mm}$ diameter photodiode was only $100 \Omega$. As introduced by Stock, the aging rate of the earliest Ge photodiode version was lower than $+/-0.5 \%$ per year and was wavelength dependent. Within the $7 \mathrm{~mm}$ diameter central part of the photodiode active area the spatial response non-uniformity was less than $+/-0.5 \%$ and increased at shorter wavelengths. Stock and others reported better spatial response uniformity for Ge photodiodes as compared to InGaAs ${ }^{8}$. They used a small integrating 
sphere in front of the InGaAs photodiode to reduce the spatial response non-uniformity. However, the sphere decreased the absolute responsivity by a factor of about 300 .

In our earlier publication ${ }^{9}$, InGaAs, Ge, and InAs photodiodes were characterized. The average internal quantum efficiency of the tested mesa epitaxial type InGaAs photodiodes was 0.975 at $1.3 \mu \mathrm{m}$. The position dependent change of the internal quantum efficiency was $+/-0.5$ $\%$. However, because of the position-dependent reflectance change, the external quantum efficiency, which was proportional to the absolute response, changed $4 \%$ as a function of position across a diameter of a windowless InGaAs photodiode. These InGaAs photodiodes were linear over three decades of radiant power as measured at the high power end $(0.8 \mathrm{~mW}$ maximum). They showed much higher shunt resistance at room temperature and higher external quantum efficiency than the few Ge and one InAs photodiodes examined in that study.

As a continuation of our previous NIR detector study ${ }^{9}$, further internal quantum efficieny measurements were made on planar diffusion InGaAs photodiodes ${ }^{10}$. The measured internal quantum efficiencies were about 0.9 at $1.15 \mu \mathrm{m}$ and $1.3 \mu \mathrm{m}$, and about 0.88 at $1.52 \mu \mathrm{m}$. The reason for these smaller values of the planar diffuse devices is that the junction was less shallow and the passivation was worse than for the mesa grown photodiodes. Also, the zinc dopant penetrates deeper than in the mesa technique, resulting in larger diode capacitance. With antireflection coating, these devices showed specular reflectances of about $2 \%$. All tested InGaAs photodiodes were p-on-n devices. The Ge photodiodes were constructed of p-type base material into which was diffused a shallow n-type junction. This construction is similar to those reported above ${ }^{7}$.

The reason for adopting the planar diffuse technology to InGaAs manufacture was to produce larger area devices with greater stability. In the mesa device, the edge of the junction extends to the edge of the wafer and is therefore not protected. At present, it seems to be more important to produce high stability than to achieve $100 \%$ internal quantum efficiency on InGaAs photodiodes, which was the goal of the previous studies ${ }^{9,10 .}$

In the present work, large area, planar structure InGaAs photodiodes with dielectric passivation and large area Ge photodiodes similar to those reported above ${ }^{7}$ are used. The diameters of the active areas are $5 \mathrm{~mm}$ for both the InGaAs and Ge photodiodes. They were all selected for high shunt resistance to obtain high sensitivity ${ }^{11}$. All photodiodes are hermetically sealed in standard packages of the same size, and cooled by two stage thermoelectric coolers. The temperature of the photodiodes is monitored by thermistors and controlled by temperature controllers $^{12}$. Since lasers are used in these measurements, the photodiode chips are tilted by $1^{\circ}$ to $2^{\circ}$ out of parallel with the package window to avoid interference. Also, the sapphire windows have 20' of wedge. The surfaces of all photodiodes are of optical quality.

\section{SCALE REALIZATION}

\subsection{Radiant power response scale}

The detector response scale derivation scheme is shown in Fig. 1. The primary standard is an electrical substitution high accuracy cryogenic radiometer (HACR) ${ }^{13}$ which measures laser power. At present, eleven laser lines are used between $320 \mathrm{~nm}$ and $950 \mathrm{~nm}$. Silicon trap detectors $^{14}$ are calibrated against the HACR at the different laser lines. Interpolation between the calibrated points gives the spectral radiant power responsivity of a trap detector in A/W between $406 \mathrm{~nm}$ and $920 \mathrm{~nm}^{14}$. The spectrally calibrated transfer standard trap detector(s) are used on the Visible Near Infrared Spectral Comparator Facility ${ }^{1}$, where working standard silicon photodiodes are calibrated against the trap detectors by using a substitution technique. The spectral power 
response scale is extended from the silicon wavelength range to the NIR range with a pyroelectic transfer detector. The relative response of the gold-black coated pyroelectric detector is determined from spectral reflectance measurements of its coating. The coating transmission is negligible. Temperature controlled working standard Ge detectors are calibrated for spectral responsivity against the known response of the pyroelectric detector. Test NIR detectors can be calibrated for spectral radiant power responsivity (in A/W) against the working standard Ge detectors between $700 \mathrm{~nm}$ and $1800 \mathrm{~nm}$.

The goal of our scale realization work is to derive the spectral irradiance and radiance response scales from the existing radiant power response scale. To achieve this goal standard quality radiometers were developed. Optical and radiometric design considerations are discussed of two different kinds of irradiance meters and a radiance meter. The characterization results of the radiance meter are discussed in this paper. The electronic characteristics of the radiometers and the detailed radiometric characterization of the irradiance meters will be discussed in consecutive papers. The scheme of the new calibration setup where the new radiometers are utilized is described in this paper. The scale realization measurements and the error budget of the new scales will be discussed in the final paper.

\subsection{Spectral irradiance and radiance response scales}

In a previous NIST project ${ }^{15}$, an irradiance meter composed of a silicon photodiode, an interference filter, an integrating sphere, and an input aperture was calibrated against a standard silicon detector using a laser beam. In this manner a detector-based irradiance scale was derived from the radiant power response of the standard silicon detector.

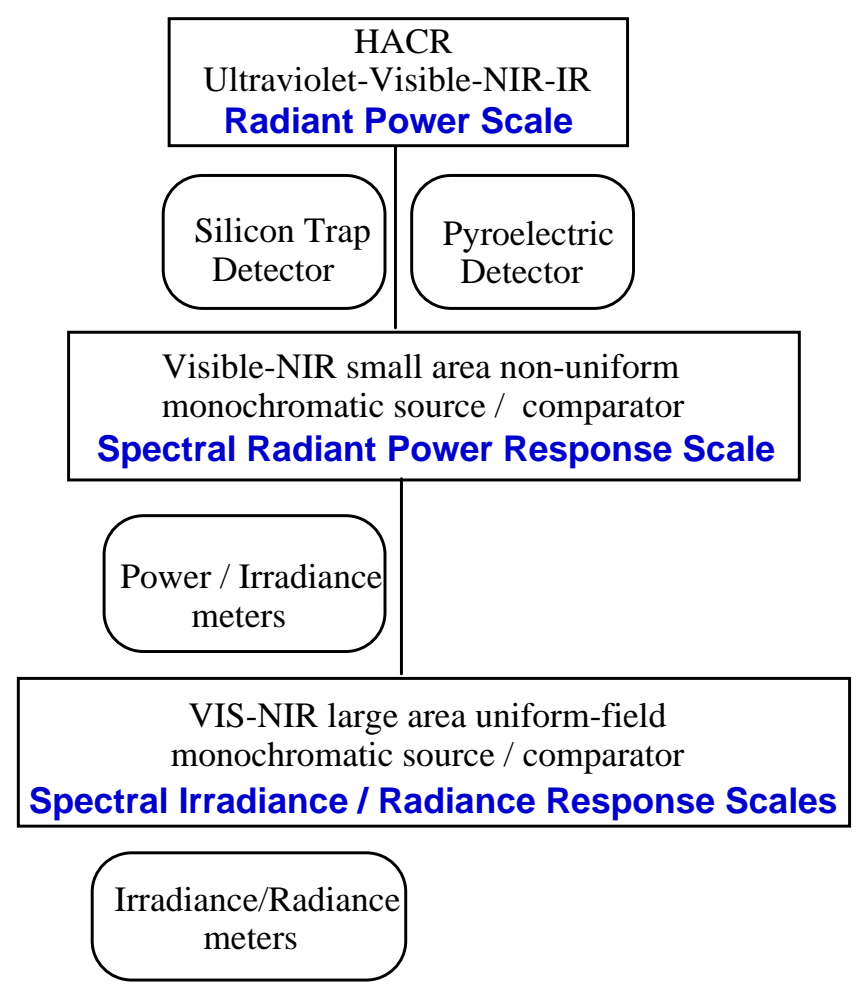

Fig. 1. Detector response scale derivation scheme 
This irradiance meter was then used to measure the spectral irradiance of two other beam geometries at the laser wavelength. The other two sources were standard spectral irradiance lamps (blackbody source-based scale) and the electron storage ring, SURF-II. The detector-based scale at the test wavelength, was $0.76 \%$ lower than the source-based scale, and $0.25 \%$ higher than the electron storage ring scale. Because of the high spatial response uniformity within the aperture area, the total uncertainty for the detector-based irradiance scale was $0.5 \%$. In contrast, the uncertainty of the source-based irradiance scale was $1.68 \%$. Throughout this paper, all uncertainty values are given as a relative expanded uncertainty with a coverage factor of $\mathrm{k}=2$. This is equivalent to an uncertainty consisting of two standard deviations about the mean of a normalized Gaussian distribution.

In our earlier report ${ }^{16}$, a technique had been described for realizing spectral irradiance scale using a large-area, high temperature, uniform black-body source and filter-radiometers that are calibrated on a detector-based system. The goal of this project was to reduce the measurement uncertainties in the present source-based spectral irradiance scale ${ }^{21}$. The filter radiometers were calibrated on the NIST Spectral Comparator Facility ${ }^{1}$, and the aperture areas were measured by flux comparison with a uniform source ${ }^{17}$. Six filter radiometers covering the visible and NIR wavelength ranges were used to measure a known irradiance source (FEL lamp). The measurements agreed with predictions to within $1 \%{ }^{18}$. Comparisons were also conducted with three NIST standard photometers, and similar agreement was observed. Since the temperature and radiance calibration of the gold point blackbody source is also detector-based ${ }^{19}$, the agreement will be improved if the input geometry of the filter radiometers is modified. The new input geometry requires a diffusing input element within the aperture area to realize the cosine function of illuminance measurements with a high accuracy.

In radiant power response calibrations, the active area of the detectors was underfilled by the monochromatic beam and the detectors measured radiant power. Since the diameter of the f/9 calibrating beam at the NIST Spectral Comparator Facility is small (i.e., it can be changed from $1.2 \mathrm{~mm}$ to a few $\mathrm{mm}$ ) and is spatially non-uniform, irradiance measuring radiometers cannot be overfilled with this beam. Otherwise, their irradiance responsivity would change as a function of detector aperture positioning. Instead, the detector area, within the limiting aperture, is mapped (scanned) with the small, non-uniform beam, and the average power responsivity, $\mathrm{s}_{\mathrm{avg}}$, is determined versus wavelength, $\lambda$. The spectral irradiance responsivity, $\mathrm{s}_{\mathrm{E}}(\lambda)$, of a silicon or NIR irradiance meter is derived from its own spatially averaged spectral radiant power responsivity:

$$
\mathrm{s}_{\mathrm{E}}(\lambda)=\mathrm{F} \cos \alpha \mathrm{s}_{\mathrm{avg}}(\lambda) \quad\left[\mathrm{A} \mathrm{\textrm {m } ^ { 2 } / \mathrm { W } ]}\right.
$$

where $\mathrm{m}^{2}$ is the unit of the known aperture area F, A is the unit of the detector output current, W is the unit of the measured beam power, and $\alpha$ is the angle of the incident beam. The angle of incidence is the angle between the normal of the aperture plane and the axis of the incident beam. If the incident beam is different from perpendicular (relative to the aperture plane), the irradiance, as measured by the irradiance meter, will change according to the cosine of the incidence angle ${ }^{20}$. Note that $\alpha$ is constant in eq. 1 . If the incident beam is not parallel, $\alpha$ is no longer a constant, but can take a range of values depending on the $\mathrm{f} / \#$. In this case the integral of $\cos \alpha$ will be measured for all incident angles (changing $\alpha$ ) of the beam.

The calibrated irradiance meter can be used as a standard to calibrate test irradiance meters by substitution in a large enough, spatially uniform monochromatic field. The large and 
uniform monochromatic fields are realized by either a collimator or an integrating sphere coupled to the output of a monochromatic radiation source.

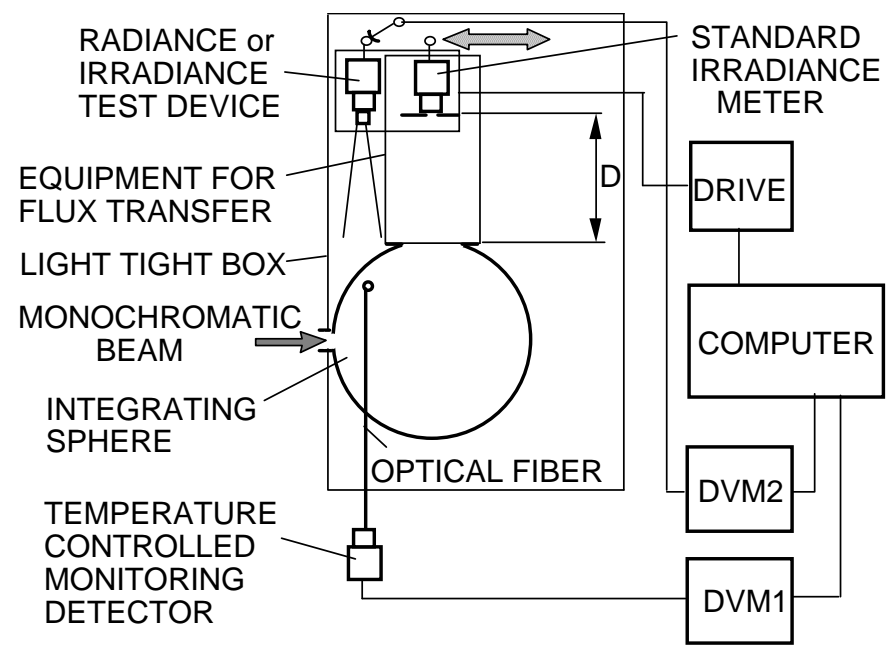

Fig. 2. Block scheme of the detector radiance/irradiance response calibration setup

Figure 2 shows the block scheme of our detector radiance/irradiance response calibration setup, which is being constructed. Instead of a traditional lamp-monochromator assembly, high intensity monochromatic sources, such as lasers or filtered lamps/arcs are to be used to produce the monochromatic beam for the integrating sphere. The radiance of the sphere wall is monitored by a two-color ( $\mathrm{Si}$ on top of InGaAs) detector which can hold the spectral radiance scale after scale realization. A standard irradiance meter is used to calibrate the monochromatic, large output area, Lambertian source of the sphere (or a plaque) for spectral radiance, $L(\lambda)$. At the NIST Spectral Comparator Facility the standard irradiance meter is calibrated first for spectral power responsivity, $s(\lambda)$, which is the wavelength dependent ratio of the detector output current, I, to the radiant power, P, as measured by the detector. A precision aperture of known area is mounted in front of the detector. If necessary, $s_{\text {avg }}(\lambda)$ can be used instead of $s(\lambda)$. The distance, $\mathrm{D}$, between the source aperture and the detector (irradiance meter) aperture is measured. The output current of the standard irradiance meter is:

$$
\mathrm{I}(\lambda)=\mathrm{s}(\lambda) \mathrm{P}(\lambda)
$$

where $\mathrm{P}(\lambda)$ is the spectral radiant power from the sphere source as measured by the standard irradiance meter. The spectral radiance can be calculated from $\mathrm{P}(\lambda)$, applying the geometric extent, $\mathrm{G}_{\mathrm{D}}{ }^{21}$ :

$$
\mathrm{L}(\lambda)=\mathrm{P}(\lambda) / \mathrm{G}_{\mathrm{D}} \quad\left[\mathrm{W} \mathrm{m}^{-2} \mathrm{sr}^{-1}\right]
$$

The geometric extent, $G_{D}$, can be given by the standard expression which describes the flux transfer between circular, parallel, and coaxial apertures with radii $\mathrm{r}_{\mathrm{S}}$ (for the source aperture) and $\mathrm{r}_{\mathrm{D}}$ (for the detector aperture), and separation, $\mathrm{D}$,

$$
\mathrm{G}_{\mathrm{D}}=\left(\pi^{2} / 2\right)\left\{\left(\mathrm{D}^{2}+\mathrm{r}_{\mathrm{S}}{ }^{2}+\mathrm{r}_{\mathrm{D}}^{2}\right)-\left[\left(\mathrm{D}^{2}+\mathrm{r}_{\mathrm{S}}{ }^{2}+\mathrm{r}_{\mathrm{D}}\right)^{2}-4 \mathrm{r}_{\mathrm{S}}^{2} \mathrm{r}_{\mathrm{D}}^{2}\right]^{1 / 2}\right\} \quad\left[\mathrm{m}^{2} \mathrm{sr}\right]
$$


As a second step, the radiance meter measures the above determined radiance of the sphere source. The spectral radiance response of the radiance meter (test device in this case) is a ratio of its output current or voltage to the measured sphere radiance versus wavelength.

Test irradiance meters can be substituted for the standard irradiance meter in the uniform monochromatic irradiance of the sphere source. This is a simple irradiance response calibration method for large area irradiance (test) meters. The same irradiance is measured by both irradiance meters. This irradiance is calculated from the irradiance response of the standard irradiance meter. The irradiance response of the test device is then determined from its output current or voltage reading and the calculated (and measured) irradiance, versus wavelength.

The response of the standard irradiance meter has to be the same for the $\mathrm{f} / 9$ calibrating beam and the beam geometry at radiance and irradiance measurements (radiation from the Lambertian source).

Properly designed NIR radiometers are the key components in realizing the above described radiometric scales for the NIR wavelength range.

\section{MECHANICAL AND OPTICAL DESIGN OF NIR RADIOMETERS}

\subsection{Radiant power meter}

Figure 3 shows the design of the photodiode-housing for radiant power measurement. The TO- 8 photodiode package is plugged into the center hole of the threaded holder. The threaded holder is limited to a FOV of $+/-30^{\circ}$ for the photodiode to block radiation from the sides. During radiant power (W) measurement, the active area of the photodiode is underfilled by the incident beam. No aperture is needed in front of the photodiode for this measurement. However, the spatial response non-uniformity of the photodiode has to be in the order of $0.1 \%$ to get repeatable radiant power responsivities (A/W) for different beam sizes and beam positions. The threaded holder can be used to mount different accessories, such as filters and/or apertures, in front of the photodiode. The heat from the bottom of the photodiode case is conducted by the copper plate to the heat sink where it is dissipated. On the side of the heat sink there is a connector for the temperature controller. The photodiode is connected to the input of the electronic unit through a connector in the adapter ring. The optical unit, which involves the adapter ring, can be detached from the electronic unit. The connector gives a direct access to both the photodiode leads and the input of the current-to-voltage converting electronics ${ }^{11}$. As a result of this design consideration, the gain linearity calibration of the signal measuring electronics can be done directly.

\subsection{Irradiance measurement}

In irradiance measurements, according to equation 1, the flux (radiant power) is measured within the aperture area of a detector. The incident flux has to overfill the aperture area. The measured irradiance (the flux collected by the aperture) depends on the angle of incidence. At a scale realization the area of the precision aperture has to be known. Test or secondary standard irradiance meters do not need apertures of known area. They are usually calibrated against 


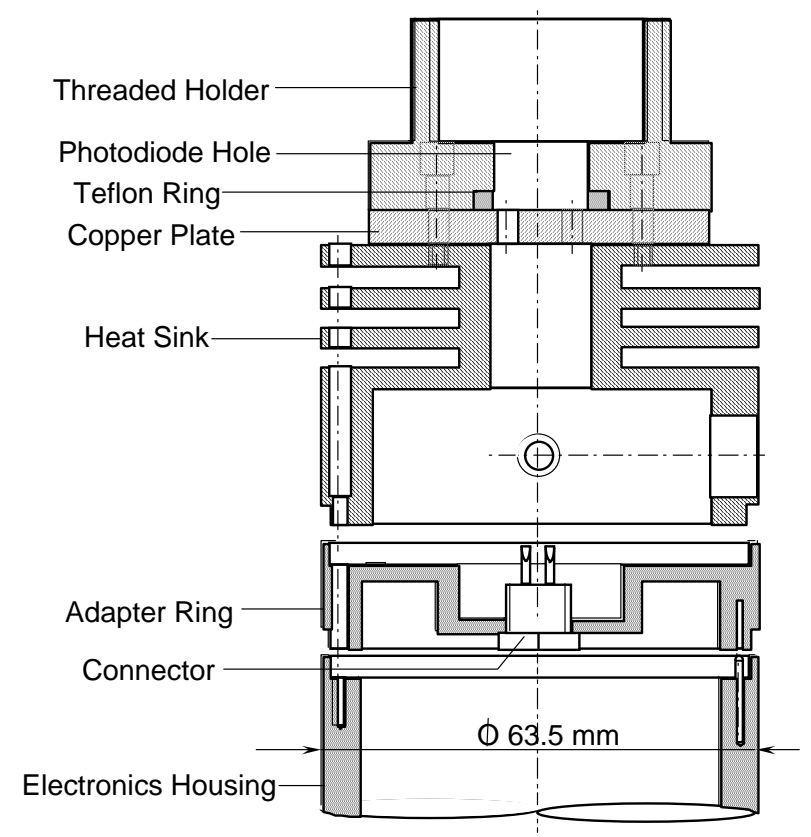

Fig. 3. Ge or InGaAs photodiode housing for radiant power measurements

standard irradiance meters, by substitution. If the field of irradiation is uniform, the spatial response uniformity of the detector does not have to be very good. In this case, the output electrical signal of the meter is determined by the average responsivity of the detector within the aperture area. The relation between the output electrical signal and the input irradiance is linear if the short circuit current of the photodiode is measured. The meters are calibrated for irradiance responsivity as a function of wavelength.

The most important requirement for an irradiance meter is to design its front geometry to satisfy the cosine law of irradiance measurements for the overall angular range of the incident flux. Figure 4.a shows a bare detector with a very thin aperture on top of it. This is the simplest and most ideal design for eliminating reflections from the aperture edge and between the front surface of the detector and the back surface of the aperture for all possible input beam geometries (from parallel to a given $\mathrm{f} / \#$ ). However, in most applications the aperture cannot be positioned on top of the bare detector. In our present work, both the Ge and the InGaAs photodiodes are located in hermetically sealed cases with windows on top. Similarly, in many applications, filters are needed between the aperture and the detector to modify the overall spectral responsivity. Figure 4.b shows the ideal situation where the incident beam is perpendicular to the aperture and the detector, and therefore there are no inter-reflections between the two in spite of a gap between them. In detector calibrations the monochromatic beam is usually not parallel. In Fig. 4.c the incident beam has a given $\mathrm{f} / \#$. In Fig. 4.d the incident beam is not normal to the aperture plane of the irradiance meter. In the last two cases there are inter-reflections between the apertures and the detectors. If filters are used and/or the detector case has a front window, additional reflections will happen ${ }^{22}$. In these situations the internal reflectance pattern depends on the beam geometry and the cosine law of irradiance measurements will work only with a decreased accuracy. However, if the irradiance meter performs accurate cosine measurement within its FOV, the absolute irradiance response can be determined with high accuracy for the different input beam geometries. 


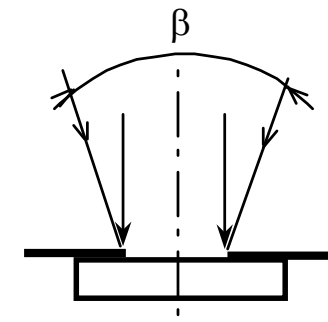

(a)

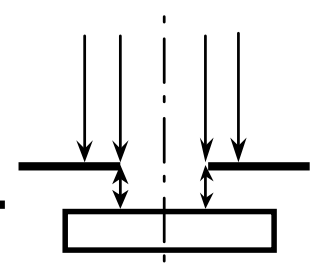

(b)

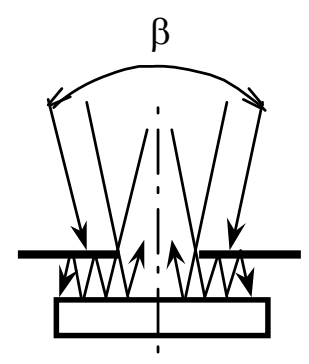

(c)

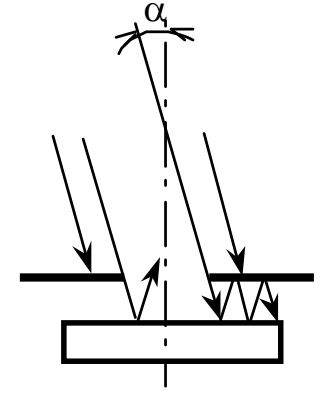

(d)

Fig. 4. Traditional input optics of irradiance meters with different incident beams

(a) Bare detector with a thin aperture on the top

(b) The incident beam is perpendicular to the plane of the aperture

(c) The incident beam has a given $\mathrm{f} / \#$

(d) The incident beam is not perpendicular to the plane of the aperture

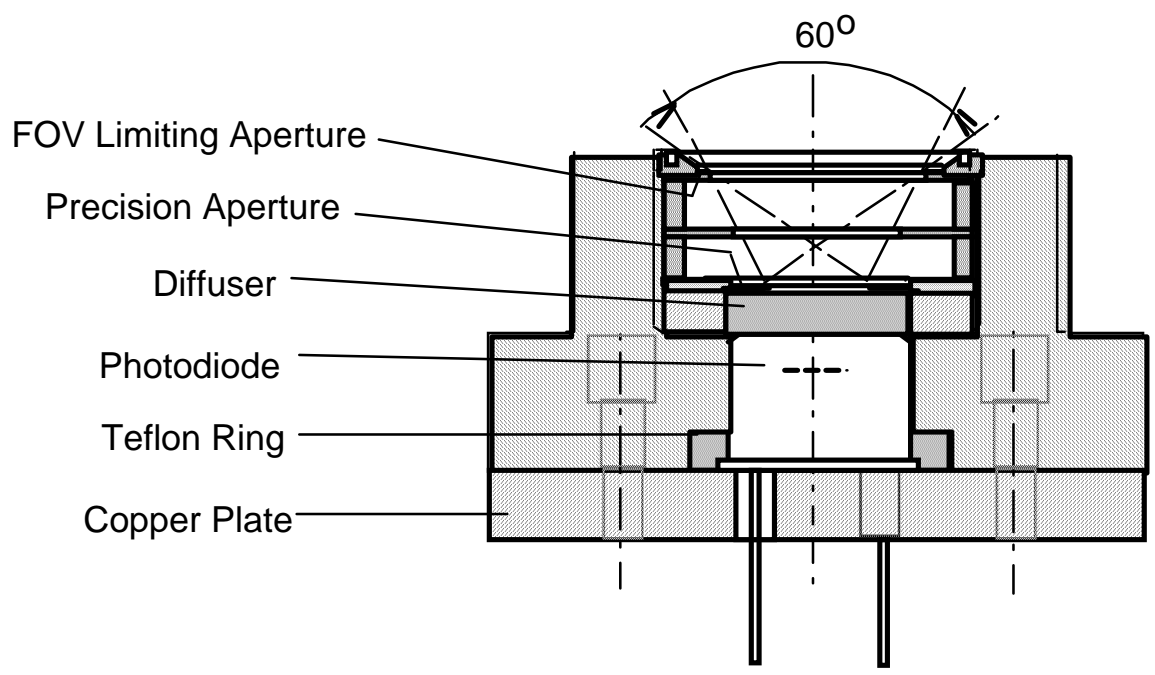

Fig. 5. Cross-section showing the input optics of a diffuser-type irradiance meter

\subsection{Diffuser-type irradiance meter}

Figure 5 shows the modified front piece (threaded holder) of the photodiode-housing for irradiance measurements. A flashed opal glass diffuser is located between the very thin aperture (black nickel coated copper) and the photodiode to eliminate internal reflections and to produce a spatially uniform surface within the aperture area. The goal is to maintain the high accuracy of the cosine function of irradiance measurements. Without the right input geometry, the shape of the cosine function could be altered greatly, resulting in low measurement accuracy. 


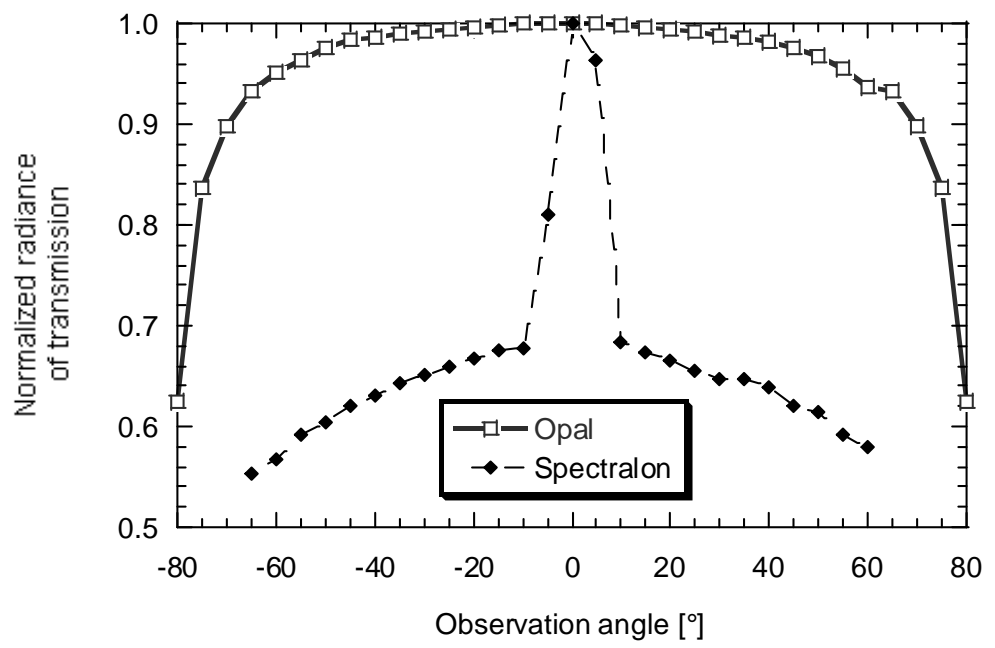

Fig. 6. BTDF of flashed opal glass and Spectralon diffusers at normal beam incidence

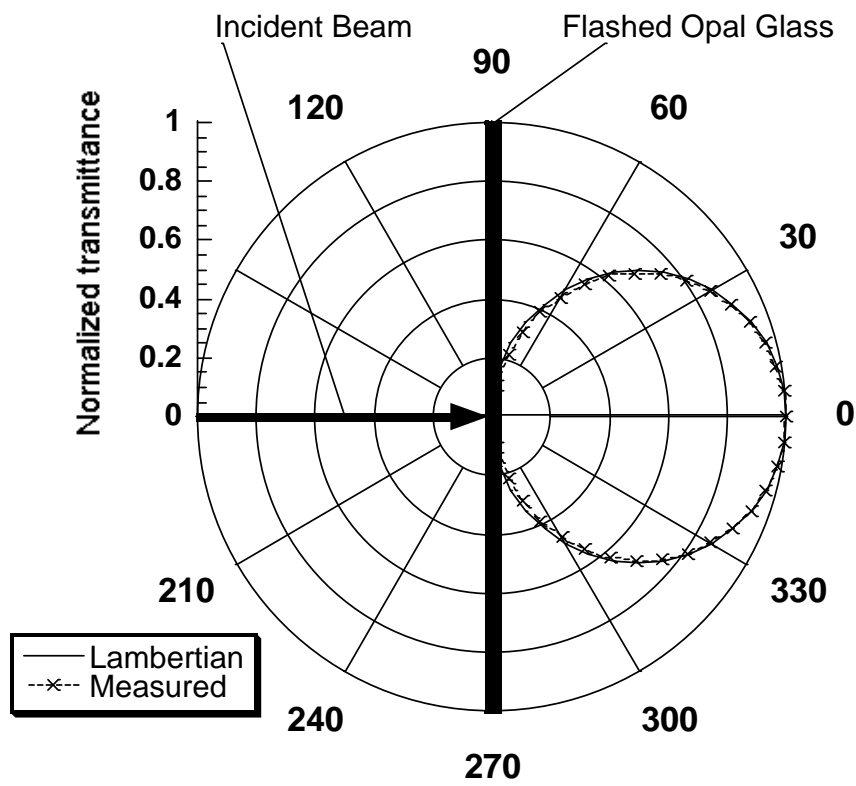

Fig. 7. Ideal Lambertian (continuous) and flashed opal glass (dashed) angular transmittance distributions

The goniometric (Bidirectional) Transmittance Distribution Function (BTDF) of two different diffusers were tested at $600 \mathrm{~nm}$, with normal beam incidence on the NIST High Accuracy Reference Reflectometer-Spectrophotometer ${ }^{23}$. The normalized radiance of transmission values for two materials as a function of observation angle are shown in Fig. 6 . The $3.2 \mathrm{~mm}$ thick Spectralon diffuser showed a large forward scatter between $+/-10^{\circ}$. The radiance of the transmittance of the $2.5 \mathrm{~mm}$ thick flashed opal glass was constant in a reasonably wide 
angular range. Figure 7 shows that the ideal Lambertian curve (solid circle) and the measured opal glass transmittance (dashed circle) curve are close to each other. Consequently, the higher quality flashed opal glass diffuser was selected for our irradiance meter developments.

As it is shown in Fig. 5, a small (5 mm diameter) photodiode can receive the Lambertian radiation from a large (16 mm diameter) opal glass diffuser which is illuminated through a large (8 $\mathrm{mm}$ diameter) aperture. By this method the photodiode measures the radiation at the center of an extended-source diffuser, where the radiance from the diffuser is constant. The beam geometry may change roughly from $\mathrm{f} / 1$ to parallel. The FOV is determined by the front aperture and the precision aperture. There is a baffle between the two apertures to minimize the measured stray radiation.

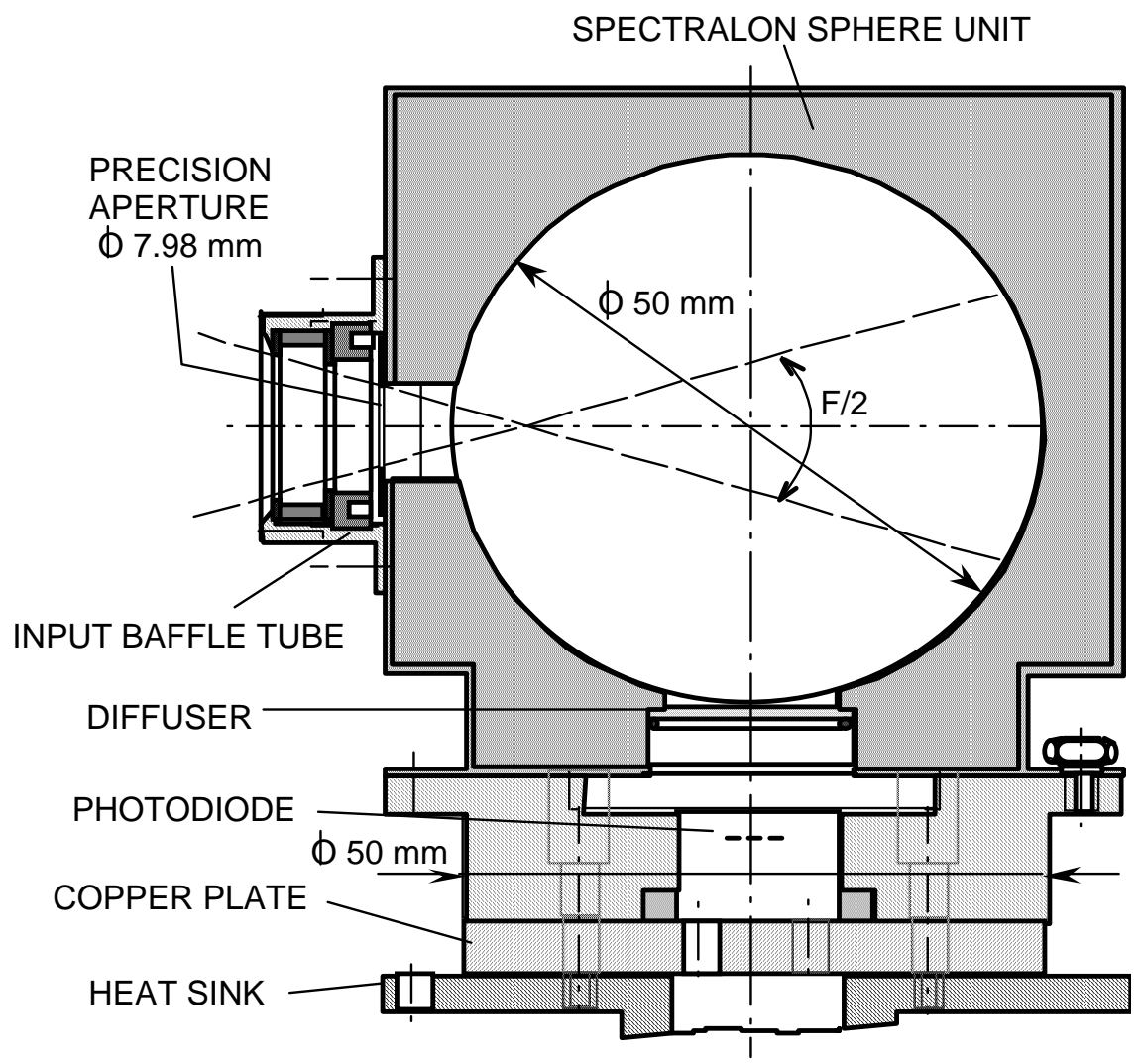

Fig. 8. Cross section of a sphere-input irradiance meter

\subsection{Sphere-type irradiance meter}

Figure 8 shows another radiometer input geometry for accurate irradiance measurements. In this sphere-input irradiance meter the diffusing element, which gives the uniform input surface within the aperture area, is a small integrating sphere. The shape of the incident beam can change between $\mathrm{f} / 2$ and parallel. The FOV is limited by the precision aperture and the front aperture of the input baffle tube. The sphere wall is made of machined Spectralon6 diffusing material, which 
has a reflectance of $99 \%$. The irradiance on any part of the sphere wall is almost the same and proportional to the input flux (radiant power) collected by the aperture. A plane-parallel diffuser, located in the exit port of the sphere, is part of the sphere wall. It is irradiated by the multiple reflections within the sphere. Its Lambertian transmitted radiation is measured by the Ge or InGaAs photodiode. The efficiency of the flux transfer between the diffuser and the photodiode ${ }^{15}$ is determined from the geometry between them. The flux coupling efficiency, $\kappa$, for a $5 \mathrm{~mm}$ diameter photodiode, an exit port diameter of $10.5 \mathrm{~mm}$, and a separation (between the diffuser and the photodiode) of $6.5 \mathrm{~mm}$, is equal to $8.5 \%$. The efficiency, $\tau$, of the $50 \mathrm{~mm}$ diameter sphere with its two ports and a diffuser (with a transmittance of $36 \%$ ) located in the exit port, is equal to $21 \%$. The overall radiant flux efficiency is $\kappa \tau=0.018$, resulting in a signal attenuation of 56. High flux must be sacrificed in order to achieve standard quality spatial response uniformity.

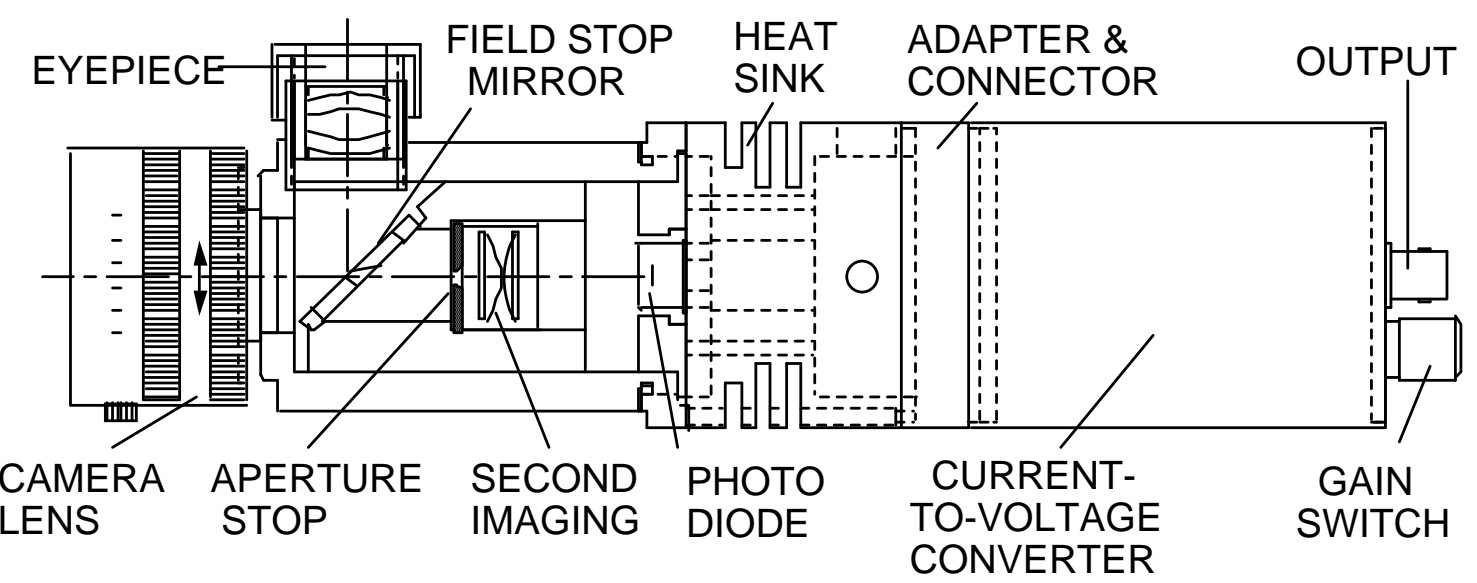

Fig. 9. Optical/mechanical scheme of a radiance optics as attached to the irradiance meter

\subsection{Radiance meter}

Radiance is a source characteristic which can be measured by radiance meters. Usually, extended sources are characterized by radiance measurements. The requirement from a standard quality radiance meter is to have a well-defined measurement angle to measure the source target area only.

Figure 9 shows the simplified optical/mechanical design of our radiance meter where the input optics are attached to the radiometer base-unit with a Bayonet mount. The beveled aperture mirror serves as a field stop, and it is positioned into the focus of the $35 \mathrm{~mm}$ focal length camera lens. Beveling is important to minimize stray radiation. This camera lens has a broad-band antireflecting (AR) coating, resulting in high transmittance in the visible and NIR ranges. The surroundings of the source target area can be viewed through the eye-piece. The target radiation is imaged to the center of the photodiode through the second imaging optics. The second imaging optics has an important role to produce a well defined FOV and a very efficient out-of-FOV blocking. The original aperture stays in front of the photodiode, and it is underfilled. There is a dominant aperture stop in front of the second imaging optics in order to keep the flux response of the optics constant for different target distances of the adjustable focus camera lens. 


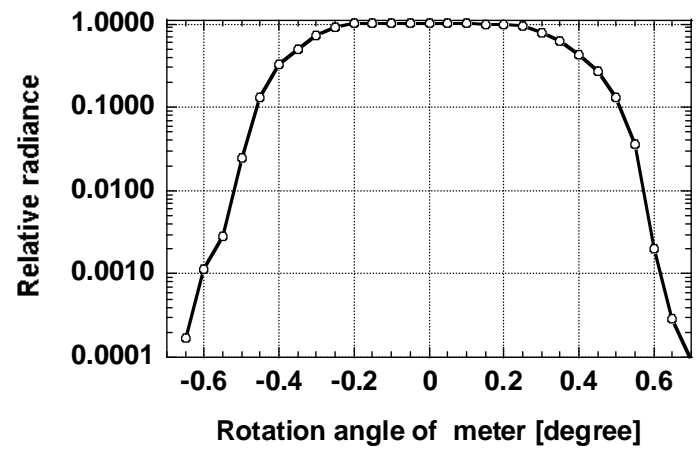

Fig. 10. Radiance measurement profile (point spread function) of a radiance meter

Figure 10 shows the point spread function of our previous generation radiance meter, similar to the one in Fig. 9, with a $50 \mathrm{~mm}$ focal length camera lens and a $0.7 \mathrm{~mm}$ diameter field stop. The plot shows the measured radiance of a small source-spot versus rotation angle of the radiance meter. The roughly $3 \mathrm{~mm}$ diameter source spot was about $2 \mathrm{~m}$ away from the radiance meter. The pivot point of the radiance meter was the center of the field stop. The figure shows that the radiance measurement angle of the meter was about $1^{\circ}$ and the out-of-FOV blocking was larger than four decades. The radiance response of the standard radiometer was measured at target distances between $1 \mathrm{~m}$ and $2 \mathrm{~m}$. Figure 11 shows that the radiance response did not change more than the uncertainty of our measurements, which was $0.1 \%$.

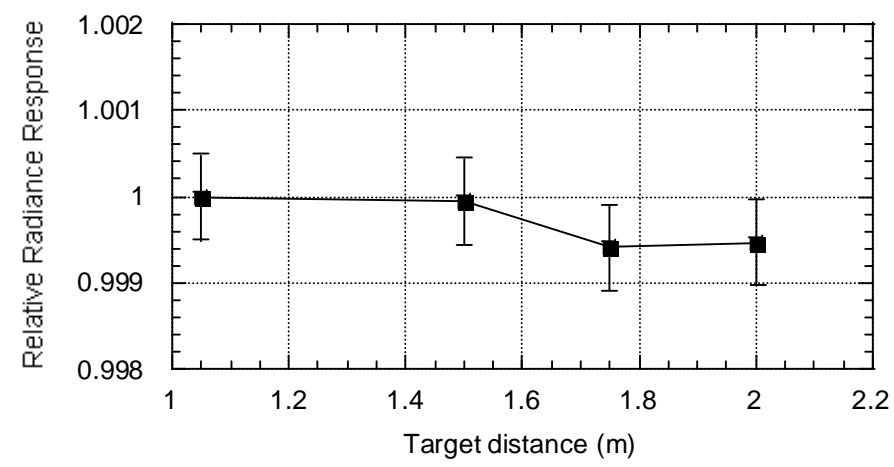

Fig. 11. Change of radiance responsivity of an adjustable focus radiance meter

\section{CONCLUSIONS}

Mechanical, optical, and radiometric design criteria of radiant power, irradiance, and radiance measuring radiometers have been discussed for high accuracy applications. The described standards quality NIR radiometers are being used to realize the NIST detector-based irradiance and radiance scales between $0.8 \mu \mathrm{m}$ and $1.8 \mu \mathrm{m}$. The principle of radiometer calibrations and the design considerations for the calibration set-up have also been discussed. The paper describes the first part of the NIR scale realization work. Design criteria for wide dynamic range DC and AC detector signal measuring electronics, electrical and radiometric NIR detector characterizations with performance comparison of Ge and InGaAs radiometers, and NIR detectorbased scale realizations with error budget determination will be described in consecutive papers. 


\section{ACKNOWLEDGEMENTS}

The author thanks Don Lynch of Reyer Corporation for the radiometer fabrication related work, George Andor of Hungarian Office of Measures for the BTDF measurements, Gyula Dezsi of Hungarian Office of Measures and Robert Saunders for participating in the construction of the radiance/irradiance calibration set-up, and Yoshi Ohno for helping in the radiance/luminance response measurements.

\section{REFERENCES}

1. T.C. Larason, S.S. Bruce, and C.L. Cromer, "The NIST High Accuracy Scale for Absolute Spectral Response from 406 nm to 920 nm,”J. Res. Natl. Inst. Stand. Technol., Vol. 101, No.2, pp. 133-140, 1996.

2. L.P. Boivin, "Diffusers in Silicon-photodiode Radiometers,” Appl. Opt., Vol. 21, No. 5, pp. 918-923, 1982.

3. J.L. Mueller, B.C. Johnson, C.L. Cromer, J.W. Cooper, J.T. McLean, S.B. Hooker, and T.L. Westphal, "The Second SeaWiFS Intercalibration Round-Robin Experiment, SIRREX-2," NASA Technical Memorandum 104566, SeaWiFS Technical Report Series, Vol. 16, pp. 36, June 1993.

4. G. Eppeldauer and Y. Ohno, "Illuminance/Luminance Standard Photometer," CORM’96, Natl. Inst. Stand. Technol., Gaithersburg, MD, May 21-23, 1996.

5. J.H. Walker, R.D. Saunders, and A.T. Hattenburg, "Spectral Radiance Calibrations," NBS Special Publication 250-1, pp. 12, 1987.

6. Identification of commercial equipment to specify adequately an experimental problem does not imply recommendation or endorsement by the National Institute of Standards and Technology nor does it imply that the equipment identified is necessarily the best available for the purpose.

7. K.D. Stock, "Internal Quantum Efficiency of Ge Photodiodes,” Appl. Opt., Vol. 27, No.1, pp.12-14, 1988.

8. K.D. Stock, R. Heine, and H. Hofer, "Influence of Inhomogeneity of NIR-Photodiodes on Calibrations at 1047 nm,” Metrologia, Vol. 28, pp. 207-210, 1991.

9. G. Eppeldauer, M. Tsudagawa, E. Zalewski, and J. Houston, "Electronic and Radiometric Characteristics of Near Infrared Photodiodes," 13th Intern. Symp. Photonic Measurements, IMEKO, Braunschweig, FRG, Proc. pp. 215-224, 1987.

10. E. Zalewski, “Characteristics of Ge and InGaAs Photodiodes,” Inst. of Physics Conf. Series, No. 92, pp. 47-54, New Developments and Applications in Optical Radiometry, International Conf. on Radiometry, NPL, London, UK, 12-13 April, 1988. 
11. G. Eppeldauer and J. Hardis, "Fourteen-decade Photocurrent Measurements with Large-area Silicon Photodiodes at Room Temperature,” Appl. Opt., Vol. 30, No. 22, pp. 30913099, 1991.

12. G. Eppeldauer, "Temperature-monitored/controlled Silicon Photodiodes for Standardization,” SPIE Proc., Volume 1479, pp. 71-77, 1991.

13. T.R. Gentile, J.M. Houston, J.E. Hardis, C.L. Cromer, and A.C. Parr, "National Institute of Standards and Technology High-Accuracy Cryogenic Radiometer," Appl. Opt. Vol. 35, No. 7, pp. 1056-1068, 1996.

14. T.R. Gentile, J.M. Houston, and C.L. Cromer, "Realization of a Scale of Absolute Spectral Response Using the NIST High Accuracy Cryogenic Radiometer," to be published in Appl. Opt.

15. A.R. Schaefer, R.D. Saunders, and L.R. Hughey, "Intercomparison Between Independent Irradiance Scales Based on Silicon Photodiode Physics, Gold-point Blackbody Radiation, and Synchrotron Radiation,” Opt. Engineering, Vol. 25, No. 7, pp. 892-896, 1986.

16. B.C. Johnson, C.L. Cromer, R.D. Saunders, G. Eppeldauer, J. Fowler, V.I. Sapritsky, and G. Dezsi, "A Method of Realizing Spectral Irradiance Based on an Absolute Cryogenic Radiometer”, Metrologia, Vol. 30, pp. 309-315, 1993.

17. J.B. Fowler and G. Dezsi, "High Accuracy Measurement of Aperture Area Relative to a Standard Known Aperture,” J. Res. Natl. Inst. Stand. Technol., Vol. 100, No. 3, pp. 277-283, 1995.

18. B.K. Tsai, B.C. Johnson, R.D. Saunders, and C.L. Cromer, "Comparison of Filter Radiometer Spectral Responsivity with the NIST Spectral Irradiance and Illuminance Scales," Metrologia, Vol. 32, pp. 473-477, 1995/96.

19. K.D. Mielenz, R.D. Saunders, and J.B. Shumaker, "Spectroradiometric Determination of the Freezing Temperature of Gold,” J. Res. Natl. Inst. Stand. Technol., Vol. 95, No. 1, pp.4967, 1990.

20. W. Budde, "Physical Detectors of Optical Radiation," Optical Radiation Measurements, Vol. 4, pp. 19, Academic Press, Inc., 1983.

21. J.H. Walker, R.D. Saunders, J.K. Jackson, and D.A. McSparron, "Spectral Irradiance Calibrations,” NBS Special Publication 250-20, pp. 7, 1987.

22. C.L. Cromer, G. Eppeldauer, J.E. Hardis, T.C. Larason, and A.C. Parr, "National Institute of Standards and Technology Detector-based Photometric Scale,” Appl. Opt. Vol. 32, No. 16, pp. 2936-2948, 1993.

23. J.E. Proctor and P.Y. Barnes, "NIST High Accuracy Reference ReflectometerSpectrophotometer,” To be published in the J. Res. Natl. Inst. Stand. Technol., 1996. 


\title{
Calibration of a pyroelectric detector at $10.6 \mu \mathrm{m}$ with the National Institute of Standards and Technology high-accuracy cryogenic radiometer
}

\author{
T. R. Gentile, J. M. Houston, G. Eppeldauer, A. L. Migdall, and C. L. Cromer
}

\begin{abstract}
The National Institute of Standards and Technology (NIST) is establishing an infrared detector calibration facility to improve radiometric standards at infrared wavelengths. The absolute response of the cryogenic bolometer that serves as the transfer standard for this facility is being linked to the NIST highaccuracy cryogenic radiometer (HACR) at a few laser wavelengths. At the $10.6-\mu \mathrm{m} \mathrm{CO}_{2}$ laser line, this link is being established through a pyroelectric detector that has been calibrated against the HACR. We describe the apparatus, methods, and uncertainties for the calibration of this pyroelectric detector.
\end{abstract}

\section{Introduction}

To meet the growing need for calibrated detectors at infrared wavelengths, the National Institute of Standards and Technology (NIST) is establishing an infrared detector calibration facility. ${ }^{1}$ The goal of this facility is to provide absolute responsivity calibrations of detectors in the $2-20 \mu \mathrm{m}$ spectral region with a relative expanded uncertainty of $\sim 4 \%$ (2 sigma). To serve as a transfer standard detector, a cryogenic bolometer has been constructed. ${ }^{2,3,4}$ The calibration of this bolometer is being linked to the NIST highaccuracy cryogenic radiometer $(\mathrm{HACR})^{5}$ at a few laser wavelengths. At the 10.6- $\mu \mathrm{m} \mathrm{CO} \mathrm{CO}_{2}$ laser line, the link to the HACR is being established through a calibrated pyroelectric detector. In this paper we describe the apparatus, methods, and uncertainties for a calibration of the pyroelectric detector against the HACR. The transfer of the calibration from the pyroelectric detector to the bolometer will be the subject of a separate paper.

The HACR is a cryogenic electrical substitution radiometer that serves as a primary standard for optical power measurements and has been used to measure radiant power at visible wavelengths with a relative standard uncertainty of $0.021 \%$. The HACR is best suited for measurements at an optical

The authors are with the National Institute of Standards and Technology, Gaithersburg, Maryland 20899.

Received 3 September 1996; revised manuscript received 2 January 1997. power level of between 0.1 and $1 \mathrm{~mW}$, but the response of the bolometer becomes nonlinear at power levels above a few microwatts. Hence an indirect calibration is performed. First a pyroelectric detector is calibrated against the HACR, and then the cryogenic bolometer is calibrated against the pyroelectric detector. This approach is also convenient because the pyroelectric detector is compact, easily portable, relatively simple to use, and operates at room temperature. Its dynamic range is suitable for bridging the range between several microwatts and 1 $\mathrm{mW}$, although a somewhat lower noise floor would be desirable. The main disadvantage of the pyroelectric detector is the spatial nonuniformity in its response. Although a thermopile detector was tested as an alternative transfer detector, we chose the pyroelectric detector because of reduced susceptibility to noise due to thermal drift.

We describe the apparatus and methods of the calibration in Section 2 and the measurement results and uncertainties in Section 3.

\section{Apparatus}

\section{A. High-Accuracy Cryogenic Radiometer}

As the HACR has been described in detail elsewhere, ${ }^{5}$ here we provide only a brief overview of the instrument. A diagram is shown in Fig. 1. The HACR is an electrical substitution radiometer operated just above the boiling point of liquid helium. An electrical substitution radiometer links a measurement of optical power to the watt by comparing the temper- 


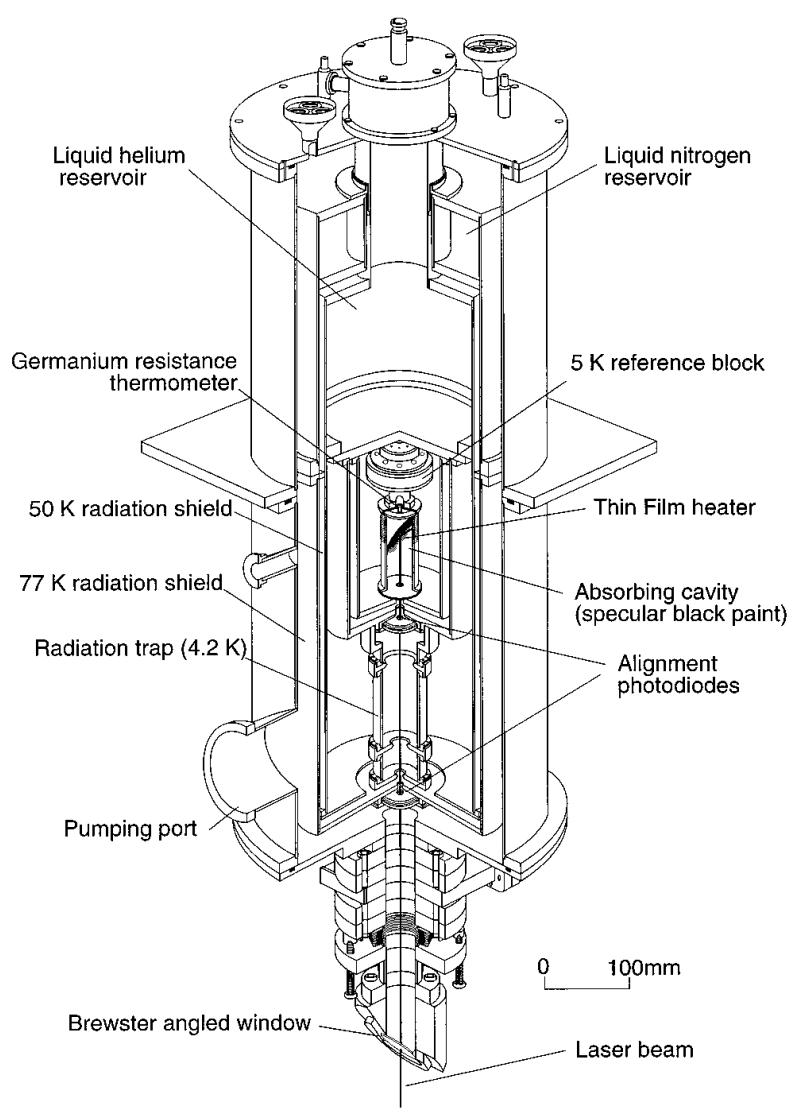

Fig. 1. Diagram of the NIST high-accuracy cryogenic radiometer.

ature rise induced in an absorbing mass by incident optical radiation to that obtained by electrical heating. The absorbing mass is a cavity designed for nearly complete absorption of laser radiation. Operation at cryogenic temperatures allows a large, highly absorptive cavity to be used without significantly increasing the time constant, and the radiative coupling of the cavity to its surroundings is reduced. These features improve the equivalence of optical and electrical heating. However, cryogenic operation also requires a vacuum Dewar, necessitating a window between the cavity and the laboratory. To reduce the reflection loss, this window is oriented at the Brewster angle, and appropriately polarized light is used. To facilitate alignment of the laser light into the cavity and to measure the amount of laser light scattered out of the beam, two sets of four annular quadrant silicon photodiodes, each $50 \mathrm{~mm}$ in diameter with a 9 -mm-diameter central aperture to pass the beam, are located along the optical path within the HACR. (Although these detectors were not directly usable with the infrared beam, they were used with a visible alignment beam that was spatially overlapped with the infrared beam.) The apertures in the quadrant photodiodes are the limiting apertures in the HACR.

\section{B. Laser System}

The laser system is shown in Fig. 2. As with the apparatus previously described for visible wavelengths, ${ }^{5}$ the primary goal is to generate a geometrically well-defined beam with stable power. The power was stabilized using an acousto-optic laser stabilizer $^{6}$ with an external feedback detector. A welldefined beam spatial profile was obtained using a spatial filter with an overfilled pinhole. Because the output power of the $\mathrm{CO}_{2}$ laser is 4 orders of magnitude higher than the level that can be used with the HACR, it was also necessary to attenuate the laser. While this could be accomplished with either attenuating filters or nearly crossed polarizers, both methods have disadvantages. A filter's attenuation could be affected by temperature rise induced by high

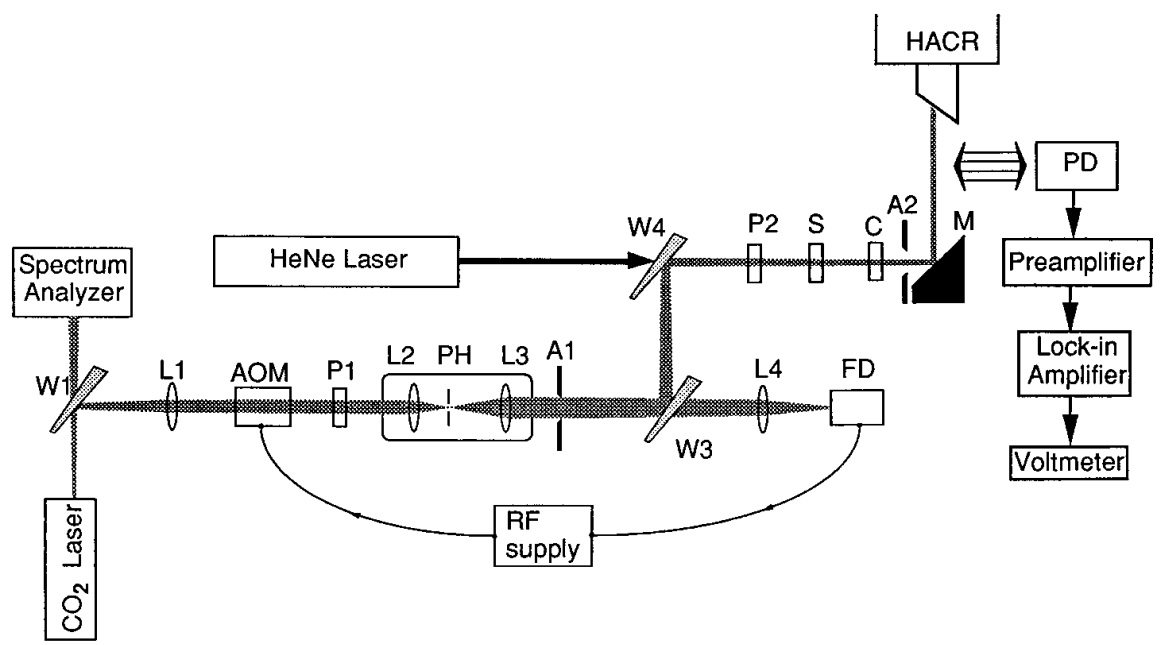

Fig. 2. Diagram of the laser system. W1, W3, W4, ZnSe-wedged windows (there is an additional wedged window W2 located just before L2 that is not shown); L1, L2, L3, L4, ZnSe lenses; AOM, acousto-optic modulator; P1, P2, polarizers; PH, pinhole; A1, A2, apertures; FD, HgCdTe feedback detector; S, shutter; C, chopper; M, steering mirror; PD, pyroelectric detector. The reflection from the steering mirror is out of the plane of the diagram. 
power levels, while crossed polarizers could degrade the polarization purity of the beam. Because of these drawbacks, most of the attenuation was accomplished by reflecting the beam from ZnSe-wedged windows instead of steering mirrors. For each window, only one of the reflected beams was used, while the other reflected beam was blocked. An attenuation factor of $\sim 300$ was obtained from the four wedged windows in the system. This approach also provided monitor beams and a method for overlapping the alignment $\mathrm{He}-\mathrm{Ne}$ beam with the $\mathrm{CO}_{2}$ beam, as described below.

The source was a $5-\mathrm{W}$, air-cooled, grating-tuned, vertically polarized $\mathrm{CO}_{2}$ laser operated with a frequency stabilizer. The laser was operated on the $P(20)$ line [wavelength equals $10.5910 \mu \mathrm{m}$ (Ref. 7)], with a $\mathrm{TEM}_{00}$ transverse mode. After exiting the laser, the diverging beam was reflected by a wedged window (W1), collimated by a ZnSe lens (L1), and passed through an acousto-optic modulator (AOM) that is part of a power stabilizer. (The beam transmitted through W1 was used to monitor the laser's output power or to determine its wavelength using a $\mathrm{CO}_{2}$ spectrum analyzer.) When $\mathrm{rf}$ power is applied to the AOM, a fraction of the laser light is deflected into a secondary beam, thereby providing control of the power in the primary beam. Farther down the beam path, a HgCdTe feedback detector (FD) sampled the beam and provided the input to the stabilizer's feedback circuit. This system maintained the power of the laser beam to within $0.1 \%$ over the course of an hour. The feedback detector was located as far downstream in the beam path as possible within the constraint of this detector's sensitivity.

After the AOM, the beam passed through a polarizer (stack of six ZnSe plates, P1) and was reflected from another wedged window (referred to as W2, but not shown). For most of the measurements this polarizer simply improved the vertical polarization of the beam, but it was also used in conjunction with a wire grid polarizer located farther downstream (P2) for relatively minor attenuation of the beam (less than a factor of 3). The beam was attenuated by rotating $\mathrm{P} 1$, while the polarizer $\mathrm{P} 2$ reestablished the vertical polarization required to minimize the reflectance from the Brewster window. The polarization of the beam incident upon P2 was nearly vertical because vertically polarized light is preferentially reflected from the wedged windows. When additional attenuation was required, a thin $12 \%$ transmitting filter (25-nm-thick layer of $\mathrm{NiCr}$ deposited on 100nm-thick substrate) was placed just before P2. Using a thin filter avoided possible interference effects and deflection of the beam.

A well-defined beam spatial profile was obtained by using a spatial filter with an overfilled pinhole. The laser light was focused with a 8-cm focal length $\mathrm{ZnSe}$ lens (L2) onto a 250- $\mu \mathrm{m}$-diameter pinhole (PH). This beam overfilled the aperture, creating an Airy diffraction pattern. (The amount of overfilling was also used to attenuate the beam by a factor of between 2.5 and 7.) The diameter of an iris (A1) was adjusted to allow only the 13 -mm-diameter central spot of the diffraction pattern to pass through. The diameter of the aperture corresponded to the location of the first minimum of the diffraction pattern, thus defining the beam diameter with minimal scattering from the aperture. A 12.5-cm focal length $\mathrm{ZnSe}$ lens (L3) produced a long beam waist between the pyroelectric detector (PD) and the limiting apertures inside the HACR dewar. At the waist, $85 \%$ of the power fell within a $3-\mathrm{mm}$ beam diameter and $99 \%$ within a $5 \mathrm{~mm}$ diameter. The beam was then reflected from another wedged window (W3), while the transmitted beam was focused by a ZnSe lens (L4) onto the feedback detector. Finally, the beam was reflected from the last wedged window (W4), where it was spatially overlapped with a visible alignment beam. The alignment beam was provided by a He-Ne laser and prepared with a similar optical system (not shown). The total attenuation of the $\mathrm{CO}_{2}$ laser beam along the optical path to the HACR was a factor of 3000 or more, reducing the $3-\mathrm{W}$ output power of the laser down to $1 \mathrm{~mW}$ or less.

The polarizer P2 was adjusted to minimize the reflection from the ZnSe Brewster window on the HACR, and a mirror $(\mathrm{M})$ reflected the laser light into the HACR. (This reflection is out of the plane of Fig. 2.) Whereas the final steering mirror could affect the polarization of the beam if the direction of the polarization were at an arbitrary angle to the plane of incidence, this effect was negligible in this system because the light was polarized parallel to the plane of incidence of the mirror. A computer-controlled shutter (S) was used to block the laser during electrical heating of the cavity and to measure the background signal from the pyroelectric detector. A chopper (C), which is required for use with the pyroelectric detector, was located after the shutter and operated at a chopping frequency of $40.0 \mathrm{~Hz}$. In addition, an 8-mm-diameter aperture (A2) located just before the final steering mirror was used to block residual scattered laser light.

The infrared beam was aligned into the HACR by being spatially overlapped with the He-Ne laser. Although the alignment of a visible beam into the HACR is usually optimized by minimizing the scattered light signal from the quadrant photodiodes, the sensitivity of this method was limited by the high level of scatter from the ZnSe Brewster window at $633 \mathrm{~nm}$. Instead, the diameter of the He-Ne beam was made to be larger than the infrared beam, in fact, nearly as large as the apertures in the annular quadrant photodiodes. This approach provided a sensitive method to align the $\mathrm{He}-\mathrm{Ne}$ beam into the HACR. After this alignment procedure, the He-Ne beam was blocked. The difference between the displacement of the $\mathrm{He}-\mathrm{Ne}$ and $\mathrm{CO}_{2}$ beams due to the $\mathrm{ZnSe}$ Brewster window is only $0.15 \mathrm{~mm}$. The alignment was tested by establishing that the temperature rise of the cavity due to the infrared beam was a maximum and not unduly sensitive to small changes in the alignment of the final steering mirror. As described below, small changes were also made in the alignment to test for 
change in the measured responsivities of the pyroelectric detectors.

\section{Pyroelectric Detectors}

Lithium tantalate detectors, coated with black paint to absorb infrared light, were calibrated against the HACR. A pyroelectric material has a permanent electric dipole moment. A change in the temperature of the material changes the magnitude of this moment, requiring a flow of charge. If a chopped source of optical power is absorbed by a pyroelectric material, the resulting time variation in its temperature will produce a measurable ac current. This current can be detected by use of a lock-in amplifier, with the chopping frequency used as the reference. ${ }^{8}$

Two $1 \mathrm{~cm} \times 1 \mathrm{~cm}$ pyroelectric detectors, ${ }^{9}$ here referred to as PD1 and PD2, were calibrated against the HACR. These particular pyroelectric detectors were chosen because of their large area. Unfortunately, as discussed below, the black paint coating supplied with the detector proved to have lower than desired absorption in the infrared. For the most part, we will describe and show data only for PD2, which is being used to calibrate the bolometer. The results for PD1 are comparable, but because of accidental damage that occurred during the course of this work, its spatial uniformity was degraded. We also calibrated a third pyroelectric detector that is part of a commercial electrical substitution pyroelectric radiometer (ECPR). This detector was calibrated with and without the electrically calibrating circuitry operational.

PD1 and PD2 were each mounted in a small aluminum box with a 13-mm-diameter entrance aperture. The boxes were mounted on a computer-controlled, motor-driven carousel, which positioned each detector in the infrared beam. Atmospheric absorption between the pyroelectric detector and the HACR is $<0.01 \%$ (Ref. 10) and was neglected. To minimize the uncertainty due to the spatial response nonuniformity of the detector, we used an algorithm for reproducibly positioning the detector in the infrared beam: For each of the two axes transverse to the beam, the detector was positioned midway between the two locations at which the detector response was $50 \%$ of its maximum value.

The spatial nonuniformity observed in the response of PD2 is shown in Fig. 3. We obtained these data by scanning the detector over a $6 \mathrm{~mm} \times 6 \mathrm{~mm}$ region in $0.5-\mathrm{mm}$ steps, using the same size beam as was used for the calibrations. For the region within $0.5 \mathrm{~mm}(2 \mathrm{~mm})$ of the center, the maximum variation in the detector response is $\pm 0.8 \%( \pm 2.6 \%)$ and is primarily along one direction. This rather substantial nonuniformity is likely to vary from detector to detector and could be improved with a better absorbing coating. We estimate the reflectance of the pyroelectric detector to be at least $25 \%$ at $10.6 \mu \mathrm{m}$, based on comparing the responsivity at $10.6 \mu \mathrm{m}$ to that measured at $633 \mathrm{~nm}$ with a calibrated visible detector. ${ }^{11}$ (The value of $25 \%$ assumes that the reflectance is close to zero at $633 \mathrm{~nm}$.) Using a half-

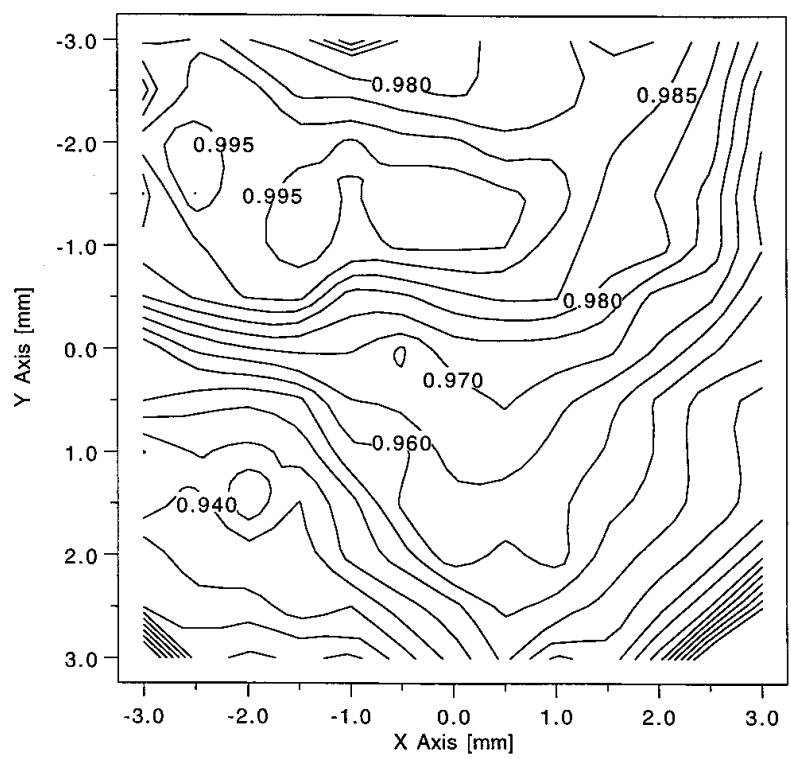

Fig. 3. Contour plot illustrating the spatial uniformity of the responsivity of pyroelectric detector PD2. These data were obtained by scanning of the 3 -mm-diameter beam ( $85 \%$ of power) in 0.5 -mm steps.

wave plate to rotate the polarization of the infrared beam, we found the polarization dependence of the detector's responsivity to be $<0.05 \%$.

The temperature dependence of the responsivity for lithium tantalate is weak. The responsivity is proportional to the ratio of the pyroelectric coefficient to the specific heat. The temperature dependence of this ratio was measured by Glass and Lines ${ }^{12}$; from their data one can estimate the expected temperature dependence of the responsivity to be $0.1 \% \mathrm{~K}^{-1}$ at room temperature. Using a temperature-controlled box, we measured $0.16 \% \mathrm{~K}^{-1}$, roughly consistent with the predicted result.

This work was carried out over a period of a few months, during which time we saw no evidence for any long-term change in the calibration of the pyroelectric detectors. However, the stability of these detectors over a period of years remains to be established.

\section{Measurement Instrumentation}

The output signal from each detector was sent to a transimpedance amplifier with a gain of $10^{8} \mathrm{~V} / \mathrm{A}$, and then to a lock-in amplifier that was sensitive to only the fundamental Fourier component of the waveform at the chopping frequency. The in-phase output of the lock-in amplifier was read by a digital voltmeter. The phase was set manually to minimize the out-ofphase signal. The component of relative standard uncertainty in the responsivity $S$ due to the uncertainty in setting the phase was $0.02 \%$.

The system, consisting of the pyroelectric detector and preamplifier, was calibrated as a unit. The responsivity was defined to be the amplitude of the ac voltage output from the preamplifier, assuming a per- 
fectly square optical waveform, for a given dc optical power that would be incident on the detector if the chopper were removed. The responsivity is given by

$$
S=V C_{d} / G C_{s} P_{L}
$$

where $V$ is the in-phase output of the lock-in amplifier in millivolts, $G$ is a correction factor for the absolute response of the lock-in, $P_{L}$ is the infrared power in $\mathrm{mW}$ as determined by the HACR, $C_{d}$ is the chopper duty cycle, and $C_{s}$ is a small correction factor associated with the deviation of the optical waveform from a perfect square wave (described below). Because the HACR has a 4-min time constant, $P_{L}$ is the average power in the chopped beam.

We determined the chopper duty cycle by measuring the ratio of the infrared power with the chopper turned on to that obtained with the chopper off. Because the pyroelectric detector responds only to chopped radiation, it was necessary to install a second chopper in the beam path. Operating the second chopper at a much higher frequency $(400 \mathrm{~Hz})$ allowed lock-in detection at the second chopper's frequency without interference from the primary chopper. The duty cycle was independently determined with the HACR and also with a $\mathrm{He}-\mathrm{Ne}$ laser and a visible detector. We obtained $C_{d}=0.4964$ with a standard uncertainty of 0.001 .

The measured responsivity of the entire system was found to change by $0.25 \% \mathrm{~Hz}^{-1}$ at the $40.0-\mathrm{Hz}$ chopping frequency. This dependence was almost entirely due to the preamplifier's $3-\mathrm{dB}$ point at 110 $\mathrm{Hz}$. (This preamplifier, originally designed for dc applications, was used simply because it could be immediately dedicated to these measurements and may be replaced in the future.) For a standard uncertainty of $\pm 0.1 \mathrm{~Hz}$ in the chopper frequency, the relative standard uncertainty in the responsivity is only $0.025 \%$.

The magnitude of the fundamental component in the output from the pyroelectric detector depends on the shape of the optical waveform, which is primarily determined by the beam size and the chopper blade geometry. The two-blade chopper has an inner diameter of $5 \mathrm{~cm}$ and an outer diameter of $11 \mathrm{~cm}$, which yields a mean chopper opening width of $6.3 \mathrm{~cm}$. Thus, for a beam diameter of $5 \mathrm{~mm}$ at the chopper, the output of the preamplifier should exhibit a rise time of only $8 \%$ of the half-period, which is due only to the chopper. (The observed rise time was roughly twice this value, owing to the limited frequency response of the preamplifier.) We have corrected the measured responsivities to the ideal conditions of a perfectly square optical waveform, which would only be obtained with an infinitely narrow beam. To determine this correction, labeled $C_{s}$ in Eq. (1), we measured the effect on the responsivity of changing the beam diameter at the chopper from $6 \mathrm{~mm}$ to under 1 $\mathrm{mm}$ and found $C_{s}=0.9980$ with a standard uncertainty of 0.0005 . (Because this correction is independent of the detector used, these measurements were performed more easily at a visible wavelength.)
Because this correction is small, the calibration is quite insensitive to the beam size and spatial profile.

To determine the correction $G$ for the absolute response of the lock-in amplifier, we used a square wave with a known amplitude. We obtained this square wave by illuminating a silicon photodiode with a chopped He-Ne laser beam. The detector's output was sent to a fast transimpedance amplifier. We determined the amplitude of the square wave by turning off the chopper and measuring the dc voltage from this amplifier with a high accuracy digital voltmeter. (The uncertainty in the measurement of dc voltage is negligible.) This measurement was performed with the lock-in amplifier on the $500-\mathrm{mV}$ range. A range-to-range correction factor was determined for the 10-mV range, which was used for most of the pyroelectric detector measurements. The total correction factor for the $10-\mathrm{mV}$ range was $G=$ 0.9023 with a standard uncertainty of 0.002 . Note that a $1-\mathrm{V}$ amplitude square wave, sent into an ideal sine-wave lock-in amplifier, produces an output of $0.9003(=4 / \pi \sqrt{2}) \mathrm{V}$. The value of $G$ for each of the other ranges that were used with the pyroelectric detectors were also measured; these correction factors were typically within $1 \%$ of 0.9003 and were measured with a relative standard uncertainty of $0.1 \%$.

\section{E. Measurement Sequence}

The details of the automated sequence for HACR measurements are described in Ref. 5. Briefly, the sequence consists of one optical measurement cycle, in which the temperature rise of the cavity due to the laser light is determined, followed by electrical heating, in which the quantity of electrical power required to reproduce this temperature is determined. The entire sequence takes $\sim 45 \mathrm{~min}$. The response of the pyroelectric detectors was measured during the electrical heating cycle.

\section{Measurement Results and Uncertainties}

\section{A. Measurement Results}

The results of several responsivity measurements of PD2 are summarized in Table 1 and shown in Fig. 4. Each data point is the mean responsivity for a given session of data acquisition, in which typically 15-20 measurements were obtained. We calibrated the detector over an infrared power range from 0.011 to $0.35 \mathrm{~mW}$, with no other deliberate change in conditions. (These data are the first seven entries in Table 1 and are shown by filled circles in Fig. 4.) As described below, this series of measurements allowed us to establish an uncertainty due to background effects. For the remaining data points, small changes were made in the measurement conditions to test the sensitivity to certain systematic effects that are discussed below. (These data are the last four values in Table 1 and are shown by open squares in Fig. 4.)

In Fig. 4, the uncertainty bar shown on each data point corresponds to the relative standard deviation 
Table 1. Measured Values of the Responsivity $S$ of the Pyroelectric Detector PD2 for a Range of Infrared Power Levels and Other Variations in the Conditions of the Calibration ${ }^{a}$

\begin{tabular}{lccc}
\hline $\begin{array}{c}P_{L} \\
(\mathrm{~mW})\end{array}$ & $\begin{array}{c}S \\
(\mathrm{mV} / \mathrm{mW})\end{array}$ & $\begin{array}{c}\sigma_{m} / S \\
(\%)\end{array}$ & $\begin{array}{c}\left(S-S_{a}\right) / S_{a} \\
(\%)\end{array}$ \\
\hline 0.3397 & 6.880 & 0.03 & -0.17 \\
0.3392 & 6.893 & 0.04 & 0.03 \\
0.3203 & 6.897 & 0.03 & 0.09 \\
0.2599 & 6.894 & 0.02 & 0.04 \\
0.07379 & 6.880 & 0.11 & -0.16 \\
0.03079 & 6.873 & 0.21 & -0.26 \\
0.01375 & 6.815 & 0.41 & -1.11 \\
1.051 & 6.904 & 0.10 & 0.18 \\
0.3395 & 6.888 & 0.02 & -0.04 \\
0.3421 & 6.924 & 0.01 & 0.48 \\
0.3368 & 6.849 & 0.04 & -0.61 \\
\hline
\end{tabular}

${ }^{a}$ The mean responsivities of the relative standard deviation of the mean, $\sigma_{m} / S$, are listed for each session of data acquisition. The percentage deviation of each responsivity from the average responsivity $\left(S_{a}=6.89 \mathrm{mV} / \mathrm{mW}\right)$ is also listed; the average is based on the data obtained at power levels above $0.2 \mathrm{~mW}$.

of the mean in the measurements of $S$ for the given session. (For the results at $1.05 \mathrm{~mW}$ and below 0.2 $\mathrm{mW}$, which were not obtained on the $10-\mathrm{mV}$ lock-in range, an additional contribution of $0.1 \%$ has been added in quadrature to yield the total uncertainty bar.) The increased standard deviation at low values of $P_{L}$ is due to comparable contributions from the noise floors of the HACR ${ }^{5}$ and the pyroelectric detector. The slight decrease in responsivity at the lowest power levels could indicate a systematic effect associated with background subtraction. This decrease was seen for both pyroelectric detectors but not for the ECPR, which suggests a possible systematic error in the calibration of the pyroelectrics. However, fitting the data to a form that models such a background error ${ }^{5}$ yielded a relative standard un-

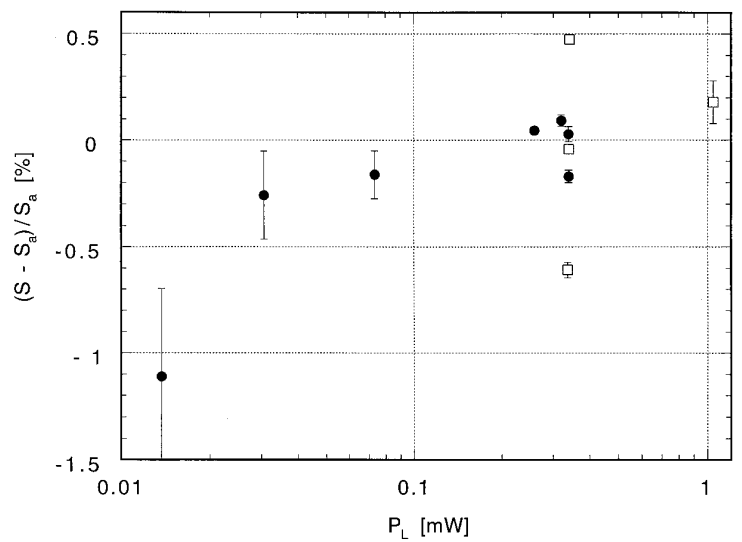

Fig. 4. Percentage deviation of each responsivity $S$ listed in Table 1 from the average responsivity $S_{a}$; the average is based on the data obtained at power levels above $0.2 \mathrm{~mW}$. The data shown by filled circles were obtained with no deliberate change in conditions to test for linearity of the detector and background effects. The data shown by open squares include changes in the conditions of the measurements that are described in the text. certainty component of $0.05 \%$ at our typical calibration power of $0.35 \mathrm{~mW}$.

We now restrict the discussion to the data obtained at power levels of $0.2 \mathrm{~mW}$ or above. For a typical session of data acquisition, the relative standard deviation in repeated measurements for either $V$ or $P_{L}$ was typically $0.15 \%$. The primary source of this variation was drift in the power of the infrared beam. For $S$ the relative standard deviation was only $0.1 \%$ or less because slow drift in the infrared power does not affect the measurement of $S$.

The data shown by open squares were obtained with small changes to the calibration conditions. These changes included removing the detector from the carousel, repeating the alignment algorithm, and small variations in the alignment of the laser beam into the HACR. The goal of these changes was to establish the reproducibility of the calibration, given the known spatial nonuniformity of the detector and the difficulty in aligning the infrared beam into the HACR. (For our work here we are primarily interested in our ability to reproducibly calibrate the detector using our alignment algorithm. The related, but separate, issue of the transfer of this calibration to the cryogenic bolometer will be discussed in another paper.)

We determined the average responsivity $\left(S_{a}=6.89\right.$ $\mathrm{mV} / \mathrm{mW}$ ) by averaging the data obtained at power levels above $0.2 \mathrm{~mW}$. The relative standard deviation of this data set is $0.31 \%$, which is substantially larger than the relative standard deviation of the mean for any given session of data acquisition. Hence the reproducibility of the calibration was affected by both unintentional and imposed changes in calibration conditions. The small relative standard uncertainty of $\pm 0.05 \%$ that is due to the typical variations of $\pm 0.3 \mathrm{~K}$ in the ambient laboratory temperature can account for only a small portion of the observed $0.31 \%$ relative standard deviation. We believe the most likely cause is the known spatial nonuniformity of the detector. From Fig. 3 we see that a change of $\pm 0.2 \mathrm{~mm}$ in the location of the detector or the beam can result in a $\pm 0.3 \%$ change in the measured responsivity. We believe that spatial uniformity could explain most of the observed variation in the measurements of $S$. We assign a type A relative standard uncertainty ${ }^{13}$ of $0.31 \%$ to the reproducibility of the calibration, which includes effects of spatial nonuniformity and beam alignment.

As a final test of the quality of the alignment into the HACR, we shifted the location of the focus farther along the beam path so that it was between the two limiting apertures in the HACR. If the beam had been slightly obstructed by these apertures, shifting the focus would cause the measured responsivity to decrease. We observed a decrease of $1.0 \%$ in the measured responsivity for detector PD2, whereas the responsivity of PD1 was nearly unchanged. This suggests that the effect was not due to alignment into the HACR but some other effect that was different for the two detectors. Changing the location of the focus did require realigning the detectors and also in- 


\begin{tabular}{lccc}
\hline \multicolumn{1}{c}{ Uncertainty Component } & Type & $\begin{array}{c}\text { Value of } \\
\text { Correction }\end{array}$ & $\begin{array}{c}\text { Component of } \\
\text { Uncertainty }(\%)\end{array}$ \\
\hline Reproducibility & $\mathrm{A}$ & & 0.31 \\
Background & $\mathrm{A}$ & & 0.05 \\
$G$ & $\mathrm{~B}$ & 0.9023 & 0.20 \\
$C_{d}$ & $\mathrm{~B}$ & 0.4964 & 0.20 \\
$C_{s}$ & $\mathrm{~A}$ & 0.9980 & 0.05 \\
Chopper frequency and lock-in phase & $\mathrm{B}$ & & 0.03 \\
Combined standard uncertainty in $P_{L}$ & $\mathrm{~B}$ & 0.9978 & 0.21 \\
Window transmittance $(T)$ & & $0.61 \mu \mathrm{W}$ & 0.18 \\
Scattered optical power $\left(P_{S}\right)$ & & 0.1 \\
\hline
\end{tabular}

${ }^{a}$ The uncertainties are identified as type A or type B relative standard uncertainties. The measured values of corrections to the data are also listed.

creased the beam size at the detectors a small amount. These changes, in conjunction with the spatial nonuniformity of the detectors, could explain the observed $1.0 \%$ shift in the responsivity for PD2. Hence we have not increased the uncertainty in the reproducibility in the calibration because of this measurement.

\section{B. Corrections for Window Transmittance and Scattered} Laser Light

The uncertainty components in these calibrations are listed in Table 2. The method used to evaluate each uncertainty component (type A or type B) is also listed. We now discuss the combined uncertainty component in the measurement of $P_{L}$ by the HACR. The infrared power $P_{L}$ is given by

$$
P_{L}=(1 / T)\left[\left(N P_{H} / A\right)+P_{S}\right] .
$$

where $P_{H}$ is the electrical heater power that yields the same temperature rise of the cavity as that produced by the infrared beam, $P_{S}$ is the estimated infrared power scattered out of the field of view of the cavity, $A$ is the absorptance of the cavity, $T$ is the transmittance of the entrance window of the HACR, and $N$ is a factor to take into account any nonequivalence between the infrared and electrical heating. For this work, the type B uncertainty in the measurement of $P_{L}$ by the HACR is dominated by the uncertainties in $T$ and $P_{S}$, which are discussed in detail below. Other HACR uncertainties discussed in Ref. 5 are less than $0.01 \%$, which is negligible for this work.

The transmittance of the window was measured on the optical table before and after the series of measurements with the HACR. Although this is a simple measurement in principle, care was required because of the nonuniformity of the available detectors. The transmittance was determined by measuring the signal on a pyroelectric detector with and without the window in the beam. Because the window translates the beam, the detector must also be translated so that the beam is still incident upon the same location on the detector. Any error in this re- location leads to an error in the measured transmittance. We measured a transmittance of $0.9993 \pm$ 0.0004 before the series of HACR measurements and $0.9962 \pm 0.0008$ afterward, where the uncertainties quoted are the standard deviations of the mean for each group of measurements (16 measurements for the before data and 7 for the after data). These transmittance measurements were separated by 4 months, and we believe that the decrease in transmittance was due to accumulation of contaminants on the window. We used the average value of $T=$ $0.9978 \pm 0.0018$ to determine $P_{L}$, where the standard uncertainty quoted is equal to the sum of half the difference between the before and after values, plus the uncertainties quoted for the before and after data, added in quadrature.

Before measuring the transmittance, the window reflectance was minimized by adjusting the polarizer P2 and the angle of incidence of the beam on the window. We minimized the reflection, rather than maximizing the transmittance, so that the procedure was the same as that performed when the window was installed in the HACR. (Because of the slow time constant of the HACR, it is difficult to maximize the transmittance by observing the temperature of the cavity.) Furthermore, we measured the reflectance to establish that the same value was obtained when the window was installed in the HACR. Using one of the pyroelectric detectors, we measured the reflectivity of the window to be $0.1 \% \pm 0.03 \%$.

Scattered laser light refers to light that is incident upon the detector to be calibrated but scattered out of the field of view of the absorbing cavity in the HACR. For visible wavelengths there is little scattered laser light, and it is measured by annular silicon photodiodes located along the optical path within the HACR. Because scattered infrared laser light cannot be measured by the photodiodes in the HACR, we estimated the correction for scattered laser light by simulating the usual trajectory of the beam into the HACR on the optical table: Just before the final steering mirror, the beam was deflected with a mirror. A window was placed in the beam where the HACR window would normally be located. To col- 
lect only the scattered light, we used a $15-\mathrm{cm}$ focallength concave mirror with a 9 -mm central aperture. This mirror was located near the position of the quadrant photodiodes in the HACR dewar, which also have 9-mm-diameter apertures. Detector PD1 was used to detect the reflected light from this mirror. We measured $P_{S}$ to be $0.18 \%$ of the incident infrared power. Most of this scattered light was not due to the window. It is difficult to establish how close this measurement is to the actual value that $P_{S}$ was during our calibrations. On the basis of the repeatability of the measurement and the sensitivity to changes in the beam alignment, we estimate an relative standard uncertainty component of $0.1 \%$ due to scattered light, about half the value of the correction itself.

\section{Conclusion}

We have calibrated a pyroelectric detector against the HACR at a wavelength of $10.6 \mu \mathrm{m}$ with a combined relative standard uncertainty of $0.48 \%$. This detector is being used to calibrate the transfer standard cryogenic bolometer for an infrared comparator facility. The calibration of the bolometer, which is being performed with a different laser system, will be discussed in another paper. The primary issue in the accuracy of the bolometer calibration is the spatial uniformity of the pyroelectric detector. Minimizing this uncertainty requires reproducing the alignment procedure and beam characteristics that were used for the HACR calibration. We believe that the transfer of this calibration can easily be done with a conservative relative standard uncertainty of $\pm 2 \%$, and with careful attention to detail, a relative standard uncertainty of $\pm 0.6 \%$ should be achievable.

We are investigating detectors with improved spatial uniformity, which can be obtained by using goldblack coatings or a light-trapping arrangement. ${ }^{14,15}$ Colleagues in our laboratory have produced gold-black coatings with less than $1 \%$ reflectance at the $\mathrm{CO}_{2}$ laser wavelength. An alternative solution is to calibrate the cryogenic bolometer directly against the HACR. This approach would require improving the sensitivity of the HACR, attenuating the beam for the bolometer by a precisely known amount, further quantifying the bolometer's nonlinearity, or some combination of these changes. Finally, work is in progress at NIST to develop a large-area silicon bolometer that could have both high sensitivity and a sufficiently large dynamic range to be used easily with the HACR.

\section{References and Notes}

1. A. Migdall, G. Eppeldauer, and C. Cromer, "IR detector spectral responsivity calibration facility at NIST," in Cryogenic
Optical Systems and Instruments VI, J. B. Heaney and L. G. Burriesci, eds., Proc. SPIE 2227, 46-53 (1994).

2. G. Eppeldauer, A. L. Migdall, and C. L. Cromer, "Characterization of a high sensitivity composite silicon bolometer," Metrologia 30, 317-320 (1993).

3. G. Eppeldauer, A. L. Migdall, and C. L. Cromer, "A cryogenic silicon resistance bolometer for use as an infrared transfer standard detector," in Thermal Phenomena at Molecular and in Cryogenic Infrared Detectors, M. Kaviany, D. A. Kaminskii, A. Majumdar, P. E. Phelan, M. M. Yovanovich, Z. M. Zhang, eds. (American Society of Mechanical Engineers, New York, 1994), Vol. 277, pp. 63-67.

4. G. Eppeldauer, A. L. Migdall, T. R. Gentile, and C. L. Cromer, "Absolute response calibration of a transfer standard cryogenic bolometer," in Photodetectors and Power Meters II, K. Murray and K. J. Kaufmann, eds., Proc. SPIE 2550, 36-46 (1995).

5. T. R. Gentile, J. M. Houston, J. E. Hardis, C. L. Cromer, and A. C. Parr, "National Institute of Standards and Technology high accuracy cryogenic radiometer," Appl. Opt. 35, 10561068 (1996).

6. Model LS-10.6, Cambridge Research and Instrumentation, Inc., 21 Erie Street, Cambridge, Mass. 02139. Certain trade names and company products are mentioned in the text or identified in an illustration in order to adequately specify the experimental procedure and equipment used. In no case does such identification imply recommendation or endorsement by the National Institute of Standards and Technology, nor does it imply that the products are necessarily the best available for the purpose.

7. R. J. Pressley, ed., Handbook of Lasers With Selected Data on Optical Technology (CRC, Cleveland, Ohio, 1971), p. 331.

8. R. W. Boyd, Radiometry and the Detection of Optical Radiation (Wiley, New York, 1983), pp. 219-223.

9. Servo Corporation of America, 111 New South Road, Hicksville, New York 11802-1490.

10. W. L. Wolfe and G. J. Zeissis, eds., Infrared Handbook, 3rd ed. (Infrared Information Analysis Center, Environmental Research Institute of Michigan, Ann Arbor, Mich., 1989), pp. 5-69.

11. T. R. Gentile, J. M. Houston, and C. L. Cromer, "Realization of a scale of absolute spectral response using the NIST high accuracy cryogenic radiometer," Appl. Opt. 35, 4392-4403 (1996).

12. A. M. Glass and M. E. Lines, "Low-temperature behavior of spontaneous polarization in $\mathrm{LiNbO}_{3}$ and $\mathrm{LiTaO}_{3}$," Phys. Rev. B 13, 180-191 (1976).

13. B. N. Taylor and C. E. Kuyatt, "Guidelines for evaluating and expressing the uncertainty of NIST measurement results," Tech. Note 1297, 2nd ed. (National Institute of Standards and Technology, Gaithersburg, Md., 1994).

14. D. J. Advena, V. T. Bly, and J. T. Cox, "Deposition and characterization of far-infrared absorbing gold black films," Appl. Opt. 32, 1136-1144 (1993).

15. R. J. Phelan, Jr., J. Lehman, and D. R. Larson, "Electrically calibrated pyroelectric detector-refinements for improved optical power measurements," in Photodetectors and Power Meters, K. J. Kaufmann, ed., Proc. SPIE 2022, 160-163 (1993). 


\title{
REALIZATION OF AN INFRARED SPECTRAL RADIANT POWER RESPONSE SCALE ON A CRYOGENIC BOLOMETER
}

\author{
Alan Migdall and George Eppeldauer \\ National Institute of Standards and Technology \\ Optical Technology Division
}

\begin{abstract}
An ambient temperature Infrared Spectral Responsivity Comparator Facility has been developed at NIST to calibrate infrared detectors and radiometers for spectral and spatial responsivities. Test detectors are substituted for working- or transfer-standard radiometers in the output beam of a monochromator. InSb working standard radiometers have been developed and characterized to hold the spectral responsivity scale between $2 \mu \mathrm{m}$ and $5.4 \mu \mathrm{m}$. The InSb working standard radiometers were calibrated against the transfer standard cryogenic bolometer that is tied to the primary standard cryogenic radiometer of NIST. The cryogenic bolometer holds the infrared power responsivity scale between $2 \mu \mathrm{m}$ and $20 \mu \mathrm{m}$. The combined standard uncertainty $(k=1)$ of the spectral power responsivity scale, as realized on the cryogenic bolometer, is $+/-0.25 \%$.
\end{abstract}

Key Words: bolometer; calibration; infrared detectors; radiometry; scale; spectral irradiance responsivity; spectral power responsivity.

\section{Introduction}

The National Institute of Standards and Technology (NIST) has developed an IR radiant power responsivity scale for the spectral range from $2 \mu \mathrm{m}$ to $20 \mu \mathrm{m}$. This scale is required for a facility that provides a detector-based calibration of IR detectors ${ }^{(1,2)}$. The scale operates at much higher sensitivities (input power levels are $10 \mu \mathrm{W}$ to $20 \mathrm{pW}$ ) than existing IR scales (between $500 \mu \mathrm{W}$ and about $0.1 \mu \mathrm{W})^{(3)}$, while using a transfer standard detector with a flat spectral responsivity over the entire spectral range. The scale has been realized on a cryogenic bolometer, which accounts for the improved sensitivity relative to a pyroelectric detector based scale. The responsivity of this bolometer has been determined by multiple ties ${ }^{(4)}$ to a primary standard, the NIST High Accuracy Cryogenic Radiometer (HACR) ${ }^{(5)}$. In addition, an independent method was used to determine the relative response of the bolometer over the entire spectral range. The uncertainty over the entire spectral range of $2 \mu \mathrm{m}$ to $20 \mu \mathrm{m}$ is typically better than $0.25 \%$, with the typical uncertainty of an individual measurement made with the bolometer being about $0.8 \%$.

Working standard InSb radiometers ${ }^{(6)}$ have been developed for the $2 \mu \mathrm{m}$ to $5.4 \mu \mathrm{m}$ wavelength range to hold the spectral responsivity scale. They are easy to use relative to the sophisticated bolometer that requires liquid He sensor-temperature and continuous computer control/monitor for responsivity change corrections ${ }^{(7)}$. Infrared test detectors with good spatial response uniformity can be calibrated against the InSb working standard radiometers in radiant power measurement mode. Spatially nonuniform infrared detectors can be used for accurate radiometric measurements when they are calibrated against the working standard InSb radiometers in irradiance measurement mode. 


\section{Spectral Responsivity of the Transfer Standard Bolometer}

To determine the radiant power responsivity of the bolometer, its relative response was determined over its entire spectral range from measurements of bolometer components. This relative response shape was then tied down at many wavelengths via $\mathrm{Si}, \mathrm{Ge}$, and pyroelectric transfer detectors to the HACR. The bolometer consists of a sapphire disk with a heat sensor on one side and a gold black absorptive coating on the other side. Incident radiation is detected as a temperature rise. Because the bolometer operates by sensing energy deposited within its absorber, its relative response is just the product of the absorptance of the bolometer's coating and the transmittance of the window of the cryogenic dewar. For more details on the device construction and characterization, see References 7, 8, and 9.

The spectral absorptance of the bolometer gold black coating was measured using a Fourier Transform Infrared (FTIR) spectrometer system with hemispherical collection optics. This allowed the total reflectance (diffuse and specular) to be measured. The results are shown in Fig. 1.

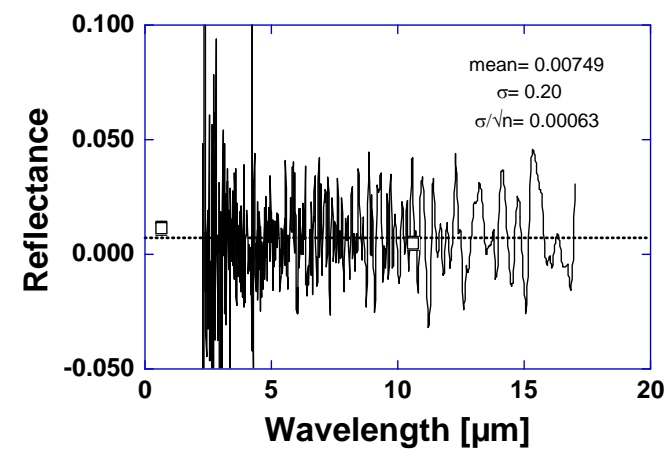

Figure 1. Total bolometer reflectance

Two additional measurements were made using laser sources. At $0.633 \mu \mathrm{m}$ and $10.6 \mu \mathrm{m}$, the reflectances were determined to be $(1.2 \pm 0.3) \%$ and $(0.5 \pm 0.3) \%$ respectively. [(The uncertainties reported in this paper are all standard uncertainties as defined in Ref. 10 (coverage factor $k=1$ ).] A linear fit to the FTIR data from $2 \mu \mathrm{m}$ to $17 \mu \mathrm{m}$ indicated a minimal slope with a value not much larger than its uncertainty. Because of this, the FTIR reflectance data were simply averaged yielding a wavelength independent reflectance value, $\mathrm{R}=0.75 \%$. This is consistent with the values and uncertainties of the two laser reflectance measurements. Because the IR termination of the reflectance data beyond $17 \mu \mathrm{m}$ was due to low FTIR sensitivity rather than a variation of gold black, it is expected that an extrapolation of this data will be valid to at least $20 \mu \mathrm{m}$.

The transmittance of the bolometer window was measured from $600 \mathrm{~nm}$ to beyond $18 \mu \mathrm{m}$. The Infrared Spectral Comparator Facility (IR SCF) was used to measure the transmittance of single $\mathrm{KBr}$ window samples from $2 \mu \mathrm{m}$ to $20 \mu \mathrm{m}$ (see Fig. 2a.). The mean size of the error bars was about $0.1 \%$ for the region from $2 \mu \mathrm{m}$ to $16 \mu \mathrm{m}$. The IR SCF was used to measure the transmittance difference of two window samples as shown in Figure 2b. The mean difference 
between the two measurements was $0.016 \%$ with a standard deviation of $0.2 \%$. These small differences allow for window replacements, while using this standard deviation as the uncertainty. These small differences also indicate that the spatial uniformity of the windows, which was not specifically tested, should be significantly below these levels.
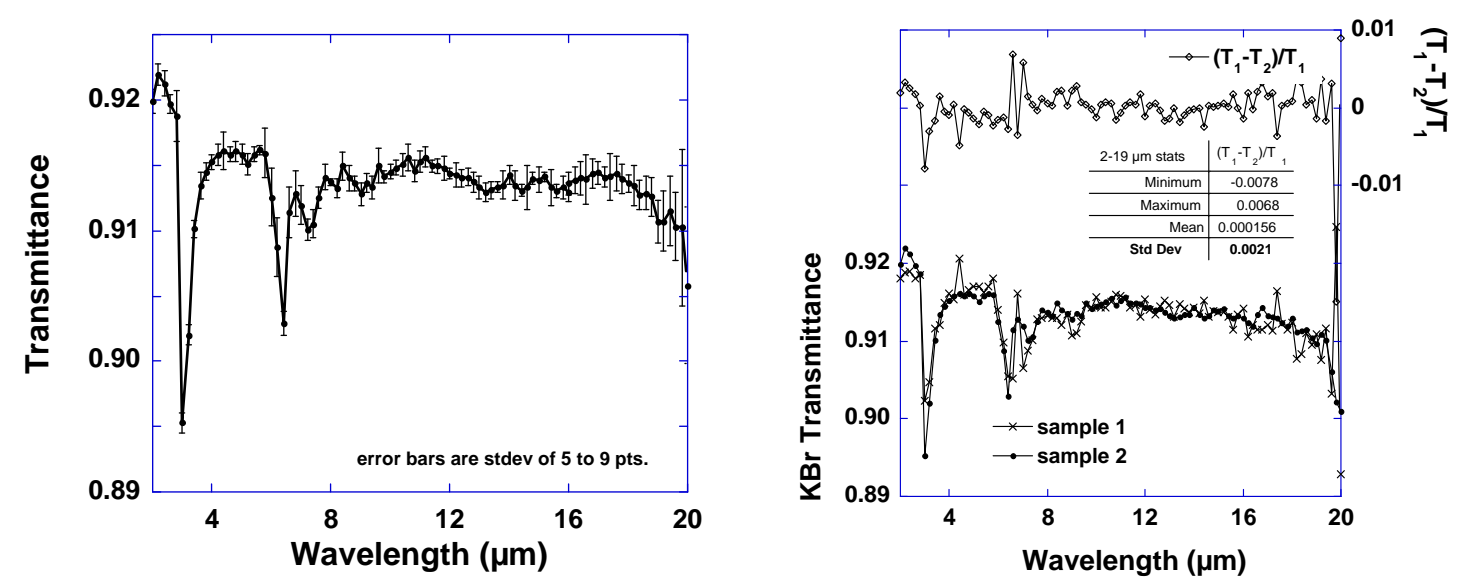

Figure 2. (a) Spectral transmittance of one and (b) two KBr windows and their relative differences.

The relative spectral response of the bolometer as shown in Figure 3 is the product of the window transmittance and the bolometer gold black absorptance. Except for a pair of $1 \%$ to $2 \%$ dips (most likely due to window coating absorption lines) the bolometer response is quite flat. The uncertainty for the $2 \mu \mathrm{m}$ to $20 \mu \mathrm{m}$ region is $0.36 \%$, the quadrature sum of the $0.2 \% \mathrm{KBr}$ transmittance uncertainty and the $0.3 \%$ gold black absorptance uncertainty. In the $0.6 \mu \mathrm{m}$ to $2 \mu \mathrm{m}$ region, the transmittance uncertainty is so small that the gold black absorptance uncertainty dominates the total uncertainty.

To convert the relative spectral curve just discussed into an absolute scale, it was necessary to determine the absolute responsivity at one, or preferably, more wavelengths. This was done by comparison to $\mathrm{Si}, \mathrm{Ge}$, and pyroelectric standard detectors, which were themselves tied to the HACR primary standard. The comparisons were made at specific wavelengths using laser sources at $0.633 \mu \mathrm{m}, 1.3 \mu \mathrm{m}$, and $10.6 \mu \mathrm{m}$ and over a continuous range from $600 \mathrm{~nm}$ to $900 \mathrm{~nm}$ using the quartz lamp and the IR SCF itself as the source. Even though the VIS/NIR calibrations are outside of the intended range of the IR SCF, those measurements are important because of their accuracy and to verify the spectral flatness of the bolometer.

The overall bolometer spectral response is shown in Fig. 4, where it is overlaid on the responsivity determined from the bolometer reflectance and window transmittance. This determination is a relative spectral curve, requiring a single scale factor to fit all of the absolute measurements. The resulting curve shown is $\mathrm{T}(1-\mathrm{R})^{*} 0.3913 \mathrm{~V} / \mu \mathrm{W}$. As can be seen from the figure, this response is a good fit, well within the uncertainties. For the VIS/NIR region the data shows excellent smoothness reflecting the quality of the Si and Ge transfer standards. 


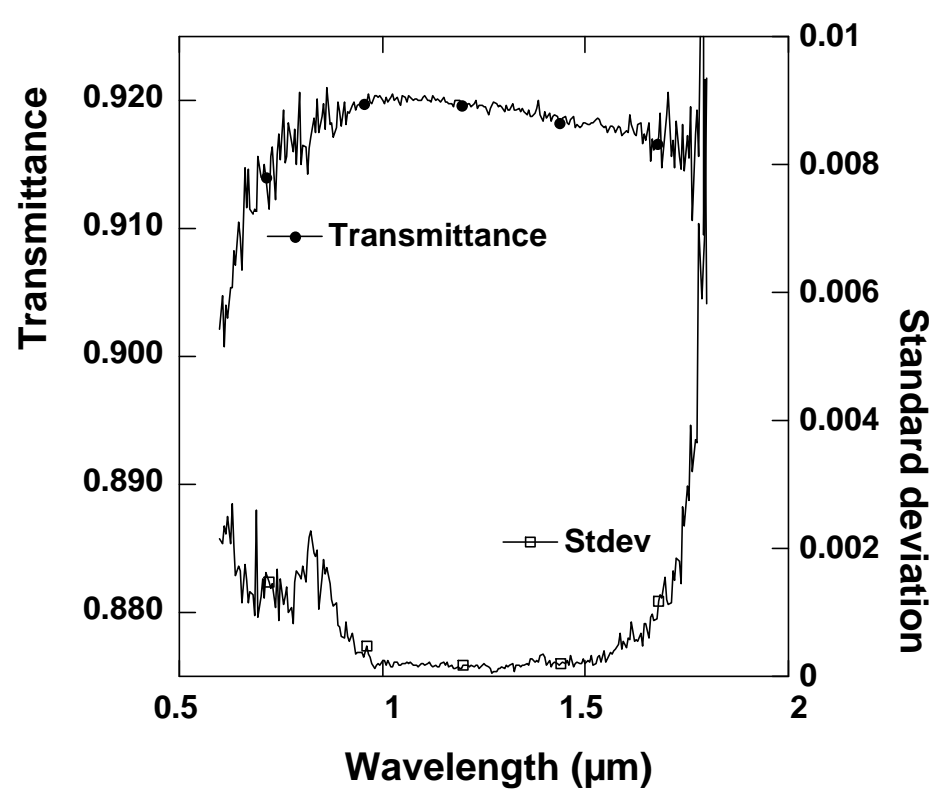

Figure 3. Transmittance of the KBr bolometer-window.

The IR region shows much higher variation due to the poorer quality of the pyroelectric transfer detector, which had much lower signal to noise ratio, poorer spatial response uniformity, and involved a more difficult calibration transfer. The overall good fit of the curve shape to the actual measurements over the $0.6 \mu \mathrm{m}$ to $11 \mu \mathrm{m}$ range indicates excellent selfconsistency. This should be emphasized as the overall data set includes three independent transfer detectors and a continuous spectral source as well as a laser source. This high level of selfconsistency increases confidence in the regions where absolute measurements were not directly made.

The overall scale factor of the relative response curve was determined by taking the ratio of the bolometer responsivity to the predicted relative response (T.(1-R)). The weighted mean of all the ratio data was $0.3913 \pm 0.00015$ (The uncertainty is the standard deviation of the mean). The weights used were the ratio uncertainty (the quadrature sum of the uncertainties of the responsivity measurements and the responsivity curve). This constant is a scale factor to convert the relative response curve to an absolute scale.

All uncertainty components were included, so the nonstatistical uncertainty correlations are ignored here. This analysis assumes a single scale factor over the entire wavelength range. To test this, the ratios of the responsivity measurements to the now absolute predicted responsivity were plotted versus wavelength and fit to a line. As shown in Fig. 5, the fit stays within $\pm 0.25 \%$ of unity indicating that any systematic tilting of the relative curve is less than this value. A second analysis was done that included some correlations in the component uncertainties with very little change of the final uncertainty ${ }^{(11)}$. 


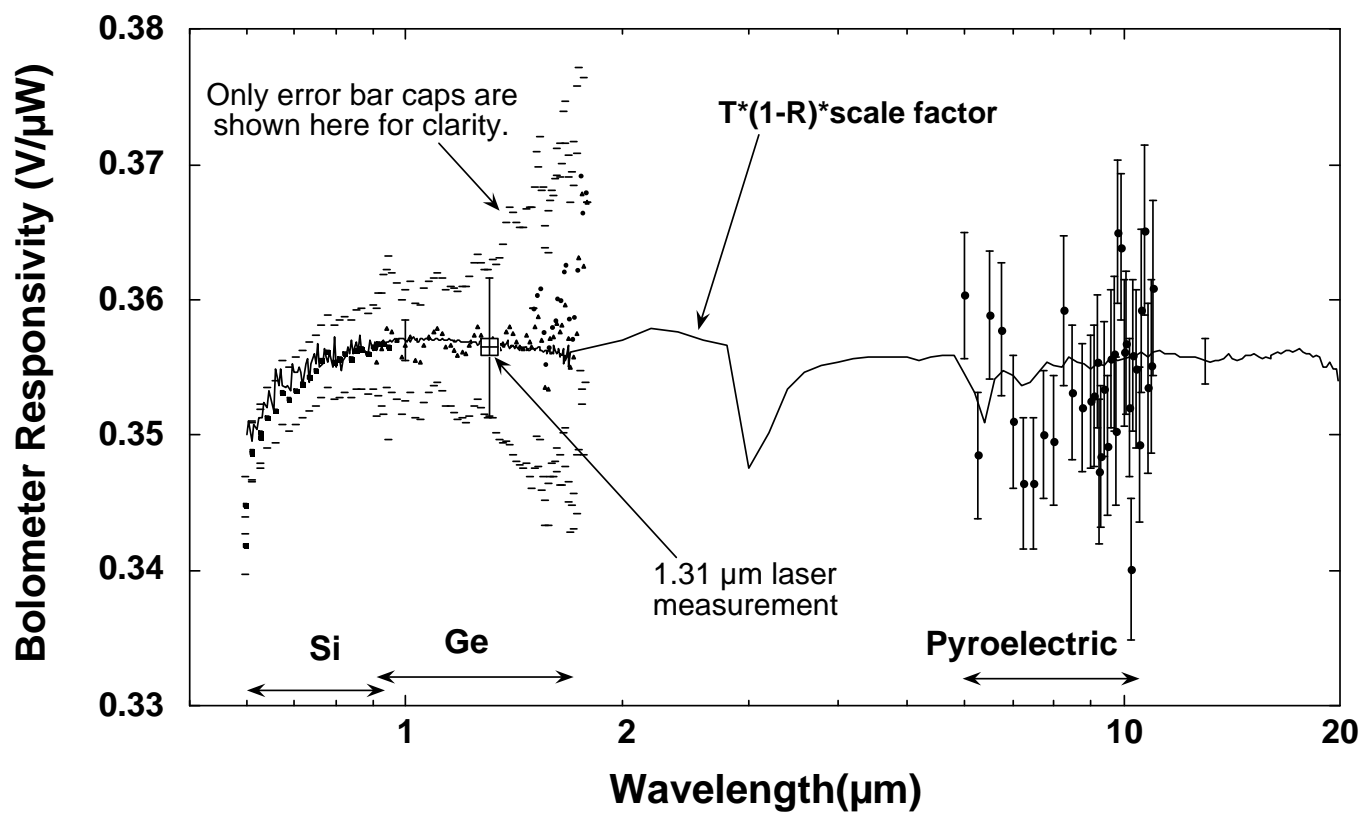

Figure 4. Radiant power responsivity of the bolometer.

The different analysis schemes support an overall uncertainty of $\pm 0.25 \%$ in the absolute responsivity scale as shown by the solid curve in Fig. 4. This uncertainty when combined with the uncertainty associated with an individual bolometer radiant power measurement at a specific wavelength (about $0.75 \%$ ) gives an overall bolometer power responsivity scale uncertainty of about $0.8 \%$. This is the uncertainty that can be expected for a bolometer measurement of radiant power made at a single wavelength within the $2 \mu \mathrm{m}$ to $20 \mu \mathrm{m}$ range of the IR SCF (assuming adequate measurement time is allowed so as not to be limited by bolometer signal noise). This uncertainty is comparable to the quoted uncertainty of a similar IR scale also set up for detector calibrations ${ }^{(3)}$.

\section{Conclusion}

NIST has developed an IR spectral responsivity scale. The radiant power responsivity scale was first implemented on a high sensitivity cryogenic bolometer between $2 \mu \mathrm{m}$ and $20 \mu \mathrm{m}$. The power responsivity scale uncertainty is comparable with the best existing IR responsivity scales. Working standard and test infrared detectors and radiometers can be calibrated against the cryogenic bolometer. 


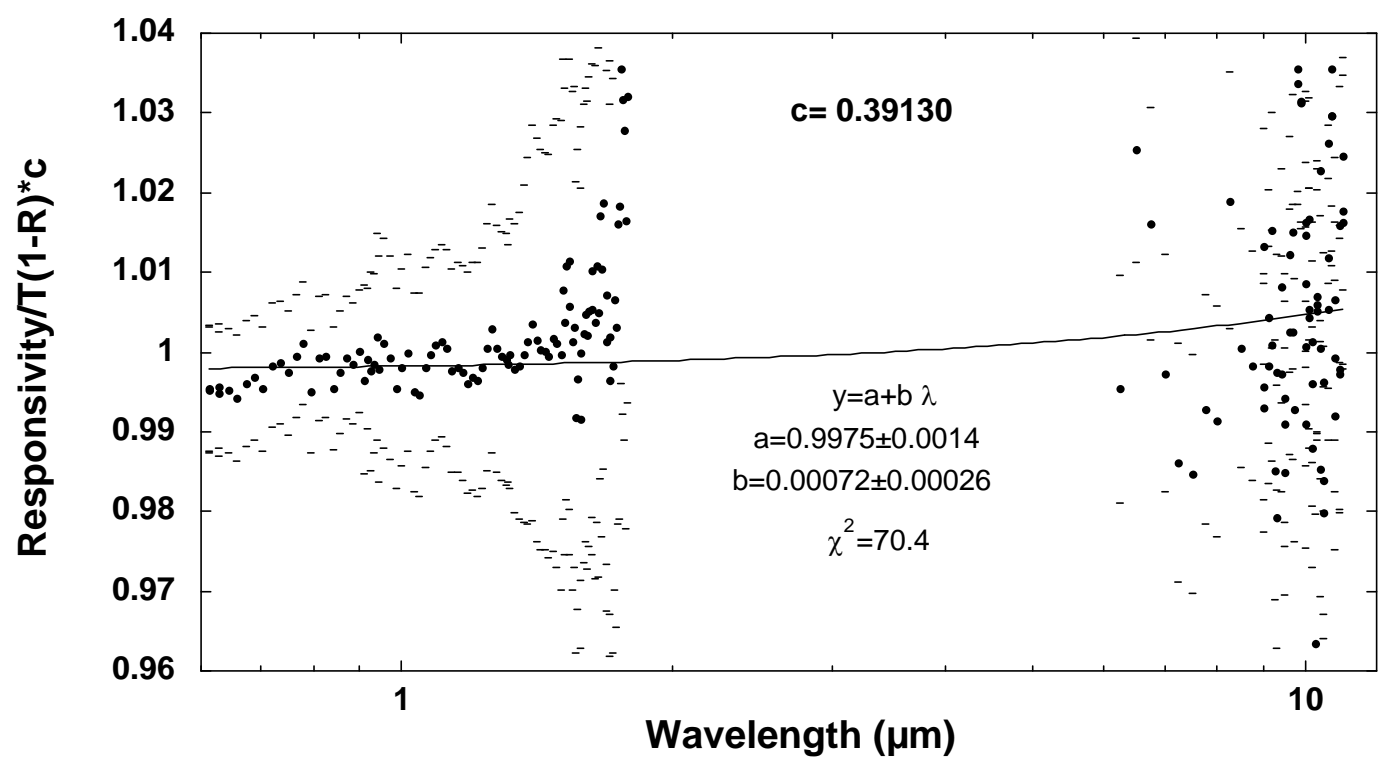

Figure 5. Ratio of the bolometer power responsivity to the scaled relative response.

\section{References}

1. Migdall, A., Eppeldauer, G., and Cromer, C., "IR Detector Spectral Responsivity Calibration Facility at NIST,” Cryogenic Optical Systems and Instruments VI, SPIE 2227, 46-53, 1994.

2. Parr, A.C., “A National Measurement System For Radiometry, Photometry, and Pyrometry Based upon Absolute Detectors, NIST Technical Note 1421, 1996.

3. Nettleton, D.H., Prior, T.R., and Ward, T.H., "Improved Spectral Responsivity Scales at the NPL, 400 nm to 20 pm,” Metrologia, Vol. 30, 425-432, 1993.

4. Migdall, A. and Eppeldauer, G., "Realization of an infrared spectral radiant power response scale on a cryogenic bolometer,” Metrologia, Vol. 35, 307-315, 1998.

5. Gentile, T.R., Houston, J.M. and Cromer, C.L., "Realization of a scale of absolute spectral response using the National Institute of Standards and Technology high-accuracy cryogenic radiometer,” Appl. Opt. 35, 1996, 4392-4403.

6. Eppeldauer, G.P., Migdall, A.L., and Hanssen, L.M., “InSb Working Standard Radiometers”, Metrologia, Vol. 35, 485-490, 1998.

7. Eppeldauer, G., Migdall, A.L., Gentile, T.R., and Cromer, C.L., “Absolute response calibration of a transfer standard cryogenic bolometer”, SPIE, Proc. Vol. 2550, 36-46, 1995.

8. Eppeldauer, G., Migdall, A.L., Cromer, C. L., “Characterization of a High Sensitivity Composite Silicon Bolometer,” Metrologia, Vol. 30, 317-320, 1993. 
9. Eppeldauer, G., Migdall, A.L., and Cromer, C.L., “A Cryogenic Silicon Resistance Bolometer for use as an Infrared Transfer Standard Detector," Thermal Phenomena at Molecular and in Cryogenic Infrared Detectors, edited by M. Kaviany et al., ASME, HTDVol. 277, New York, NY, 63-67, 1994.

10. Taylor, B.N. and Kuyatt, C.E., Guidelines for Evaluating and Expressing the Uncertainty of NIST Measurement Results, Natl. Inst. Stand. Technol. (U.S.), Tech. Note 1297, 1994.

11. Migdall, A.L., Eppeldauer, G.P., NIST Measurement Services: Spectroradiometric Detector Measurements: Part III-Infrared Detectors, NIST Special Publication 250-42, 1998. 


\title{
SPECTRAL POWER AND IRRADIANCE RESPONSIVITY CALIBRATION OF InSb WORKING STANDARD RADIOMETERS
}

\author{
George Eppeldauer and Miklos Racz \\ National Institute of Standards and Technology \\ Optical Technology Division \\ Gaithersburg, Maryland 20899 \\ * Guest researcher from the Hungarian Academy of Sciences \\ Research Institute for Technical Physics and Materials Science ${ }^{1}$
}

\begin{abstract}
New, improved performance, InSb power/irradiance meters have been developed and characterized to hold the National Institute of Standards and Technology (NIST) spectral responsivity scale between $2 \mu \mathrm{m}$ and $5.1 \mu \mathrm{m}$. The InSb radiometers were calibrated against the transfer standard cryogenic bolometer that is tied to the primary standard cryogenic radiometer of NIST. The InSb radiometers serve as easy-to-use working standards for routine spectral power and irradiance responsivity calibrations. The spectral irradiance responsivities were derived from the spectral power responsivities utilizing the measured area of the apertures in front of the InSb detectors.
\end{abstract}

\section{Introduction}

High sensitivity infrared radiometer standards can eliminate signal level problems caused by the weak outputs of commonly used thermal sources. An accurate infrared spectral responsivity scale has been realized on pyroelectric detectors at the National Physical Laboratory. ${ }^{1}$ The responsivity calibration of an InSb transfer standard detector has been reported for metrological applications between $1 \mu \mathrm{m}$ and $3 \mu \mathrm{m} .^{2}$ The new infrared spectral responsivity scale developed at NIST has been realized on a cryogenic bolometer ${ }^{3}$ that operates at much higher sensitivities (input power levels are $10 \mu \mathrm{W}$ to $20 \mathrm{pW}$ ) than pyroelectric detector based scales (between $500 \mu \mathrm{W}$ and $1 \mu \mathrm{W}$ ).

Working standard InSb radiometers ${ }^{4}$ have been developed for the $2 \mu \mathrm{m}$ to $5.4 \mu \mathrm{m}$ wavelength range to hold the spectral responsivity scale. They are easy to use relative to the sophisticated cryogenic bolometer ${ }^{5}$ that requires liquid $\mathrm{He}$ sensor-temperature and continuous computer control/monitor for responsivity-change corrections. ${ }^{6}$ Design, characterization, and spectral power responsivity calibration of the improved performance second-generation InSb radiometers is discussed in this paper. Realization of a new spectral irradiance responsivity scale is described also for the $2 \mu \mathrm{m}$ to $5.1 \mu \mathrm{m}$ wavelength range. Infrared test detectors with good spatial response uniformity can be calibrated against the InSb working standard radiometers in radiant power measurement mode. Spatially nonuniform infrared detectors can be used for accurate radiometric measurements when they are calibrated against the working standard InSb radiometers in irradiance measurement mode.

\section{Mechanical structure of the InSb radiometers}

\footnotetext{
${ }^{1}$ Current position is with the Konkoly Observatory, Budapest, Hungary
} 
Following the first-generation $4 \mathrm{~mm}$ diameter InSb detectors ${ }^{4}$ second generation InSb radiometers have been developed in cooperation with EG\&G Judson. ${ }^{7}$ Large area and selected high-shuntresistance detectors are mounted in the $77 \mathrm{~K}$ dewars. These InSb detectors were designed to minimize the problem of flashing ${ }^{4}$ even when exposed to short wavelength optical radiation. Precision apertures of $6.4 \mathrm{~mm}$ diameter are mounted in all dewars in front of the $7 \mathrm{~mm}$ diameter InSb photovoltaic detectors. Long-snout and short-snout dewars have been developed for different applications. In the long-snout version, as shown in Figure 1, a $17^{\circ}$ field-of-view (FOV) limiter is realized with a cold second aperture positioned $50 \mathrm{~mm}$ away from the precision aperture. The advantage of this arrangement is that laser beams can be measured if a wedged Dewar-window is used to avoid interference and to produce a large enough separation between the primary and reflected beams in the plane of the precision aperture. Also, the background rejection is higher here than in the short-snout Dewar because of the $31^{\circ}$ partial (clipped) FOV. The separation between the two apertures is only $7.2 \mathrm{~mm}$ in the short-snout Dewar. This unit is used only for non-coherent radiation measurements where easy alignment is an issue. The background produced signal is higher in the short-snout unit because of the $90^{\circ}$ overall FOV (the unvignetted FOV remained $17^{\circ}$ ). Optical filters can be mounted inside the cylindrical holder of the cold FOV limiter. These radiometers can be used in both radiant power and irradiance measurement modes.

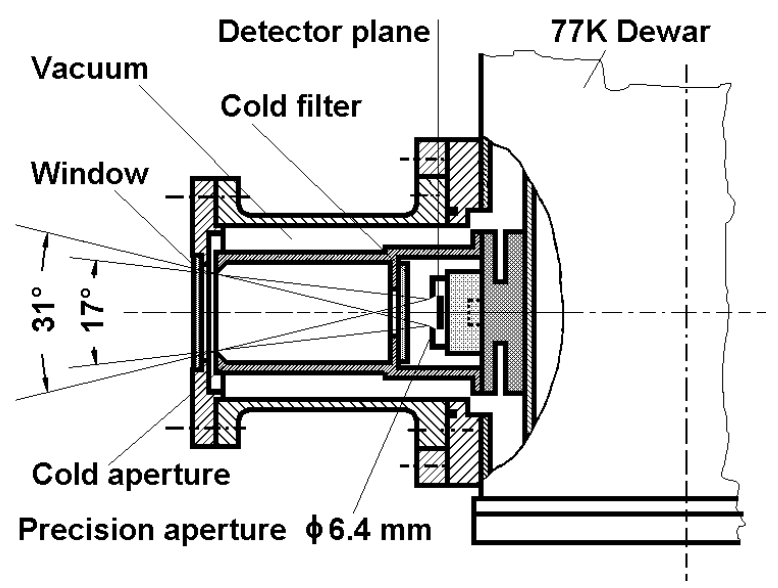

Fig. 1. Mechanical-optical construction of the second generation (long-snout) InSb radiometer.

\section{DC background and AC noise test of the InSb radiometers}

The current-to-voltage converter gains of the InSb radiometers were designed to achieve a DC thermal background-induced output voltage of less than $5 \mathrm{~V}$.

An infrared collimator was used for AC noise tests. The infrared collimator was built from a variable temperature blackbody (BB) radiator, a $25 \mathrm{~cm}$ diameter reflecting (collimating) mirror, and a detector stage. An aperture wheel, a chopper, and then a shutter (this was the closest to the $\mathrm{BB})$ were located in front of the BB cavity. The output voltage from the current-to-voltage converter of the InSb radiometer was measured with a lockin amplifier.

The noise floor of the second-generation InSb radiometers was tested with a $40 \mathrm{~Hz}$ chopping frequency while the shutter was closed. From 10 measurements, a 6.7 pA standard deviation was calculated for the current noise of the broadband InSb-1 radiometer when the electrical bandwidth 
was $0.5 \mathrm{~Hz}$. This corresponds to a noise equivalent photocurrent of $5 \mathrm{pA} / \mathrm{Hz}^{1 / 2}$. The InSb-1 radiometer is a short-snout unit without any cold filter. This is our most frequently used working standard.

For very high sensitivity measurements, the background rejection of the long-snout InSb radiometer was further increased by installing a cold filter (Model BBP-3200-3590-D from Spectrogon US, Inc.) $)^{7}$ with a bandpass of $3.2 \mu \mathrm{m}$ to $3.6 \mu \mathrm{m}$ (FWHM). The current-to-voltage gain could be increased from the $3 \times 10^{4} \mathrm{~V} / \mathrm{A}$ maximum of the short-snout unit to $10^{6} \mathrm{~V} / \mathrm{A}$ where the DC background-caused output voltage was $1.6 \mathrm{~V}$. A noise equivalent power (NEP) of 0.22 $\mathrm{pW} / \mathrm{Hz}^{1 / 2}$ was obtained at the peak responsivity of the filtered InSb radiometer. The dynamic (signal) range was six orders of magnitude at the $10^{6} \mathrm{~V} / \mathrm{A}$ gain.

\section{Calibration facility and procedure}

All measurements were performed on the Ambient Infrared Spectral Comparator Facility (IRSCF). ${ }^{8}$ The optical-mechanical scheme of the facility is shown in Fig. 2.

The light sources were a $100 \mathrm{~W}$ tungsten halogen lamp from $2 \mu \mathrm{m}$ to $3.65 \mu \mathrm{m}$, and a $45 \mathrm{~W}$ ceramic glower from $3.65 \mu \mathrm{m}$ to $5.7 \mu \mathrm{m}$. The sources were imaged to the entrance aperture of the prism-grating double monochromator using spherical and flat mirrors. A chopper was positioned in front of the entrance aperture. Either $1 \mathrm{~mm}$ or $2 \mathrm{~mm}$ diameter apertures could be used. An input iris was mounted on the spherical mirror to adjust the solid angle of the beam entering the monochromator. The flat mirror was removable using a kinematic mount. The bandpass of the monochromator was about $1 \%$ of the selected wavelength. Laser beams could be introduced into the monochromator when the flat mirror was removed. The laser inputs were used either for wavelength calibration or to scan detectors with a small spot for high resolution spatial response tests. Order sorting filters were used at the exit aperture of the monochromator to improve the out-of-band blocking of the prism (KRS-5) pre-disperser of the monochromator. Off-axis paraboloid mirrors imaged the output beam to the detectors. The $\mathrm{f} / \#$ of the output beam could be controlled with another iris. The detectors measured the total radiant power of the incident monochromatic beam (focussed into a $2 \mathrm{~mm}$ diameter spot on the detector surface) in underfilled mode. The kinematic mount of the bolometer was held by a separate stage where it could be tilted rotated, and shifted. Three InSb radiometers could be aligned on a second detector stage which was mounted on the computer moved Z (vertical) translation stage. Both detector stages were mounted on the computer controlled Y (horizontal) translation stage.

Spatial response scans were made along the horizontal and vertical diameters of all detectors before measurements to determine the location of the beam spot in the center of the detector.

The calibration procedure included a number of spectral scans in different wavelength intervals. During a scan, the computer set the wavelength, moved the bolometer into the beam where the test points ${ }^{8}$ and the output signal were measured, then the first InSb radiometer was moved into the same beam where its output signal was measured as well. During the responsivity scale derivation, a large enough number of measured points and scans were selected to make the statistical evaluation easier. After all InSb radiometers were measured at a selected wavelength, the scan was repeated at a $25 \mathrm{~nm}$ or $50 \mathrm{~nm}$ increment. 


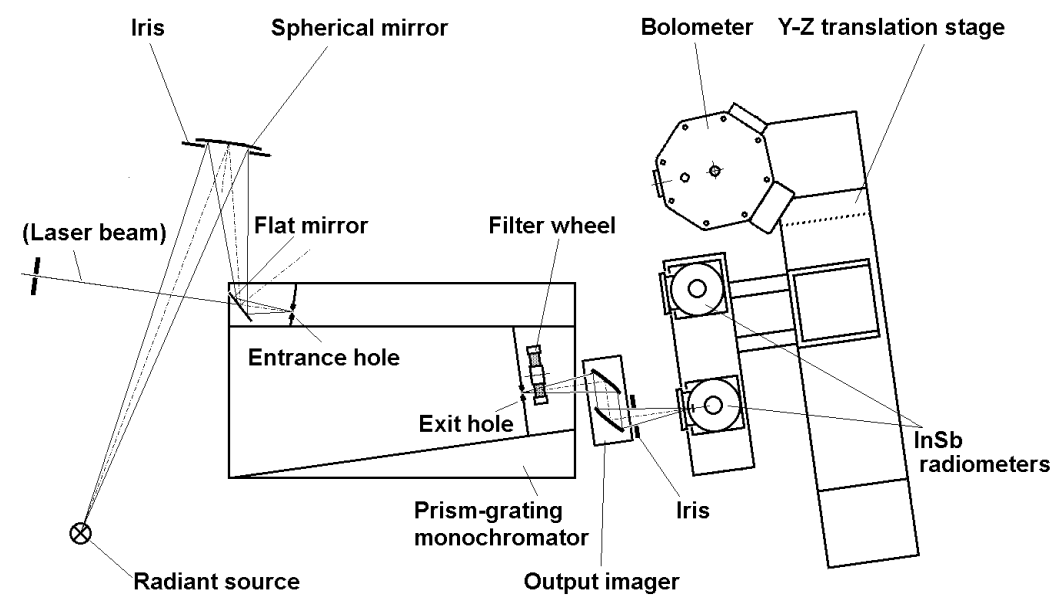

Fig. 2. Optical-mechanical scheme of the Ambient Infrared Spectral Comparator Facility

\section{Measurement results}

Those electronic and radiometric characteristics of the second generation InSb radiometers were measured which could influence the performance of the spectral responsivity calibrations.

\subsection{Frequency dependent signal responsivity}

The frequency dependence of the InSb radiometer responsivity was measured to allow response corrections for different chopping frequencies in different applications. Usually, the DC signal responsivity is reported. The measured responsivity curves of the InSb-1 radiometer are shown in Fig. 3 at transimpedances of $10 \mathrm{k} \Omega, 20 \mathrm{k} \Omega$, and $30 \mathrm{k} \Omega$. The $30 \mathrm{k} \Omega$ feedback resistor was used in the responsivity calibrations at the IRSCF. The lowest $3 \mathrm{~dB}$ roll-off frequency was about $400 \mathrm{~Hz}$. The roll-off frequencies were determined from curve fits to a single time constant equivalent circuit of a low-pass RC filter. ${ }^{9}$ The chopping frequency (for a given application) should be selected to be $40 \mathrm{~Hz}$ or less to keep the operating point on the plateau of the responsivity curves.

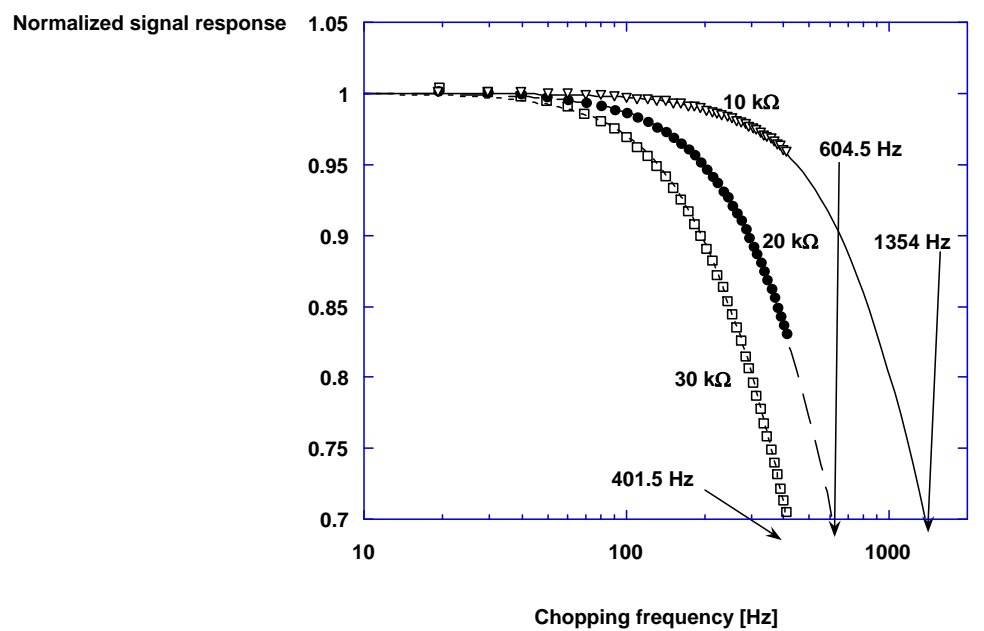

Fig. 3. Frequency-dependent signal responsivity curves of the InSb-1 radiometer at different gains. 


\subsection{Spatial response}

Horizontal and vertical scans were made through the detector centers with $2 \mathrm{~mm}$ diameter beam spots. The goal of these measurements was to check the spatial response uniformity of the detectors. The scans were made to find the plateau for power responsivity measurement and also to calculate an uncertainty component for the uncertainty budget. Beam clipping was avoided by working in the center of the measured plateau.

Figure 4 shows the horizontal and vertical response scans of the InSb-1 radiometer. The response difference is shown for both scans relative to the response in the detector center. The scans appear to show that the degree of non-uniformity is $0.36 \%$ along a vertical diameter calculated as the standard deviation from seven points on the plateau. The non-uniformity along the perpendicular (horizontal) diameter was much lower. The average run-to-run standard deviation for the seven points was $0.05 \%$.

\subsection{Spectral power responsivity}

The measured spectral power responsivity curves of the second generation InSb-1 and the first generation InSb-0 radiometers are shown in Fig. 5. The curves were calculated from a number of spectral responsivity scans performed in a few weeks period. The responsivity at each wavelength was determined from the average of at least four substitution measurements against the transfer standard cryogenic bolometer. Both the transfer- and working-standards were underfilled by the incident monochromatic radiation. The graph also shows the $100 \%$ external quantum efficiency ${ }^{10}$ (EQE) (discussed below). The second generation detector has improved spectral responsivity. A roughly 5\% dip at $4.2 \mu \mathrm{m}$ and a smaller dip at $2.7 \mu \mathrm{m}$ were caused by atmospheric absorption because the length of the optical path was different for the InSb detectors as compared to the transfer standard bolometer. The dips at $4.2 \mu \mathrm{m}$ are shown with thinner lines. Only the monochromator was purged. The other small dips at $3.2 \mu \mathrm{m}$ were caused by absorption inside of the InSb Dewars. Dip-free responsivity curves could be obtained by either extending the purged beam-passes or applying responsivity corrections. The right-side Y-axis combines the relative uncertainties $(k=1)$ of the two detector spectral responsivity measurements. Uncertainties are not shown for the $4.2 \mu \mathrm{m}$ absorption band where high accuracy calibrations are not suggested. Increased uncertainties can be seen at the other absorption bands. The low uncertainties reflect the small changes (drift) during a short substitution measurement at a selected wavelength. The uncertainties of the InSb-0 detector are lower because of the better spatial response nonuniformity.

\subsection{Spectral irradiance responsivity}

The spectral irradiance responsivity of the working standard radiometers was determined to calibrate test detectors with large spatial response non-uniformity. The response non-uniformity of these test detectors can be averaged out if they are calibrated in irradiance measurement mode where both the standard and the test irradiance meters are overfilled with a uniform field of radiation.

The spectral irradiance responsivity of the standard meter is equal to the product of the spectral power responsivity and the area of the precision aperture in front of the detector. The second generation InSb irradiance meters were built and used for two different applications. In the first one, the broad-band detector was utilized as a working standard in a short-snout Dewar. In the 
second application, a cold filter with a band-pass from $3.2 \mu \mathrm{m}$ to $3.6 \mu \mathrm{m}$ (FWHM) was used in a long-snout Dewar to measure the very low irradiance levels in the infrared collimator (mentioned in Section 3). This irradiance mode working standard was used to calibrate the infrared collimator and it also measured the area of very small apertures (in the aperture wheel of the collimator) radiometrically relative to a large (mechanically measured) reference aperture. The details of the collimator calibration and its aperture area measurements are beyond the scope of this paper. The power levels measured on the collimator were lower than $0.1 \mathrm{~mW}$ where the detector non-linearity is much less than $1 \% .^{2,4}$ At $10^{6} \mathrm{~V} / \mathrm{A}$ gain and $800{ }^{\circ} \mathrm{C}$ BB temperature, the signal-to-noise ratio of this irradiance meter was $2 \times 10^{3}$ when the smallest collimator aperture (0.2 mm diameter) was tested.

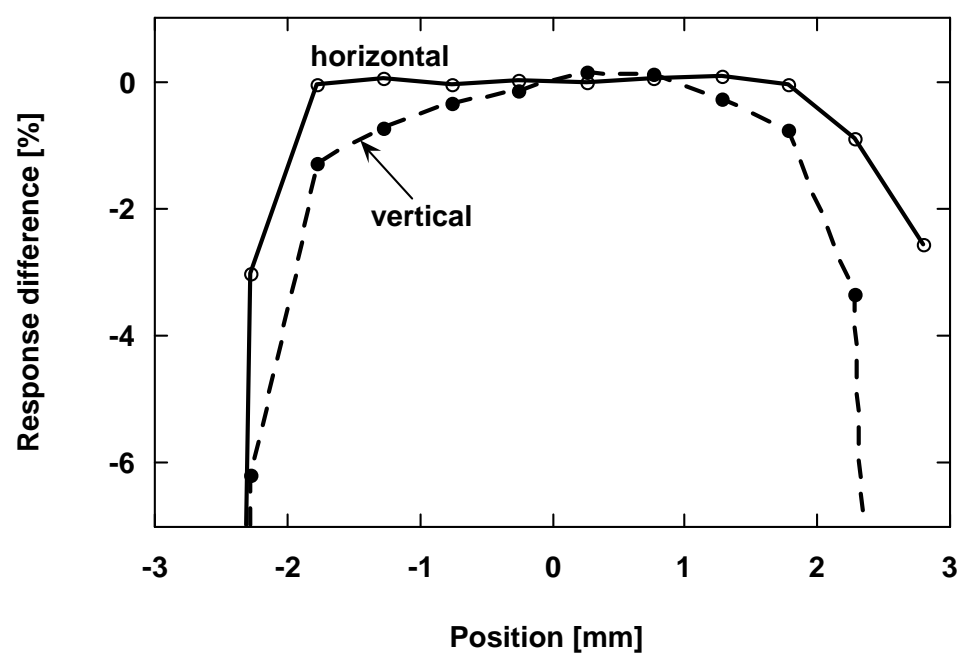

Fig. 4. Horizontal and vertical response scans of the InSb-1 radiometer. The curves show the response differencies relative to the response in the detector center.

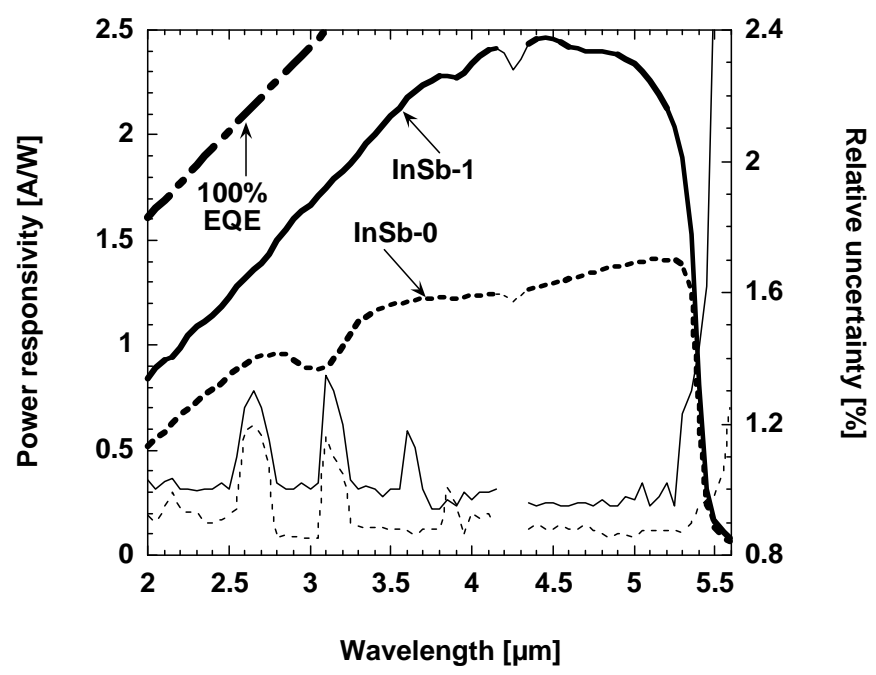

Fig. 5. Spectral responsivity curves of the first generation (InSb-0) and second generation (InSb-1) radiometers are shown. The responsivities measured at the $4.2 \mu \mathrm{m}$ absorption band are shown where the curves become thinner. The thoretical limit of the responsivity is the $100 \%$ EQE line. The two curves at the bottom are the uncertainties (scaled on the right-hand $\mathrm{Y}$ axis). 


\subsection{External quantum efficiency}

The external quantum efficiency is EQE $=1239.48 * s / \lambda$ where $s$ is the responsivity and $\lambda$ is the wavelength. Also, EQE $=(1-\rho) *$ IQE where $\rho$ is the reflectance and IQE is the internal quantum efficiency equal to the ratio of the number of collected electrons to the number of photons absorbed by the detector. The EQE of the second generation InSb detectors was almost twice that of the first generation devices. The highest EQE of 75\% was calculated between $3.6 \mu \mathrm{m}$ and 3.7 $\mu \mathrm{m}$ for the InSb-1 detector. Both radiometer generations had sapphire windows with a transmittance of about $90 \%$ at $3 \mu \mathrm{m}$. The InSb-1 radiometer was equipped with a $1.8 \mu \mathrm{m}$ cut-on (cold) filter. This filter has a transmittance of $90 \%$ to $95 \%$ for wavelengths longer than $2 \mu \mathrm{m}$. From the measured (and calculated) EQE and the signal loss caused by the window and the coldfilter, a reflectance of about $10 \%$ can be estimated for the InSb-1 detector. The IQE is close to unity. The InSb-0 (first generation) radiometer was not filtered.

\section{Uncertainties}

The uncertainties were evaluated and expressed according to the guidelines of the NIST Technical Note $1297^{11}$ and the ANSI/NCSL Z540-2-1997 U.S. Guide to the Expression of Uncertainty in Measurement (GUM). ${ }^{12}$ The modelling of the measurand (the spectral power responsivity) and its measurement procedure have been discussed in detail in the NIST Special Publication 250-42, ${ }^{8}$ where the uncertainty components were evaluated for the spectral responsivity determination of the transfer standard cryogenic bolometer.

In the present example, the power responsivity scale was derived from the cryogenic bolometer to the working standard InSb-1 radiometer. The responsivity scale uncertainty of the bolometer dominates the uncertainty budget of the InSb working standard. The additional uncertainty sources of this scale derivation are summarized in Table 1 . The uncertainty components that contribute to the overall uncertainty are described below.

The uncertainty components, caused by the spatial response non-uniformity of the bolometer and InSb detector were calculated from spatial response scans as the standard deviation of the measured data points. All the utilized data points were located on the plateau of the response curves measured along the detector diameters, indicating that the incident beam was not clipped by the input optics of the radiometers. Because of the limitations in the vertical moving of the bolometer stage, only horizontal scans were made on the bolometer. The standard deviation from the horizontal scan represented the spatial response non-uniformity of the bolometer in the uncertainty budget.

The measurement noise was obtained from the standard deviation of the average responsivity. The InSb radiometer was substituted for the bolometer at least four times at each wavelength. The measurement noise includes the signal changes due to component instabilities (e.g. source fluctuations) and wavelength dependent (random) uncertainties of the monochromator. The longterm drift of the source did not produce any additional uncertainty component because the detector substitution at each wavelength took care of this problem. Also, the chopper duty-factor (open-to-close ratio) related uncertainty components could be neglected because the same chopper was used for both the bolometer and the InSb radiometer.

The frequency instability of the chopper produced an uncertainty component in the frequency dependent responsivity corrections. The InSb radiometer responsivity correction was determined from the data fits of multiple $3 \mathrm{~dB}$ signal roll-off measurements at different photocurrent-to- 
voltage gains. The photocurrent-to-voltage gains were measured against a standard current source (calibrator). The gain correction uncertainty includes the gain calibration uncertainty and the gain instabilities as well.

Table 1

Uncertainties of power responsivity calibration with the working standard InSb-1 radiometer outside of the atmospheric and Dewar absorption bands

\begin{tabular}{|c|c|c|}
\hline \multirow[b]{2}{*}{ Uncertainty origin } & \multicolumn{2}{|c|}{ Uncertainty $(k=1)[\%]$} \\
\hline & Type $A^{11}$ & Type B \\
\hline Infrared spectral responsivity scale (on the bolometer) ${ }^{8}$ & & 0.8 \\
\hline Bolometer spatial response non-uniformity & & 0.24 \\
\hline InSb-1 spatial response non-uniformity (horizontal) & & 0.05 \\
\hline InSb-1 spatial response non-uniformity (vertical) & & 0.36 \\
\hline Noise of the bolometer responsivity measurement & 0.19 & \\
\hline Noise of the InSb-1 radiometer responsivity measurement & 0.15 & \\
\hline Responsivity correction of the InSb- 1 radiometer to $0 \mathrm{~Hz}$ & & 0.07 \\
\hline Bolometer preamplifier gain correction ${ }^{8}$ & & 0.18 \\
\hline InSb-1 radiometer gain correction & & 0.07 \\
\hline Lockin amplifier gain correction ${ }^{8}$ & & 0.12 \\
\hline $\begin{array}{l}\text { Bolometer responsivity corrections (temperature, bias, } \\
3 \mathrm{~dB} \text { roll-off measurements. and non-linearity) }\end{array}$ & 02 & \\
\hline InSb response non-linearity ${ }^{2,4}$ & & 0.1 \\
\hline Wavelength shift of the monochromator from $2 \mu \mathrm{m}$ to $3.7 \mu \mathrm{m}$ & & 0.3 \\
\hline Wavelength shift of the monochromator from $3.7 \mu \mathrm{m}$ to $5.1 \mu \mathrm{m}$ & & 0.1 \\
\hline & & \\
\hline Relative combined standard uncertainty from $3.7 \mu \mathrm{m}$ to $5.1 \mu \mathrm{m}$ & & \\
\hline
\end{tabular}

Table 2

Uncertainties of irradiance responsivity calibration with the working standard InSb-1 radiometer

\begin{tabular}{lcc}
\hline \multicolumn{1}{c}{ Uncertainty origin } & \multicolumn{2}{c}{ Uncertainty [\%] } \\
& Type A & Type B \\
& & 1.00 \\
$\begin{array}{l}\text { Power responsivity of the InSb-1 radiometer } \\
\text { Aperture area measurement of the InSb-1 radiometer } \\
\text { Directional responsivity of the InSb-1 radiometer within } \mathrm{f} / 4\end{array}$ & 0.18 & 0.075 \\
\hline Relative combined standard uncertainty $(k=1)$ & \multicolumn{2}{c}{1.024} \\
\hline
\end{tabular}

The uncertainty components originating from the lockin amplifier gain calibrations, the bolometer temperature, bias voltage, $3 \mathrm{~dB}$ roll-off frequency, and non-linearity corrections were discussed in our previous publication. ${ }^{3,8}$ The uncertainty component caused by the InSb response nonlinearity was taken from previously published results on similar InSb detectors. ${ }^{2,4}$ 
The uncertainty budget was determined for two wavelength ranges because the effect of the $5 \mathrm{~nm}$ maximum possible wavelength shift of the monochromator caused higher uncertainty between 2 $\mu \mathrm{m}$ and $3.7 \mu \mathrm{m}$ as compared to the uncertainty component between $3.7 \mu \mathrm{m}$ and $5.1 \mu \mathrm{m}$. We have not specified any responsivity uncertainties between $4.2 \mu \mathrm{m}$ and $4.3 \mu \mathrm{m}$. High accuracy measurements should not be done (without purging) within this wavelength band where the atmospheric absorption is very high. All the uncertainty components in Table 1 are specified outside of the absorption bands. The roughly 1\% uncertainty components within the $2.7 \mu \mathrm{m}$ and $3.2 \mu \mathrm{m}$ absorption bands produced peaks in the combined uncertainties in Fig. 5.

Table 2 is an extension of Table 1 for irradiance responsivity measurements using the same working standard (InSb-1) radiometer. The aperture area was calculated from the average of several diameter measurements. The diameters were measured with a collimated beam geometry on an optical microscope. The aperture is positioned less than $1 \mathrm{~mm}$ away from the detector. The International Commission on Illumination (CIE) recommended ${ }^{13}$ directional error $\mathrm{f}_{2}(\varepsilon)$ was calculated from directional (angular) response measurements of the InSb radiometer. The $\mathrm{f}_{2}(\varepsilon)$ was smaller than $0.5 \%$ between $-7^{\circ}$ and $7^{\circ}$ which is roughly the angular range of the $\mathrm{f} / 4$ incident beam. The standard deviation of the directional responses within this angular range was $0.18 \%$. This uncertainty component is used for unknown source distribution if the angular range of the source is within $\mathrm{f} / 4$.

\section{Conclusion}

High electronic and radiometric performance InSb radiometers have been developed, characterized, and calibrated to hold the spectral power and irradiance responsivity scales between $2 \mu \mathrm{m}$ and $5.1 \mu \mathrm{m}$. The relative uncertainty $(k=1)$ of the responsivity scales maintained by the working standard radiometers is about $1 \%$. Test infrared radiometers can be calibrated against the working standard InSb radiometers in both power and irradiance measurement modes.

\section{References}

1. Nettleton, D.H., Prior, T.R., and Ward, T.H., "Improved Spectral Responsivity Scales at the NPL, $400 \mathrm{~nm}$ to $20 \mu \mathrm{m}$,” Metrologia, Vol. 30, 425-432, 1993.

2. L-P Boivin, Properties of indium antimonide detectors for use as transfer standards for detector calibrations, Appl. Opt. Vol. 37, pp. 1924-1929, 1998.

3. A. Migdall and G. Eppeldauer, "Realization of an Infrared Spectral Radiant Power Response Scale on a Cryogenic Bolometer”, Metrologia, Vol. 35, p. 307-315, 1998.

4. G.P. Eppeldauer, A.L. Migdall, and L.M. Hanssen, "InSb Working Standard Radiometers," Metrologia, Vol. 35, p. 485-490, 1998.

5. G. Eppeldauer, A. L. Migdall, and C. L. Cromer, "A cryogenic silicon resistance bolometer for use as an infrared transfer standard detector," in HTD-Vol. 277, pp. 63-67, Thermal Phenomena at Molecular and in Cryogenic Infrared Detectors, M. Kaviany, D. A. Kaminski, A. Majumdar, P. E. Phelan, M.M. Yovanovich, and Z. M. Zhang, eds. (The American Society of Mechanical Engineers, New York), Book No. H00908-1994. 
6. G. Eppeldauer, A.L Migdall., T.R. Gentile, and C.L Cromer., “Absolute response calibration of a transfer standard cryogenic bolometer," in Proc. Vol. 2550, Photodetectors and Power Meters II, K. Muray and K. J. Kaufmann, eds. SPIE, pp. 36-46, 1995.

7. Identification of commercial equipment to specify adequately an experimental problem does not imply recommendation or endorsement by the National Institute of Standards and Technology nor does it imply that the equipment identified is necessarily the best available for the purpose.

8. A.L. Migdall and G.P. Eppeldauer, Spectroradiometric Detector Measurements: Part III Infrared Detectors, NIST Special Publication 250-42, US Government Printing Office, Washington, DC, 1998.

9. G.P. Eppeldauer, Noise-Optimized Silicon Radiometers, J. Res. Natl. Inst. Stand. Technol. Vol. 105, pp.209-219, 2000.

10. T.C. Larason, S.S. Bruce, and A.C. Parr, Spectroradiometric Detector Measurements: Part I Ultraviolet Detectors and Part II - Visible and Near-Infrared Detectors, NIST Special Publication 250-41, pp. 35, US Government Printing Office, Washington, DC, 1998.

11. B.N. Taylor and C.E. Kuyatt, Guidelines for Evaluating and Expressing the Uncertainty of NIST Measurement Results, National Institute of Standards and Technology, Tech. Note 1297, US Government Printing Office, Washington, DC, 1994.

12. ANSI/NCSL Z540-2-1997, American National Standard for Expressing Uncertainty - U.S. Guide to the Expression of Uncertainty in Measurement, National Conference of Standards Laboratories, Boulder, CO, 1998.

13. CIE Publ. No. 53 (TC-2.2), Methods of Characterizing the Performance of Radiometers and Photometers, Central Bureau of the CIE, A-1033 Vienna, P.O.Box 169, Austria, 1982. 


\title{
Spectral responsivity determination of a transfer-standard pyroelectric radiometer
}

\author{
George Eppeldauer, Miklos Racz* and Leonard Hanssen \\ Optical Technology Division \\ National Institute of Standards and Technology (NIST) \\ Gaithersburg, Maryland 20899-8441 \\ *Guest Researcher at NIST from the Hungarian Academy of Sciences, \\ now at Konkoly Observatory, Budapest H-1121, Hungary
}

\begin{abstract}
A NIST-developed pyroelectric radiometer was characterized and calibrated to extend the NIST high accuracy spectral responsivity scale from the visible range to the ultraviolet (UV) and infrared (IR). The transmission of the gold-black coated $\mathrm{LiNbO}_{3}$ pyroelectric material is negligibly small, therefore the absorptance, equal to (1-reflectance), is proportional to the responsivity of the detector. The spectral total reflectance of the coating was measured with integrating spheres and spectrophotometers to determine the relative spectral responsivity from the UV to the IR. The relative spectral responsivity was converted into absolute spectral power and irradiance responsivities by measuring the total power in a $442 \mathrm{~nm}$ stabilized laser beam. The reference device for absolute calibration was a Si trap-detector calibrated against the primary standard cryogenic radiometer. The calibrations were repeated with 31 months separation. A $0.3 \%$ long-term shift in the reflectance was measured between $800 \mathrm{~nm}$ and $19 \mu \mathrm{m}$. The $28 \%$ reflectance at $10.6 \mu \mathrm{m}$ increased the $0.1 \%$ spatial responsivity non-uniformity in the visible (where the reflectance was $0.4 \%$ ) to $8 \%$. The spectral power and irradiance responsivity scales of the pyroelectric radiometer have been realized between $250 \mathrm{~nm}$ and $2.5 \mu \mathrm{m}$ with a relative standard uncertainty of less than $0.34 \%$ (coverage factor $k=1$ ).
\end{abstract}

Keywords: absolute responsivity, gold-black coating, infrared, irradiance, pyroelectric detector, radiant power, radiometer, reflectance, relative response, transfer standard, ultraviolet

\section{INTRODUCTION}

The subject of the present work is to extend the ambient-background detector-based responsivity scale of NIST to a wide wavelength range with improved uncertainty. The present NIST responsivity scale uncertainty is about $1 \%^{1}$ in the $2 \mu \mathrm{m}$ to $20 \mu \mathrm{m}$ range [1, 2] and close to $1 \%$ in the $250 \mathrm{~nm}$ to $400 \mathrm{~nm}$ range [3]. The uncertainty from $920 \mathrm{~nm}$ to $1.8 \mu \mathrm{m}$ increases from $0.5 \%$ to $1.8 \%$, respectively [4]. In the reference (silicon) spectral range, between $406 \mathrm{~nm}$ and $920 \mathrm{~nm}$, the responsivity scale uncertainty is only $0.03 \%$ [5]. A new facility is being developed at NIST for Spectral Irradiance and Radiance Responsivity Calibrations with Uniform Sources (SIRCUS) [6]. The purpose of this facility is to decrease the uncertainty gap between the reference scale and the extended scales. In order to achieve this goal, a group of improved performance transfer standard radiometers is being developed. The SIRCUS facility operates in the $250 \mathrm{~nm}$ to $2500 \mathrm{~nm}$ range. This range is determined by the useful reflectance coverage of the Spectralon coating of the integrating sphere sources and the spectral coverage of the monitor and working standard silicon and extended InGaAs photodiodes. The design and most of the radiometric and electronic characterizations of a high performance $\mathrm{LiNbO}_{3}$ pyroelectric radiometer have already been published [7]. We describe here the radiometric characterizations and calibrations needed to extend the high accuracy responsivity scale to the $250 \mathrm{~nm}$ to $2500 \mathrm{~nm}$ range. The gold-black reflectance measurements were made

\footnotetext{
${ }^{1}$ All uncertainties discussed in this paper are relative standard uncertainties with a coverage factor of one. Property of the United States Government. Not subject to copyright.
} 
between $250 \mathrm{~nm}$ and $19 \mu \mathrm{m}$. The reflectance measurements were repeated after 31 months to check the long-term stability of the transfer standard radiometer and the repeatability of the spectral reflectance measurements. Spatial responsivity tests are described at $10.6 \mu \mathrm{m}$ where the high reflectance of the coating increases the spatial non-uniformity of responsivity. For the highest accuracy responsivity scale extension, a group of pyroelectric radiometers will be used. Here, the scale extension work is described only for the $\mathrm{LiNbO}_{3}$ pyroelectric transfer standard radiometer.

\section{SPECTRAL REFLECTANCE MEASUREMENTS}

The transmission of the gold-black coated detector is negligibly small, therefore the absorptance, equal to 1-reflectance, is proportional to the responsivity of the detector. We measured the spectral near-normal (directional-hemispherical) reflectance of the gold-black coated detector to determine the relative spectral responsivity.

The spectral reflectance of the gold-black coated pyroelectric detector had already been measured with a modified Perkin-Elmer ${ }^{2}$ spectrophotometer between $800 \mathrm{~nm}$ and $1.8 \mu \mathrm{m}$ [7]. Similar measurements were made on the same detector with a Cary-5E spectrophotometer between $250 \mathrm{~nm}$ and $830 \mathrm{~nm}$. In both cases, the spectral reflectance was compared to a white polytetrafluoroethylene (PTFE) reflectance standard. The zero reflectance was obtained when a light trap replaced the pyroelectric detector at the sample port of the integrating sphere. The integrating sphere collected the diffuse radiation reflected back from the detector surface. The monochromatic input beam was directed to the surface of the detector at a $6^{\circ}$ angle of incidence. The $8 \mathrm{~mm}$ diameter detector-aperture was removed during the reflectance measurements to avoid beam clipping. The two spectral reflectance curves, measured on the different spectrophotometers, are shown in Fig. 1.

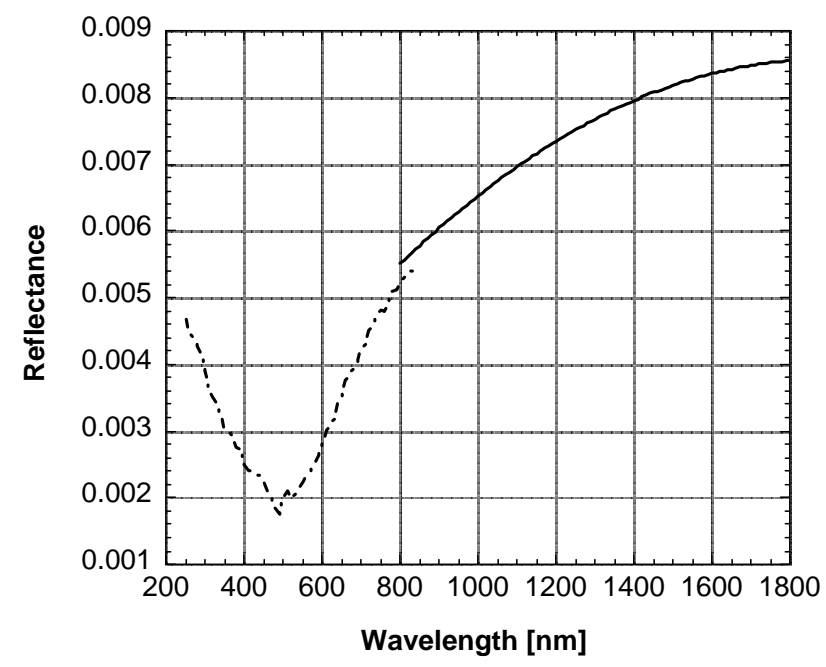

Fig. 1. Spectral reflectance of the gold-black coated pyroelectric detector.

The dashed curve was measured on a Cary-5E spectrophotometer.

The solid curve was measured on a modified Perkin-Elmer spectrophotometer.

\footnotetext{
2 Certain commercial equipment, instruments, or materials are identified in this paper to foster understanding. Such identification does not imply recommendation or endorsement by the National Institute of Standards and Technology, nor does it imply that the materials or equipment are necessariliy the best available for the purpose.
} 
As the measurement was noisy between $800 \mathrm{~nm}$ and $1800 \mathrm{~nm}$, a curve-fit is shown in that range. The uncertainty of the reflectance measurements was $0.1 \%$ between $250 \mathrm{~nm}$ and $1600 \mathrm{~nm}$ and it increased to $0.23 \%$ at $1800 \mathrm{~nm}$. These measurements were made in September, 1999.

In order to extend the high accuracy responsivity scale to the infrared (IR) range, the absolute directionalhemispherical reflectance of the $\mathrm{LiNbO}_{3}$ pyroelectric detector was measured between $1 \mu \mathrm{m}$ and $19 \mu \mathrm{m}$. The IR reflectance measurements were made on a Fourier Transform (FT) spectrometer in both September 1999 and April 2002. The April 2002 results are shown in Fig. 2 to illustrate that the reflectance is higher than $50 \%$ at $19 \mu \mathrm{m}$. A Quartz beam splitter and a tungsten halogen lamp source was used in the FT spectrometer for the $1 \mu \mathrm{m}$ to $3.3 \mu \mathrm{m}$ wavelength range. A Ge coated $\mathrm{KBr}$ beam splitter with a ceramic glow-bar source was used for wavelengths longer than $3.3 \mu \mathrm{m}$. The uncertainty of the IR reflectance measurements ranges from $0.1 \%$ near 0.01 reflectance to $1.5 \%$ at 0.5 reflectance.

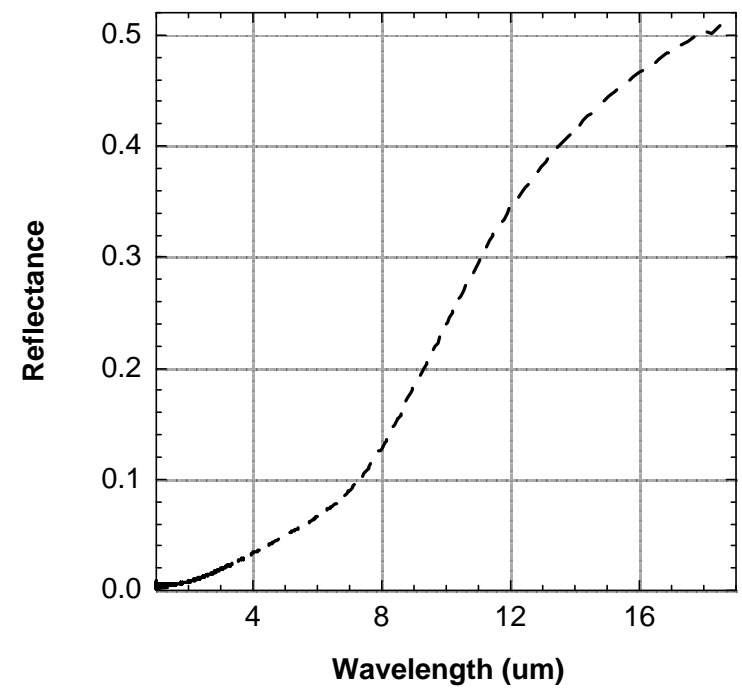

Fig. 2. IR spectral reflectance of the gold-black coated pyroelectric detector. The beam splitter and the source of the FT spectrometer were changed at $3.3 \mu \mathrm{m}$.

\section{LONG TERM STABILITY OF SPECTRAL ABSORPTANCE}

The spectral absorptance (1-reflectance) values of the September, 1999 and the April and June 2002 measurements are summarized in Fig. 3 between $250 \mathrm{~nm}$ and $3.5 \mu \mathrm{m}$. The measurements were made with a large time separation to check the long-term stability of the gold-black coated detector and the repeatability of the calibrations. The 1-reflectance curves are shown only up to $3.5 \mu \mathrm{m}$ to show the measured differences on a more magnified scale. The FT spectrometer measurements start at $1 \mu \mathrm{m}$. The noise in all September 1999 measurements is averaged out. The April 2002 measurements started at $500 \mathrm{~nm}$ to check the changes up to $1.5 \mu \mathrm{m}$. The noise at $800 \mathrm{~nm}$ increased due to a detector change (from photomultiplier tube to PbS detector) in the spectrophotometer. The noise in the FT measurement (April 2002) at the $1 \mu \mathrm{m}$ region was caused by the poor signal-to-noise ratio. The graph shows a $0.3 \%$ shift in the 1-reflectance values between the September 1999 and the April 2002 measurements for wavelengths longer than $800 \mathrm{~nm}$. The results obtained with the two independent reflectance measurement methods in April 2002 agreed within $0.1 \%$ in the $1 \mu \mathrm{m}$ to $1.5 \mu \mathrm{m}$ range where they overlap. The measured shift at $500 \mathrm{~nm}$ was negligibly small. In order to check for possible changes in the UV, the reflectance was measured from $325 \mathrm{~nm}$ to $500 \mathrm{~nm}$ in June 2002. As the same $0.3 \%$ shift was measured for wavelengths longer than 800 
nm using two different methods, the reason of the shift was the change in the gold-black coated detector. The measured change in the $325 \mathrm{~nm}$ to $500 \mathrm{~nm}$ range was less than $0.1 \%$.

\section{SPATIAL RESPONSIVITY IN THE INFRARED}

In our September 1999 measurements, the reflectance of the gold-black coated $\mathrm{LiNbO}_{3}$ pyroelectric detector was less than $1 \%$ between $800 \mathrm{~nm}$ and $1800 \mathrm{~nm}$ [7]. The spatial response non-uniformity was $+/-0.1 \%$ at $633 \mathrm{~nm}$. High reflectance can increase the spatial response non-uniformity resulting in a large uncertainty in radiant power measurements where the detector is underfilled by the incident beam.

In order to determine the useful wavelength range of a responsivity scale extension with the $\mathrm{LiNbO}_{3}$ pyroelectric radiometer, the non-uniformity of the spatial responsivity was measured at a wavelength where the reflectance of the gold-black coating was much higher than $1 \%$. Figure 4 shows the result of a spatial responsivity scan at $10.6 \mu \mathrm{m}$ where the reflectance is about $28 \%$. The scanning beam diameter was $0.4 \mathrm{~mm}$. The contour plot shows an $8 \%$ maximum-to-minimum change in the responsivity. The reflectance at $633 \mathrm{~nm}$ was about $0.4 \%$ in September 1999 [7]. The $28 \%$ reflectance at $10.6 \mu \mathrm{m}$ is a factor of 70 higher. This is the reason that the non-uniformity of the spatial responsivity increased from the $0.1 \%$ level to $8 \%$. We can conclude that the increase in the non-uniformity of the spatial responsivity is proportional to the increase of the reflectance and the proportionality factor is one.

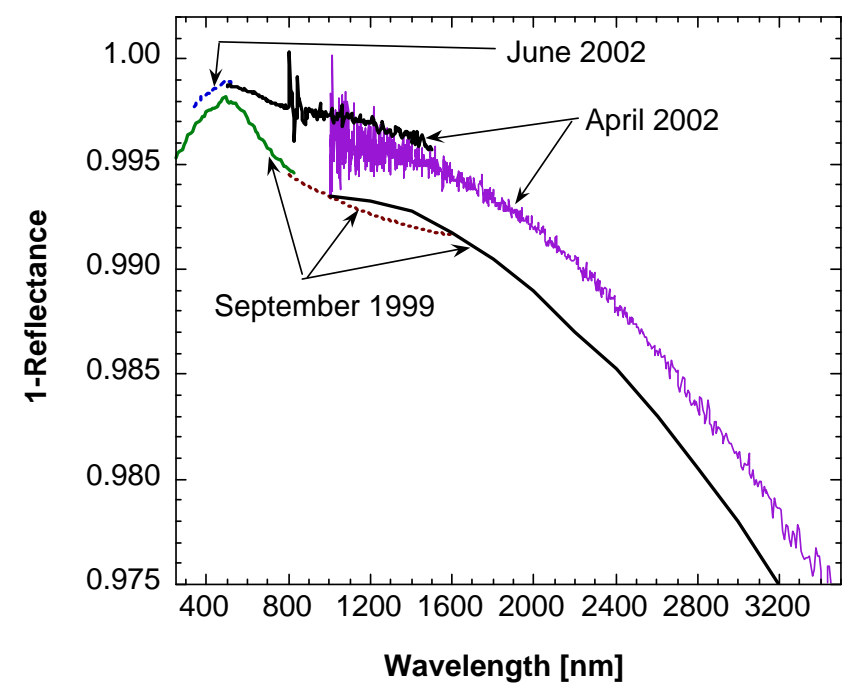

Fig. 3. The absorptance (1-reflectance) versus wavelength of the gold-black coated pyroelectric detector measured at two different times using two different measurement setups.

If we do not improve the absorptance (e.g. by multiple reflections on the detector surface or with coatings of lower reflectance in the IR), the upper wavelength limit for high accuracy scale extension will be $2.5 \mu \mathrm{m}$. The reflectance at this wavelength is $1.27 \%$, about 3 times higher than the $633 \mathrm{~nm}$ value. The uncertainty component at $2.5 \mu \mathrm{m}$, originating from the non-uniformity of the spatial responsivity, will be about $0.3 \%$, equal to the 31 months reflectance change measured on the gold-black coated pyroelectric detector.

\section{SPECTRAL RESPONSIVITY}

The updated 1-reflectance curve is shown on the left Y axis of Fig. 5. This curve includes the April 2002 measurement results between $500 \mathrm{~nm}$ and $2.5 \mu \mathrm{m}$. Since the long-term reflectance change was negligibly small between $325 \mathrm{~nm}$ and $500 \mathrm{~nm}$, the $250 \mathrm{~nm}$ to $500 \mathrm{~nm}$ results measured in September 1999, are also 
included in the curve. The small step at $1.5 \mu \mathrm{m}$ shows the very small difference between the Cary-5E and the FT reflectance measurements made in April 2002. The power responsivity scale is shown on the right $\mathrm{Y}$ axis. The absolute tie point is shown with an open triangle at $442 \mathrm{~nm}$. The tie point was obtained from radiant power measurement on October 1, 1999 directly after the relative spectral responsivity determination. The detector substitution method was used on the SIRCUS facility. Both the pyroelectic radiometer and the reference TS07 silicon (reflectance type) trap detector measured the same $23.6 \mu \mathrm{W}$ total power in a collimated (and chopped) HeCd laser beam. The power of the $442 \mathrm{~nm}$ beam was controlled to a constant value using a laser beam stabilizer. The trap detector was calibrated against the primary standard cryogenic radiometer for radiant power responsivity. The $442 \mathrm{~nm}$ responsivity of the pyroelectric radiometer was $1.010 \times 10^{-7} \mathrm{~A} / \mathrm{W}$ at $10^{9} \mathrm{~V} / \mathrm{A}$ gain and $10.1 \mathrm{~Hz}$ chopping frequency. The duty cycle of the chopped radiation was $50 \%$.

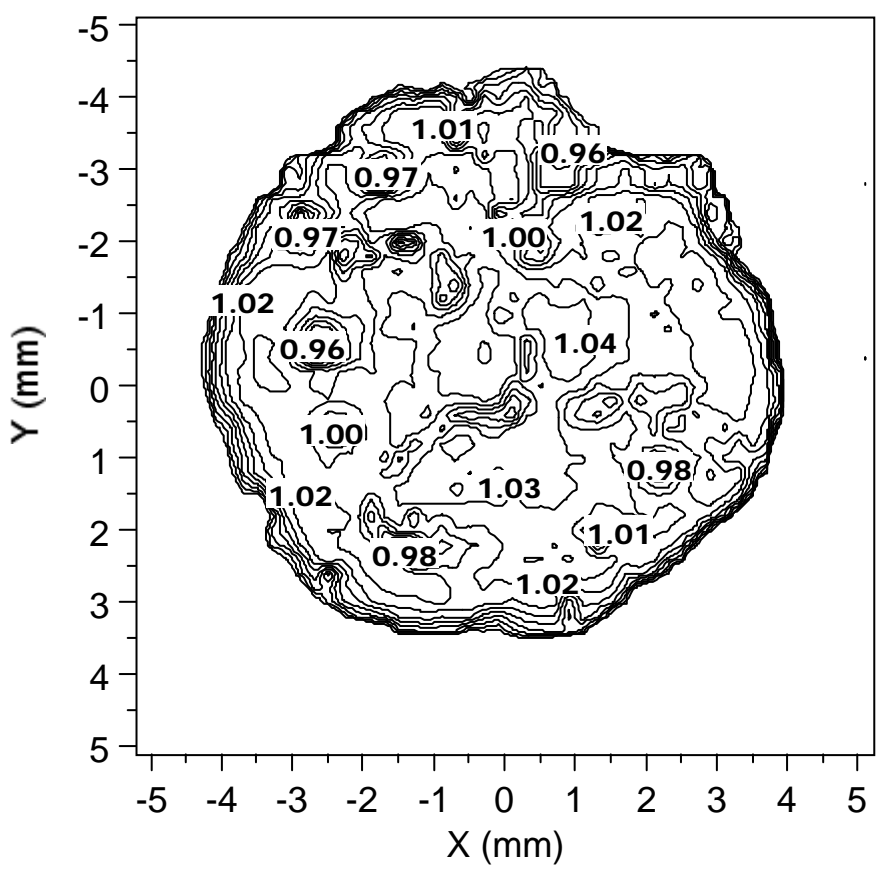

Fig. 4. Spatial response uniformity contour-plot of the gold-black coated $\mathrm{LiNbO}_{3}$ pyroelectric radiometer at $10.6 \mu \mathrm{m}$. The scanning beam diameter was $0.4 \mathrm{~mm}$.

The temperature of the pyroelectric detector was monitored by a thermistor. As the temperature coefficient of the pyroelectric detector responsivity is $0.15 \% /{ }^{\circ} \mathrm{C}$ [7], the responsivity can be corrected for different measurements. The corrected responsivity will be:

$$
\mathrm{R}_{\mathrm{m}}=f \mathrm{R}_{\mathrm{c}}
$$

where $R_{c}$ is the pyroelectric radiometer responsivity at the calibration temperature $T_{c}$ and $f$ is a correction factor. The $f$ correction factor can be calculated from the temperature coefficient of the detector responsivity and the $\Delta \mathrm{T}$ temperature difference:

$$
f=1+(0.0015 \Delta \mathrm{T})
$$

where $\Delta \mathrm{T}=\mathrm{T}_{\mathrm{c}}-\mathrm{T}_{\mathrm{m}} \cdot \mathrm{T}_{\mathrm{m}}$ is the temperature of the detector in a measurement after the responsivity calibration. $\Delta \mathrm{T}$ is determined from thermistor resistance measurements at calibration and then at a given application. The thermistor was attached to the pyroelectric detector [7] and its resistance was previously calibrated versus temperature. 
The pyroelectric radiometer has a $60 \mathrm{~Hz}$ roll-off in the responsivity when the highest gain is selected [7]. Accordingly, the responsivity at a $10 \mathrm{~Hz}$ chopping frequency is not constant. For rotating choppers the frequency has to be controlled to a constant value. If the frequency changes, responsivity corrections are needed to minimize frequency dependent responsivity changes. Better stability was obtained when the rotating chopper was substituted by a tuning fork chopper. The tuning fork chopper was tuned to a stable $10.1 \mathrm{~Hz}$ and frequency dependent responsivity corrections were not needed.

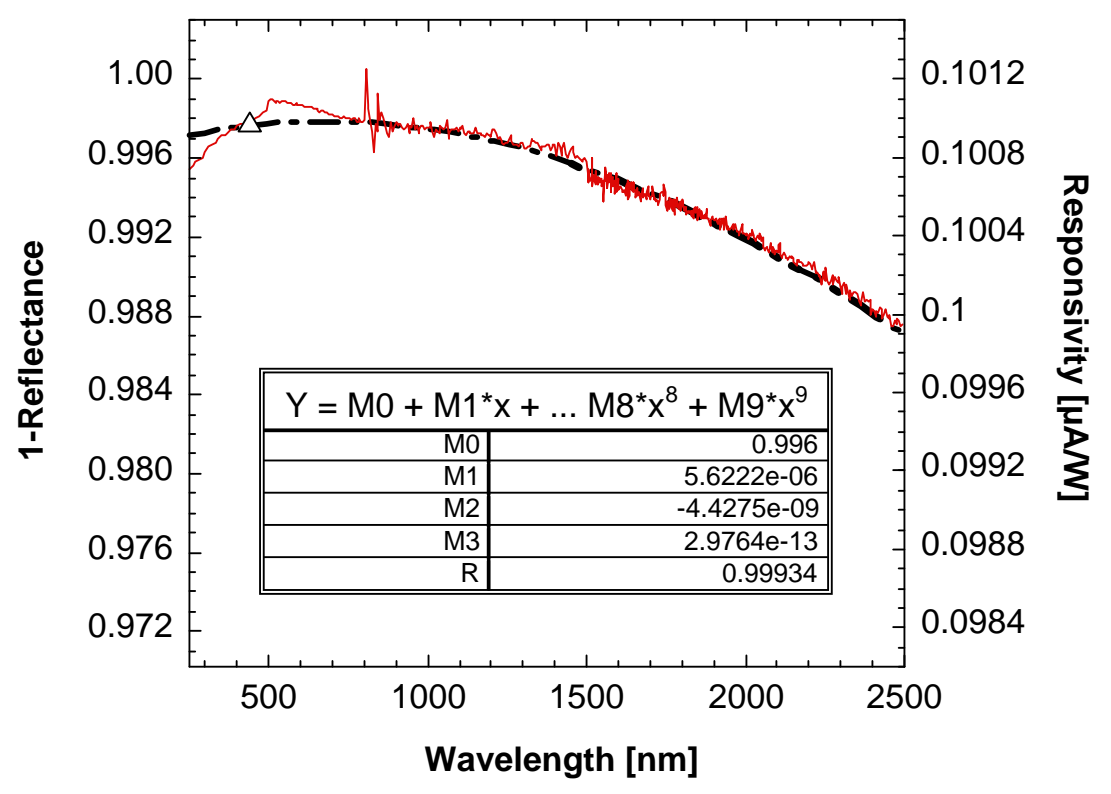

Fig. 5. The April 2002 spectral power responsivity scale of the $\mathrm{LiNbO}_{3}$ pyroelectric detector.

The uncertainty of the trap detector responsivity was $0.05 \%$ [6]. The dashed line in Fig. 5 is a third order polynomial fit to the 1-reflectance curves. The overall responsivity variation of the pyroelectric detector was about $1 \%$ between $250 \mathrm{~nm}$ and $2.5 \mu \mathrm{m}$.

The uncertainty budget of the April 2002 spectral responsivity scale as extended by the $\mathrm{LiNbO}_{3}$ pyroelectric radiometer is shown in Table 1 . The relative combined standard uncertainty of the absolute and relative responsivity calibrations is $0.15 \%$. The non-uniformity of the spatial responsivity was wavelength dependent. The largest overall combined uncertainty of $0.34 \%$ was dominated by the $0.3 \%$ spatial non-uniformity in the responsivity of the pyroelectric detector at $2.5 \mu \mathrm{m}$. The overall combined uncertainty was $0.18 \%$ in the ultraviolet and $0.25 \%$ between $1 \mu \mathrm{m}$ and $2 \mu \mathrm{m}$.

For irradiance responsivity measurements, a black-Nickel coated Copper aperture was mounted in front of the pyroelectric detector. The $0.1 \mathrm{~mm}$ thick aperture was very close to the detector to minimize unwanted signal increase caused by reflections between aperture and detector. The area of the aperture was measured twice. The average area was $49.962 \mathrm{~mm}^{2}$ with an uncertainty of $0.036 \%$. The irradiance responsivity is the product of the aperure area and the spectral power responsivity. The irradiance responsivity at the $442 \mathrm{~nm}$ tie point was $5.05 \times 10^{-6} \mathrm{~A} \mathrm{~mm}^{2} / \mathrm{W}$. The overall combined uncertainties of the irradiance responsivity scale realization are similar to the overall combined uncertainties of the radiant power responsivity scale realization. 
The long-term changes in the extended spectral responsivity scale, maintained by the $\mathrm{LiNbO}_{3}$ pyroelectric transfer standard radiometer, are shown in Table 2 . The $0.3 \%$ long-term change of the spectral responsivity is an uncertainty component that can be separated from the scale realization uncertainties. This component can be decreased by more frequent responsivity scale realizations.

\section{FUTURE SCALE EXTENSION TO THE INFRARED}

Our near-future goal is to extend the high accuracy spectral responsivity scale up to $20 \mu \mathrm{m}$. The high accuracy IR scale is needed for the SIRCUS-IR facility we have started to develop recently.

One way to keep the dominant uncertainty component, the non-uniformity of the spatial responsivity, small is to mount a reflecting dome to the front of the detector. The dome can produce multiple reflections for the incident beam resulting in lower reflectance loss and higher signal absorption. The other way to improve the spatial uniformity is to make better coatings. Earlier made gold-black coatings, selected for low IR reflectance, showed less than $1 \%$ reflectance even at $20 \mu \mathrm{m}$ [2]. Using one or both of the two methods, the uncertainty component caused by the non-uniformity of the spatial responsivity can be significantly decreased.

We have started realizing the high accuracy spectral responsivity scale from $200 \mathrm{~nm}$ to $20 \mu \mathrm{m}$ using three pyroelectric transfer standard radiometers. Two $\mathrm{LiTaO}_{3}$ pyroelectric radiometers equipped with reflecting domes are being developed in addition to the $\mathrm{LiNbO}_{3}$ pyroelectric radiometer discussed in this paper.

Table 1

Uncertainty budget of the April 2002 spectral power/irradiance responsivity scales

Uncertainty factor

Absolute responsivity calibration:

Trap detector spectral power responsivity

Trap detector repeatability

Trap current-to-voltage gain

Laser beam instability

Pyroelectric detector gain

Pyroelectric detector temperature dependent

response (at $0.1^{\circ} \mathrm{C}$ change)

Pyroelectric detector frequency dependent response

with $0.025 \mathrm{~Hz}$ change at $10.1 \mathrm{~Hz}$ and $10^{9} \mathrm{~V} / \mathrm{A}$ gain
Type A

[\%]

0.04

0.02

0.015

0.07

Relative responsivity calibration:

Spectral reflectance measurements

0.1

Relative combined standard uncertainty

0.15

\begin{tabular}{lll}
\hline Pyroelectric detector non-uniformity of spatial responsivity: & & 0.1 \\
$250 \mathrm{~nm}$ to $1 \mu \mathrm{m}$ & 0.2 & 0.18 \\
$1 \mu \mathrm{m}$ to $2 \mu \mathrm{m}$ & 0.3 & 0.25 \\
$2 \mu \mathrm{m}$ to $2.5 \mu \mathrm{m}$ & & 0.34 \\
\hline Irradiance responsivity: & 036 & 0.19 \\
Aperture area & & 0.05 \\
Multiple reflections between aperture and detector & & 0.26 \\
at $2.5 \mu \mathrm{m}$ (worst case) & & 0.34 \\
$250 \mathrm{~nm}$ to $1 \mu \mathrm{m}$ & & \\
$1 \mu \mathrm{m}$ to $2 \mu \mathrm{m}$ & & \\
$2 \mu \mathrm{m}$ to $2.5 \mu \mathrm{m}$ & & \\
\hline
\end{tabular}


Table 2

Long-term (31 months) responsivity changes

for wavelengths longer than $800 \mathrm{~nm}$

from $500 \mathrm{~nm}$ to $800 \mathrm{~nm}$

for wavelengths shorter than $500 \mathrm{~nm}$
$0.3 \%$

0.1 to $0.3 \%$

$0.1 \%$

Using three transfer standard devices, individual long term response changes can be minimized by using the average responsivity of the group. The dominant uncertainty component of the total uncertainty will come from the spectral reflectance measurements. According to our preliminary measurements, the reflectance with a dome and improved coating can be less than $0.25 \%$ at $19 \mu \mathrm{m}$. The small non-uniformity of the spatial responsivity, caused by the low reflectance, will result in an uncertainty component of less than $0.1 \%$. At present, the temperature of the $\mathrm{LiNbO}_{3}$ pyroelectric detector is monitored for temperature dependent responsivity corrections. The temperature of the $\mathrm{LiTaO}_{3}$ pyroelectric detectors is controlled to a constant value. Accordingly, the temperature dependent uncertainty of the responsivity will be much smaller than $0.1 \%$. The signal will be chopped with a tuning-fork chopper to minimize frequency dependent responsivity changes. This is an important requirement for high signal-gain selections where the signal roll-off of the photocurrent meters is at low (about $60 \mathrm{~Hz}$ ) frequencies. Our realistic goal is to achieve an overall responsivity scale uncertainty of about $0.15 \%$ outside of the $406 \mathrm{~nm}$ to $920 \mathrm{~nm}$ reference range.

\section{CONCLUSIONS}

The NIST high accuracy spectral responsivity scale from the $406 \mathrm{~nm}$ to $920 \mathrm{~nm}$ reference range was extended to a wider range of $250 \mathrm{~nm}$ to $2500 \mathrm{~nm}$. The extension was made with a NIST developed high performance $\mathrm{LiNbO}_{3}$ pyroelectric transfer standard radiometer. As the transmission of the detector goldblack coating was negligibly small, the absorptance, equal to 1-reflectance, was proportional to the relative responsivity of the radiometer. Spectral reflectance measurements were made with an uncertainty of $0.1 \%$. The measurements were made twice, with 31 months separation. During this time, an $0.3 \%$ constant reflectance decrease was obtained between $800 \mathrm{~nm}$ and $19 \mu \mathrm{m}$. The $0.1 \%$ non-uniformity of the spatial responsivity at $633 \mathrm{~nm}$, where the reflectance is $0.4 \%$, increased to $8 \%$ at $10.6 \mu \mathrm{m}$ where $28 \%$ reflectance was measured. The large non-uniformity of the spatial responsivity limited the power responsivity scale extension to $2.5 \mu \mathrm{m}$. The relative spectral responsivity was converted into spectral power and irradiance responsivities by making an absolute tie point at $442 \mathrm{~nm}$, where no long-term reflectance change was measured. In contrast to the $0.5 \%$ to $1.8 \%$ uncertainty of the present NIST (extended) responsivity scales, the uncertainty of the new wavelength-extended scales was less than $0.34 \%$.

As a continuation of the above scale realization work, the high accuracy spectral responsivity scale is being extended to $20 \mu \mathrm{m}$ using two additional NIST developed $\mathrm{LiTaO}_{3}$ pyroelectric radiometers. The two new radiometers have improved gold-black coatings and are equipped with reflecting domes to further increase absorption and uniformity of spatial responsivity. While the temperature of the $\mathrm{LiNbO}_{3}$ detector was monitored, the $\mathrm{LiTaO}_{3}$ detectors are temperature controlled to minimize temperature dependent response changes. The group average will be used to decrease the effect of individual response changes. The expected uncertainty of the planned responsivity scale extension is about $0.15 \%$ from $200 \mathrm{~nm}$ to $20 \mu \mathrm{m}$.

\section{ACKNOWLEDGEMENTS}

The authors thank John Lehman for developing the high performance pyroelectric detectors, Haimei Gong for participating in the spatial responsivity characterizations, Edward Early and Simon Kaplan for their 
participation in the spectral reflectance measurements. This work was supported by the U. S. Air Force Metrology under contract numbers CCG-461 and CCG-439.

\section{REFERENCES}

1. Eppeldauer G. and Racz M., Spectral power and irradiance responsivity calibration of InSb workingstandard radiometers, Applied Optics, Vol. 39, No. 31, p. 5739-5744, 2000.

2. Migdall A. and Eppeldauer G., Realization of an Infrared Spectral Radiant Power Response Scale on a Cryogenic Bolometer, Metrologia, Vol. 35, p. 307-315, 1998.

3. Shaw P-S., Larason T. C., Gupta R, Brown S. W., Vest R. E., and Lykke K. R., The new ultraviolet spectral responsivity scale based on cryogenic radiometry at Synchrotron Ultraviolet Radiation Facility III, Rev, Sci. Instr., Vol. 72, No. 5, p. 2242-2247, 2001.

4. Shaw P-S., Larason T. C., Gupta R, Brown S. W., and Lykke K. R., Improved near-infrared spectral responsivity scale, J. Res. NIST, Vol. 105, No. 5, p. 689-700, 2000.

5. Gentile T. R., Houston J. M., and Cromer C. L., Realization of a scale of absolute spectral response using the NIST high accuracy cryogenic radiometer, Appl. Opt. Vol. 35, No. 22, p. 4392-4403, 1996.

6. Brown S. W., Eppeldauer G. P., and Lykke K. R., NIST Facility for Spectral Irradiance and Radiance Response Calibrations with a Uniform Source, Metrologia, Vol. 37, p. 579-582, 2000.

7. Lehman J., Eppeldauer G., Aust J. A., and Racz M., Domain-engineered pyroelectric radiometer, Applied Optics, Vol. 38, No. 34, p. 7047-7055, 1999. 


\title{
Spectral irradiance responsivity measurements between $1 \mu \mathrm{m}$ and $5 \mu \mathrm{m}$
}

\author{
G. P. Eppeldauer, J. P. Rice, J. Zhang, and K. R. Lykke \\ Optical Technology Division \\ National Institute of Standards and Technology \\ Gaithersburg, Maryland, 20899-8441
}

\begin{abstract}
Infrared radiometers for irradiance measurement have been developed at the National Institute of Standards and Technology (NIST). These high performance irradiance meters are used to realize and maintain the spectral irradiance responsivity scale between $1 \mu \mathrm{m}$ and $5 \mu \mathrm{m}$. They are also working standards that disseminate the infrared irradiance responsivity scale to other institutions and facilities. Both design considerations and responsivity scale transfer to the irradiance meters are discussed. The radiometers were calibrated for spectral irradiance responsivity on the new NIST Infrared Facility for Spectral Irradiance and Radiance Responsivity Calibrations using Uniform Sources (IR-SIRCUS). The spectral irradiance responsivity calibrations described are derived from absolute cryogenic radiometers.
\end{abstract}

\section{INTRODUCTION}

In addition to InGaAs, Ge [1, 2], and InSb [3] working standard radiometers developed earlier, extended-InGaAs (EIGA) radiometers have been developed at the National Institute of Standards and Technology (NIST) to fill a gap between $1.8 \mu \mathrm{m}$ and $2 \mu \mathrm{m}$ in the infrared (IR) spectral responsivity scale. The radiometers can measure irradiance from about $0.8 \mu \mathrm{m}$ to $5.1 \mu \mathrm{m}$. The InGaAs irradiance meter was calibrated earlier [4] against the NIST detector spectral power responsivity scale [5] using the raster scan method [6]. The EIGA radiometer has been calibrated against an Electrical Substitution Bolometer (ESB) [7] that was calibrated against an electrical substitution type absolute cryogenic radiometer (ACR) using silicon trap detectors [8]. The InSb radiometers were calibrated earlier against a cryogenic bolometer [9] that was calibrated against the primary standard cryogenic radiometer (HACR) [10-12] of NIST.

At present, on the Infrared Facility for Spectral Irradiance and Radiance Responsivity Calibrations using Uniform Sources (IR-SIRCUS) [13], spectral irradiance responsivity measurements are performed between $1 \mu \mathrm{m}$ and $5 \mu \mathrm{m}$ with an extended uncertainty $(k=2)$ of $3 \%$ using the above infrared radiometers. The irradiance responsivity scale transfers to both the EIGA radiometer and selective InSb radiometers are described below.

\section{DESIGN OF THE EXTENDED-InGaAs RADIOMETER}

The EIGA radiometer can measure irradiance between $1 \mu \mathrm{m}$ and $2.5 \mu \mathrm{m}$. The design of the radiometer head that includes the input optics and housing is shown in Fig. 1. The $3 \mathrm{~mm}$ diameter photodiode is located in a sealed case. The case has a borosilicate window on the front. The window, a flashed opal glass (transmitting) diffuser, and a thin aperture are glued together (at the edges) and are plugged into an aluminum holder. There is a thread on the front side of the holder that makes it possible to either apply a protecting cap or to screw the whole radiometer head into a flange located on the top of the sphere source. The holder and the bottom surface of the photodiode-case are attached to a copper plate that delivers the dissipated heat to a heat sink. A thermo-electric (TE) cooler and a thermistor are also located inside of the photodiode-case to apply temperature controlled cooling for the photodiode. The leads from the TE cooler and the thermistor are connected to a 4-pin connector at the bottom of the aluminum housing. A temperature controller can be connected to this connector through a shielded cable. The operating temperature of the photodiode is $-20{ }^{\circ} \mathrm{C}$. The photodiode leads are connected to a BNC connector located also on the bottom plate of the housing. The preamplifier (discussed below) can be connected to the BNC connector using a short coaxial cable. The housing of the radiometer head is electrically shielded. 


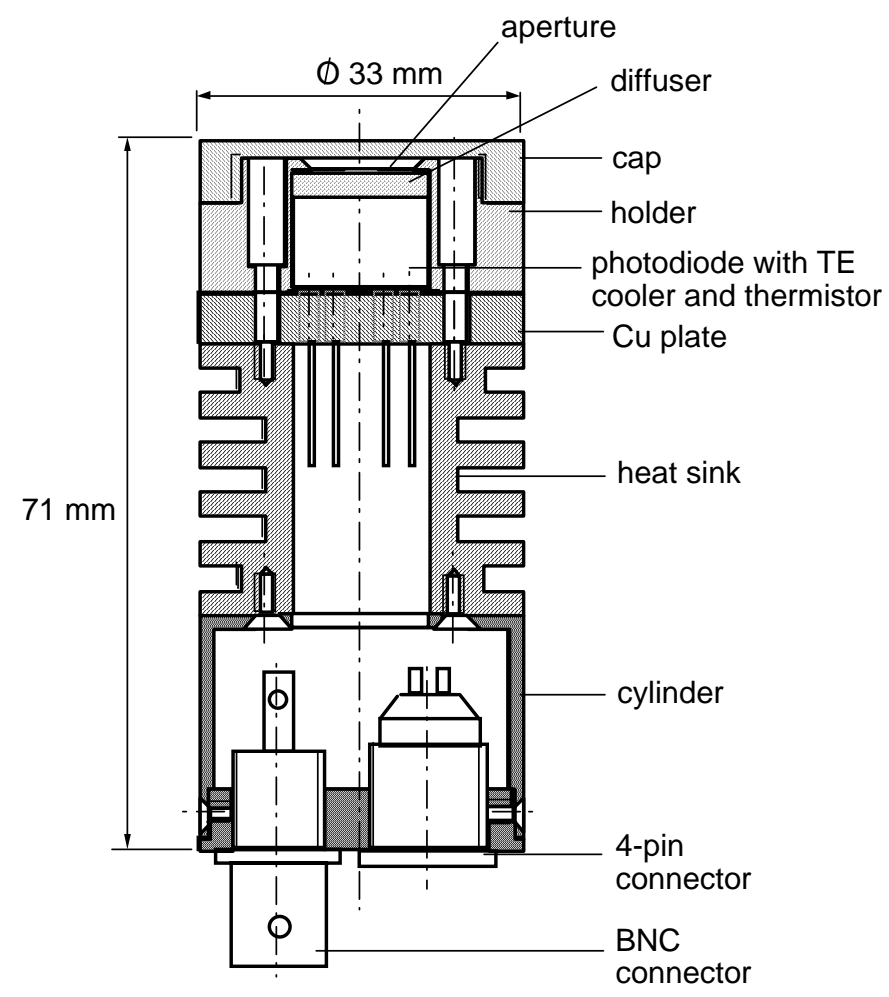

Fig. 1. Housing of the extended-InGaAs radiometer head.

\section{ELECTRONIC DESIGN CONSIDERATIONS}

A short-circuit current measuring preamplifier was needed that has a small enough input resistance for the low shunt resistance EIGA photodiode. It was also necessary to measure chopped radiation when the irradiance responsivity scale was derived from the ESB. The simplified circuit diagram of the photodiode current meter is shown in Fig. 2.

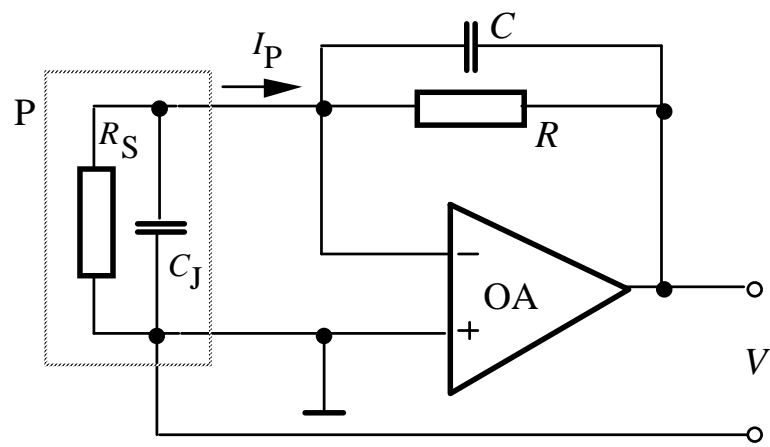

Fig. 2. Simplified circuit diagram of the EIGA photodiode preamplifer.

The photocurrent $I_{\mathrm{P}}$ of photodiode P is measured by the current-meter. The current-to-voltage conversion in the current meter is realized by an operational amplifier OA that has a feedback impedance $Z$ (parallel connected feedback resistor $R$ and capacitor $C$ ). The OA has a low-frequency (DC) open-loop gain of about $A=10^{6}$. The OA output voltage $V$ is attenuated to its input by $A$, resulting in a very small voltage drop on photodiode $\mathrm{P}$. The photodiode impedance $Z_{\mathrm{P}}$ is the 
parallel connection of its shunt resistance $R_{\mathrm{S}}$ and junction capacitance $C_{\mathrm{J}}$. The input impedance of the current meter will be [14]:

$$
Z_{\mathrm{I}}=\frac{Z}{A}=\frac{R}{A} \frac{1}{(1+j \omega C R)}=\frac{1}{\frac{A}{R}+j \omega A C}
$$

where $\omega$ is the angular frequency. The rewritten form of Eq. (1) shows that the input impedance of the current meter can be calculated as the parallel connection of the down-transformed $R$ and the up-transformed $C$ :

$$
\left(Z_{\mathrm{I}}\right)^{-1}=\left(\frac{R}{A}\right)^{-1}+\left(\frac{1}{j \omega A C}\right)^{-1}
$$

The requirement for short-circuit current measurements is:

$$
Z_{\mathrm{I}}<<Z_{\mathrm{P}}
$$

The shunt impedance of the photodiode determines not only the linearity of photocurrent measurements but also the voltage gain (amplification) for the input drift and noise of the amplifier [15]. Also, the shunt resistance can dominate the uncertainty of the photocurrent-to-voltage conversion [16]:

$$
\frac{V}{I_{\mathrm{P}}}=R \frac{1}{1+G^{-1}}
$$

where $G$ is the loop gain. The photocurrent-to-voltage conversion $R$, which is the signal gain at zero frequency, will be accurate only if $G>>1$. When chopped radiation is measured, this condition must be achieved for the signal (chopping) frequency.

The loop gain, which is the product of $A$ and $\beta$ (the feedback attenuation), can be written as [14]:

$$
G=A_{0} \beta_{0} \frac{1}{1+j \omega \tau_{\mathrm{i}}} \frac{1+j \omega \tau}{1+j \omega \tau_{2}}
$$

where $A_{0}$ is the $0 \mathrm{~Hz}$ (DC) open-loop voltage gain of the OA and $\beta_{0}=R_{\mathrm{S}} /\left(R_{\mathrm{S}}+R\right)$ is the DC feedback attenuation. The inverse feedback attenuation $1 / \beta$ is equal to the voltage gain for the input noise and drift. If a photodiode with high $R_{\mathrm{S}}$ is selected, the feedback attenuation $\beta_{0}$ will be close to unity, resulting in high $G$ and low voltage gain for noise and drift. The feedback resistors $R$ of the OA can change in a wide range, from $1 \mathrm{k} \Omega$ to $100 \mathrm{M} \Omega$ to measure photocurrent in a wide dynamic range.

The shunt resistance of the $3 \mathrm{~mm}$ diameter EIGA photodiode is only a few hundred on $\mu$ at room temperature. At the operating temperature of $-20^{\circ} \mathrm{C}$, we measured $25 \mathrm{k} \Omega$ and $40 \mathrm{k} \Omega$ shunt resistances for two photodiodes, respectively. The junction capacitance was $C_{\mathrm{J}}=1.8 \mathrm{nF}$. $C_{\mathrm{J}}$ will be increased significantly by the parallel connected, up-transformed feedback capacitor $A C$.

In short-circuit current measurements, when photodiode with low junction capacitance is selected, the measurement time constant is determined by the feedback impedance:

$$
\tau=\frac{\left(C_{\mathrm{J}}+A C\right) R}{A}=R C .
$$


As a design example, in Eq. 5, the differentiating time constant $\tau$ can cancel the integrating time constant $\tau_{2}$. This is called full frequency compensation. The time constant $\tau_{2}=R_{\mathrm{S}} C_{\mathrm{J}}$ when $R>>R_{\mathrm{S}}$ [14]. In our case, for $R_{\mathrm{S}}=25 \mathrm{k} \Omega$, $\tau_{2}=45 \mu \mathrm{s}$, and for $R_{\mathrm{S}}=40 \mathrm{k} \Omega, \tau_{2}=72 \mu \mathrm{s}$.

At the maximum gain of $R=10^{8} \Omega$, the input resistance, equal to $R_{\mathrm{I}}=100 \Omega$, will result in a non-linearity in the photocurrent measurement of $0.4 \%$ for $R_{\mathrm{S}}=25 \mathrm{k} \Omega$ and $0.25 \%$ for $R_{\mathrm{S}}=40 \mathrm{k} \Omega$. At $R=10^{7} \Omega$, the non-linearity will decrease to significantly less than $0.1 \%$.

As $A C>>C_{\mathrm{J}}$ for $C_{\mathrm{J}}=1.8 \mathrm{nF}$, a measurement time constant (for full frequency compensation) of $\tau=R C=72 \mu \mathrm{s}$ could be obtained at $R=10^{8} \Omega$ if $C$ was equal to $0.7 \mathrm{pF}$. However, it is difficult to achieve such a low parallel capacitance. A realistic stray (parallel) capacitance in a well designed circuit layout is about $2 \mathrm{pF}$. Consequently, only partial frequency compensation can be achieved at the highest signal gain of $R=10^{8} \Omega$, which will result in a $3 \mathrm{~dB}$ roll-off frequency of 0.8 $\mathrm{kHz}$. (No external capacitor will be connected parallel to $R=10^{8} \Omega$.) However, at $R=10^{7} \Omega$, the $45 \mu \mathrm{s}$ (or $72 \mu \mathrm{s}$ ) compensating $R C$ time constant can be achieved. $C=4.5 \mathrm{pF}$ will result in a signal $3 \mathrm{~dB}$ roll-off frequency of $3.5 \mathrm{kHz}$. After this full compensation, the dominating integrating time constant of $G$ will be $\tau_{\mathrm{i}}$ from the $f_{\mathrm{i}}=10 \mathrm{~Hz}$ roll-off frequency of the OA open-loop voltage gain A. This roll-off frequency is very convenient to obtain high $G$ for all $R$ at a chopping frequency of about $10 \mathrm{~Hz}$. Usually, $R$ is changed in decade steps to cover a wide dynamic signal range. The parallel $C$ capacitors can be increased in decade steps (from $5 \mathrm{pF}$ at $10^{7} \Omega$ ) with the decade step decrease of $R$ (from $10^{7} \Omega$ ) to perform frequency compensations for all $R$. Care should be made at low $R$ selections where the starting condition of $R>>R_{\mathrm{S}}$ cannot be achieved anymore. At these low gain selections, the full frequency compensations can be achieved only when $\tau_{2}$ is correctly calculated from the non-simplified equation [14]. Otherwise, partial frequency compensations will be performed that are still adequate to perform high enough $G$ at the signal (chopping) frequency at these signal gain selections.

The OA has to be selected to satisfy the noise, drift, input current, and bandwidth requirements [16].

\section{IRRADIANCE RESPONSIVITY SCALE TRANSFER TO $2.5 \mu \mathrm{m}$}

The reference spectral irradiance responsivity scale from a silicon trap detector that was tied to an ACR was transferred to a cryogenically cooled ESB. The ESB operates at liquid helium temperature and uses a chopper [7]. In the open position of the chopper, the optical radiation overfills the $5 \mathrm{~mm}$ diameter precision aperture of the ESB. This radiation is converted into heat on the gold-black-coated silicon bolometer. Through the action of an electronic servo loop, this heat is equalized with the electrical heat applied to the same bolometer in the closed position of the chopper. In equilibrium, the electrical heat power (output signal) is equal to the radiant power originating from the irradiance (input signal) of the overfilled aperture.

The relative spectral responsivity of the ESB was determined from two spectral measurements. The directionalhemispherical spectral reflectance of the gold black coated bolometer surface was measured with an integrating sphere [17]. The absorption of the bolometer, equal to one minus reflectance, is proportional to its responsivity. The spectral absorption of the bolometer was multiplied by the spectral transmittance of the ESB dewar window to obtain the overall relative spectral responsivity. Three tie points from a silicon trap detector between $738 \mathrm{~nm}$ and 820 nm were measured. One of these (at $790.4 \mathrm{~nm}$ ) was used to convert the relative spectral responsivity into absolute spectral irradiance responsivity. The other two were used to assess the uncertainty component due to choice of tie point wavelength. Figure 3 shows the spectral irradiance responsivity of the ESB with the tie point at $790.4 \mathrm{~nm}$. Curve fits were applied to the measured relative spectral response data. An $8^{\text {th }}$ order polynomial fit was applied below $1800 \mathrm{~nm}$ and a $4^{\text {th }}$ order polynomial fit was used above. The broad, smooth variations are caused by the spectral transmittance change of the coated $\mathrm{KBr}$ window of the ESB dewar.

For the ESB irradiance measurements, a $15 \mathrm{~Hz}, 50 \%$ duty-cycle chopper was used to modulate the laser beam introduced into an integrating sphere. The sphere exit port was used as a uniform monochromatic irradiance source. Both the ESB and the trap detector measured the irradiance from the sphere source. For the trap-detector measurements, the chopper was stopped in its open position and the measurements were made in DC mode. The demodulated DC output of the ESB and the DC output of the trap-detector were read out by a digital voltmeter. 


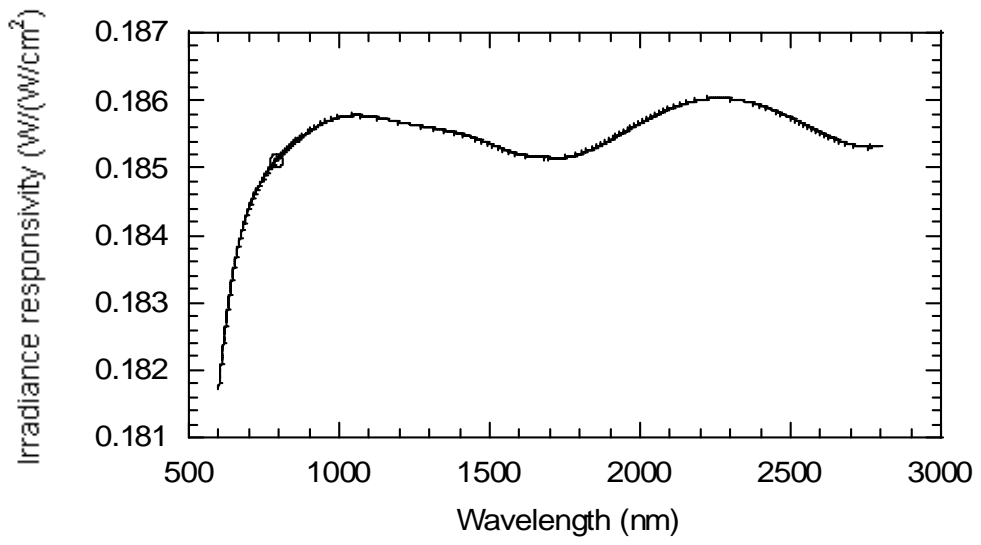

Fig. 3. ESB spectral irradiance responsivity

The ESB responds to the amplitude of the chopped radiation, which is the DC level that is present when the chopper is stopped. Thus, no chopper correction needs to be applied to the ESB output signal. Since the trap-detector was used in DC mode, no chopper correction needs to be applied to the trap-detector responsivity either.

For the tie points, irradiance responsivity measurements of the ESB and trap-detector to the same sphere radiant intensity, $I_{0}$, were made and recorded. The radiant intensity of the sphere is independent of which detector is viewing the sphere:

$$
I_{\mathrm{o}}=E_{\mathrm{t}} d_{\mathrm{t}}^{2}=E_{\mathrm{e}} d_{\mathrm{e}}^{2}
$$

where $E_{\mathrm{e}}$ is the irradiance on the ESB, $d_{\mathrm{t}}$ is the separation between source and trap-detector apertures, and $d_{\mathrm{e}}$ is the separation between source and ESB apertures. The irradiance $E_{\mathrm{t}}$ at the trap-detector is known from the measured output signal $Y_{\mathrm{t}}$, the known responsivity $\mathrm{s}_{\mathrm{t}}$, the gain $G_{\mathrm{t}}$, and the aperture area $A_{\mathrm{t}}$ of the trap detector:

$$
E_{\mathrm{t}}=\frac{Y_{\mathrm{t}}}{G_{\mathrm{t}} s_{\mathrm{t}} A_{\mathrm{t}}}
$$

Combining Eqs. (7) and (8) leads to the measurement equation for the ESB irradiance responsivity:

$$
s_{\mathrm{e}}=\frac{P_{\mathrm{e}}}{E_{\mathrm{e}}}=\frac{P_{\mathrm{e}}}{Y_{\mathrm{t}}} G_{\mathrm{t}} s_{\mathrm{t}} A_{\mathrm{t}} \frac{d_{\mathrm{e}}^{2}}{d_{\mathrm{t}}^{2}}
$$

where $P_{\mathrm{e}}$ is the ESB output signal (electric power). In practice, $s_{\mathrm{e}}$ is given in $\mathrm{W} / \mathrm{Wcm}^{-2}, P_{\mathrm{e}}$ in $\mathrm{W}$ (the product of the measured V and A of the ESB electrical heater), $E_{\mathrm{e}}$ in W/cm ${ }^{2}, Y_{\mathrm{t}}$ in V, $G_{\mathrm{t}}$ in V/A, $s_{\mathrm{t}}$ in A/W, $A_{\mathrm{t}}$ in $\mathrm{cm}^{2}, d_{\mathrm{e}}$ and $d_{\mathrm{t}}$ in $\mathrm{cm}$.

The spectral irradiance responsivity from the ESB was transferred to the EIGA\#2 working standard radiometer. The irradiance responsivity scale transfer is shown below.

Both the ESB and the detector-under-test (DUT, EIGA\#2 in this case) measured the same radiant intensity from the laser-illuminated integrating sphere at the IR-SIRCUS facility. The chopping frequency was again $15 \mathrm{~Hz}$. Similarly to Eq. (7): 


$$
I_{\mathrm{o}}=E_{\mathrm{e}} d_{\mathrm{e}}^{2}=E_{\mathrm{d}} d_{\mathrm{d}}^{2}
$$

where subscript d stands for DUT.

Earlier, the ESB responsivity was referenced to the amplitude of the chopped radiation. In the present scale transfer, both the DUT and the monitor were photodiodes. Since they were chopped during measurements, a chopper correction must be applied to reference their values to the amplitude of the chopped radiation. That is for the ESB,

$$
P_{\mathrm{e}}=s_{\mathrm{e}} E_{\mathrm{e}}
$$

but for the DUT, as read out by a lock-in amplifier,

$$
Y_{\mathrm{d}}=\frac{\sqrt{2}}{\pi} s_{\mathrm{d}} G_{\mathrm{d}} E_{\mathrm{d}}
$$

where $Y_{\mathrm{d}}$ is the output signal of the DUT (EIGA\#2). The pre-factor appears because the lock-in amplifier indicates the rms value of the fundamental Fourier sine-wave component [18] of a $50 \%$ duty-cycle square-wave applied to its input. $G_{\mathrm{d}}$ is the gain of the DUT current-to-voltage converter.

Combining Eqs. (10) through (12) leads to the measurement equation for the EIGA\#2 irradiance responsivity:

$$
s_{\mathrm{d}}=\frac{\pi}{\sqrt{2}} \frac{Y_{\mathrm{d}}}{P_{\mathrm{e}}} s_{\mathrm{e}} \frac{d_{\mathrm{d}}^{2}}{G_{\mathrm{d}} d_{\mathrm{e}}^{2}}
$$

Since the same monitor photodiode was used during both the ESB and the DUT output signal measurements, and output signal ratios were consistently used at IR-SIRCUS, we use a form of the measurement equation written in terms of output signal ratios:

$$
s_{\mathrm{d}}=\frac{\pi}{\sqrt{2}} \frac{Y_{\mathrm{d}} / Y_{\mathrm{m}}}{P_{\mathrm{e}} / Y_{\mathrm{m}}} s_{\mathrm{e}} \frac{d_{\mathrm{d}}^{2}}{G_{\mathrm{d}} d_{\mathrm{e}}^{2}}
$$

where $Y_{\mathrm{m}}$ represents the monitor output signal. The spectral irradiance responsivity of the EIGA\#2 was derived from the ESB in the overall wavelength range.

The spectral irradiance responsivity of the EIGA\#2 working standard is shown in Fig. 4. Interpolations were applied between the measured data points to obtain a continuous curve. Cubic spline interpolation was applied above $1800 \mathrm{~nm}$ and smooth-type interpolation was used for the shorter wavelength range (using KaleidaGraph Data Analysis and Graphic Presentation [19] software). The effect of the atmospheric absorptions bands on the spectral responsivity of the EIGA\#2 cannot be seen because the separations $d_{\mathrm{d}}$ and $d_{\mathrm{e}}$ were roughly equal during the responsivity scale transfer.

At present, there are not enough data points for the overall wavelength range to perform accurate responsivity interpolation everywhere. The largest gap is between $1989 \mathrm{~nm}$ and $2253 \mathrm{~nm}$ where the measurements were switched from using the signal output to the idler output of an optical parametric oscillator (OPO) based on a periodically-poled lithium niobate (PPLN) crystal. This OPO was pumped by a $1.064 \mu \mathrm{m}$ quasi-cw (220 MHz, $7 \mathrm{ps)} \mathrm{mode-locked}$ $\mathrm{Nd}: \mathrm{YVO}_{4}$ laser and the wavelength was tuned by changing the grating period and the temperature of the crystal. Also, the interpolation is poor around $2.4 \mu \mathrm{m}$ where the slope of the responsivity change is steep. 
The relative expanded uncertainty (coverage factor $k=2$ ) of the ESB spectral irradiance responsivity calibration) is $0.6 \%$. The uncertainty budget that applies only for the irradiance responsivity tie points of the working standard irradiance meter EIGA\#2 is shown in Table 1.

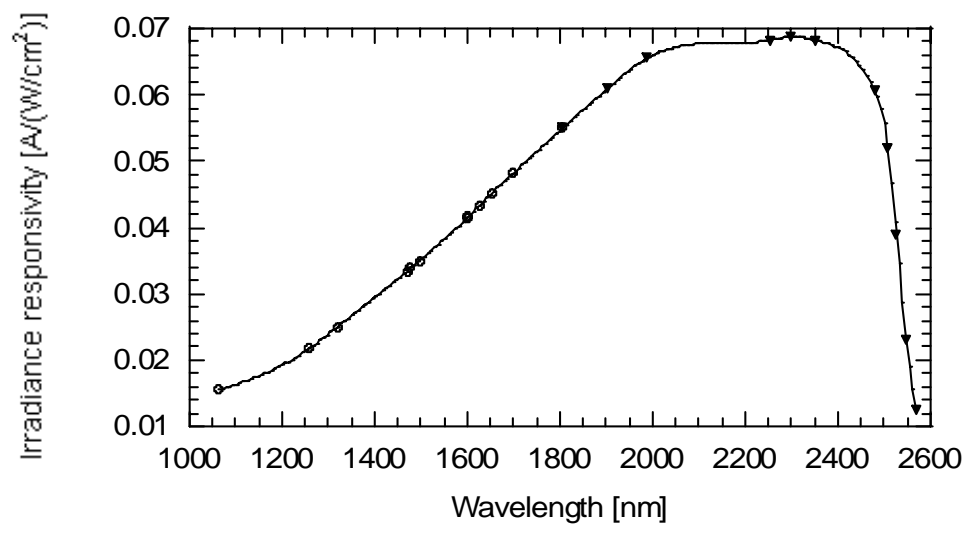

Fig. 4. Spectral irradiance responsivity of the EIGA\#2 working standard.

The uncertainty of the ESB output signal / monitor output signal dominated the uncertainty budget in Table 1 because of the different time constants in the ESB and the monitor-detector.

Table 1

Uncertainty budget of the irradiance responsivity tie points of the working standard EIGA\#2 irradiance meter

Uncertainty component

Type A Type B

[\%] [\%]

Absolute responsivity calibration:

$\begin{array}{ll}\text { ESB position } & 0.23 \\ \text { DUT position } & 0.3\end{array}$

ESB irradiance responsivity

0.3

ESB output signal/Monitor output signal

0.89

DUT output signal/Monitor output signal $\quad 0.37$

DUT lockin amplifier measurement

0.13

Relative combined standard uncertainty

$1.1 \%$

Relative expanded uncertainty with $k=2$

$2.2 \%$

\section{IRRADIANCE RESPONSIVITY MEASUREMENTS TO $5 \mu \mathrm{m}$}

Selective InSb radiometers were calibrated for spectral irradiance responsivity on the IR-SIRCUS as well. The irradiance source this time was a $2.5 \mathrm{~cm}$ diameter Infragold-LF coated integrating sphere with an exit-port diameter of $6 \mathrm{~mm}$. The output beam from the PPLN OPO was tunable between $1466 \mathrm{~nm}$ and $4920 \mathrm{~nm}$. The beam was always chopped at $13 \mathrm{~Hz}$ before the entrance of the sphere source. Two baffles were located between the sphere source and the moving stage 
where the reference and test (selective InSb) radiometers were mounted. The reference detector was the InSb1 irradiance meter calibrated earlier against a transfer standard cryogenic bolometer [9] that was calibrated against the HACR. The radiometers were placed at $\approx 1.1 \mathrm{~m}$ from the sphere source. The baffles minimized the stray radiation on the detectors. A monitor radiometer was mounted in front of the first baffle close to the optical axis but outside of the baffle opening. The output voltages from the current-to-voltage converters of the reference, test, and monitor radiometers were measured by sine-wave measuring digital lock-in amplifiers. The three lock-in amplifiers were attached to the computer of the IRSIRCUS facility.

The selective InSb radiometers were calibrated against the InSb1 radiometer using the substitution method described above for the EIGA\#2 calibration. The difference between this and the above substitution method is that here both the reference and test radiometers are measured by the same kind of lock-in amplifier while above only the test radiometer (EIGA\#2) signal was measured with a lock-in amplifier and the ESB was not. In this substitution, the lock-in gain factors are cancelled and the pre-factor (in Eqs. 13 and 14) is not needed in the measurement equation. In all measurements, both the test and reference radiometer signals were divided by the signal of the monitor detector. The ratio measurements were repeated 20 times at each wavelength to reduce the uncertainty contribution from the noise in the detector signals.

The irradiance responsivity of the test detector is:

$$
s_{\mathrm{d}}=\frac{Y_{d}}{Y_{r}} s_{\mathrm{r}} \frac{G_{r} d_{\mathrm{d}}^{2}}{G_{\mathrm{d}} d_{\mathrm{r}}^{2}}
$$

where subscript $r$ stands for reference and subscript $d$ for DUT.

The irradiance responsivity determination is made at each laser wavelength using this measurement equation.

The spectral irradiance responsivity curves of the selective InSb radiometers are shown in both linear (left vertical axis) and logarithmic (right vertical axis) scales in Figs. 5 to 7. The dominant uncertainty of the spectral responsivity determinations originated from the effect of laser intensity noise. The relative expanded uncertainty $(k=2)$ of the InSb spectral irradiance responsivities is $3 \%$ at all wavelengths. The uncertainty is determined from the $2.05 \%(k=2)$ responsivity uncertainty of the InSb1 reference irradiance meter [9], and additional uncertainty components. The additional components are the residuals of the source intensity fluctuations, photocurrent measurement uncertainty, gain switching uncertainty, stray radiation of irradiance measurements, length (detector-to-source separation) measurements, wavelength measurements, interpolation uncertainties, and background dominated detector noise.

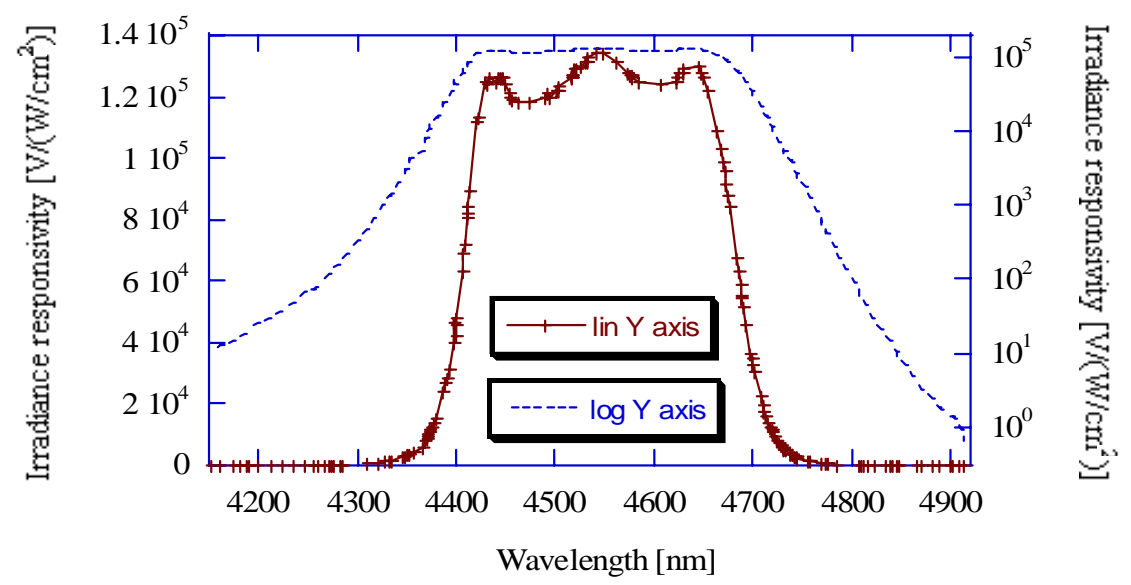

Fig. 5. Spectral irradiance responsivity of InSb\#5 at $2 \times 10^{5} \mathrm{~V} / \mathrm{A}$ gain 
The EIGA\#2 was calibrated against the InSb1 reference radiometer at $2.35 \mu \mathrm{m}$ to compare the above two different irradiance responsivity scales (where they overlap). The expanded relative uncertainty of this responsivity determination was $3 \%$ (same as for the selective InSb irradiance meters). The responsivity obtained was $2.6 \%$ higher than the $0.0682 \mathrm{~A} /\left(\mathrm{Wcm}^{-2}\right)$ EIGA\#2 responsivity derived from the ESB. This responsivity deviation is in accordance with the 2.2 $\%$ (in Table 1) and $3 \%$ relative expanded uncertainties of the two different EIGA\#2 responsivity determinations.

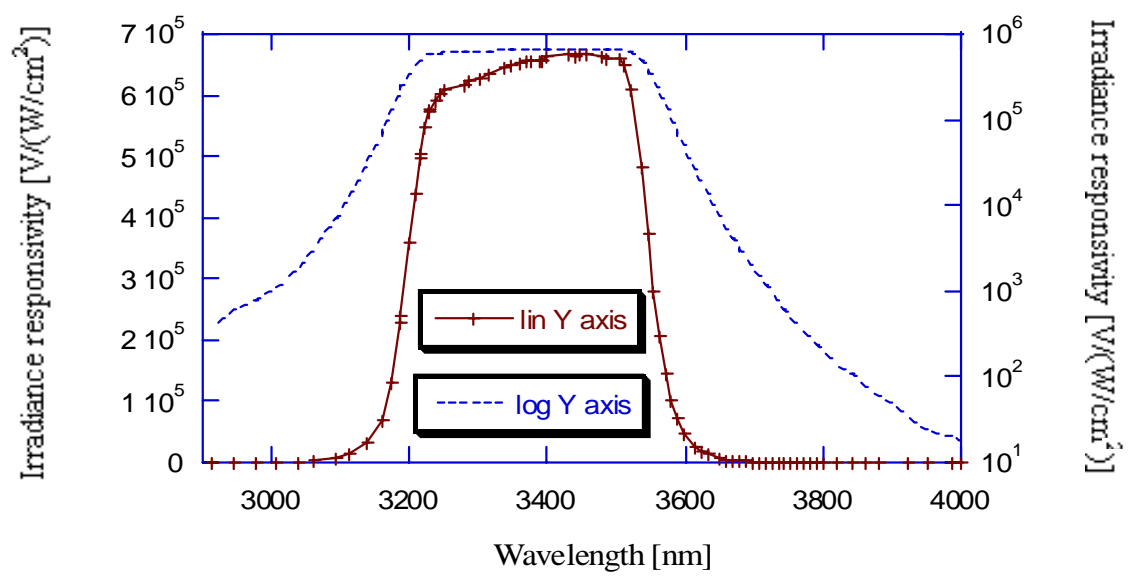

Fig. 6. Spectral irradiance responsivity of InSb\#6 at gain $10^{6}$ V/A

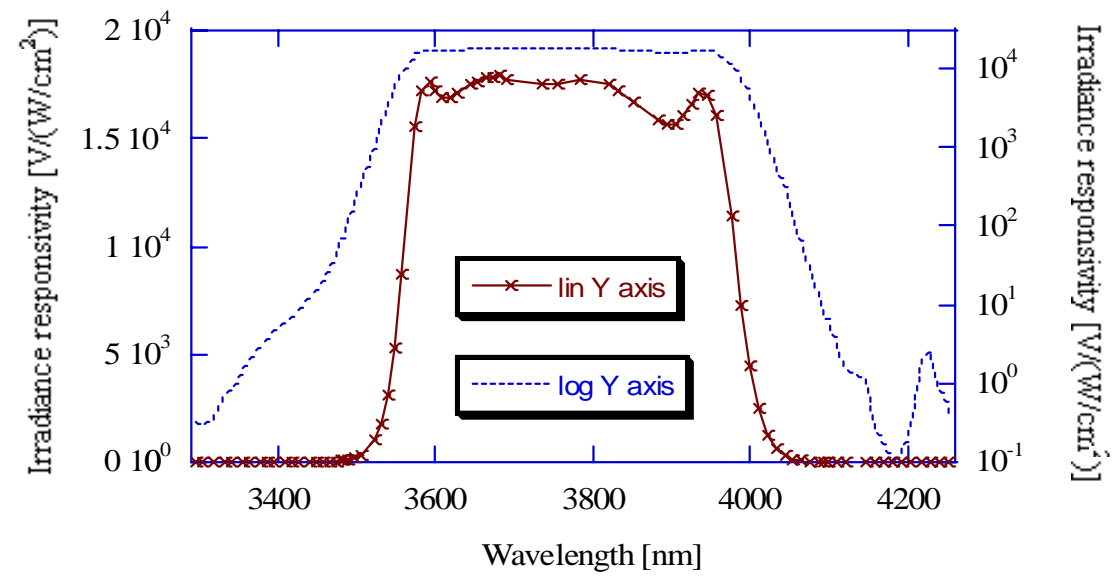

Fig. 7. Spectral irradiance responsivity of InSb\#7 at gain $2.94 \times 10^{4} \mathrm{~V} / \mathrm{A}$.

\section{CONCLUSIONS}

The NIST spectral irradiance responsivity scale from the $405 \mathrm{~nm}$ to $920 \mathrm{~nm}$ wavelength range has been extended to $5 \mu \mathrm{m}$ using improved performance irradiance meters. The transfer standard ESB was calibrated against the ACR through a silicon trap detector to convert its relative spectral irradiance responsivity into absolute. The ESB irradiance responsivity scale was transferred to the EIGA\#2 working standard radiometer. The relative expanded uncertainty $(k=2)$ of the irradiance responsivity at the calibration wavelength (tie) points is $2.2 \%$ on the EIGA\#2 radiometer. Spectrally selective InSb irradiance meters were calibrated with $3 \%$ uncertainty $(k=2)$ against the reference InSb1-new irradiance meter that 
has a traceable responsivity to the HACR. The different responsivity scales were compared at $2.35 \mu \mathrm{m}$ by calibrating the EIGA\#2 against both the ESB and the InSb1-new radiometer. The two scales were different by $2.6 \%$ at $2.35 \mu \mathrm{m}$ where they overlap. Work is being continued to extend the spectral irradiance responsivity scale of the IR-SIRCUS facility further in the infrared with decreased uncertainty.

\section{REFERENCES}

1. Eppeldauer, G., Electronic characteristics of Ge and InGaAs radiometers. SPIE proceedings, 1997. 3061-97: p. 833838.

2. Eppeldauer, G., Near Infrared Radiometer Standards. SPIE proceedings, 1996. 2815: p. 42-54.

3. Eppeldauer, G.P., Migdall, A.L., and Hanssen, L.M., InSb Working Standard Radiometers. Metrologia, 1998. 35: p. 485-490.

4. Eppeldauer, G.P., Racz, M., and Larason, T.C., Optical characterization of diffuser-input standard irradiance meters. SPIE Proc., 1998. 3573: p. 220-224.

5. Larason, T.C., Bruce, S.S., and Parr, A.C., Spectroradiometric detector measurements. NIST Special Publication, 1998. 250-41.

6. Cromer, C.L., et al., The NIST detector-based luminous intensity scale. J. Res. NIST, 1996. 101(2): p. 109-132.

7. Rice, J.P., An electrically substituted bolometer as a transfer-standard detector. Metrologia, 2000. 37: p. 433-436.

8. Eppeldauer, G.P. and Lynch, D.C., Opto-mechanical and electronic design of a tunnel-trap Si- radiometer. J. Res. NIST, 2000. 105(6): p. 813-828.

9. Eppeldauer, G.P. and Racz, M., Spectral Power and irradiance responsivity calibration of InSb working standard radiometers. Appl. Opt., 2000. 39(31): p. 5739-5744.

10. Gentile, T.R., et al., National Institute of Standards and Technology high-accuracy cryogenic radiometer. Applied Optics, 1996. 35(7): p. 1056-1068.

11. Gentile, T.R., et al., Calibration of a pyroelectric detector at $10.6 \mu \mathrm{m}$ with the NIST High-Accuracy Cryogenic Radiometer. Applied Optics, 1997. 36: p. 3614-3621.

12. Migdall, A.L., and G.P. Eppeldauer, Realization of an IR spectral radiant power response scale on a cryogenic radiometer. Metrologia, 1998. 35: p. 307-315.

13. Lykke, K.R., et al., Development of a monochromatic, uniform source facility for calibration of radiance and irradiance detectors from 0.2 to 12 microm. Metrologia, 1998. 35: p. 479-484.

14. Eppeldauer, G., Chopped Radiation Measurement With Large Area Si Photodiodes. J. Res. NIST, 1998. 103: p. 153162.

15. Eppeldauer, G. and Hardis, J.E., Fourteen-decade photocurrent measurements with large-area silicon photodiodes at room temperature. Applied Optics, 1991. 30: p. 3091-3099.

16. Eppeldauer, G., Noise-optimized silicon radiometers. J. Res. NIST, 2000. 105(2): p. 209-219.

17. Eppeldauer, G.P., Racz, M., and Hanssen, L.M., Spectral responsivity determination of a transfer-standard pyroelectric radiometer. SPIE Proceedings, 2002. 4818: p. 118-126.

18. Model SR830 DSP Lock-In Amplifier, Manual, Rev. 1.4. Stanford Research Systems, Inc., 1995.

19. Disclaimer, The mention of certain commercial products in this paper is for information purposes only and does not constitute an endorsement of the product by the authors or their institutions. 


\title{
Development and calibration of pyroelectric radiometer standards at NIST
}

\author{
G. P. Eppeldauer, J. Zeng, and L. M. Hanssen \\ Optical Technology Division \\ National Institute of Standards and Technology \\ Gaithersburg, Maryland 20899
}

\begin{abstract}
The reference spectral power responsivity scale of NIST is being extended from the silicon range to the infrared (IR) using pyroelectric radiometers. Two transfer standard pyroelectric radiometers have been developed at NIST. The main design consideration was to obtain only a minimal increase in the measurement uncertainty during the responsivity scale extension. Domain engineered $\mathrm{LiNbO}_{3}$ and regular $\mathrm{LiTaO}_{3}$ pyroelectric detectors were used in the two radiometers. Both detectors are gold-black coated and temperature controlled. Reflecting domes are attached to the radiometer inputs to decrease the reflectance loss and to improve the spatial uniformity of responsivity in the infrared. Four commercial pyroelectric detectors have been added to the group and used as working standards. The relative spectral responsivity of all pyroelectric detectors was determined from spectral reflectance measurements. The radiant power responsivity tie points were derived from Si trap and single element detectors traceable to the NIST reference responsivity scale. The pyroelectric radiometers have been characterized for frequency and temperature dependent responsivity, noise, spatial non-uniformity of responsivity, angular responsivity, and linearity. The expanded (relative) uncertainty of the spectral power responsivity calibrations ranged between $0.5 \%$ and $1.2 \%(k=2)$ within the $1 \mu \mathrm{m}$ to $19 \mu \mathrm{m}$ range.
\end{abstract}

\section{INTRODUCTION}

Infrared detector standards are being developed for the 2 to $2.5 \mu \mathrm{m}$ [1], the 3 to $5 \mu \mathrm{m}$ [2], [3], and the 8 to $12 \mu \mathrm{m}$ [4] atmospheric windows where the absorption lines will not affect the uncertainty of the responsivity measurements. The present work describes the development of pyroelectric transfer standard radiometers and also the use of commercially available pyroelectric detectors.

The pyroelectric transfer standard radiometers were primarily developed to extend the reference radiant power responsivity scale from the end of the silicon wavelength range at $1 \mu \mathrm{m}$ to the 8 to $12 \mu \mathrm{m}$ range (and beyond) with the lowest possible measurement uncertainty. It was shown earlier that gold-black coated domain engineered $\mathrm{LiNbO}_{3}$ pyroelectric detectors can be applied for responsivity measurements with about $0.1 \%(k=1)$ responsivity uncertainty in the near infrared range [5]. As increased reflectance in the longer wavelength infrared results in increased nonuniformity of the spatial responsivity [1], reflecting domes are mounted at the inputs to minimize reflection losses [6], [7]. The commercial pyroelectric detectors are used as working standard radiometers to propagate the NIST infrared responsivity scale to different applications.

\section{RADIOMETER DESIGN}

The radiometers were designed and selected with optimum radiometric and electronic characteristics to minimize the increase in the uncertainty of spectral responsivity associated with transfer calibrations.

\subsection{Transfer standard radiometers}

The two transfer standard radiometers contain 1-cm diameter pyroelectric detector elements with gold-black coatings used to convert optical power into temperature change and then into current. In Radiometer \#2, a domain-engineered $\mathrm{LiNbO}_{3}$ detector and in Radiometer \#1, a regular $\mathrm{LiTaO}_{3}$ detector without domain engineering were used [5]. Each detector was extended to a radiometer. As it is shown in Fig. 1, the detector-case is attached to a thermoelectric (TE) cooler/heater and a thermistor, mounted inside of the detector case, is used as a temperature sensor. Using a temperature 
controller, the temperature of the detector is controlled to a constant value slightly above room temperature. The gold coated reflecting dome has a hemisphere shape, with an internal diameter of $33 \mathrm{~mm}$. It is mounted above the $20^{\circ}$ tilted pyroelectric detector. The dome has a $4 \mathrm{~mm}$ diameter opening at the optical axis. The figure shows how the specular components from a parallel incident radiation at the two ends of the input beam diameter are reflected back to the active area of the detector. A current-to-voltage converter with multiple signal-gain selections is mounted in an Aluminum box. The box is attached to the radiometer Aluminum housing using a connector. The overall housing of the radiometer is electrically shielded to minimize $60 \mathrm{~Hz}$ pickup. A photo of the radiometer is shown in Fig. 2.

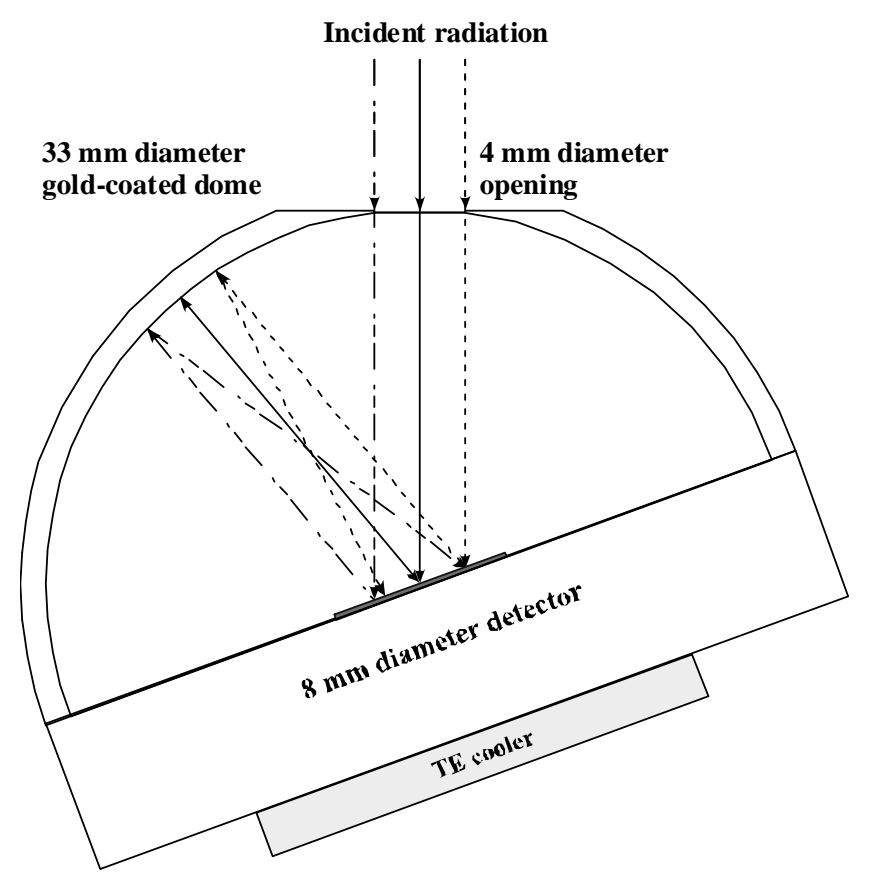

Fig. 1. Input scheme of the transfer standard pyroelectric radiometer.

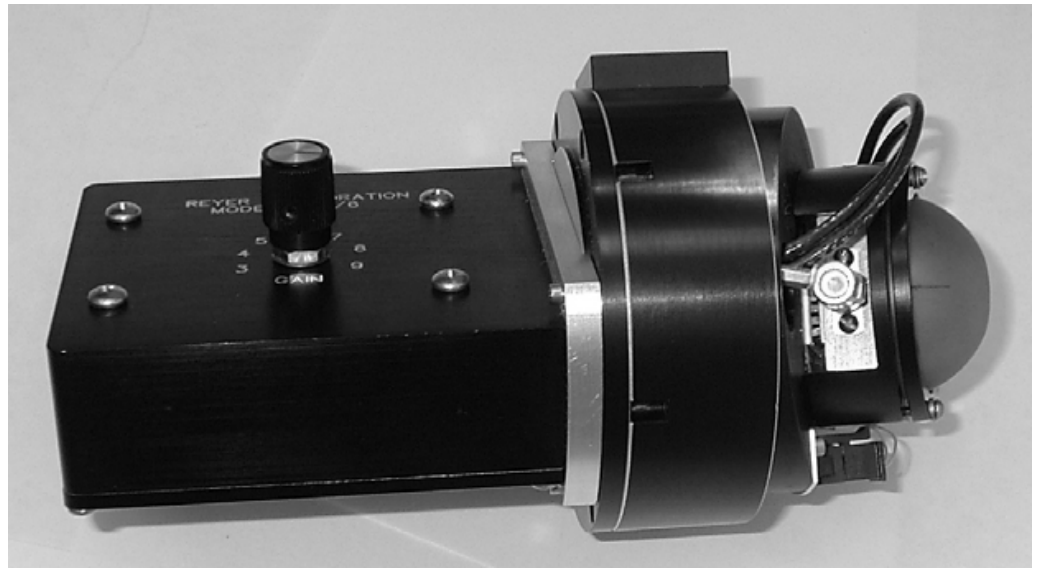

Fig. 2. Photo of the transfer standard pyroelectric radiometer (the top cover is removed) 


\subsection{Working standard radiometers}

Four commercial pyroelectric detectors are used as working standards. These working standards propagate the NIST infrared responsivity scale to different field applications. All four detectors are mounted in a housing where the detector temperature is monitored. The preamplifier is located inside the detector can where a fixed $10 \mathrm{G} \Omega$ feedback resistor is connected to an operational amplifier to convert the detector current into an output voltage. The detectors are coated with a black paint to convert optical radiation changes into temperature changes.

\section{RADIOMETER CHARACTERIZATION}

A set of characterizations were performed on the radiometers to establish the uncertainties associated with the radiometer's responsivity during both calibration and the calibration-transfer to other radiometers. The frequency- and temperature-dependent responsivity, signal-gain stability, and noise characteristics were measured in the Ambient Infrared Detector Characterization Facility. The Infrared Detector Evaluation Facility (IDEF) was used for measurement of the spatial non-uniformity of responsivity, linearity, angular responsivity, absolute responsivity, stability, and repeatability. The Infrared Total Integrated Scatter Instrument (ITIS) was used to measure the reflectance at laser wavelengths. The Fourier Transform Infrared Spectrophotometry Facility (FTIS) was used to measure the spectral reflectance of the coated detectors to determine the relative spectral responsivity.

\subsection{Characterization of the transfer standard radiometers}

The detector-dome combination acts as a trap to provide near unity absorptance across the entire wavelength range of use. Accordingly, the spatial uniformity of responsivity at long wavelengths is significantly improved over the bare detector case. The spatial scan results obtained for Radiometers \#1 and \#2 at $10.6 \mu \mathrm{m}$ are shown in Fig. 3. The size of the scanning spot was $0.2 \mathrm{~mm}$. The maximum-to-minimum spatial non-uniformity of responsivity is less than $0.6 \%$ within a $1.5 \mathrm{~mm}$ diameter center area of both radiometers. Without the reflecting dome, the spatial non-uniformity of responsivity was found to be $10 \%$ to $15 \%$ at $10.6 \mu \mathrm{m}$.
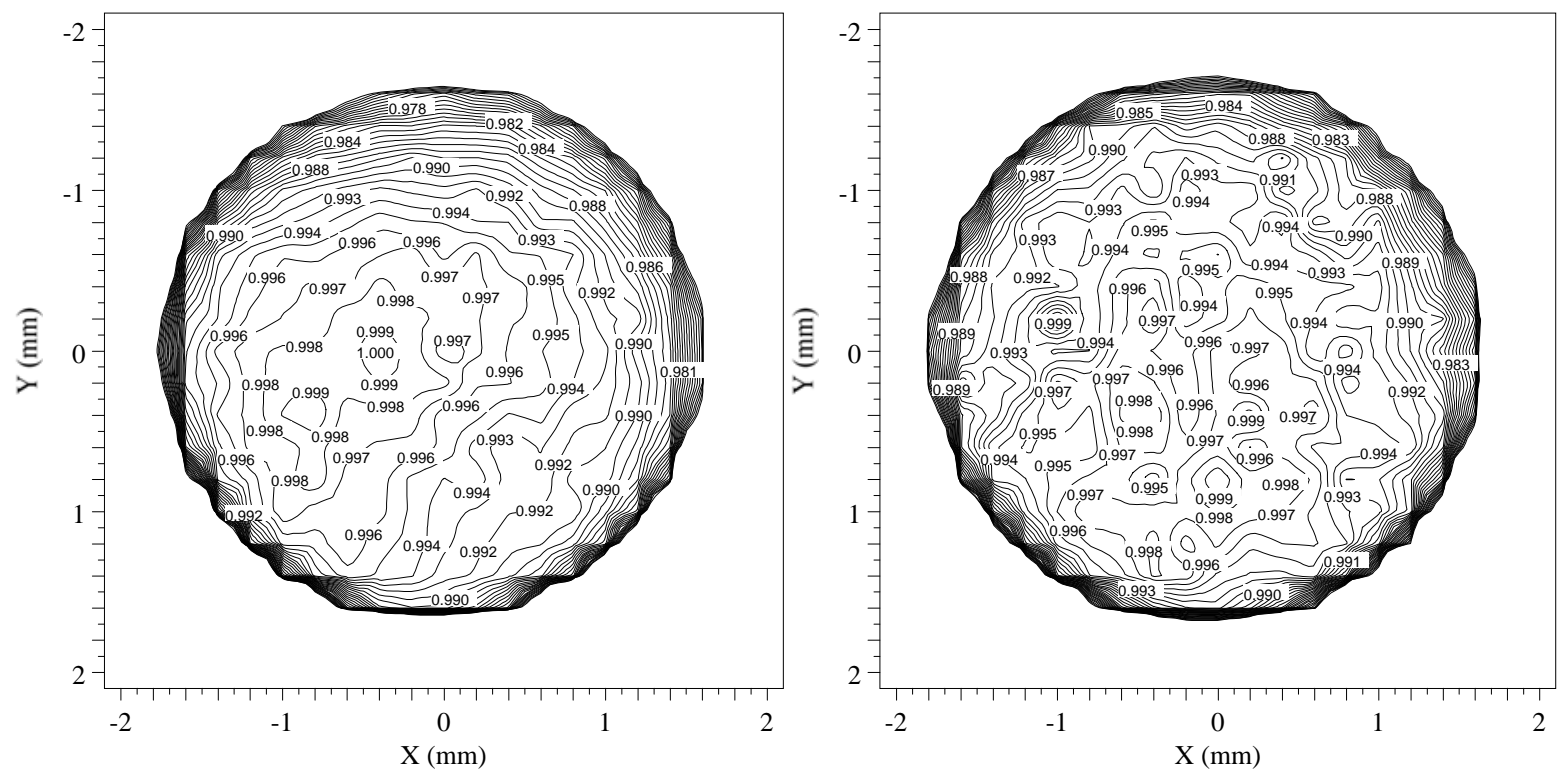

Fig. 3. Spatial (normalized) uniformity of responsivity of the dome-equipped transfer standard pyroelectric Radiometers \#1 (left plot) and \#2 (right plot) at $10.6 \mu \mathrm{m}$. 
The noise equivalent power (NEP) is one of the most important characteristics of pyroelectric radiometers that are used to measure $1 \mu \mathrm{W}$ signal levels from monochromators. Therefore, the noise characteristics of the two transfer standard radiometers were tested. Fig. 4 shows the measured output total noise of the domain engineered Radiometer (\#2) on the left $\mathrm{Y}$ axis. The $1 / \mathrm{f}$ noise of the operational amplifier is the dominating noise up to a gain of $10^{6} \mathrm{~V} / \mathrm{A}$. At higher gain levels the resistor (parallel connected detector resistance and feedback resistance) noise dominates the output noise of the radiometer. The background produced photocurrent and its noise are negligibly small because of the very low responsivity (output photocurrent per input radiant power) of the pyroelectric detector (about six orders of magnitude lower than that of silicon photodiodes). This means that pyroelectric detectors do not operate in background limited condition as most of the high responsivity infrared detectors do. Because of the very low detector responsivity, the signal power to be measured has to be very high. While radiant power levels of $1 \mathrm{pW}$ with a signal-to-noise $(\mathrm{S} / \mathrm{N})$ ratio of 100 or higher can be measured with silicon photodiodes, the radiant power for pyroelectric detectors must be about six orders of magnitude higher to get similar photocurrents and $\mathrm{S} / \mathrm{N}$ ratios. The noise equivalent current (NEC) is calculated as the ratio of the output total noise voltage to the signal gain. The NEC is shown on the right $Y$ axis. The noise measurements were made both in the dark and at $1.5 \mathrm{pA}$ signal current originating from the chopped signal of a ceramic glower. The chopping frequency was $10.5 \mathrm{~Hz}$ and the lock-in time constant was $1 \mathrm{~s}$. Each data point was obtained as the standard deviation of the mean from 20 measurements. The same output total noise voltage (as in Fig. 4) was measured for both cases with a relative measurement uncertainty of $25 \%(k=1)$. The NEC at a signal gain of $10^{9}$ $\mathrm{V} / \mathrm{A}$ is $6.5 \mathrm{fA}$. An NEP of $20 \mathrm{nW}$ can be calculated as the ratio of the $6.5 \mu \mathrm{V}$ output total noise (at $10^{9} \mathrm{~V} / \mathrm{A}$ ) to the 326 V/W responsivity of this radiometer at $1.32 \mu \mathrm{m}$ and a $1 \mathrm{~s}$ lock-in time constant. The radiometer responsivity was measured by substitution against a Ge detector standard in a measurement of total power in the beam from a $1.32 \mu \mathrm{m}$ diode laser. The $1 \mathrm{~s}$ time constant corresponds to a frequency bandwidth of $0.16 \mathrm{~Hz}$. The NEP expressed for a $1 \mathrm{~Hz}$ electrical bandwidth is:

$$
\mathrm{NEP}=20 \mathrm{nW} \times(1 / 0.16)^{1 / 2}=50 \mathrm{nW} / \mathrm{Hz}^{1 / 2}
$$

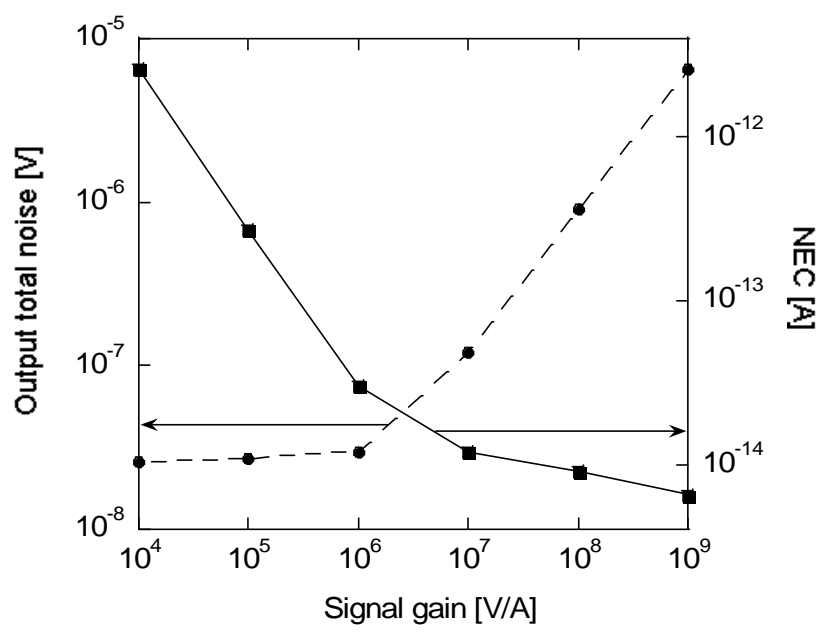

Fig. 4. Noise characteristics of the domain engineered $\mathrm{LiNbO}_{3}$ pyroelectric Radiometer \#2.

The change of the NEP at a signal-gain of $10^{9} \mathrm{~V} / \mathrm{A}$ versus lock-in time constant is shown in Fig. 5 for the regular $\mathrm{LiTaO}_{3}$ Radiometer \#1. The graph shows that the NEP decreases from $13 \mathrm{nW}$ at a $1 \mathrm{~s}$ lock-in time constant to $3 \mathrm{nW}$ at a $10 \mathrm{~s}$ time constant. The large decrease in the NEP was obtained because of the $766 \mathrm{~V} / \mathrm{W}$ responsivity of Radiometer \#1 at a gain of $10^{9} \mathrm{~V} / \mathrm{A}$, which is more than two times higher than that of the domain engineered Radiometer \#2 of 326 $\mathrm{V} / \mathrm{W}$. (The pyroelectric coefficient of $\mathrm{LiNbO}_{3}$ is smaller than that of $\mathrm{LiTaO}_{3}$ and the domain engineered detector is about four times thicker than the regular $\mathrm{LiTaO}_{3}$.) 
The frequency dependent responsivity of the radiometers was measured at a gain of $10^{9} \mathrm{~V} / \mathrm{A}$. The measured $3 \mathrm{~dB}$ upper roll-off frequency was $76 \mathrm{~Hz}$ for both transfer standards. This frequency was determined by the $2.1 \mathrm{pF}$ stray capacitance connected in parallel with the $1 \mathrm{G} \Omega$ feedback resistor. In order to have an operating point on the plateau of the frequency dependent responsivity curve, the feedback resistor cannot be increased to a higher value than $10^{9} \Omega$. In this case, chopping frequency of about $10 \mathrm{~Hz}$ can be used. Fig. 4 shows that the NEC (and hence the NEP) will decrease if the feedback resistor can be further increased. A feedback resistor of $10 \mathrm{G} \Omega$ could be used if the stray capacitance was decreased by an order of magnitude. In this case, sub $10 \mathrm{nW}$ NEPs could be achieved with a $1 \mathrm{~s}$ lock-in time constant.

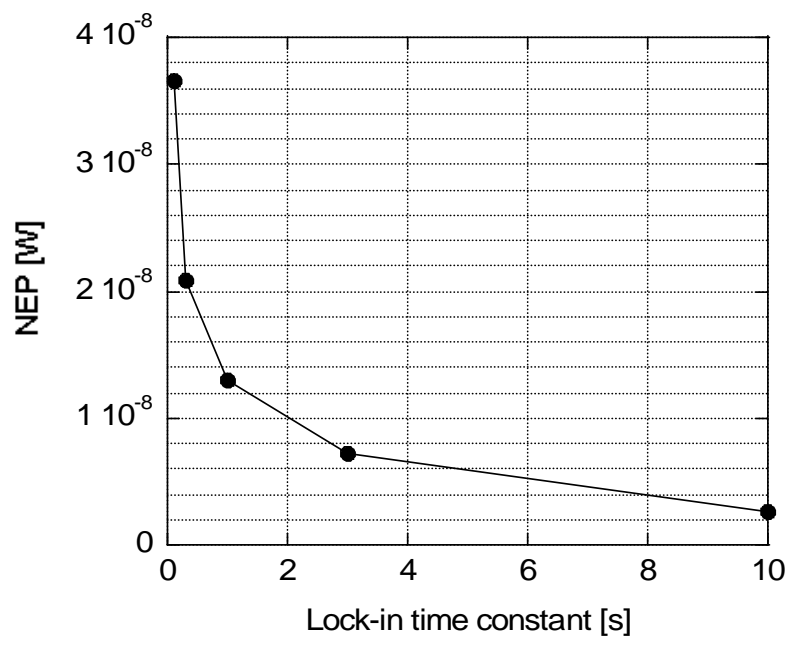

Fig. 5. NEP of the regular $\mathrm{LiTaO}_{3}$ pyroelectric Radiometer \#1 at a signal-gain of $10^{9} \mathrm{~V} / \mathrm{A}$ versus lock-in time constant.

For the two domed pyroelectric transfer standards, the results of the spatial non-uniformity of responsivity measurements together with the reflectance measurement results obtained on the ITIS and FTIS facilities are summarized in Table 1. The FTIS measurement provides the spectral dependence of the reflectance, whereas the ITIS measurement provides a more accurate (absolute reflectance) result, at a single wavelength. The table shows that $0.1 \%$ to $0.2 \%$ reflectance levels were measured on the domed detectors at $10.6 \mu \mathrm{m}$ with the two different methods. The FTIR reflectance measurement indicated no wavelength dependence (leading to the spectrally flat responsivity seen in Fig. 10). The low reflectance of the domed detector in the infrared range results in the low ( $0.5 \%$ to $0.6 \%)$ spatial nonuniformity of responsivity at $10.6 \mu \mathrm{m}$ (as can also be seen in Fig. 3) for both domed detectors.

Table 1. Experimental results of spatial non-uniformity of responsivity and reflectance measurements of the regular pyroelectric Radiometer \#1 and the domain engineered pyroelectric Radiometer \#2 at $10.6 \mu \mathrm{m}$

\begin{tabular}{|c|c|c|c|c|}
\hline \multicolumn{2}{|c|}{$\begin{array}{c}\text { Transfer standard } \\
\text { pyroelectric } \\
\text { radiometers }\end{array}$} & $\begin{array}{c}\text { Spatial } \\
\text { non-uniformity } \\
\text { of responsivity }\end{array}$ & $\begin{array}{c}\text { Spectral reflectance } \\
\text { (from FTIS) }\end{array}$ & $\begin{array}{c}\text { Reflectance } \\
\text { (from ITIS) }\end{array}$ \\
\hline$\# 1$ & Bare & $15 \%$ (max-min) & $16 \%$ & - \\
\hline$\# 1$ & w/Dome & $<0.6 \%$ & - & $-18 \%$ \\
\hline$\# 2$ & Bare & - & $7 \%$ & - \\
\hline$\# 2$ & w/Dome & $<0.5 \%$ & $0.1 \%$ & $0.15 \%$ \\
\hline
\end{tabular}


To obtain the detector element absorptance, we also need to include loss sources other than reflectance: absorption by the dome of the detector reflected radiation, and radiation absorbed outside of the detector. We assume that the goldcoated dome reflectance is $98.5 \pm 0.5 \%$. Ray-tracing results indicate that the radiation absorbed outside of the detector is $<0.05 \%$ and $<0.02 \%$, for Radiometers \#1 and \#2, respectively. Using these measurement results, the absorptance at $10.6 \mu \mathrm{m}$ is $99.6 \%$ and $99.8 \%$, for Radiometers \#1 and \#2, respectively.

\subsection{Characterization of the working standard radiometers}

All four working standard radiometers have been fully characterized to operate them under application conditions so that the measurement uncertainty in use will not be significantly higher than when calibrated. The characterizations help the users to determine the optimum spot size of the incident beam, chopping frequency, and temperature dependent responsivity corrections for different applications.

The radiometers were tested for frequency dependent responsivity at the $10^{10} \mathrm{~V} / \mathrm{A}$ (fixed) signal-gain. They measured the radiation from a ceramic glower from chopper frequencies ranging between $4.5 \mathrm{~Hz}$ and $200 \mathrm{~Hz}$. The curve-fit to the measured data is shown for one of the test radiometers in Fig. 6. The fit equation is:

$$
y=m_{1} \frac{\sqrt{1+\left(\frac{M_{0}}{m_{4}}\right)^{2}}}{\sqrt{\left[1+\left(\frac{M_{0}}{m_{2}}\right)^{2}\right]\left[1+\left(\frac{M_{0}}{m_{3}}\right)^{2}\right]}}
$$

where $y$ is the signal gain (signal output / signal input), $M_{0}$ is the frequency, $m_{1}$ is the magnitude (amplitude) of the DC output voltage, $m_{4}$ is a roll-on frequency produced by a differentiating time constant, $m_{2}$ and $m_{3}$ are roll-off frequencies resulting from integrating time constants. The graph shows that the responsivity roll-on originating from $m_{4}$ of the black paint can cancel the responsivity roll-off produced by $m_{2}$ of the current-to-voltage converter. The result is a $109 \mathrm{~Hz}$ upper roll-off frequency originating from the remaining $m_{3}$ time constant produced by the paint coating.

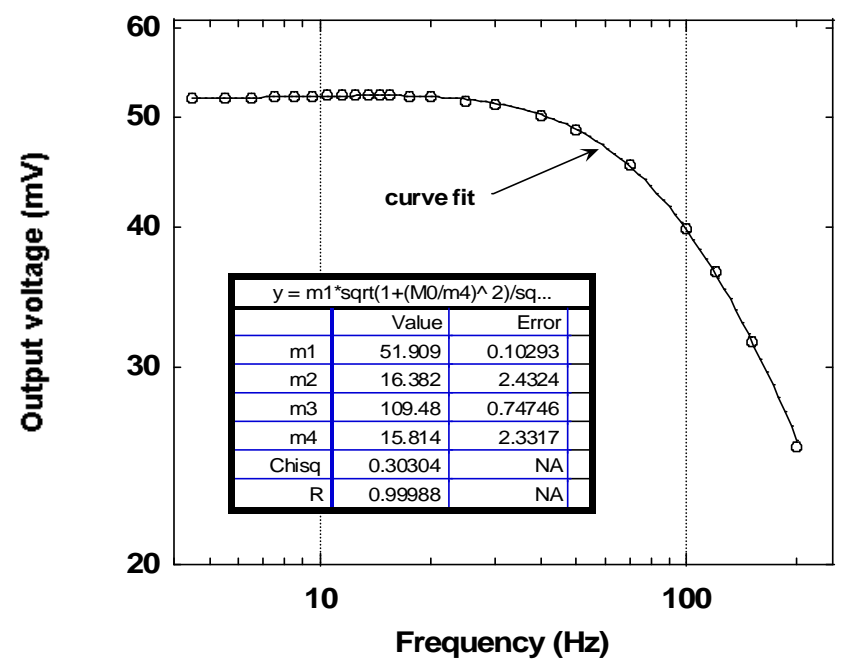

Fig. 6. Frequency dependent responsivity of a working standard pyroelectric radiometer at a signal (current) gain of $10^{10} \mathrm{~V} / \mathrm{A}$. 
The spatial non-uniformity of responsivity was measured at $10.6 \mu \mathrm{m}$. The results in Fig. 7 show that the detector has a roughly $1 \%$ spatial variation of responsivity in the center area which makes it possible to use it in radiant power measurement mode (where the detector is underfilled by the incident radiation) with low uncertainty. The incident radiation should avoid the detector corners, where the responsivity is highly non-uniform because of the contacts.

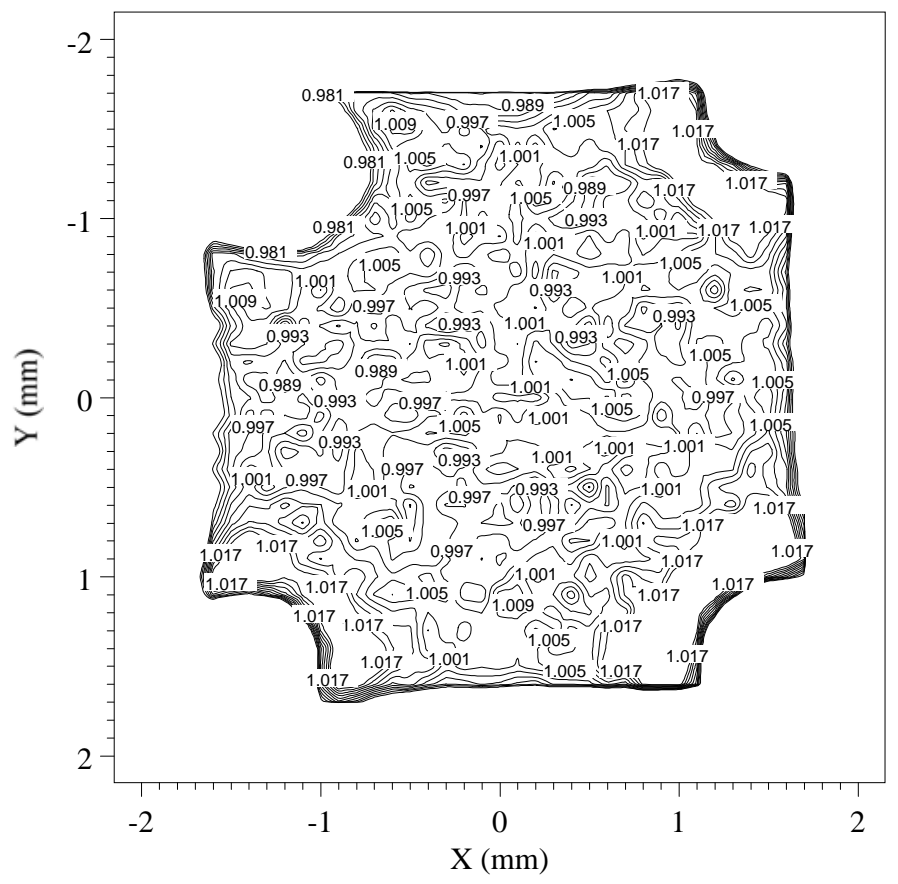

Fig. 7. Spatial non-uniformity of responsivity of one of the paint coated pyroelectric detectors at $10.6 \mu \mathrm{m}$. The responsivity is highly non-uniform at the corners because of the contacts.

In order to determine the correct responsivity of the radiometers for all future applications, the temperature coefficient of responsivity (the relative responsivity change versus temperature) was measured for all four working standard radiometers. The radiometers were located in a temperature controlled box and measured the stable broad-band radiation from a tungsten lamp (located outside of the box) at different detector (box) temperatures. The slope of the output voltages versus temperature data points (from a linear fit) was divided by the output voltage at the predicted temperature reading of the future absolute responsivity calibration. A $0.19 \% /{ }^{\circ} \mathrm{C}$ temperature coefficient of responsivity was obtained for three detectors and $0.26 \% /{ }^{\circ} \mathrm{C}$ was obtained for one detector. The relative expanded uncertainty of these measurements was $20 \%(k=2)$.

The noise floor of the working standard radiometers was measured in the dark at a chopping frequency of $10.5 \mathrm{~Hz}$, with a lock-in integrating time constant of $2 \mathrm{~s}$. The standard deviation was calculated from 32 data points. The calculated noise equivalent photocurrent ranged between $2 \mathrm{fA}$ and $4 \mathrm{fA}$. With a $10^{-6} \mathrm{~A} / \mathrm{W}$ nominal responsivity at $10.6 \mu \mathrm{m}$, a noise equivalent power (NEP) of 2 to $4 \mathrm{nW}$ was obtained for the working standard radiometers using the $2 \mathrm{~s}$ time constant.

The angular responsivity of the test detectors was measured to obtain quantitative information about responsivity changes when the incident radiation geometry is varied. This enables corrections to be made for any specific geometry, such as for the converging beam from a monochromator. The $10.6 \mu \mathrm{m}$ laser beam, in both $s$ and then $p$ polarization states, was focused to the working standard radiometers with a biconvex lens. The working standard radiometers were rotated about the detector center (coincident with the input beam). The working standard radiometer output signal was 
divided by the signal of the monitor detector. The ratios were recorded versus angle of rotation. Fig. 8 shows the angular response for one of the working standard radiometers at $10.6 \mu \mathrm{m}$. The angular response change for the average $s$ and $p$ polarizations was less than $0.5 \%$ within a $+/-15^{\circ}$ angular range.

A radiometer should have constant radiant power responsivity when the power level of the incident radiation changes. The working standard radiometers were measured from the maximum allowed radiant power level down to the low end limit, determined by the signal-to-noise ratio at the test radiometer output.

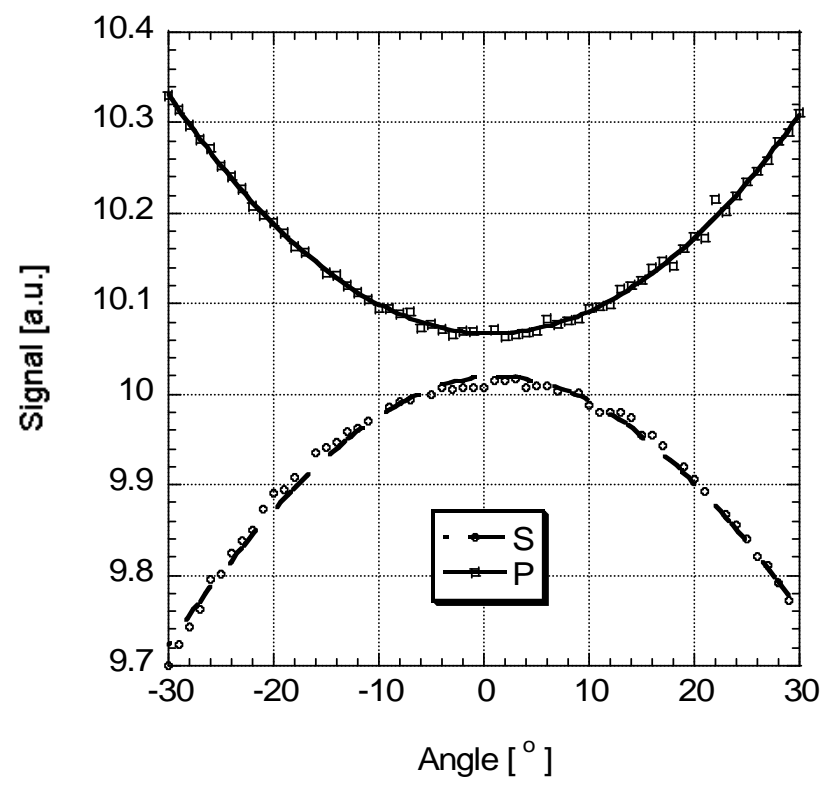

Fig. 8. The angular response of the working standard Radiometer P8 at $10.6 \mu \mathrm{m}$.

The response linearity of two working standard radiometers was measured using a collimated beam from a $\mathrm{CO}_{2}$ laser (tuned to $10.6 \mu \mathrm{m}$ ). The linearity measurement results are shown for both working standard radiometers in Fig. 9. The beam power was adjusted to different power levels at which measurements were made with the same filter, moved in and out of the beam and the ratio or filter transmittance calculated. A constant transmittance (which is proportional to the responsivity) indicates the linearity of the detector response versus incident radiant power. The beam power was changed using ND filters and a polarizer attenuator. The maximum power with the filter out of the laser beam was $3 \mathrm{~mW}$ (shown in Fig. 9 with the large open circle). The results show that the working standard radiometers had linear response (constant responsivity) within a power range larger than three orders of magnitude. The small signal-to-noise ratios limited the linearity tests to a minimum incident power of about $0.1 \mu \mathrm{W}$. 


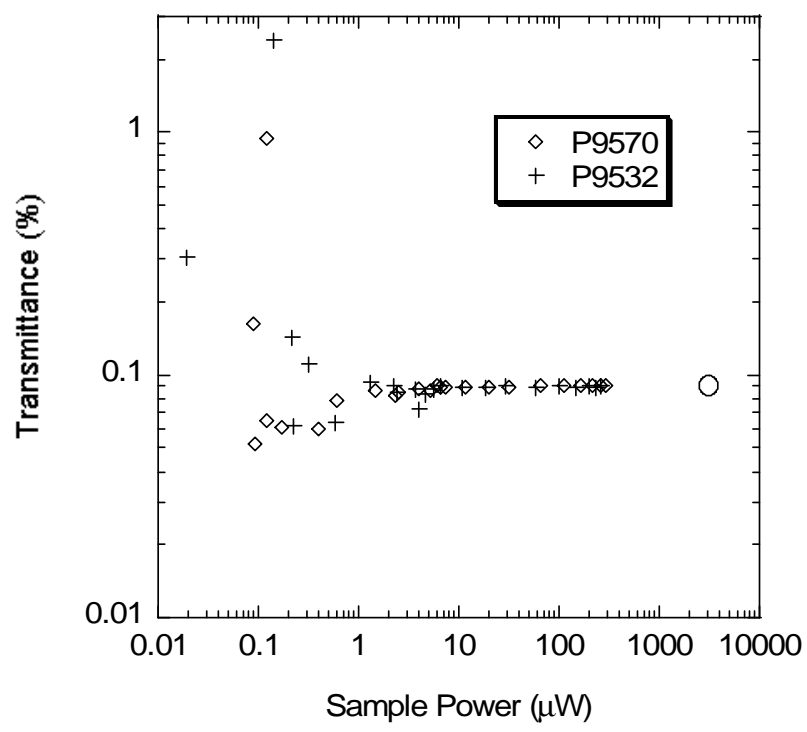

Fig. 9. Linearity measurement results of two working standard radiometers at $10.6 \mu \mathrm{m}$.

\section{CALIBRATION OF PYROELECTRIC RADIOMETERS}

The spectral power responsivities of the transfer and working standard pyroelectric radiometers were determined using the same calibration facilities and similar procedures.

The facility for Spectral Irradiance and Radiance Responsivity Calibrations using Uniform Sources (SIRCUS) made it possible to improve the uncertainty of monochromator-based spectral radiant power responsivity measurements and to extend the calibrations from power mode to irradiance [2] and radiance measurement modes [8]. The high beam power and stability of the tunable IR lasers of the SIRCUS facility makes it possible to calibrate a wide range of IR detectors and radiometers for spectral power, irradiance, and radiance responsivity.

After determination of the relative spectral responsivities using the ITIS and the FTIS facility results, radiant power responsivity tie points were made at a few wavelengths using the IDEF facility to convert the relative spectral responsivities into absolute. The tie points have been derived recently from a Si-trap detector and earlier from a single element Ge photodiode, both traceable to the NIST reference responsivity scale [9]. During the calibration of the first two working standard radiometers, more tie points have been derived from an earlier developed $\mathrm{LiNbO}_{3}$ pyroelectric radiometer standard [5] and also from a single element $\mathrm{LiTaO}_{3}$ pyroelectric transfer detector (PD2) calibrated against the primary standard cryogenic radiometer at $10.6 \mu \mathrm{m}[10]$.

The responsivity tie points were determined using the detector substitution method. The standard detector, having power responsivity $s_{S}$, is placed into the laser beam, with its output current $y_{\mathrm{S}}$ measured. A test detector is then placed into the same beam, with its output current $y_{T}$ measured. The power responsivity of the test detector is then given by

$$
s_{\mathrm{T}}=\frac{y_{\mathrm{T}}}{y_{\mathrm{s}}} \cdot s_{\mathrm{s}}
$$

The signals $y_{S}$ and $y_{T}$ are corrected for the drift of the source intensity by using the monitor detector signal. All measurements were performed at an ambient temperature of $25^{\circ} \mathrm{C} \pm 1{ }^{\circ} \mathrm{C}$. 


\subsection{Transfer standard radiometer responsivities}

The spectral power responsivity of the transfer standard pyroelectric Radiometer \#2 at a signal gain of $10^{9} \mathrm{~V} / \mathrm{A}$ is shown as an example in Fig. 10 between $1 \mu \mathrm{m}$ and $18 \mu \mathrm{m}$. The relative spectral responsivity function was determined from spectral reflectance measurements on the FTIR. The noise at the short wavelength end of each scan was caused by the small signal-to-noise ratios. The responsivity tie points are shown with open squares and a dot in the center. The absorptances at $10.6 \mu \mathrm{m}$ (see Section 3.1) were used to determine the $10.6 \mu \mathrm{m}$ tie point relative to the $785 \mathrm{~nm}$ reference tie point. The relative expanded $(k=2)$ uncertainties are shown with error bars. The uncertainty of the $785 \mathrm{~nm}$ tie point propagated to $10.6 \mu \mathrm{m}$ together with additional uncertainty components, shown in Table 2, increased the relative expanded uncertainty from $0.46 \%$ to $0.58 \%(k=2)$. This is the dominating uncertainty component for the overall spectral power responsivity function of the pyroelectric transfer standard Radiometer \#2.

\subsection{Working standard radiometer responsivities}

As the working standard radiometers propagate the NIST detector responsivity scales to field applications, the increase in the responsivity uncertainty during the scale transfer has to be minimized. For the first two radiometers, a redundant responsivity scale transfer was developed to obtain low and reliable uncertainty of spectral power responsivity.

The initial redundant responsivity scale transfer was made prior to the final calibration of the transfer standard Radiometers \#1 and \#2, when the Si-trap detector was not available for a low uncertainty tie point at the short wavelength end. The calibration results of the first two working standard pyroelectric radiometers are shown in Fig. 11. The plot shows the FTIS and ITIS determined relative spectral responsivity curves and the (absolute) power

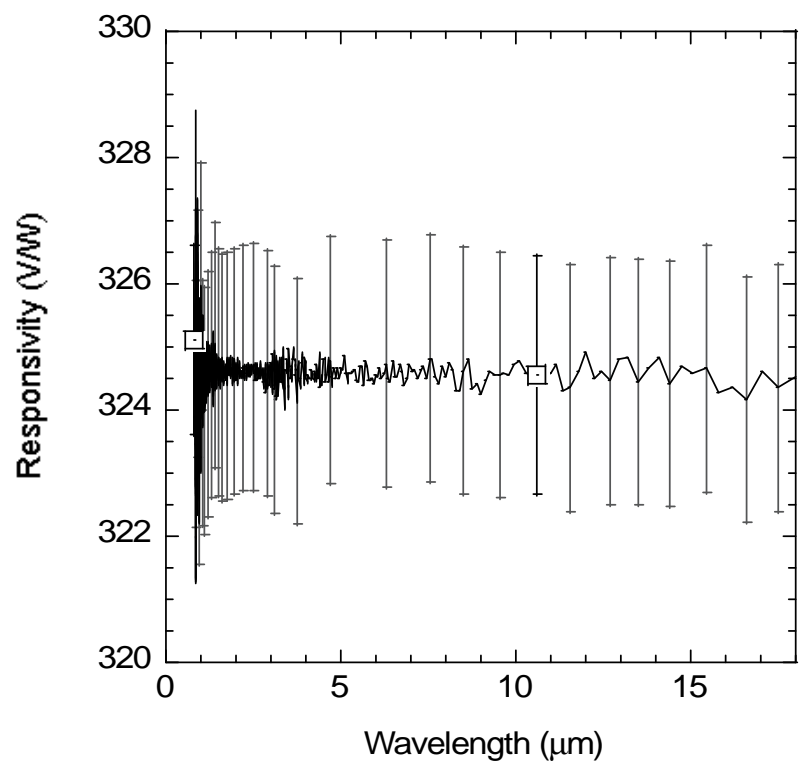

Fig. 10. Spectral power responsivity of the pyroelectric transfer standard Radiometer \#2 at $10^{9} \mathrm{~V} / \mathrm{A}$. 


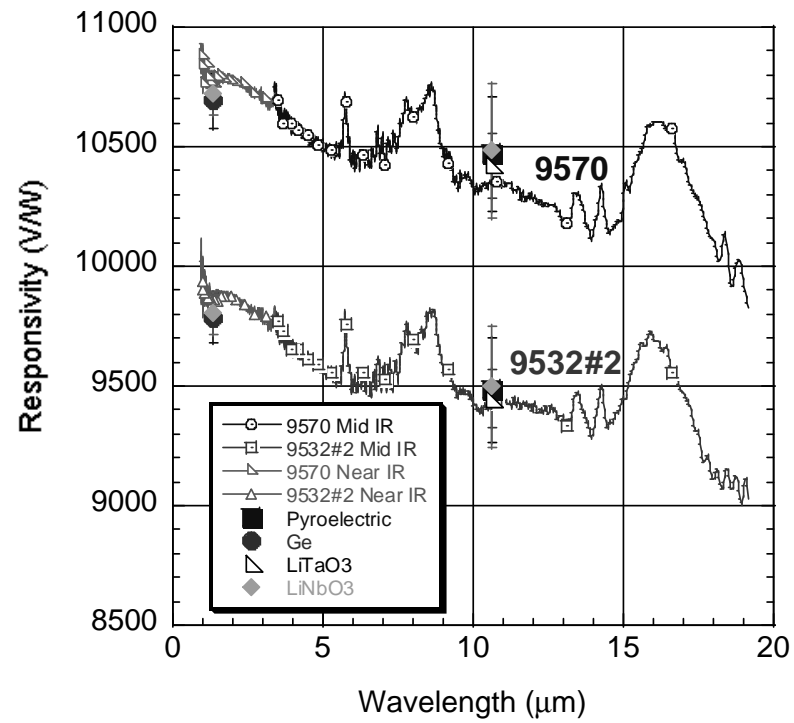

Fig. 11. Spectral power responsivities of the previously calibrated working standard Radiometers 9570 and $9532 \# 2$ at $10^{10}$ V/A signal gains.

responsivity (tie) points with error bars (corresponding to the expanded uncertainties) obtained from laser measurements at $1.32 \mu \mathrm{m}$ and $10.6 \mu \mathrm{m}$. At $1.32 \mu \mathrm{m}$ the working standard pyroelectric radiometers were compared to both Ge and pyroelectric $\left(\mathrm{LiNbO}_{3}\right)$ transfer standard radiometers. At $10.6 \mu \mathrm{m}$ the same working standard radiometers were compared to three transfer standard radiometers (pyroelectric $\mathrm{LiTaO}_{3}, \mathrm{LiNbO}_{3}$, and another $\mathrm{LiTaO}_{3}$ ). The spectral curves are "tied" to the $1.32 \mu \mathrm{m}$ and $10.6 \mu \mathrm{m}$ responsivity points: i.e. they fall within the error bars at the overlap. The error bars represent the expanded uncertainties. For the $1 \mu \mathrm{m}$ to $19 \mu \mathrm{m}$ spectral range, this was $2.7 \%(k=2)$.

The other two working standard pyroelectric radiometers have been calibrated recently. These radiometers have tie points against a Si-trap detector (at $785 \mathrm{~nm}$ ) and the transfer standard Radiometers \#1 and \#2 at $10.6 \mu \mathrm{m}$. The spectral power responsivity of the pyroelectric working standard Radiometer P8 is shown as an example in Fig. 12. Its $10.6 \mu \mathrm{m}$ tie point was derived from the transfer standard Radiometer \#2 using the detector substitution method. The responsivity is given in $\mathrm{V} / \mathrm{W}$ at a signal-gain of $10^{10} \mathrm{~V} / \mathrm{A}$, where the voltage was derived from the DC (un-chopped) responsivity of the Si-trap detector. The combined relative expanded uncertainties, that include the relative uncertainty of the spectral reflectance measurements of about $0.3 \%(k=2)$, are also shown with error bars in Fig. 12. 


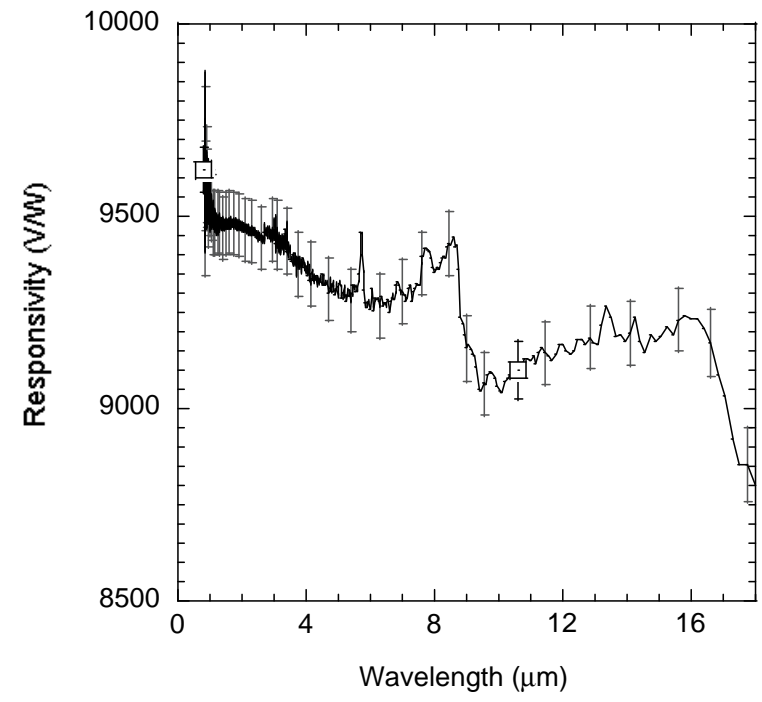

Fig. 12. Spectral power responsivity of the pyroelectric working standard Radiometer P8 at a signal-gain of $10^{10} \mathrm{~V} / \mathrm{A}$.

In applications, where these pyroelectric radiometers are used as working standards, the incident beam power at a given wavelength can be calculated from the reading of the lock-in amplifier (attached to the output of the working standard radiometer) divided by the responsivity at the given wavelength. This beam power will be measured by the test devices in a given application.

The uncertainty budget of the spectral power responsivity determination (as an example) for the working standard Radiometer P8 is included in Table 2 at both $785 \mathrm{~nm}$ and $10.6 \mu \mathrm{m}$. The relative expanded uncertainty of the working standard Radiometer P8 is $0.6 \%(k=2)$ at the $785 \mathrm{~nm}$ and $0.8 \%(k=2)$ at the $10.6 \mu \mathrm{m}$ tie points. As the uncertainties of the new responsivity tie points were improved, the combined uncertainties of the two newer working standards are about three times lower than the uncertainties of the first two working standards discussed above.

Table 2. Uncertainty budget as an example for the transfer standard pyroelectric Radiometer \#2 and the working standard pyroelectric Radiometer P8 at $785 \mathrm{~nm}$ and $10.6 \mu \mathrm{m}$.

\begin{tabular}{|c|c|c|c|c|}
\hline \multirow{2}{*}{$\begin{array}{c}\text { Uncertainty component, } \\
\text { Type }\end{array}$} & \multicolumn{2}{|c|}{ Transfer Standard Radiometer \#2 } & \multicolumn{2}{c|}{ Working Std. Radiometer P8 } \\
\cline { 2 - 5 } & $\mathbf{7 8 5} \mathbf{~ n m}$ & $\mathbf{1 0 . 6} \mathbf{~ m}$ & $\mathbf{7 8 5} \mathbf{~ n m}$ & $\mathbf{1 0 . 6} \boldsymbol{\mu \mathbf { m }}$ \\
\hline Si-trap detector, B & $0.20 \%$ & $0.23 \%$ & $0.20 \%$ & $0.30 \%$ \\
\hline Signal variations, A & $0.09 \%$ & $0.05 \%$ & $0.04 \%$ & $0.10 \%$ \\
\hline Spatial non-uniformity, B & $0.06 \%$ & $0.09 \%$ & $0.20 \%$ & $0.20 \%$ \\
\hline Angular (0-8 ${ }^{\circ}$ ) resp. change, B & $0.10 \%$ & $0.10 \%$ & $0.10 \%$ & $0.10 \%$ \\
\hline Temperature changes, B & $0.02 \%$ & $0.02 \%$ & $0.02 \%$ & $0.02 \%$ \\
\hline Lock-in measurements, B & $0.10 \%$ & $0.10 \%$ & $0.10 \%$ & $0.10 \%$ \\
\hline TIS and ray-trace, B & & $0.08 \%$ & & \\
\hline Relative combined std. uncertainty & $0.23 \%$ & $0.29 \%$ & $0.3 \%$ & $0.4 \%$ \\
\hline $\begin{array}{c}\text { Relative expanded uncertainty } \\
(k=2)\end{array}$ & $0.46 \%$ & $0.58 \%$ & $0.6 \%$ & $0.8 \%$ \\
\hline
\end{tabular}




\section{CONCLUSIONS}

Two transfer standard pyroelectric radiometers have been developed at NIST. As a result of the enhanced absorption from the addition of a reflecting dome above the detector, the Radiometers \#1 and \#2 have approximately constant radiant power responsivities with less than a variation of $0.4 \%$ and $0.2 \%$, respectively, between $1 \mu \mathrm{m}$ and $11 \mu \mathrm{m}$, and estimated to be approximately $0.5 \%$ for Radiometer \#2 out to $18 \mu \mathrm{m}$. Additional radiometers with the same design, given knowledge of the bare detector reflectance at longer wavelengths, will not require spectral calibration. One responsivity tie point with low uncertainty is enough to perform the radiometer calibration for the above wavelength range. Four commercial pyroelectric detectors were also calibrated for spectral power responsivity. These radiometers are used as working standards in field applications. They have structured spectral responsivities due to the paint coating. Therefore, spectral reflectance measurements are needed to determine their relative spectral responsivities. A Si tunnel trap detector with a $0.2 \%$ relative expanded uncertainty $(k=2)$ was the reference radiometer for both the transfer and the working standard pyroelectric radiometer calibrations.

The domed transfer standard pyroelectric radiometers were used to give the $10.6 \mu \mathrm{m}$ responsivity tie point for the second set of working standard pyroelectric radiometers with a relative expanded uncertainty of about $0.6 \%(k=2)$. The combined relative expanded uncertainties of the recently calibrated working standard pyroelectric radiometers were about $1 \%(k=2)$ between $1 \mu \mathrm{m}$ and $18 \mu \mathrm{m}$.

\section{ACKNOWLEDGEMENT}

The authors thank John Lehman for developing the high performance pyroelectric detectors used in the transfer standard radiometers described in this paper.

\section{REFERENCES}

1. $\quad$ Eppeldauer, G.P., Racz, M., and Hanssen, L.M., Spectral responsivity determination of a transfer-standard pyroelectric radiometer. SPIE Proceedings, 2002. 4818: p. 118-126.

2. $\quad$ Eppeldauer, G.P., et al., Spectral irradiance responsivity measurements between $1 \mu \mathrm{m}$ and $5 \mu \mathrm{m}$. SPIE proceedings, 2004. 5543(Society of Photo-Optical Instrumentation Engineers): p. 248-257.

3. $\quad$ Eppeldauer, G.P. and Racz, M., Spectral Power and irradiance responsivity calibration of InSb working standard radiometers. Appl. Opt., 2000. 39(31): p. 5739-5744.

4. Gong, H., Hanssen, L.M., and Eppeldauer, G.P., Spatial and angular responsivity measurements of photoconductive HgCdTe LWIR radiometers. Metrologia, 2004. 41: p. 161-166.

5. $\quad$ Lehman, J., et al., Domain-engineered pyroelectric radiometer. Appl. Opt., 1999. 38(34): p. 7047-7055.

6. Day, G.W., Hamilton, A., and Pyatt, K.W., Spectral reference detector for the visible to 12- micrometer region; convenient, spectrally flat. Appl. Opt., 1976. 15(7): p. 1865-1868.

7. Fox, N.P., Prior, T.R., and Theocharous, E., Radiometric calibration of infrared detectors and thermal imaging systems. SPIE Proceedings, 1995. 2474: p. 229-237.

8. $\quad$ Eppeldauer, G.P., et al., Realization of a spectral radiance responsivity scale with a laser-based source and $\mathrm{Si}$ radiance meters. Metrologia, 2000. 37: p. 531-534.

9. $\quad$ Larason, T.C., Bruce, S.S., and Parr, A.C. Spectroradiometric detector measurements. NIST Special Publication, 1998. 250-41.

10. Gentile, T.R., et al., Calibration of a pyroelectric detector at $10.6 \mu \mathrm{m}$ with the NIST High-Accuracy Cryogenic Radiometer. Appl. Opt., 1997. 36: p. 3614-3621. 


\title{
Extension of the NIST infrared spectral responsivity scale to routine monochromator-based calibrations using improved-NEP pyroelectric detectors
}

\author{
G. P. Eppeldauer, J. Zeng, H. W. Yoon, B. Wilthan, T. C. Larason, and L. M. Hanssen \\ National Institute of Standards and Technology \\ Gaithersburg, Maryland USA
}

\begin{abstract}
Routine NIST spectral responsivity calibrations are needed for the infrared range. Low NEP pyroelectric radiometers have been developed for traditional monochromator applications to extend the responsivity scale to the infrared. After NEP tests, the best pyroelectric detectors were converted to transfer-standard radiometers. The relative spectral responsivities were determined from spectral reflectance measurements of the organic black detector-coatings. The absolute tie points were measured against a domed pyroelectric radiometer standard and a sphere-input extended-InGaAs transfer standard radiometer. A single grating monochromator has been adapted to the calibration facility and used to characterize and calibrate infrared detectors.
\end{abstract}

\section{Introduction}

Since responsivity calibrations at the National Institute of Standards and Technology (NIST) Spectral Irradiance and Radiance Responsivity Calibrations using Uniform Sources (SIRCUS) [1] facility are slow and expensive, a revisit of the traditional monochromator source applications was necessary. However, the noise-equivalent-power (NEP) of traditionally used pyroelectric detectors is too high for wide-wavelength-range measurements at the outputs of regular monochromators. Also, application of low NEP cryogenic bolometers is complicated, slow, and expensive [2]. Instead, improved NEP pyroelectric detectors with $5 \mathrm{~mm}$ detector-diameter have been developed to obtain high signal-to-noise ratios (SNR) at the output of regular monochromators [3]. The low-NEP pyroelectric detectors were extended to user friendly transfer standard radiometers that can extend the responsivity scale to the long-wave IR with low measurement uncertainty. Though the improved NEPs are about one order of magnitude lower than that of traditional pyroelectric detectors, they are still high enough not to obtain any measurable signal contribution from the background radiation and the stray light of the monochromator. Traditionally used InSb, HgCdTe, and other IR detectors can be calibrated with low responsivity-uncertainty against the new pyroelectric transfer standard in the $0.3 \mu \mathrm{W}$ to $\sim 1 \mu \mathrm{W}$ output beam of traditional monochromators. While the calibrations are performed in this limited power (signal) range, the traditionally used (very low-NEP) IR detectors (such as quantum detectors) will extend the spectral responsivity calibrations to low signal levels.

\section{Transfer standard pyroelectric radiometer}

For spectral responsivity scale extension and monochromator use, eight, low-NEP pyroelectric hybrid detectors have been characterized and selected. They were built from 
$100 \mu \mathrm{m}$ thick $\mathrm{LiTaO}_{3}$ crystals and utilized a frequency compensation method [3]. The $5 \mathrm{~mm}$ diameter active area of the detectors was coated with organic black paint. The paint introduced a differentiating time-constant that cancelled the large integrating time constant of the photocurrent-to-voltage converter (attached to the pyroelectric detector) [3]. The paint also introduced an integrating time-constant which is much smaller than the original (cancelled) integrating time-constant. As a result, the upper $3 \mathrm{~dB}$ roll-off frequency could be extended by a factor of five (to about $100 \mathrm{~Hz}$ ) at a signal-gain of $10^{10} \mathrm{~V} / \mathrm{A}$ which is an order of magnitude higher than the maximum signal-gain without this frequency compensation. Most of the tested pyroelectric detectors were extended to radiometers where the temperature of the detectors was monitored. One pyroelectric detector was extended to a radiometer where the detector temperature is stabilized at $26{ }^{\circ} \mathrm{C}$ using thermoelectric heating/cooling. The picture of this transfer standard radiometer head is shown in Fig. 1.

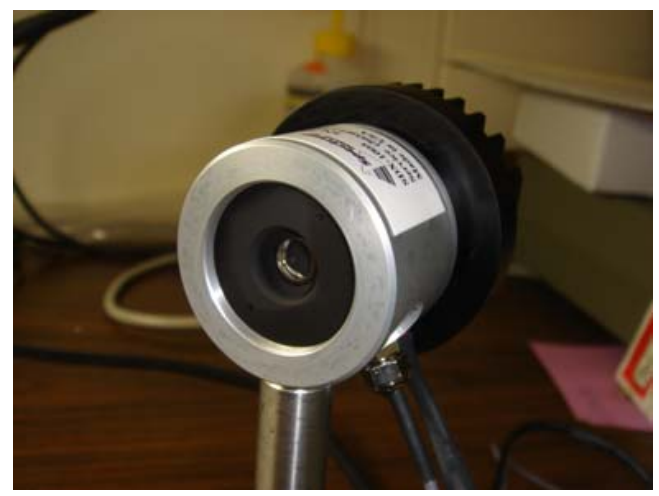

Fig. 1. A temperature-controlled pyroelectric transfer-standard radiometer head.

\section{Tests of the pyroelectric transfer standard radiometer}

The output beam-power of a single-grating monochromator together with the output signal-to-noise ratio (SNR) of the pyroelectric transfer-standard radiometer was tested to evaluate the achievable responsivity uncertainties. The arrangement of the monochromator-based setup is shown in Fig. 2.

An alignment laser, a blackbody source for single beam operation, a ceramic glower, and a tungsten-halogen lamp can be alternately selected with a rotating mirror. The selected beam is imaged to the entrance slit of the monochromator though order sorting filters. The total power in the output beam of the monochromator is measured by the reference and test detector using the detector substitution method.

The output beam-power of the monochromator changed between $0.3 \mu \mathrm{W}$ and $1.6 \mu \mathrm{W}$ within the $900 \mathrm{~nm}$ to $2600 \mathrm{~nm}$ wavelength interval of a grating blazed at $1.25 \mu \mathrm{m}$. The standard deviation changed between $0.2 \%$ and $0.4 \%$ between $1000 \mathrm{~nm}$ and $2500 \mathrm{~nm}$. This is equivalent to a signal-to-noise ratio (SNR) between 500 and 250 in the output signal of the radiometer [3]. This is the radiant power range where traditionally used InSb, MCT and other IR detectors can be calibrated against the pyroelectric transfer standard. 


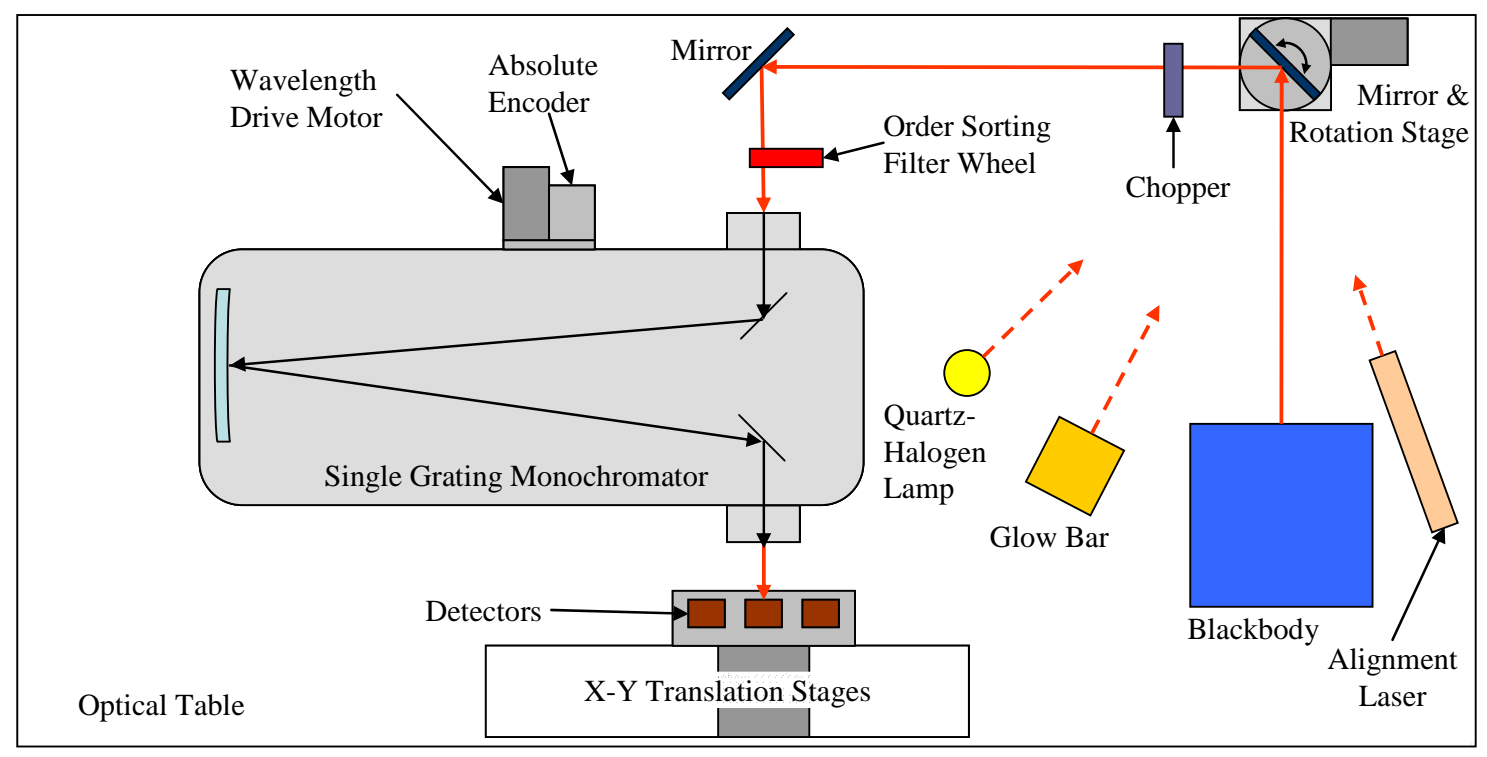

Fig. 2. Monochromator based detector responsivity calibrator.

For the best pyroelectric detectors, less than $4 \mathrm{nW} / \mathrm{Hz}^{1 / 2}$ NEPs were measured which is an order of magnitude smaller than the NEP of our earlier developed (traditional) pyroelectric detector standard [4]. The NEPs of the eight tested pyroelectric radiometers ranged between $3 \mathrm{nW} / \mathrm{Hz}^{1 / 2}$ and $10 \mathrm{nW} / \mathrm{Hz}^{1 / 2}$ [3]. The test results of the temperature controlled transfer-standard pyroelectric radiometer are shown in Table 1 . The NEP was calculated as the ratio of the standard deviation (noise) of the output voltage (from twenty data points) to the radiometer responsivity. The responsivity value includes the $10^{10} \mathrm{~V} / \mathrm{A}$ gain of the current meter attached to the pyroelectric detector. A $785 \mathrm{~nm}$ responsivity tie point was made against a silicon trap detector and a $10.6 \mu \mathrm{m}$ tie point was derived from our previously developed and calibrated dome-input pyroelectric radiometer [4]. These tie point derivations were made at a chopping frequency of $10.5 \mathrm{~Hz}$. The frequency dependent responsivity of the pyroelectric transfer standard has been measured to determine the upper $3 \mathrm{~dB}$ roll-off frequency and to apply responsivity corrections for different signal frequencies in different applications. The result of the frequency dependent responsivity measurement for the transfer standard is shown in Fig. 3. A three time-constant curve fit [4] was applied to the measured data after normalizing the data at low frequencies. The $3 \mathrm{~dB}$ upper roll-off frequency is about $100 \mathrm{~Hz}$, a decade higher than the suggested chopping frequency.

Table 1.

Test results of the transfer standard pyroelectric radiometer.

\begin{tabular}{|c|c|}
\hline Standard deviation in dark & $9.4 \times 10^{-6} \mathrm{~V}$ \\
\hline NEP (with 1 s time constant) & $4.7 \mathrm{nW}$ \\
\hline $3 \mathrm{~dB}$ upper roll-off frequency & $100 \mathrm{~Hz}$ \\
\hline Responsivity, $785 \mathrm{~nm}, 10.5 \mathrm{~Hz}$ & $5059 \mathrm{~V} / \mathrm{W}$ \\
\hline Responsivity, $10.6 \mu \mathrm{m}, 10.5 \mathrm{~Hz}$ & $4995 \mathrm{~V} / \mathrm{W}$ \\
\hline
\end{tabular}




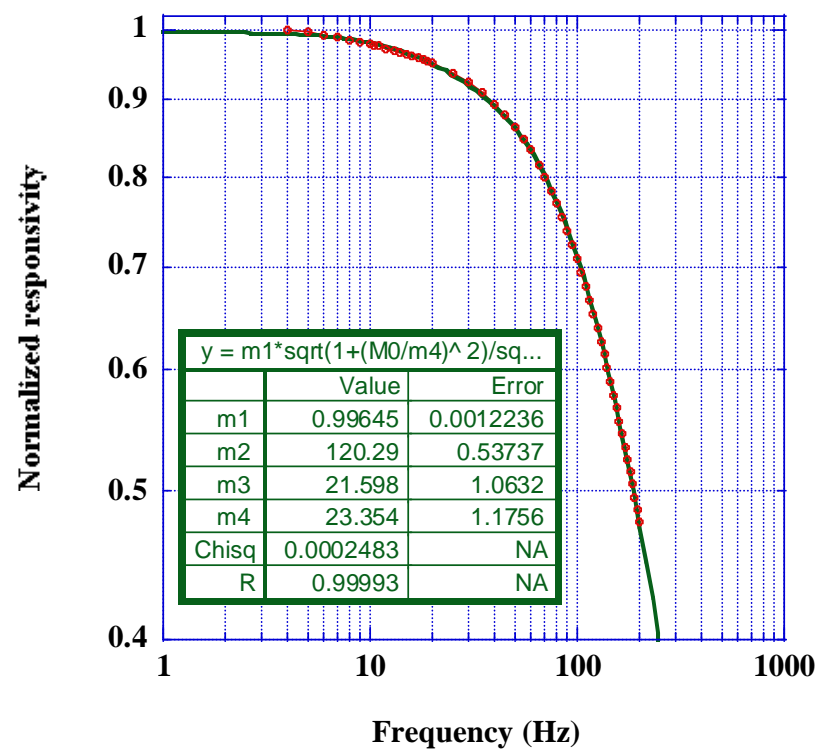

Fig. 3. Frequency dependent responsivity of the pyroelectric transfer standard.

\section{Calibration of the pyroelectric transfer standard radiometer}

The spectral reflectance of the organic black coated transfer standard detector was measured on the NIST FTIR Spectrometer Facility. The spectral reflectance of this paintcoated detector is structured versus wavelength. The relative spectral responsivity of the detector was determined first. The spectral reflectance measurement results are shown in Fig. 4. The reflectance varied from $3.5 \%$ to $5.5 \%$ between $1.5 \mu \mathrm{m}$ and $14.5 \mu \mathrm{m}$. The expanded uncertainty $(k=2)$ of the spectral reflectance measurements is shown in the same graph. This reflectance uncertainty includes the noise and the signal loss due to the detector can. The loss is a systematic uncertainty component which will have a minimal contribution to the responsivity uncertainty budget since an absolute tie point to the relative spectral reflectance curve will take care of this problem. Since the responsivity is proportional to 1-reflectance, the expanded responsivity uncertainty component from the relative spectral reflectance is $0.5 \%(k=2)$ between $2.5 \mu \mathrm{m}$ and $13 \mu \mathrm{m}$.

The $10.6 \mu \mathrm{m}$ tie point was derived from the previously developed domed pyroelectric radiometer [4]. The expanded uncertainty of the $10.6 \mu \mathrm{m}$ tie points is $0.8 \%(k=2)$.

The monochromator-based tie point was made against a sphere-input extended-InGaAs (EIGA) radiometer. The EIGA radiometer was calibrated against an electrical substitution cryogenic radiometer using the detector substitution method [5]. Both radiometers measured the same radiant power from intensity stabilized tunable lasers. Since the laser power levels were high, the EIGA radiometer was operated in a DC signal measurement mode. The calibrations were made between $1 \mu \mathrm{m}$ and $2.5 \mu \mathrm{m}$ with an expanded uncertainty of $0.4 \%(k=2)$. The tie point at the monochromator-based facility was at 
$1790 \mathrm{~nm}$. This tie point calibration was made at $0.5 \mu \mathrm{W}$ radiant power output-radiation of the monochromator. The other (longer wavelength) tie points from the monochromatorbased spectral measurements were not used because of the smaller signal-to-noise ratios. The radiation was chopped with $12 \mathrm{~Hz}$ and the radiometer (both reference and test) output signals were measured by a sine-wave measuring lock-in amplifier. This power level was selected to avoid non-linearity errors in the calibrations of the pyroelectric detectors.

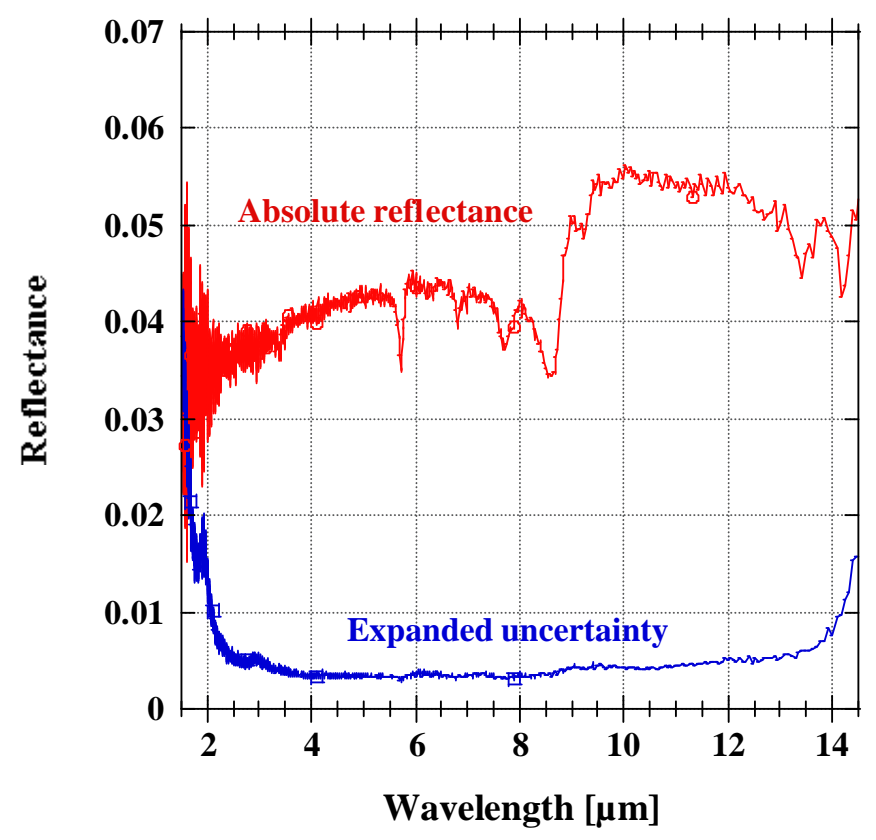

Fig. 4. Spectral reflectance of the pyroelectric transfer standard detector.

Before the tie point calibration, the frequency dependent responsivity of the EIGA sphere-detector was measured. Also, the signal attenuation of the pyroelectric transfer standard was checked between $12 \mathrm{~Hz}$ and $10.5 \mathrm{~Hz}$. The signal attenuation of the EIGA sphere-radiometer was $0.3 \%$ over-compensated by the increased signal responsivity of the pyroelectric radiometer at $10.5 \mathrm{~Hz}$ (compared to $12 \mathrm{~Hz}$ ) where the spectral responsivities are reported. The $0.3 \%(k=2)$ over compensation has been corrected and this uncertainty component is not included in the overall uncertainty budget. The reading of the sine-wave measuring lock-in amplifier was multiplied by 2.221 to obtain the correct responsivity value for the tie point. From three repeats of the absolute tie-point measurements of the pyroelectric transfer standard, an expanded measurement uncertainty of $0.5 \%(k=2)$ was obtained. This uncertainty component is noise dominated.

Figure 5 shows the measured spatial uniformities of the pyroelectric transfer standard at two different wavelengths. The roughly $2 \%$ maximum-to-minimum non-uniformity in the center-area at $785 \mathrm{~nm}$ increased by about $50 \%$ at $10.6 \mu \mathrm{m}$. The increase in the spatial non-uniformity is associated with the $\sim 50 \%$ higher reflectance at $10.6 \mu \mathrm{m}$. According to 
Fig. 5, the uncertainty component that originates from the spatial non-uniformity of responsivity is wavelength dependent. If the detector center-area is used and the incident beam diameter is between $1 \mathrm{~mm}$ and $2 \mathrm{~mm}$ and positioned into the detector center, the spatial non-uniformity uncertainty component can be decreased to $0.5 \%(k=2)$.
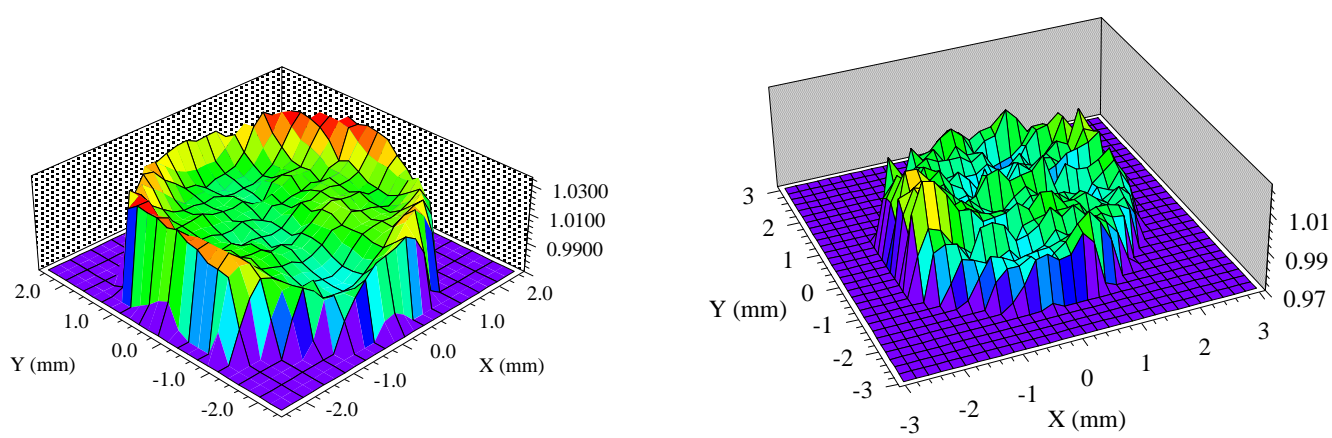

Fig. 5. Spatial uniformity plots of the transfer standard at $785 \mathrm{~nm}$ and $10.6 \mu \mathrm{m}$.

The responsivity uncertainty budget of the pyroelectric transfer standard radiometer at $10.5 \mathrm{~Hz}$ signal frequency from $2.5 \mu \mathrm{m}$ to $13 \mu \mathrm{m}$ is shown in Table 2. The expanded uncertainty at $2 \mu \mathrm{m}$ is $1.3 \%(k=2)$ because of the increased noise in the spectral reflectance measurements. Because of the same reason, the expanded uncertainty at $14 \mu \mathrm{m}$ increased to $1.2 \%(k=2)$.

The spectral radiant power responsivity of the pyroelectric transfer standard radiometer is shown in Fig. 6. In addition to the $10.6 \mu \mathrm{m}$ tie point, the tie point of the sphere-input EIGA radiometer is also shown in Fig. 6. The individual error bars represent the expanded uncertainties $(k=2)$ of the tie points. The sphere-EIGA related error bar (full square) illustrates the overall expanded uncertainty summarized in Table 2.

\section{Spectral responsivity propagation from the pyroelectric transfer standard}

The scale from the transfer-standard pyroelectric radiometer can be transferred to other infrared test detectors using the detector substitution method. The substitution is performed in the $0.3 \mu \mathrm{W}$ to $1.6 \mu \mathrm{W}$ radiant power range using a detector-stage at the output of the single monochromator in Fig. 2. The monochromator has four gratings to continuously cover the spectral range to $24 \mu \mathrm{m}$. The total-power in the output beam of the monochromator is measured by under-filling both the reference and the test detector(s). A stable $1000 \mathrm{C}^{0}$ blackbody source is imaged to the entrance slit of the monochromator to make wide consecutive wavelength-scans for both the test and the reference (transfer standard) detectors. The measurements also can be performed in twobeam mode where the signal of the test or reference detector is simultaneously divided by the signal from a monitor detector. 


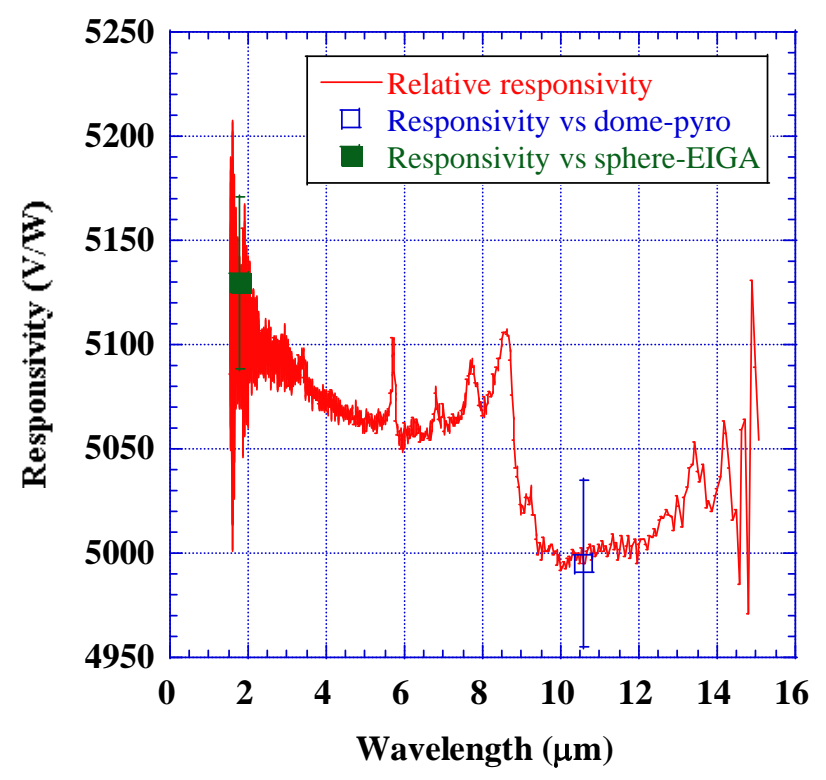

Fig. 6. Spectral responsivity of the pyroelectric transfer standard radiometer.

Traditional IR detectors, such as InSb and MCT detectors can be calibrated against the pyroelectric transfer standard to extend the radiant power range to low signal levels. All infrared responsivity measurements on the monochromator-based facility are performed in AC mode using individual lock-in amplifiers for the reference, test, and monitor detectors.

Table 2. Uncertainty budget $(k=2)$ for the spectral responsivity of the pyroelectric transfer standard at $10.5 \mathrm{~Hz}$ from $2.5 \mu \mathrm{m}$ to $13 \mu \mathrm{m}$.

\begin{tabular}{|l|c|c|}
\hline \multicolumn{1}{|c|}{ Uncertainty factor } & Type & $\begin{array}{c}\text { Uncertainty } \\
(k=2)[\%]\end{array}$ \\
\hline Tie point absolute responsivity calibration: & & \\
\hline Sphere-EIGA radiometer responsivity & $\mathrm{A}$ & 0.4 \\
\hline Pyroelectric radiometer gain & $\mathrm{B}$ & 0.04 \\
\hline Pyroelectric temperature dependence & $\mathrm{B}$ & 0.04 \\
\hline Pyroelectric radiometer frequency dependence & $\mathrm{B}$ & 0.08 \\
\hline Pyroelectric spatial non-uniformity of responsivity & $\mathrm{B}$ & 0.5 \\
\hline Pyroelectric noise dominated repeatability & $\mathrm{B}$ & 0.5 \\
\hline Combined expanded uncertainty & & 0.82 \\
\hline Relative responsivity calibration: & & \\
\hline Spectral reflectance measurement & $\mathrm{A}$ & 0.5 \\
\hline Overall expanded uncertainty & & $\mathbf{0 . 9 6}$ \\
\hline
\end{tabular}




\section{Conclusions}

Traditional pyroelectric detectors need high incident radiant power, such as stabilized tunable lasers to obtain high signal-to-noise ratios for low uncertainty measurements. The laser-used spectral responsivity measurements are time consuming and expensive. Development of low-NEP pyroelectric radiometers was needed to use traditional monochromators for routine spectral responsivity calibrations in the infrared. Characterizations and calibration of a recently developed low-NEP pyroelectric transfer standard radiometer is described in detail. This transfer standard can be used at radiant power levels higher than $0.3 \mu \mathrm{W}$ to obtain $1 \%(k=2)$ responsivity uncertainties. It is operated in a limited power range (to a couple of $\mu \mathrm{W}$ ) to avoid non-linearity errors in the pyroelectric detector. Traditional InSb, HgCdTe, and other very low-NEP infrared detectors can be calibrated against the pyroelectric transfer standard at the monochromator output to extend the spectral responsivities to sub $\mathrm{pW}$ radiant power levels. The new monochromator based responsivity scale can be used to $14 \mu \mathrm{m}$.

\section{References}

1. S. W. Brown, G. P. Eppeldauer, and K. R. Lykke, Facility for Spectral Irradiance and Radiance Responsivity Calibrations using Uniform Sources, Applied Optics, Vol. 45, No. 32, p. 8218-8237, 2006.

2. G. Eppeldauer, A. L. Migdall, and C. L. Cromer, A cryogenic silicon resistance bolometer for use as an infrared transfer standard detector, 6th AIAA/ASME Thermophysics and Heat Transfer Conference, June 1994, Colorado Springs, CO. Proc. HTD-Vol. 277, Thermal Phenomena at Molecular and in Cryogenic Infrared Detectors, p. 63-67 (Book No. H00908), 1994.

3. G. P. Eppeldauer, J. Zeng, and H. W. Yoon, Low-NEP pyroelectric radiometer standards, SPIE Proc. Vol. 6940, pp. 694036-1 to 694036-8, 2008.

4. G. P. Eppeldauer, J. Zeng, and L. M. Hanssen, Development and calibration of pyroelectric radiometer standards at NIST, SPIE Proc. Vol. 6201, pp. 620119-1 to 620119-12, 2006.

5. G. P. Eppeldauer and H. W. Yoon, Short wave infrared radiometers design and characterizations, SPIE Proc. Vol. 6542, pp. 65420O-1 to 65420O-9, 2007. 
RESPONSIVITY CALIBRATION METHODS FOR 365 nm IRRADIANCE METERS

\author{
T. C. Larason, S. W. Brown, G. P. Eppeldauer, and K. R. Lykke \\ National Institute of Standards and Technology ${ }^{1}$ \\ Gaithersburg, MD 20899-8441, USA
}

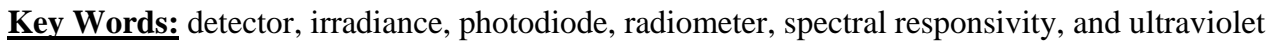

\begin{abstract}
$\underline{\text { Abstract }}$
Two detector-based responsivity calibration methods have been compared at the National Institute of Standards and Technology (NIST) for ultraviolet (UV) irradiance meters in the $365 \mathrm{~nm}$ spectral region. Both methods are based on an electrical substitution High Accuracy Cryogenic Radiometer (HACR), but utilize different facilities and transfer standards. One facility is a monochromator-based, spectral power responsivity measuring system utilizing an aperture | scanning method, while the second is a tunable laser and integrating sphere source_system using a light-trapping silicon transfer detector with a known aperture area. The first reported comparison of these two fundamentally different methods agreed to $1 \%$ to $2 \%$ near the peak and long wavelength side of the bandpass curves which is comparable to their expanded $(k=2)$ uncertainties.
\end{abstract}

\title{
I. INTRODUCTION
}

UV irradiance meters have a broad range of industrial applications from semiconductor manufacturing (photolithography) to nondestructive testing to curing inks and coatings used in high volume printing. Increasing industrial application of UV irradiance measurements has led to a steady increase in the number of calibration requests to NIST for UV irradiance meters, particularly those used to measure $365 \mathrm{~nm}$ radiation. The measured signal from a given irradiance meter is the integral of the source distribution and the meter spectral responsivity,

$$
i=\int_{\lambda} E(\lambda) \cdot S(\lambda) \cdot \mathrm{d} \lambda
$$

where $i$ is the signal, $E(\lambda)$ is the spectral irradiance from the source measured and $S(\lambda)$ is the spectral irradiance responsivity of the meter. Knowledge of the spectral irradiance responsivity of a meter is critical for high accuracy measurements of sources with different spectral power distributions. This is especially critical if more than one model of irradiance meter (i.e., different responsivities) is used in the measurement chain.

NIST currently has two facilities for measuring the spectral responsivity of UV irradiance meters: the Ultraviolet Spectral Comparator Facility (UV SCF) [1] and the facility for Spectral Irradiance and Radiance Responsivity Calibrations with Uniform Sources (SIRCUS) [2]. Diagrams of these facilities are shown in Figures 1 and 2, respectively. The irradiance scale of each facility is based on the High Accuracy Cryogenic Radiometer (HACR), the NIST primary standard cryogenic radiometer. While similar methods have been reported elsewhere [3, 4] this is the first direct comparison of these two fundamentally different techniques.

A number of $365 \mathrm{~nm}$ irradiance meters with different optical diffusers and spectral responsivities were measured in both NIST facilities and their responsivities compared to verify the irradiance calibration uncertainties of the UV SCF and to refine the SIRCUS irradiance calibration procedures. The results of this intercomparison are shown here for two representative irradiance meters. One irradiance meter, designated IM\#1, was a commercial device with an approximately $50 \mathrm{~nm}$ bandpass (FWHM). The second, IM\#2, was a NIST designed transfer standard with an approximately $25 \mathrm{~nm}$ bandpass (FWHM).

\footnotetext{
${ }^{1}$ Optical Technology Division, Physics Laboratory, Technology Administration, U.S. Department of Commerce. Official contribution of the National Institute of Standards and Technology, not subject to copyright in the United States.
}

Page 1 


\section{Preprint}

IEEE TRANSACTIONS ON INSTRUMENTATION AND MEASUREMENT, VOL. 50, NO. 2, APRIL 2001

Light Tight Enclosure

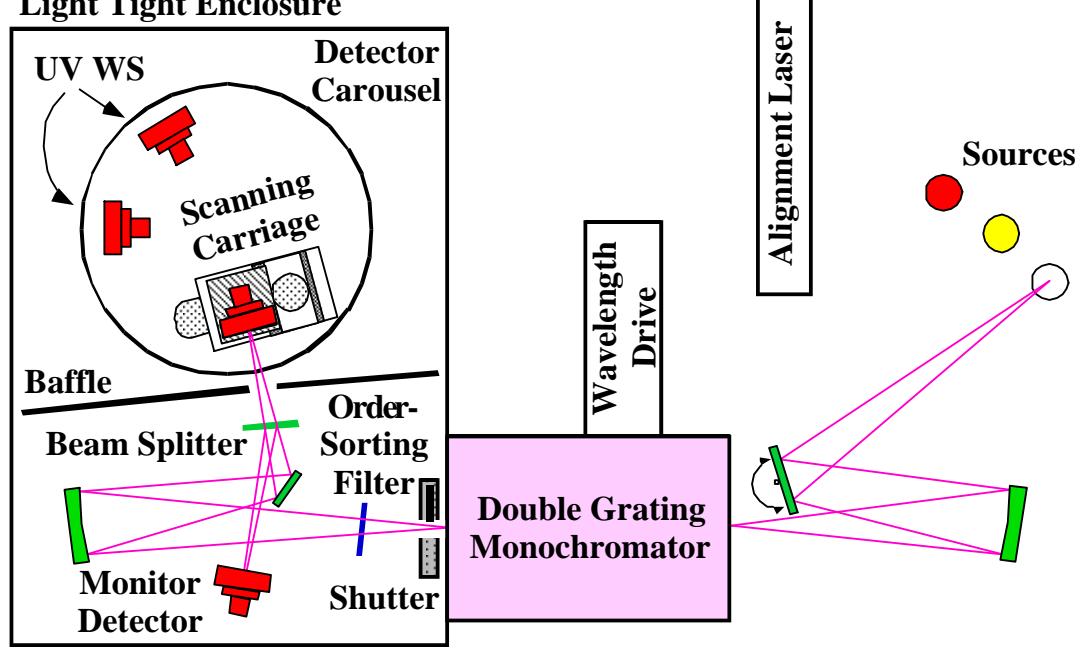

Figure 1. Ultraviolet Spectral Comparator Facility (UV SCF) Diagram. The irradiance meter-under-test is mounted on the scanning carriage. Spectral responsivity comparison is accomplished by rotating the detector carousel to place one of the UV working standards (WS) into the beam. The order-sorting filter removes $2^{\text {nd }}$ order light beyond $400 \mathrm{~nm}$.

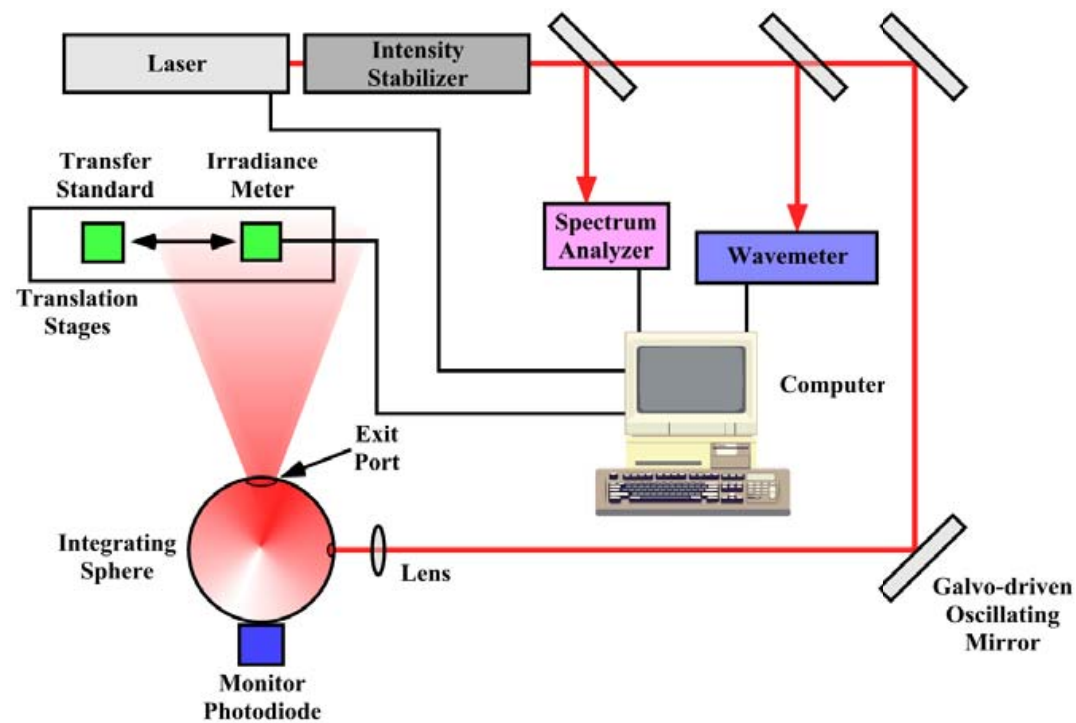

Figure 2. Spectral Irradiance and Radiance Responsivity Calibrations with Uniform Sources (SIRCUS) Diagram. The irradiance meter calibration is performed by direct substitution with the irradiance transfer standard.

\section{UV SCF MEASUREMENTS}

The first facility, the UV SCF, is a monochromator-based system developed in the early 1990s to measure the absolute spectral responsivity and uniformity of photodetectors in the $200 \mathrm{~nm}$ to $500 \mathrm{~nm}$ spectral region. The principal component is a computer controlled $1 / 4 \mathrm{~m}$ focal length, $f / 5$, double grating monochromator with a $3 \mathrm{~nm}$ bandpass. A variety of sources (typically an argon arc) can be selected using a computer controlled turning mirror to align the source with the spherical mirror that focuses the light onto the monochromator's entrance slit. The argon arc and monochromator operate as a tunable monochromatic source. The typical entrance and exit slits are circular $1.5 \mathrm{~mm}$ apertures. A shutter is located just after the exit slit. Inside a light tight box, a rotary stage is used to place an irradiance meter or silicon working standard at the focal plane. The exit aperture is imaged onto the detectors resulting in an oval beam of axis diameters $2.0 \mathrm{~mm}$ and $2.5 \mathrm{~mm}$. The beam was centered on, and underfilled the irradiance meter apertures and working standard detectors. A pair of orthogonal linear positioning stages translates the irradiance meters for both 


\section{Preprint}

IEEE TRANSACTIONS ON INSTRUMENTATION AND MEASUREMENT, VOL. 50, NO. 2, APRIL 2001

alignment to the optical axis and scanning for uniformity measurements. The stages' travel range is $50 \mathrm{~mm}$ with a manufacturer-specified resolution of $0.1 \mu \mathrm{m}$ and an accuracy of $0.25 \mu \mathrm{m}$ per $25 \mathrm{~mm}$. Each detector is mounted to allow its position to be adjusted along the optical axis for focusing. A gimbal mount allows for the rotation and tilt of each detector to be adjusted for perpendicular alignment to the optical axis. The output current of each detector is converted to a voltage by a transimpedance amplifier and measured by a digital voltmeter. A beam splitter directs approximately $10 \%$ of the beam to a silicon monitor photodiode. Simultaneously measuring the monitor and detector signals compensates for any source fluctuations.

The absolute spectral power responsivity of the irradiance meters was determined from $300 \mathrm{~nm}$ to $400 \mathrm{~nm}$ in $1 \mathrm{~nm}$ increments by direct substitution comparisons to the silicon photodiode working standards. The effective aperture area of each irradiance meter was determined by scanning the monochromator output beam over the irradiance meter's entrance aperture in $0.2 \mathrm{~mm}$ increments to simulate a uniform irradiance. This method has been used by NIST since 1991 [5]. It has been shown [6] that the effective aperture area is the ratio of the total signal, $i$, summed over the scanned area (total irradiance responsivity) to the product of the total beam power, $\Phi[\mathrm{W}]$, and the average spectral power responsivity, $s(\lambda)$, within the active area of the aperture,

$$
A=\frac{\sum i(x, y) \Delta x \Delta y}{\Phi \cdot s(\lambda)}
$$

where $\Delta \mathrm{x}$ and $\Delta \mathrm{y}$ are the small steps taken to completely overscan the aperture. The spectral irradiance responsivity was the product of the effective aperture area and the spectral power responsivity [7].

The aperture scanning method can be applied to irradiance meters with non-uniform spatial responsivities. Figure 3 shows the different spatial responsivity uniformities of the two irradiance meters. The large differences are due to different input geometries and diffuser materials. The data are from the UV SCF aperture scans at $0.2 \mathrm{~mm}$ steps.

The expanded uncertainty for the UV SCF measurements varies with wavelength, is a minimum at the peak responsivity, and increases at other wavelengths due primarily to wavelength uncertainties and the decreasing signal-tonoise ratio. The expanded uncertainty $(k=2)$ for both irradiance meters at $365 \mathrm{~nm}$ was $\approx 2 \%$. The uncertainties increased in regions of low responsivity to $\approx 10 \%$. These higher uncertainties do not contribute significantly to the overall measurement uncertainty of broadband sources.

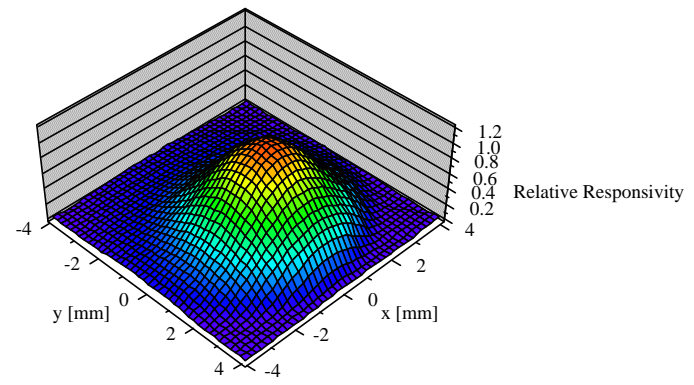

(a)

Figure 3. Surface (3D) plot of typical $365 \mathrm{~nm}$ detectors having different input geometries and diffuser materials. Figure 3a shows Irradiance Meter \#1 and Figure 3b is Irradiance Meter \#2.

\section{SIRCUS MEASUREMENTS}

Recently a second facility, the SIRCUS, has been developed. The SIRCUS employs a variety of tunable lasers (Ti:Sapphire and dye lasers) at both fundamental and frequency doubled wavelengths to cover the spectral range from $275 \mathrm{~nm}$ to $1000 \mathrm{~nm}$. The output of the laser was sent through an intensity stabilizer and a portion into a wavemeter. From the intensity stabilizer, the beam was sent through a lens into a $5 \mathrm{~cm}$ diameter integrating sphere. The $8 \mathrm{~mm}$ diameter exit port of the sphere approximated a point source, producing a uniform irradiance on the detectors. A beam splitter and monitor silicon photodiode were used to correct for source fluctuations during the measurements. A 


\section{Preprint}

IEEE TRANSACTIONS ON INSTRUMENTATION AND MEASUREMENT, VOL. 50, NO. 2, APRIL 2001

computer controlled linear positioning stage positioned the detectors in front of the integrating sphere exit port at a distance of $25 \mathrm{~cm}$.

The spectral power responsivity of the silicon trap irradiance transfer standard was measured directly against the HACR. The transfer standard was equipped with a known-area aperture. The radiant intensity of the sphere was calculated from the transfer standard power responsivity, aperture area, and sphere to transfer standard distance. The irradiance meters were substituted for the transfer standard in the uniform irradiance of the integrating sphere source. The laser was tuned between $368 \mathrm{~nm}$ and $385 \mathrm{~nm}$ with roughly $2.5 \mathrm{~nm}$ steps. The uncertainty for the SIRCUS results was $\approx 1 \%$, mainly due to the measurement reproducibility.

\section{RESULTS AND DISCUSSION}

The spectral irradiance responsivity, in A $\mathrm{mm}^{2} / \mathrm{W}$, of the two irradiance meters is presented in Figures 4 and 5.

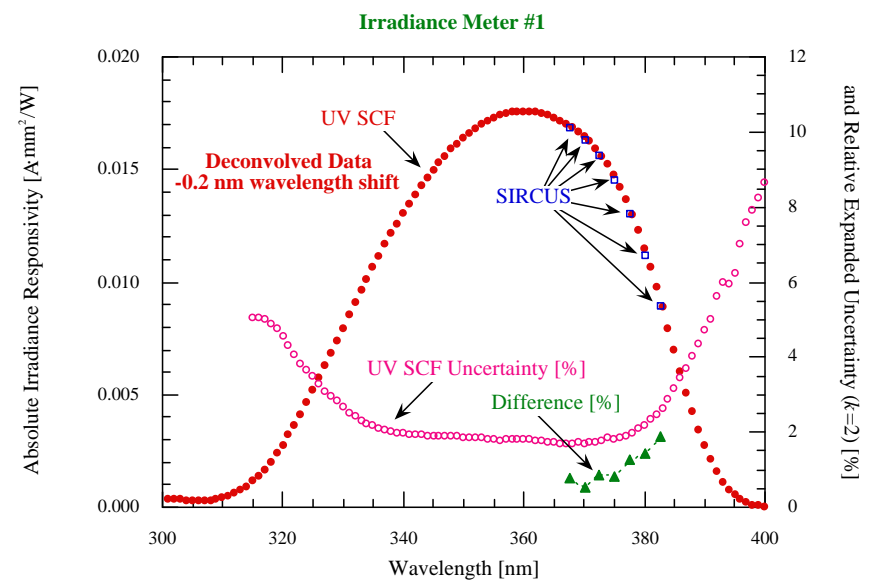

Figure 4. Plot of irradiance responsivity, UV SCF relative expanded $(k=2)$ uncertainties, and relative differences for the two methods for Irradiance Meter \#1. Irradiance Meter \#1 is a typical commercial $365 \mathrm{~nm}$ detector with an $\approx 50 \mathrm{~nm}$ bandpass (FWHM).

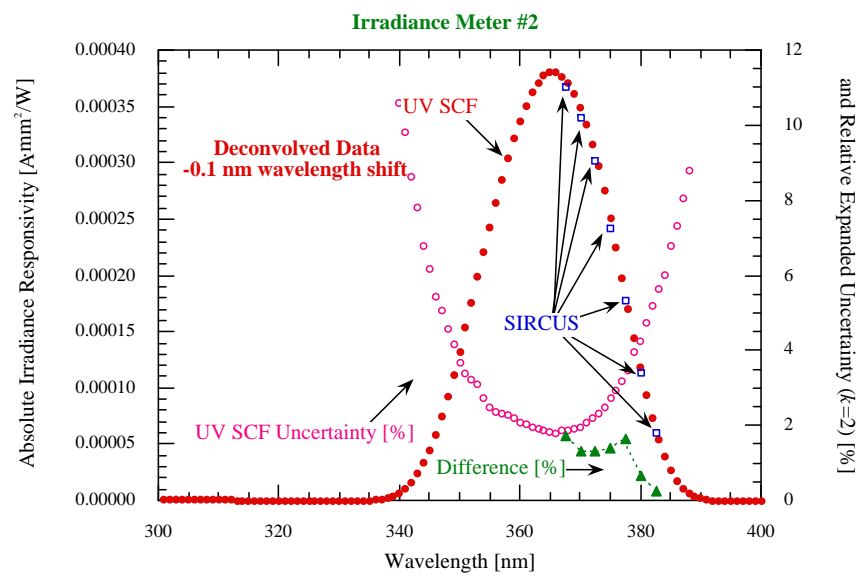

Figure 5. Plot of irradiance responsivity, UV SCF relative expanded $(k=2)$ uncertainties, and relative differences for the two methods for Irradiance Meter \#2. Irradiance Meter \#2 is a NIST designed transfer standard with a $\approx 25 \mathrm{~nm}$ bandpass (FWHM). 
The relative expanded uncertainty $(k=2)$ in the SCF measurements and the relative difference between the SCF and SIRCUS measurements are also shown in Figures 4 and 5. The two methods using the UV SCF and SIRCUS agreed to the $1 \%$ to $2 \%$ level in the peak portion of the bandpass curves as shown in Figures 4 and 5 . This is within the expanded $(k=2)$ uncertainties for the responsivity measurements. The difference increases at the lower values of the responsivity to just greater than the expanded uncertainties. The UV SCF wavelength uncertainty is larger than that of SIRCUS and may be a factor in the observed differences. Since the UV SCF data were taken in $1 \mathrm{~nm}$ intervals and the monochromator bandpass is $3 \mathrm{~nm}$, the data were deconvolved using the monochromator slit-function. A $0.1 \mathrm{~nm}$ to $0.2 \mathrm{~nm}$ wavelength shift in the deconvoluted UV SCF data brings the measurements between these two facilities into agreement on the $1 \%$ to $2 \%$ level over the entire spectral range. The calibration of the monochromator wavelength scale was checked with spectral line lamps and showed agreement to better than $0.1 \mathrm{~nm}$ over this spectral region. The wavelength shift was $-0.2 \mathrm{~nm}$ for meter \#1 and $-0.1 \mathrm{~nm}$ for meter \#2. One would expect the same shift for each irradiance meter if the wavelength scale were indeed the cause of the differences observed. A hypothesized cause is that the effective aperture area changes with wavelength. Thus, it may be necessary to scan the aperture not only at the peak responsivity, but also on either side of the peak. A definitive answer to the observed difference is currently under investigation.

The two independent methods discussed both have advantages and disadvantages. The UV SCF can scan spectrally over a wide range easily but is limited by low flux levels, the relatively wide spectral bandwidth for filter cutoff regions, and the extremely long time intervals ( $4 \mathrm{~h}$ to $6 \mathrm{~h}$ ) needed for the spatial scanning of the aperture. The SIRCUS facility has better wavelength accuracy, higher flux, faster measurement comparison (only a few minutes per data point since there is no spatial scanning), and most importantly, lower uncertainty. The lower uncertainty of the SIRCUS facility gives an independent verification of the aperture scanning method. The major disadvantage of the SIRCUS is the number of measurements needed to trace the responsivity of an irradiance meter. This is especially true over a broad wavelength range where several lasers may be needed.

Because of the labor-intensive nature of the SIRCUS measurements, a third method is proposed combining the best features of the previous two methods. The UV SCF would measure the relative spectral responsivity over a wide wavelength range, then the SIRCUS facility would measure a relatively small number of "tie" points at selected wavelengths in the center of the irradiance meter bandpass, in the tails, and in the "cut-off” regions. Measurements using this method would benefit from the lower uncertainties of the SIRCUS facility measurements and the broad spectral coverage of the UV SCF all within a fraction of the time required by the first two methods discussed here.

\section{CONCLUSION}

NIST can provide $365 \mathrm{~nm}$ irradiance meter spectral responsivity measurements to support a variety of industrial and laboratory applications using either a monochromator-based facility or tunable laser and integrating sphere facility. The agreement between these two fundamentally different methods in this first reported comparison is $1 \%$ to $2 \%$ in the peak portion of the bandpass curves which is comparable to their expanded $(k=2)$ uncertainties. The SIRCUS uncertainties are lower than the UV SCF with the potential for $0.1 \%$ uncertainties in the future. This will greatly reduce the calibration uncertainties NIST provides for UV irradiance meters in the future.

\section{References}

[1] T. C. Larason, S. S. Bruce, and A. C. Parr, Spectroradiometric Detector Measurements: Part I - Ultraviolet Detectors and Part II - Visible to Near-Infrared Detectors, Natl. Inst. Stand. Technol., Spec. Publ. 250-41, U.S. Government Printing Office, Washington, D.C., 1998.

[2] S. W. Brown, G. P. Eppeldauer, and K. R. Lykke, "NIST Facility for Spectral Irradiance and Radiance Response Calibrations with a Uniform Source,” Metrologia, vol. 37, pp. 579-582, 2000.

[3] C. A. Schrama and H. Reijn, "Novel calibration method for filter radiometers," Metrologia, vol. 36, pp. 179-182, 1999.

[4] T. M. Hunt, N. P. Fox, W. S. Hartree, and N. M. Durant, "Evaluating the performance of filter radiometers as a means of improving the uncertainty of ultraviolet measurements,” Metrologia, vol. 35, pp. 345-351, 1998.

[5] J. M. Bridges and C. L. Cromer, "Final Report on Calibration and Characterization of I-Line Exposure Meters," SEMATECH, 2706 Montopolis Drive, Austin, TX 78741, SEMATECH Report 91090678A-ENG, October 17, 1991.

[6] C. L. Cromer, G. Eppeldauer, J. E. Hardis, T. C. Larason, Y. Ohno, and A. C. Parr, "The NIST detector-based luminous intensity scale,” J. Res. Natl. Inst. Stand. Technol., vol. 101, pp. 109-132, 1996.

[7] G. P. Eppeldauer, M. Racz, and T. C. Larason, “Optical Characterization of Diffuser-Input Standard Irradiance Meters,” in Proc. Int. Soc. Opt. Eng., vol. 3573, pp. 220-224, 1998. 


\title{
Realization of a spectral radiance responsivity scale with a laser-based source and Si radiance meters
}

George P. Eppeldauer, Steven W. Brown, Thomas C. Larason, Miklos Racz , and Keith R. Lykke National Institute of Standards and Technology Optical Technology Division Gaithersburg, Maryland 20899

${ }^{*}$ Guest researcher from the Hungarian Academy of Sciences Research Institute for Technical Physics and Materials Science Principal Contact: George P. Eppeldauer

Phone: 301-975-2338; Fax: 301-869-5700

Email: geppeldauer@nist.gov

\begin{abstract}
A spectral radiance responsivity (SRR) scale derived from the spectral irradiance responsivity scale has been realized on the newly developed NIST facility for Spectral Irradiance and Radiance Responsivity Calibrations with Uniform Sources (SIRCUS). The scales were directly derived from the primary standard cryogenic radiometer using transfer standard irradiance trap detectors and laser-based uniform sources. Standards quality silicon radiance meters have been developed to hold the SRR scale. The radiance meters were characterized and optimized for sizeof-source effects. The optimized meters were calibrated for SRR against the monochromatic uniform source. In addition to the SIRCUS calibrations, preliminary tests were made on the Spectral Comparator Facility (SCF) of NIST as well. These relative SRR tests were made in both imaging and non-imaging measurement modes. The results of the different SRR calibrations are compared and evaluated.
\end{abstract}




\section{Introduction}

The high accuracy of primary standard cryogenic radiometers [1] can be utilized only if improved quality radiometers are developed and calibrated directly against the primary standard. To achieve this goal, we are developing a reference (high accuracy) spectral irradiance and radiance responsivity calibration facility [2]. This facility utilizes uniform sources using integrating spheres and lasers $[3,4,5]$ to obtain lower scale uncertainties compared to traditional monochromators [6]. This paper describes how the irradiance responsivity scale is propagated to the radiance responsivity scale on SIRCUS. Design and the basic radiometric characterizations of a broad-band working standard Si radiance meter is discussed. This high performance radiance meter will hold the high accuracy SRR scale. Arrangements and considerations are discussed for accurate relative and absolute SRR measurements.

\section{Radiance responsivity scale derivation}

Figure 1 shows the scale propagation from the NIST High Accuracy Cryogenic Radiometer (HACR) to the SRR scale level. Si trap detectors are calibrated against the HACR at several laser lines. The response interpolation is done between $406 \mathrm{~nm}$ and $920 \mathrm{~nm}$ based on the physical model of the Hamamatsu 1337 Si photodiodes [7, 8]. The aperture area in front of the trap detector is measured with high accuracy using point source geometry [9]. The irradiance responsivity of the transfer standard Si trap detector is equal to its power responsivity times the aperture area.

The SRR scale is derived from the spectral irradiance responsivity scale. First, the radiant intensity of the uniform source is determined from the detector irradiance responsivity and the distance between detector and source apertures. In this case, the distance is large (about $1 \mathrm{~m}$ ), therefore the source is used as a point source. The radiance within the source aperture (at a laser wavelength) is the ratio of the radiant intensity to the source aperture area. When calibrating the radiance meter against the Lambertian (uniform) source, its target area (the measured spot) has 
to be within the source aperture (of $5 \mathrm{~cm}$ diameter). The radiance responsivity of the meter is the ratio of its electrical output signal to the measured source radiance.

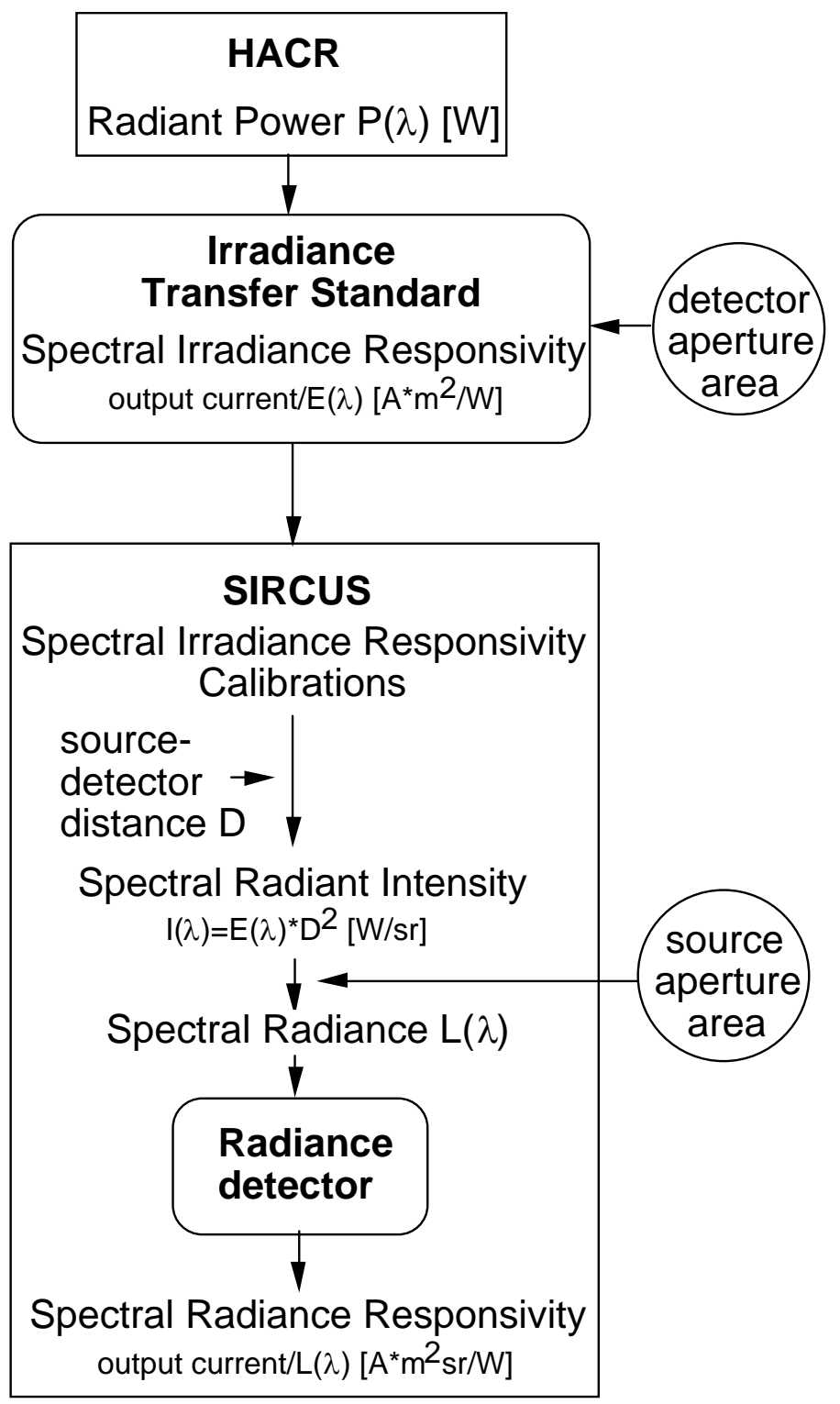

Figure 1. SRR scale derivation.

\section{Si radiance meter design}

Figure 2 shows the design of the transfer standard Si radiance meter. The input optics are attached to the radiometer base unit. A beveled aperture in the mirror serves as a field stop and it is positioned into the focus of the camera 
lens. The surroundings of the source target area can be viewed through the eye-piece. The target radiation is imaged to the center of the Si photodiode through a second imaging optics producing a well defined field-of-view (FOV) and a very efficient out-of-target blocking. The aperture stop in front of the second imaging system keeps the flux response of the optics constant for different target distance (lens focus) adjustments. In order to eliminate interferences, we replaced the original plane-parallel window of the Si photodiode with a ground glass diffuser of high transmittance.

\section{Characterization of the Si radiance meter}

Figure 3 shows the angular responsivity of the Si radiance meter. The measured source was a $1000 \mathrm{~W}$ tungsten halogen lamp equipped with a $5 \mathrm{~mm}$ aperture $4 \mathrm{~cm}$ away from the lamp. The distance between the radiometer and the source aperture was about $2.5 \mathrm{~m}$. The radiometer was rotated in the horizontal plane around a pivot point in the center of its camera lens. This scan was repeated after a $90^{\circ}$ rotation of the radiometer around its axis to obtain the angular responsivity in the perpendicular (vertical) plane as well. The radiance measurement angle is $0.6^{\circ}$ (FWHM) and the out-of-target blocking (the signal at zero rotation angle ratioed to the signals at different rotation angles) is 2

$\mathrm{x} 10^{3}$ at $0.5^{\circ}, 10^{4}$ at $1.5^{\circ}$ and higher than $10^{5}$ at $5^{\circ}$. The vertical and horizontal scans resulted in similar results 


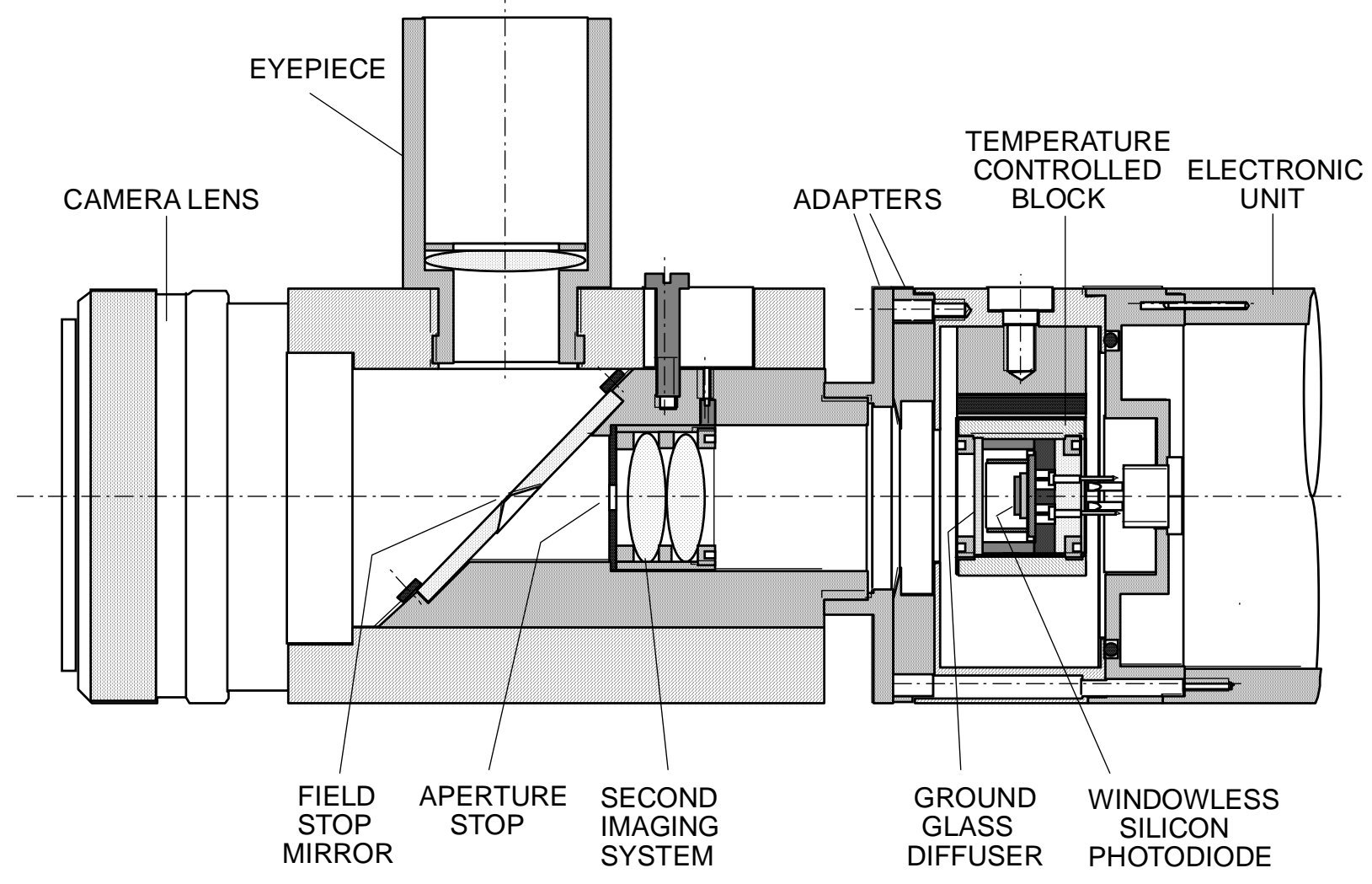

Figure 2. Working standard Si radiance meter.

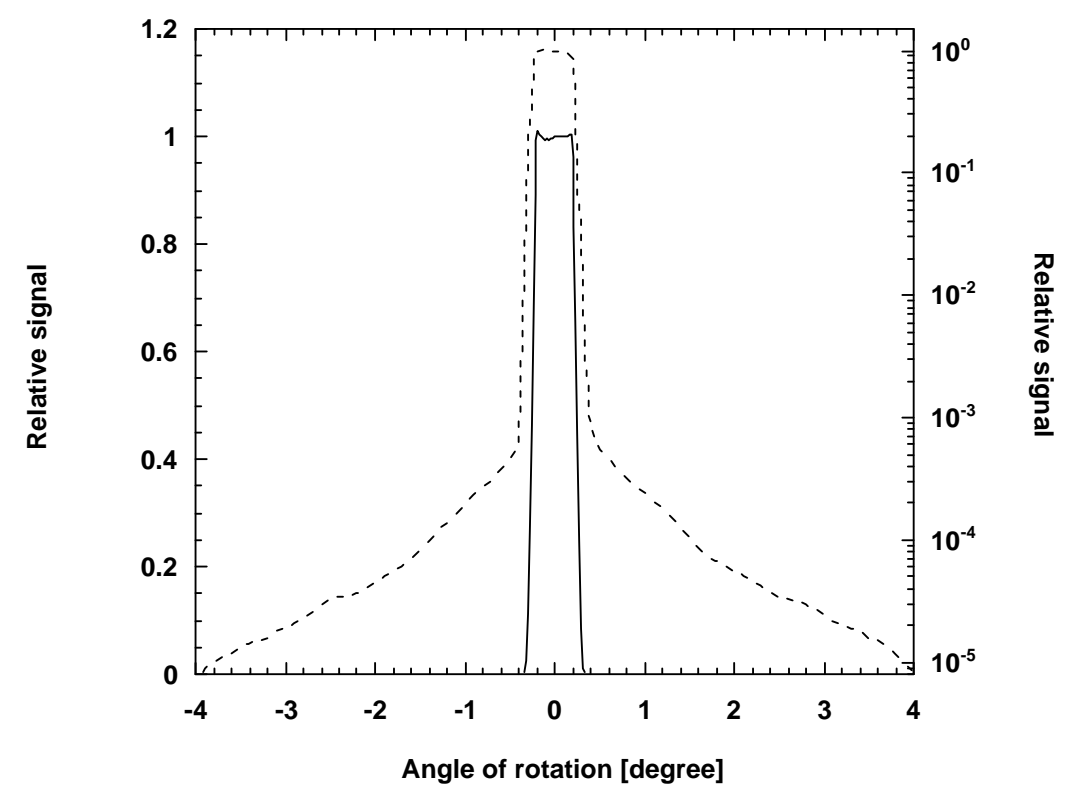

Figure 3. Angular responsivity of the Si radiance meter. Horizontal response (dashed line, logarithmic scale) 
and vertical response (solid line, linear scale)

\section{Absolute spectral radiance responsivity}

The absolute SRR was measured on the SIRCUS using a $20 \mathrm{~cm}$ diameter Spectralon [8] coated integrating sphere. The sphere was irradiated by 98 laser lines of different wavelengths between $400 \mathrm{~nm}$ and $920 \mathrm{~nm}$. The measurement steps followed the scale derivation chart in Figure 1. The solid line in Figure 5 shows the absolute SRR of the working standard radiance meter.

\section{Uncertainties}

The uncertainty budget of the SIRCUS radiance responsivity calibrations is summarized in Table 1 . Type A and Type B uncertainties [10] are shown for both the present (preliminary) and future calibrations. The source radiance uncertainties are separated from the uncertainties of the radiance transfer to test radiance meters. We plan to decrease the present (uncorrected) relative combined standard uncertainty ( $k=1)$ of $0.21 \%$ to about $0.05 \%$ after improving the uncertainties as indicated in the Table.

\section{Relative spectral radiance responsivity comparison to the SCF}

Independent relative SRR measurements were made on the SCF. We made two preliminary tests only with two different beam geometries. The first one was a simple, non-imaging method which can be used for high quality radiance meters where a second imaging optics projects the beam always into the same position on the detector. In this case, the target spot of the radiance meter was not defined. These test results are shown in Figure 5 (non-imaged SCF curves). With normalization at $705 \mathrm{~nm}$, the obtained relative radiance responsivity curve agrees with the results of the absolute (SIRCUS) radiance responsivity measurements within $1 \%$ between $475 \mathrm{~nm}$ and $920 \mathrm{~nm}$. The repeatability uncertainty was equal to or less than $0.25 \%$ from three relative spectral responsivity scans made with different (repeated) alignments. The relative standard uncertainties $(\mathrm{k}=1)$ of the SCF measurements are $0.78 \%$ at 
$400 \mathrm{~nm}$ and $0.11 \%$ from $450 \mathrm{~nm}$ to $900 \mathrm{~nm}$ in power responsivity mode [6] and $0.94 \%$ at $400 \mathrm{~nm}$ and almost constant at $0.54 \%$ in the visible up to $925 \mathrm{~nm}$ in irradiance responsivity mode [11].

Table 1

Relative combined standard uncertainty $(\mathrm{k}=1)$ of the SIRCUS present radiance responsivity calibrations [\%] (future calibration uncertainties are shown with italic)

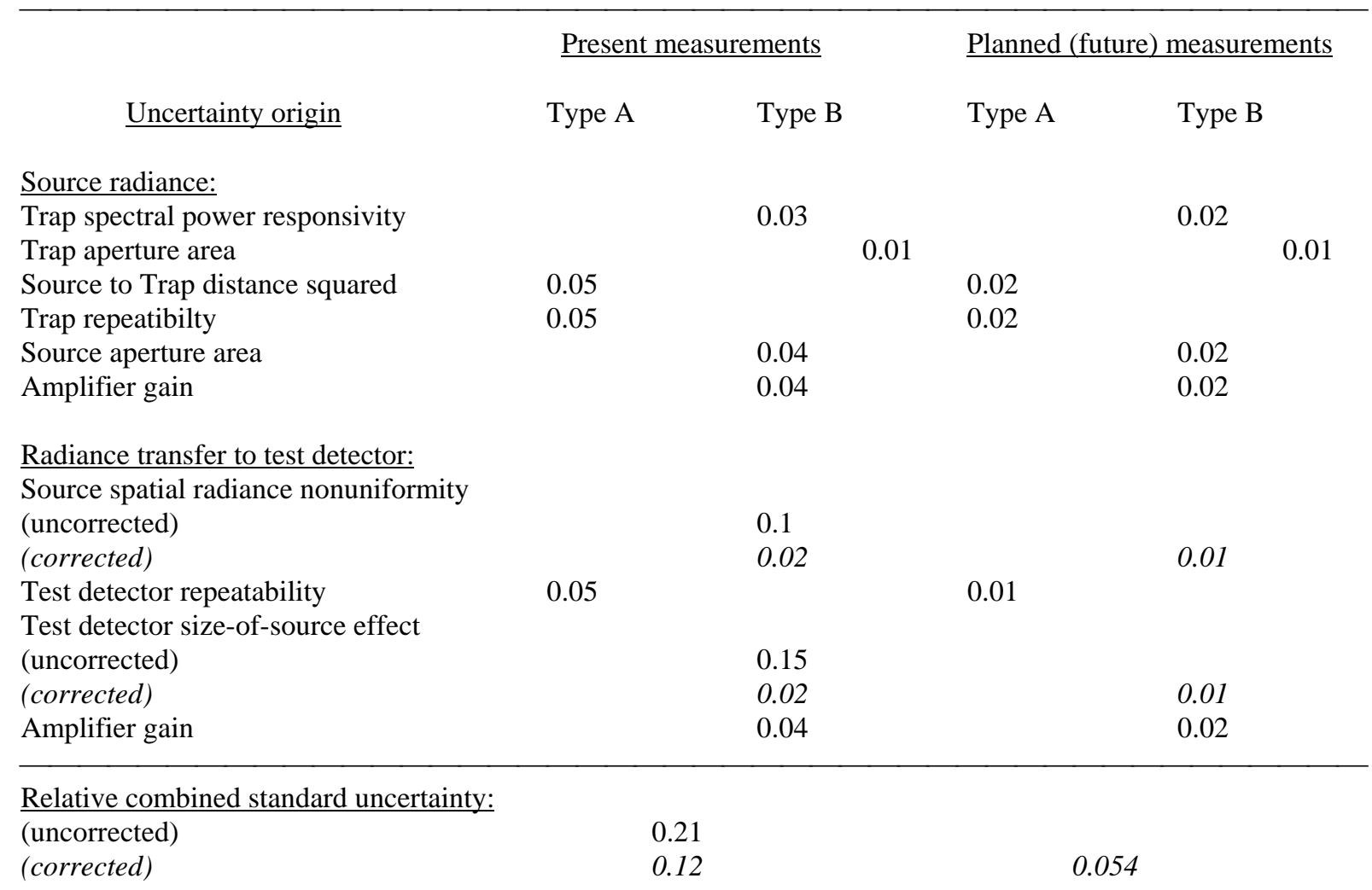

Because the non-imaging method is not correct for radiance responsivity measurements, we are suggesting here an imaging type test method. We made a preliminary test using this second method where the standard arrangement of the SCF was modified to measure simple radiance meters where the detector may have spatially non-uniform responsivity and no second imaging optics are used. The modification is shown in Figure 4 . The f/9 converging beam from the monochromator, before the two lenses, produces a $1.1 \mathrm{~mm}$ diameter spot (which is on the surface of the power measuring detector in the standard SCF arrangement). In the modification, a camera lens of $35 \mathrm{~mm}$ focal length is used as a relay lens to magnify this spot into a $15 \mathrm{~mm}$ diameter image, inside of an achromatic collector lens of $350 \mathrm{~mm}$ focal length. The corrected lens collects the radiation exiting from the relay lens and projects it into the center of the camera lens of the radiance meter. The distance of the radiance meter from the collector lens has to 
be adjusted until the diameter of the target spot of the radiance meter is equal to the diameter of the reference detector which is positioned directly after the collector lens. The reference detector has to be circular (e.g. a $1 \mathrm{~cm}^{2}$ silicon detector). In the eyepiece of the radiance meter, the black (measured) spot has to be in the center of the 15 mm diameter image inside of the collector lens. The relative radiance responsivity of the meter will be the power responsivity of the reference detector times the ratio of the radiance meter output signal to the output signal of the reference detector.

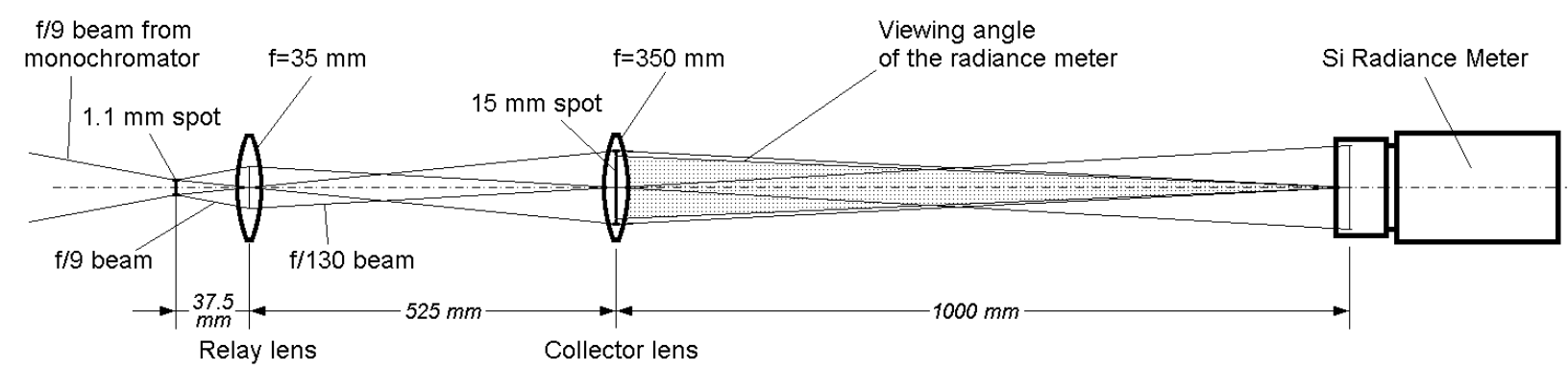

Figure 4. Optical arrangement for radiance meter relative responsivity measurement.

In our preliminary test, an $18 \mathrm{~mm}$ by $18 \mathrm{~mm}$ reference $\mathrm{Si}$ detector was used and the above described target-spot and detector-diameter match was not achieved. Because of lens chromatic abberations, the size of the magnified spot inside of the collector lens was wavelength dependent. The lenses were optimized for the visible range. Also, the irradiance distribution within the monochromator output spot depends on the wavelength. These problems caused relatively large uncertainties for wavelenghts shorter than $420 \mathrm{~nm}$. In Figure 5, where the radiance responsivity measurement results are summarized, the normalization was made at $705 \mathrm{~nm}$, the peak responsivity of the photodiode, resulting in about $2.5 \%$ discrepancy (relative to the absolute radiance reponsivity) at $450 \mathrm{~nm}$ (imaged SCF curves).

The two relative radiance responsivity curves are different not only because of the non-optimized arrangement of the imaged SCF measurements (as mentioned above), but also because the transmittance of the anti-reflecting coating can be different for the different incident angles of the two cases. A systematic relative radiance responsivity uncertainty was measured at $420 \mathrm{~nm}$. This problem, which requires further examination, significantly 
increased the responsivity deviation of the non-imaged SCF measurements relative to the SIRCUS results for wavelengths shorter than $475 \mathrm{~nm}$.

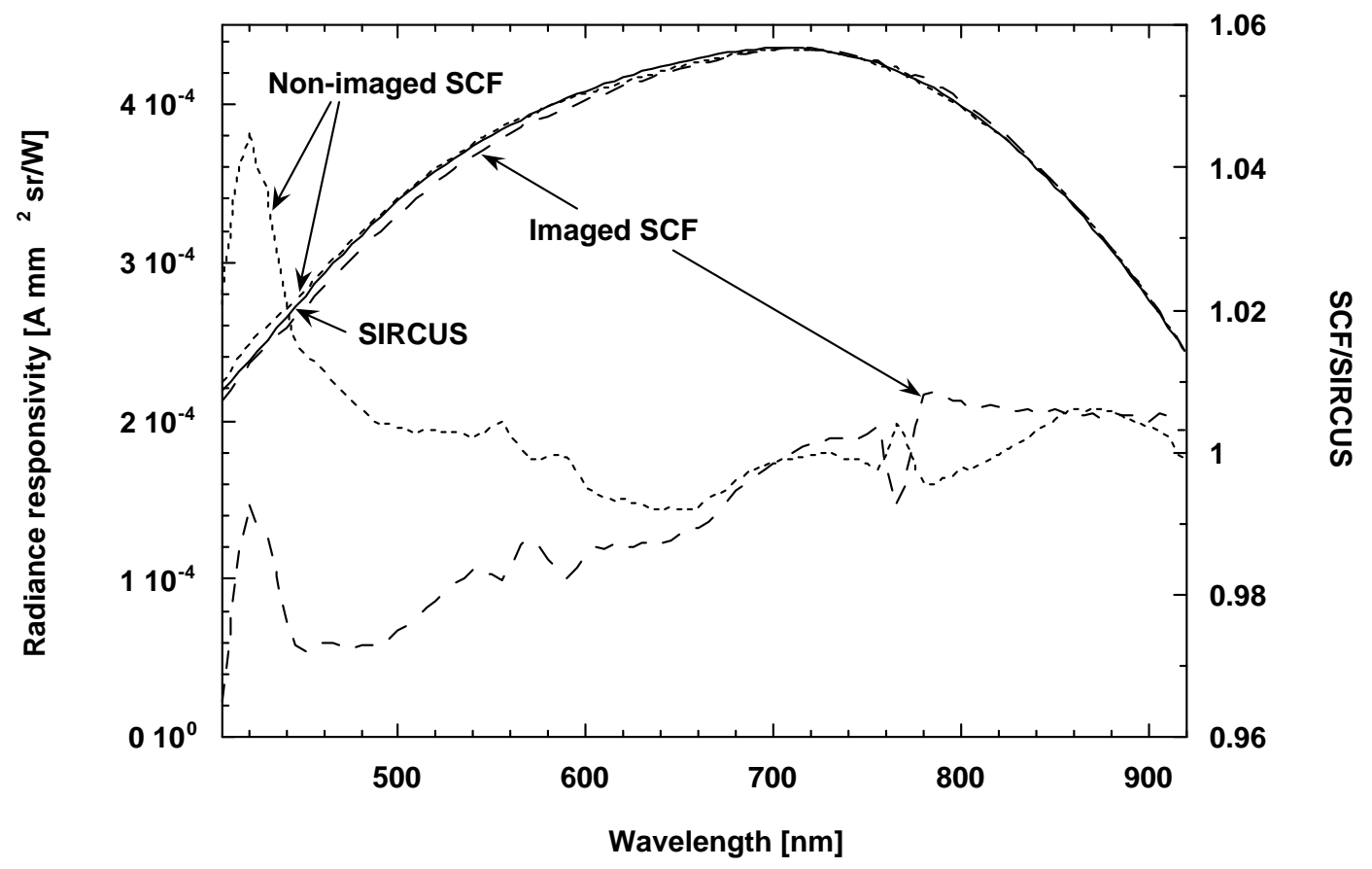

Figure 5. SRR of the NIST Radiance Meter \#303. The relative radiance responsivity curves were measured on the SCF in both imaging and non-imaging modes and normalized to the absolute responsivity as measured on the SIRCUS. The normalizations were made at $705 \mathrm{~nm}$. The responsivity ratios are shown relative to the SIRCUS results.

\section{Conclusion}

SRR calibrations were performed on the preliminary SIRCUS facility. The $0.21 \%$ relative combined standard uncertainty $(\mathrm{k}=1)$ of the first radiance responsivity measurements (which is 3-4 times better than the SCF irradiance responsivity uncertainty in the same wavelength range) showed the dominating uncertainty components. We could 
also estimate the achievable uncertainties and the necessary improvements (finalization) of the facility. Comparison of the SIRCUS responsivity measurements to the traditional monochromator based SCF measurements showed that systematic errors in the SCF SRR measurements can be verified. The SIRCUS, with the final control and measurement system, including a variety of transfer- and working-standard radiometers and improved correction and calibration procedures, will serve as a high accuracy reference calibration facility for radiometry, photometry, and colorimetry.

\section{Acknowledgements}

The authors thank the Air Force Metrology for funding this project.

\section{References}

[1] T.R. Gentile, J.M. Houston, J.E. Hardis, C.L. Cromer, and A.C. Parr, "National Institute of Standards and Technology high accuracy cryogenic radiometer,” Appl. Opt. 35 (7), 1056-1068, 1996.

[2] S.W. Brown, G.P. Eppeldauer, and K.R. Lykke, "NIST Facility for Spectral Irradiance and Radiance Response Calibrations with a Uniform Source,” Newrad'99, Madrid, Spain.

[3] K.R. Lykke, P.S. Shaw, L.M. Hanssen, and G.P. Eppeldauer, “ Development of a Monochromatic Uniform Source Facility for Calibration of Radiance and Irradiance Detectors from $0.2 \mu \mathrm{m}$ to $12 \mu \mathrm{m}$,” Metrologia, 36, p. 141-146, 1999.

[4] K.D. Mielenz, R.D. Saunders, and J.B. Shumaker, "Spectroradiometric determination of the freezing temperature of gold,” J. Res. Natl. Stand. Technol. 95, 49-67, 1990.

[5] V.E. Anderson, N.P. Fox, and D.H. Nettleton, "Highly stable, monochromatic and tunable optical radiation source and its application to high accuracy spectrophotometry,” Applied Optics, 31, No. 4, 536-545, 1992. 
[6] T.C. Larason, S.S. Bruce, A.C. Parr, “Spectroradiometric Detector Measurements: Part I-Ultraviolet Detectors and Part II-Visible to Near-Infrared Detectors,” National Institute of Standards and Technology Special Publication 250-41, 1998.

[7] T.R. Gentile, J.M. Houston, and C.L. Cromer, "Realization of a scale of absolute spectral response using the National Institute of Standards and Technology high-accuracy cryogenic radiometer,” Appl. Opt. 35, No. 22, 4392-4403, 1996.

[8] Identification of commercial equipment to specify adequately an experimental problem does not imply recommendation or endorsement by the National Institute of Standards and Technology nor does it imply that the equipment identified is necessarily the best available for the purpose.

[9] J.B. Fowler, R.S. Durvasula, and A.C. Parr, "High-accuracy aperture-area measurement facilities at the National Institute of Standards and Technology,” Metrologia, 35, No. 4. 497-500, 1998.

[10]B.N. Taylor and C.E. Kuyatt, “Guidelines for Evaluating and Expressing the Uncertainty of NIST Measurement Results,” Natl. Inst. Stand. Technol., Tech. Note 1297, 1994.

[11] G. Eppeldauer, M Racz, and T. Larason, “Optical characterization of diffuser-input standard irradiance meters,” SPIE 3573, 220-224, 1998. 


\title{
UNCERTAINTIES OF SPECTRAL RESPONSIVITY MEASUREMENTS
}

\author{
G. P. Eppeldauer, G. Sauter ${ }^{1}$, and J. L. Gardner ${ }^{2}$
}

\author{
National Institute of Standards and Technology \\ Gaithersburg, Maryland, USA \\ ${ }^{1}$ PTB, Braunschweig, Germany \\ ${ }^{2}$ National Measurement Institute, Lindfield, Australia
}

\begin{abstract}
Uncertainty estimates of detector spectral responsivity measurements are discussed. A model describes independent (uncorrelated) uncertainty components at a given wavelength based on the measurement equation for test detector calibrations. Systematic (correlated) components across wavelength are described briefly for spectrally integrated responsivity measurements. Propagation of the random and systematic components is discussed.
\end{abstract}

\section{INTRODUCTION}

The uncertainty analysis of spectral responsivity measurements should follow the "Guide to the Expression of Uncertainty in Measurement" (GUM) [1]. A Technical Report within the TC2-48 Technical Committee of the International Committee on Illumination $(\mathrm{CIE})$ is being prepared to make recommendations for spectral responsivity measurements of detectors, radiometers, and photometers. The goal of the present work is to prepare a chapter for the CIE TC2-48 Technical Report on the uncertainty determination of spectral responsivity measurements using the GUM recommendations.

Starting with the measurement equation, a model is described to evaluate the radiant power responsivity determination of a test detector at a given wavelength. Uncertainty in the measured value arises from the uncertainty of the spectral responsivity of the standard (reference) detector, and from uncertainties in the elements of the correction factor used to evaluate the radiant power responsivity of the test detector from the measurement (calibration) transfer. Following the description of contributions that are independent of wavelength, systematic (correlated) uncertainty components in the reference detector are described and propaga- tion of the components will be discussed for spectrally integrated measurements.

\section{THE MEASUREMENT PROCESS}

The relative standard uncertainty of spectral responsivity values can be derived from the measurement equation of the detector substitution method. The substitution method transfers the radiometric responsivity of a standard detector to a test detector that has similar properties to the standard detector. When the standard detector measures the constant input flux (the total radiant power in the incident beam) $\Phi$, its output signal $I_{\mathrm{S}}$ is:

$$
I_{S}=S_{S} \cdot \Phi
$$

where $s_{S}$ is the known responsivity of the standard detector. In the following step, the test detector is substituted for the standard detector and measures the same radiant power in the same arrangement:

$$
I_{\mathrm{T}}=s_{\mathrm{T}} \cdot \Phi
$$

where $I_{\mathrm{T}}$ is the output signal of the test detector and $s_{\mathrm{T}}$ is the unknown radiant power responsivity of the test detector that can be calculated from Eqs. (1) and (2):

$$
s_{\mathrm{T}}=s_{\mathrm{S}} \cdot \frac{I_{\mathrm{T}}}{I_{\mathrm{S}}}
$$

When a monitor detector is used to decrease fluctuations in the radiant power, the modified measurement equation will be:

$s_{\mathrm{T}}=s_{\mathrm{S}} \cdot \frac{I_{\mathrm{T}} / I_{\mathrm{MT}}}{I_{\mathrm{S}} / I_{\mathrm{MS}}}=s_{\mathrm{S}} \cdot \frac{R_{\mathrm{T}}}{R_{\mathrm{S}}}$

where instead of the electrical output signals $I_{\mathrm{T}}, I_{\mathrm{S}}$ their ratios to the simultaneously measured monitor output signals $I_{\mathrm{MT}}, I_{\mathrm{MS}}$ are used: $R_{\mathrm{T}}=I_{\mathrm{T}} / I_{\mathrm{MT}}, R_{\mathrm{S}}=I_{\mathrm{S}} / I_{\mathrm{MS}}$. 


\section{MODEL AT A GIVEN WAVELENGTH}

In Eq. (4) the radiant power responsivity of the test detector is calculated as a product of at least three factors. If we ignore the measurement errors of the electrical signals, the relative standard uncertainty in the spectral radiant power responsivity of the test detector can be written as

$u_{\text {rel }}\left(s_{\mathrm{T}}\right)=\left[u_{\text {rel }}^{2}\left(s_{\mathrm{S}}\right)+u_{\text {rel }}^{2}\left(R_{\mathrm{T}}\right)+u_{\text {rel }}^{2}\left(R_{\mathrm{S}}\right)\right]^{1 / 2}$

In the brackets, the first contribution is the relative standard measurement uncertainty calculated from the certificate of the reference detector and the operational conditions during transfer. The other two contributions come from the measurement transfer.

Note that at present, calibration certificates do not report correlation coefficients or uncorrelated and correlated components separately. While measurements at the one wavelength do not depend on these correlations, they become important when combining values at different wavelengths in spectrally integrated components; this is discussed in Section 4 below.

The effective radiant power responsivity $s_{\mathrm{S}}$ of the reference detector requires a small correction for a possible temperature deviation $\Delta T$ (relative to the temperature during calibration), and for a relative nonuniformity $\beta_{\mathrm{S}}$ :

$\mathrm{S}_{\mathrm{S}}=\mathrm{S}_{\mathrm{S}}^{\prime} \cdot\left(1+\alpha_{\mathrm{S}} \cdot \Delta T+\beta_{\mathrm{S}}\right)$

where $\alpha_{\mathrm{S}}$ is the relative temperature coefficient for the responsivity. The relative standard measurement uncertainty of the radiant power responsivity $s_{S}^{\prime}$ of the reference detector is calculated from the expanded measurement uncertainty $U\left(s_{\mathrm{S}}^{\prime}\right)$ and the coverage factor $(k=2)$ given in the calibration certificate when the responsivity $s_{S}^{\prime}$ was measured at temperature $T_{\mathrm{C}}$ and is given by

$u_{\text {rel }}\left(s_{S}^{\prime}\right)=U\left(s_{S}^{\prime}\right) /\left(2 s_{S}^{\prime}\right)$.

The temperature deviation $\Delta T=T_{\mathrm{S}}-T_{\mathrm{C}}$ is calculated from the temperature $T_{\mathrm{C}}$ (nominal value, no uncertainty contribution) and the measured ambient temperature $T_{\mathrm{S}}$ during use. Often the standard deviation $s\left(T_{\mathrm{S}}\right)$ of repeated thermometer readings is smaller than its resolution, which is an interval $\pm \operatorname{res}\left(T_{\mathrm{S}}\right)$ with rectangular probability distribution. The temperature is measured with the thermometer certified with an (absolute) expanded measurement uncertainty $U\left(T_{S}\right)=\delta T$ for a $(k=2)$ coverage interval and gives the standard variance $(\delta T / 2)^{2}$. The standard variance of the temperature deviation from the three contributions is:

$u^{2}(\Delta T)=\left(\frac{\delta T}{2}\right)^{2}+s^{2}\left(T_{\mathrm{S}}\right)+\frac{\operatorname{res}^{2}\left(T_{\mathrm{S}}\right)}{3}$

The relative temperature coefficient $\alpha_{\mathrm{S}}$ is measured separately and the value can be determined with an associated relative standard measurement uncertainty $u\left(\alpha_{\mathrm{S}}\right)$ or taken from the related literature.

Finally, the relative non-uniformity $\beta_{\mathrm{S}}$ has a zero value (it is included in the responsivity value) but its variation contributes to the responsivity measurement uncertainty within an interval $\pm \Delta s_{S}$, having a rectangular probability distribution. Therefore, the standard variance is calculated as $u^{2}\left(\beta_{\mathrm{S}}\right)=\Delta s_{\mathrm{S}}^{2} / 3$.

After these preparations and the two assumptions $1>>\left|\alpha_{\mathrm{S}} \cdot \Delta T\right|+\left|\beta_{\mathrm{S}}\right|$ and no correlations between the quantities, the relative combined variance associated to the responsivity of the standard detector can be written:

$$
\begin{aligned}
u_{\mathrm{rel}}^{2}\left(\mathrm{~s}_{\mathrm{S}}\right)= & u_{\mathrm{rel}}^{2}\left(\mathrm{~s}_{\mathrm{S}}^{\prime}\right)+u^{2}\left(\beta_{\mathrm{S}}\right)+\alpha_{\mathrm{S}}^{2} \cdot u^{2}(\Delta T)+ \\
& u^{2}\left(\alpha_{\mathrm{S}}\right) \cdot \Delta T^{2}
\end{aligned}
$$

If the temperature at the time of measurement is the same as that at the time of calibration, $\Delta T=0$. In this case, from second order terms, the factor $\Delta T^{2}$ has to be replaced by the associated standard variance $u^{2}(\Delta T)$.

For the test detector, similar contributions have to be taken into consideration. The corrected value of the radiant power responsivity $s_{T}^{\prime}$ of the test detector can be calculated for the ambient temperature $T_{\mathrm{C}}$ and for zero contribution of its own nonuniformity:

$$
\mathrm{s}_{\mathrm{T}}=\mathrm{s}_{\mathrm{T}}^{\prime} \cdot\left(1+\alpha_{\mathrm{T}} \cdot \Delta T+\beta_{\mathrm{T}}\right)
$$


Similarly to the considerations before, the associated relative standard measurement uncertainty of $s_{\mathrm{T}}$ can be determined assuming small corrections $1>\left|\alpha_{\mathrm{T}} \cdot \Delta T\right|+\left|\beta_{\mathrm{T}}\right|$ and no correlation. Then the relative variance can be written:

$$
\begin{aligned}
u_{\text {rel }}^{2}\left(\mathrm{~s}_{\mathrm{T}}\right)= & u_{\mathrm{rel}}^{2}\left(\mathrm{~s}_{\mathrm{T}}^{\prime}\right)+u^{2}\left(\beta_{\mathrm{T}}\right)+\alpha_{\mathrm{T}}^{2} \cdot u^{2}(\Delta T)+ \\
& u^{2}\left(\alpha_{\mathrm{T}}\right) \cdot \Delta T^{2}
\end{aligned}
$$

The combination of Eq. (4) with the corrections in Eq. (6) and (10) give a new model for the radiant power responsivity evaluation:

$$
\mathrm{s}_{\mathrm{T}}^{\prime}=\mathrm{s}_{\mathrm{S}}^{\prime} \cdot\left[\frac{1+\alpha_{\mathrm{S}} \cdot \Delta T+\beta_{\mathrm{S}}}{1+\alpha_{\mathrm{T}} \cdot \Delta T+\beta_{\mathrm{T}}}\right] \cdot \frac{I_{\mathrm{T}} / I_{\mathrm{MT}}}{I_{\mathrm{S}} / I_{\mathrm{MS}}}
$$

and taking the earlier assumptions into account (first order approach), the bracket is simplified to $\left[1+\left(\alpha_{\mathrm{S}}-\alpha_{\mathrm{T}}\right) \cdot \Delta T+\beta_{\mathrm{S}}-\beta_{\mathrm{T}}\right]$. This approach directly shows that for very similar test and reference detectors the temperature and spatial uniformity errors will cancel out.

The four photocurrents in Eq. (12), also contribute to the measurement uncertainty. The assumptions for the photocurrent measurements are:

A. The same amplifier and Digital Voltmeter (DVM) are used for both the test and reference detectors assuming that the gain and range settings are valid for both photocurrents.

B. With the test detector in place and shutter open, a series of $n \geq 20$ repeated readings are taken simultaneously for both the test $\left(I_{\mathrm{T}}^{\prime}\right)$ and the monitor $\left(I_{\mathrm{MT}}^{\prime}\right)$ detectors.

C. With the above conditions (especially for the amplifier gain and DVM range) but with the shutter closed, "dark measurements" $\left(I_{\text {TO }}^{\prime}, I_{\text {MTO }}^{\prime}\right)$ are taken.

D. With the reference detector in place and shutter open, $n$ repeated readings are taken simultaneously for both the reference $\left(I_{\mathrm{S}}^{\prime}\right)$ and monitor ( $\left.I_{\mathrm{MS}}^{\prime}\right)$ detectors.

E. Under the same conditions, but with the shutter closed, "dark measurements" ( $\left.I_{\text {so }}^{\prime}\right)$, $\left(I_{\text {MSO }}^{\prime}\right)$ are taken.

Mean values like $\overline{I_{T}^{\prime}}$ and its related standard deviations of the mean $s\left(\overline{I_{T}^{\prime}}\right)$ are calculated for all the measured quantities and the two ratios: $R_{\mathrm{T}}^{\prime}=I_{\mathrm{T}}^{\prime} / I_{\mathrm{MT}}^{\prime} ; R_{\mathrm{S}}^{\prime}=I_{\mathrm{S}}^{\prime} / I_{\mathrm{MS}}^{\prime}$. In principle, instead of these ratios, the dark current corrected ratios should be averaged. However, if the dark current is small and the measurement setup is fairly stable, then the measurement uncertainty evaluation can be simplified:

$R_{\mathrm{T}}=\frac{1}{n} \sum_{i} \frac{l_{\mathrm{T}, i}^{\prime}-\bar{l}_{\mathrm{T}, i}^{\prime}}{I_{\mathrm{MT}, i}^{\prime}-\bar{l}_{\mathrm{MT}, i}^{\prime}} \approx \bar{R}_{\mathrm{T}}^{\prime} \cdot\left(1-\frac{\bar{l}_{\mathrm{T} 0}^{\prime}}{\bar{l}_{\mathrm{T}}^{\prime}}+\frac{\overline{I_{\mathrm{MTO}}^{\prime}}}{\bar{l}_{\mathrm{MT}}^{\prime}}\right)$

The ratio for the reference detector readings can be calculated similarly. Finally, the double ratio in Eq. (4) will be:

$\frac{R_{\mathrm{T}}}{R_{\mathrm{S}}} \approx \frac{\overline{R_{\mathrm{T}}^{\prime}}}{\overline{R_{\mathrm{S}}^{\prime}}} \cdot\left(1+\frac{\overline{l_{\mathrm{SO}}^{\prime}}}{\overline{l_{\mathrm{S}}^{\prime}}}-\frac{\overline{l_{\mathrm{T} 0}^{\prime}}}{\overline{l_{\mathrm{T}}^{\prime}}}+\frac{\overline{l_{\mathrm{MTO}}^{\prime}}}{\overline{l_{\mathrm{MT}}^{\prime}}}-\frac{\overline{l_{\mathrm{MSO}}^{\prime}}}{\overline{l_{\mathrm{MS}}^{\prime}}}\right)$

Usually, there are four contributions to the measurement uncertainty from a series of repeated readings of a radiometer: the standard deviations of the two mean values for light and dark readings; the calibration factor, that cancels out in Eq.(14); and the resolution $\pm \Delta l$ of the DVM. For all photocurrents, the standard measurement uncertainties are calculated similarly:

$u\left(\bar{l}_{\text {so }}^{\prime}\right)=\sqrt{s^{2}\left(\bar{l}_{\text {so }}^{\prime}\right)+(\Delta l / \sqrt{3})^{2}}$

If a common amplifier is used for both the standard and the test detectors, then the currents $\bar{l}_{\mathrm{S} 0}^{\prime}, \bar{l}_{\mathrm{T} 0}^{\prime}$ for dark (and offset) measurements are the same, as are their measurement uncertainties. As they are determined in a series, one after the other, there is no statistical correlation, even if recorded by the same amplifier.

Finally, the model to evaluate the radiant power responsivity of the test detector is calculated from the combination of Eqs. (12) and (14) with the higher orders omitted:

$$
\begin{aligned}
& s_{\mathrm{T}}^{\prime}=s_{\mathrm{S}}^{\prime} \cdot \frac{\overline{R_{\mathrm{T}}^{\prime}}}{\overline{R_{\mathrm{S}}^{\prime}}} \cdot \text { corr } \\
& \text { corr }=\left[\begin{array}{l}
1+\left(\alpha_{\mathrm{S}}-\alpha_{\mathrm{T}}\right) \cdot \Delta T+\beta_{\mathrm{S}}-\beta_{\mathrm{T}}+ \\
\frac{\bar{l}_{\mathrm{S} 0}^{\prime}}{\overline{l_{\mathrm{S}}^{\prime}}}-\frac{\bar{l}_{\mathrm{T} 0}^{\prime}}{\bar{l}_{\mathrm{T}}^{\prime}}+\frac{\overline{l_{\mathrm{MTO}}^{\prime}}}{\bar{l}_{\mathrm{MT}}^{\prime}}-\frac{\overline{l_{\mathrm{MS}}^{\prime}}}{\overline{l_{\mathrm{MS}}^{\prime}}}
\end{array}\right]
\end{aligned}
$$

All the contributions in the brackets are very small corrections and the associated relative standard measurement uncertainty is often 
negligible. In a reduced form, the relative standard measurement uncertainty associated to the radiant power responsivity is combined from four dominant contributions:

$u_{\text {rel }}\left(s_{\mathrm{T}}^{\prime}\right)=\sqrt{u_{\text {rel }}^{2}\left(s_{\mathrm{S}}^{\prime}\right)+u_{\text {rel }}^{2}\left(\bar{R}_{\mathrm{T}}^{\prime}\right)+u_{\text {rel }}^{2}\left(\bar{R}_{\mathrm{S}}^{\prime}\right)+u_{\text {rel }}^{2}(\text { corr })}$

The principles in the measurement equation of Eq. (16) are valid for each single wavelength $\lambda$. Accordingly, all quantities are wavelength dependent:

$s_{\top}^{\prime}(\lambda)=s_{S}^{\prime}(\lambda) \cdot \frac{\overline{R_{T}^{\prime}}(\lambda)}{\overline{R_{S}^{\prime}}(\lambda)} \cdot \operatorname{corr}(\lambda)$

The correction factor includes contributions (originating from the measurement transfer) from temperature deviation $\Delta T$, relative temperature coefficient of responsivities $\alpha_{\mathrm{S}}, \alpha_{\mathrm{T}}$, relative spatial non-uniformity of responsivities $\beta_{\mathrm{S}}, \beta_{\mathrm{T}}$, and dark (offset) currents $\bar{l}_{\mathrm{SO}}^{\prime}, \bar{l}_{\mathrm{T} 0}^{\prime}, \bar{l}_{\mathrm{MTO}}^{\prime}, \bar{l}_{\mathrm{MSO}}^{\prime}$ of the standard, test, and monitor detectors, all of which are independent of wavelength (or their changes are very small between neighbouring wavelengths where the measurement transfer is made).

The ratios $\overline{R_{T}^{\prime}}(\lambda), \overline{R_{S}^{\prime}}(\lambda)$ are formed from output signals $I_{\mathrm{T}}(\lambda), I_{\mathrm{S}}(\lambda)$ with simultaneously measured monitor output signals $I_{\mathrm{MT}}(\lambda), I_{\mathrm{MS}}(\lambda)$ using the correlation between the related photocurrents to eliminate noise from possible fluctuations of the source. Usually this is totally independent of wavelength.

Typically, the contributions for the determination of any single responsivity value and the associated uncertainty, are uncorrelated (at the given wavelength where the measurement transfer is made). These significant independent uncertainty components are added in quadrature. In case of independent variables, their covariances are zero. The quadrature sum can be applied to all random components at a given wavelength.

One component that may correlate the test and standard measurements is that of wavelength uncertainty. Random wavelength setting errors are correlated between test and standard measurements if the wavelength is set and the test and standard detectors measured; they are not correlated if the spectral range is swept independently for the test detector and standard detectors.

\section{SPECTRALLY INTEGRATED RESPONSIVITY}

The values representing the responsivity function $s_{S}^{\prime}(\lambda)$ listed in a calibration certificate may be calculated from realizations totally independent for the different wavelengths. In this case, the uncertainty components may not be correlated. However in practice, the responsivity function of the reference detector - usually a trap detector calibrated with a laser beam at a limited number of wavelengths - is interpolated based on the physical knowledge (quantum efficiency) or mathematical smoothness and results in correlations. Similarly, the responsivity functions of detectors, calibrated with a trap detector, are corrected by mathematical procedures for wavelength setting, for errors due to output slit function or to smooth the values from wavelength to wavelength. All these procedures produce function values with uncertainties that are correlated. The correlations do not affect the individual values but become important if spectrally integrated quantities are calculated. Calibration transfer from the primary standard radiometer can also introduce correlated uncertainties to a working reference standard, due to systematic offsets or scaling factors common to all measurement wavelengths. Calibration certificates usually state the total uncertainty at a given wavelength and rarely distinguish between effects that are random and systematic (correlated) between wavelengths.

At the highest level (primary or secondary calibration laboratory) measurements, when combining spectral values over different wavelengths, it is usually the systematic components that determine the measurement uncertainty. For example. in a photodiode radiant power responsivity calibration, such systematic components (at all wavelengths) are the absolute responsivity of the reference detector, repeatability with beam position, and photodiode amplifier gain.

The integrated responsivity of a detector when it measures a broad-band light-source can be written as 


$$
R=\sum_{n} s_{n} P_{n}
$$

where $P_{\mathrm{n}}$ is the spectral power distribution of the light-source and $s_{n}$ is the measured spectral power responsivity of the detector all at the $n$th (equally-spaced) wavelength. The detector is usually optically filtered to obtain a known spectral power responsivity function in a well defined wavelength interval.

The relative uncertainty of $R$ has to be calculated. Uncertainties in $S_{\mathrm{n}}$ are independent of those in $P_{\mathrm{n}}$ and hence

$$
u^{2}(R)=\sum_{n}\left(s_{n}^{2} u^{2}\left(P_{n}\right)+P_{n}^{2} u^{2}\left(s_{n}\right)\right)
$$

Often the spectral distribution of the source is a defined quantity (e.g. CIE Standard IIluminant $A$ ) and its values carry no uncertainty. In the following discussion we assume that source uncertainty is negligible; if required, the principles used to propagate uncertainties from the spectral responsivity values to that of the integrated response are identical for those of the source uncertainties.

As seen in Eqs. (3) and (4), determination of spectral responsivity is a transfer from a reference standard to the device under test,

$$
S_{T, n}=S_{S, n} t_{n}
$$

where $n$ denotes wavelength and $t$ is the transfer ratio at the nth wavelength. Uncertainties in spectral responsivity of the test detector arise from those of the standard and from the transfer process; these are treated independently and then added in quadrature.

\subsection{Uncertainties of the standard detector}

From Eq. (21),

$$
u\left(s_{T, n}\right)=t_{n} u\left(s_{S, n}\right)=\frac{S_{T, n}}{S_{S, n}} u\left(s_{S, n}\right)
$$

where the uncertainties in spectral responsivity of the reference detector are obtained from the calibration certificate. These may be complex, depending on the method of generating the primary standard. In such cases, a full propagation using covariances between the reference values is required $[1,2]$ - these must be sought from the supplier of the reference standard.

Some certificates provide total random component uncertainties and total correlated uncertainties at each wavelength. Where the ratio (systematic component : total uncertainty) of these components is approximately constant through the spectral range of interest, the correlation coefficient $r$ is also approximately constant and given by the square of the ratio and effective uncertainties that are random and fully-correlated between wavelengths are readily determined. The effective uncertainty in spectral responsivity that is random between wavelengths is then given by

$$
u_{R}\left(s_{n}\right)=\sqrt{1-r} u\left(s_{n}\right)
$$

and the total contribution of random effects to the uncertainty in the integrated spectral quantity is given by

$$
u_{R}^{2}(R)=(1-r) \sum P_{n}^{2} u^{2}\left(s_{n}\right)
$$

The effective uncertainty in spectral responsivity that is fully correlated between wavelengths is given by

$$
u_{s}\left(s_{n}\right)=\sqrt{r} u\left(s_{n}\right)
$$

The total contribution of the systematic effects is given by the linear sum:

$$
u_{S}(R)=r \sum P_{n} u\left(s_{n}\right)
$$

Alternatively, where the systematic and random components are given at each wavelength, or their wavelength dependence is given as a scaling of some parameter, each systematic component may be propagated separately as a linear sum, and the total random components propagated in quadrature.

The total uncertainty contribution of the reference standard is the quadrature sum of the systematic and random components.

A further complication is that spectral responsivity values for the reference standard may require interpolation to the wavelength values of the measurement. Interpolation 
introduces correlations and these must be taken into account when propagating the uncertainties of the standard detector. A simple process that avoids these complications is to interpolate the transfer ratio to the wavelengths at which the reference standard is calibrated and propagate uncertainties in the integral sum (including the change in wavelength spacing) using only those values [3].

\subsection{Uncertainties of transfer process}

Each effect contributing to uncertainty in the transfer can be classified as independent and either random between wavelengths or fully-correlated between wavelengths. These effects can also be separately determined for the numerator or denominator of the transfer ratio, as shown in Eqs. (3) and (4), and combined either linearly or in quadrature depending on whether the effects are correlated or random between test and reference signals at the one wavelength.

Once the uncertainty in the transfer ratio and hence its uncertainty in spectral responsivity

$$
u_{e}\left(s_{T, n}\right)=s_{S, n} u_{e}\left(t_{n}\right)
$$

is determined for each effect at each wavelength, its contribution to the integral sum is calculated. For random components,

$$
u_{e}^{2}(R)=\sum P_{n}^{2} u_{e}^{2}\left(s_{n}\right)
$$

For systematic components, uncertainties in the values of spectral responsivity at different wavelengths are generally positively correlated, and the contribution to the integral sum is given by

$$
u_{e}(R)=\sum P_{n} u_{e}\left(s_{n}\right)
$$

However some effects (e.g. wavelength offsets) can produce correlations that are positive or negative between wavelength pairs. These effects are properly handled by attaching a sign, that of the sensitivity coefficient for the effect, to the uncertainty in the transfer ratio. For example, consider the transfer ratio shown in Eq. (21). In the presence of a wavelength uncertainty fully correlated between test and reference, the signed uncertainty in the transfer ratio test:standard signals at the $n$th wavelength is given by $u_{e, s}\left(t_{n}\right)=\left(\frac{1}{R_{S, n}} \frac{\partial R_{T, n}}{\partial \lambda}-\frac{R_{T, n}}{R_{S, n}{ }^{2}} \frac{\partial R_{S, n}}{\partial \lambda}\right) u(\lambda)$

The signed uncertainty in spectral responsivity for this effect at the $n$th wavelength is then given by combining Eqs. (27) and (30):

$$
u_{e, s}\left(S_{T, n}\right)=\frac{s_{R, n}}{R_{S, n}}\left(\frac{\partial R_{T, n}}{\partial \lambda}-t_{n} \frac{\partial R_{S, n}}{\partial \lambda}\right) u(\lambda)
$$

This expression can be positive or negative depending on the slopes of the test and reference signals with respect to wavelength. The propagated uncertainty for a wavelength offset applicable at all wavelengths, including these mixed correlations, is found by the linear sum

$$
u_{e}(R)=\sum P_{n} u_{e, s}\left(s_{T, n}\right)
$$

Detailed discussions and examples for uncertainty contributions of different correlated combinations can be found in [2].

\section{CONCLUSION}

When reporting spectral responsivity measurement uncertainties, the total uncertainty contribution of effects systematic over wavelengths should be given at each wavelength, instead of the total uncertainty as is the usual practice.

\section{REFERENCES}

1. International Organization for Standardization, "Guide to the Expression of Uncertainty in Measurement", Geneva 1993

2. J. L. Gardner, Chapter 6: Uncertainty estimates in radiometry, Experimental Methods in the Physical Sciences, 41 291-325, Elsevier Inc. 2005.

3. J. L. Gardner, Uncertainties in interpolated spectral data, J. Res. Natl. Inst. Stand. Technol. 108 69-78 (2003) 


\title{
Realization of the National Institute of Standards and Technology detector-based spectral irradiance scale
}

\author{
Howard W. Yoon, Charles E. Gibson, and Patricia Y. Barnes
}

\begin{abstract}
A detector-based spectral irradiance scale has been realized at the National Institute of Standards and Technology (NIST). Unlike the previous NIST spectral irradiance scales, the new scale is generated with filter radiometers calibrated for absolute spectral power responsivity traceable to the NIST highaccuracy cryogenic radiometer instead of with the gold freezing-point blackbody. The calibrated filter radiometers are then used to establish the radiance temperature of a high-temperature blackbody (HTBB) operating near $3000 \mathrm{~K}$. The spectral irradiance of the HTBB is then determined with knowledge of the geometric factors and is used to assign the spectral irradiances of a group of 1000-W free-electron laser lamps. The detector-based spectral irradiance scale results in the reduction of the uncertainties from the previous source-based spectral irradiance scale by at least a factor of 2 in the ultraviolet and visible wavelength regions. The new detector-based spectral irradiance scale also leads to a reduction in the uncertainties in the shortwave infrared wavelength region by at least a factor of $2-10$, depending on the wavelength. Following the establishment of the spectral irradiance scale in the early 1960 s, the detector-based spectral irradiance scale represents a fundamental change in the way that the NIST spectral irradiance scale is realized. (C) 2002 Optical Society of America
\end{abstract}

OCIS codes: $120.3930,120.3940,120.4800,120.5630$.

\section{Introduction}

The development and use of cryogenic radiometers have led the trend toward decreasing uncertainties in the radiometric and the photometric scales because of improvements in the absolute power measurements. ${ }^{1,2}$ Furthermore, detector-based scales often can be maintained with shorter measurement chains and lower uncertainties than source-based scales. For example, the photometric scale at the National Institute of Standards and Technology (NIST) has been detector based and traceable to the NIST highaccuracy cryogenic radiometer (HACR) since $1993,{ }^{3}$ and the implementation of the detector-based photometric scale resulted in a factor of 2 reduction in the expanded uncertainties compared with the previous source-based photometric scale. ${ }^{4}$ Because the radio-

The authors are with the Division of Optical Technology, National Institute of Standards and Technology, Mail Stop 8441, Gaithersburg, Maryland 20899. H. W. Yoon's e-mail address is hyoon@nist.gov.

Received 7 January 2002; revised manuscript received 6 June 2002.

0003-6935/02/285879-12\$15.00/0

(C) 2002 Optical Society of America metric quantities of spectral radiance and spectral irradiance require measurements at many different wavelengths compared with the single measurement in the assignment of a photometric quantity, the establishment of a detector-based spectroradiometric scale has proven more time-consuming.

The spectral radiance and the radiance temperature scale at NIST have all been based on the absolute radiometric determination of the freezing temperature of gold, ${ }^{5}$ and the spectral irradiance scale is in turn realized from the spectral radiance scale. ${ }^{6} \quad$ As shown in Fig. 1(a), during the process of scale realization, the radiance temperature at 655 $\mathrm{nm}$ is assigned to a vacuum tungsten-filament lamp (gold-point lamp) operated to match the radiance temperature of the gold freezing-temperature blackbody. The radiance temperature of a tungsten lamp operated at a higher temperature $(1530 \mathrm{~K})$ is in turn determined with the gold-point lamp, and the radiance temperature of a variable-temperature blackbody (VTBB) is assigned. The VTBB has a small 2-mm-diameter opening, and the estimated emissivity of the VTBB is $0.9990 \pm 0.0005$ based on the geometry and temperature uniformity. ${ }^{7}$ The spectral radiance of tungsten-filament lamps and integrating sphere sources is assigned by use of the 


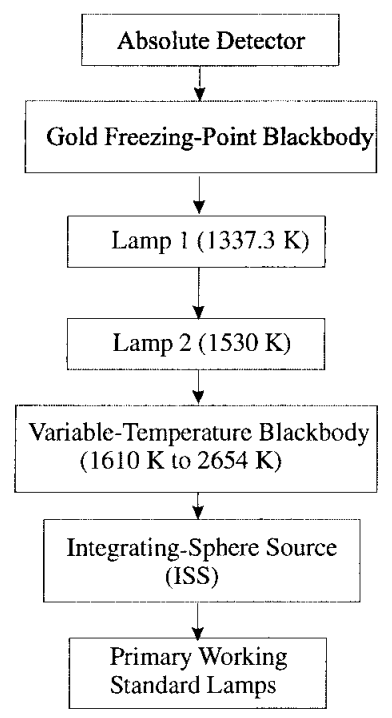

(a)

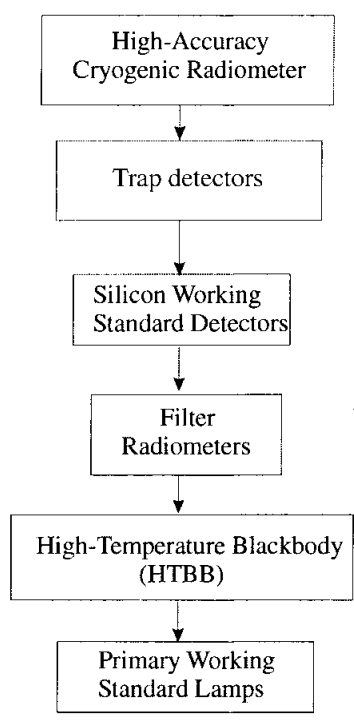

(b)
Fig. 1. (a) Measurement chain used to derive the source-based spectral irradiance scale used in the 1990 and 1992 scale realizations. (b) The measurement chain used in the new detector-based spectral irradiance scale of 2000.

VTBB; and typically, the temperature of the VTBB is changed to match the spectral radiance of the sources to minimize the effects that are due to the nonlinear responsivities of the detectors. For spectral irradiance realizations, the spectral radiance of an integrating-sphere source (ISS) is assigned, and the ISS is fitted with a precision aperture for use as a source of known spectral irradiance to calibrate a set of 1000-W free-electron laser (FEL) lamps.

The source-based spectral irradiance scale realization procedure is long and involves many separate steps to assign the spectral irradiance of a set of lamps. The primary reason for the long calibration chain is the low spectral radiance output of the gold freezing temperature blackbody at $1337.33 \mathrm{~K} .{ }^{8}$ Because the 1000-W FEL lamps have spectral shapes similar to that of a $3000 \mathrm{~K}$ blackbody, the radiant output of the intermediate transfer artifacts must be progressively increased to eventually match the spectral irradiance output of the 1000-W FEL lamp. Furthermore, a major contribution to the final uncertainties in the spectral irradiance scale is due to the temporal drift of the ISS and the low signal of the ISS, especially in the shortwave infrared region. The temporal drift of the ISS also depended on the wavelength region, resulting in greater temporal changes in the ultraviolet wavelength region. The spectral irradiance scale was difficult to realize at many different wavelengths because of a possible temporal drift resulting from the additional time needed for realizations.

In the past decade, new developments in the construction of high-temperature blackbodies (HTBBs) made from pyrolytic graphite have allowed prolonged $(>100 \mathrm{~h})$ operations at or above $3000 \mathrm{~K} .{ }^{9}$ Further- more, at NIST the uncertainties in the detector power responsivity scale were reduced with the advent of the HACR as the primary standard for spectral power responsivity calibrations. The advantages of the new detector-based scale realization are evident in Fig. 1(b), with a much shorter chain from the cryogenic radiometer to the primary working standards compared with the measurement chain shown in Fig. 1(a). In the realization of the detector-based scale, the filter radiometers (FRs) are used to determine the temperature of the HTBB, and then the spectral irradiance output of the HTBB is used to directly calibrate the primary working standard lamps.

In this paper we describe the realization of the NIST detector-based spectral irradiance scale. The FRs used to determine the radiance temperature of the HTBB are characterized, and the agreement between the absolute detector-based radiance temperatures are compared with those found by use of the International Temperature Scale of 1990 (ITS-90). ${ }^{10}$ The agreement between the detectorbased and the gold freezing-temperature-based spectral radiances is also shown to verify that the HTBB spectral radiance does not deviate significantly from a single-temperature Planck radiance distribution. From the assignment of the radiance temperature with the FRs, the spectral irradiance of a set of FEL lamps is determined. The detectorbased and the gold freezing blackbody-based spectral irradiances are compared, and the uncertainties of the detector-based spectral irradiance scale are discussed.

\section{Experimental Approach}

\section{A. Radiance Temperature Determinations with Filter} Radiometers

A schematic of the measurements is shown in Fig. 2. Both the spectroradiometer and the FRs reside on a common moving optical table. The FRs are first used to determine the radiance temperature of the HTBB; and thus with knowledge of the radiance temperature, the spectral irradiance responsivity of the spectroradiometer is assigned by use of a precision aperture on the integrating-sphere receiver (ISR) with the knowledge of the distance between the ISR and the precision aperture in front of the blackbody. The spectral irradiance of the 1000-W quartz tungsten-halogen lamp (type FEL) is then assigned by use of the spectral irradiance responsivity of the spectroradiometer.

The FRs are constructed with broadband filters and silicon detectors that are both temperature stabilized as shown in Fig. 3. A precision aperture is placed in front of the filter-detector package, and the aperture area is measured separately in the NIST High-Accuracy Aperture Measurement Facility to a relative combined expanded $(k=2)$ uncertainty of $0.01 \% .{ }^{11}$ The precision apertures attached to the FRs range from 4.0 to $4.4 \mathrm{~mm}$ in diameter. The detectors are calibrated for absolute spectral power responsivity on the NIST Spectral Comparator Fa- 
Spectral Irradiance Measurement

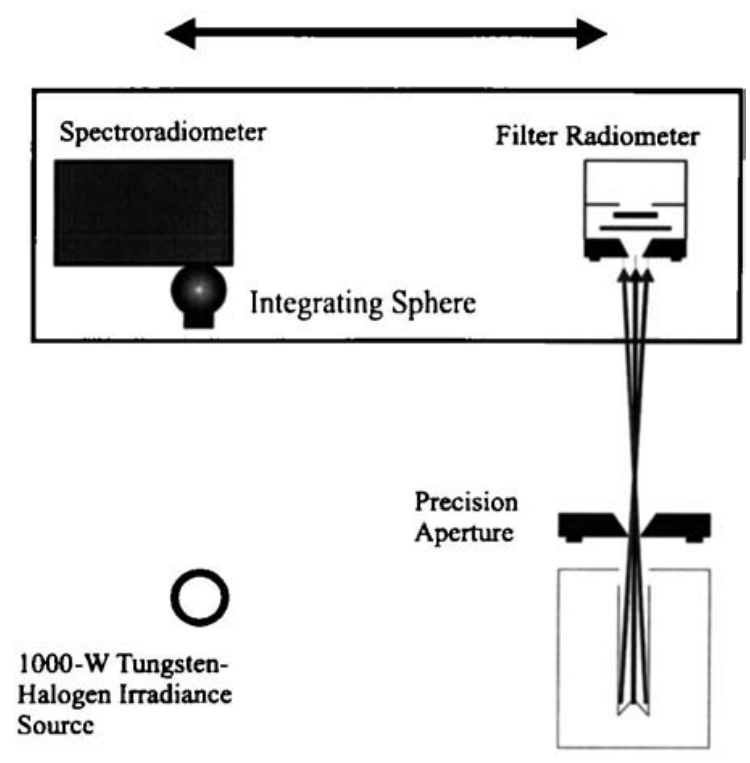

High-Temperature Blackbody Source

Fig. 2. Schematic of the laboratory setup used for the spectral irradiance scale realization showing the positions of the HTBB, the FRs, the spectroradiometer, and the FEL lamp.

cility (SCF), ${ }^{12}$ and the power responsivity is converted to spectral irradiance responsivity from knowledge of the aperture area. A total of three detectors are used, and the absolute spectral power responsivities are shown in Fig. 4. Both filter radiometers FR2 and FR4 have photopic response filters resulting in a broad spectral responsivity. The FR3 has greater spectral responsivity to shorter wavelengths then the other two radiometers, with a drop in responsivity near $400 \mathrm{~nm}$. The broadband radiometers were chosen for increased long-term stability, as compared with narrowband interference filters. ${ }^{13}$

The radiance temperature of the HTBB is found with a measurement equation, which describes the optical flux transfer with circular and coaxial source

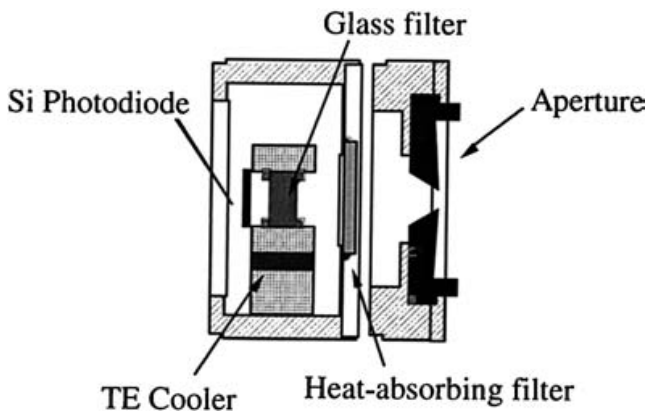

Fig. 3. Schematic of the filter radiometers used for the absolute temperature determination of the HTBB.

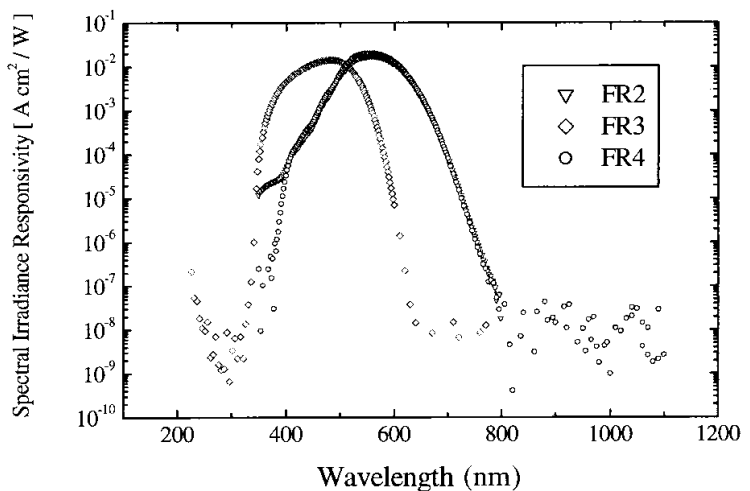

Fig. 4. Absolute spectral irradiance responsivities of the FRs used in the scale realization. Both filter radiometers FR2 and FR4 are constructed with broadband photopic response filters that differ in responsivity only slightly below $400 \mathrm{~nm}$.

and receiver apertures. ${ }^{14}$ For a FR, the signal $s$ is given by

$$
S=\frac{G \pi r_{\mathrm{BB}}^{2} \pi r^{2}(1+\delta)}{D^{2}} \epsilon \int R(\lambda) L(\lambda, T) d \lambda,
$$

where $\epsilon$ is the emissivity and $R$ is the absolute spectral power responsivity. The geometric factors $D$ and $\delta$ are given by $D^{2}=d^{2}+r^{2}+r_{\mathrm{BB}}^{2}$ and $\delta=$ $r^{2} r_{\mathrm{BB}}^{2} / D^{4}$, where $r_{\mathrm{BB}}$ and $r$ are the radius of the blackbody aperture and the FR apertures, respectively, and $d$ is the distance between the FR aperture and the blackbody aperture. $G$ is the preamplifier gain and $L$ is the spectral radiance found by use of the Planck radiation law. The radiance temperatures are found when we iteratively change the blackbody temperature in Eq. (1) until an exact match of the calculated signal to the measured signal is found.

The uncertainties of the radiance temperatures found with the FRs are primarily determined by the uncertainties in the spectral power responsivity. Figure 5(a) plots the expanded uncertainties of the SCF in the spectral power responsivity measurements. The lowest uncertainties are maintained in the visible wavelength region from 400 to $900 \mathrm{~nm}$ where the spectral power responsivity of the Si-trap transfer detector can be smoothly interpolated between the discrete laser calibration wavelengths of the HACR. The uncertainties of the spectral power responsivity can be converted directly to uncertainties in temperature by use of the derivative of the Wien approximation with respect to temperature:

$$
\frac{\Delta L}{L}=\frac{c_{2}}{\lambda} \frac{\Delta T}{T^{2}}
$$

is the relationship between the uncertainty in radiance $L$ and the uncertainty in blackbody temperature $T ; c_{2}$ is the second Planck's constant, 1.4387752 $\mathrm{cm} / \mathrm{K}$; and $\lambda$ is the wavelength. The Wien approximation is used to simplify the handling of equations. Under these experimental conditions, no appreciable 

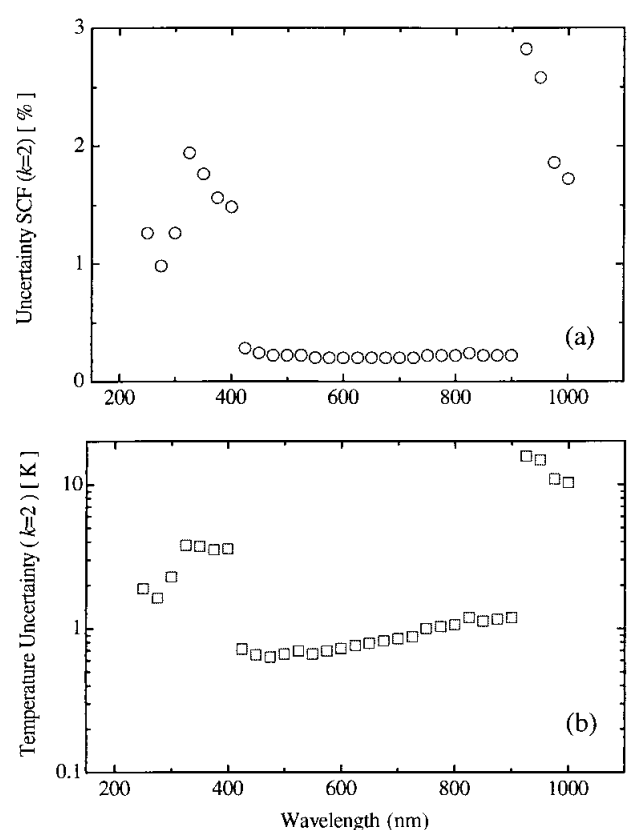

Fig. 5. (a) Expanded uncertainties of the NIST SCF for spectral power responsivity showing the low expanded uncertainties $(0.22 \%)$ between 400 and $900 \mathrm{~nm}$. (b) The uncertainties in the spectral power responsivity converted to temperature uncertainties by use of the derivative of the Wien approximation at $3000 \mathrm{~K}$.

differences exist between Eq. (2) and the similar differential equation found when we use Planck's radiance law in the wavelength between 250 and 2400 $\mathrm{nm}$. Because of the changes in the shape of the blackbody radiance with temperature as described in the Planck radiance law, the temperature uncertainties are both dependent on temperature as well as wavelength. The corresponding uncertainties in radiance temperatures are plotted in Fig. 5(b) for a blackbody temperature of $3000 \mathrm{~K}$, where the lowest temperature uncertainties exist in the spectral region between 400 to $900 \mathrm{~nm}$.

\section{B. Description of the Facility for Automated Spectroradiometric Calibrations}

All the experimental research described in this paper was performed in the Facility for Automated Spectroradiometric Calibrations (FASCAL), with the exception of the spectral power responsivity measurements of the FRs. ${ }^{6}$ Briefly, the detector system consists of a prism-grating double monochromator with custom-modified entrance and exit optics. A simplified schematic of FASCAL is shown in Fig. 2. The light is collected with an off-axis spherical mirror with either an integrating receiver for spectral irradiance or a flat plane mirror for spectral radiance. The measurements from 250 to $900 \mathrm{~nm}$ were performed with a photomultiplier tube in dc measurement mode. We performed the measurements from 800 to $2400 \mathrm{~nm}$ using an InGaAs detector in photovoltaic mode using a mechanical light chopper at 290 $\mathrm{Hz}$ with phase-sensitive detection. A significant im- provement to the FASCAL detection system is due to the replacement of the $\mathrm{PbS}$ detector $\left[D^{*}=4 \times 10^{11}\right.$ $\left.\left(\mathrm{cm} / \mathrm{Hz}^{1 / 2}\right) / \mathrm{W}\right]$ with the extended InGaAs detector $\left[D^{*}=2 \times 10^{12}\left(\mathrm{~cm} / \mathrm{Hz}^{1 / 2}\right) / \mathrm{W}\right]$, resulting in a standard deviation of signals $<0.05 \%$ when a $1000-\mathrm{W}$ FEL lamp is measured from 800 to $2300 \mathrm{~nm}$. The spectrometer and the detector enclosure are also continuously purged with air filtered to remove $\mathrm{CO}_{2}$ and $\mathrm{H}_{2} \mathrm{O}$, thus improving the long-term stability of the detector system.

\section{Description of the High-Temperature Blackbody}

One of the technological advances that makes possible the realization of the detector-based spectral irradiance scale is the development of pyrolytic graphite blackbodies, enabling extended operations near $3000 \mathrm{~K}$ without rapid degradation of the cavity. The HTBB used in this study consists of pyrolytic graphite rings with an inner diameter of $24 \mathrm{~mm}$ stacked to form a cavity with a depth of $14.5 \mathrm{~cm}$. The cavity bottom consists of an inverted cone with an apex angle of approximately $150^{\circ}$, and the radiance at the backside of the cavity bottom is imaged through an opening at the rear of the cavity by use of a fused-silica lens onto a temperature-stabilized photometer for operation in closed-loop configuration. The lens also serves to seal the rear of the blackbody cavity to atmosphere. The current of the HTBB power supply is adjusted with a control loop to maintain a constant output from the photometer. Although the spectral irradiance in the ultraviolet wavelength region could be increased with higher temperatures, the HTBB was operated near $2950 \mathrm{~K}$ to avoid possible changes in the spectral emissivity of the blackbody that are due to the sublimation of the graphite. ${ }^{15}$ Because the graphite rings expand approximately $10 \%$ from room temperature to near $3000 \mathrm{~K}$, the current was increased at $0.1 \mathrm{~A} / \mathrm{s}$ to nearly $510 \mathrm{~A}$ at $2950 \mathrm{~K}$ to allow gradual expansion of the cavity. The HTBB was also turned off in a similar manner to avoid thermophysical shocks.

Figure 6 is a plot of the radiance temperatures measured during a single day of measurements from 9:30 to 14:00 with FR2, FR3, and FR4. The feedback detector is a temperature-stabilized Si photodiode with a photopic response filter. The optical feedback is found to stabilize the HTBB to $\pm 0.0004 \mathrm{~V}$ at a full signal of $4.4 \mathrm{~V}$. Figure 6 shows that the HTBB temperature was stable to $<0.5 \mathrm{~K}$ over the duration of the 5 -h measurement. The expanded uncertainties of the temperatures are shown for the FR2 temperatures that overlap the temperatures measured with FR3 and FR4. The three FRs agree in radiance temperature determinations to $<0.5 \mathrm{~K}$, in agreement with the combined expanded uncertainty in temperature of $0.86 \mathrm{~K}$ at $2950 \mathrm{~K}$.

Each of the FR measurements is an independent determination of temperature when a primary thermometer is used. A primary thermometer is distinguished from a secondary thermometer in that the temperature is obtained by use of a primary thermometer without use of any adjustable parameters. ${ }^{16}$ 


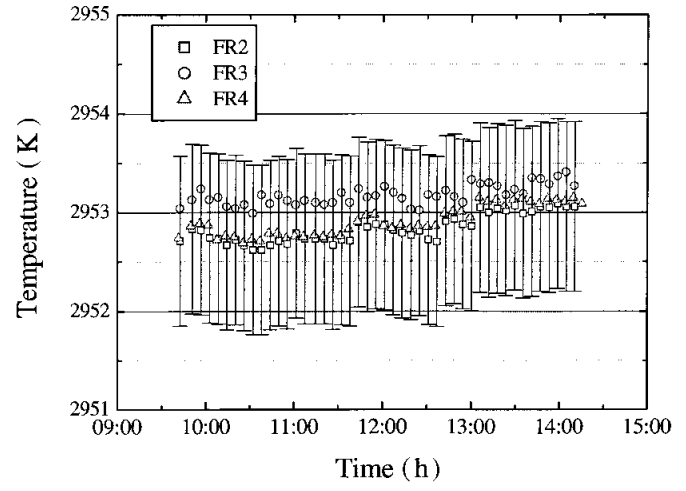

Fig. 6. Temporal stability measurement of radiance temperatures with the FRs. The expanded uncertainties in temperature of $\pm 0.86 \mathrm{~K}$ are shown for FR2. The temporal drift in the HTBB temperature is less than $0.5 \mathrm{~K}$ over the duration of the nearly 5 -h monitoring time.

The temperature of the HTBB increased at most by 1 $\mathrm{K}$ over $20 \mathrm{~h}$ of continuous operation with constant optical feedback control by use of the optical output from the rear of the cavity. The slight increase in temperature over time is possibly attributable to the opening at the rear of the cavity that gets smaller from carbon deposits at the edges of the opening. The HTBB was operated with an open cavity with continuous $0.5-1 / \mathrm{min}$ purge of argon from the rear of the housing and the argon exiting through the front opening of the cavity.

\section{Verification of the High-Temperature Blackbody as a Planckian Radiator}

To determine the spectral emissivity of the HTBB, the spectral radiance of the HTBB was measured from 250 to $2400 \mathrm{~nm}$ at discrete wavelengths by comparison with a VTBB with a high emissivity. Any temperature nonuniformity of the cavity would lead to deviations of the HTBB spectral radiance from a single-temperature Planck radiance. For these comparisons, the temperature of the VTBB was set at $2687 \mathrm{~K}$, and the temperature of the HTBB was set at $2950 \mathrm{~K}$. Although the temperatures are different, the radiance ratios did not differ even at $250 \mathrm{~nm}$, where the differences are greatest, by more than a factor of 7. Furthermore, prior to these measurements, the linearity of the spectroradiometer was measured at various wavelengths with the portable Beamconjoiner ${ }^{17}$ and was found to be linear under these experimental conditions. The spectral radiance determinations were performed before and after spectral irradiance transfer to a set of 1000-W FEL lamps. The checks of the HTBB as a Planckian radiator were performed to verify that the HTBB did not change in the spectral distribution because the FRs are used to measure over a relatively broad spectral region.

The spectral radiance measurements of the HTBB were performed with the aperture in front of the spherical input mirror with the spectroradiometer reduced to $4 \mathrm{~cm}$ in diameter, resulting in $F / 25$ focused at the opening of the HTBB at the plane of the water-cooled aperture. The reduction in the opening of the spherical input mirror resulted in a circular target of $12 \mathrm{~mm}$ in diameter at the bottom of the HTBB cavity. To keep the same target area constant for the measurements, in both the spectral irradiance and the spectral radiance modes, the HTBB was mounted on a motorized translation stage for control of the distance between the HTBB and the measuring instruments. If possible, the same area at the rear of the HTBB should be viewed for all measurements to avoid errors from the possible spatial nonuniformity of the HTBB.

We determined the temperature of the VTBB using spectral radiance ratios at $655 \mathrm{~nm}$ to a vacuum tungsten-filament lamp maintained at the freezing temperature of gold as prescribed in the ITS-90. The spectral radiance of the HTBB was determined at discrete wavelengths by comparison with the VTBB and also determined from the radiance temperature found with the FRs. The radiance temperatures of the HTBB found with the FRs were in agreement with those found with the ITS-90 ratios to within $0.5 \mathrm{~K}$ in all cases in which such comparisons were performed. We compared the respective blackbodies using spectral radiance ratios:

$$
L_{\lambda, \mathrm{HTBB}}=L_{\lambda, \mathrm{VTBB}} f_{\lambda} \frac{S_{\lambda, \mathrm{HTBB}}}{S_{\lambda, \mathrm{VTBB}}} .
$$

The $L_{\lambda}$ and the $S_{\lambda}$ denote the spectral radiance and the measured spectral radiance response of the HTBB and the VTBB, and $f_{\lambda}$ is the possible linearity correction to the response ratios. The spectral radiances of the HTBB were found to conform to a singletemperature Planck radiance law to within the combined uncertainties of the measurements as shown in Fig. 7. Figure 7 shows the percent differences from a single-temperature Planck radiance law with the temperature of the HTBB determined with the FRs and the spectral radiances determined with the VTBB. The errors bars on the individual comparisons correspond to the expanded uncertainty of $0.86 \mathrm{~K}$ at $2943.4 \mathrm{~K}$ converted to a spectral radiance uncertainty by use of the derivative of the Wien approximation from Eq. (2). As expected for comparison of two blackbodies, the differences in the spectral radiance become smaller with increasing wavelength, but there are no discernible increasing spectral differences toward the shorter wavelengths, as would be expected if either of the temperature assignments of the respective blackbodies were in error. The differences between the detector-based radiance temperatures and the source-based determinations also agree with the previous measurements, which also showed differences of $<0.5 \%$ from 250 to 1000 nm. ${ }^{18}$

\section{E. Spectral Irradiance Transfer to 1000-W FEL Lamps}

The spectral irradiance assignment of twelve 1000-W FEL working standard lamps occurred over a two- 


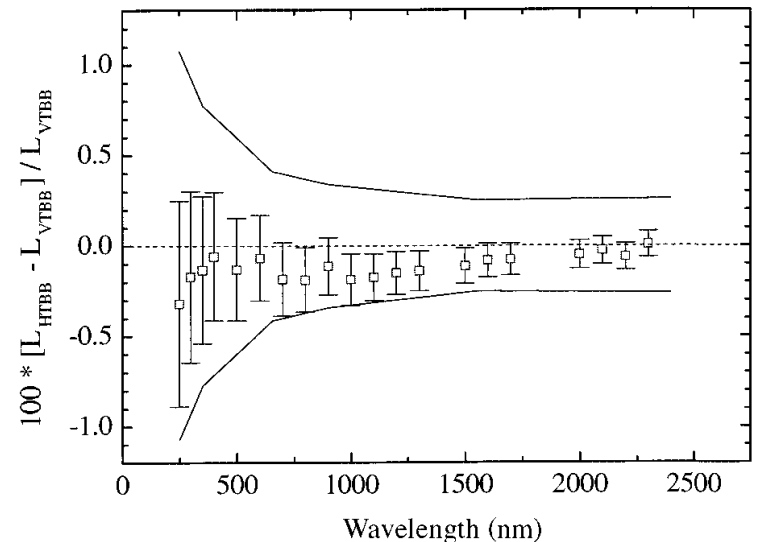

Fig. 7. Spectral radiance differences of the HTBB from a singletemperature Planck radiance as assigned by use of the FRs to the spectral radiances assigned by use of the VTBB. The error bars indicate the $0.86 \mathrm{~K}$ expanded temperature uncertainty at $2950 \mathrm{~K}$ converted to spectral radiance uncertainties, and the continuous curves indicate the expanded uncertainties of the spectral radiance scale.

week period from 28 September to 12 October 2000. The spectral irradiances of a group of three lamps were assigned at one time, and the measurements were separated into the ultraviolet and the visible wavelength region (250-900 nm), and the shortwave infrared region $(800-2400 \mathrm{~nm})$. Because there are four separate FEL lamp measurement stations, each of the measurements of individual lamps was performed in a different station by use of different shunt resistors, power supplies, and lamp mounts. Each of the lamp stations was checked for possible position effects, and no perceptible effects from station-tostation variations were found.

The HTBB was turned on at least $2 \mathrm{~h}$ before use and was temperature-stabilized by the optical feedback. The temperature of the HTBB was assigned by use of the FRs, and the spectral irradiance responsivity of the spectroradiometer was assigned by use of the known spectral irradiance of the HTBB. All the spectral irradiance responsivity transfers to the spectroradiometer were done at a distance of $43.406 \mathrm{~cm}$ $( \pm 0.005 \mathrm{~cm})$ from the HTBB aperture to the $1-\mathrm{cm}^{2}-$ area aperture of the ISR, resulting in a $24.7-\mathrm{mm}$ diameter circular target area at the cavity bottom. The spectral irradiance responsivity of the spectroradiometer is assigned by use of the known spectral irradiance of the HTBB at the input aperture of the ISR:

$$
E_{\lambda, \mathrm{HTBB}}=\frac{L(\lambda, T) \pi r_{\mathrm{BB}}^{2}}{D^{2}},
$$

with the measurement parameters as defined in Eq. (1).

After the spectral irradiance responsivity assignment of the spectroradiometer, the spectral irradiance of a group of three FEL lamps was assigned with each lamp measured in three separate source posi-

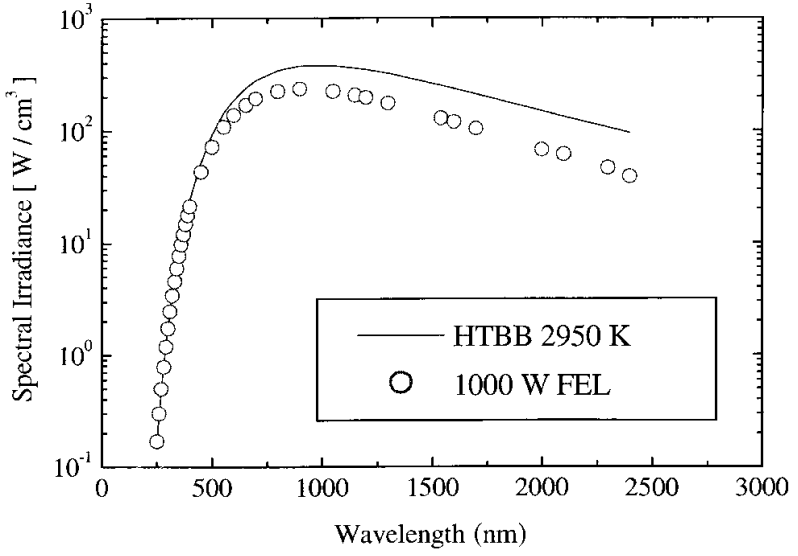

Fig. 8. Spectral irradiance of the HTBB at $2950 \mathrm{~K}$ with the measurement parameters as described with the spectral irradiances of a typical 1000-W FEL lamp. The measurement parameters were chosen to closely match the signals of both sources.

tions. The spectral irradiance of each lamp was assigned by

$$
E_{\lambda, \mathrm{FEL}}=E_{\lambda, \mathrm{HTBB}} f_{\lambda} \frac{S_{\lambda, \mathrm{FEL}}}{S_{\lambda, \mathrm{HTBB}}},
$$

where $E_{\lambda, \mathrm{FEL}(\mathrm{HTBB})}$ is the spectral irradiance of the FEL lamp (HTBB), $S_{\lambda, \text { FEL(HTBB) }}$ is the net signal from the FEL lamp (HTBB) measured with the spectroradiometer, and $f_{\lambda}$ is the linearity correction factor for the comparison. With use of a shutter, the background signals were subtracted from the total signals to obtain the net signals. The distance of the HTBB from the ISR was also chosen such that the same gain factors could be used to measure both the FEL and the HTBB. Figure 8 is a plot of the calculated spectral irradiances of the 1000-W FEL along with the HTBB at $2950 \mathrm{~K}$ with the limiting aperture of 9.9933 $\mathrm{mm}( \pm 0.0002 \mathrm{~mm})$ in diameter and the ISR aperture of $11.2838 \mathrm{~mm}( \pm 0.0002 \mathrm{~mm})$ in diameter separated by a distance of $43.406 \mathrm{~cm}( \pm 0.005 \mathrm{~cm})$. At all wavelengths, Fig. 8 shows that the HTBB has a greater spectral irradiance than the FEL lamp. Although the spectral irradiance changes by a factor $>10^{3}$ from 250 to $2400 \mathrm{~nm}$, the HTBB/FEL signal ratio does not exceed 2.5. The close match of the irradiances was chosen to minimize possible errors resulting from signal-to-noise ratio, nonlinearity, and stray-light effects.

\section{Uncertainty Analysis of the Detector-Based Spectral Irradiance Scale}

The discussion of the uncertainties associated with the detector-based spectral irradiance scale realization is separated into parts. Because the absolute radiance temperature determination of the HTBB is performed with FRs with peak spectral responsivities near $550 \mathrm{~nm}$, the expanded uncertainties in the spectral irradiance measurement at $550 \mathrm{~nm}$ is discussed first. The spectral irradiance uncertainties are converted into temperature uncertainties by use of the 
Table 1. Expanded Component Uncertainties in the Spectral Irradiance Measurement with the FR at $550 \mathrm{~nm}$

\begin{tabular}{ccc}
\hline $\begin{array}{c}\text { Source of Uncertainty } \\
(550 \mathrm{~nm})\end{array}$ & $\begin{array}{c}\text { Fractional } \\
\text { Uncertainty }\end{array}$ & $\begin{array}{c}\text { Expanded } \\
\text { Uncertainty } \\
(\%)\end{array}$ \\
\hline $\begin{array}{c}\text { Spectral power responsivity, } \\
\text { FR } \\
\begin{array}{c}\text { Solid-angle factor, } \\
\text { HTBB/FR }\end{array}\end{array}$ & $\frac{\Delta R}{R}$ & 0.22 \\
$\begin{array}{c}\text { Area of detector aperture } \\
\text { Area of HTBB aperture } \\
\text { Measurement precision, } \\
\text { HTBB/FR }\end{array}$ & $\frac{\Delta A}{A}$ & 0.026 \\
$\begin{array}{l}\text { Amplifier gain } \\
\text { Combined expanded } \\
\text { uncertainty }(k=2)\end{array}$ & $\frac{\Delta A}{A}$ & 0.02 \\
\hline
\end{tabular}

derivative of the Wien approximation. The Wien approximation is used because the measurements are performed at short wavelengths from the peak wavelength of the $3000 \mathrm{~K}$ Planck spectral radiance. The uncertainties of the spectral irradiances of the primary working standards are assigned. Finally, the expanded uncertainties of the issued standards are assigned with additional uncertainty added to account for the temporal changes of the working standard lamps.

The uncertainties associated with the detector- based temperature determinations of the HTBB can be found from the uncertainty analysis of Eq. (1) as shown in Table 1. The individual components are listed in descending order of importance, with the uncertainty that is due to the spectral power responsivity dominating. Additional sources of uncertainty from the alignment and aperture area measurements are small. The expanded spectral irradiance responsivity uncertainty at $550 \mathrm{~nm}$ of $0.26 \%$ leads to an expanded temperature uncertainty of 0.86 $\mathrm{K}$ at $2950 \mathrm{~K}$.

Table 2 describes the additional uncertainties associated with the HTBB spectral irradiance including the components that are due to spectral emissivity, spatial uniformity, and temporal stability. The first row shows the expanded uncertainty of the spectral irradiance that is due to the $0.86 \mathrm{~K}$ expanded temperature uncertainty at $2950 \mathrm{~K}$. The uncertainties that are due to the spectral emissivity and the spatial uniformity of the HTBB are then assigned. The temporal stability of the HTBB was measured and also constantly monitored by use of the optical feedback at the rear of the cavity. The uncertainties in the alignment of the apertures and in the distance measurements are included in the geometric factor uncertainty.

The detectors used in the spectroradiometers have shown better than $0.1 \%(k=2)$ stability during the spectral irradiance measurements. However, the increase of the uncertainty at $2400 \mathrm{~nm}$ because the stability of the spectroradiometer responsivity is due to the performance of the InGaAs detector. A substantial contribution to the total uncertainty comes

Table 2. Expanded Uncertainties of the Detector-Based 2000 Spectral Irradiance Scales of the Primary Working Standards and the Issued Standards

\begin{tabular}{|c|c|c|c|c|c|c|c|c|}
\hline \multirow[b]{2}{*}{ Component } & \multicolumn{8}{|c|}{ Wavelength (nm) } \\
\hline & 250 & 350 & 655 & 900 & 1600 & 2000 & 2300 & 2400 \\
\hline HTBB spectral emissivity & 0.1 & 0.1 & 0.1 & 0.1 & 0.1 & 0.1 & 0.1 & 0.1 \\
\hline HTBB spatial uniformity & 0.1 & 0.1 & 0.1 & 0.1 & 0.1 & 0.1 & 0.1 & 0.1 \\
\hline $\begin{array}{l}\text { Geometric factors in irradiance } \\
\text { transfer }\end{array}$ & 0.1 & 0.1 & 0.1 & 0.1 & 0.1 & 0.1 & 0.1 & 0.1 \\
\hline $\begin{array}{l}\text { Spectroradiometer responsivity } \\
\text { stability }\end{array}$ & 0.1 & 0.1 & 0.1 & 0.1 & 0.1 & 0.1 & 0.1 & 0.5 \\
\hline Wavelength accuracy $(0.1 \mathrm{~nm})$ & 0.58 & 0.38 & 0.18 & 0.005 & 0.011 & 0.013 & 0.011 & 0.011 \\
\hline $\begin{array}{l}\text { Lamp/spectroradiometer } \\
\text { transfer }\end{array}$ & 0.1 & 0.1 & 0.1 & 0.1 & 0.1 & 0.1 & 0.1 & 0.1 \\
\hline Lamp current stability & 0.08 & 0.06 & 0.03 & 0.02 & 0.02 & 0.01 & 0.01 & 0.01 \\
\hline $\begin{array}{l}\text { Uncertainty of the primary } \\
\text { working standards, total } \\
(k=2)\end{array}$ & 0.85 & 0.60 & 0.36 & 0.28 & 0.24 & 0.24 & 0.23 & 0.54 \\
\hline Lamp-to-lamp transfer & 0.1 & 0.1 & 0.1 & 0.1 & 0.1 & 0.1 & 0.1 & 0.1 \\
\hline $\begin{array}{l}\text { Long-term stability of working } \\
\text { standards }\end{array}$ & 1.31 & 0.94 & 0.50 & 0.36 & 0.20 & 0.16 & 0.14 & 0.14 \\
\hline $\begin{array}{l}\text { Uncertainty of the issued } \\
\text { standards, total }(k=2)\end{array}$ & 1.56 & 1.12 & 0.63 & 0.47 & 0.33 & 0.31 & 0.29 & 0.57 \\
\hline
\end{tabular}

${ }^{a}$ Uncertainty $(k=2)(\%)$. 


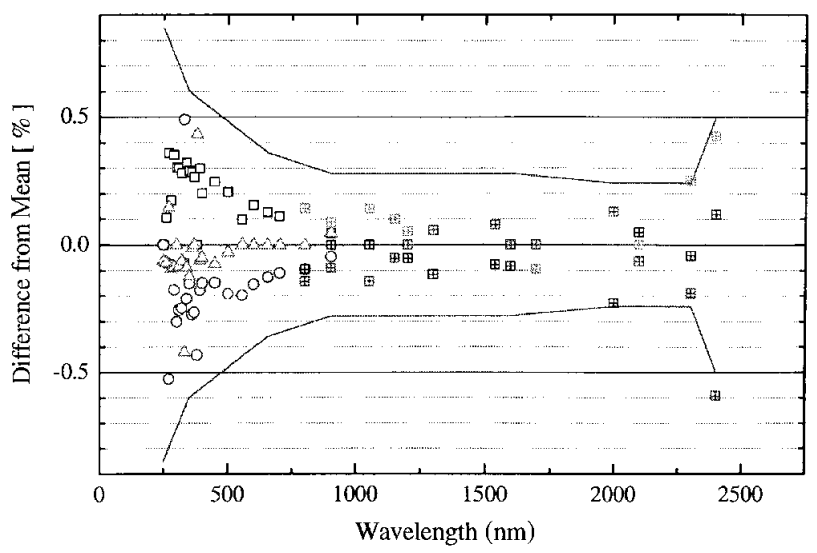

Fig. 9. Percent differences from the mean of three separate spectral irradiance scale realizations performed on four separate days. The differences are shown with the assigned expanded uncertainties of the working standards for the detector-based spectral irradiance scale.

from the uncertainty in the absolute wavelength. The wavelength drive of the prism-grating spectroradiometer has an absolute optical encoder attached to the shaft, and piecewise continuous polynomial corrections are applied to achieve \pm 0.05 -nm standard uncertainty in the wavelength. For determination of the uncertainties on the issued standards, additional components arising from the lamp-to-lamp transfer and long-term stability of the working standards are added. A substantial increase in the total uncertainties arises from the possible temporal drift of the working standards. All lamp standards are subjected to a screening procedure for temporal drift, and lamps that change by more than $0.5 \%$ at $650 \mathrm{~nm}$ over the duration of 24 operational hours are rejected from further evaluation as possible standards. ${ }^{6}$ The temporal drift is found to be inversely proportional to the wavelength and is accordingly larger at the shorter wavelengths.

To determine whether the uncertainties were correctly estimated, data from separate independent scale realizations of a FEL lamp (F210) were analyzed. Figure 9 shows the percent differences from the mean in the detector-based spectral irradiance of the lamp with three independent assignments from 250 to $900 \mathrm{~nm}$ and from three other separate assignments from 800 to $2400 \mathrm{~nm}$ over four separate days. The spectral irradiance assignments show that the three independent scale realizations for a particular wavelength region are all within the assigned $(k=2)$ expanded uncertainties of the 2000 spectral irradiance scale. The temperature of the HTBB was determined on each of the days during the realization, and the spectral irradiance responsivity was assigned approximately $30 \mathrm{~min}$ after a temperature assignment by use of the FRs. The separate spectral irradiance assignments are all within the expanded uncertainties. The slight upturn in the uncertainties at $2400 \mathrm{~nm}$ is due to the rapidly decreasing spectral responsivity of the InGaAs detector near the
Table 3. Comparison of the Expanded Uncertainties in the Spectral Irradiance Scales of the 1990 and 2000 Scale Realizations

Expanded Uncertainties in Spectral Irradiance

\begin{tabular}{ccc} 
Wavelength & \multicolumn{2}{c}{$(\%)(k=2)$} \\
\cline { 2 - 3 }$(\mathrm{nm})$ & 1990 & 2000 \\
\hline 250 & 1.8 & 1.56 \\
350 & 1.1 & 1.12 \\
655 & 0.9 & 0.63 \\
900 & 1.1 & 0.47 \\
1600 & 1.4 & 0.33 \\
2300 & 3.8 & 0.29 \\
2400 & 4.4 & 0.57 \\
\hline
\end{tabular}

bandgap energy of the material. The spectral shape of the expanded uncertainty follows the inverse wavelength dependence expected for a scale based on a temperature determination of a HTBB.

\section{Comparison of the Detector- and Source-Based Spectral Irradiance Scales}

Table 3 shows the total expanded uncertainties of the spectral irradiance scales from the 1990 and the 2000 realizations. Figure 10 shows that the detectorbased spectral irradiance scale results in the reduction in the uncertainties in the 250-900-nm wavelength regions by a factor of 2 and a greater reduction up to a factor of 10 in the uncertainties from the 900 - to $2400-\mathrm{nm}$ wavelength region. The reduction in the uncertainties is primarily due to the elimination of the ISS that was used as a transfer artifact to obtain spectral irradiance from the spectral radiance assignment. The spectral irradiance of the ISS was lower than that of the 1000-W FEL and was especially difficult to measure in the infrared wavelength region with the previous $\mathrm{PbS}$ detector, resulting in the large uncertainties. The detectorbased spectral irradiance utilizes the HTBB, which has greater spectral irradiance than the 1000-W FEL lamp at all wavelengths, and the improved InGaAs detector in the infrared further reduces the measure-

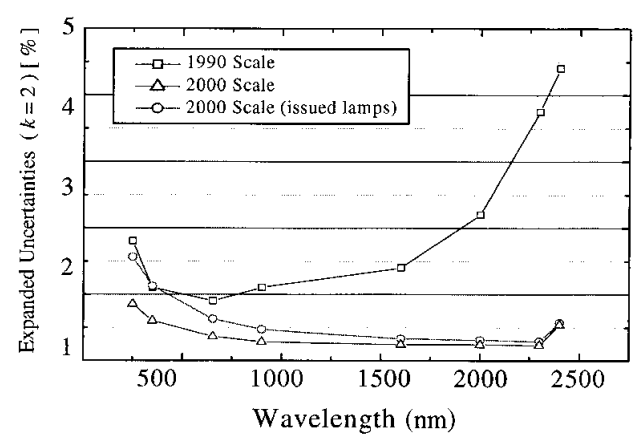

Fig. 10. Comparison of the expanded uncertainties of the 1990 scale realization along with the expanded uncertainties of the 2000 scale realization. The expanded uncertainties of the issued lamps are greater because of the additional component of the long-term temporal stability of the working standards. 


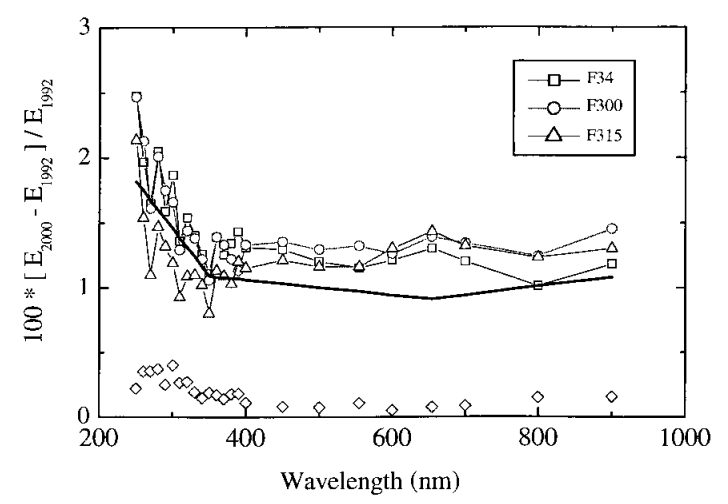

(a)

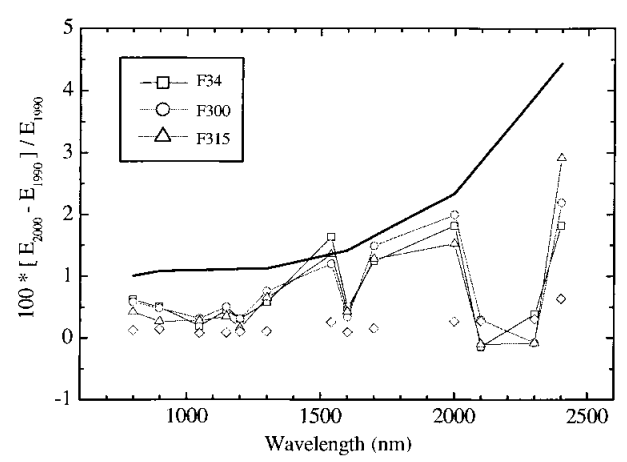

(b)

Fig. 11. (a) Comparison from 250 to $900 \mathrm{~nm}$ of the spectral irradiance assigned to the check standard lamps in 1992 with the detector-based spectral irradiance assignments. The expanded uncertainties assigned in 1990 are shown as the dark curve. The standard deviations of the mean of the three lamps are shown as open diamonds, indicating the good agreement between the three lamps. (b) A comparison from 800 to $2400 \mathrm{~nm}$ of the three check standard lamps of the 1990 spectral irradiance assignment with the detector-based spectral irradiance assignment. The standard deviations of the mean of the three lamps are shown as open diamonds along with the expanded uncertainties of the 1990 scale shown by the dark curve.

ment uncertainties. The spectral shape of the uncertainties also has the characteristic $1 / \lambda$ dependence of the decrease in uncertainties with increasing wavelength that can be seen in Eq. (2).

The differences in the spectral irradiance between the source-based and the detector-based spectral irradiance scale are shown in Fig. 11(a) for a set of check standard lamps (F34, F300, F315) in the wavelength region from 250 to $900 \mathrm{~nm}$ and in the wavelength region from 800 to $2400 \mathrm{~nm}$ in Fig. 11(b). At NIST, a set of lamps (working standard lamps) are used for routine calibrations, and another set of lamps (check standard lamps) are set aside to verify that the working standard lamps did not drift. The check standards were used approximately twice a year to verify that the working standard lamps did not changed; thus the total operational hours on the check standards are typically much less than the operational hours of the working standards.

In Fig. 11(a) the comparison of the detector-based scale with the source-based scale shows that the difference of the NIST spectral irradiance assignments is greater than their assigned expanded uncertainties by $\leq 0.5 \%$. The sawtooth oscillations that can be seen in the source-based spectral irradiance evident at $<400 \mathrm{~nm}$ is correlated in all the lamps and is evident to a lesser extent at longer wavelengths. The correlated oscillations are attributed to the experimental procedure used in the previous spectral irradiance scale realization of 1992 . In the realization of 1992, the ISS was assigned a spectral radiance at a particular wavelength, and the spectral irradiance of a set of three lamps was assigned immediately by use of the spectral irradiance of the ISS. Thus the spectral irradiance at each wavelength is realized separately and independently; and especially in the ultraviolet region, the oscillations could arise from a possible temporal drift of the ISS. Our ability to maintain the spectral irradiance over ten years using a set of stable lamps is shown by the agreement among lamps F34, F300, and F315 as compared with the detector-based spectral irradiance scale. The standard deviation of the mean found when we used the percent differences of the three lamps from the detector-based scale is plotted in Fig. 11(a) with the open diamonds and shows that the lamps have stayed together as a group to $<0.5 \%$ from 1992 .

In the shortwave infrared wavelength region, the source-based spectral irradiance scale from 800 to $2400 \mathrm{~nm}$ was last realized from the gold freezingpoint blackbody in 1990. A new spectral irradiance scale realization was attempted in 1992 from 250 to $2400 \mathrm{~nm}$ with a more stable ISS, but the scale realization in the infrared region was not completed because of the insufficient spectral irradiance of the 1992 ISS in the infrared and the low signal-to-noise ratio from the $\mathrm{PbS}$ detector. The discontinuity at 800 and $900 \mathrm{~nm}$ for a common lamp is due to the separate scale realizations performed in 1990 and in 1992. For assignment of spectral irradiance of the issued lamps, the different spectral irradiance values were averaged to obtain a single value at those wavelengths. As in the comparisons from 250 to $900 \mathrm{~nm}$, the check standard lamps remained close together as a group with their spectral irradiances much better than $<0.5 \%$ as shown in Fig. 11(b) by the open diamonds at the bottom of the figure. Two noticeable drops in the irradiances are observed at 1600 and $2100 \mathrm{~nm}$ in the comparison of the scales. The Consultative Committee on Photometry and Radiometry (CCPR) comparison of $1990^{19}$ also showed that the $1 \%$ drop at $1600 \mathrm{~nm}$ and the nearly $2 \%$ drop at 2100 $\mathrm{nm}$ were seen in all comparisons with the other countries, indicating that the two spectral features were inherent to the NIST spectral irradiance scale of 1990. The differences of the detector-based spectral irradiance scale to the source-based scale of 1990 show that the assignment of the spectral irradiances were within their stated uncertainties since 1990.

These comparisons to the check standards should reflect the differences between the new detectorbased scale and the scale realized in 1992, and more 
importantly, the scale realized in 1990 before and after the international comparison of spectral irradiance organized by the CCPR. If the differences seen can be extrapolated to the CCPR comparisons in 1990, then the NIST spectral irradiance scale for the comparison would have been approximately $1 \%$ low in the visible and approximately $2 \%$ low in the ultraviolet wavelength region. The large variations in the spectral irradiance scales of the individual countries preclude discerning whether the differences found between the detector-based scale and the 1990 scale could be detected in the international intercomparison.

\section{Discussion}

Because of the long history of the spectral irradiance scale at NIST and the resulting wide usage of the FEL lamps issued by NIST, the one most important result is the relationship between the spectral irradiances from the detector-based scale realization of 2000 and the previously assigned spectral irradiances. Figures 12(a) and 12(b) show the means of the spectral irradiances of the three working standards used to assign FEL lamps to the detector-based spectral irradiance scale along with the expanded uncertainties of the 1992 scale realization. In the wavelength region from 250 to $900 \mathrm{~nm}$, only one point, at $250 \mathrm{~nm}$, falls beyond the $0.5 \%$ of the expanded $(k=2)$ uncertainties; and if the uncertainties are increased to $3 \sigma(k=3)$, then all the values are within uncertainties. In the infrared region from 900 to $2400 \mathrm{~nm}$, only three values exceed the expanded uncertainties by more than $0.5 \%$, and again all the differences fall within the $3 \sigma(k=3)$ uncertainties.

Because we have shown that the radiance temperature scale and the spectral radiance scale at NIST are both in agreement with the detector-based scales within the combined uncertainties, what are the reasons for the observed discrepancies in the spectral irradiance scales? In the infrared wavelength region from 900 to $2400 \mathrm{~nm}$, the spectral irradiances of the check standard lamps are within the assigned expanded uncertainties, although the irradiances were assigned in 1990. The larger deviations of the working standards can be attributed to the longer operational hours and reveal that the spectral irradiances of the working standards should have been verified against the check standards at shorter time intervals.

Some of the differences from 250 to $900 \mathrm{~nm}$ that can be seen in Fig. 12(a) can also be attributed to temporal drift of the working standards because of longer operational hours, but the comparison with the check standards shows that much of the differences between the detector-based scale and the scale realized in 1992 are also apparent in lamps that have not been utilized extensively. The spectral irradiance scales of 1990 and 1992 are both dependent on the radiance temperature and the spectral radiance scales. Because both the radiance temperature scales and the spectral radiance scales were found to

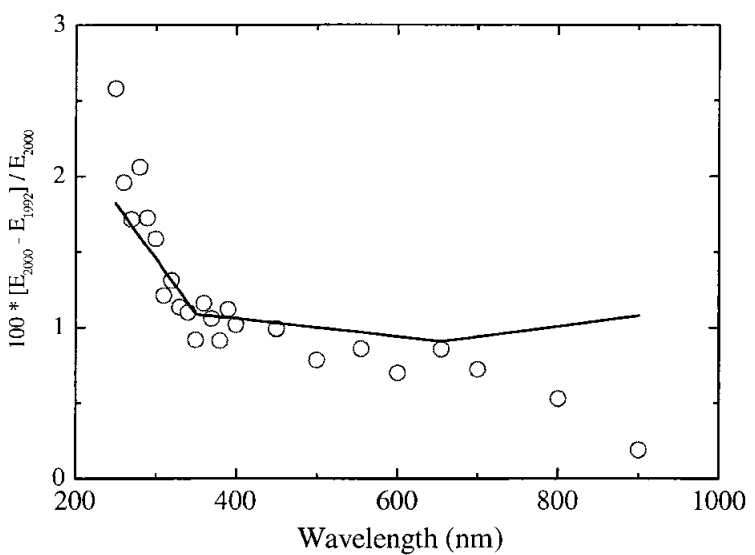

(a)

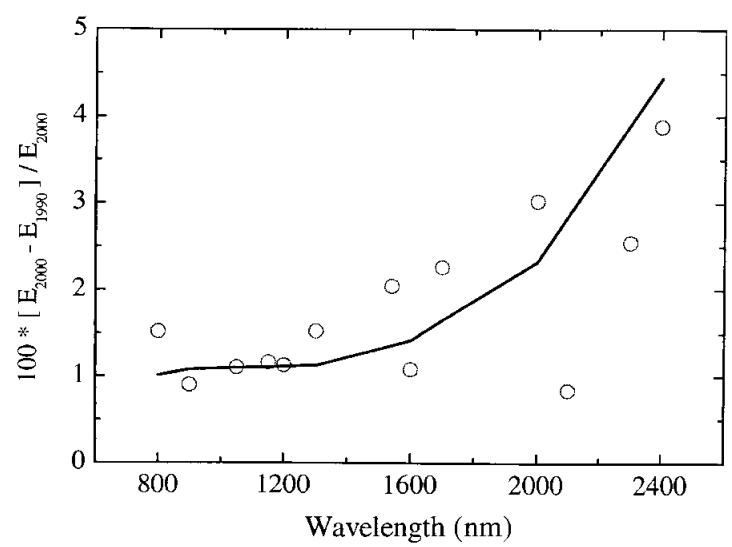

(b)

Fig. 12. (a) Comparison from 250 to $900 \mathrm{~nm}$ of the difference of the average of the spectral irradiances assigned in 1992 of three working standards F234, F302, and F210 to the detector-based spectral irradiances. The curve indicates the expanded uncertainties of the 1992 spectral irradiance scale. (b) A comparison from 800 to $2400 \mathrm{~nm}$ of the working standard lamps of the differences of the 1990 spectral irradiance scale to the detector-based scale. The curve indicates the expanded uncertainties of the 1990 spectral irradiance assignments.

be in agreement with the detector-based scale within the combined uncertainties, possible differences between the source-based and the detector-based spectral irradiances scales could have arisen in the process of deriving the spectral irradiance scale from the spectral radiance scale.

The long time period between the spectral irradiance scale realizations of 1992 and 2000 partly results from the experimental difficulties in the realizations of a spectral irradiance scale with the gold freezing-point blackbody. The need for numerous transfer artifacts and systematic corrections that are due to the less than optimal performance of these artifacts prohibited frequent scale realizations. The new detector-based spectral irradiance scale realization relies only on an accurate radiance temperature determination of a HTBB whose adherence to the Planck radiance law 
was verified by comparison with a blackbody with a known high emissivity. Initially, the detectorbased spectral irradiance scale realizations will be performed on an annual basis in FASCAL. With sufficient evidence that the spectral irradiance scale can be maintained on working standard lamps without increases in the errors that are due to longterm drift, the scale realizations will be performed on a less frequent basis.

In the past scale realizations, spectral irradiance assignments at additional wavelengths have been difficult because of the lengthy procedure needed for the realizations. The temporal drift of the transfer artifacts used in the realization resulted in the limited number of wavelengths of the spectral irradiance assignments of the working standards. Because the HTBB used in the detector-based spectral irradiance scale is temporally stable with a drift of $<1 \mathrm{~K}$ in $20 \mathrm{~h}$ at $3000 \mathrm{~K}$, spectral irradiance assignments of working standards are possible at many additional wavelengths if desired by the optical measurement community. These spectral irradiance calibrations at much finer wavelength intervals can help to resolve issues such as the optimal interpolation algorithm for use in the calibration of spectroradiometers. ${ }^{20}$

\section{Effect of the Changes That Are Due to the Detector-Based Spectral Irradiance Scale}

The greatest changes of the detector-based spectral irradiance scale realization result from the lower uncertainties, enabling measurements to be made with higher accuracy and confidence. However, if the dominant sources of the total measurement uncertainties were not due to the intrinsic scale uncertainties of the transfer artifact, then it would be difficult to utilize the lower uncertainties achieved with the detector-based scale. In addition, the small differences between the detector-based and the sourcebased spectral irradiance scales are within the expanded uncertainties of many measurement systems, including the spectroradiometers used in the ultraviolet monitoring of terrestrial solar spectral irradiance. ${ }^{21}$

In the photometric community, possible changes could arise in the calibration of sources for color temperature. ${ }^{22}$ At NIST, color temperatures are assigned in the Photometric Calibration Facility by use of the relative spectral irradiance of FEL lamps. Because the spectral shapes of the source-based and the detector-based spectral irradiances in the wavelength region from 350 to $700 \mathrm{~nm}$ differ at most by $<0.5 \%$ [Fig. 12(a)], the changes to the color temperature assignments will be negligible.

\section{Conclusion}

The realization of the NIST detector-based spectral irradiance scale was described. The realization is based on the absolute detector-based radiance temperature determinations of a HTBB and is now traceable to the HACR instead of a fixed-point blackbody. The differences in the spectral irradiances between the gold-point blackbody-based and the detectorbased scales are discussed, and the differences are found to agree within the combined uncertainties of the measurements. The detector-based scale results in the reduction of the uncertainties from 250 to $2400 \mathrm{~nm}$ with the greatest reduction occurring in the spectral range from 1000 to $2400 \mathrm{~nm}$ with reductions by almost a factor of 10 . The new detector-based spectral irradiance scale realization also corrects the correlated deviations from a smooth Planck radiance law that existed in the old spectral irradiance scale. Finally, the differences in the way that the scale is derived represent a fundamental change in the scale realization of spectroradiometric sources, and these results are only the beginning of the implementation of the detector-based source scales at NIST.

The authors gratefully acknowledge the help and encouragement of B. Carol Johnson, Robert Saunders, and Al Parr of the Optical Technology Division at all stages of this project. The authors also acknowledge the assistance of Tom Larason and George Eppeldauer in the development and characterization of the filter radiometers used in the scale realization.

\section{References}

1. A. C. Parr, "A national measurement system for radiometry, photometry, and pyrometry based upon absolute detectors," NIST Tech. Note 1421 (National Institute of Standards and Technology, Gaithersburg, Md., 1996).

2. T. R. Gentile, J. M. Houston, and C. L. Cromer, "Realization of a scale of absolute spectral response using the National Institute of Standards and Technology high-accuracy cryogenic radiometer," Appl. Opt. 35, 4392-4403 (1996).

3. C. L. Cromer, G. Eppeldauer, J. E. Hardis, T. C. Larason, and A. C. Parr, "National Institute of Standards and Technology detector-based photometric scale," Appl. Opt. 32, 2936-2948 (1993).

4. Y. Ohno, "Improved photometric standards and calibration procedures at NIST," J. Res. Natl. Inst. Stand. Technol. 102, 323-331 (1997).

5. K. D. Mielenz, R. D. Saunders, A. C. Parr, and J. J. Hsia, "The 1990 NIST scales of thermal radiometry," J. Res. Natl. Inst. Stand. Technol. 95, 621-629 (1990).

6. J. H. Walker, R. D. Saunders, J. K. Jackson, and D. A. McSparron, "Spectral irradiance calibrations," NBS Measurement Services SP 250-20 (National Bureau of Standards, Gaithersburg, Md., 1987).

7. H. J. Kostkowski, D. E. Erminy, and A. T. Hattenburg, "Highaccuracy spectral radiance calibration of tungten-strip lamps," Adv. Geophys. 14, 111-127 (1970).

8. K. D. Mielenz, R. D. Saunders, and J. B. Shumaker, "Spectroradiometric determination of the freezing temperature of gold,” J. Res. Natl. Inst. Stand. Technol. 95, 49-67 (1990).

9. V. I. Sapritsky, "Black-body radiometry," Metrologia 32, 411427 (1995-1996).

10. H. Preston-Thomas, “The ITS-90," Metrologia 27, 3-10 (1990).

11. J. B. Fowler, R. D. Saunders, and A. C. Parr, "Summary of the high-accuracy aperture-area measurement capabilities at the NIST," Metrologia 37, 621-623 (2000).

12. T. C. Larason, S. S. Bruce, and A. C. Parr, "Spectroradiometric detector measurements: Part I-Ultraviolet detectors and Part II-Visible to near-infrared detectors," NIST Measurement Services SP 250-41 (National Institute of Standards and Technology, Gaithersburg, Md., 1998). 
13. P. Sperfeld, J. Metzdorf, S. Gala Yousef, K. D. Stock, and W. Möller, "Improvement and extension of the blackbody-based spectral irradiance scale," Metrologia 35, 267-271 (1998).

14. H. W. Yoon and C. E. Gibson, "Determination of radiance temperatures using detectors calibrated for absolute spectral power response," in Proceedings of the Seventh International Symposium on Temperature and Thermal Measurements in Industry and Science, J. Dubbeldam and M. J. de Groot, eds. (Edauw and Johannisen, The Netherlands, 1999), pp. 737-742.

15. P. Sperfeld, S. Galal-Yousef, J. Metzdorf, B. Nawo, and W. Moller, "The use of self-consistent calibrations to recover absorption bands in the black-body spectrum," Metrologia 37, 373-376 (2000).

16. T. J. Quinn, Temperature (Academic, London, 1990).

17. A. Thompson and H. M. Chen, "Beamcon III: a linearity measurement instrument for optical detectors," J. Res. Natl. Inst. Stand. Technol. 99, 751-755 (1994).
18. H. W. Yoon and C. E. Gibson, "Comparison of the absolute detector-based spectral radiance assignment with the current NIST-assigned spectral radiance of tungsten strip lamps," Metrologia 37, 429-432 (2000).

19. J. H. Walker, R. D. Saunders, J. K. Jackson, and K. D. Mielenz, "Results of a CCPR intercomparison of spectral irradiance measurements by national laboratories," J. Res. Natl. Inst. Stand. Technol. 96, 647-668 (1991).

20. L. K. Huang, R. P. Cebula, and E. Hilsenrath, "New procedure for interpolating NIST FEL lamp irradiances," Metrologia 35, 381-386 (1998).

21. G. Bernhard and G. Seckmeyer, "Uncertainty of measurements of spectral solar UV irradiance,” J. Geophys. Res. 104, 14321-14345 (1999).

22. Y. Ohno, "Photometric calibrations," NIST Measurement Services SP 250-37 (National Institute of Standards and Technology, Gaithersburg, Md., 1997). 


\section{The NIST Detector-Based Luminous Intensity Scale}

C. L. Cromer, G. Eppeldauer, J. E. Hardis ${ }^{1}$, T. C. Larason, Y. Ohno, and A. C. Parr

National Institute of Standards and Technology,

Gaithersburg, MD 20899-0001

\author{
The Système International des Unités (SI) \\ base unit for photometry, the candela, \\ has been realized by using absolute detec- \\ tors rather than absolute sources. This \\ change in method permits luminous inten- \\ sity calibrations of standard lamps to be \\ carried out with a relative expanded uncer \\ tainty (coverage factor $k=2$, and thus a \\ 2 standard deviation estimate) of $0.46 \%$, \\ almost a factor-of-two improvement. A \\ group of eight reference photometers has \\ been constructed with silicon photodi- \\ odes, matched with filters to mimic the \\ spectral luminous efficiency function for \\ photopic vision. The wide dynamic range \\ of the photometers aid in their calibra-
}

\begin{abstract}
tion. The components of the photometers were carefully measured and selected to reduce the sources of error and to provide baseline data for aging studies. Periodic remeasurement of the photometers indicate that a yearly recalibration is required. The design, characterization, calibration, evaluation, and application of the photometers are discussed.
\end{abstract}

Key words: calibration; candela; illuminance; lumen; luminous intensity; lux; measurement; photometer; photometry; scale; standards; units.

Accepted: December 11, 1995

\section{Introduction}

Traditionally, standardization in photometry was a discipline driven by primary light sources, first candles, then flames [1], carbon-filament lamps, and, beginning in 1948, blackbody radiators operated at the freezingpoint temperature of molten platinum [2]. The latter marked a turning point, because the platinum-point blackbody, valued for its reproducibility and universality compared with the earlier alternatives, was the first standard photometric source with radiometric properties that could be readily calculated, in principle.

Over time, dissatisfaction with platinum-point blackbody standards grew. For the few national laboratories that had them, they were difficult to maintain. They operated at a temperature of little technological interest

${ }^{1}$ To whom correspondence should be sent, jhardis@ nist.gov. [taken first as $2045 \mathrm{~K}$, later $2042 \mathrm{~K}$, on the International Practical Temperature Scale of 1968 (IPTS-68)], and the applicability of this broadband radiation to spectroradiometry was poor. In 1975, Blevin and Steiner [3], reflecting the mood of the period, made two proposals. They sought first to redefine the photometric base unit in a manner to fix its relationship with other Système International des Unités (SI) base units, such as the meter and the ampere. Second, they argued that the photometric base unit should be changed from the candela to the lumen, considering the close relationship between luminous flux (lumen, $\mathrm{lm})^{2}$ and radiometric power measurements (watt, W).

\footnotetext{
${ }^{2}$ As an aid to the reader, the appropriate coherent SI unit in which a quantity should be expressed is indicated in parenthesis when the quantity is first introduced.
} 
After additional study and due consideration, in 1979 the 16th Conférence Générale des Poids et Mesures (CGPM) adopted the first of these proposals. They abrogated the definition of the candela (originally called the new candle) first adopted by the 8th Conférence Générale in 1948, and redefined it as follows [4]:

The candela is the luminous intensity, in a given direction, of a source that emits monochromatic radiation of frequency $540 \times 10^{12}$ hertz and that has a radiant intensity in that direction of $(1 / 683)$ watt per steradian.

The 1979 redefinition of the candela permitted diverse methods to be used in deriving luminous intensity scales. All the methods also rely on the principles governing photometry as compiled by the Bureau International des Poides et Mesures (BIPM) for the Comité Consultatif de Photométrie et Radiométrie (CCPR) [5]. These include the Commission Internationale de L'Éclairage (CIE) spectral luminous efficiency function for photopic (cone) vision, $V(\lambda)$, which relates visual sensitivities at different wavelengths [6]. (The lone frequency of $540 \times 10^{12} \mathrm{~Hz}$ mentioned in the definition has a wavelength of $555.016 \mathrm{~nm}$ in standard air, which for almost all purposes can be taken to be $555 \mathrm{~nm}$ without affecting the accuracy of a real measurement.)

Since the redefinition, national standards laboratories [7-14] and other research facilities [15] have been free to realize the candela by use of whatever radiometric means they found most suitable. Most have used detectors that were equipped with filters that were designed to match their spectral responsivity to the $V(\lambda)$ function. At NIST [then the National Bureau of Standards (NBS)] the luminous intensity scale remained based on a standard source, a blackbody radiator operating at the freezing-point temperature of molten gold (the gold point) [7].

As shown in Fig. 1, the blackbody radiation at the gold point (1337.58 K on IPTS-68) was used to calibrate a variable temperature blackbody, which provided the NBS scale of spectral radiance [16]. From this the spectral irradiance scale was derived [17]. The luminous intensity scale was derived through spectral irradiance measurements of selected lamps forming a primary reference group, which maintained the candela with respect to the spectral irradiance scale. A secondary reference group of lamps, calibrated against the primary group, was used for routine candela calibrations.

All the measurements in this lineage compared a light source with another light source. The final measurement uncertainty of $0.8 \%$ (2 standard deviation estimate) [18] contained a relatively large component from the uncertainty in the gold-point temperature at the top of

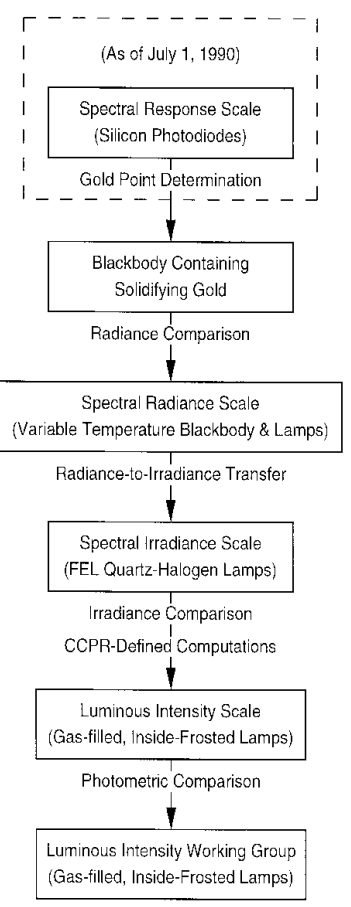

Fig. 1. Calibration chain for luminous intensity prior to the present study.

the chain (comparing IPTS-68 with thermodynamic temperature), and it was further limited by the longterm behavior of the incandescent lamps that were used.

In 1990, the introduction of the new International Temperature Scale (ITS-90) caused changes. The gold point was redefined as $1337.33 \mathrm{~K}$ [19], which caused the NIST luminous intensity scale to shift, depending on the color temperature of the source, by approximately $0.35 \%$ [20]. More important, NIST revised its procedures to decouple the spectral radiance scale from ITS90. NIST now considers the gold-point temperature to be a measured rather than a defined quantity. While the current NIST measurement of $1337.33 \mathrm{~K} \pm 0.23 \mathrm{~K}$ (restated from ' $3 \sigma$ "' to $k=2$ ) [21] is in exact agreement with ITS-90, the new policy allows for the possibility of future scale revisions as experimental information becomes available. The current NIST gold-point temperature of $1337.33 \mathrm{~K}$ is detector based. That is, the result follows from measurements using absolute radiometric detectors, a silicon photodiode and an electrically calibrated radiometer.

The purpose of this paper is to describe the considerable simplification that results by realizing the candela against the detector base directly. We expand upon our previous reports on this subject [22,23], giving more details behind the new NIST scale for luminous intensity 
and discussing our experience with it. The benefits of this conversion include reduced uncertainty in our calibration services and the additional flexibility to provide new calibration services for detector-based devices.

\section{Experimental Approach \\ 2.1 Mathematical Framework}

The photometric analog of power in radiometry is luminous flux, $\Phi_{\mathrm{v}},(\mathrm{lm})$, where

$$
\Phi_{\mathrm{v}}=K_{\mathrm{m}} \int_{\lambda} \Phi_{\mathrm{e}}(\lambda) V(\lambda) \mathrm{d} \lambda,
$$

where $\Phi_{\mathrm{e}}(\lambda)$ is the spectral radiant flux of the light $(\mathrm{W} / \mathrm{nm})$ and $K_{\mathrm{m}}$ is the proportionality constant in the definition of the candela. While a strict reading of the definition gives $K_{\mathrm{m}}=683.002 \mathrm{~lm} / \mathrm{W}$ [6], for almost all purposes it is taken to be $683 \mathrm{~lm} / \mathrm{W}$ without affecting the accuracy of any real measurement.

A photometer is a device that can be used to help measure such a flux. Typically, it has an output current ${ }^{3}$ I (ampere, A), where

$$
I=\int_{\lambda} \Phi_{\mathrm{e}}(\lambda) s(\lambda) \mathrm{d} \lambda
$$

where $s(\lambda)(\mathrm{A} / \mathrm{W})$ is its spectral responsivity. It is advantageous to factor

$$
s(\lambda)=s(555) s_{\mathrm{n}}(\lambda),
$$

where $s(555)(\mathrm{A} / \mathrm{W})$ is the value of $s(\lambda)$ at $555 \mathrm{~nm}$. This emphasizes the similarity of $s_{\mathrm{n}}(\lambda)$ to $V(\lambda)$, both dimensionless functions that are normalized at $555 \mathrm{~nm}$. It also permits the overall uncertainty of the spectral responsivity scale to be associated with one number, $s(555)$, with the function $s_{\mathrm{n}}(\lambda)$ consisting of relative measurements only.

The luminous responsivity [24] of the photometer is $s_{\mathrm{v}}(\mathrm{A} / \mathrm{lm})$, where

$$
s_{\mathrm{v}}=\frac{I}{\Phi_{\mathrm{v}}}=\frac{s(555)}{K_{\mathrm{m}}} \frac{\int_{\lambda} \Phi_{\mathrm{e}}(\lambda) s_{\mathrm{n}}(\lambda) \mathrm{d} \lambda}{\int_{\lambda} \Phi_{\mathrm{e}}(\lambda) V(\lambda) \mathrm{d} \lambda} .
$$

For a perfect photometer, $s_{\mathrm{n}}(\lambda)$ would equal $V(\lambda)$, and its luminous responsivity would be independent of the power distribution of the light. In practice, this approach

\footnotetext{
${ }^{3}$ Current is used as an example; the output might be a voltage instead.
}

requires knowing $\Phi_{\mathrm{e}}(\lambda)$ in order to calculate a spectral mismatch correction factor

$$
F=\frac{\int_{\lambda} \Phi_{\mathrm{e}}(\lambda) V(\lambda) \mathrm{d} \lambda}{\int_{\lambda} \Phi_{\mathrm{e}}(\lambda) s_{\mathrm{n}}(\lambda) \mathrm{d} \lambda} .
$$

In general, the closer $s_{\mathrm{n}}(\lambda)$ is to $V(\lambda)$, the better $F$ will be known for the same incertitude about $\Phi_{\mathrm{e}}(\lambda)$.

Figure 2 illustrates the application of such a photometer to luminous intensity measurement. In Fig. $2 \mathrm{a}$, it is supposed that the photometer intercepts a beam of light, and that all the light illuminates only a portion of the active area of the photometer. In this case, the photometer would have an output current $I$ from which the luminous flux of the beam could be determined, presuming that $s(\lambda)$ is sufficiently invariant from point to point over the active area:

$$
\Phi_{\mathrm{v}}=\frac{K_{\mathrm{m}} F I}{s(555)}
$$

In Fig. 2b, it is further supposed that the photometer is fitted with an aperture of precisely known area. Then, if the light is not confined to a small spot but rather overfills the aperture uniformly, the photometer would have an output current $I$ that is proportional to the illuminance $E_{\mathrm{v}}$ (lumen per square meter, lux, lx) on the aperture. For an aperture area $A$ (square meter, $\mathrm{m}^{2}$ ), (a)

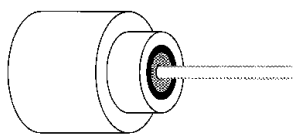

(c) (b)

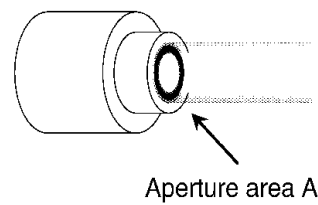

Aperture area $A$

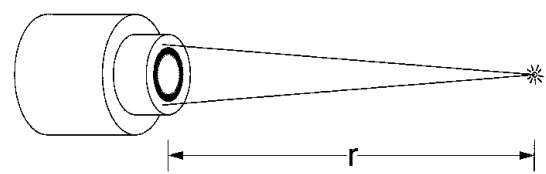

Fig. 2. Application of a photometer to luminous intensity measurement as a progression. (a) When the light beam underfills the entrance aperture, the photometer measures luminous flux $(\mathrm{lm})$, the photometric analog to radiant power. The responsivities of our detectors were tested in at least seven positions, as shown. (b) When the light beam overfills the entrance aperture, the photometer measures illuminance (lx). (c) When the photometer is used with a point light source at a distance, the aperture area and the distance to the source combine to define a solid angle. The photometer then measures the luminous intensity (cd) of the source. 


$$
E_{\mathrm{v}}=\frac{K_{\mathrm{m}} F I}{S(555) A} .
$$

Figure 2c shows the overall geometry for luminous intensity measurement. A point light-source a distance $r$ from the plane of the aperture and lying on the normal to its center would have a luminous intensity $I_{\mathrm{v}}$ (lumen per steradian, candela, cd), where

$$
I_{\mathrm{v}}=\frac{K_{\mathrm{m}} F I r^{2}}{s(555) A}
$$

The applicability of these geometric prerequisites to real measurements is explored below.

\subsection{Description of the Photometers}

To measure photometric quantities and to maintain the luminous intensity scale at NIST, a group of eight photometers has been developed. Many laboratories have used absolute detectors such as electrically calibrated thermal detectors and self-calibrated silicon photodiodes to realize the candela. We chose to use calibrated silicon photodiodes because of their wider dynamic range and simplicity of operation. The photo- meters contain specially selected silicon photodiodes with $V(\lambda)$ matching filters, as well as the electronics to implement the high-sensitivity, wide-dynamic-range circuit previously described [25]. With an integration time of $1.67 \mathrm{~s}$, a measurement bandwidth of $0.3 \mathrm{~Hz}$, and an amplifier gain of $10^{11} \mathrm{~V} / \mathrm{A}$, the output voltage noise in these devices corresponds to $\approx 1 \mathrm{fA}$ of photocurrent. This important feature of the NIST detectors permits precise measurement of $s_{\mathrm{n}}(\lambda)$ even in the regions where its values are small.

Figure 3 depicts the photometer design. The silicon photodiode, the $V(\lambda)$ correcting-filter package, and a precision aperture are mounted in the front piece of a cylindrical housing. A PTFE ${ }^{4}$ disk of low electrical conductivity supports the photodiode; small pin-terminals in the disk form a socket. The $V(\lambda)$ filter is glued to a holder and is positioned close to the photodiode. On the front side of the filter, the precision aperture is glued to a holder. This holder is carefully machined so that its front surface, the frontmost surface of the photometer, is $3.00 \mathrm{~mm}$ from the plane of the aperture knife edge. All these components are marked in a manner that permits us to preserve their orientation during disassembly and reassembly.

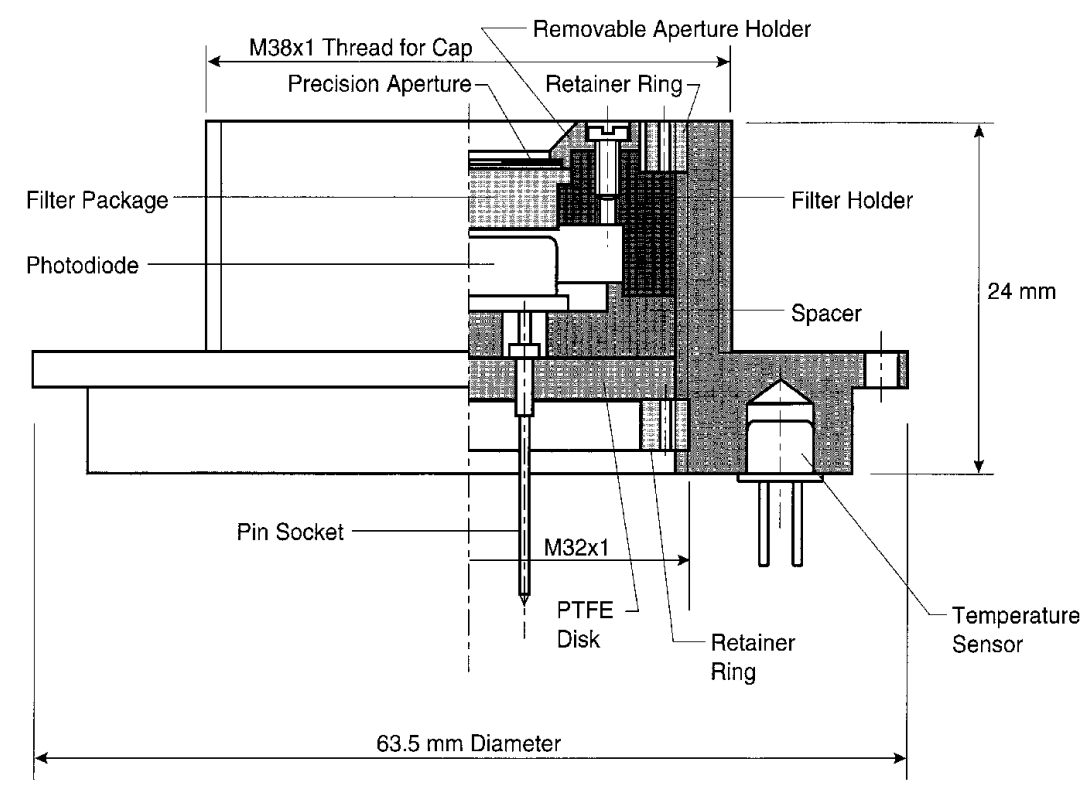

Fig. 3. Photometer design. A filter modifies the spectral responsivity of a silicon photodiode to replicate as closely as possible the 1924 CIE spectral luminous efficiency function for phototopic vision.

\footnotetext{
${ }^{4}$ PTFE, polytetrafluoroethylene, is more commonly known as Teflon, which is a brand name for such materials.
} 
The cylindrical housing itself, which extends back from the front piece shown in Fig. 3, contains an amplifier that also acts as a photocurrent-to-voltage converter. A switch selects the transimpedance gain of the amplifier, decade values from $10^{4} \Omega$ through $10^{10} \Omega$. (Photometers 1 and 2 also have $10^{11} \Omega$ ranges.) The characteristics of the filter and photodiode change with temperature, so the operating temperature of the photometer is monitored by a sensor inserted in the front wall of the housing [26]. The housing contains all additional components necessary for signal and temperature outputs; it is lighttight and acts as an electrical shield.

\subsection{The New Luminous Intensity Scale}

It is simpler to realize the candela by this approach, diagramed in Fig. 4. The luminous intensity scale is derived by measuring $s(\lambda)$ of each photometer in the group directly against the NIST spectral responsivity scale. The spectral responsivity scale is derived from comparative measurements against absolute radiometric detectors; at the time of the initial study, $100 \%$ quantum efficient detectors [27] were the basis of the scale. Today, the scale is based on cryogenic radiometry ${ }^{5}$ [28]. With the application of the $V(\lambda)$ curve in Eq. (5), and the application of the geometric definitions in Eq. (8), the candela is determined. Additionally, since the photometers do not age in use as rapidly as lamps do, an additional step to form a working group of photometers for routine use is unnecessary.

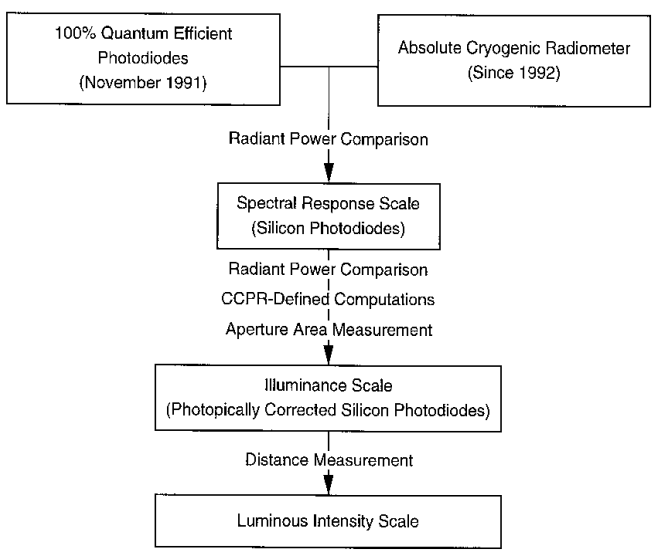

Fig. 4. Calibration chain for luminous intensity as revised by the present study.

\footnotetext{
${ }^{5}$ The photometers cannot be compared directly against the cryogenic radiometer since the radiometer requires a laser light source. The parallel surfaces of the optical elements in the photometers might cause errors due to interference effects with such illumination.
}

\section{Characterization of the Photometers 3.1 Instrumentation and General Procedures}

The principal apparatuses used to study the photometers and their components are shown in Fig. 5. They comprise the Spectral Comparator Facility (SCF), which holds the NIST spectral responsivity scale referenced in Figs. 1 and 4. An ultraviolet (UV) instrument spans $200 \mathrm{~nm}$ to $400 \mathrm{~nm}$; a visible/near-infrared (IR) instrument spans $350 \mathrm{~nm}$ to $1800 \mathrm{~nm}$. A detector under test is held in a carriage that can be translated under computer control. Any point on the active area of the detector can be positioned at the focus of a nearly circular spot, $1.1 \mathrm{~mm}$ or $1.5 \mathrm{~mm}$ in diameter for the visible or UV system, respectively. The carriage also holds reference detectors that serve as secondary standards and that are measured alternately with the device being tested. Compensation for changes in the light source during the course of the measurement is made by using the signal from a monitor detector. The computer controls the monochromator, which has a bandpass of $4 \mathrm{~nm}$ for this spot size and a spectral standard uncertainty of $\pm 0.2 \mathrm{~nm}$ [29]. The apparatuses typically deliver a few microwatts of optical power to the detector.

Before the photometers were assembled, the SCF was used to study their components, both to diagnose systematic effects and as the basis for aging studies. When the spectral responsivity of an individual photodiode or a photometer (the photodiode, filter, and aperture together) was measured, the device itself was mounted on the carriage. For the spectral transmittance of a filter alone to be determined, the filter was held on the carriage, but a photodiode behind it was not. (Filter transmittance is the ratio of the apparent detector responsivity with and without the filter interposed in the beam.) In this case, the photodiode was tilted to prevent interreflections.

Care was taken to insulate thermally the devices from the carriage, which heats up during use because of its stepping motors. The ambient temperature during measurement was monitored; when applicable, the temperature circuitry of the device under test was used. This permitted a direct comparison between the temperatures at calibration and use. Generally, variations in ambient temperature were held within $\pm 1{ }^{\circ} \mathrm{C}$ during the course of a measurement.

In addition to the optical calibrations performed at the SCF, the transimpedance gains of the photometer amplifiers were calibrated electrically. With this procedure, the photodiode is replaced by a computer-controlled voltage source, $V_{\mathrm{IN}}$, and a resistor substitution box in series. Unlike the internal resistors $R_{\mathrm{f}}$ built into the photometer heads, the external resistors $R_{\text {EXT }}$ are easily remeasured. (As explained in Ref. [25], $R_{\mathrm{f}}$ is the trans- 


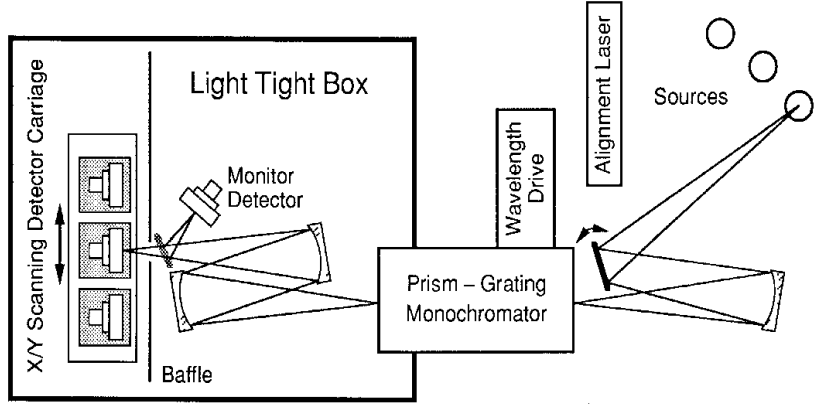

(a)

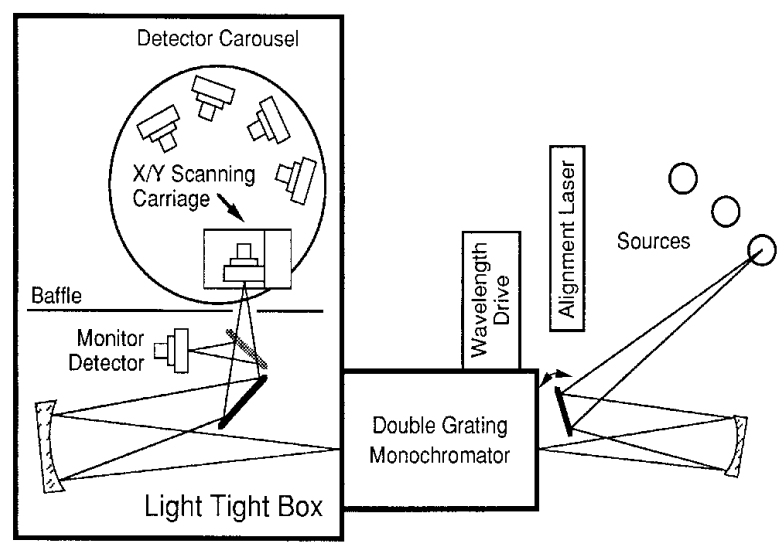

(b)

Fig. 5. Facility used to calibrate the photometric detectors: (a) with visible and IR radiation, (b) with UV radiation.

impedance gain of the amplifier.) For many combinations of internal and external resistors (as selected by the photometer gain switch and the substitution box, respectively), the output of the photometer, $V_{\text {OUT }}$, is measured for a series of $V_{\mathrm{IN}}$. The linear coefficient of this dependence, as obtained from a least-squares fit, is equal to the corresponding $R_{\mathrm{f}} / R_{\text {EXT. }}$. This permits the individual values of $R_{\mathrm{f}}$ to be determined with a relative expanded uncertainty of $<0.01 \%$ by data fitting. Calibrations on the SCF, reported in the unit volt per watt for an individual photometer gain-switch setting, can be transferred between different settings when these data are used.

\subsection{Photodiodes}

For this project we used Hamamatsu S1226 and S1227 series photodiodes ${ }^{6}$ [30]. They were selected for

\footnotetext{
${ }^{6}$ Certain commercial equipment, instruments, or materials are identified in this paper to foster understanding. Such identification does not imply recommendation or endorsement by the National Institute of Standards and Technology, nor does it imply that the materials or equipment identified are necessarily the best available for the purpose.
}

the largest shunt resistance that the manufacturer could provide, $2.5 \mathrm{G} \Omega$ to $7.0 \mathrm{G} \Omega$, in order to minimize noise and drift in the circuit [25]. This type of photodiode has less infrared sensitivity than some others, which is advantageous for photometry. As a consequence, their infrared response is more temperature dependent than the alternatives. We used quartz rather than glass or resin windows, since we found that the former had less surface scatter. S1227-1010BQ photodiodes having $1 \mathrm{~cm}^{2}$ area were used in Photometers 1 and 2 because they contained larger $V(\lambda)$ filters. The other six photometers used S1226-8BQ $0.3 \mathrm{~cm}^{2}$ photodiodes, with the exception of Photometer 4, which contained an S1227-66BQ. (The only difference was in the case.)

Figure 6 shows the absolute spectral responsivity of three of these photodiodes, at one spot in their centers, as measured at the SCF. The dashed curve is the measurement of Photodiode 1. Photodiode 2 behaved similarly. The solid and dotted curves, measurements of Photodiodes 7 and 8 respectively, bound the responsivity curves of the remaining photodiodes. 


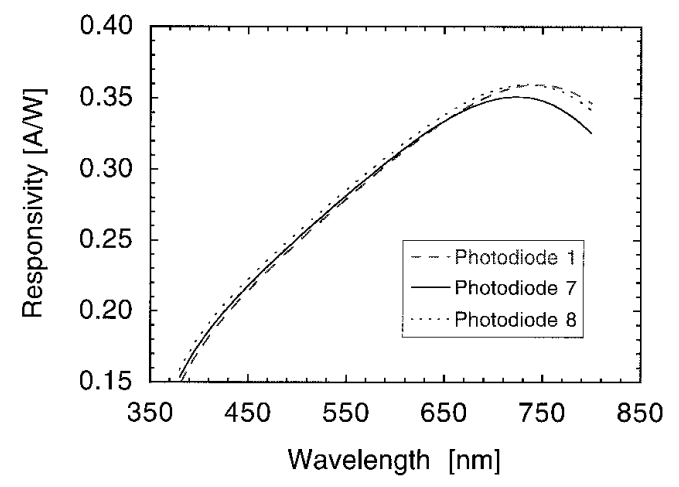

Fig. 6. Absolute responsivity of the silicon photodiodes used in the detectors. The dashed curve is Photodiode 1, type S1227-1010BQ. Photodiode 2 , of the same type, matches very closely. The solid curve is Photodiode 7 and the dotted curve is Photodiode 8, both of type S1226-8BQ. All other photodiode curves are bounded by the latter two and are similarly shaped. The relative standard uncertainty of $0.3 \%$ is commensurate with the curve widths.

The eight photodiodes were chosen after screening many more for uniformity over their active areas, particularly the portion that would be visible through an aperture. Uniformity maps such as the one shown in Fig. 7 for Photodiode 2 were made for each device. To construct a uniformity map, the photodiode responsivity was measured on the SCF on a grid of points $0.5 \mathrm{~mm}$ apart at three different wavelengths. Mathematica [31] was used to generate surface plots. Typically, the great- est responsivity was at the edge of the photodiode, as in Fig. 7 where the most sensitive spot is the lower right corner. The responsivities over the interior "bowl" of the selected photodiodes were generally constant to better than $0.2 \%$.

The change in photodiode responsivity due to a change in temperature is shown in Fig. 8. Six photodiodes, most of which were included among the final eight, were tested in a temperature-controlled housing. At each wavelength, the spectral responsivities of the six were measured at the SCF at $25^{\circ} \mathrm{C}, 30^{\circ} \mathrm{C}$, and $35^{\circ} \mathrm{C}$. Figure 8 shows the average of the six results, the linear temperature dependence as determined through leastsquares fitting. For the wavelengths of most interest in photometry, $400 \mathrm{~nm}$ to $700 \mathrm{~nm}$, the temperature dependence of the photodiode responsivity was $<0.03 \% /{ }^{\circ} \mathrm{C}$.

\subsection{Filters}

We obtained layered, colored glass filters from various sources to benefit from the experience that this diversity offers. Filters 1 and 2 were provided through the courtesy of the National Research Council of Canada (NRC), Filter 3 was provided courtesy of the National Physical Laboratory of the U.K. (NPL), and Filters 4 to 8 were manufactured by PRC Krochmann (PRC)[32]. Such filters are individually made to achieve a good realization of the $V(\lambda)$ function. First, the glasses are carefully chosen $[8,33]$, and then the thicknesses of the individual glass layers are determined

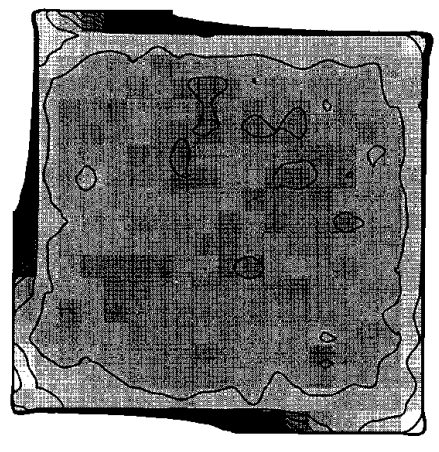

$530 \mathrm{~nm}$

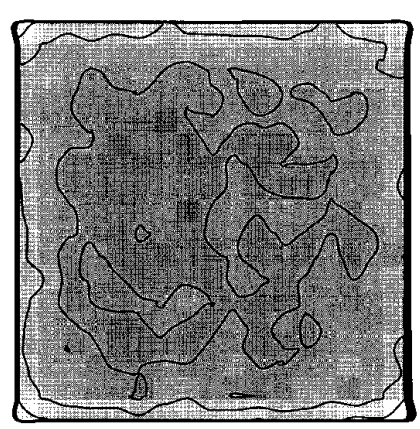

$555 \mathrm{~nm}$

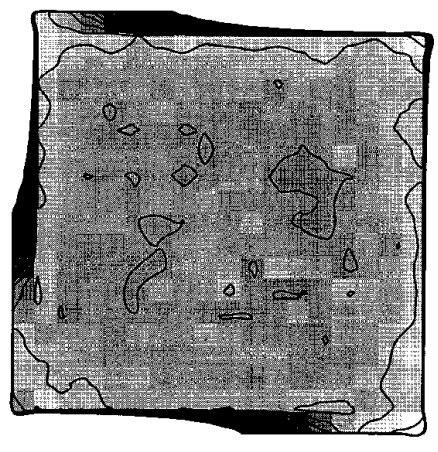

$580 \mathrm{~nm}$

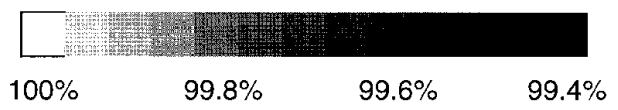

Fig. 7. Responsivity map of a typical photodiode used in this study. The responsivity of a photodiode (A/W) was measured while scanning a monochromatic probe beam over the surface. This photodiode, which was used in Photometer 2 , is $1 \mathrm{~cm}$ on a side. The grey scale shows the responsivity at a point, referenced to the greatest value on the device (100\%). The contours indicate changes of $0.05 \%$ in responsivity. 


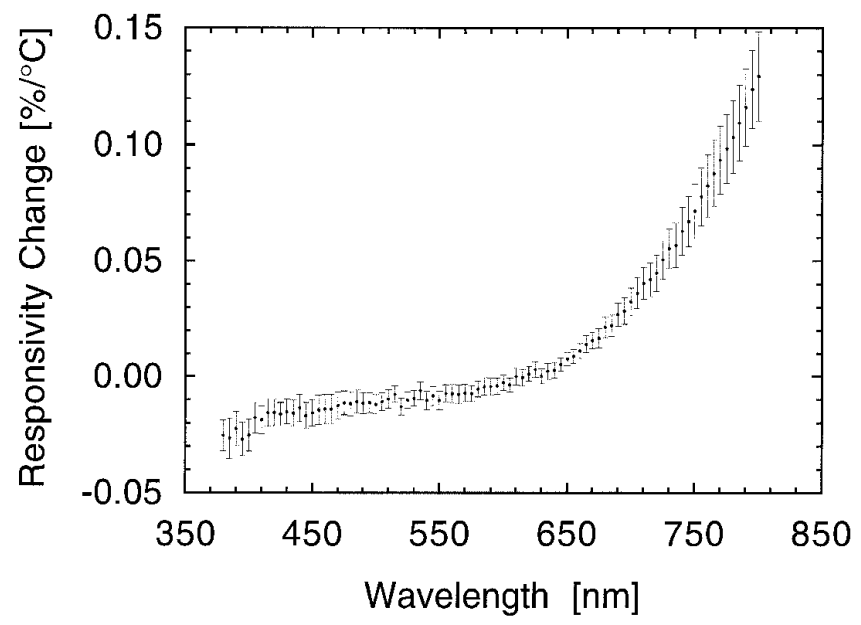

Fig. 8. Temperature dependence of the silicon photodiodes at $30^{\circ} \mathrm{C}$. Responsivities of six photodiodes of the types used in this work were measured at $25^{\circ} \mathrm{C}, 30{ }^{\circ} \mathrm{C}$, and $35^{\circ} \mathrm{C}$. The plot shows the linear change in responsivity, as a fraction of their nominal values, averaged over the six photodiodes. Individual variations among the six generally agreed within the measurement noise. The error bars represent the statistically estimated standard deviation, from the sample of six.

through an iterative procedure including repeated polishing and transmittance measurements. Filters 1 and 2 were originally designed to match QED-200 trap detectors; Filter 3 was designed to match Centronics OSD 300-5 photodiodes. Filters 4 to 8 were optimized to match our type of silicon photodiode.

While spectral match is important, so that Eq. (5) is insensitive to $\Phi_{\mathrm{e}}(\lambda)$, other important filter properties include the spatial uniformity, birefringence, and temperature dependence. Filters 4 to 8 were selected from among 24 candidates after visual inspection. Filters with obvious dislocations, scratches, bubbles, and other optical defects were rejected. The remaining filters were screened for uniformity by scanning them with a whitelight spot $1.5 \mathrm{~mm}$ to $2.0 \mathrm{~mm}$ in diameter. Those with the sharpest and largest changes were eliminated.

Since the filters are composed of dissimilar layers cemented together, any resulting strains might cause birefringence or a polarization-dependent transmittance. (The light from a monochromator during calibration is partially polarized.) To verify the absence of such a problem, representative filters were tested. A plane polarizer was interposed between the photometers and a lamp operating at approximately $2856 \mathrm{~K}$. No change in signal above noise was noted as the photometer was rotated, limiting the potential error to $0.01 \%$. Nevertheless, candidate filters that showed the greatest birefringence were also rejected.
After selecting the most promising filters, more detailed diagnostics were performed. Transmittance measurements were made in $5 \mathrm{~nm}$ intervals, and at many positions on the filters to determine their spatial uniformity. Hexagonal patterns were used, consisting of 37 spots for the larger filters ( 1 and 2), and 7 spots for the smaller ( 3 to 7 ). Figure 9 shows the average transmittances of all spots measured on representative filters, using the SCF. Figure 9a compares representative filters from the different sources; others from a common source would be indistinguishable on the graph. However, Filter 8 was from a different batch and provided a better spectral match than the other PRC filters. The small difference between it and the others is highlighted in Fig. 9b.

Figure 10 shows the variation among the measurements at the different spots, expressed as the scatter of the measurements. Scatter in excess of the measurement noise (the heavy curves) represents non-uniformity in the filter transmittance. Figure $10 \mathrm{~b}$ provides a striking illustration of how the individual layers in these filters contribute differently at different wavelengths. Below $525 \mathrm{~nm}$, the change in transmittance between Filters 5 and 8 (seen in Fig. 9b) is well correlated with the improved uniformity of Filter 8.

Of particular concern is the temperature dependence of the filter transmittance. Figure 11 shows representative data obtained by using a commercial spectrophotometer equipped with a sample heater. A $3 \mathrm{~mm}$ by $10 \mathrm{~mm}$ 


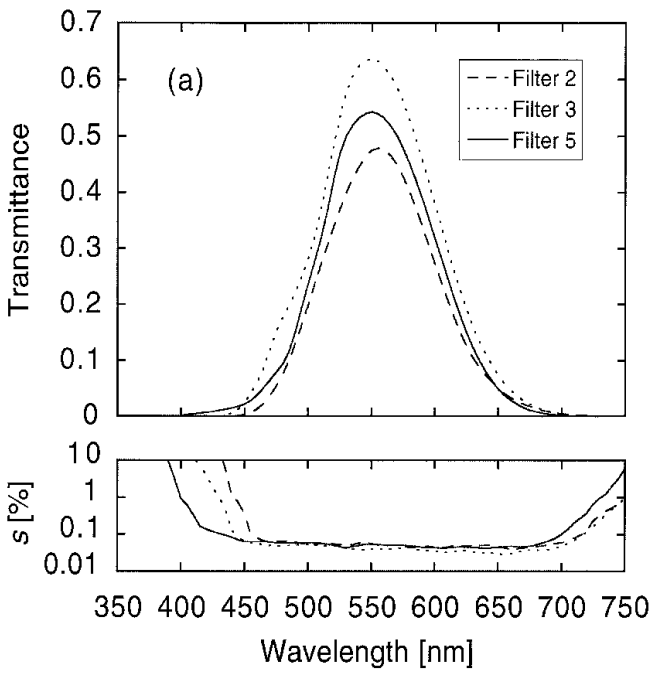

Fig. 9a. Transmittance of the matching filters used in the detectors. The standard deviation of the measurements, as the percent of the signal, is shown. Representative samples of the filters from the three sources: Filter 2, NRC, dashed curve; Filter 3, NPL, dotted curve; Filter 5, PRC, solid curve.

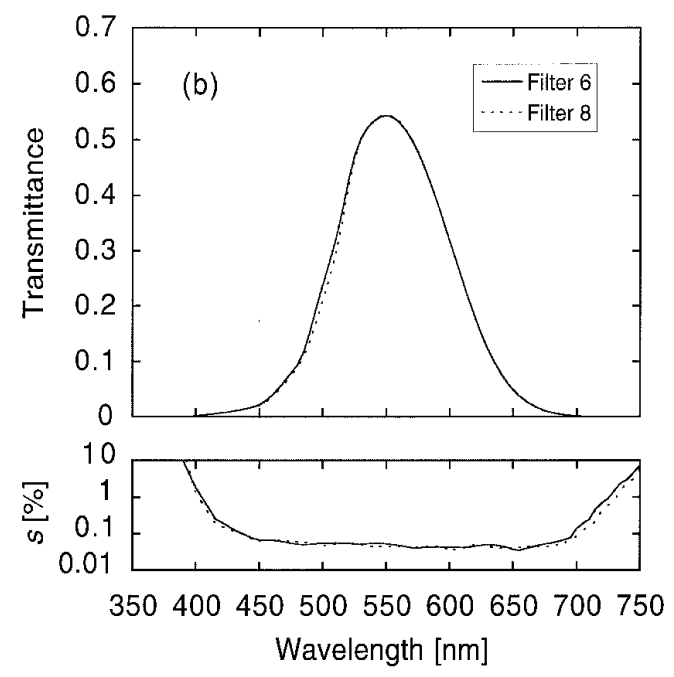

Fig. 9b. Transmittance of the matching filters used in the detectors. The standard deviation of the measurements, as the percent of the signal, is shown. Comparison of the two batches of PRC filters: Filter 6 , first batch, solid curve; Filter 8 , second batch, dotted curve.

probe beam was used. For Filter 3, this data is consistent with the filters discussed in Ref. [8]. This data is also consistent with the broadband temperature dependence of the complete photometers, which is discussed in detail below.

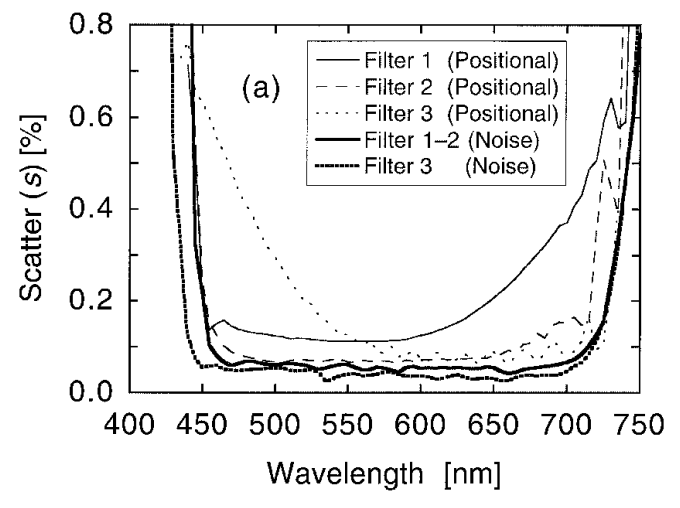

Fig. 10a. Transmittance uniformity of the matching filters, comparing several positions on the filters. The variation between the measurements is given by their standard deviations from their means. NRC and NPL filters. The heavy curves are the limiting measurement noise: solid for NRC, broken for NPL. Filter 1 (NRC) is the light solid curve; Filter 2 (NRC) is the light dashed curve; Filter 3 (NPL) is the light dotted curve.

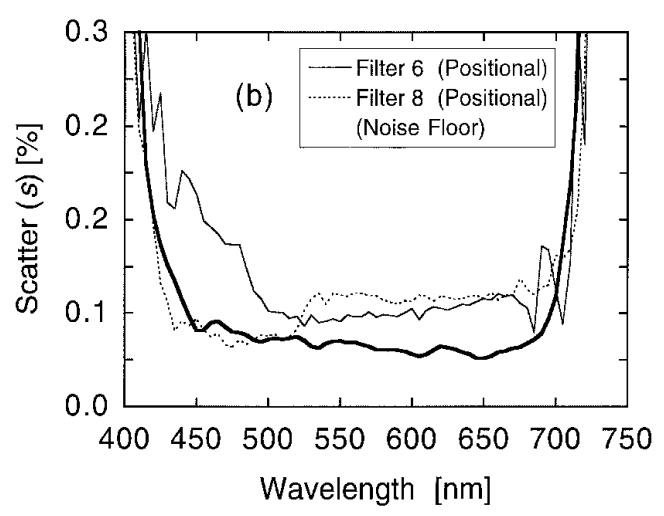

Fig. 10b. Transmittance uniformity of the matching filters, comparing several positions on the filters. The variation between the measurements is given by their standard deviations from their means. PRC filters. The heavy curve is the measurement noise. Filter 6 (first batch) is the light solid curve, and is typical of the others in the batch. Filter 8 (second batch) is the light dashed curve.

\subsection{Apertures}

The photometers were fitted with precision apertures, nominally $0.5 \mathrm{~cm}^{2}$ for Photometers 1 and 2 , and $0.1 \mathrm{~cm}^{2}$ for Photometers 3 to 8 . They were electroformed out of nickel-clad copper and given a black, nickel finish. The fabrication and properties of similar apertures are discussed in Ref. [34]. Most important to us is the resultant knife-edge from this process, sharp and without burrs. However, such apertures may depart from circularity. 


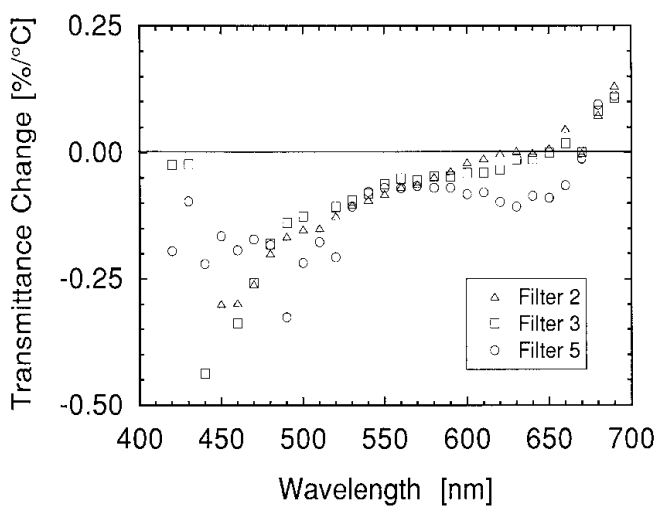

Fig. 11. Temperature dependence of the $V(\lambda)$ matching filters. Transmittances at $23{ }^{\circ} \mathrm{C}$ and $33{ }^{\circ} \mathrm{C}$ were measured using a Cary 2390 spectrophotometer. The small differences plotted are of the same magnitude as the uncertainties in the measurements - this data is shown to illustrate the overall trend. $\triangle$, Filter 2 (NRC); $\square$, Filter 3 (NPL); $\bigcirc$, Filter 5 (PRC).

The Precision Engineering Division at NIST measured and certified the areas using a View Engineering Precis 3000 vision-based measuring machine [35]. After a pass was made to find the approximate center of the aperture, 720 radii were measured from the center to the lip at $0.5^{\circ}$ angular intervals. The measurements were not sensitive to the method of lighting the aperture (i.e., different forms of front and back lighting). The area was estimated from these radii by a polygonal approximation. The combined standard uncertainties of the radii measurement and the area estimation were given as $0.02 \%$ for the larger apertures and $0.05 \%$ for the smaller. Since the coefficient of linear thermal expansion for copper is $\approx 0.0017 \% /{ }^{\circ} \mathrm{C}$, temperature corrections were unnecessary.

\subsection{Assembled Photometers}

After the photodiode, filter, and apertures were individually tested, they were assembled into photometers as shown in Fig. 3. The advantage to calibrating the components assembled is that internal reflections and scattering have similar effects during both calibration and use. The essential role of the SCF is to calibrate the spectral responsivity $s(\lambda)$ of the photometers to determine $s$ (555) [Eq. (3)] and $F$ [Eq. (5)]. The small output spot from the SCF can be positioned at various places within the aperture.

The first attempt at calibrating the photometers was to measure $s(\lambda)$ at seven positions within the aperture, comprising the vertices and center of a regular hexagon. (Photometers 1 and 2 were measured at 37 positions, which formed a larger, regular hexagonal pattern.) The average over these positions was taken to be $s(\lambda)$ for the photometer as a whole. However, consistency among the photometer calibrations was improved by a factor of two by using the following method.

$s(\lambda)$ was first measured at $5 \mathrm{~nm}$ intervals at one position near the center of the aperture of each photometer. Data from representative photometers are shown in Fig. 12a. Of particular importance in these data is the degree of IR and UV suppression, the latter including both transmission and fluorescence signals.

However, a correction was needed because $s(\lambda)$ varied over the aperture area. The spectral responsivity of each photometer, relative to the center point, was determined at $50 \mathrm{~nm}$ intervals on a fine, rectangular mesh of points. For the larger apertures (Photometers 1 and 2) the step size was $0.25 \mathrm{~mm}$; for the smaller apertures (Photometers 3 to 8 ) the step size was $0.2 \mathrm{~mm}$. Measurements that were not affected by the aperture edge were averaged.

Figure $12 \mathrm{~b}$ shows such data, the ratio of the average responsivity to the responsivity of the center spot. Polynomial fits are made to these data in order to interpolate between them. This permits us to estimate the average responsivity, given the center point responsivity, at all wavelengths. After application to the data in Fig. 12a, the final spectral responsivities for representative photometers are shown in Fig. 12c. The scatter given in the lower part of the figure is only the statistical noise of measuring $s(\lambda)$ at the center. Additional uncertainties also apply, and they are discussed below. During the calibration process the temperature of a photometer was monitored using its built-in thermometer. Variations were generally held to $\pm 1^{\circ} \mathrm{C}$. The average temperature was recorded for each photometer to be used for temperature dependence corrections.

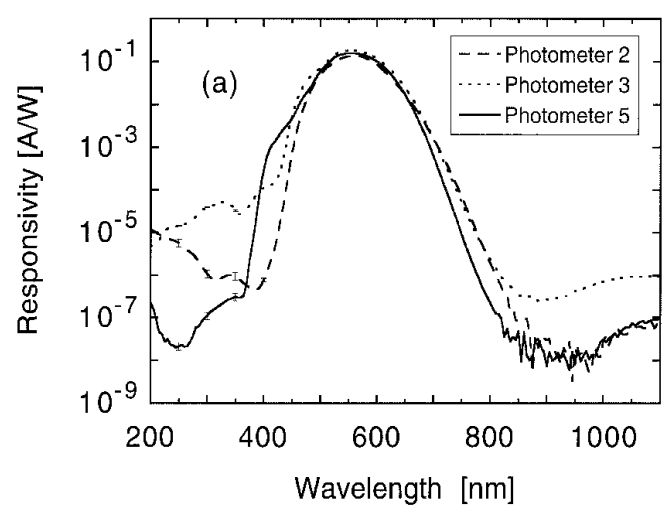

Fig. 12a. Responsivity of the filtered photodiode packages with emphasis on their behavior in the UV and IR. One spot in the center of the aperture is probed. The measurement uncertainty at this spot is commensurate with the width of the curve in the visible, with the apparent scatter of the data in the IR, and shown by error bars in the UV. Representative packages: Photometer 2, NRC, dashed curve; Photometer 3, NPL, dotted curve; Photometer 5, PRC, solid curve. 


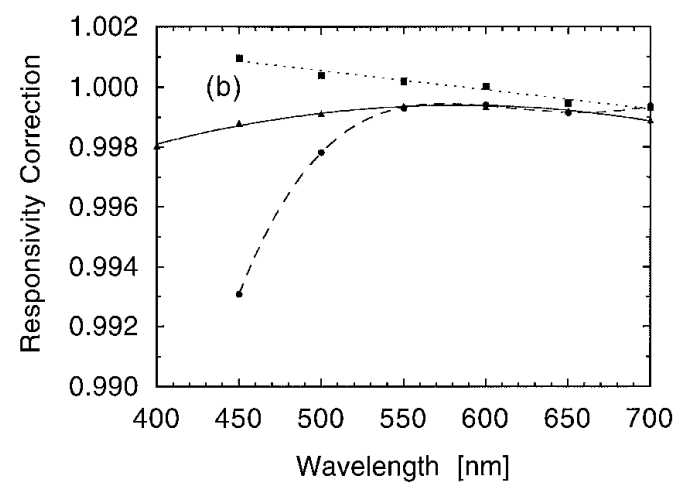

Fig. 12b. Comparison of responsivity at the center spot with the average of many spots over the face of the aperture. Data taken at 50 $\mathrm{nm}$ intervals are interpolated by polynomial fits. The correction factor converts the responsivity at the center to the average responsivity over the face of the aperture. The curves are as in Fig. 12a.

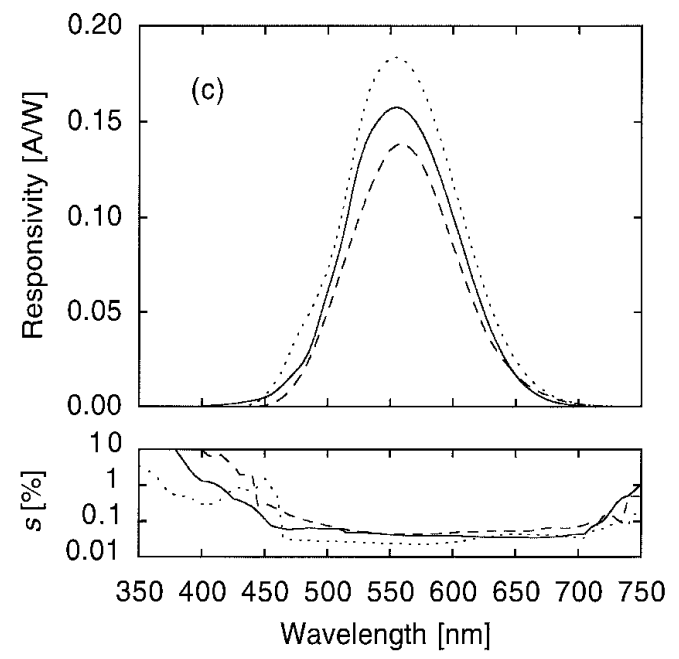

Fig. 12c. Responsivity of the filtered photodiode packages. The curves are as in Fig. 12a, after the corrections in Fig. 12b have been applied. The standard deviation of the measurements is shown below.

Figure 13 shows the mesh of spectral responsivity measurements in more detail. Photometer 3 provides a striking illustration of how spatial nonuniformities may be associated with the individual glass layers in a filter, each affecting a particular wavelength band. This data also helps to estimate the systematic error that might arise if the aperture is not fully and uniformly illuminated during a measurement.

$s(\lambda)$ varies with the temperature of the photodiode and the filter, as shown in Figs. 8 and 11. We measured the overall temperature effect by operating representative photometers at elevated temperatures. Figure 14 diagrams the experimental setup. A photometer was placed in a heated, plastic foam box and left to reach thermal equilibrium overnight. It was illuminated in the normal manner by an inside-frosted lamp of the type formerly used at NIST for luminous intensity calibrations. The lamp had a color temperature $\approx 2856 \mathrm{~K}$. A temperature-controlled monitor detector with a $V(\lambda)$ filter was used to compensate for the variation in lamp output from lighting to lighting.

Figure 15 shows the results. The luminous responsivity of the photometers decreased with increasing temperature, as measured with each photometer's built-in thermometer. As expected, the data form clusters that depend on the filter construction. Therefore, all data concerning filters from the same source are considered together and fit to a common line. Compared with the value when the photometer was unheated, the responsivity of Photometer 3 decreased by $0.049 \% /{ }^{\circ} \mathrm{C}$, the responsivities of Photometers 1 and 2 decreased by $0.063 \% /{ }^{\circ} \mathrm{C}$, and the rest decreased by $0.088 \% /{ }^{\circ} \mathrm{C}$. The standard uncertainty of these results is $<0.002 \% /{ }^{\circ} \mathrm{C}$. The temperature effect would be different when measuring sources with other spectral compositions.

Direct comparison of these results with the data of Fig. 11 is difficult because of the large uncertainties in the latter. Nevertheless, the spectral temperature dependence presented in Figs. 8 and 11 corresponds to broadband changes (as above) of $0.08 \% /{ }^{\circ} \mathrm{C}, 0.06 \% /{ }^{\circ} \mathrm{C}$, and $0.10 \% /{ }^{\circ} \mathrm{C}$, respectively. The largest discrepancy is for Photometer 3. Ref. [8] gives an independent measurement of $0.12 \% /{ }^{\circ} \mathrm{C}$ for a similar photometer.

Pertinent aspects of the photometers are summarized in Table 1. As explained in Ref. [25], the higher the shunt resistance of the photodiode, the better can be the signal-to-noise ratio of the circuit. A limiting photocurrent noise $\approx 1 \mathrm{fA}$ in Photometers 1 and 2 corresponds to a sensitivity limit $\approx 10^{-7} \mathrm{~lx}$. Besides the spectral correction factor $F$, a traditional metric of the match of $s_{\mathrm{n}}(\lambda)$ to $V(\lambda)$ is $f_{1}^{\prime}$ [24], which is also shown in the table.

\subsection{Illuminance Uncertainty}

Following Eq. (7), the relative combined standard uncertainty, $u_{\mathrm{c}, \mathrm{r}}$, of the illuminance responsivity $I / E_{\mathrm{v}}$ of the photometers arises from the standard uncertainties of $s(555), F$, and $A$. They are summarized in Table 2. By adopting the terminology of the BIPM [36] and ISO [18], the uncertainties are categorized as Type A, meaning those that were evaluated from the statistics of repeated measurements; and Type B, meaning those that were not (such as estimates of possible systematic effects based on scientific judgment). These uncertainties are reported in relative (that is, fractional) form, as percentages, because of the way the uncertainties scale and combine in Eq. (7). 
$500 \mathrm{~nm}$
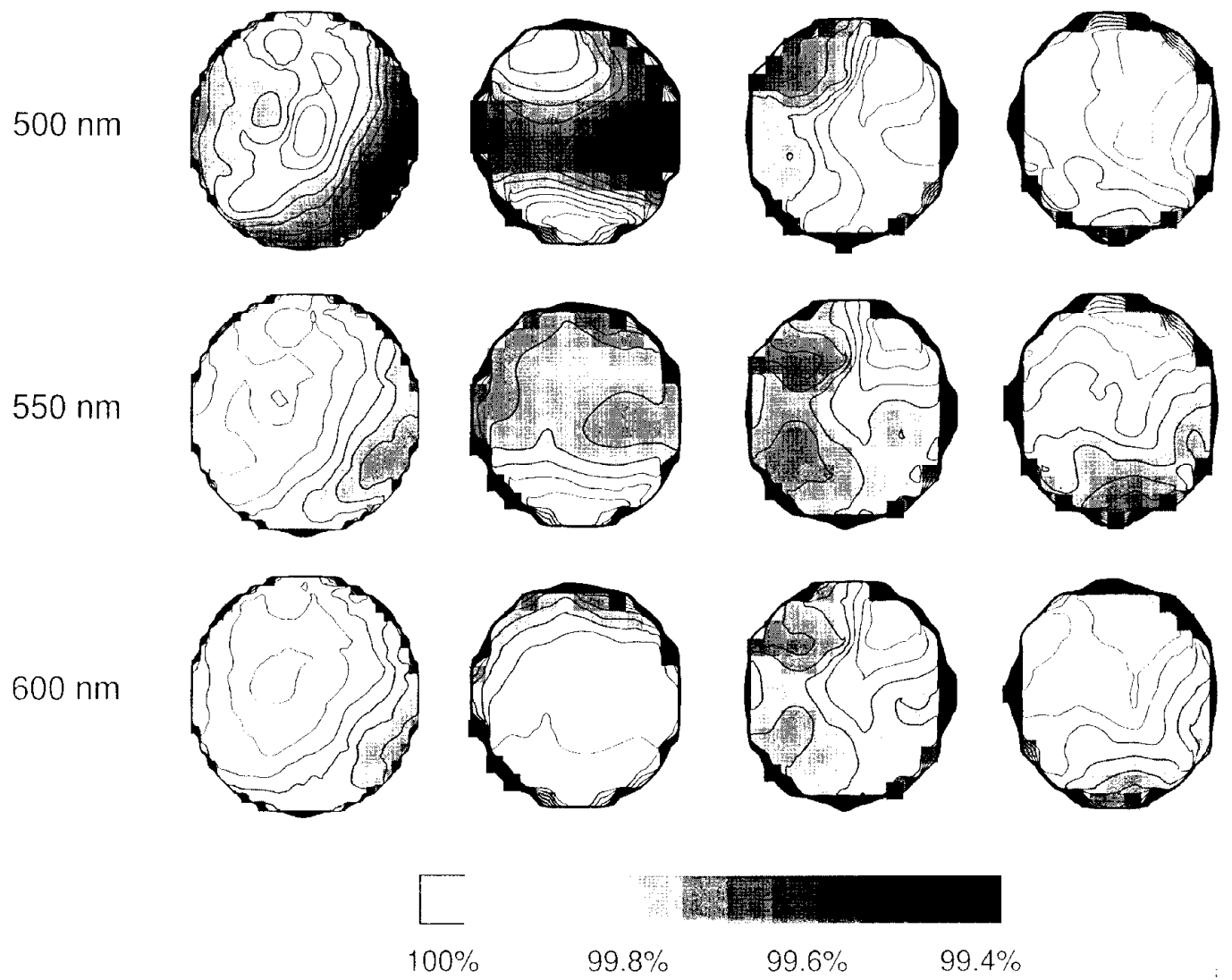

$99.6 \%$

$99.4 \%$

Fig. 13. Responsivity map of representative photometers. The responsivities (A/W) of the photometers were measured while scanning a monochromatic probe beam over the aperture area. Photometer $2 \mathrm{had}$ an aperture diameter of $7.98 \mathrm{~mm}$; the others had a diameter of $3.57 \mathrm{~mm}$. The grey scale shows the responsivity at a point, referenced to the greatest value measured $(100 \%)$. The contours indicate changes of $0.05 \%$ in responsivity.

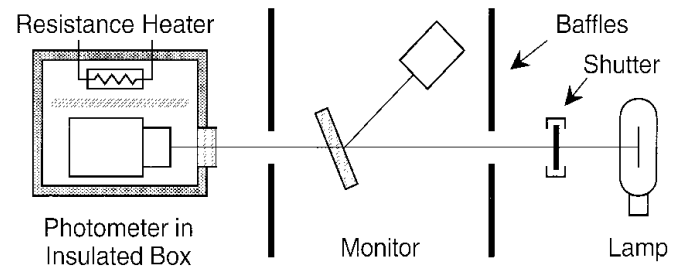

Fig. 14. Arrangement to determine the overall temperature dependence of the photometers. The photometer was allowed to reach thermal equilibrium overnight in an insulated box also containing a resistance heater. The photometric responsivity of the photometer was then measured, using a temperature-controlled detector to compensate for variations in the reference lamp.
The principal uncertainty in $s(555)$ is that of the NIST spectral responsivity scale. The currently accepted relative standard uncertainty of $0.11 \%$ [37] arises largely from the uncertainty in the absolute spectral responsivity of silicon photodiode trap detectors, with smaller additional contributions resulting from comparisons between the trap detectors and the working standards. The uncertainty that arose from random effects in comparing the photometers with the scale, obtained by averaging the standard uncertainties shown in Fig. 12c for the eight photometers, is $0.04 \%$.

Calculation of $F$ [Eq. (5)] requires knowledge or presumption of the spectral distribution of the source, $\Phi_{\mathrm{e}}(\lambda)$. Since the photometers are normally illuminated by an incandescent lamp operating with a color temper- 


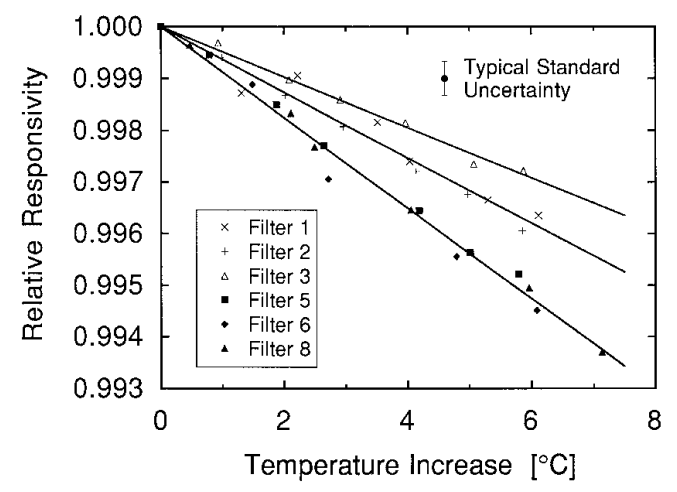

Fig. 15. Temperature dependence of photometer luminous responsivity when viewing a broadband source at $2856 \mathrm{~K}$. Photometer number (filter source): $\triangle, 3$ (NPL); $\times, 1$ (NRC); +, 2 (NRC); $\mathbf{\square}, 5$ (PRC); $\bullet$, $6(\mathrm{PRC}) ; \boldsymbol{\Delta}, 8(\mathrm{PRC})$. Linear fits include all data from each filter source.

ature of $2856 \mathrm{~K}$ (CIE Source-A), we begin by presuming Planckian distributions. Following Eq. (3), only the uncertainty of $s_{\mathrm{n}}(\lambda)$ relative to the NIST scale matters. The statistical noise of the responsivity measurements is shown in Fig. 12c. After adding their effects in quadrature, the resultant uncertainty of the $\Phi_{\mathrm{e}}(\lambda)$-weighted integral in $F$ is $0.01 \%$. (This result presumes that the possible error that is accounted for under the $0.11 \%$ spectral responsivity scale uncertainty is uniform for all wavelengths. If it varies with wavelength, the possible error in $F$ may be greater than $0.01 \%$. Nevertheless, for the purpose of analysis of the combined uncertainty in illuminance calibration, this effect is accounted for by the spectral responsivity scale uncertainty already in the budget.)

An uncertainty is also introduced from the correction polynomials, which do not pass directly through the data points in Fig. 12b, and which slightly differ in additional ways from the exact correction functions. This is, at worst, a $0.01 \%$ effect. Further, there is an uncertainty as to how well the aperture averages are computed.
Patches of area within the apertures that were near the center were covered approximately five times by the probe beam. Portions near the rim of the aperture were covered no more than once. This center-weighting would tend to bias the average if the responsivity varied radially, which Fig. 13 shows to be the case at $500 \mathrm{~nm}$ for Photometers 2 and 3. While the uncertainties due to nonuniform responsivity are difficult to quantify, given the typical magnitudes shown in Figs. 12b and 13, we estimate that the nonuniformity causes an additional $0.02 \%$ relative standard uncertainty in determining $s(\lambda)$.

When an actual lamp is used, its color temperature may be other than the desired $2856 \mathrm{~K}$ or its spectrum may be other than true Planckian. Figure 16 shows the sensitivity of $F$ to variations in blackbody temperature for the different types of filters used. For an uncertainty in the temperature of $\pm 10 \mathrm{~K}$, the uncertainty in $F$ amounts to no more than $0.02 \%$. To quantify the nonPlanckian effect, we measured the spectral irradiance of five inside-frosted lamps of the type formerly issued by NIST for luminous intensity standards. While their correlated color temperatures were $\approx 2850 \mathrm{~K}$, their distribution temperatures were within $3 \mathrm{~K}$. Equation (5) was evaluated for each photometer and for each lamp using its actual spectra, and the results were no more than $0.02 \%$ greater than when presuming a $2856 \mathrm{~K}$ blackbody.

The evaluation of $F$ does not include infrared and ultraviolet response beyond the domain of $V(\lambda)$. However, each is a potential problem. Evaluation of Eq. (2) using the spectral responsivity data of Fig. 12a shows that the infrared response $(800 \mathrm{~nm}$ to $1100 \mathrm{~nm})$ is less than $0.003 \%$ of the signal for a $2856 \mathrm{~K}$ radiator. Ultraviolet response ( $200 \mathrm{~nm}$ to $400 \mathrm{~nm}$ ) is less than $0.002 \%$.

Two experimental factors characteristic of the SCF affect the responsivity calibration through both $s(555)$ and $F$. First, the integral in Eq. (2) is dependent on the wavelength calibration of the SCF. Numerical simulation using the responsivities of the photometers (Fig. $12 \mathrm{c}$ ) and $2856 \mathrm{~K}$ blackbody sources shows that $F / s(555)$

Table 1. Summary of the photometers

\begin{tabular}{clccccc}
\hline \hline Photometer & Photodiode & $\begin{array}{c}\text { Shunt resistance } \\
(\mathrm{G} \Omega)\end{array}$ & Filter source & $\begin{array}{c}\text { Calibration } \\
(\mathrm{nA} / \mathrm{lx})\end{array}$ & $\begin{array}{c}F \\
(2856 \mathrm{~K})\end{array}$ & $\begin{array}{c}f_{1}{ }^{\prime} \\
(\%)\end{array}$ \\
\hline 1 & S1227-1010BQ & 5 & NRC & 10.116 & 1.002 & 6.00 \\
2 & S1227-1010BQ & 5.2 & NRC & 10.067 & 1.003 & 5.97 \\
3 & S1226-8BQ & 7 & NPL & 2.821 & 0.954 & 7.26 \\
4 & S1227-66BQ & 6.6 & PRC & 2.350 & 0.990 & 2.55 \\
5 & S1226-8BQ & 7 & PRC & 2.335 & 0.989 & 2.35 \\
6 & S1226-8BQ & 7 & PRC & 2.331 & 0.990 & 2.37 \\
7 & S1226-8BQ & 7 & PRC & 2.341 & 0.987 & 2.79 \\
8 & S1226-8BQ & 4.3 & PRC & 2.334 & 1.000 & 1.43 \\
\hline
\end{tabular}


Table 2. Uncertainty budget for illuminance calibration

\begin{tabular}{|c|c|c|c|}
\hline \multirow{2}{*}{\multicolumn{2}{|c|}{ Source of uncertainty }} & \multicolumn{2}{|c|}{$\begin{array}{c}\text { Relative } \\
\text { standard uncertainty }(\%)\end{array}$} \\
\hline & & \multirow[t]{2}{*}{ Type A } & \multirow[t]{2}{*}{ Type B } \\
\hline$s(555)$ & & & \\
\hline & Spectral responsivity scale & & 0.11 \\
\hline & Comparison of photometer with scale & 0.04 & \\
\hline \multicolumn{4}{|l|}{$F$} \\
\hline & Measurement scatter (noise) & 0.01 & \\
\hline & Data fitting procedure & 0.01 & \\
\hline & Residual non-uniformity within aperture & 0.02 & \\
\hline & Color temperature of lamp $( \pm 10 \mathrm{~K})$ & & 0.02 \\
\hline & Planckian approximation for lamp & & 0.02 \\
\hline & Infrared leakage & & 0.003 \\
\hline & Ultraviolet leakage and fluorescence & & 0.002 \\
\hline \multicolumn{4}{|c|}{ Correlated $s(555)$ and $F$} \\
\hline & Wavelength calibration & & 0.04 \\
\hline & Numerical aperture & & 0.05 \\
\hline \multicolumn{4}{|l|}{$A$} \\
\hline & Aperture area (as certified, small apertures) & & 0.05 \\
\hline \multicolumn{4}{|c|}{ Additional } \\
\hline & Temperature variation & & 0.03 \\
\hline & Polarization sensitivity & & 0.01 \\
\hline & Electrical current-to-voltage conversion & 0.003 & \\
\hline & Responsivity nonlinearity & & 0.001 \\
\hline & Other & & 0.12 \\
\hline \multirow{2}{*}{\multicolumn{2}{|c|}{$\begin{array}{l}\text { Combined standard uncertainty } \\
\text { Expanded uncertainty }(k=2)\end{array}$}} & \multicolumn{2}{|c|}{0.19} \\
\hline & & \multicolumn{2}{|c|}{0.39} \\
\hline
\end{tabular}

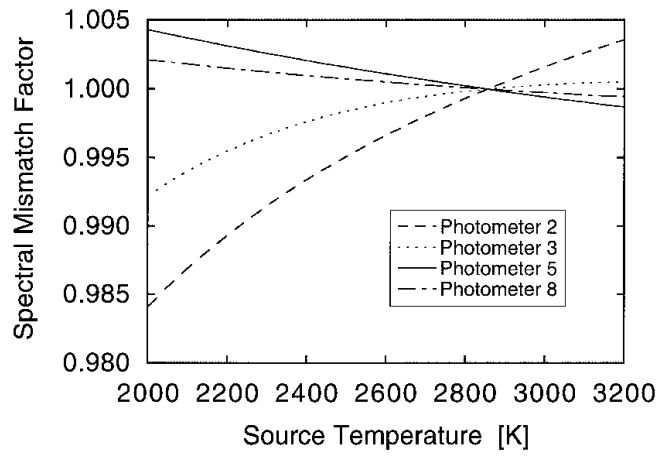

Fig. 16. Effect on photometer calibration when sources at different temperatures $T$ are viewed. The required correction is reported as $F(T) / F(2856 \mathrm{~K})$. Representative packages: Photometer 2, dashed curve; Photometer 3, dotted curve; Photometer 5, solid curve; Photometer 8 , dash-dot curve.

varies by $0.69 \% / \mathrm{nm}$ of offset. The wavelength calibration uncertainty of $0.2 \mathrm{~nm}$ leads to an uncertainty of $0.14 \%$ in the calibration of the photometer.
Second, $s(\lambda)$ measurements can be affected by the angular convergence (to a focus) of the probe spot. The optical density of the filter would appear too large when a light ray from the monochromator intersects it obliquely, giving an erroneously low value of $s(\lambda)$. While the photometer is aligned normal to the beam axis within a few milliradians by retroreflecting the alignment laser shown in Fig. 5, the lamp sources are focused using $f / 9$ optics, which have a maximal angle of incidence of $55 \mathrm{mrad}$. Presuming the sole effect of the filter is absorption, excluding front-surface reflection, the proportionately longer path length at that angle for the data in Fig. 9 would bias the integral in Eq. (2) by $0.20 \%$ (Photometers 1 and 2, the worst case). The actual bias would be less, considering the distribution of angles within the ray bundle and the reflection that was ignored. Since the bias varies as $\theta^{2}$, a uniform distribution of rays would give an overall bias of $0.10 \%$.

To mitigate these two effects and to improve accuracy, we used both the SCF and the NIST Reference Spectrophotometer [38] to measure the transmittance of the $V(\lambda)$ filters. Comparison of the data, matching peak 
position and shape, indicated that the two sources of bias on the SCF fortuitously canceled each other. The residual uncertainty in the responsivity caused by the wavelength scale is $0.04 \%$, and that caused by the SCF optics is $0.05 \%$.

The Precis 3000 aperture area measurements, for Eq. (7), are given in Table 3, and their uncertainty is included in Table 2. While these measurements were made while the apertures were detached, we also sought to confirm their behavior when they were installed in the photometers. For this, we used the SCF. Consider the output light beam from the monochromator as having a principle axis and an irradiance $B\left(x^{\prime}, y^{\prime}\right)\left(\mathrm{W} / \mathrm{m}^{2}\right)$ in a plane more-or-less perpendicular to this axis, its coordinate origin at the intersection point. The photometers were mounted on the $x-y$ carriage, in order to position the probe beam axis at point $(x, y)$ in the aperture plane. If $s(x, y)$ is the responsivity $s(\lambda)$ of the photometer at $(x, y)$ with a wavelength setting $\lambda$ of the monochromator, the total signal from the photometer

$$
I(x, y)=\iint_{-\infty}^{\infty} s\left(x+x^{\prime}, y+y^{\prime}\right) B\left(x^{\prime}, y^{\prime}\right) \mathrm{d} x^{\prime} \mathrm{d} y^{\prime} .
$$

Using the $x-y$ carriage, the probe beam can be scanned over the photometer in fine steps, and the output summed, approximating

$$
\begin{gathered}
\iint_{-\infty}^{\infty} I(x, y) \mathrm{d} x \mathrm{~d} y= \\
\iiint \int_{-\infty}^{\infty} s\left(x+x^{\prime}, y+y^{\prime}\right) B\left(x^{\prime}, y^{\prime}\right) \mathrm{d} x^{\prime} \mathrm{d} y^{\prime} \mathrm{d} x \mathrm{~d} y \\
=\iint_{-\infty}^{\infty}\left[\iint_{-\infty}^{\infty} s\left(x+x^{\prime}, y+y^{\prime}\right) \mathrm{d} x \mathrm{~d} y\right] B\left(x^{\prime}, y^{\prime}\right) \mathrm{d} x^{\prime} \mathrm{d} y^{\prime} \\
=\iint_{-\infty}^{\infty} s(x, y) \mathrm{d} x \mathrm{~d} y \iint_{-\infty}^{\infty} B\left(x^{\prime}, y^{\prime}\right) \mathrm{d} x^{\prime} \mathrm{d} y^{\prime} .
\end{gathered}
$$

(The separation follows after transforming the inner integral, $x \rightarrow x-x^{\prime}$.) The first integral on the right is the product of the aperture area and the average photometer responsivity within that area. It is the important quantity for any sort of irradiance measurement instrument, including the photometer described in Eq. (7). The second integral is just the total beam power $B(\mathrm{~W})$. Given an independent determination of the average $s(\lambda)$ within the active area of the aperture, by completely overscanning the aperture with small step size $\Delta x$ and $\Delta y$, the aperture area $A$ is given by

$$
A=\frac{\sum I(x, y) \Delta x \Delta y}{B s(\lambda)} .
$$

This fine scanning was, in fact, the exercise reported in connection with Figs. 12b and 13. Such area computations, averaged over wavelength, are also shown in Table 3. The uncertainty due to the scatter of the data of different wavelengths is shown as well.

It is clear that there is an unresolved discrepancy between the two methods. It cannot be accounted for solely by temperature variations, the residual uncertainty in the average responsivity, or the reliability of the displacement measurements $\Delta x$ and $\Delta y$. Numerical modeling indicates that a small portion of it may arise from reflections and scattering within the photometer, where the back side of the aperture traps light that would otherwise escape. The discrepancy does not cast doubt on the actual aperture areas, as the Precis 3000 measurements differed on average by only $0.01 \%$ from independent measurements made by the aperture manufacturer. Either the problem lies in this second method of determining areas, or there may be an unaccounted aspect of the photometers themselves. An additional uncertainty component of $0.12 \%$ is included in the uncertainty budget to account for this and other possible influences.

Table 3. Aperture area measurements

\begin{tabular}{ccrc}
\hline \hline $\begin{array}{c}\text { Photometer } \\
\text { number }\end{array}$ & $\begin{array}{c}\text { Precis 3000 } \\
\left(\mathrm{cm}^{2}\right)\end{array}$ & $\begin{array}{c}\text { SCF } \\
\left(\mathrm{cm}^{2}\right)\end{array}$ & $\begin{array}{c}\text { Ratio } \\
\text { SCF/Precis }\end{array}$ \\
\hline 1 & $0.500044(1 \pm 0.02 \%)$ & $0.500492(1 \pm 0.03 \%)$ & 1.0009 \\
2 & $0.499756(1 \pm 0.02 \%)$ & $0.501015(1 \pm 0.04 \%)$ & 1.0025 \\
3 & $0.099964(1 \pm 0.05 \%)$ & $0.100298(1 \pm 0.08 \%)$ & 1.0033 \\
4 & $0.100065(1 \pm 0.05 \%)$ & $0.100534(1 \pm 0.05 \%)$ & 1.0047 \\
5 & $0.100042(1 \pm 0.05 \%)$ & $0.100375(1 \pm 0.02 \%)$ & 1.0033 \\
6 & $0.099969(1 \pm 0.05 \%)$ & $0.100345(1 \pm 0.05 \%)$ & 1.0038 \\
7 & $0.100065(1 \pm 0.05 \%)$ & $0.100399(1 \pm 0.06 \%)$ & 1.0033 \\
8 & $0.099857(1 \pm 0.05 \%)$ & $0.100206(1 \pm 0.06 \%)$ & 1.0035 \\
& & Average $(3$ to 8$)$ & 1.0037 \\
& & &
\end{tabular}


Additional small uncertainties arise from the method of temperature-correcting the photometers $(0.03 \%)$, from potential polarization selectivity of the photometers $(0.01 \%)$, and from the electrical calibration of the amplifier $(0.003 \%)$. There is also an uncertainty in the calibration due to a potential nonlinear response of the photometers, that is, whether the output voltage remains proportional to the illuminance for disparate values of the same. We presume that the answer is spectrally independent, or at least insensitive to the color temperature of an incandescent lamp that is attenuated by "neutral" density filters. Figure 17 shows the results of a linearity test on a typical photometer using the beam conjoiner method previously described [39]. During calibration, the photocurrent peak (at $555 \mathrm{~nm}$ ) is typically $10^{-6}$ to $10^{-7} \mathrm{~A}$. Clearly, nonlinearity effects contribute an error of less than $0.001 \%$.

\section{Realization of the Candela 4.1 Photometry Bench}

The application of a photometer, measuring illuminance, to the luminous intensity determination of a light source [Eq. (8)] is facilitated by the optical bench shown in Fig. 18. The base consists of three $1.8 \mathrm{~m}(6 \mathrm{ft})$ long, $46 \mathrm{~cm}$ (18 in) thick, steel optical tables with a regular array of tapped holes. Upon it, rigid telescope mounts and upright, marked fiducial plates define the reference axes. The longitudinal axis runs parallel to rails upon which a carriage glides, holding a photometer. A support with cross hairs is substituted for the photometer to align the carriage and rails; lateral alignment within \pm 2 $\mathrm{mm}$ is achieved at the end opposite the telescope. By substituting a flat mirror for the photometer and by viewing the telescope in itself, orthogonality is ensured to within $5 \mathrm{mrad}$. A lamp being measured is mounted on another carriage, which permits it to be placed at the intersection of the reference axes. With a side-viewing telescope, the lamp filament is aligned to the plane defined in combination with the vertical fiducial mark. (When frosted lamps are measured, such as the type previously issued by NIST as luminous intensity standards, a model is aligned rather than the lamp itself. The model contains additional fiducial marks both to set the filament plane and to locate the filament within that plane [7].)

The lamp is powered by a constant-current source, which is set under computer control with a resolution of $0.15 \mathrm{~mA}$. The current is independently monitored across an air-cooled, Leeds \& Northrup 4360, $0.1 \Omega$ precision shunt resistor [40], which is calibrated at NIST under operating conditions with a standard uncertainty of $0.002 \%$. The proper operating current for the color temperature of interest is determined by repeated measurements using a diode-array-type spectroradiometer. Additionally, the computer monitors the lamp voltage and the photometer signal and temperature, and it operates the shutter under programmed control.

The apparatus in Fig. 18 is covered by a plastic box lined with black velvet. Surfaces within the box, to the maximum extent possible, are either painted black or covered with black cloth. A baffled chimney above the lamp permits convective cooling without introducing

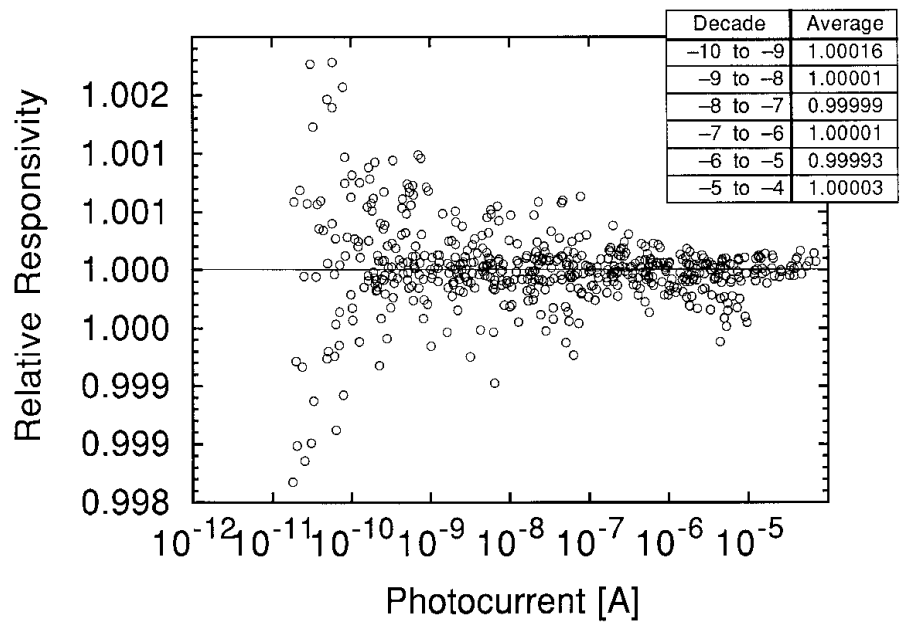

Fig. 17. Relative responsivities of Photometer 2 as measured with the beam conjoiner at various input powers. 


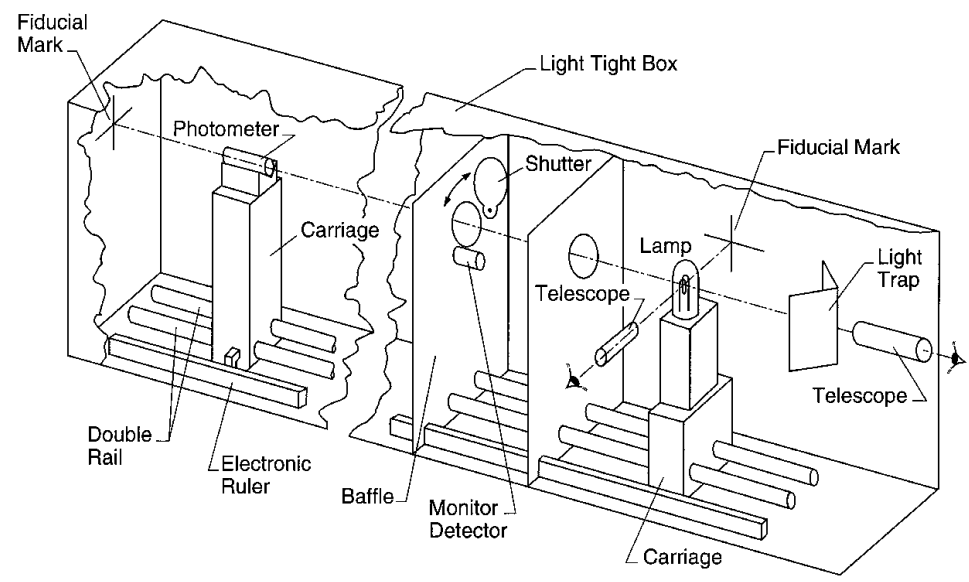

Fig. 18. New NIST photometry bench.

stray light. A light trap is interposed in front of the longitudinal telescope during operation to minimize the light that is reflected back at the photometer. (The side telescope is blocked by black cloth.)

To estimate the magnitude of stray light resulting from reflections and scattering, an additional photometer was used concurrently during testing and evaluation. It was placed outside the area illuminated through the baffles, but near, and oriented in the same general manner as, the photometer being used for measurement. With various arrangements, the stray light was consistently $<0.03 \%$ of the signal. To estimate the stray light originating near the lamp, we covered the side of the lamp towards the photometer. This signal was $<0.001 \%$ of the original. The box attenuated the ambient light from the laboratory by a factor on the order of $10^{6}$.

\subsection{Lamp-to-Photometer Distance}

The position of the photometer carriage is monitored by a computer-readable, absolute linear encoder with a resolution of $0.013 \mathrm{~mm}$. The distance $r$ between the photometer and the transverse reference axis, and a lamp filament, is fixed by sliding an attachment on the photometer carriage into the view of the telescope so that the zero position can be noted. The accuracy of the encoder was checked with a $2.75 \mathrm{~m}(9 \mathrm{ft})$ vernier caliper by moving the photometer carriage to various positions and measuring its distance mechanically from the telescope mount as well as electronically. These repeated measurements had a consistency between the methods of $0.18 \mathrm{~mm}$, which we take to be the uncertainty in determining the distance. In actuality most of this scatter was associated with the use of the large caliper, and it will not affect photometric measurements. A standard uncertainty of $0.18 \mathrm{~mm}$ in separation corresponds to a relative standard uncertainty in luminous intensity of $0.01 \%$ when the photometer is $3.6 \mathrm{~m}$ from the lamp at the far end of the bench.

More significantly, a lamp is not the point source envisioned in Fig. 2c. The size of the radiating volume requires that $I_{\mathrm{v}}$ in Eq. (8) be taken as the asymptotic value at large $r$. Typical inside-frosted lamps calibrated at NIST are tubular with a radius of $5 \mathrm{~cm}$ and extend 10 $\mathrm{cm}$ below the center of the filament, which is $5 \mathrm{~cm}$ below the top of the lamp. Less important is the transverse extent of the radiating and scattering surfaces, away from the longitudinal axis. At a distance of $2 \mathrm{~m}$ to the photometer, a lateral displacement of $10 \mathrm{~cm}$ by a point source would decrease its reading by only $0.38 \%$ $(0.25 \%$ because of the increased distance and $0.13 \%$ because of the increased angle of incidence). In comparison, a $5 \mathrm{~cm}$ longitudinal displacement of a point source would affect the reading by $5 \%$. Clearly the model is most sensitive to the longitudinal location of the origin of the light.

For this study, the automation afforded by computerized instrumentation and data analysis permitted us to make rapid measurements with the photometer at many distances from the lamp. In this way, an effective origin of the light was found as the best-fit offset $r_{\mathrm{o}}$ in the expression

$$
E_{\mathrm{v}}=\frac{I_{\mathrm{v}}}{\left(r-r_{\mathrm{o}}\right)^{2}},
$$

given the measured illuminance $E_{\mathrm{v}}$ as a function of $r$. (Similarly, the best-fit luminous intensity $I_{\mathrm{v}}$ can be derived.) 
Five inside-frosted lamps were measured in this fashion, each with two randomly chosen photometers. The intensity of the lamp was monitored during these measurements by a stationary, unfiltered, temperature-controlled silicon photodiode. It was exposed to the lamp through a fiber-optic cable, the other end of which was mounted on the second baffle where shown in Fig. 18. The photodiode assembly itself was shadowed from direct radiation from the lamp. This data was used to compensate the output of the moving photometer for variations in the lamp intensity. Equation (12) was best fit by including only data taken with $r$ between $270 \mathrm{~cm}$ and $370 \mathrm{~cm}$, the maximum of the apparatus.

Typical offsets of $0.50 \mathrm{~cm} \pm 0.15 \mathrm{~cm}$ were found for NIST inside-frosted lamps, with a systematic tendency for the offset to decrease by $\approx 0.15 \mathrm{~cm}$ after a lamp had been burning for $\approx 1 \mathrm{~h}$. This may be attributed in part to imperfect compensation by the monitor if the spectral distribution of the lamp was changing, particularly in the infrared. Surprisingly, similar offsets of $0.3 \mathrm{~cm} \pm$ $0.2 \mathrm{~cm}$ were found in a set of five, unfrosted Osram WI $41 / \mathrm{G}$ lamps. However, part of this $(<0.2 \mathrm{~cm})$ can be attributed to the shape and thickness of the glass envelope, which, acting as a diverging lens, displaces the apparent position of the filament.

The uncertainty of $r$ in Eq. (8) is dependent both on the physical measurement of distance and on the applicability of the model Eq. (8) represents, that is, on how one wishes to treat the issue of the effective origin of the light. To ignore it means including a potential systematic error in $r$; to measure it means using up precious hours of a standard lamp's life. For the purpose of defining the new NIST scale of luminous intensity, we presume that the offset is determined and applied, either for the lamp being measured or from a collection of lamps of similar construction. The relative combined standard uncertainty of $r, u_{\mathrm{c}, \mathrm{r}}(r)$, is then dominated by the uncertainty in the offset distance, typically $0.11 \mathrm{~cm}$ in our measurements. At $r=3.7 \mathrm{~m}$, the corresponding relative uncertainty in luminous intensity is $0.06 \%$.

\subsection{Self-Consistency of Photometer Group}

The calibration errors due to random causes can be established for the photometers by measuring the same luminous intensities with all of them, under the same conditions. This was done with a group of five insidefrosted standard lamps, and the results are shown in Table 4. Some photometers gave results consistently above or below the group average for every lamp. This is because what were random effects during calibration become "frozen", into the responsivity assignment for each photometer. However, we can average out this variation by applying correction factors to the original calibrations in order to bring the set of calibrations into self-consistency. Such correction factors are given in the table.

The correction factors are calculated by modeling each entry in Table 4 as the product of a true luminous intensity for the lamp in that column (five unknowns) and a correction factor for the true photometer responsivity in that row (eight unknowns). These 13 values are derived by data fitting; the full procedure will be published separately. In effect, each photometer calibration is compared with the average of them all, and each is slightly adjusted such that the adjusted values do not bias the group average. Strictly, the normalization condition for the correction factors is that their product must be 1 . The results show that the random effects that arose during the calibration of the photometer responsivities affected the calibrations, on average, by $0.15 \%$. The residuals after the data fit show that the random error in making each luminous intensity measurements for the table had a relative standard deviation of $0.02 \%$.

Table 4. Self-consistency check of photometer group. The luminous intensity (cd) of five lamps are determined with the eight photometers built to realize the scale. Each value was measured three times; the typical scatter was $0.02 \%$ of the mean. The experimental standard deviations of the eight measurements of the lamps, with the different photometers, are given at the bottom. The correction coefficient is explained in the text.

\begin{tabular}{ccccccc}
\hline \hline \multirow{2}{*}{ Photometer } & \multicolumn{7}{c}{ Lamp identification number } \\
& 4975 & 4976 & 4977 & 4978 & 4979 & Correction coefficient \\
\hline 1 & 705.94 & 707.29 & 680.34 & 708.69 & 708.67 & 0.9980 \\
2 & 706.56 & 707.53 & 680.92 & 709.28 & 709.04 & 0.9987 \\
3 & 707.60 & 709.08 & 681.70 & 710.42 & 710.48 & 1.0004 \\
4 & 708.37 & 709.74 & 682.66 & 711.02 & 711.02 & 1.0014 \\
5 & 707.25 & 708.40 & 681.27 & 709.74 & 709.99 & 0.9997 \\
6 & 708.20 & 709.78 & 682.85 & 711.11 & 710.63 & 1.0012 \\
7 & 706.32 & 707.52 & 680.39 & 708.75 & 708.94 & 0.9984 \\
8 & 708.79 & 710.31 & 683.22 & 711.46 & 711.53 & 1.0021 \\
& & & & & & $0.15 \%$ \\
\hline
\end{tabular}


The scatter in Table 4 can be reduced to $0.11 \%$ by using the aperture areas measured by the SCF found in Table 3 for Eq. (8), but this may be deceiving. Photometers 1 and 2 not only have the larger (hence better known) aperture areas, they also require the most severe uniformity corrections (Fig. 12b); this indicates a potential bias in this alternative.

The same experiment was repeated with a set of five Osram WI 41/G lamps. The correction factors were found to be the same within $0.05 \%$, except for Photometer 6 , which was different by $0.1 \%$. The residuals had a relative standard deviation of $0.06 \%$. Since the inside-frosted lamps appeared to be better behaved, we henceforth apply the correction factors in Table 4 to the calibrations in Table 1 for routine use of individual photometers. The additional consistency between the groups of two different types of lamps was most encouraging.

The result that the calibrations of a set of photometers had an actual random standard deviation of $0.15 \%$ may be compared with Table 2 . Random influences noted in the Table 2 uncertainty budget (those of Type A, and some fraction of the uncertainties in aperture area and temperature) together amount to a relative standard uncertainty $\approx 0.06 \%$. The difference is surprising, and is perhaps the result of $1 / f$ noise in one of the measurement steps. However, in the end the conclusion of Table 2 is still meaningful. The random component of each photometer after averaging (the self-consistency correction) would have a relative standard deviation of $(0.15 / \sqrt{8}) \%$, which is also $\approx 0.06 \%$. That is, the combined relative standard uncertainty in Table 2 should be taken as applicable following the self-consistency step just described.

\subsection{Uncertainty Budget for Luminous Intensity Measurements}

In Table 5 the uncertainties for luminous intensity measurements of inside-frosted lamps are summarized. The starting point is the uncertainty budget in Table 2; $u_{\mathrm{c}, \mathrm{r}}$ for the illuminance responsivity of a photometer, $0.19 \%$, carries over directly and becomes the dominant uncertainty in this budget. The measurement noise contributes $0.02 \%$, as explained in Sec. 4.3.

The photometers are operated through three cycles of exposure and darkness. Each period of exposure or darkness is $\approx 3 \mathrm{~s}$, including settling time and an integration time of $1.67 \mathrm{~s}$ for the output voltage measurement. This provides sufficient noise reduction, yet is sufficiently quick to obviate worry about heating the filter because of optical absorption, a mechanism that would not be detected by the temperature probe. While a precise model would depend on detailed knowledge about the construction of the filters, we can demonstrate an order-of-magnitude estimate. Presuming that all power dissipated from a $500 \mathrm{~W}$ lamp is radiated, at a distance $>2 \mathrm{~m}$ the irradiance is $<4 \mathrm{~mW} / \mathrm{cm}^{2}$. Taking a typical specific heat of glass to be $\approx 1700 \mathrm{~mJ} /\left(\mathrm{K} \cdot \mathrm{cm}^{3}\right)$ and an optical depth of a temperature sensitive, thermally insulated, totally adsorbing layer to be $\approx 0.1 \mathrm{~cm}$, a $3 \mathrm{~s}$

Table 5. Uncertainty budget for luminous intensity measurements

\begin{tabular}{|c|c|c|}
\hline \multirow[b]{2}{*}{ Source of uncertainty } & \multicolumn{2}{|c|}{$\begin{array}{c}\text { Relative } \\
\text { standard uncertainty }(\%)\end{array}$} \\
\hline & Type A & Type B \\
\hline \multicolumn{3}{|l|}{ Illuminance Responsivity } \\
\hline Scale uncertainty from Table 2 & & 0.19 \\
\hline Measurement noise & 0.02 & \\
\hline Filter absorption & & 0.006 \\
\hline \multicolumn{3}{|l|}{ Lamp to Photometer Distance } \\
\hline Size and construction of lamp & & 0.06 \\
\hline Physical distance measurement & 0.01 & \\
\hline \multicolumn{3}{|l|}{ Geometrical } \\
\hline Photometer transverse placement & & a \\
\hline Photometer orthogonality & & 0.002 \\
\hline \multicolumn{3}{|l|}{ Lamp Operation } \\
\hline Current regulation & & 0.03 \\
\hline Aging (per hour) & & 0.1 \\
\hline Combined standard uncertainty & \multicolumn{2}{|c|}{0.23} \\
\hline Expanded uncertainty $(k=2)$ & \multicolumn{2}{|c|}{0.46} \\
\hline
\end{tabular}

${ }^{\mathrm{a}}$ Too small to list. 
exposure would raise the temperature of this layer by $\approx 0.07 \mathrm{~K}$. These severe assumptions show that the influence of absorption on one measurement is $<0.006 \%$. While any short-term drift of the photometer cannot be attributed to absorption by the filter at these power levels, errors might arise at higher irradiances or with longer integration times. (Possible tracking errors of the thermometer in an environment with a slowly changing ambient temperature were taken into account in the Table 2 uncertainty budget.)

The uncertainties of the photometer to lamp distance, $r$ in Eq. (8), are discussed in detail in Sec. 4.2. There is a $0.06 \%$ relative standard uncertainty in luminous intensity measurements resulting from the difference between the geometric and effective position of the lamp filament. The relative standard uncertainty caused by the electronic ruler is $<0.01 \%$.

The various geometrical uncertainties make negligible contributions to the overall uncertainty. A transverse misalignment of the photometer by $\pm 2 \mathrm{~mm}$ would affect the measurement by only a few parts in $10^{7}$. A nonorthogonality to the longitudinal axis of $5 \mathrm{mrad}$ would affect the measurement by $<0.002 \%$. Clearly the geometrical prerequisites of Eq. (8) are met. The angles of incidence on the photometer from the extended source are much less than those encountered during illuminance calibration, and this would tend only to reduce the possible systematic error in numerical aperture already accounted for.

NIST originally elected to use inside-frosted lamps as luminous intensity standards because measurement results were less affected by small changes in the orientation of the lamps [41]. Variations of $<0.2 \%$ were reported for misorientations in pitch (about the vertical lamp axis) of less than $\pm 2^{\circ}$. Similarly, the fine-grained frosting aids in generating uniform illuminance in the far field, in the neighborhood of the photometer. We believe that any remaining local variations in illuminance will not contribute to possible measurement error beyond those already accounted for in connection with the spatial averaging of the responsivity of the photometers. Errors that may arise because of the differences in lamp orientation between NIST and other laboratories are beyond the scope of this paper.

At the operating point, marginal fractional changes in lamp current cause magnified fractional changes in lamp output by factors of 6 to $8[42,8]$. Since the nominal current of an inside-frosted lamp is $3 \mathrm{~A}$, the 0.15 $\mathrm{mA}$ resolution in the current control implies a luminous stability of $0.02 \%$. The $0.002 \%$ calibration relative standard uncertainty of the shunt resistor implies a reproducibility in output of $0.016 \%$. Together these imply a relative standard uncertainty component resulting from lamp current measurement of $0.03 \%$.
Before luminous intensity measurements were made, the lamp currents were ramped slowly up to the operating point, and the lamps were allowed an equilibration time of at least $10 \mathrm{~min}$. Nevertheless, it is important to remember that lamps change with age rather than reach a stable equilibrium. Figure 19a shows the behavior of three types of lamps over the course of $2 \mathrm{~h}$ of operation. The scatter in the data, or noise, was discussed in connection with Table 4. Figure $19 \mathrm{~b}$ demonstrates that the effect spans separate lamp lightings. The gaps in the data correspond to ramping and equilibration periods during which no data were taken. While Fig. 19a shows that the lamps changed most rapidly for an additional 20 min to 30 min after the initial warm-up period (as noted above in connection with the determination of $r_{\mathrm{o}}$ ), permanent changes in luminous intensity of $0.1 \% / \mathrm{h}$ contraindicate long equilibration times and are a severe limitation on a calibration service requiring lamps as transfer standards. More recently, modified FEL 1000 W quartz-halogen lamps were further tested for suitability as photometric transfer standards, and they were shown to be stable to within $0.2 \%-0.6 \%$ over $60 \mathrm{~h}$ of operation [43].

\subsection{Comparison of New and Old Scales}

Before this study the last full realization of the old luminous intensity scale (Fig. 1) occurred in 1985 in connection with the international intercomparison of such scales [44]. At that time the NBS candela was found to be $0.58 \%$ smaller than the world mean. (That is, lamps calibrated at NBS were given higher candela values than the average.) Of this, $0.35 \%$ was later removed with the adoption of ITS-90 [20], making the NIST scale $0.23 \%$ smaller than the world mean.

Encouraging early results by Andor and Zalewski in 1988 [45] showed that a detector-based candela gave results $0.07 \%$ larger than the world mean. This was determined by measuring the primary lamp group with the prototype photometers similar to those reported in this study. Based on this and other indirect evidence, in Ref. [22] we concluded that the new scale realization described in this study did not cause a significant scale shift in comparison with the uncertainty of the old scale, and that it was perhaps on the order of $0.3 \%$.

While studies continue at NIST to validate this result, additional confirming evidence has recently become available. In 1985, the luminous intensity scale of Germany maintained at the Physikalisch-Technische Bundesanstalt (PTB) was found to be $0.32 \%$ larger than the world mean [44]. A comparison of the new NIST scale with the PTB scale [46] showed that the scale difference narrowed from $0.9 \%$ in 1985 to $0.2 \%$ in 1993 . This implies that the new NIST scale is $0.12 \%$ larger than the 


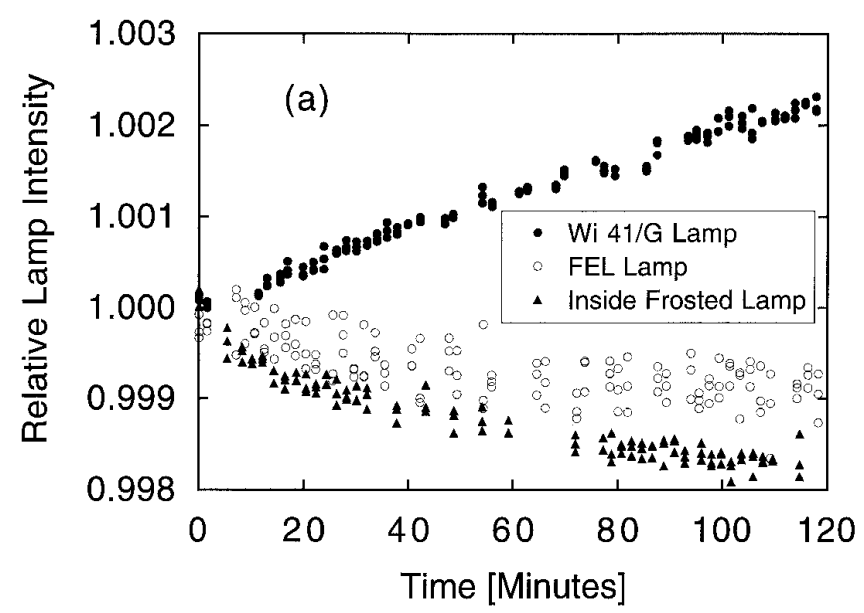

Fig. 19a. Drift and noise in the output of representative standard lamps during one lighting of an: Osram Wi 41/G lamp, O; FEL lamp, O; and Inside-frosted T-20 lamp, $\boldsymbol{\Delta}$.

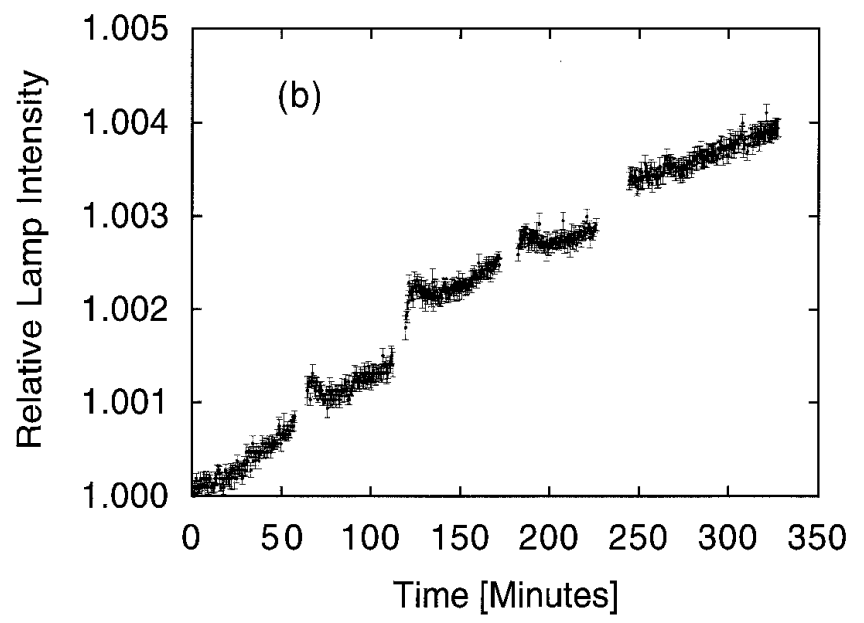

Fig. 19b. Drift and noise in the output of representative standard lamps during five consecutive lightings of the Osram lamp.

1985 world mean, a $0.35 \%$ shift from the old NIST scale with the ITS-90 correction applied. Additionally, the Országos Mérésügyi Hivatal (OMH) in Hungary has maintained a scale based on the BIPM lamp group that holds the 1985 world mean. Preliminary data from a comparison of the new NIST scale with the OMH scale implies that the NIST scale is $0.03 \%$ smaller than the world mean, a $0.2 \%$ shift from the old NIST scale. Another international intercomparison is planned for 1995 [47].

\subsection{Long-Term Stability of the Standard Photometers}

The calibration procedure described in Secs. 3.5 and 4.3 has been repeated twice to test the stability of the calibration result shown in Table 1. The results are shown in Table 6. For the purpose of comparison, the data are adjusted to correspond to a uniform temperature of $298 \mathrm{~K}$ and normalized to the calibration values in Table 1. The data shows that the group average changed by $<0.1 \%$ in their first year, and then by an additional $0.4 \%$ in the subsequent 2 years. 
Table 6. Photometer calibration stability

\begin{tabular}{ccccc}
\hline \hline & \multicolumn{3}{c}{ Relative illuminance responsivity } & \\
Photometer & Nov. 1991 & Nov. 1992 & Dec. 1994 & After cleaning \\
\hline 1 & 1.0000 & 0.9998 & 0.9939 & 1.0032 \\
2 & 1.0000 & 0.9996 & 0.9875 & 1.0056 \\
3 & 1.0000 & 0.9960 & 0.9926 & \\
4 & 1.0000 & 0.9991 & 0.9964 & \\
5 & 1.0000 & 0.9988 & 0.9976 & \\
6 & 1.0000 & 0.9999 & 0.9977 & \\
7 & 1.0000 & 1.0010 & 0.9987 & \\
8 & 1.0000 & 0.9997 & 0.9969 & \\
& & & & \\
\hline Average & 1.0000 & 0.9992 & 0.9952 & \\
\hline
\end{tabular}

One reason for this change appeared to be a surface film that had developed on the exterior side of the glass filters on Photometers 1 and 2. These filters were wiped gently with dry lens tissue, and their photometers were recalibrated. Indeed, their values shifted significantly. The average drift of Photometers 3 to 8 remained $\approx 0.11 \% / \mathrm{yr}$.

\section{Conclusion}

Two major goals have been reached. A luminous intensity scale has been derived with detectors, and in a simpler and more direct manner than before. In the process the uncertainty of lamp calibration has been reduced.

This change also puts NIST on good footing for future improvements. The principal uncertainties in the illuminance calibration, the uncertainty of the spectral responsivity scale and the uncertainty in the aperture area, will be reduced significantly by ongoing research and development in our Division. We can expect to reduce the smaller uncertainties as well by improvements in measurement technique. A $0.2 \%$ relative expanded uncertainty $(k=2)$ in illuminance measurement appears to be achievable.

Based on our experience, we believe that the detectorbased scale will prove more durable and stable than the lamp-based scale. Nevertheless, yearly recalibration of the standard photometers will be required to maintain the accuracy of the scale, and frosted FEL lamps hold promise as an improved vehicle for disseminating the scale.

This study is of particular benefit for those many applications where illuminance needs to be measured directly, including imaging (such as photography) and ergonomics, where the effects of lighting rather than the light sources themselves matter. The standard photometers have enabled NIST to expand its range of services to include the calibration of luminance meters and illuminance meters [48]. In the field, secondary-standard illuminance meters can be used to calibrate other illuminance meters by substitution, eliminating the need for a long optical bench. Further, the standard photometers have been applied to realize a detector-based geometrically total luminous flux scale for the measurement of lamps [49]. This important development brings the benefits of this study to the lighting industry, for which total luminous flux is perhaps the most important measurable quantity.

While traditional photometry has always involved standard light sources, e.g., lamps in recent decades, detector-based standardization permits smaller uncertainties and often simpler procedures. Unlike lamps the photometers require no large power supplies, and they are useful over a wide dynamic range. Photometry benches need not be long to provide for $1 / r^{2}$ attenuations. Well-characterized photometers should prove especially useful for the calibration of modern, nonincandescent light sources, including self-luminous displays. (Care needs to be taken to know the spectrum of the source.) Stable photometers also permit the incidental use of lamps during calibration procedures without regard to their long-term stability. With standards-quality lamps difficult to procure, this alternate technology merits particular attention.

\section{Acknowledgments}

Many people, staff and visitors, contributed much to this project. We thank Ronald Wilkinson for many skilled photometric measurements, Joel Fowler and Patrick Tobin for much assistance in the design and construction of the new bench, H. Sun for many filter characterizations and extensive study of the temperature dependence of the photometers, Ambler Thompson and Sally Bruce for stewardship and operation of the beam conjoiner, John Jackson for measuring the spectral irra- 
diance of lamps at FASCAL, Gyula Dézsi and Georg Sauter for hospitality and fellowship in the tasks of comparing the luminous intensity scales, Donna Bell and Jason Hoffman for able assistance in many respects, Robert Saunders for many helpful discussions, and Klaus Mielenz for support and encouragement throughout the project.

\section{References}

[1] J. W. T. Walsh, Chap. 1 in Photometry, 3rd Ed., Constable \& Company, London (1958).

[2] Comptes Rendus des Séances de la Neuvième Conférence Générale des Poids et Mesures (Bureau International des Poids et Mesures, Paris, 1948), session 9, p. 53.

[3] W. R. Blevin and B. Steiner, Redefinition of the Candela and the Lumen, Metrologia 11, 97-104 (1975).

[4] Comptes Rendus des Séances de la $16^{\mathrm{e}}$ Conférence Générale des Poids et Mesures (Bureau International des Poids et Mesures, F-92312 Sèvres, Cedex, France, 1979), session 16, p. 100; see also P. Giacomo, News from the BIPM, Metrologia 16, 55-61 (1980). (Corrected English translation: Metrologia 17, 74 (1981).)

[5] G. Wyszecki, W. R. Blevin, K. G. Kessler, and K. D. Mielenz, Principles Governing Photometry (Bureau International des Poids et Mesures, F-92312 Sèvres, Cedex, France, 1983); see also Principles Governing Photometry, Metrologia 19, 97-101 (1983).

[6] The Basis of Physical Photometry, Publ. 18.2 (Commission Internationale de L'Éclairage, Paris, 1983). (Currently available through the U. S. National Committee of the CIE, c/o Mr. Thomas M. Lemons, TLA-Lighting Consultants, Inc., 7 Pond Street, Salem, MA 01970-4819.)

[7] R. L. Booker and D. A. McSparron, Photometric Calibrations, Natl. Bur. Stand. (U.S.), Spec. Publ. 250-15 (1987).

[8] T. M. Goodman and P. J. Key, A radiometric realization of the candela, NPL Report QU 75 (National Physical Laboratory, Teddington, UK, February 1986); T. M. Goodman and P. J. Key, The NPL Radiometric Realization of the Candela, Metrologia 25, 29-40 (1988).

[9] L. P. Boivin, A. A. Gaertner, and D. S. Gignac, Realization of the New Candela (1979) at NRC, Metrologia 24, 139-152 (1987).

[10] C. Carreras and A. Corrons, Absolute spectroradiometric and photometric scales based on an electrically calibrated pyroelectric radiometer, Appl. Opt. 20, 1174-1177 (1981).

[11] Z. Gao, Z. Wang, D. Piao, S. Mao, and C. Yang, Realization of the Candela by Electrically Calibrated Radiometers, Metrologia 19, 85-92 (1983).

[12] J. L. Gardner, Recent International Intercomparison of Basic Lighting Standards, Lighting in Australia 7(4), 21-24 (1987).

[13] V. I. Sapritski, A New Standard for the Candela in the USSR, Metrologia 24, 53-59 (1987); V. I. Sapritskii, National Primary Radiometric Standards of the USSR, Metrologia 27, 53-60 (1990).

[14] V. Jediny, J. Krempasky, J. Zatkovic, and P. Nemecek, Luminous intensity measurement according to the new definition of the candela, Cesk. Cas. Fyz. A 38(6), 601-611 (1988).

[15] G. Eppeldauer, Longterm changes of silicon photodiodes and their use for photometric standardization, Appl. Opt. 29, 22892294 (1990).
[16] J. H. Walker, R. D. Saunders, and A. T. Hattenburg, The NBS Scale of Spectral Radiance, Metrologia 24, 79-88 (1987); J. H. Walker, R. D. Saunders, and A. T. Hattenburg, Spectral Radiance Calibrations, Natl. Bur. Stand. (U.S.), Spec. Publ. 250-1 (1987).

[17] J. H. Walker, R. D. Saunders, J. K. Jackson, and D. A. McSparron, Spectral Irradiance Calibrations, Natl. Bur. Stand. (U.S.), Spec. Publ. 250-1 (1987).

[18] The present study follows, to the extent possible, the ISO Guide to the Expression of Uncertainty in Measurement, International Organization for Standardization, Geneva, Switzerland (1993). Since 1994, NIST policy has been to conform to the Guide in reporting its activities, using an expanded uncertainty coverage factor (as defined in the Guide) of $k=2$. Prior work at NIST was generally reported with " $3 \sigma$ " uncertainties. For consistency in this paper, when a standard uncertainty of the present study is compared with an earlier result, the latter is restated to a " $1 \sigma$ ", basis. Earlier results are restated to a " $2 \sigma$ " basis when the context calls for an expanded uncertainty; see also B. N. Taylor and C. E. Kuyatt, Guidelines for Evaluating and Expressing the Uncertainty of NIST Measurement Results, Natl. Inst. Stand. Technol. Note 1297, 2nd ed. (1994).

[19] H. Preston-Thomas, The International Temperature Scale of 1990 (ITS-90), Metrologia 27, 3-10 (1990). (Erratum, ibid. p. 107.)

[20] K. D. Mielenz, R. D. Saunders, A. C. Parr, and J. J. Hsia, The 1990 NIST Scales of Thermal Radiometry, J. Res. Natl. Inst. Stand. Technol. 95, 621-629 (1990).

[21] K. D. Mielenz, R. D. Saunders, and J. B. Shumaker, Spectroradiometric Determination of the Freezing Temperature of Gold, J. Res. Natl. Inst. Stand. Technol. 95, 49-67 (1990).

[22] C. L. Cromer, G. Eppeldauer, J. E. Hardis, T. C. Larason, and A. C. Parr, National Institute of Standards and Technology Detector-Based Photometric Scale, Appl. Opt. 32(16), 2936-2948 (1993).

[23] Y. Ohno, C. L. Cromer, J. E. Hardis, and G. Eppeldauer, The Detector-Based Candela Scale and Related Photometric Calibration Procedures at NIST, J. Illum. Eng. Soc. 23(1), 89-98 (1994).

[24] Methods of Characterizing the Performance of Radiometers and Photometers, Publ. 53, Commission Internationale de L'Éclairage, Paris (1982). (See Ref. [6] for availability.)

[25] G. Eppeldauer and J. E. Hardis, Fourteen-decade photocurrent measurements with large-area silicon photodiodes at room temperature, Appl. Opt. 30, 3091-3099 (1991).

[26] G. Eppeldauer, Temperature Monitored/Controlled Silicon Photodiodes for Standardization, in Surveillance Technologies, S. Gowrinathan, R. J. Mataloni, and S. J. Schwartz, eds., Proc. Soc. Photo-Opt. Instrum. Eng. 1479, 71-77 (1991).

[27] E. F. Zalewski and C. R. Duda, Silicon photodiode device with $100 \%$ external quantum efficiency, Appl. Opt. 22, 2867-2873 (1983).

[28] J. M. Houston, C. L. Cromer, J. E. Hardis, and T. C. Larason, Comparison of the NIST High Accuracy Cryogenic Radiometer and the NIST Scale of Detector Spectral Response, Metrologia 30, 285-290 (1993). T. C. Larason, S. S. Bruce, and C. L. Cromer, The NIST High Accuracy Scale for Absolute Spectral Response from $406 \mathrm{~nm}$ to $920 \mathrm{~nm}$, J. Res. Natl. Inst. Stand. Technol. 101, 133 (1996).

[29] The characteristics of the same model of monochromator are described in R. D. Saunders and J. B. Shumaker, Apparatus function of a prism-grating double monochromator, Appl. Opt. 25, 3710-3714 (1986).

[30] Hamamatsu Corporation, 360 Foothill Road, P. O. Box 6910, Bridgewater, NJ 08807-0910.

[31] Mathematica software is a product of Wolfram Research, 100 Trade Center Dr., Champaign, IL 61820-7237. 
[32] The authors extend special thanks to Phil Boivin, National Research Council of Canada, and David Nettleton, National Physical Laboratory of Great Britain, for kindness and generosity in supplying some of the filters used in this project. We also thank G. Czibula, PRC Krochmann (Geneststrasse, 6, D-1000 Berlin 62 , Germany), for cooperation and assistance in developing the additional filters.

[33] G. Czibula, Producing a Detector with Predetermined Spectral Responsivity, presented at the International Measurement Confederation 10th International Symposium of the Technical Committee on Photon-Detectors, 20-22 Sept. 1982, Berlin (OMIKK Technionform, Budapest, 1983) pp. 189-199.

[34] T. M. Goodman, J. E. Martin, B. D. Shipp, and N. P. Turner, The Manufacture and Measurement of Precision Apertures, in Proceedings of the Second International Conference on New Developments and Applications in Optical Radiometry, M. P. Fox and D. H. Nettleton, eds., Vol. 92 of Institute of Physics Conference Series, Institute of Physics, Bristol, UK (1989) pp. 121-128.

[35] View Engineering, 1650 N. Voyager Ave., Simi Valley, CA 93063-3385.

[36] P. Giacomo, News from the BIPM, Metrologia 17, 69-74 (1981); see also H. H. Ku, Uncertainty and Accuracy in Physical Measurements, Natl. Inst. Stand. Technol. Spec. Publ. 805 (1990).

[37] C. L. Cromer, A New Spectral Response Calibration Method using a Silicon Photodiode Trap Detector, presented at the 1991 Measurement Science Conference.

[38] K. L. Eckerle, J. J. Hsia, K. D. Mielenz, and V. R. Weidner, Regular Spectral Transmittance, Natl. Bur. Stand. (U.S.), Spec. Publ. 250-6 (1987).

[39] R. D. Saunders and J. B. Shumaker, Automated radiometric linearity tester, Appl. Opt. 23, 3504-3506 (1984).

[40] Leeds \& Northrup, 351 N. Sumneytown Pike, North Wales, PA 19454-2531.

[41] E. F. Zalewski, A. R. Schaefer, K. Mohan, and D. A. McSparron, Optical Radiation Measurements: Photometric Instrumentation and Research (1970 to 1971), Natl. Bur. Stand. (U.S.), Tech. Note 594-2 (1972) pp. 22-33.

[42] Incandescent Lamps, Publ. TP-110, General Electric Company, Nela Park, Cleveland, OH (1964).

[43] Y. Ohno and J. K. Jackson, Characteristics of Modified FEL Quartz-Halogen Lamps for Photometric Standards, Metrologia (in press).

[44] J. Bonhoure, Metrologia 24, 157-162 (1987); see also Rapport de la $11^{\mathrm{e}}$ Session, Comité Consultatif de Photométrie et Radiométrie, Bureau International des Poids et Mesures, F-92312 Sèvres Cedex, France (1986).

[45] G. Andor and E. F. Zalewski, (personal communication).

[46] Y. Ohno and G. Sauter, 1993 Intercomparison of Photometric Units Maintained at NIST (USA) and PTB (Germany), J. Res. Natl. Inst. Stand. Technol. 100, 227-239 (1995).

[47] Rapport de la $12^{\text {e }}$ Session, Comité Consultatif de Photométrie et Radiométrie, Bureau International des Poids et Mesures, F-92312 Sèvres Cedex, France (1990).

[48] NIST Calibration Services Users Guide 1995, Natl. Inst. Stand. Technol. Spec. Publ. 250 (1995).

[49] Y. Ohno, Realization of NIST 1995 Luminous Flux Scale using Integrating Sphere Method, J. Illum. Eng. Soc. 25(1), 13-22 (1996).

\begin{abstract}
About the authors: Albert Parr serves as Chief of the Optical Technology Division of the NIST Physics Laboratory. Christopher Cromer manages the Optical Sensor Group in the Optical Technology Division. Yoshihiro Ohno is the project leader in the Optical Technology Division for photometry and provides luminous intensity and other photometric calibrations. G. Eppeldauer, J. E. Hardis, and T. C. Larason are scientists in the Optical Technology Division. The National Institute of Standards and Technology is an agency of the Technology Administration, U. S. Department of Commerce.
\end{abstract}




\section{Spectral Response Based Calibration Method of Tristimulus Colorimeters}

\section{George Eppeldauer}

National Institute of Standards and Technology, Gaithersburg, MD 20899-0001

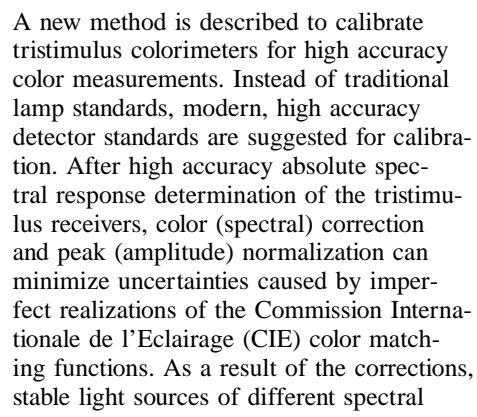

A new method is described to calibrate tristimulus colorimeters for high accuracy color measurements. Instead of traditional lamp standards, modern, high accuracy detector standards are suggested for calibration. After high accuracy absolute spectral response determination of the tristimulus receivers, color (spectral) correction and peak (amplitude) normalization can minimize uncertainties caused by imperfect realizations of the Commission Internationale de l'Eclairage (CIE) color matching functions. As a result of the corrections, stable light sources of different spectral

power distributions can be measured with an accuracy dominated by the sub tenths of a percent uncertainty of novel spectral response determinations.

Key words: chromaticity coordinates; color calibration; color temperature; colorimetry; detector standard; photometry; spectral response; tristimulus values.

Accepted: August 19, 1998

Available online: http://www.nist.gov/jres

\section{Introduction}

Tristimulus colorimetry is based on light measurement using three or more receivers with spectral responsivities matched to the Commission Internationale de l'Eclairage (CIE) $\bar{x}(\lambda), \bar{y}(\lambda)$, and $\bar{z}(\lambda)$ color matching functions [1]. To achieve accurate measurements for a large variety of light sources, the spectral matches should be as close as possible to the color matching functions. The receivers are usually realized with silicon photodiodes and attached filter packages [2]. Usually, the spectral mismatch between the realized and the color matching functions give the dominant uncertainties in tristimulus color measurements.

At present, tristimulus colorimeters are calibrated with standard lamps. The calibration of the most frequently used color temperature standard lamps is derived from source-based spectral irradiance scales. NIST reported a $0.67 \%$ relative expanded uncertainty $(k=2)^{1}$ for the disseminated spectral irradiance standard lamps in the visible range and a relative $0.59 \%$ long-term reproducibility [3]. Research is being conducted at NIST to decrease the $0.67 \%$ uncertainty by a factor of three still using standard lamps [4]. The accuracy of the standard lamp influences the photometric [receiver matched to $\bar{y}(\lambda)$ ] accuracy of the tristimulus colorimeter. The wavelength dependent (e.g., burning time caused) changes of the standard lamp influence the colorimetric accuracy of the tristimulus meter.

Several commercially available colorimeters were compared for accuracy by measuring nine different laser lines (saturated colors) by Berns et al. [6]. The differences between the theoretical and the measured chromaticity coordinates were reported. The lowest rms

\footnotetext{
${ }^{1}$ Throughout this paper uncertainties are given as relative expanded uncertainties with a coverage factor $k=2$ unless otherwise stated [5].
} 
errors in $x$ and $y(0.0042$ and 0.0051 , respectively) were observed on a tristimulus colorimeter employing partial (mosaic) filters. The errors are expected to decrease to a large extent for white light sources, such as tungsten lamps. The reported errors were dominated by spectral response deviations (mismatch) of the receivers relative to the CIE recommended color matching functions. The uncertainties of the calibrating standard lamps also contributed to the results.

In contrast to source standards, primary standard detectors (cryogenic radiometers) can measure optical (radiant) power with an uncertainty of $10^{-4}[7,8]$. Certain type silicon detectors, in a light-trap arrangement, can be calibrated against primary standard radiometers using several intensity stabilized lasers [9]. With interpolation between the laser lines, a $0.06 \%$ uncertainty in spectral responsivity was reported on light-trap Si detectors between $406 \mathrm{~nm}$ and $920 \mathrm{~nm}$ [10]. This uncertainty is about one fourth of the uncertainties reported for traditional monochromator based detector spectral response measurements [11].

Achievable total uncertainty of spectral transmittance measurements on high quality color filters is reported to be $2 \times 10^{-4}$ [12].

A narrow-band filter-radiometer was calibrated for spectral irradiance response in the visible region against a trap detector with an uncertainty of $0.07 \%$ [13] for measured irradiance. In that report, the dominant uncertainty components were the uncertainties of the trap detector response, $0.036 \%$, the area of the trap aperture, $0.034 \%$, and the wavelength reproducibility, $0.026 \%$.

If the uncertainties of the receiver response measurements are very small, the spatial response non-uniformities of the filter-detector packages could limit the accuracy of the tristimulus measurements. This problem can be avoided if apertures are used in front of the filter-detector packages and the apertures are overfilled with the uniform field of the (point) source to be measured. The area of the apertures can be measured with an uncertainty of $0.026 \%$ [14].

The motivation behind the development of a detectorbased calibration method for tristimulus colorimeters was to utilize the significantly lower uncertainty of new detector standards compared with traditional lamp standards. The goal of the method described in this paper is to determine broad-band calibration factors for all receivers in a tristimulus colorimeter to minimize measurement uncertainty. If the spectral response determination of the receivers is accurate, application of the calibration factors for different source distributions will result in high colorimetric accuracy. This multiple receiver method is an extension of the single receiver spectral mismatch correction used in our detector-based illuminance scale realization $[15,16]$.

\section{Theoretical Basis}

In order to determine $x, y$ chromaticity coordinates of a light source, the CIE tristimulus values of the source are to be obtained by

$$
\begin{gathered}
X=k \int_{\lambda} S(\lambda) \bar{x}(\lambda) \mathrm{d} \lambda \\
Y=k \int_{\lambda} S(\lambda) \bar{y}(\lambda) \mathrm{d} \lambda \\
Z=k \int_{\lambda} S(\lambda) \bar{z}(\lambda) \mathrm{d} \lambda,
\end{gathered}
$$

where $S(\lambda)$ is the spectral power distribution of the source to be measured; $\bar{x}(\lambda), \bar{y}(\lambda)$, and $\bar{z}(\lambda)$ are the 1931 CIE color-matching functions; and $k$ is a normalization factor. In practice, $\bar{x}(\lambda)$ is realized by two receivers, $\bar{x}_{\mathrm{L}}(\lambda)$ and $\bar{x}_{\mathrm{S}}(\lambda)$ :

$\bar{x}_{\mathrm{S}}(\lambda)=0$ and $\bar{x}_{\mathrm{L}}(\lambda)=\bar{x}(\lambda)$ if the wavelength is longer than $504 \mathrm{~nm}$, and

$\bar{x}_{\mathrm{L}}(\lambda)=0$ and $\bar{x}_{\mathrm{S}}(\lambda)=\bar{x}(\lambda)$ if the wavelength is shorter than $504 \mathrm{~nm}$.

(Note that the subscripts L and S mean long and short, respectively.)

The CIE tristimulus value $X$ can be written as:

$$
X=X_{1}+X_{2},
$$

where

$$
\begin{aligned}
& X_{1}=k \int_{\lambda} S(\lambda) \bar{x}_{\mathrm{L}}(\lambda) \mathrm{d} \lambda, \text { and } \\
& X_{2}=k \int_{\lambda} S(\lambda) \bar{x}_{\mathrm{S}}(\lambda) \mathrm{d} \lambda .
\end{aligned}
$$

$Y$ in Eq. (1) will give an absolute photometric quantity (e.g., in lux) [15] if

$$
k=K_{\mathrm{m}}=683 \mathrm{~lm} / \mathrm{W} .
$$

The measured photodiode output currents of the four separate receivers are

$$
\begin{gathered}
I_{X 1}=\int_{\lambda} S(\lambda) s_{X \mathrm{~L}}(\lambda) \mathrm{d} \lambda \\
I_{X 2}=\int_{\lambda} S(\lambda) s_{X S}(\lambda) \mathrm{d} \lambda \\
I_{Y}=\int_{\lambda} S(\lambda) s_{Y}(\lambda) \mathrm{d} \lambda \\
I_{Z}=\int_{\lambda} S(\lambda) s_{Z}(\lambda) \mathrm{d} \lambda .
\end{gathered}
$$

where $s_{X \mathrm{~L}}(\lambda), s_{X S}(\lambda), s_{Y}(\lambda)$, and $s_{Z}(\lambda)$ are the absolute spectral responsivities of the receivers.

When measuring a light source of known spectral power distribution $S(\lambda)$, the receiver calibration factors can be determined from the ratio of Eq. (1) to Eq. (4): 


$$
\begin{gathered}
k_{X 1}=\frac{X_{1}}{I_{X 1}}=\frac{K_{\mathrm{m}} \int_{\lambda} S(\lambda) \bar{x}_{\mathrm{L}}(\lambda) \mathrm{d} \lambda}{\int_{\lambda} S(\lambda) s_{X \mathrm{~L}}(\lambda) \mathrm{d} \lambda} \\
k_{X 2}=\frac{X_{2}}{I_{X 2}}=\frac{K_{\mathrm{m}} \int_{\lambda} S(\lambda) \bar{x}_{\mathrm{S}}(\lambda) \mathrm{d} \lambda}{\int_{\lambda} S(\lambda) s_{X \mathrm{~S}}(\lambda) \mathrm{d} \lambda} \\
k_{Y}=\frac{Y}{I_{Y}}=\frac{K_{\mathrm{m}} \int_{\lambda} S(\lambda) \bar{y}(\lambda) \mathrm{d} \lambda}{\int_{\lambda} S(\lambda) s_{Y}(\lambda) \mathrm{d} \lambda} \\
k_{Z}=\frac{Z}{I_{Z}}=\frac{K_{\mathrm{m}} \int_{\lambda} S(\lambda) \bar{z}(\lambda) \mathrm{d} \lambda}{\int_{\lambda} S(\lambda) s_{Z}(\lambda) \mathrm{d} \lambda} .
\end{gathered}
$$

By normalizing the color matching functions to their peak values, the receiver calibration factors can be written as:

$$
\begin{aligned}
k_{X 1} & =\frac{1.06291 K_{\mathrm{m}} F_{X 1}}{s_{X \mathrm{~L}}(599)} \\
k_{X 2} & =\frac{0.3501 K_{\mathrm{m}} F_{X 2}}{s_{X \mathrm{~S}}(442)} \\
k_{Y} & =\frac{K_{\mathrm{m}} F_{Y}}{s_{Y}(555)} \\
k_{Z} & =\frac{1.78297 K_{\mathrm{m}} F_{Z}}{s_{Z}(446)}
\end{aligned}
$$

by introducing the color correction factors:

$$
\begin{gathered}
F_{X 1}=\frac{\int_{\lambda} S(\lambda) \bar{x}_{\mathrm{Ln}}(\lambda) \mathrm{d} \lambda}{\int_{\lambda} S(\lambda) s_{X \mathrm{Ln}}(\lambda) \mathrm{d} \lambda} \\
F_{X 2}=\frac{\int_{\lambda} S(\lambda) \bar{x}_{\mathrm{Sn}}(\lambda) \mathrm{d} \lambda}{\int_{\lambda} S(\lambda) s_{X \mathrm{Sn}}(\lambda) \mathrm{d} \lambda} \\
F_{Y}=\frac{\int_{\lambda} S(\lambda) V(\lambda) \mathrm{d} \lambda}{\int_{\lambda} S(\lambda) s_{Y \mathrm{n}}(\lambda) \mathrm{d} \lambda} \\
F_{Z}=\frac{\int_{\lambda} S(\lambda) \bar{z}_{\mathrm{n}}(\lambda) \mathrm{d} \lambda}{\int_{\lambda} S(\lambda) s_{\mathrm{Zn}}(\lambda) \mathrm{d} \lambda},
\end{gathered}
$$

where $s_{\mathrm{XL}}(599), s_{X S}(442), s_{Y}(555)$, and $s_{Z}(446)$ are the absolute responses of the realized receivers at the peak wavelengths of the color matching functions; and $s_{X \mathrm{Ln}}(\lambda), s_{X \mathrm{Sn}}(\lambda), s_{Y \mathrm{n}}(\lambda)$, and $s_{\mathrm{Zn}}(\lambda)$ are the relative responses of the realized receivers normalized also at the peak wavelengths of the color matching functions. The peak wavelengths of the realized receivers are not necessarily equal to the peak wavelengths of the color matching functions.

A color correction factor will be unity if the normalized channel response is equal to the normalized CIE color matching function.
Once the tristimulus colorimeter is calibrated for $k_{X 1}$, $k_{X 2}, k_{Y}$, and $k_{Z}$, the tristimulus values of a test light source can be measured as

$$
\begin{aligned}
& X^{\prime}=X_{1}{ }^{\prime}+X_{2}{ }^{\prime} \quad \text { where } X_{1}{ }^{\prime}=k_{X 1} I_{X 1}{ }^{\prime} \text { and } X_{2}{ }^{\prime}=k_{X 2} I_{X 2}{ }^{\prime} \\
& Y^{\prime}=k_{Y} I_{Y}{ }^{\prime} \\
& Z^{\prime}=k_{Z} I_{Z}{ }^{\prime}
\end{aligned}
$$

where $I_{X 1}{ }^{\prime}, I_{X 2}{ }^{\prime}, I_{Y}{ }^{\prime}$, and $I_{Z}{ }^{\prime}$ are the measured output currents of the receivers.

The calibration procedure can be applied to various measurement geometries (e.g., illuminance, luminance, luminous flux, or luminous intensity) depending on the units in which $s_{X \mathrm{~L}}(599), s_{X S}(442), s_{Y}(555)$, and $s_{Z}(446)$ are expressed.

\section{Achievable Accuracy}

In order to obtain the highest color measurement accuracy, the receiver calibration factors are to be redetermined for all $S(\lambda)$ source distributions to be measured. The spectral mismatch of the receivers, relative to the CIE functions, should be small to allow for relatively large uncertainties when determining $S(\lambda)$. It was shown in an earlier work [16] that the change of $F_{Y}$ with a high quality spectral match of $f_{1}{ }^{\prime}=1.43 \%$ [2], was $0.1 \%$ for a color temperature change from $2600 \mathrm{~K}$ to $3200 \mathrm{~K}$ of a Planckian radiator. With a lower quality spectral match of $f_{1}{ }^{\prime}=3.4 \%$, which is typical for the red and blue receivers, the change in $F_{Y}$ would be larger, still allowing for a large enough uncertainty of $S(\lambda)$. The final $S(\lambda)$ for tungsten lamps, which are more or less similar to Planckian radiators [17], can be obtained by iterating the Planckian function (at different temperatures) and the tristimulus measurements, until the highest color measurement accuracy is reached. For other types of sources with smoothly varying spectral power distribution (e.g., many kinds of paints, color tiles, etc.), $S(\lambda)$ can be measured with a low accuracy spectroradiometer, and the color measurement accuracy still remains high.

According to the references in the Introduction, the presently achievable relative expanded uncertainty of absolute spectral response determinations is on the order of $0.1 \%$. The uncertainty of relative spectral response measurements can be lower because of the smaller number of uncertainty components. The uncertainties of the tristimulus values propagate to the uncertainties of the chromaticity coordinates. The chromaticity coordinates can be calculated from the tristimulus values via 


$$
x=\frac{X}{X+Y+Z}, \quad y=\frac{Y}{X+Y+Z} .
$$

Now, assume that the relative uncertainties of $X, Y$, and $Z$ are all $0.1 \%$. For a Standard Illuminant $\mathrm{A}$, where $x=0.4476$ and $y=0.4074$, the worst case scenario $(\Delta X=+0.1 \%, \Delta Y=-0.1 \%$, and $\Delta Z=0 \%)$ shows a chromaticity coordinate change of $\cong 0.0004$ for both $x$ and $y$, resulting in $\mathrm{a} \cong 10 \mathrm{~K}$ change in the correlated color temperature. These expanded uncertainties are similar to those reported for the source-based NIST color temperature scale [18]. With improvements of spectral response determinations (as suggested below), the accuracy of detector-based tristimulus color measurements can be further increased.

\section{Suggested Realizations}

The described detector-based calibration method can be applied to the calibration of existing tristimulus colorimeters where the spectral response of the receivers can be measured. The achievable color measurement accuracy will depend on the uncertainty of the spectral response measurements.

Standard quality tristimulus colorimeters can be constructed using high accuracy detector standards such as trap detectors. The advantage of using silicon trap detector standards is that the spectral response of the trap detector can be determined directly against a primary standard cryogenic radiometer [10]. Further advantages of trap detectors are relative response non-uniformities of less than $0.02 \%$ [19] and very low reflectance in the visible wavelength range [9], especially for transmission type versions [20, 21]. The filter packages can be measured separately if they are used with transmission-type trap detectors because of zero back reflection from these detectors.

We have started developing a new facility to calibrate illuminance measuring photometers and tristimulus colorimeters against irradiance measuring trap detectors. These trap detectors will be calibrated against the cryogenic radiometer in the power (detector is underfilled by the laser beam) measurement mode. The trap detectors are equipped with precision apertures and will measure the well-collimated radiation (within the aperture) from point sources to obtain the highest accuracy. The point sources are being realized with small integrating spheres illuminated by tunable lasers. The aperture areas are also measured with point sources. The accuracy of our cryogenic radiometer is being improved to achieve radiant power measurements with an uncertainty of between $0.01 \%$ and $0.02 \%$. The expected spectral irradiance response uncertainty of the irradi- ance trap detectors (for point sources) is about $0.03 \%$. All of our existing illuminance-type meters can be calibrated against the irradiance trap detectors with substitution in the uniform field of the tunable monochromatic point source. After spectral response calibrations, the described correction method will be applied. A tristimulus colorimeter calibrated this way will have a chromaticity coordinate measurement uncertainty (expanded but not relative) of about 0.0003 . The accuracy of matrix corrected tristimulus colorimeters [22, 23, 24] can also be improved if they are calibrated against the suggested response corrected reference tristimulus colorimeters. Further analysis is suggested to establish a relationship between the $f_{1}{ }^{\prime}$ of the receivers and the colorimetric accuracy when spectrally structured and changing source spectral power distributions (e.g., color TV monitors) are measured.

\section{Conclusion}

The significant decrease of uncertainties in detector spectral response measurements in the past 5 years motivated the development of a detector-based calibration method for tristimulus colorimeters.

A method for color (spectral mismatch) correction and peak (amplitude) normalization of color measuring tristimulus receivers has been developed. Broad-band calibration factors, based on spectral response measurements of the receivers, can be determined to minimize the color measurement uncertainties caused by the imperfect receiver response realizations. The method utilizes the lower uncertainty of new detector standards relative to traditional lamp standards. The calibration factors can be determined for sources of different spectral power distributions.

Application of the described detector-based calibration method will result in chromaticity coordinate expanded uncertainties (not relative) of less than 0.001 . This corresponds to a color temperature measurement accuracy equal to or better than that of presently used primary lamp standards.

\section{Acknowledgments}

The author thanks Y. Ohno and M. Racz for their helpful advice.

\section{References}

[1] Publication CIE No 15.2 (1986). Central Bureau of the CIE, A-1033 Vienna, P.O. Box 169, Austria.

[2] Publication CIE No 69 (1987). Central Bureau of the CIE, A1033 Vienna, P.O.Box 169, Austria, pp. 5, 9. 
[3] J. H. Walker, R. D. Saunders, J. K. Jackson, and D. A. McSparron, Spectral irradiance calibrations. NBS Special Publication 250-20 (1987).

[4] B. K. Tsai, Developments in the New Spectral Irradiance Scale at the National Institute of Standards and Technology, NCSL Workshop and Symposium, Session 3E (1996) pp. 325-341.

[5] B. N. Taylor and C. E. Kuyatt, Guidelines for Evaluating and Expressing the Uncertainty of NIST Measurement Results, NIST Technical Note 1297 (1994).

[6] R. S. Berns, M. E. Gorzynski, and R. J. Motta, CRT Colorimetry, Part II: Metrology, Color Res. Appl. 18 (3), 315-325 (1993).

[7] J. E. Martin, N. P. Fox, and P. J. Key, Metrologia 21, 147-155 (1985).

[8] K. D. Stock and H. Hofer, PTB primary standard for optical radiant power: transfer-optimized facility in the clean-room center, Metrologia 32(6), 545-549 (1996).

[9] T. R. Gentile, J. M. Houston, J. E. Hardis, C. L. Cromer, and A. C. Parr, National Institute of Standards and Technology high accuracy cryogenic radiometer, Appl. Opt. 35, 1056-1068 (1996).

[10] T. R. Gentile, J. M. Houston, and C. L. Cromer, Realization of a scale of absolute spectral response using the NIST high accuracy cryogenic radiometer, Appl. Opt. 35, 4392-4403 (1996).

[11] T. C. Larason, S. S. Bruce, and C. L. Cromer, The NIST High Accuracy Scale for Absolute Spectral Response from $406 \mathrm{~nm}$ to 920 nm, J. Res. Natl. Inst. Stand. Technol. 101(2), 133-140 (1996).

[12] K. L. Eckerle, J. J. Hsia, K. D. Mielenz, and V. R. Weidner, NBS Special Publication 250-6, Regular Spectral Transmittance (1987) p. 34.

[13] R. Friedrich, J. Fischer, and M. Stock, Accurate calibration of filter radiometers against a cryogenic radiometer using a trap detector, Metrologia 32(6), 509-513 (1996).

[14] E. Ikonen and P. Toivanen, A new optical method for high-accuracy determination of aperture area (preprint).

[15] C. L. Cromer, G. Eppeldauer, J. E. Hardis, T. C. Larason, and A. C. Parr, National Institute of Standards and Technology Detector-based Photometric Scale, Appl. Opt. 32(16), 2936-2948 (1993).

[16] C. L. Cromer, G. Eppeldauer, J. E. Hardis, T. C. Larason, Y. Ohno, and A. C. Parr, The NIST Detector-Based Luminous Intensity Scale, J. Res. Natl. Inst. Stand. Technol. 101, 109-132 (1996).

[17] Y. Ohno and M. Navarro, Improved Photometric and Colorimetric Calibrations at NIST, Proc. of the NCSL Workshop and Symposium, Session 1E (1997) pp. 99-110.

[18] Y. Ohno, Photometric Calibrations, NIST Special Publication 250-37 (1997).

[19] R. Kohler, R. Goebel, M. Stock, and R. Pello, An international comparison of cryogenic radiometers, in Optical Radiation Measurements III, J. M. Palmer, ed., Proc. SPIE2815 (1996) pp. 22-30.

[20] J. L. Gardner, Transmission trap detectors, Appl. Opt. 33(25), 5914-5918 (1994).

[21] T. Kubarsepp, P. Karha, and E. Ikonen, Characterization of a polarization-independent transmission trap detector, Appl. Opt. 36(13), 2807-2812 (1997)

[22] G. Eppeldauer and J. Schanda, Colorimeter with matrix transformation, AIC Conference on Color Dynamics, Budapest, Hungary, Proc. (1976) pp. 403-413.

[23] E 1455-92, Standard Practice for Obtaining Colorimetric Data from a Visual Display Unit Using Tristimulus Colorimeters, ASTM Standards on Color and Appearance Measurement, 4th Ed. (Available from ASTM, 100 Barr Harbor Dr., Wet Conshohocken, PA 19428-2959.)
[24] Y. Ohno and J. E. Hardis, Improved Matrix Method for Tristimulus Colorimetry of Displays, AIC Color'97, Kyoto, May 26-30, 1997.

About the author: George Eppeldauer is a Ph.D. Electronics Engineer in the Optical Technology Division of the NIST Physics Laboratory. The National Institute of Standards and Technology is an agency of the Technology Administration, U.S. Department of Commerce. 


\title{
Design and characterization of a photometer-colorimeter standard
}

\author{
George P. Eppeldauer and Miklós Rácz
}

\begin{abstract}
A photometer and tristimulus colorimeter has been developed at the National Institute of Standards and Technology (NIST) to realize a color scale. A novel construction was developed to implement the spectral-responsivity-based scale with small uncertainty. The new device can be used as a reference illuminance and luminance meter as well. Temperature-controlled filter combinations, with 5-8 layers in one package, are used to match the responsivity of a silicon tunnel-trap detector to the CIE colormatching functions with small spectral mismatch values $\left(f_{1}{ }^{\prime}\right)$. Design considerations to extend the tunnel-trap detector with replaceable single and double apertures and changeable filter combinations are described. The design and fabrication of the filter packages and the dependence of the $f_{1}{ }^{\prime}$ values on the thickness of the filter layers are discussed. The colorimeter was characterized for angular, spatial, and spectral responsivity. An improved preamplifier can convert current to voltage in an 11-decade dynamic range with $0.01 \%$ uncertainty. (C) 2004 Optical Society of America
\end{abstract}

OCIS codes: $\quad 040.5160,330.1730,120.4800,120.5240$.

\section{Introduction}

In our earlier publication ${ }^{1}$ a method was suggested for calibrating tristimulus colorimeters against detector standards instead of traditional lamp standards. In this new calibration method, spectral corrections are applied to the broadband calibration factors of the tristimulus channels of a colorimeter. As a result of the spectral and amplitude corrections, the imperfect realizations of the Commission Internationale de l'Eclairage (CIE) $\bar{x}(\lambda), \bar{y}(\lambda)$, and $\bar{z}(\lambda)$ color-matching functions can be compensated. It is the uncertainty of the spectral responsivity measurement of the channels that will dominate the uncer-

G. P. Eppeldauer (george.eppeldauer@nist.gov) is with the National Institute of Standards and Technology, Optical Technology Division, Gaithersburg, Maryland 20899. M. Rácz is with the Konkoly Observatory, Hungarian Academy of Sciences, P.O. Box 67, 1525 Budapest, Hungary, and the Hungarian Academy of Sciences, Research Institute for Technical Physics and Materials Science, Magyar Tudomanyos Akademia (Hungarian Academy of Sciences), Muszaki Fizikai es Anyagtudomanyi Kutatointezet (Research Institute for Technical Physics and Material Science), P.O. Box 49, 1525 Budapest, Hungary, and was a National Institute of Standards and Technology guest researcher when this research was begun.

Received 30 July 2003; revised manuscript received 17 February 2004; accepted 18 February 2004.

0003-6935/04/132621-11\$15.00/0

(C) 2004 Optical Society of America tainty of tristimulus color measurements. The present paper describes the design considerations and characterizations of a special tristimulus colorimeter construction with which the spectral responsivity of the colorimeter channels can be determined with extremely low uncertainty.

To make it possible to measure a light source with a known spectral power distribution $S(\lambda)$, the channel calibration factors can be determined from the ratio of the CIE tristimulus values to the measured output currents of the four channels:

$$
\begin{aligned}
& k_{X_{1}}= \frac{X_{1}}{I_{X_{1}}}=\frac{K_{m} \int_{\lambda} S(\lambda) \bar{x}_{1}(\lambda) \mathrm{d} \lambda}{\int_{\lambda} S(\lambda) s_{X_{1}}(\lambda) \mathrm{d} \lambda}=\frac{1.06291 K_{m} F_{X_{1}}}{s_{X_{1}}(599)}, \\
& k_{X_{2}}=\frac{X_{2}}{I_{X_{2}}}=\frac{K_{m} \int_{\lambda} S(\lambda) \bar{x}_{2}(\lambda) \mathrm{d} \lambda}{\int_{\lambda} S(\lambda) s_{X_{2}}(\lambda) \mathrm{d} \lambda}=\frac{0.3501 K_{m} F_{X_{2}}}{s_{X_{2}}(442)}, \\
& k_{Y}=\frac{Y}{I_{Y}}=\frac{K_{m} \int_{\lambda} S(\lambda) \bar{y}(\lambda) \mathrm{d} \lambda}{\int_{\lambda} S(\lambda) s_{Y}(\lambda) \mathrm{d} \lambda}=\frac{K_{m} F_{Y}}{s_{Y}(555)}, \\
& k_{Z}=\frac{Z}{I_{Z}}=\frac{K_{m} \int_{\lambda} S(\lambda) \bar{z}(\lambda) \mathrm{d} \lambda}{\int_{\lambda} S(\lambda) s_{Z}(\lambda) \mathrm{d} \lambda}=\frac{1.78297 K_{m} F_{Z}}{s_{Z}(446)} .
\end{aligned}
$$

As $\bar{x}(\lambda)$ has two lobes, it is realized by two channels, $\bar{x}_{1}(\lambda)$ and $\bar{x}_{2}(\lambda)$. The right-hand side of the equations was obtained by normalizing the color-matching 
functions to their peak values and introducing the color correction factors:

$$
\begin{gathered}
F_{X_{1}}=\frac{\int_{\lambda} S(\lambda) \bar{x}_{1 n}(\lambda) \mathrm{d} \lambda}{\int_{\lambda} S(\lambda) s_{X_{1 n}}(\lambda) \mathrm{d} \lambda}, \\
F_{X_{2}}=\frac{\int_{\lambda} S(\lambda) \bar{x}_{2 n}(\lambda) \mathrm{d} \lambda}{\int_{\lambda} S(\lambda) s_{X_{2 n}}(\lambda) \mathrm{d} \lambda}, \\
F_{Y}=\frac{\int_{\lambda} S(\lambda) V(\lambda) \mathrm{d} \lambda}{\int_{\lambda} S(\lambda) s_{Y n}(\lambda) \mathrm{d} \lambda}, \\
F_{Z}=\frac{\int_{\lambda} S(\lambda) \bar{z}_{n}(\lambda) \mathrm{d} \lambda}{\int_{\lambda} S(\lambda) s_{Z n}(\lambda) \mathrm{d} \lambda},
\end{gathered}
$$

where $s_{X_{1}}(599), s_{X_{2}}(442), s_{Y}(555)$, and $s_{Z}(446)$ are the absolute responsivities of the realized channels at the peak wavelengths of the color-matching functions, $\bar{x}_{1 n}(\lambda), \bar{x}_{2 n}(\lambda)$, and $\bar{z}_{n}(\lambda)$ are the color-matching functions normalized to one at their peaks, $V(\lambda)$ is the spectral luminous efficiency function for photopic vision (also normalized to one at its peak), and $s_{X_{1 n}}(\lambda)$, $s_{X_{2 n}}(\lambda), s_{Y n}(\lambda)$, and $s_{Z n}(\lambda)$ are the relative spectral responsivities of the realized channels normalized to one at their peak wavelengths.

After the tristimulus colorimeter is calibrated for $k_{X_{1}}, k_{X_{2}}, k_{Y}$, and $k_{Z}$, the tristimulus values of a test light source can be measured as

$$
\begin{aligned}
& X^{\prime}=X_{1}{ }^{\prime}+X_{2}{ }^{\prime}, \quad \text { where } X_{1}{ }^{\prime}=k_{X_{1}} I_{X_{1}}{ }^{\prime}, X_{2}{ }^{\prime}=k_{X_{2}} I_{X_{2}}{ }^{\prime}, \\
& Y^{\prime}=k_{Y} I_{Y}{ }^{\prime}, \\
& Z^{\prime}=k_{Z} I_{Z}{ }^{\prime},
\end{aligned}
$$

where $I_{X_{1}}{ }^{\prime}, I_{X_{2}}{ }^{\prime}, I_{Y}{ }^{\prime}$, and $I_{Z}{ }^{\prime}$ are the measured output currents of the tristimulus channels.

This calibration procedure can be applied to either illuminance or luminance measurement geometry by use of the relevant units of $s_{X_{1}}(599), s_{X_{2}}(442), s_{Y}(555)$, and $s_{Z}(446)$ in the equations.

According to the above procedure, the photometric and colorimetric measurement uncertainties will depend only on the uncertainty of the spectral responsivity determination of the colorimeter channels. An advantage of the method is that accurate color and photometric measurements can be made even if the CIE functions are poorly realized (with large spectral mismatch) in the channels of a colorimeter. For perfect realizations, the color of any source distributions could be measured accurately with one set of calibration factors shown in Eq. (1). In this case, all the color correction factors in Eq. (2) would become unity. We have decided to keep the CIE $f_{1}{ }^{\prime}$ (spectral mismatch) values ${ }^{2}$ of the realized channels small so that one set of calibration factors can be used for sources with similar spectral distributions. Also, rough knowledge of the source distribution is enough to perform accurate color measurements. Even with small $f_{1}^{\prime}$ values, an additional set of calibration factors will be needed to measure very different source distributions, such as gas discharge lamps or display monitors.
Our goal was to design and fabricate a photometercolorimeter for which the spectral responsivity of the channels can be determined with an expanded uncertainty $(k=2)$ of $0.1 \%$ or less. ${ }^{3} \quad$ A $0.1 \%$ change in the spectral irradiance responsivity of any one channel will result in a chromaticity coordinate change of 0.0002 for both $x$ and $y$ (for a Planckian radiator) and a change in the illuminance responsivity of $0.1 \%$. These expected colorimeter uncertainties are equivalent to the uncertainties of current primary lamp standards. While intensity and spectral distribution of lamp standards may change significantly with burning time, the responsivity change of colorimeter channels can be much smaller versus time.

In order to satisfy the above expectations, a silicon tunnel-trap detector is used in our colorimeter standard. This detector does not have back reflections to the filters, and its spectral responsivity can be determined with an uncertainty of $0.1 \%$. The four filter combinations that modify the spectral responsivity of the trap detector (to be similar to the CIE functions) needed individual design and fabrication to achieve small $f_{1}^{\prime}$ values. The small $f_{1}^{\prime}$ values make it possible to avoid frequent change (redetermination) of the channel calibration factors even for significant changes in the measured source distribution. Temperature stabilization of the filter combinations (packages) is necessary to eliminate changes in their spectral transmittances.

The photometric and tristimulus color scale realizations have been studied. 4 The photometercolorimeter, when equipped with different filters, can be used as a radiometric standard as well. We have also studied the application of the filter-trap radiometer for ultraviolet and visible calibrations. ${ }^{5}$

\section{Trap-Detector-Based Photometer-Colorimeter}

The silicon tunnel-trap detector we developed recently ${ }^{6}$ was extended by a temperature-controlled filter wheel with four selectable filter combinations, a removable luminance measuring tube, and an improved current-to-voltage converter. The details of the trap-detector extension are discussed below. The goal was to develop a versatile tunnel-trapdetector-based photometer-colorimeter for which the channel spectral responsivities can be determined with low uncertainties.

\section{A. Silicon Tunnel-Trap Detector}

A significant advantage of a tunnel-trap detector is that no light is reflected back from the detector to the input optics (such as filters and aperture). Consequently, the filter combinations and the trap detector can be calibrated either separately or as a combined package. The nonabsorbed light (i.e., light transmitted through the trap) can be easily measured to apply the physical model of internal quantum efficiency for responsivity determination. ${ }^{7}$ The other advantage of a trap detector is that the uniformity of spatial responsivity can be high if the internal quantum efficiency of the photodiodes is uniform over the active area. These features can make well-designed 


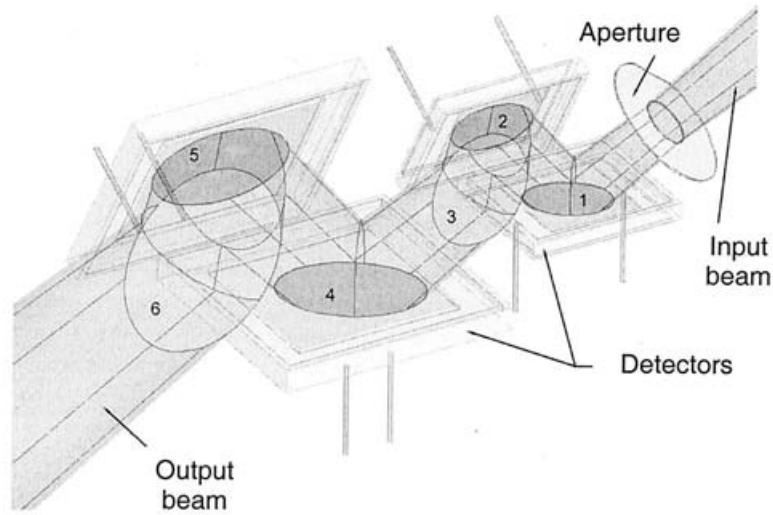

Fig. 1. Beam propagation in the triangular tunnel-trap detector. The converging incident beam is focused to the aperture.

and precisely fabricated trap detectors suitable for accurate measurement of beam power.

The scheme of a recently designed tunnel-trap detector is shown in Fig. 1. Two $10 \mathrm{~mm} \times 10 \mathrm{~mm}$ and four $18 \mathrm{~mm} \times 18 \mathrm{~mm}$ silicon photodiodes, equivalent to the Hamamatsu S1337 model, ${ }^{8}$ were packed in a light-trapping arrangement. We used this photodiode type to utilize the physical model of internal quantum efficiency for spectral responsivity extrapolation and interpolation. ${ }^{7}$ This photodiode model has a high (close to unity) and spatially uniform internal quantum efficiency. The beam propagates in the tunnel-trap detector from the first photodiode (behind the aperture) to the last (sixth) photodiode. Photodiodes 3 and 6 are not shown for better illustration. The output beam from the trap detector can be utilized for alignment or for detector transmittance measurements in spectral responsivity determination. A second light trap can be attached to the trap-detector output to absorb transmitted radiation and to minimize ambient stray light.

The implementation of the scheme of Fig. 1 is shown in Fig. 2. The photodiodes are mounted in a triangular tunnel. Choosing different size of photodiodes allowed the aperture area and the field of view (FOV) to be maximized. Temperature control for the photodiodes was not needed because the temperature coefficient of responsivity of the photodiodes is close to zero in the visible range.

This trap detector is suitable for both radiant power and irradiance measurements. In power mode measurements, the aperture is underfilled, and in irradiance mode measurements the aperture is overfilled by the incident radiation. In power mode measurements, the incident beam must be focused to the front surface of the aperture. Radiance or luminance can be measured by attaching a radianceluminance measuring tube (see below) to the front of the trap detector.

\section{B. Trap Detector Extended with a Filter Wheel}

The inside arrangement of the extended trap detector is illustrated in Fig. 3 . The incident beam (originat-

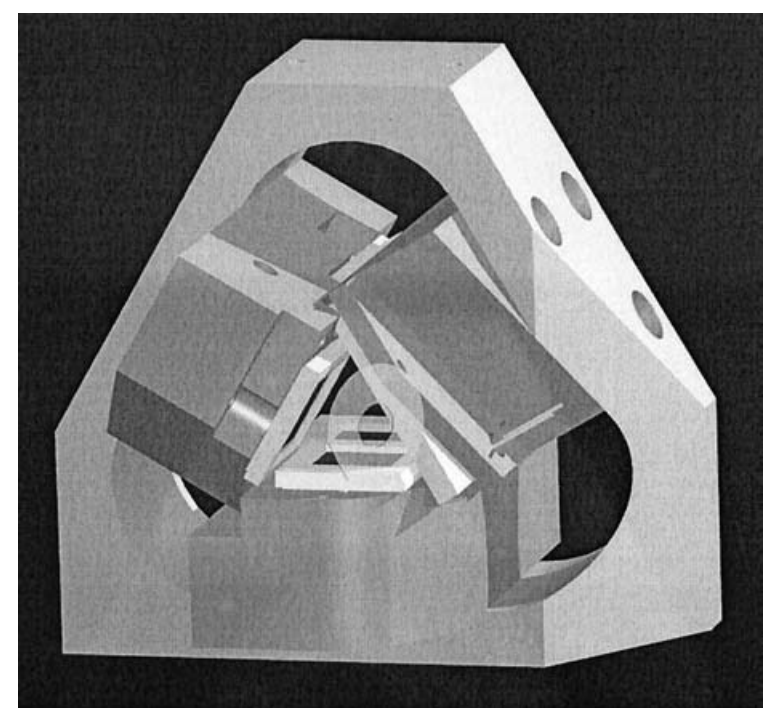

Fig. 2. Construction of the triangular tunnel-trap detector. The aperture is drawn to be semitransparent in the figure.

ing from a baffled point source) overfills the common aperture mounted on the front panel of the cover box. The aperture front surface and the front surface of the cover box are in the same plane. This is the reference plane of the photometer-colorimeter for illuminance mode measurements. The replaceable filter combinations (packages) are mounted in a temperature-controlled filter wheel. The wheel can be heated slightly above room temperature with a thermoelectric cooler-heater. This regulated temperature will ensure stable spectral transmittances for the filters without condensation. The wheel has five well-defined positions with filter-mounting holes. The filter combinations are mounted in the first four holes. The last hole in the fifth position is empty for

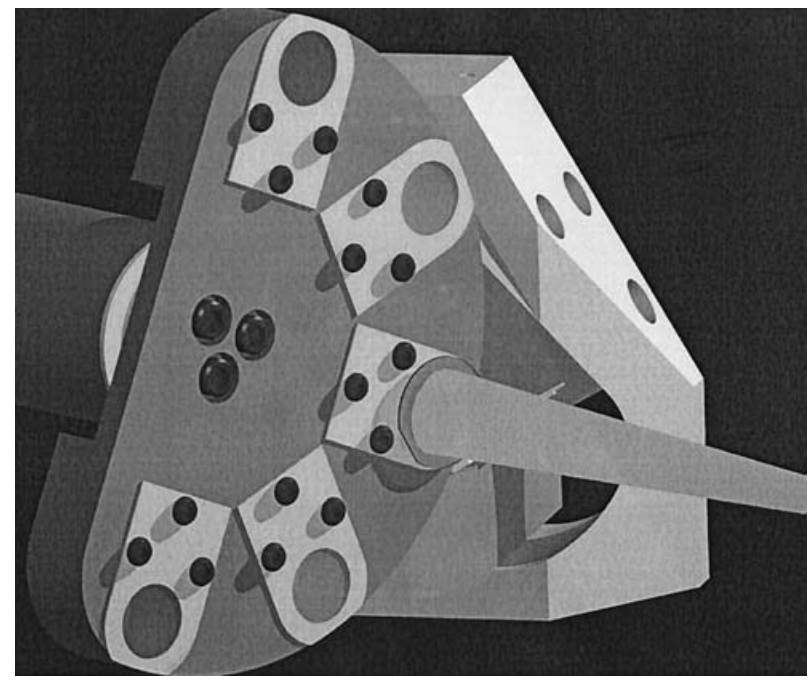

Fig. 3. Filter wheel between the aperture and the trap detector. The outside edge of the aperture can be seen where the incident beam from a baffled point source overfills the aperture. 


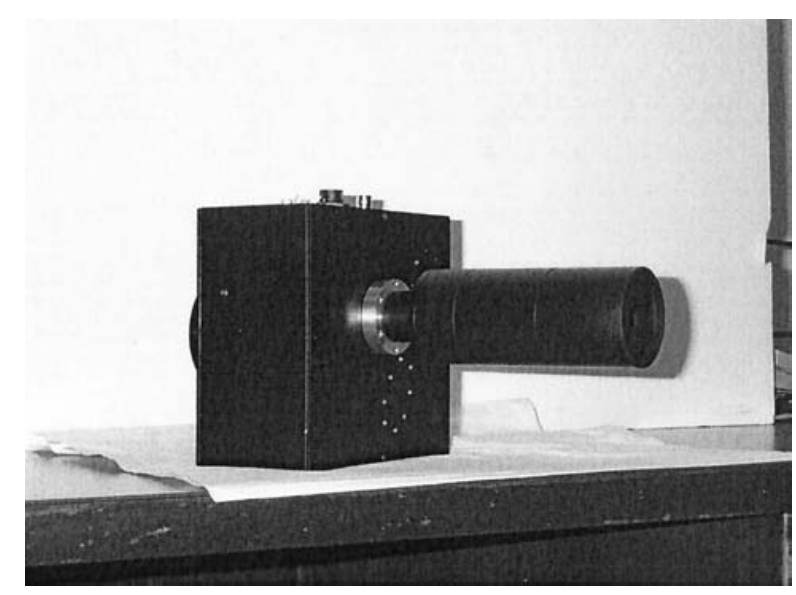

Fig. 4. Picture of the photometer-colorimeter equipped with a radiance-luminance measuring tube.

spectral responsivity determination of the trap detector itself.

The filter wheel is located between the aperture and the front end of the tunnel-trap detector. A rotating knob is mounted on the shaft of the wheel and located on the back of the cover box. The length of the filter combinations is limited to $10 \mathrm{~mm}$ by the thickness of the filter wheel. The space for the wheel, between the aperture and the trap detector, is $\sim 12 \mathrm{~mm}$. This separation did not decrease the FOV of the meter.

\section{Radiance-Luminance Measuring Tube}

While illuminance (or irradiance) can be measured with one aperture, luminance (or radiance) mode measurements need two apertures. An input tube with two baffles between the apertures can be attached to the front of the photometer-colorimeter for radiance and luminance mode measurements. The aperture in the front plate of the housing and the front aperture of the tube will determine the radiance (luminance) measurement angle. Figure 4 shows the picture of the photometer-colorimeter when it is equipped with the radiance-luminance measuring tube.
The FOV scheme of the radiance-luminance measuring tube is shown in Fig. 5 . The diameter of the trap-detector (illuminance measuring) aperture is $d$. The diameter of the front aperture is $D$.

The radiant power $\Phi_{d}$ entering the trap detector in radiance measurement mode is

$$
\Phi_{d}=L_{t} \omega_{m} A_{d},
$$

where $L_{t}$ is the radiance of the target surface measured by the meter, $\omega_{m}$ is the viewing solid angle (measurement angle) of the meter, and $A_{d}$ is the (acceptance) area of the trap-detector aperture. Using the symbols of Fig. 5,

$$
\begin{aligned}
& A_{d}=d^{2} \pi / 4, \\
& \omega_{m}=2 \pi[1-\cos (\gamma / 2)] .
\end{aligned}
$$

When $D=11.29 \mathrm{~mm}$, and the separation between the two apertures is $s=165 \mathrm{~mm}$, the nominal (or effective) viewing angle can be calculated:

$$
\gamma=2 \tan ^{-1}(D / 2 s)=3.919^{\circ} \text {. }
$$

From the above values and for $d=5 \mathrm{~mm}$,

$$
\begin{aligned}
& A_{d}=0.1964 \mathrm{~cm}^{2}, \\
& \omega_{m}=0.003674 \mathrm{sr} .
\end{aligned}
$$

When the trap detector (calibrated for power responsivity) measures radiant power, the radiance of the target surface can be calculated from Eq. (4). Determination of $\omega_{m}$ from the nominal viewing angle $\gamma$ is an accurate approximation when $A_{d}$ is small. If we integrate the radiant power reaching the detector (from a uniform source of $L_{t}=$ constant radiance) for an ideal $\gamma$ angle $(d=0)$ and the real case $(d>0)$, using the same detector aperture $\left(A_{d}\right)$, the integrated difference (ideal radiance minus real radiance) will be $-0.012 \%$ for $d=5 \mathrm{~mm}$ and $-0.006 \%$ for $d=3.5$ $\mathrm{mm}$. These systematic radiance (luminance) measurement errors are very small. For a detector aperture of $d>5 \mathrm{~mm}$, the integrated difference can be utilized as a correction factor for the measured radiance (luminance). A detailed analysis with the achievable uncertainties of radiance measuring tubes

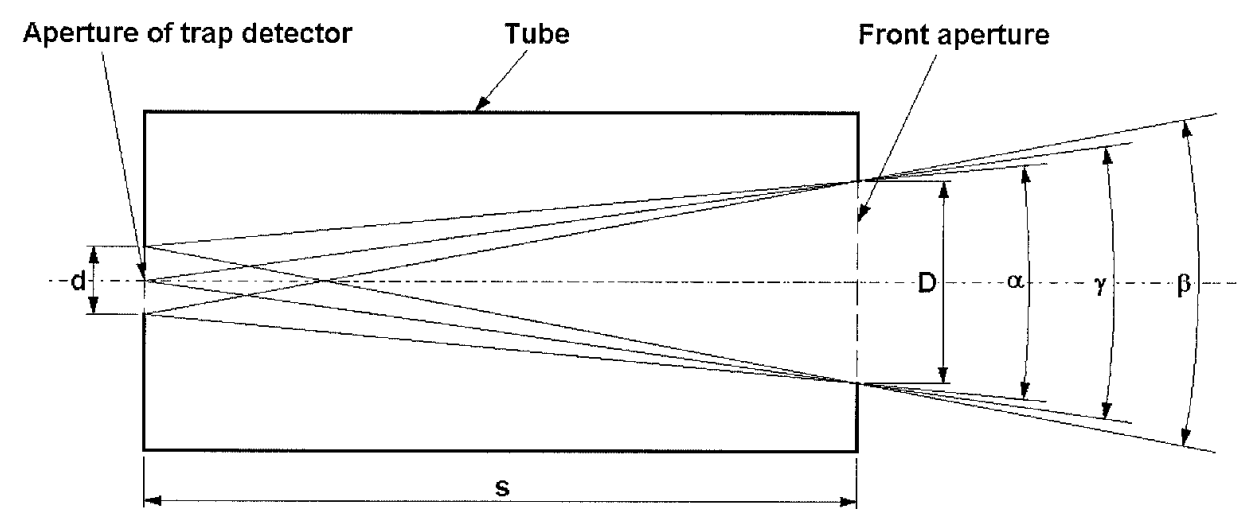

Fig. 5. FOV design of radiance-luminance measurement. 


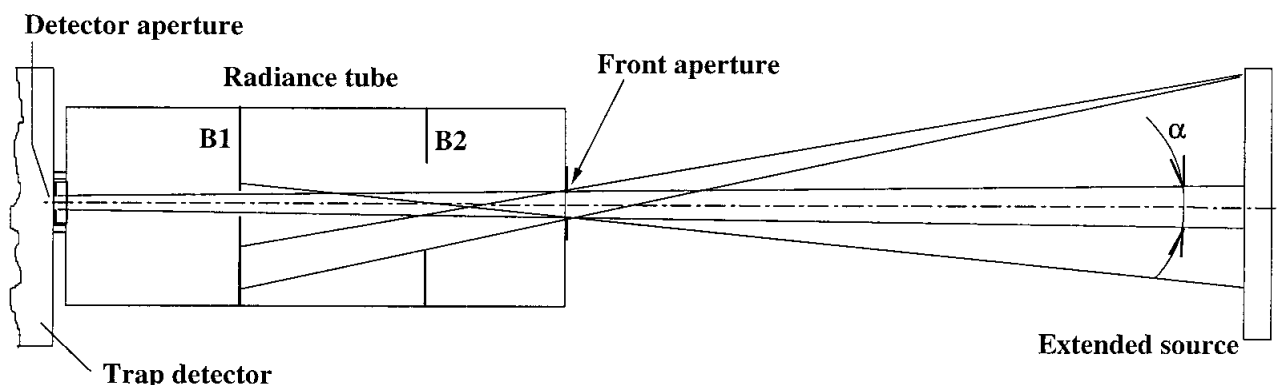

Fig. 6. Baffle (B1, B2) arrangement inside the radiance-luminance tube and FOV and out-of-FOV rays of an extended source measurement.

with different input geometries will be discussed in a following paper.

The unvignetted FOV of the radiance (luminance) measuring tube is

$$
\alpha=2 \tan ^{-1} \frac{D-d}{2 s} .
$$

For the above geometry (with $d=5 \mathrm{~mm}$ ), $\alpha$ is $2.18^{\circ}$. The full radiance-measurement angle is

$$
\beta=2 \tan ^{-1} \frac{D+d}{2 s} .
$$

For the above tube design, $\beta=5.65^{\circ}$, which is smaller than the $8^{\circ} \mathrm{FOV}$ of the trap detector (discussed below). With this design, the unvignetted target spot diameter (the plateau of the output signal) will be $40 \%$ of the full spot diameter seen by the radianceluminance meter.

The design of the baffle arrangement inside the tube is shown separately in Fig. 6 to illustrate the separation of rays from an extended source. Baffles $\mathrm{B} 1$ and B2 minimize the effect of stray light and reject radiation from light sources outside the FOV. For simplicity, three rays are shown outside the two FOV rays (producing $\alpha$ ) to illustrate this situation for a large extended source.

For the above tube design, B1 had a diameter of 8.0 $\mathrm{mm}$, and it was located at a distance not too far from the detector aperture to avoid any decrease (by clipping) in the FOV. This baffle was fabricated with the same procedure as the other two FOV-limiting apertures. They were all made of copper, coated with black nickel. The aperture thickness is 0.15 $\mathrm{mm}$. The position of B2 is not critical. It has a larger diameter and stops radiation entering the front aperture at large incidence angles.

As an alternative to the geometrical radiance (or radiance responsivity) determination described above, the radiance responsivity of the photometercolorimeter can be determined from radiance measurement of a calibrated sphere source. The radiant intensity of the sphere source can be determined against an irradiance meter standard (such as another trap detector) when the trap detector and source separation is large enough to take the source as a point source. The source radiance measured by the photometer-colorimeter will be equal to the ratio of the radiant intensity and the output area of the source. ${ }^{9}$ The radiance responsivity will be the ratio of the output signal of the photometer-colorimeter and the source radiance.

The above tube design can be utilized in measurements for which the target spot (determined by the full radiance measurement angle) of the photometercolorimeter can be positioned within the radiating surface of the source to be measured. A calibration source should be spatially uniform (such as a sphere exit port) to yield low measurement uncertainties.

\section{Spatial Characteristics}

Three spatial characteristics were measured to verify the design expectations in the three measurement modes. The uniformity of spatial responsivity was measured in flux (power) mode with the trap detector underfilled by an incident beam scanned over the aperture. The angular responsivity was measured in illuminance mode with the aperture of the trap detector overfilled by the incident radiation. The FOV was measured in radiance mode to determine the measurement angle and the out-of-target blocking of the photometer-colorimeter for luminance measurements.

\section{A. Spatial Uniformity of Responsivity}

To minimize uncertainty in flux measurement mode, a good spatial uniformity of responsivity is needed from the trap detector. The spatial uniformity was tested by scanning the incident beam over the active area of the tunnel-trap detector. The $f / 9$ output beam ( $6.4^{\circ}$ full angle) of a monochromator was focused on the plane of the removed aperture. The diameter of the beam spot was $1.1 \mathrm{~mm}$. The beam was not clipped by any of the six photodiodes inside the trap detector. The scan was made with $0.5-\mathrm{mm} /$ step increments. The wavelength of the beam was $500 \mathrm{~nm}$. The $0.05 \%$ nonuniformity contours are shown in Fig. 7. The real acceptance area is at least $0.55 \mathrm{~mm}$ (but not more than $1.05 \mathrm{~mm}$ ) larger than the measured uniform area (shown in the plot). Accordingly, the maximum diameter of the trap detector aperture is $5 \mathrm{~mm}$. 


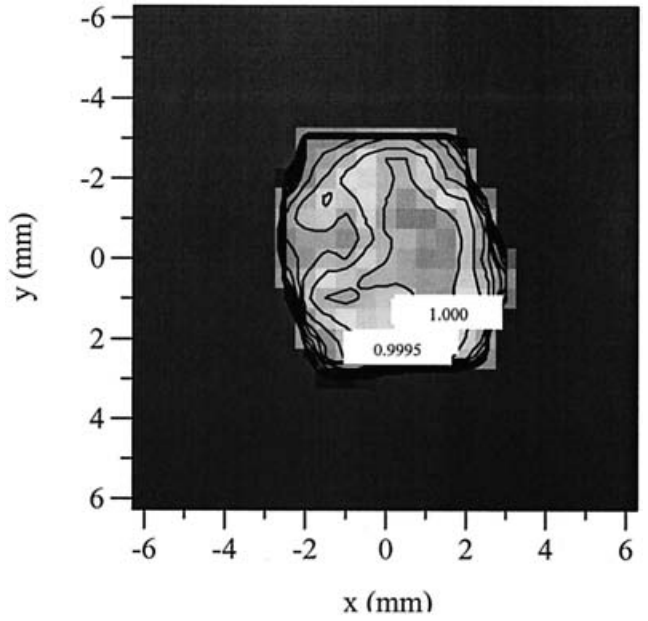

Fig. 7. Nonuniformity of spatial responsivity of the tunnel trap detector. The $0.05 \%$ contours are shown at a wavelength of 500 $\mathrm{nm}$. The detector was moved by $0.5-\mathrm{mm} / \mathrm{step}$ increments, and the beam spot diameter was $1.1 \mathrm{~mm}$.

\section{B. Angular Responsivity}

The normalized angular responsivities of the tunneltrap detector, when equipped with a $3.5-\mathrm{mm}$ diameter aperture, were measured in two perpendicular planes. The results are shown in Fig. 8.6 During the horizontal scan, the trap detector was rotated in the horizontal plane around its aperture center. It measured the radiation from a luminous intensity lamp (considered a point source) at a separation of $3 \mathrm{~m}$. Thereafter, the radiometer was rotated $90^{\circ}$ around its optical axis and the vertical scan was done in the same way as the horizontal one. The graph shows that the deviation of the realized angular responsivities from the expected cosine function is less than $0.02 \%$ within a $5^{\circ} \mathrm{FOV}$ and $\sim 0.05 \%$ at $8^{\circ} \mathrm{FOV}$. Accordingly, the allowed maximum convergence (full)

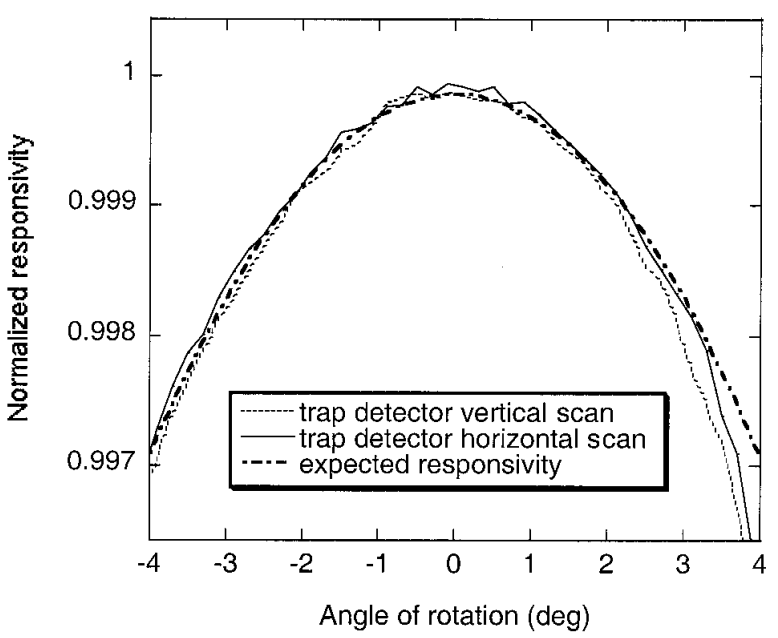

Fig. 8. Normalized angular responsivities of the tunnel-trap detector when equipped with a $3.5-\mathrm{mm}$ diameter aperture. The expected (cosine) responsivity is shown as well.

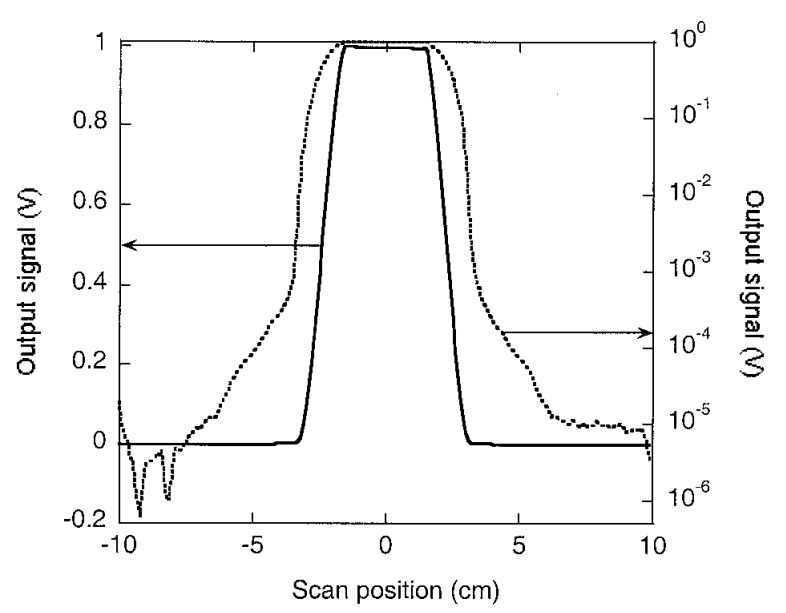

Fig. 9. Point-spread response of the radiance measuring trap detector on both linear and logarithmic scales.

angle of an incident beam is $8^{\circ}$. In Subsection 2.C, the largest beam convergence angle is $\beta=5.65^{\circ}$.

The measurement results discussed above verified the optical and mechanical design expectations for the tunnel trap detector.

\section{Radiance-Luminance Measurement FOV}

The point-spread function of the trap detector with the radiance-luminance tube was measured with a small incandescent lamp of $\sim 5-\mathrm{mm}$ diameter at a distance of $1 \mathrm{~m}$. The lamp was mounted in an optical axis and the photometer-colorimeter was moved in a plane perpendicular to that optical axis. The filter combinations were moved away from the input of the trap detector, and the open channel was measured. The measurement results are shown in Fig. 9 on both linear and logarithmic scales. As a result of the optimized input geometry of the radianceluminance tube, an out-of-field blocking of more than 4 orders of magnitude was measured.

\section{Tristimulus Filters}

The four channels of the photometer-colorimeter are spectrally matched to the $\bar{x}_{1}(\lambda), \bar{x}_{2}(\lambda), \bar{y}(\lambda)$, and $\bar{z}(\lambda)$ color-matching functions by individually realizing four filter combinations that are alternately used with the tunnel-trap detector. The responsivity of a channel is the trap-detector spectral responsivity multiplied by the spectral transmittance of the selected filter combination.

\section{A. Design of the Filter Combinations}

Different spectral transmittances were needed from the filter combinations to modify the spectral responsivity of the trap detector according to the CIE colormatching functions. Our goal was to achieve the smallest possible CIE $f_{1}{ }^{\prime}$ values (discussed in Subsection 4.C) for the realized tristimulus channels. The achievable $f_{1}^{\prime}$ value is limited by several factors, such as the availability of certain filter types, the thickness limit of the filter combination (package), the thick- 
ness uncertainty of the individual layers during fabrication, temperature differences between design and applications, and the uncertainty of the spectral transmittance determinations.

All possible color filters that seemed to be promising for the realization of the four filter combinations were purchased. Unfortunately, the number of commercially available filter types is decreasing. The fabrication of many filter types has been discontinued because of the decreasing demand in the market. A database was generated from the spectral transmittance measurements of the collected filters. The most suitable filter types (models) were chosen for the tristimulus filter combinations. Filters were purchased only from Schott and Hoya. ${ }^{8}$

The thicker (longer) the filter combination, the lower the transmittance. The maximum thickness of a filter combination was limited to $10 \mathrm{~mm}$ because of the space available between the aperture and the front end of the trap detector. The peak transmittance maximization of the individual filter combinations was a secondary concern because of the wide signal (dynamic) range of the trap detector. The peak transmittance of each filter combination was limited to a minimum of $1.5 \%$.

In each filter combination, after optimization for the spectral transmittance, five to eight filter disks (layers) were cemented together. The ends of all combinations were evenly aligned to the front surface of the filter wheel (facing the aperture). All filterdisk diameters were $9.5 \mathrm{~mm}$. This diameter, with the $10 \mathrm{~mm}$ maximum thickness of a combination, was a good compromise to avoid limiting the original FOV (acceptance angle) of the trap detector.

The optimum thickness of all filters within a filter combination was calculated with an interactive program. A similar optimization method, to obtain the best spectral fit, has already been published. ${ }^{10}$ After the initial filter type and thickness selections, the program finds a local minimum of the $f_{1}{ }^{\prime}$ value by individually changing the thickness of the other filter layers inside of the same package. The $f_{1}{ }^{\prime}$ optimization is an iterative method where the filters are remeasured several times for spectral transmittance after they are polished to the desired thickness calculated by the computer program. As the uncertainty of our filter thickness realization was $3 \mu \mathrm{m}$, the fabrication of the filter combination (the first filter realization) started with the thinnest filter. Several pieces of the thinnest filters were made (polished) and then the thickness of each piece was measured. The filter that gave the best results was selected for the combination, and the thickness of the other layers (in the same combination) was recalculated. The less critical filter of the combination was calculated last. The program continuously displayed the peak transmittance and the total thickness of the filter combination being optimized. The uncertainty of the spectral transmittance measurements was $0.2 \%$.

The spectral responsivity of the filter-detector combination was calculated with the following equation:

$S_{n}(\lambda)=(1-\rho) \exp \left[\log _{10} S_{d}(\lambda)+\sum_{i} t_{i} \frac{\log _{10} \frac{\tau_{i}^{m}(\lambda)}{1-\rho}}{t_{i}^{m}}\right]$,

where

$\lambda$ is wavelength,

$\rho$ is the surface reflection,

$S_{d}(\lambda)$ is the spectral responsivity of the detector,

$i$ is the filter index,

$t_{i}$ is the thickness of the $i$ th filter in the package,

$\tau_{i}^{m}(\lambda)$ is the measured spectral transmittance of the $i$ th filter,

$t_{i}^{m}$ is the measured thickness of the $i$ th filter,

$m$ superscript shows that the value was measured.

The surface (Fresnel) reflection depends on the refractive index of the filter material. The refractive indices of the filters are similar to one another with a significant wavelength dependence in the visible range. The internal transmittance of the filter layers dominates the spectral transmittance of the realized filter combination. For all separate filters and cemented filter combinations, a $\rho=0.08$ was used in the calculations. Ultraviolet and infrared blocking filters were used to attenuate the incident radiation for wavelengths shorter than $360 \mathrm{~nm}$ and longer than $830 \mathrm{~nm}$. The blocking was necessary for all filter combinations because of the broad sensitivity range of the silicon trap detector.

To yield optical-quality filter combinations, both sides of the individually made filters were polished. The individual filters were glued together with optical cement that has constant spectral transmittance in the visible range (Lens Bond Optical Cement, Type F65, Modified RD3-74 from Summers Laboratories, Inc.). The refractive index of the optical cement is very similar to that of the glass filters and should almost eliminate internal reflections.

\section{B. Responsivity of the Realized Filter Combinations}

The spectral power responsivities of the colorimeter channels were determined to characterize the filter combinations for $f_{1}{ }^{\prime}$ values. The spectral irradiance responsivity calibrations of the channels will be performed later on the combined filter-detector channels. The four channels with a common aperture at the front will be calibrated against an irradiance mode reference trap detector when both measure the same irradiance from a monochromatic (point) source. Irradiance measuring reference trap detectors are available with $0.1 \%$ responsivity uncertainty. The calibrations will be made at the National Institute of Standards and Technology (NIST) Spectral Irradiance and Radiance Responsivity Calibrations with Uniform Sources (SIRCUS) facility with an irradiance responsivity uncertainty of close to $0.1 \%$. 


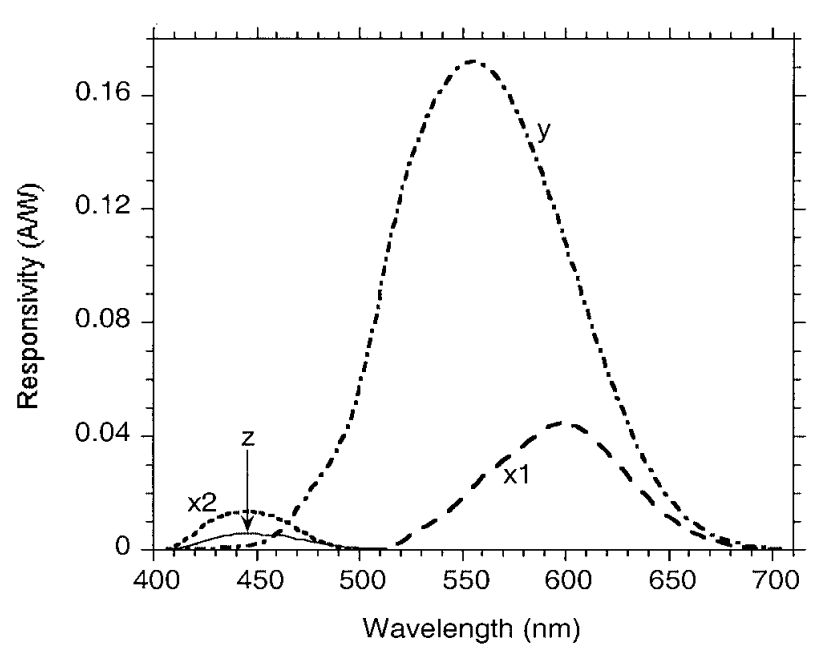

(a)

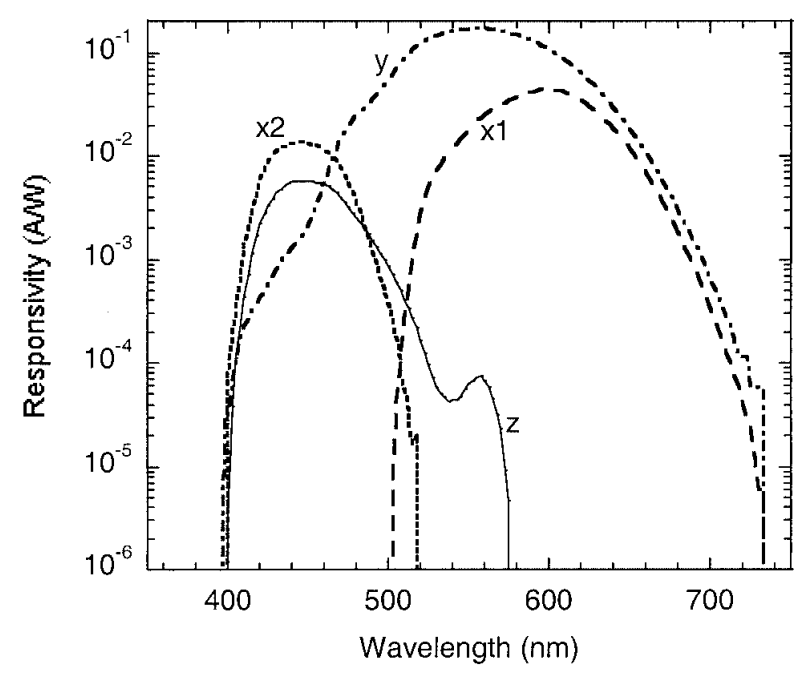

(b)

Fig. 10. Absolute responsivities of the realized tristimulus channels. Spectral responsivity on (a) a linear scale and (b) a logarithmic scale.

The photometer-colorimeter calibrations will be described in a future publication.

The absolute spectral responsivities of the realized channels are shown in Fig. 10(a) on a linear scale and Fig. 10(b) on a logarithmic scale. The logarithmic graph shows the sharp cutoff of the filters at the sides of the responsivity curves. These spectral responsivities were determined from piecewise measurements. The filter transmittances were measured separately in the $360-830 \mathrm{~nm}$ wavelength range with 5-nm increments. The measurements were made with a prism-grating spectrometer. The spectral responsivity of the trap detector was derived from another trap detector calibrated against the primary standard cryogenic radiometer. ${ }^{7}$ The CIE-

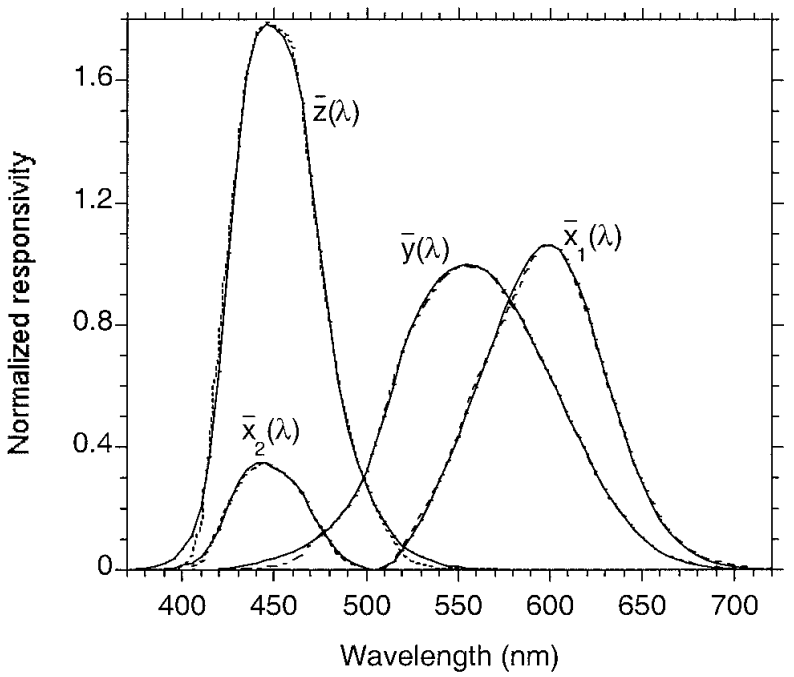

Fig. 11. $\operatorname{CIE} \bar{x}(\lambda), \bar{y}(\lambda)$, and $\bar{z}(\lambda)$ color-matching functions (solid curves) and the realized and then normalized channel responsivities (dashed curves).

defined color-matching functions and the realized and then normalized channel responsivities are shown in Fig. 11.

\section{Spectral Mismatch of the Channels}

The spectral mismatch of the realized channel responsivities relative to the CIE standard functions can be calculated from the above determined channel responsivities. The spectral responsivity mismatch of a realized channel relative to the CIE defined function can be described by the CIEdefined spectral mismatch index ${ }^{2} f_{1}{ }^{\prime}$. According to the definition, $f_{1}{ }^{\prime}$ can be used to define the degree of spectral mismatch of photometers when the goal is to equalize the realized spectral responsivity curve to the $V(\lambda)$ function.

According to the definition,

$$
\begin{aligned}
f_{1}{ }^{\prime} & =\frac{\int_{o}^{\infty}\left|\mathrm{s}^{*}(\lambda)_{\mathrm{rel}}-V(\lambda)\right| \mathrm{d} \lambda}{\int_{o}^{\infty} \mathrm{V}(\lambda) \mathrm{d} \lambda} \times 100 \%, \\
s^{*}(\lambda)_{\mathrm{rel}} & =\frac{\int_{o}^{\infty} S(\lambda)_{A} V(\lambda) \mathrm{d} \lambda}{\int_{o}^{\infty} \mathrm{S}(\lambda)_{\mathrm{A}} \mathrm{s}(\lambda)_{\mathrm{rel}} \mathrm{d} \lambda} s(\lambda)_{\mathrm{rel}}
\end{aligned}
$$

where $s^{*}(\lambda)_{\text {rel }}$ is called the normalized relative spectral responsivity, $S(\lambda)_{A}$ is the spectral distribution of the Standard Illuminant $\mathrm{A}^{11}$ (used in the measurement), $V(\lambda)$ is the spectral luminous efficiency of photopic vision, and $s(\lambda)_{\text {rel }}$ is the relative spectral responsivity of the realized channel. Here we extended the $\operatorname{CIE} f_{1}{ }^{\prime}$ definition from the original photometric channel to all four tristimulus channels by substituting the $\bar{x}_{1}(\lambda), \bar{x}_{2}(\lambda), \bar{y}(\lambda)$, and $\bar{z}(\lambda)$ colormatching functions for the $V(\lambda)$ function.

A mismatch of $f_{1}{ }^{\prime}=1.39 \%$ has been reported ${ }^{12}$ for a $V(\lambda)$ function realization fabricated with a similar (sandwich layer) method. This $f_{1}{ }^{\prime}$ can be misleading, because the wavelength increments were $10 \mathrm{~nm}$ 


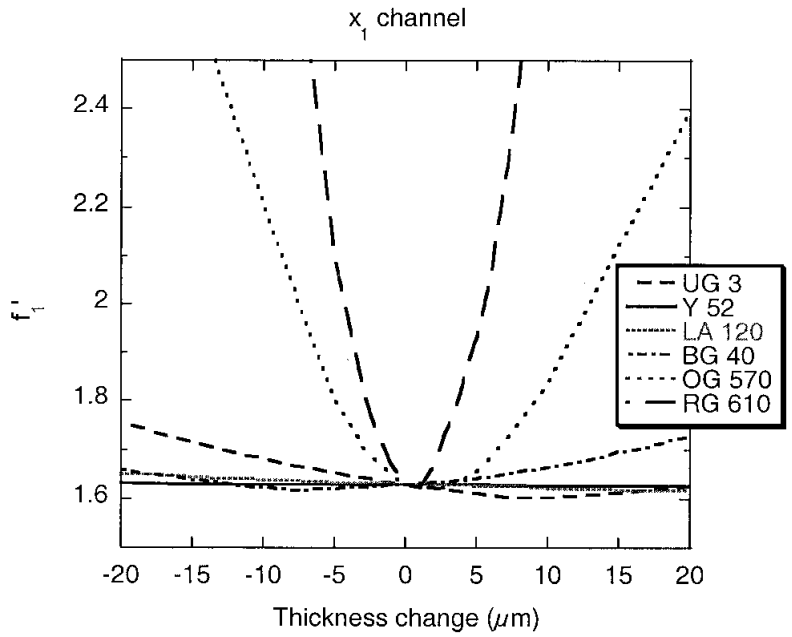

(a)

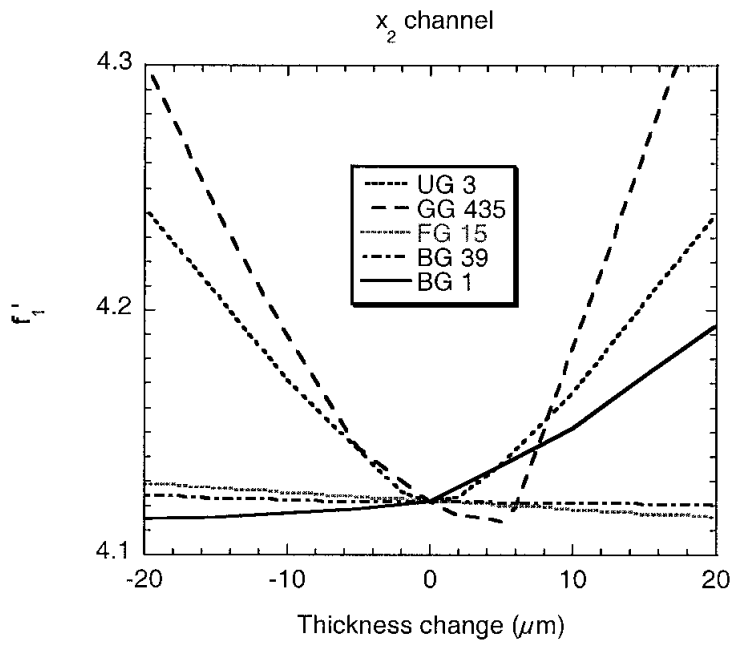

(b)

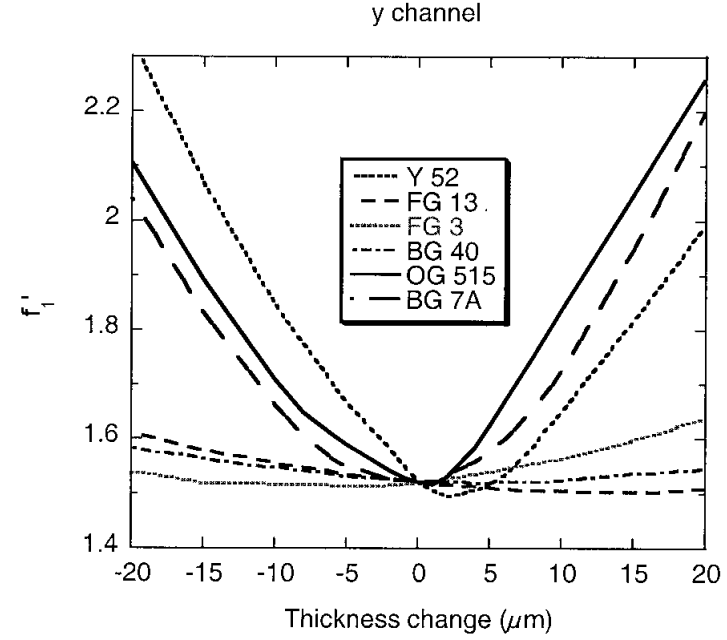

(c)

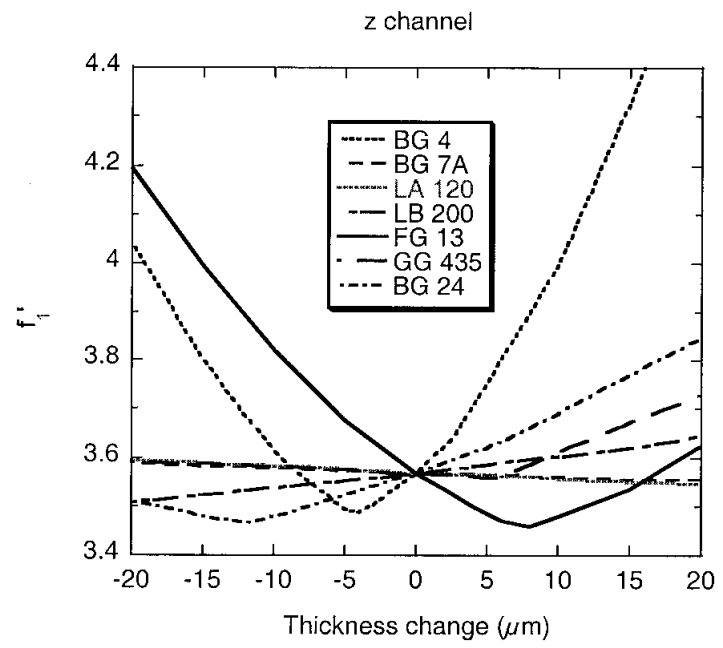

(d)

Fig. 12. $f_{1}{ }^{\prime}$ value dependence of the four channels as functions of the thickness change of the filter layers.

in a shorter wavelength range of $400-750 \mathrm{~nm}$. In the present work the design-related transmittance measurements and the $f_{1}{ }^{\prime}$ optimizations were made between 360 and $830 \mathrm{~nm}$ with 5 -nm increments. These measurements followed the CIE definitions for the color-matching functions. However, our final filter combination measurements were made between 380 and $780 \mathrm{~nm}$ with 5 -nm increments.

\section{Analysis of the $f_{1}{ }^{\prime}$ Values for Fabrication}

It was an important design criterion to understand how the $f_{1}^{\prime}$ values of the realized channels depend on the thickness changes of the individual filter layers inside of a filter combination. Figure 12 shows the changes of the $f_{1}^{\prime}$ values for partial thickness changes of the layers for all of the realized channels. The graphs show that the fabrication of the thinnest filters is the most critical (see Table 1). This was the reason that we started the layer fab- rications within a filter combination with the thinnest filters.

The filter models of the four realized filter combinations with the designed (calculated) and realized thickness of the layers and combinations are summarized in Table 1. Table 1 shows that the fabricated thickness of the filter layers can be equal to or very different from the calculated (designed) values. The allowed thickness deviation was dependent on filter type and filter combination. We controlled the layer polishing tolerances according to the results of Fig. 12. The calculated (designed) and realized $f_{1}{ }^{\prime}$ and peak transmittance $\left(\tau_{\max }\right)$ values are also shown in the table. The $f_{1}^{\prime}$ values were calculated from the spectral responsivities of the channels after the realization of the filter combinations. The channel responsivities were determined from separate transmittance (for the filter combinations) and responsivity (for the trap-detector) measurements. 
Table 1. Filter Types with Designed (Calculated) and Realized Thickness of Layers, Spectral Mismatch Values, and Maximum Transmittances for the Four Filter Combinations

\begin{tabular}{|c|c|c|c|c|c|}
\hline \multirow[b]{2}{*}{ Filter } & \multicolumn{2}{|c|}{ Thickness $(\mu \mathrm{m})$} & \multirow[b]{2}{*}{ Filter } & \multicolumn{2}{|c|}{ Thickness $(\mu \mathrm{m})$} \\
\hline & Calculated & Realized & & Calculated & Realized \\
\hline $\mathrm{X}_{1}$ & & & & & \\
\hline BG $40^{a}$ & 3100 & 3093 & $\mathrm{BG} 1^{a}$ & 3120 & 3149 \\
\hline OG $570^{a}$ & 54 & 60 & $\mathrm{BG} 39^{a}$ & 2028 & 2031 \\
\hline $\mathrm{RG} 610^{a}$ & 42 & 42 & $\mathrm{FG} 15^{a}$ & 4236 & 4250 \\
\hline $\mathrm{UG} 3^{a}$ & 1215 & 1143 & GG $435^{a}$ & 170 & 169 \\
\hline LA $120^{b}$ & 1556 & 1728 & $\mathrm{UG} 3^{a}$ & 162 & 148 \\
\hline $\mathrm{Y} 52^{b}$ & 2850 & 2531 & Total & 9716 & 9747 \\
\hline Total & 8817 & 8597 & $f_{1}^{\prime}$ & $3.83 \%$ & $4.12 \%$ \\
\hline$f_{1}^{\prime}$ & $1.55 \%$ & $1.63 \%$ & $\tau_{\max }$ & $3.41 \%$ & $3.36 \%$ \\
\hline$\tau_{\max }$ & $8.15 \%$ & $8.93 \%$ & & & \\
\hline $\mathrm{Y}$ & & & & & \\
\hline $\mathrm{BG} 7 \mathrm{~A}^{a}$ & 288 & 297 & $\mathrm{BG} 4^{a}$ & 376 & 375 \\
\hline $\mathrm{BG} 40^{\circ}$ & 2623 & 2585 & $\mathrm{BG} 7 \mathrm{~A}^{a}$ & 644 & 433 \\
\hline $\mathrm{FG} 3^{a}$ & 63 & 63 & $\mathrm{BG} 24^{a}$ & 1945 & 1857 \\
\hline $\mathrm{FG} 13^{a}$ & 1506 & 1563 & $\mathrm{BG} 39^{a}$ & 2028 & 2028 \\
\hline OG $515^{a}$ & 43 & 44 & $\mathrm{FG} 13^{a}$ & 676 & 654 \\
\hline $\mathrm{Y} 52^{b}$ & 120 & 115 & GG $435^{a}$ & 153 & 156 \\
\hline Total & 4643 & 4667 & LA $120^{b}$ & 2820 & 2969 \\
\hline$f_{1}^{\prime}$ & $1.47 \%$ & $1.58 \%$ & LB $200^{b}$ & 1226 & 1265 \\
\hline$\tau_{\max }$ & $38.04 \%$ & $36.43 \%$ & Total & 9868 & 9737 \\
\hline & & & $f_{1}^{\prime}$ & $3.31 \%$ & $3.57 \%$ \\
\hline & & & $\tau_{\max }$ & $1.65 \%$ & $1.79 \%$ \\
\hline
\end{tabular}

${ }^{a}$ Schott filter.

${ }^{b}$ Hoya filter.

\section{E. Spectral Transmittance Differences}

As a result of our rigorous filter fabrication procedure, the relative transmittance curves of the filter combinations are very similar to the designed transmittance curves. However, the differences between the realized (and designed) curves and the CIE functions are significantly larger. The performed transmittance differences are illustrated in Fig. 13 for the

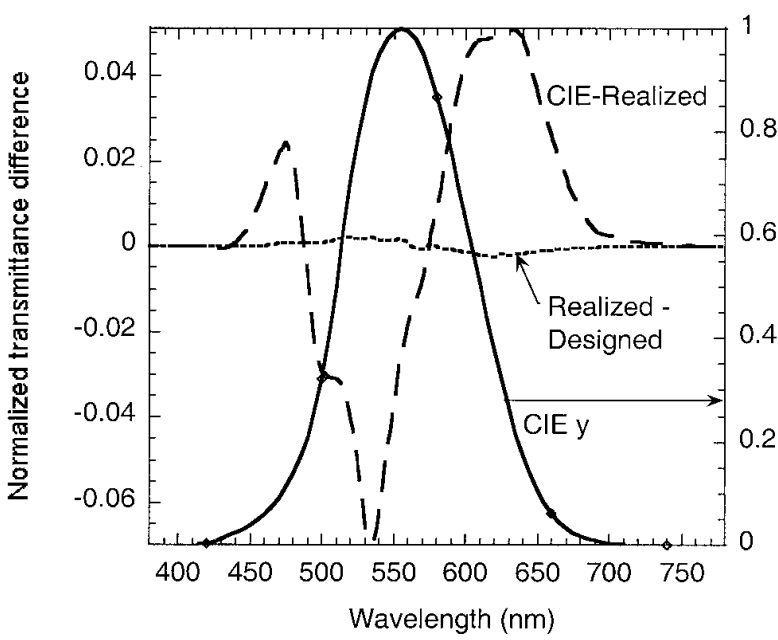

Fig. 13. Transmittance differences (left $Y$ axis) of the realized photometric channel relative to the CIE and designed (normalized) spectral transmittances. The CIE function is shown to illustrate where the significant differences are. photometric channel. The calculated transmittance was normalized to the measured values. The CIE function is shown to illustrate where the significant differences are. A highest transmittance difference (between the realized function and the CIE function) of 0.07 was obtained at $535 \mathrm{~nm}$. The spectral transmittance differences relative to the CIE functions were dominated by the availability of filter components. Table 1 shows that the maximum (peak) transmittance, $\tau_{\max }$, values after the realizations were randomly different for the four filter combinations. This variation was caused by the fabrication thickness tolerance that we allowed for the thick filter layers.

The Table also shows that the $f_{1}{ }^{\prime}$ values of the fabricated filter combinations are all somewhat higher than the designed values. The increase was obtained for a few reasons. The largest change was caused by the temperature difference between the design and final transmittance measurements. The $f_{1}{ }^{\prime}$ values depend on the temperature because the cut-on edges of the filters, used on the shortwavelength sides of the transmittance curves, are temperature dependent. During the design measurements the temperature of the filters was not controlled. The lack of temperature-controlled filter holders made the design measurements simpler and faster. The transmittance of the realized filter combinations was measured at $26{ }^{\circ} \mathrm{C}$ in the temperaturecontrolled filter wheel. Here temperature stabilization was necessary to achieve high stability. The temperature of the filter wheel was controlled to $\sim 3{ }^{\circ} \mathrm{C}$ above room temperature to avoid condensation. The increase in the $f_{1}{ }^{\prime}$ values can be less if the filters are measured and used at the same temperature (for both design and applications).

Also, significant $f_{1}^{\prime}$ changes were obtained because of the limited sensitivity of the transmittancemeasuring spectrophotometer. Sometimes filter transmittances had to be calculated from transmittances measured with different filter thicknesses and limited signal resolution. The thin filters needed special attention. The transmittance of a thin filter has to be measured, because it cannot be calculated from the transmittance measurement of the same thick filter. Also, when thin filters of large diameter are to be made, they should be cemented to a transparent glass substrate before polishing. The thick substrate (with spectrally constant transmittance) helps avoid cracks and can make polishing and thickness measurements easier. The final transmittance of a filter combination will be a product of the spectral transmittances of the five to eight individual layers, the glass substrate, and the optical cement between the layers.

Without careful filter combination design and fabrication, the $f_{1}^{\prime}$ can be several percent or higher. With the careful design and fabrication procedure described above, the $f_{1}{ }^{\prime}$ of the $\bar{y}(\lambda)$ and $\bar{x}_{1}(\lambda)$ channels was less than $2 \%$ for the $380-780 \mathrm{~nm}$ wavelength range with $5-\mathrm{nm}$ increments. The implementation of the blue channels is more difficult because of the 
smaller number of suitable filters available for this range. Eight filters were needed for the $\bar{z}(\lambda)$ channel to achieve an $f_{1}{ }^{\prime}=3.57 \%$. An $f_{1}{ }^{\prime}$ of $\sim 4 \%$ seems to be the best realization for the $\bar{x}_{2}(\lambda)$ channel.

\section{Photocurrent Measurement Uncertainty}

The design of the photocurrent-to-voltage converter for the silicon tunnel-trap detector has already been published. ${ }^{6}$ The improvement introduced in the present design resulted in an uncertainty reduction in the current-to-voltage conversion for all signal gains. We used feedback resistors with $0.01 \%$ uncertainties of their decadic nominal values and temperature coefficients of 10 parts in $10^{6} /{ }^{\circ} \mathrm{C}$. Accordingly, calibrations for the individual signal gains were not necessary anymore. The dynamic signal range of the photometercolorimeter is 11 decades in dc measurement mode with a noise-equivalent photocurrent of $32 \mathrm{fA}$ at a bandwidth of $0.3 \mathrm{~Hz}$.

\section{Conclusion}

A new photometer and tristimulus colorimeter has been developed to implement the first detector-based tristimulus color scale we published recently. ${ }^{1}$ The design considerations of a silicon tunnel-trap detector that was extended by a temperature-controlled filter wheel, an aperture, a removable luminance measuring tube, and an improved current-to-voltage converter have been described. Four filter combinations were made to implement the CIE colormatching functions with $f_{1}{ }^{\prime}$ values whose small size constitutes a benchmark for subsequent work. The main goal was to realize a colorimeter in which the channel spectral responsivities could be determined with uncertainties of $0.1 \%$.

The characterization results on the uniformity of spatial responsivity, angular responsivity, FOV, and out-of-field blocking verified the optical and mechanical design expectations. Photocurrents from the trap detector could be measured with an uncertainty of $0.01 \%$ in a dynamic range of 11 decades. The spectral transmittance of the realized filter combinations and the spectral responsivity of the trap detector were measured to determine the $f_{1}{ }^{\prime}$ values of the realized channels. An analysis showed the $f_{1}{ }^{\prime}$ dependence on the thickness change of the filter layers inside of a filter combination. As a result of the rigorous filter realization procedure, the $f_{1}{ }^{\prime}$ values of the realized channels were dominated by the limited number and availability of suitable filter components. Very small $f_{1}^{\prime}$ values were realized to allow us to use the same set of calibration factors for the colorimeter channels and to obtain small color measuring uncertainties even when the spectral distribution of the measured source is different (e.g., when it changes) from the distribution used to determine the calibration factors. The colorimeter can measure sources with all kinds of spectral distribution. A new set of calibration factors will be needed only for very different source distributions, such as discharge lamps or displays (cathode ray tube or liquid crystal).

The described design and realization consider- ations, the results of measurements and characterizations, and the planned and available calibration methods, devices, and setup promise a real possibility for determining the spectral irradiance and radiance responsivities of the colorimeter channels with the expected $0.1 \%$ uncertainty. After calibration, the reference photometer-colorimeter will be used to propagate NIST realized detector-based scales to field-level applications. We hope to discuss the spectral irradiance responsivity calibration of the channels and the determination of the calibration factors for different source distributions in a future paper.

The authors thank Don Lynch of Reyer Corporation for the mechanical fabrication and filter wheel design, Thomas Larason for the detector spatial response uniformity test, Steve Brown for the pointspread response test, Edward Early for measuring the spectral transmittance of the filter packages, and David Wilmering and John Fuller for fabricating the filter packages.

\section{References and Notes}

1. G. Eppeldauer, "Spectral response based calibration method of tristimulus colorimeters," J. Res. Natl. Inst. Stand. Technol. 103, 615 (1998).

2. Publication CIE No. 69 (Central Bureau of the CIE, Vienna, 1987).

3. All uncertainties discussed in this paper are relative expanded uncertainties with a coverage factor of two.

4. G. P. Eppeldauer, S. W. Brown, C. C. Miller, and K.R. Lykke, "Improved accuracy photometric and tristimulus-color scales based on spectral irradiance responsivity," in Proceedings of the Twenty-Fifth Session of the CIE (Central Bureau of the CIE, Vienna, 2003), Vol. 1, pp. D2-30-D2-33.

5. D. W. Allen, G. P. Eppeldauer, S. W. Brown, E. A. Early, B. C. Johnson, and K. R. Lykke, "Calibration and characterization of trap detector filter radiometers," in Earth Observing Systems VIII, W. L. Barnes, ed., Proc. SPIE 5151, 471-479 (2003).

6. G. P. Eppeldauer and D. C. Lynch, "Opto-mechanical and electronic design of a tunnel-trap Si-radiometer,” J. Res. Natl. Inst. Stand. Technol. 105, 813-828 (2000).

7. T. R. Gentile, J. M. Houston, and C. L. Cromer, "Realization of a scale of absolute spectral response using the NIST high accuracy cryogenic radiometer," Appl. Opt. 35, 4392-4403 (1996).

8. The mention of certain commercial products in this paper is for information purposes only and does not constitute an endorsement of the product by the authors or their institutions.

9. G. P. Eppeldauer, S. W. Brown, T. C. Larason, M. Racz, and K. R. Lykke, "Realization of a spectral radiance responsivity scale with a laser-based source and Si radiance meters," Metrologia 37, 531-534 (2000).

10. G. Czibula, "Producing a detector with predetermined spectral responsivity," in Proceedings of the International Measurement Confederation (IMEKO) 10th International Symposium of the Technical Committee on Photon Detectors (IMEKO Secretariat, Budapest, 1982), Vol. 1, pp. 189-199.

11. Publication CIE No. 15.2 (Central Bureau of the CIE, Vienna, 1986).

12. J. P. Makai, G. Czibula, and J. Schanda, "The importance of the spectral responsivity correction in case of photoelectrical elements," in Proceedings of the International Measurement Confederation (IMEKO) 11th International Symposium of the Technical Committee on Photon Detectors (IMEKO Secretariat, Budapest) (1984), pp. 215-225. 


\title{
NEW PHOTOMETER STANDARDS FOR LOW UNCERTAINTY ILLUMINANCE SCALE REALIZATION
}

\author{
G. P. Eppeldauer, C. C. Miller, and Y. Ohno \\ National Institute of Standards and Technology, USA
}

\begin{abstract}
Improved performance photometers have been developed at the National Institute of Standards and Technology (NIST) to utilize the low spectral responsivity uncertainty of spectral irradiance responsivity measurements performed on the facility of Spectral Irradiance and Radiance Responsivity Calibrations using Uniform Sources (SIRCUS). Based on the improved design of the transfer standard photometers, a lower uncertainty detector-based photometric scale is being realized with an uncertainty goal of $0.1 \%(\mathrm{k}=2)$. The existing photometer standards that maintain the present NIST illuminance unit can be calibrated against the new transfer standards. In addition, new generation working standard photometers have been developed to utilize the extended group policy to better monitor and correct responsivity changes. The working standards propagate the new reference scales from the transfer standards with a minimal increase in measurement uncertainty. Design issues of the new photometers and the improved scale realization and transfer are discussed.
\end{abstract}

Keywords: detector-based calibration, illuminance, photometry, spectral responsivity, standard

\section{INTRODUCTION}

The present NIST illuminance unit is maintained on a group of eight photometer standards with a relative expanded uncertainty $(k=2)$ of $0.39 \%$ [1]. Several components in the uncertainty budget are related to the determination of the photometer relative spectral responsivities using the traditional lamp/monochromator system of the NIST Spectral Comparator Facility (SCF). The dominant uncertainties originate from the converging beam geometry, the small spot size on the photometers, wavelength errors, aperture area measurement error, and the spatial non-uniformity of the detector and filter combination. A significant long-term uncertainty component originates from stain settlement (build-up of a layer) on the front surface of a filter package that decreases the responsivity of the photometer. The standard photometers, built in 1990, cannot be calibrated on the SIRCUS facility because the coherence in the laser-based sphere sources results in $10 \%$ level interference fringes in the output signals. Instead, new generation transfer standard photometers with optimized optical, photometric, and electronic characteristics have been developed. The improved scale is realized on the new transfer standard photometers. The old photometer standards, together with the new generation working standard photometers, can be calibrated against the new transfer standard photometers by measuring the illuminance from a $2856 \mathrm{~K}$ color temperature standard lamp. The working standard photometers will be recalibrated yearly against the transfer standards and their long term stability will be monitored. The extended group of working standards is used for routine photometer calibrations and photometric scale dissemination.

\section{TRANSFER STANDARDS}

The transfer standards were designed such that it is the uncertainty of the spectral irradiance responsivity calibrations that determines the photometric scale uncertainty and not limitations in the performance of the photometers themselves. New filter combinations have been designed with a minimum allowed thickness of $4.5 \mathrm{~mm}$ to avoid interference fringes. They are individually fabricated to closely match the responsivity of the silicon detector to the $\operatorname{CIE~} V(\lambda)$ function [2]. The filter combinations are in a filter wheel located between the common detector and aperture. The wheel temperature is controlled at $25{ }^{\circ} \mathrm{C}$ where the spectral mismatch errors were minimized.

Two types of transfer standard photometers were designed and built. The first type, as shown in Fig. 1, uses windowless silicon photodiodes in a tunnel trap configuration [3]. The cut-outs were made for better illustration. The long-term stability of this photometer is regularly monitored to see if any responsivity changes happen due to either stain settlement on the filter surfaces or 
because the photodiodes are exposed to the ambient air. A few tenths of a percent changes have been observed by us earlier on open Si trap detectors at $400 \mathrm{~nm}$. The changes gradually decreased and became negligibly small at $500 \mathrm{~nm}$. In this tunnel-trap detector, light is not reflected back to the filters.

In the improved (newer) transfer standard, a single-element silicon photodiode is used which has a sealed wedged-window to avoid fringes. The picture of the new transfer standard (with the front panels removed) is shown in Fig. 2. The front panels are removed for better illustration. The photo shows the light tight box with the detector and the filter-wheel inside. The photocurrent meter is attached to the side of the box. The current measuring electronics are matched to the electronic characteristics of the photodiode for low-noise performance over a signal dynamic range of 12 decades. The uncertainty of the current-to-voltage conversion is $0.02 \%(k=2)$.

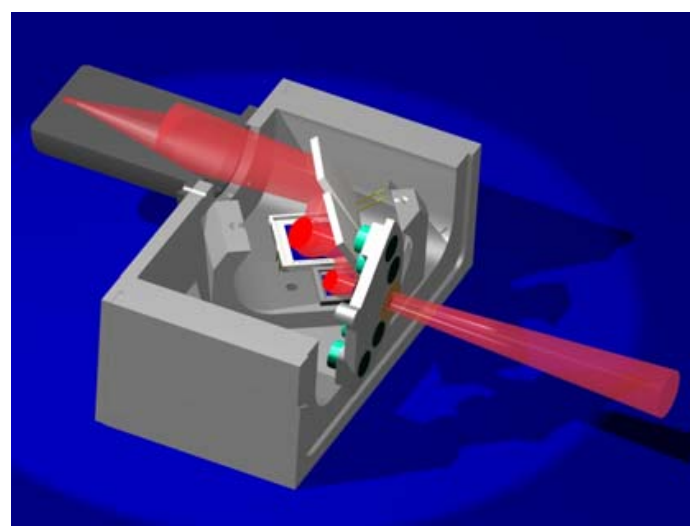

Fig. 1. The filter-trap transfer standard.

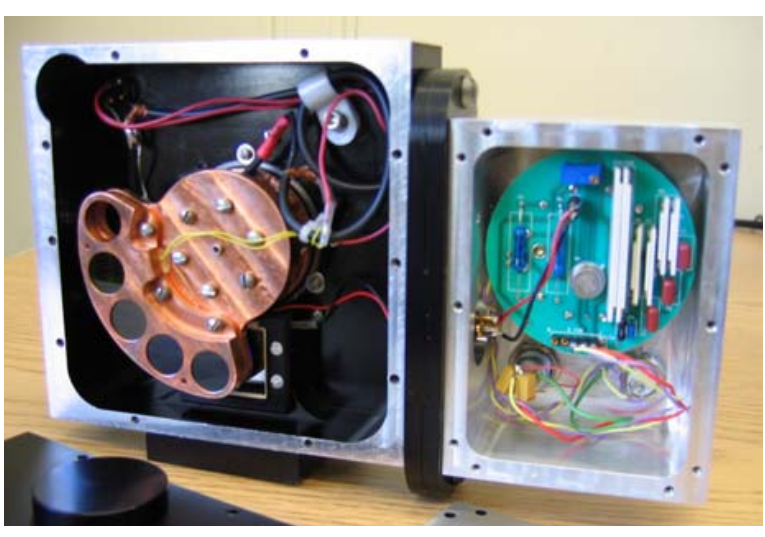

Fig. 2. The new generation transfer standard

\section{WORKING STANDARDS}

The illuminance responsivities of the present working standard photometers have been determined yearly based on their spectral power responsivity measurements at the SCF. Figure 3 shows the long-term changes normalized to unity in 1991. Yearly scale realizations were made to determine the actual responsivities based on the SCF scale realizations. The thick line shows a $0.3 \%$ maximum responsivity change for the group average of photometers 4,6 , and 8 . The front surfaces of the filters in photometers 1 and 2 were cleaned first in 1994. The other photometers were not cleaned until 2006, when the front surfaces of all photometer filters were cleaned. The results show that the long term stability of the individual photometers can be improved to +/$0.1 \%$ if the front surfaces of the filters are cleaned on a regular basis. Change of photometer 5 after 2003 is due to repairs.

Ten-year long changes of $0.02 \%$ were measured at the SCF on silicon photodiodes sealed with fused silica windows used in these working standards. It can be concluded that change-free photometer standards can be made if the photometer head is sealed with a fused silica window. The stability of the working standards should be matched to the lower uncertainty of the SIRCUS-based scale to obtain an overall uncertainty improvement in the disseminated scale. A package design was applied for both the old and the new working standard photometers to obtain a uniform temperature distribution for the filter combination and the silicon photodiode. The spectral transmittance of the $V(\lambda)$ curve is temperature dependent. Also, the applied infrared-suppressed silicon photodiode has a significant temperature coefficient of

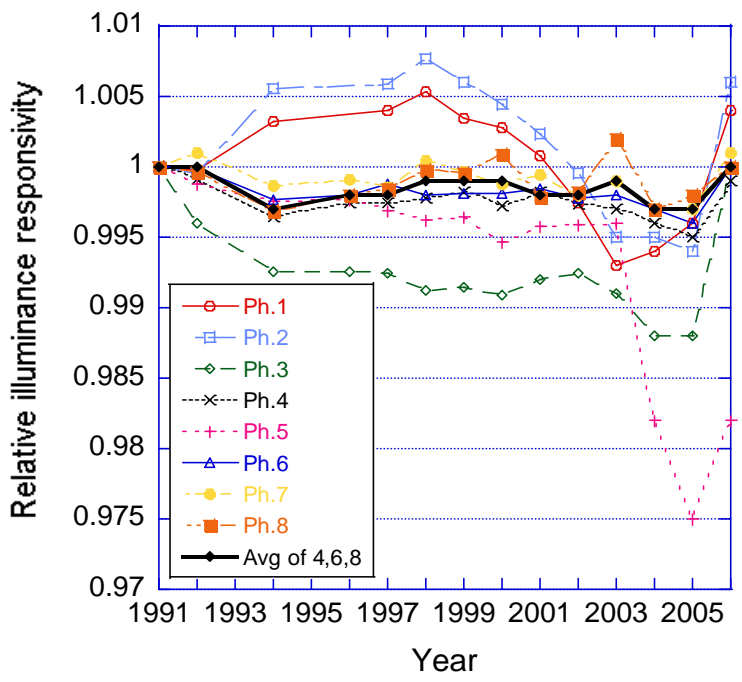

Fig. 3. Long-term changes of the old photometers. All photometers were cleaned in 2006 and Ph.1 and Ph.2 were cleaned in 1994 and 1997. 


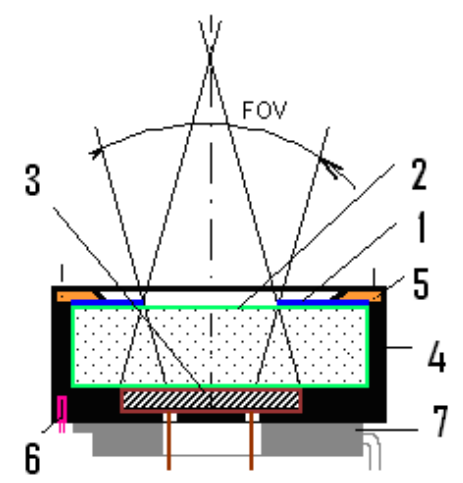

Fig. 4. Structure of a photometer package

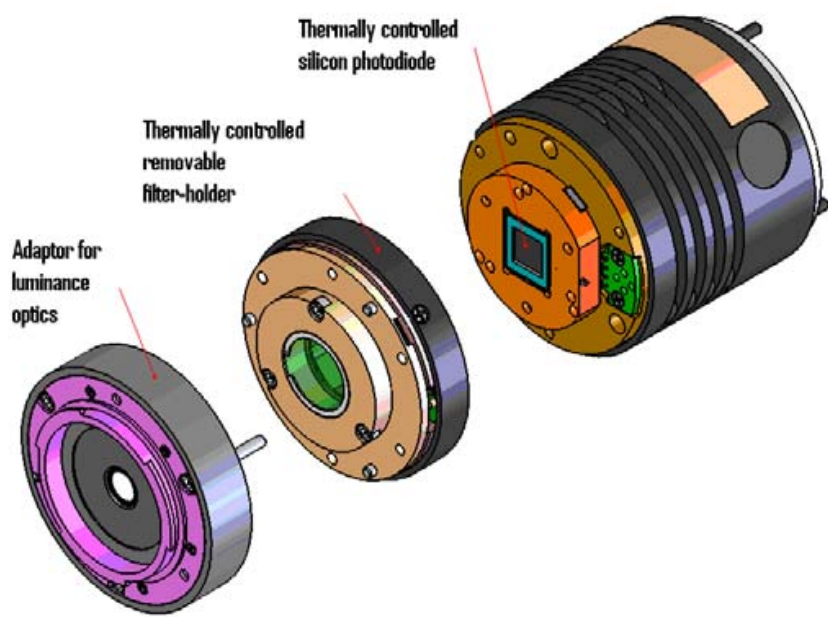

Fig. 5. A new generation working standard.

responsivity at wavelengths longer than $630 \mathrm{~nm}$. These two components are mounted in a metal housing, and the uniform temperature is monitored in the existing (old) photometers [1], and stabilized in the new working standards. As shown in Fig. 4, the aperture (1), the filter combination (2), and the photodiode (3) are close to each other inside of a Copper pot (4). While there is a small gap between the photodiode and the filter, the very thin aperture is mounted on the front surface of the filter to avoid inter-reflections. In order to simplify cleaning the front surface of the filter, the aperture is fixed in a holder (5) that can be easily removed and relocated into the same position using an asymmetric screw arrangement. A temperature sensor (6) is plugged into the copper pot. A ring shape thermoelectric cooler (7) is mounted to the bottom of the copper pot in the new working standards to keep the temperature constant, just above the ambient.

The field-of-view (FOV) of the illuminance meter depends on the diameter of the aperture and the photodiode active area. It is always kept small (e.g. $11^{\circ}$ ) to reject ambient light as well as reflections and stray light from a test source. The base unit, as shown on the right side of Fig. 5, can be equipped with a bayonet mount at the front to attach an input optic for luminance measurement. In this example, the filter can be removed; therefore its temperature is independently controlled from the temperature of the photodiode. The photocurrent meter is located inside of another cylindrical housing attached to the bottom of the base unit. The electronic characteristics of the current-to-voltage converter are matched to the high shunt resistance of the silicon photodiode to obtain low output noise and wide signal range.

\section{RESPONSIVITIES}

The spectral irradiance responsivities of the transfer standards were determined against two irradiance-measuring reference trap detectors. As an example, the spectral irradiance responsivity of one of the transfer standards, as measured on the SIRCUS, is shown in Fig. 6. The left- $Y$ axis shows the measured data on a linear scale. The right- $Y$ axis is a logarithmic scale to show the out-of-band responsivity of the realized $\mathrm{V}(\lambda)$ curve.

The illuminance responsivity $s_{e}$, which is the ratio of the photometer output current to the illuminance measured by the photometer, was determined from the SIRCUS measured spectral irradiance responsivity $s_{E}(\lambda)$ and the incident irradiance $E(\lambda)$ from a CIE Standard Illuminant A source:

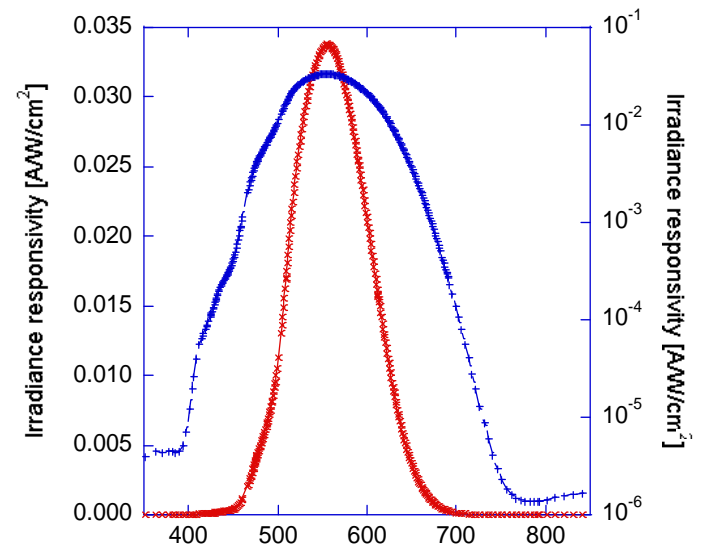

Fig. 6. Spectral irradiance responsivity of the filter-trap transfer standard. 


$$
s_{\mathrm{e}}=\frac{\int s_{\mathrm{E}}(\lambda) E(\lambda) d \lambda}{K_{\mathrm{m}} \int V(\lambda) E(\lambda) d \lambda}
$$

where $K_{\mathrm{m}}$ is the maximum spectral luminous efficacy, $683 \mathrm{Im} / \mathrm{W}$ and $\lambda$ is the wavelength.

The illuminance responsivity has also been measured against the SCF-based scale. The illuminance responsivity of the new generation transfer standard was measured against the average illuminance responsivity of a selected group of the old photometer standards. The photometers measured the same illuminance from a $2856 \mathrm{~K}$ color temperature lamp. The preliminary ratio of the SIRCUS-based illuminance responsivity to the SCF-based illuminance responsivity at gain $10^{8}$ VIA is $\approx_{1.004}$. The relative expanded uncertainty of the SIRCUS-based illuminance responsivity is estimated to be $0.1 \%(k=2)$. This preliminary uncertainty is dominated by a constant $0.08 \%$ irradiance responsivity difference in the visible wavelength range between the two reference trap detectors used to calibrate the transfer standards. Also, the ratio obtained at the previous photometric scale comparison in 2003, using the filter-trap transfer standard, was 1.0038. At present, the data evaluation for the filter-trap transfer standard is still being done. There will be further comparisons of the two scales after the annual SCF-based calibration of the old photometers.

\section{CONCLUSIONS}

The results of a research work to improve the uncertainty of the NIST detector-based photometric scale are presented. Transfer standard illuminance meters have been developed and calibrated on the SIRCUS facility with decreased spectral irradiance responsivity uncertainty. In order to decrease the long-term responsivity changes of the old (presently used) working standard photometers that maintain the NIST photometric scale, the old group has been extended with new-generation photometers. The extended group can be calibrated against the new transfer standards. Repeated scale realizations since 1991 verified that the long term responsivity changes of the photometers are caused by the development of stain layers at the front surface of the $V(\lambda)$ filter packages. The instabilities of the old photometer standards could be decreased to within $+/-0.1 \%$ of their original (clean) responsivities after the front surfaces of the filters were cleaned. It was suggested that photometer working standards, free of responsivity changes (without any cleaning), can be made if the front of the photometer head is sealed with a fused silica window. The working standard group policy was utilized to determine and then to minimize the long term responsivity changes. Using the SIRCUS-based lower scale uncertainty and the minimized long-term change in the average responsivity of a selected working standard group, the uncertainty of routine illuminance meter calibrations can be reduced by about a factor of two.

\section{ACKNOWLEDGEMENTS}

The authors thank R. Bousquet, S. Brown, and K. Lykke for help with the SIRCUS measurements.

\section{REFERENCES}

1. Cromer, C.L., et al., The NIST detector-based luminous intensity scale. J. Res. NIST, 1996. 101(2): p. 109-132.

2. CIE, The Basis of Physical Photometry. Publication CIE Central Bureau, A-1033 Vienna, P. O. Box 169, Austria, 1983. 18.2.

3. Eppeldauer, G.P. and D.C. Lynch, Opto-mechanical and electronic design of a tunnel-trap Siradiometer. J. Res. Nat'l. Inst. Stand. Technol., 2000. 105(6): p. 813-828.

Authors:

George Eppeldauer

National Institute of Standards and Technology

Gaithersburg, Maryland 20899 USA

Phone: 301-975-2338

e-mail: george.eppeldauer@nist.gov
Cameron Miller

National Institute of Standards and Technology

Gaithersburg, Maryland 20899 USA

Phone: 301-975-4713

e-mail: ccmiller@nist.gov

Yoshi Ohno 
Eppeldauer, et al. New photometer standards...

National Institute of Standards and Technology

Gaithersburg, Maryland 20899 USA

Phone: 301-975-2321

e-mail: ohno@nist.gov 


\title{
IMPROVED ACCURACY PHOTOMETRIC AND TRISTIMULUS-COLOR SCALES BASED ON SPECTRAL IRRADIANCE RESPONSIVITY
}

\author{
George P. Eppeldauer, Steven W. Brown, C. Cameron Miller, and Keith R. Lykke \\ National Institute of Standards and Technology \\ Optical Technology Division \\ Gaithersburg, Maryland, 20899 USA
}

\begin{abstract}
A new spectral irradiance and radiance responsivity calibration with uniform sources (SIRCUS) facility has been developed at the National Institute of Standards and Technology (NIST) that can calibrate radiometers in irradiance measurement mode with expanded uncertainties $(k=2)$ of $0.15 \%$. A reference tristimulus colorimeter/photometer has been developed to reduce the uncertainty in the NIST photometric units and to realize a detector-based color scale. A novel, spectrally tunable, solid-state source has also been developed to transfer the illuminance responsivity and tristimulus color scales from the reference colorimeter/photometer to test photometers and colorimeters that measure light sources with specific spectral distributions. By setting the transfer source distribution to approximate a particular target distribution, test photometers and colorimeters, without knowing their relative spectral responsivities, can be calibrated against the reference instrument with almost no spectral mismatch errors resulting in low photometric and color measurement uncertainties.
\end{abstract}

Keywords: colorimetry, detector-based calibration, illuminance, irradiance, photometry, spectral responsivity, standard, transfer-source, tristimulus

\section{INTRODUCTION}

Improvements in detector technology over the past decade have opened a new era in the field of radiometric and photometric calibrations. Lower measurement uncertainties can be achieved with modern detector standards than with traditionally used source standards. Consequently, fundamental photometric and radiometric scales at NIST are currently realized and maintained using detector-based standards [1, 2]. The detector-based approach is being extended to colorimetry as well [3]. In the present NIST photometric units, a major source of uncertainty is the spectral responsivity measurements of the photometers using the NIST Spectral Comparator Facility (SCF) [4] that utilizes a monochromator and a lamp.

A calibration facility for Spectral Irradiance and Radiance Responsivity Calibrations using Uniform Sources (SIRCUS) has been developed at NIST to overcome the limitations of the SCF, in particular for calibrating irradiance and radiance meters $[5,6]$. The facility introduces the output from tunable lasers into integrating spheres, producing a monochromatic, high-power uniform source. The responsivity scale is transferred from the NIST High-Accuracy Cryogenic Radiometer [7] and maintained on irradiance standard 6-element transmission trap detectors [8]. Their spectral power responsivity is determined using a cryogenic electrical substitution radiometer, and their irradiance responsivity is derived by placing a calibrated aperture at the input plane. The SIRCUS facility has several advantages over conventional lamp-monochromator systems to realize photometric and colorimetric scales. It provides high-power, low wavelength uncertainty, and the ability to produce a monochromatic Lambertian radiation that enables calibration of instruments directly for irradiance or radiance responsivity.

The overall relative combined expanded $(k=2)$ uncertainty for the NIST illuminance unit realization using the SCF is currently $0.39 \%$ [1]. There are several components in the uncertainty budget related to the determination of the photometer spectral responsivity in the SCF [1]. These uncertainty components can be significantly reduced or eliminated by realizing the illuminance scale on SIRCUS.

It is expected that the standard uncertainty in the spectral responsivity scale will be reduced from $0.11 \%$ (measured on the SCF) to $0.075 \%$ due to the reduction of transfer steps from the absolute cryogenic radiometer. The CIE spectral luminous efficiency, $V(\lambda)$, is a strong function of wavelength. The SIRCUS wavelength scale, with uncertainties reduced to $0.001 \mathrm{~nm}$ from the current SCF's wavelength uncertainty of $0.1 \mathrm{~nm}$, will reduce the relative standard uncertainty for this component from $0.04 \%$ to less than $0.001 \%$. A significant set of uncertainty components results from the geometry of the SCF output beam. The SCF uses $f / 9$ optics to focus the light to a $1 \mathrm{~mm}$ spot that is used to calibrate the photometers. The photometers are used in a far field condition. SIRCUS can calibrate photometers in a geometrical configuration identical to the application geometry. In addition, since SIRCUS calibrates the detector in irradiance mode instead of power mode, the uncertainty of the aperture area and the uncertainty due to the non-uniform spatial response of the photometer will be eliminated. The combined uncertainty from these components related to measurement geometry can be reduced from $0.12 \%[1]$ to less than $0.01 \%$. The overall relative expanded uncertainty $(k=2)$ for the NIST illuminance unit realization is expected to be reduced to $0.24 \%$, a $40 \%$ reduction in the 
uncertainty in the current scale, when the new illuminance scale is realized. The new illuminance scale will lead to an expected overall relative expanded uncertainty of $0.27 \%(k=2)$ for the NIST candela.

Similar reductions in the uncertainty of the color scale may be expected as well. Typically, a $0.1 \%$ uncertainty in the spectral irradiance responsivity of any one channel of the tristimulus colorimeter will result in a change of 0.0004 in the $x, y$ chromaticity coordinates (for a Planckian radiator). These expected colorimeter uncertainties are equivalent to the uncertainties of the current primary lamp standards. Though it may be challenging to further reduce color uncertainties with the detector-based calibration, it leaves future possibility with further improvement of the spectral irradiance responsivity uncertainty.

The SIRCUS facility is used to calibrate primary photometers and colorimeters spectrally. Generally, the scale is transferred from the primary reference instruments to test photometers and colorimeters by direct substitution against incandescent lamp sources (typically operated at $2856 \mathrm{~K}$ ). Test instruments are often used to measure sources with markedly different spectral distributions, e.g. discharge lamps, displays, light emitting diodes (LEDs), etc. Because the instrument's responsivity does not exactly match the $V(\lambda)$ or the color-matching functions, correction factors need to be applied to the instruments to maintain low uncertainty [9]. For such a spectral mismatch correction, however, the relative spectral responsivity of the test instruments must be known. In many cases, such data are not available and it would be often too costly for users to measure them. To avoid such difficulties, test instruments with unknown relative spectral responsivity can be calibrated with standard photometers/colorimeters (with known relative spectral responsivity) using the particular light source to be measured by the test instrument. It is not practical to maintain artifacts of all possible types of specialty sources at NIST. To solve this problem, we have developed a spectrally tunable transfer source based on light emitting diodes (LEDs) [10]. This source is designed to mimic the spectral distributions of both traditional and special test sources. It will be used to propagate the NIST reference scales to field level photometers and colorimeters with small increases in the uncertainties.

\section{REFERENCE TRISTIMULUS COLORIMETER/PHOTOMETER}

Improved performance photometer and tristimulus colorimeter standards were developed using temperaturestabilized, filtered single-element and tunnel-trap silicon detectors to utilize the very low uncertainties of the new SIRCUS spectral responsivity scales [5]. Four filter combinations with the trap detector implement the CIE color matching functions [11]. The spectral mismatch of the realized channels is characterized by the CIE $f_{1}^{\prime}$ values [12]. The realized $f_{1}$ values are $1.6 \%$ for the $\bar{x}_{1}(\lambda)$ and $V(\lambda), 3.6 \%$ for the $\bar{Z}(\lambda)$, and $4.1 \%$ for the $\bar{X}_{2}(\lambda)$ channels. The filter combinations are mounted in a temperature controlled (heated) filter wheel placed between the trap detector and the front aperture. The aperture plane is the reference plane of the illuminance mode photometer/colorimeter. A current-to-voltage converter can be plugged to the top of the light tight box of the photometer/colorimeter. Electronic and radiometric characterizations, including current-to-voltage conversion uncertainty and spatial, angular, and spectral responsivity, showed that the prototype photometer/colorimeter had satisfied the design expectations and it will be possible to determine the channel spectral irradiance responsivities with $0.1 \%$ relative standard uncertainty.

A single photometer has also been developed where the photodiode and the filter combination are mounted inside of a temperature-controlled copper block. Care was taken in the design - and the front surface of the $V(\lambda)$ filter was sanded - to avoid interference fringes in the SIRCUS calibrations. The $f_{1}^{\prime}$ was $1.85 \%$. An aperture mounted in front of the filters gives the reference plane of irradiance and illuminance measurements.

\section{SPECTRAL RESPONSIVITY MEASUREMENTS}

Depending on the application, a variety of different integrating spheres are used on SIRCUS. For irradiance responsivity calibrations, the source is a $2.5 \mathrm{~cm}$ diameter sphere with variable precision exit-apertures that mimics the beam geometry of luminous intensity lamp measurements. In this case, the interreflection effects within a photometer will not change from calibration to application. Also, because of the uniform irradiance, the non-uniformity of spatial responsivity of detectors, filters, and diffusers will not contribute to the uncertainty of measurements. Corrections for bandpass will not be necessary, as the bandwidth from the lasers is negligible. Comparisons of the spectral responsivities determined on the SIRCUS and the SCF are done on a regular basis to minimize uncertainties.

\section{ILLUMINANCE RESPONSIVITY}

The photometer illuminance responsivity is the ratio of the photometer output current to the illuminance measured by the photometer:

$$
s_{E i}=\frac{\int_{\lambda} E(\lambda) S_{E}(\lambda) d \lambda}{K_{m} \int_{\lambda} E(\lambda) V(\lambda) d \lambda}
$$


where $E(\lambda)$ is the incident spectral irradiance on the photometer, $V(\lambda)$ is the CIE spectral luminous efficiency, $K_{m}$ is the maximum spectral luminous efficacy, $683 \mathrm{Im} / \mathrm{W}, s_{E}(\lambda)$ is the spectral irradiance responsivity of the photometer as measured on the SIRCUS, and $\lambda$ is the wavelength.

The broad-band (illuminance) calibration factor of the photometer is:

$$
C_{c a l}=\frac{1}{S_{E i}},[\mid x / A]
$$

\section{CALIBRATION FACTORS FOR THE TRISTIMULUS COLORIMETER}

Similar to the above illuminance responsivity calibration of a single photometer, the channel calibration factors of the tristimulus colorimeter can be determined from the ratio of the CIE tristimulus values to the measured output currents of the four channels:

$$
\begin{aligned}
& k_{X 1}=\frac{X_{1}}{I_{X 1}}=\frac{K_{m} \int_{\lambda} E(\lambda) \bar{X}_{1}(\lambda) d \lambda}{\int_{\lambda} E(\lambda) s_{X 1}(\lambda) d \lambda}=\frac{1.06291 K_{m} F_{X 1}}{s_{X 1}(599)} \quad k_{X 2}=\frac{X_{2}}{I_{X 2}}=\frac{K_{m} \int_{\lambda} E(\lambda) \bar{X}_{2}(\lambda) d \lambda}{\int_{\lambda} E(\lambda) s_{X 2}(\lambda) d \lambda}=\frac{0.3501 K_{m} F_{X 2}}{s_{X 2}(442)} \\
& k_{Y}=\frac{Y}{I_{Y}}=\frac{K_{m} \int_{\lambda} E(\lambda) V(\lambda) d \lambda}{\int_{\lambda} E(\lambda) s_{Y}(\lambda) d \lambda}=\frac{K_{m} F_{Y}}{s_{Y}(555)} \quad k_{Z}=\frac{Z}{I_{Z}}=\frac{K_{m} \int_{\lambda} E(\lambda) \bar{Z}(\lambda) d \lambda}{\int_{\lambda} E(\lambda) s_{Z}(\lambda) d \lambda}=\frac{1.78297 K_{m} F_{Z}}{s_{Z}(446)}
\end{aligned}
$$

The right side of the equations was obtained by normalizing the $\bar{x}_{1}(\lambda), \bar{x}_{2}(\lambda), V(\lambda)$, and $\bar{z}(\lambda)$ functions to their peak values and introducing the color correction factors for all tristimulus channels:

$$
\begin{array}{rlrl}
F_{X 1} & =\frac{\int_{\lambda} E(\lambda) \bar{X}_{1 n}(\lambda) d \lambda}{\int_{\lambda} E(\lambda) s_{X 1 n}(\lambda) d \lambda} & F_{X 2} & =\frac{\int_{\lambda} E(\lambda) \bar{X}_{2 n}(\lambda) d \lambda}{\int_{\lambda} E(\lambda) s_{X 2 n}(\lambda) d \lambda} \\
F_{Y}=\frac{\int_{\lambda} E(\lambda) V(\lambda) d \lambda}{\int_{\lambda} E(\lambda) s_{Y n}(\lambda) d \lambda} & F_{Z}=\frac{\int_{\lambda} E(\lambda) \bar{Z}_{n}(\lambda) d \lambda}{\int_{\lambda} E(\lambda) s_{Z n}(\lambda) d \lambda}
\end{array}
$$

where $s_{X_{1}}(599), s_{X_{2}}(442), s_{Y}(555)$, and $s_{Z}(446)$ are the absolute irradiance responsivities of the realized channels at the peak wavelengths of the color matching functions, $\bar{x}_{1 \mathrm{n}}(\lambda), \bar{x}_{2 \mathrm{n}}(\lambda)$, and $\bar{Z}_{\mathrm{n}}(\lambda)$ are the color matching functions normalized to one at their peaks, $s_{X 1 n}(\lambda), s_{X 2 n}(\lambda), s_{Y_{n}}(\lambda)$, and $s_{Z n}(\lambda)$ are the relative spectral irradiance responsivities of the realized channels normalized to one at their peak wavelengths.

After the tristimulus colorimeter is calibrated for $k_{x 1}, k_{x 2}, k_{y}$, and $k_{z}$, the tristimulus values of a test light source can be measured as

$$
\begin{aligned}
& X^{\prime}=X_{1}{ }^{\prime}+X_{2}^{\prime}, \\
& Y=k_{Y} I_{Y}^{\prime}, \text { and } \quad Z^{\prime}=k_{Z} I_{Z}^{\prime}=k_{X 1} I_{X 1}{ }^{\prime} \text { and } X_{2}{ }^{\prime}=k_{X 2} I_{X 2}{ }^{\prime}
\end{aligned}
$$

where $I_{X l^{\prime}}, I_{X 2^{\prime}}, I_{Y^{\prime}}$, and $I_{z^{\prime}}$ are the measured output currents of the tristimulus channels. This calibration procedure can be applied to either illuminance or luminance measurement geometry using the relevant units of $s_{X 1}(599), s_{X 2}(442), s_{Y}(555)$, and $s_{Z}(446)$ in the equations.

From the procedures described above, the photometric and colorimetric measurement uncertainties will depend only on the uncertainty of the spectral responsivity determination of the channels. An advantage of the method is that accurate color and photometric measurements can be made even if the CIE functions are poorly realized (with large spectral mismatch in the channels).

If the CIE $f_{1}^{\prime}$ (spectral mismatch) values of the realized channels are small, the color correction factors can be kept very close to unity even if the source distribution changes (between the calibration source and the test source) are significant. However, even with small $f_{1}^{\prime}$ values, an additional set of calibration factors may be needed to accurately measure very different source distributions, such as gas discharge lamps or display monitors. In this case, rough knowledge of the source distribution is enough to derive correction factors enabling accurate photometric and color measurements. This is the principle behind the development of a spectrally tunable source.

\section{SCALE DERIVATIONS USING A TUNABLE OUTPUT LED TRANSFER SOURCE}

By setting the transfer source distribution equal, or very similar, to that of the test source to be measured, the photometric and color scales can be transferred from the primary standard photometers and colorimeters calibrated on SIRCUS to field meters with minimal increase in uncertainties. In our recently developed 
spectrally tunable solid-state source [10] the radiant flux from a sufficient number of light emitting diodes (LEDs) with different spectral distributions is introduced into an integrating sphere. A reference spectroradiometer is mounted on the sphere that measures the radiance (luminance) at the sphere exit port. The LEDs are individually controlled by adjustable current sources and are tuned - according to the spectroradiometer reading - to approximate a target source distribution. Standard and test photometers and colorimeters then measure the irradiance from the source at a given distance. The source can be rapidly tuned to match any desired spectral distribution. Customers no longer need to send the source artifact (e.g. discharge lamp or display), only its spectral distribution(s). Knowledge of the relative spectral responsivity of the test instruments is not needed when they are calibrated against the reference instruments by measuring the transfer source tuned to the source distribution to be measured. In this case, the spectral mismatch errors are very small resulting in low measurement uncertainties.

The initial prototype source was built using a total of 40 LED's, 4 LED's each with 10 different spectral distributions. While this is a transfer source, in principle only requiring that the radiance be stable over the course of a measurement, it is desirable to know and understand the radiometric characteristics of the LEDs. This knowledge will impact the ultimate design of the source. LED's were tested for stability and dependence on drive current. The effect of LED seasoning for luminance and chromaticity was studied. Also, the luminance, chromaticity, and spectral distribution changes versus LED drive current were analyzed for representative red, green, and blue LEDs [10]. The prototype source demonstrated the feasibility of a spectrally tunable LED-based source.

Simulations showed that a 40-channel source could approximate the CIE standard illuminant A within $0.1 \%$ and the D65 within $5 \%$ deviation over the visible spectral range from $380 \mathrm{~nm}$ to $780 \mathrm{~nm}$. Consequently, we have started the development of a 40 -channel source. The source has 4 LED heads, with 10 channels per head. There are typically 3 LEDs per channel, though for certain spectral regions only low power LEDs are available and in those cases additional LEDs are added to the channel.

\section{SUMMARY}

Recently performed irradiance responsivity calibrations with expanded uncertainties $(k=2)$ of $0.15 \%$ motivated the development of a detector-based tristimulus colorimetric scale and the improvement of the present NIST photometric units. A reference tristimulus colorimeter/photometer has been developed to implement the first detector-based color scale and to study the uncertainty decrease of illuminance responsivity calibrations on the new SIRCUS facility. A novel, spectrally tunable, solid-state source was developed to transfer the high accuracy illuminance responsivity and tristimulus color scales from the reference tristimulus colorimeter/photometer to working standard and field photometers and tristimulus meters. By setting the transfer source distribution to approximate a particular target distribution, the photometric and color scales can be transferred to field meters with minimal increase in uncertainties.

\section{REFERENCES}

[1] Cromer, C. L., Eppeldauer, G., Hardis, J. E., Larason, T. C., Ohno, Y., and Parr, A. C., The NIST DetectorBased Luminous Intensity Scale, J. Res. of the NIST, Vol. 101, No 2, p.109-132, 1996.

[2] Yoon, H. W., Gibson, C. E., and Barnes, P. Y., Realization of the National Institute of Standards and Technology detector-based spectral irradiance scale, Appl. Opt. Vol. 41, No. 28, 5879-5890, 2002.

[3] Eppeldauer G., Spectral Response Based Calibration Method of Tristimulus Colorimeters, J. Res. of the NIST Vol. 103, No. 6. 615-619, 1998.

[4] Larason, T. C., Bruce, S. S., and Parr, A. C., Spectroradiometric Detector Measurements: Part I-Ultraviolet Detectors and Part II-Visible to Near-Infrared Detectors, NIST Special Publ. 250-41, 1998.

[5] Brown, S. W., Eppeldauer, G. P., and Lykke, K. R., NIST Facility for Spectral Irradiance and Radiance Response Calibrations with a Uniform Source, Metrologia, Vol. 37, 579-582, 2000.

[6] Eppeldauer, G. P., Brown, S. W., Larason, T. C., Racz, M., and Lykke, K. R., Realization of a spectral radiance responsivity scale with a laser-based source and Si radiance meters, Metrologia, Vol. 37, 531-534, 2000.

[7] Gentile, T. R., Houston, J. M., Hardis, J. E., Cromer, C. L., and Parr, A. C., National Institute of Standards and Technology high-accuracy cryogenic radiometer, App. Opt. Vol. 35, No. 7, 1056-1068, 1996.

[8] Eppeldauer, G. P. and Lynch, D. C., Opto-mechanical and electronic design of a tunnel-trap Si-radiometer, J. Res. Natl. Inst. Stand. Technol. Vol. 105, No. 6, p. 813-828, 2000.

[9] Brown, S. W., Zong, Y., and Ohno, Y., NIST Calibrations of Colorimeters for Display Measurements, Information Display, Vol. 15, 30-34, 1999.

[10] Brown, S. W., Santana, C., and Eppeldauer, G. P., Development of a Tunable LED-based Colorimetric Source, J. Res. NIST, Vol. 107, No. 4, 363-371, 2002.

[11] Publication CIE No. 15. 2 (1986), Central Bureau of the CIE, A-1033 Vienna, P.O. Box 169, Austria.

[12] Publication CIE No. 69 (1987), Central Bureau of the CIE, A-1033 Vienna, P.O. Box 169, Austria. 


\title{
Realization and application of a detector-based tristimulus color scale at the National Institute of Standards and Technology, USA
}

\author{
G. P. Eppeldauer, S. W. Brown, K. R. Lykke, and Y. Ohno \\ National Institute of Standards and Technology, Optical Technology Division \\ Gaithersburg, Maryland (USA) \\ Corresponding author: G. P. Eppeldauer (george.eppeldauer@nist.gov)
}

\begin{abstract}
The recently introduced detector-based calibration method for tristimulus colorimeters has been realized. The NIST reference colorimeter has been calibrated for spectral irradiance responsivity with an uncertainty of $0.15 \%$ (coverage factor $k=2$ ) resulting in a chromaticity uncertainty of 0.0004 $(k=2)$ in the $x$ and $y$ chromaticity coordinates when a CIE Illuminant A source is measured. The color scale is realized and maintained by reference colorimeters rather than reference lamps. The color measurement uncertainty increases when sources other than Illuminant A are measured due to spectral mismatch errors of the channels. Variable reference source models have been developed so that the reference source for the colorimeter can be automatically matched to the measured test source of certain types. A model using Planckian radiation with a variable temperature has been tested and proved to be effective in eliminating the mismatch errors for incandescent lamps. Another model based on primary colors of a display has also been tested and shown to be effective.
\end{abstract}

\section{INTRODUCTION}

A reference tristimulus colorimeter has been developed at the National Institute of Standards and Technology (NIST) to implement the recently developed tristimulus colorimeter calibration method based on detector standards [1, 2]. The reference tristimulus colorimeter was designed such that the combined uncertainty of the color and photometric measurements is dominated by the uncertainty of the spectral responsivity calibrations. The spectral responsivities of the four colorimeter channels are closely matched to the CIE color matching functions (the $\mathrm{f}_{1}$ ' values are $1.6 \%$ for the $\mathrm{Y}$ and $\mathrm{X}_{1}, 3.6 \%$ for the $\mathrm{Z}$, and $4.1 \%$ for the $\mathrm{X}_{2}$ channels) to minimize additional uncertainties in color measurement. The channel spectral irradiance responsivities were determined with the lowest possible uncertainty using our laser-based facility to produce uniform monochromatic irradiance [3].

Based on the spectral irradiance responsivity of the channels, a colorimetric scale has been realized and maintained by the reference colorimeters instead of standard lamps. The detector-based color scale can be calculated for any given spectral distribution of a reference source, which can be either theoretical or real. Generally, CIE Illuminant A is used as the reference source. In this case, the uncertainty of measurement increases when sources other than Illuminant $\mathrm{A}$ are measured due to inherent spectral mismatch of the colorimeter channels (to the CIE functions). To avoid spectral mismatch errors, an approximate spectral distribution of the test source is needed. A need for a spectroradiometer for this purpose would negate the benefit of tristimulus colorimeters.

To solve this problem, a variable reference source model has been developed. This method, applied to certain types of source, avoids or reduces the spectral mismatch errors without knowledge of the spectral distribution of the test source. The spectral distribution of a model, used as the reference source in the colorimeter calibration equation (see below), is adjusted based on the color temperature or tristimulus values of the test source as measured by the colorimeter. A model using Planckian radiation at a variable temperature has been tested and shown to be effective for the measurement of incandescent lamps. A model based on the spectral distribution of each primary color of a liquid crystal display (LCD) has also been tested and shown to be effective for display color measurements. 


\section{DETECTOR-BASED CALIBRATION OF TRISTIMULUS COLORIMETERS}

The spectral irradiance responsivities of the four colorimeter channels were determined on the new NIST facility for Spectral Irradiance and Radiance Responsivity Calibrations using Uniform Sources (SIRCUS) [3]. The measurement is traceable to an absolute cryogenic radiometer that maintains the NIST primary unit for optical power.

The continuously tunable output from several different tunable lasers was consecutively introduced into a small integrating sphere to produce uniform, monochromatic irradiance [3] at the reference planes of the tristimulus colorimeter and the reference trap detector. The spectral irradiance responsivity scale from the trap detector was transferred to the channels of the tristimulus colorimeter using the detector substitution method. The colorimeter signal gain was $10^{8} \mathrm{~V} / \mathrm{A}$ for all channels. The results are shown in Fig. 1; cubicspline fits were applied for interpolation between the laser-measured data points.

The responsivity transfer in the uniform monochromatic irradiance makes the calibration

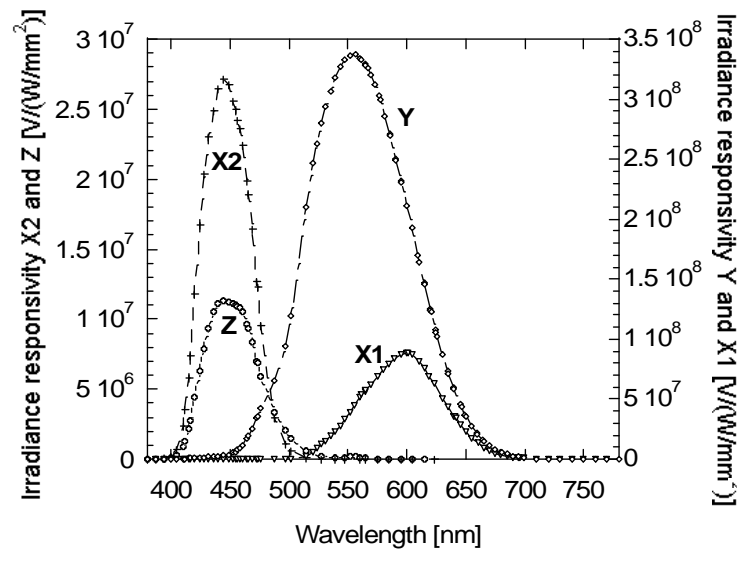

Figure 1: SIRCUS measured spectral irradiance responsivities of the reference tristimulus meter. and application beam geometries equal.

Furthermore, the uncertainty components caused by the changing internal reflectance patterns in the colorimeter (between calibration and applications) and spatial non-uniformities of the channel responsivities are reduced compared to lamp-monochromator-based calibrations. The relative combined expanded uncertainty $(k=2)$ of the spectral irradiance responsivity transfer to the colorimeter was $0.15 \%$.

The low responsivity uncertainty made it possible to accurately calibrate the colorimeter channels for responsivity to tristimulus values. The channel broad-band (spectrally integrated) calibration factors have been calculated [2]:

$$
\begin{array}{ll}
k_{\mathrm{X} 1}=\frac{X_{1}}{I_{\mathrm{X} 1}}=\frac{\mathrm{K}_{\mathrm{m}} \int_{\lambda} E(\lambda) \bar{X}_{1}(\lambda) d \lambda}{\int_{\lambda} E(\lambda) s_{\mathrm{X} 1}(\lambda) d \lambda} & k_{\mathrm{X} 2}=\frac{X_{2}}{I_{\mathrm{X} 2}}=\frac{\mathrm{K}_{\mathrm{m}} \int_{\lambda} E(\lambda) \bar{X}_{2}(\lambda) d \lambda}{\int_{\lambda} E(\lambda) s_{\mathrm{X} 2}(\lambda) d \lambda} \\
k_{\mathrm{Y}}=\frac{Y}{I_{\mathrm{Y}}}=\frac{\mathrm{K}_{\mathrm{m}} \int_{\lambda} E(\lambda) V(\lambda) d \lambda}{\int_{\lambda} E(\lambda) s_{\mathrm{Y}}(\lambda) d \lambda} & k_{\mathrm{Z}}=\frac{Z}{I_{\mathrm{Z}}}=\frac{\mathrm{K}_{\mathrm{m}} \int_{\lambda} E(\lambda) \bar{Z}(\lambda) d \lambda}{\int_{\lambda} E(\lambda) s_{\mathrm{Z}}(\lambda) d \lambda}
\end{array}
$$

where $E(\lambda)$ is the relative spectral distribution of the reference source, $\bar{x}_{1}(\lambda), \bar{x}_{2}(\lambda), V(\lambda)$, and $\bar{z}(\lambda)$ are the CIE color matching functions, $K_{m}$ is the maximum spectral luminous efficacy, $683 \mathrm{~lm} / \mathrm{W}, s_{\mathrm{X} 1}(\lambda), s_{\mathrm{X} 2}(\lambda), s_{\mathrm{Y}}(\lambda)$, and $s_{\mathrm{Z}}(\lambda)$ are the channel spectral irradiance responsivities, and $\lambda$ is the wavelength. Both the responsivity measurements and the integrals were made between $360 \mathrm{~nm}$ and $1000 \mathrm{~nm}$ (the responsivity limit of silicon detectors). The calibration factors obtained for CIE Illuminant A are: $k_{\mathrm{Y}}=2.036 \mathrm{~lx} / \mathrm{V}, k_{\mathrm{X} 1}=8.359, k_{\mathrm{X} 2}=8.769$, and $k_{\mathrm{Z}}=108.96$. The tristimulus values of test light sources were determined using these calibration factors:

$$
X^{\prime}=X_{1}{ }^{\prime}+X_{2}^{\prime} \text {, where } X_{1}^{\prime}=k_{\mathrm{X} 1} I_{\mathrm{Xl}}{ }^{\prime} \text { and } X_{2}^{\prime}=k_{\mathrm{X} 2} I_{\mathrm{X} 2}, Y^{\prime}=k_{\mathrm{Y}} I_{\mathrm{Y}^{\prime}} \text {, and } Z^{\prime}=k_{\mathrm{Z}} I_{\mathrm{Z}}{ }^{\prime}
$$

where $I_{\mathrm{Xl}}{ }^{\prime}, I_{\mathrm{X} 2}{ }^{\prime}, I_{\mathrm{Y}^{\prime}}$, and $I_{\mathrm{Z}}{ }^{\prime}$ are the output signals of the channels for a given test source.

The $0.15 \%$ responsivity uncertainty propagates to the tristimulus values of the measured test sources and result in uncertainties in the $x, y$ chromaticity coordinates of 0.0004 when a CIE Illuminant A source is measured. 


\section{VARIABLE REFERENCE SOURCE MODEL}

The low uncertainty of spectral responsivity measurements and the small spectral mismatch of the channel responsivities to the CIE color matching functions makes it possible to provide an accurate color scale to a tungsten lamp realizing CIE Illuminant A. However, when sources other than Illuminant A are measured, spectral mismatch errors are inevitable even with well-matched channel spectral responsivities. With the developed NIST colorimeter, the chromaticity errors for Planckian radiation from $2300 \mathrm{~K}$ to $3200 \mathrm{~K}$ are within 0.001 . The errors for light sources other than tungsten lamps are estimated to be within 0.003 in $x, y$.

An advantage of the detector-based calibration method is that the calibration factors of the colorimeter can be determined for any spectral distribution including theoretical models. The error will be significantly reduced if an approximate spectral distribution of the test source is used as $E(\lambda)$ in Eqs. (1). However, use of a spectroradiometer for this purpose will negate the benefit of tristimulus colorimeters. An attempt has been made to use spectral distribution models to reduce or eliminate spectral mismatch errors with no prior information of the spectrum of the test source.

A model using Planckian radiation with a variable temperature $T[\mathrm{~K}]$ has been introduced and tested. $E(\lambda)$ in Eqs. (1) is given by

$$
E(\lambda)=k \lambda^{-5}\left[\exp \left(c_{2} / \lambda T\right)-1\right]^{-1} \quad\left(c_{2}=1.4388 \times 10^{-2} \mathrm{~m} \cdot \mathrm{K}, k \text { : normalization constant }\right)
$$

The calibration factors in Eqs. (1) are initially calculated for $T=2856 \mathrm{~K}$ (CIE Illuminant A). Then a test lamp is measured with the colorimeter and the color temperature $T_{1}$ is obtained. Next, $T_{1}$ is replaced in Eqs. (1) and the calibration factors in Eqs. (1) are recalculated, and the color quantities of the test source are recalculated. With this recalculation, the CCT (and thus the spectral distribution) of the reference source is nearly equal to that of the test source, realizing the condition of strict substitution. One time recalculation removes most of the errors for incandescent lamps, but two or three iterations can remove the mismatch error completely. There are only small remaining errors due to the small deviation of the spectral distribution of the test source from the theoretical Planckian curve. The residual errors for a quartz halogen lamp at color temperatures of $2300 \mathrm{~K}$ to $3200 \mathrm{~K}$ were found to be negligible $(<0.00002$ in $x, y)$ for our NIST reference colorimeter.

This model works perfectly for Planckian sources, and is also effective to some extent for other general sources, depending on the relative spectral responsivity of the channels.

Another model has been tested for display calibration. The spectral distribution of displays are very similar within each type of display, therefore their spectral distribution can be modeled. A model for a display is prepared as:

$$
E(\lambda)=k_{\mathrm{B}} S_{\mathrm{B}}(\lambda)+k_{\mathrm{G}} S_{\mathrm{G}}(\lambda)+k_{\mathrm{R}} S_{\mathrm{R}}(\lambda)
$$

where $S_{B}(\lambda), S_{G}(\lambda), S_{R}(\lambda)$ are the relative spectral distribution of each primary color in a typical LCD or CRT. The mixing factors $k_{\mathrm{B}}, k_{\mathrm{G}}, k_{\mathrm{R}}$ are initially determined so that $E(\lambda)$ gives a white point at $6500 \mathrm{~K}$ and a test display is measured with the colorimeter. Based on the first tristimulus values measured, the mixing factors $k_{\mathrm{B}}, k_{\mathrm{G}}, k_{\mathrm{R}}$ are recalculated to give $E(\lambda)$ the same chromaticity coordinates as the measured values of the test source. Based on the new $E(\lambda)$, the color quantities of the test source are recalculated. A single iteration is sufficient. Additional iterations did not converge in our test. Using this process, the spectral distribution of the reference source $E(\lambda)$ is adjusted to be nearly equal to the test source. Although the

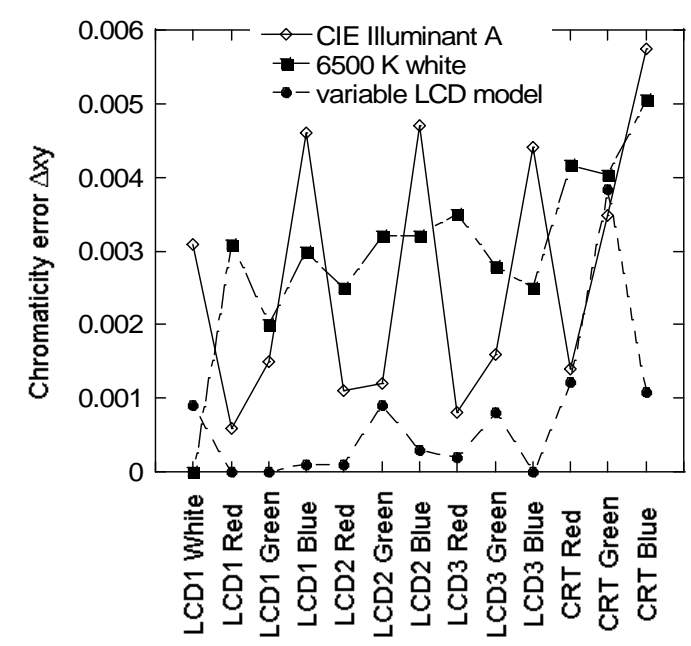

Figure 2: Chromaticity error $\Delta x y=\sqrt{\Delta x^{2}+\Delta y^{2}}$ in the measurement of various display colors due to spectral mismatch of the NIST colorimeter, with various reference sources including the variable LCD model. 
spectral distribution of each primary color of the measured display may not be exactly the same, they are very similar within each type of display, and most of the spectral mismatch errors are removed for a given type of display, regardless of the color measured. As a demonstration, Fig. 2. shows a simulation of measurements of three different LCD displays and one CRT, where the reference source model is based on the primary color spectra of LCD1. The results are shown for three cases where displays are measured with colorimeter calibration using Illuminant A, LCD1 white (6500 K), and the variable LCD model. The results for LCD1, shown as zero for all colors, are for verification. The results for LCD2 and LCD3, having slightly different spectra from LCD1, show that errors are reduced by an order of magnitude and kept within 0.001 in $\Delta x y$. The results for CRT, having very different spectra, are not improved as much.

\section{TRANSFER CALIBRATION}

Test colorimeters can be calibrated against the detector-based reference colorimeter under illumination by a reference (transfer) source. For a simple case, a CIE illuminant A source is used, but when the test colorimeter is used to measure other various sources, the spectral mismatch error can be significant. To avoid such errors, the transfer calibration needs to be done with the same type of source that is to be measured by the test colorimeter (strict substitution). If the source is one of the types described in the reference source models above (incandescent lamp, display), then such a real source can be used for calibration transfer. For other source types, the transfer calibration needs to be performed with a known approximate spectral distribution $E_{\mathrm{a}}(\lambda)$ of the source to be measured. The reference colorimeter is calibrated with $E_{\mathrm{a}}(\lambda)$ replaced in Eqs. (1), and determines the tristimuls values $X_{\mathrm{r}}, Y_{\mathrm{r}}, Z_{\mathrm{r}}$ of the source. The test colorimeter is calibrated against the $X_{\mathrm{r}}, Y_{\mathrm{r}}, Z_{\mathrm{r}}$ values. Such transfer calibrations for various sources can be done effectively by using a spectrally tunable source (STS) as reported in Ref. 4 . The STS is set to simulate a given test source spectrum $E_{\mathrm{m}}(\lambda)$, and the test colorimeter is calibrated against the reference colorimeter under that illumination. The STS can produce as many spectra as needed, and transfer calibration can be done for each type of source with small spectral mismatch errors, without knowing the spectral responsivity of the test colorimeter.

\section{CONCLUSIONS}

The NIST reference tristimulus colorimeter has been calibrated for absolute spectral irradiance responsivity on our tunable-laser-based spectral calibration facility. The four channels of the colorimeter were calibrated with an uncertainty of $0.15 \%$ (coverage factor $k=2$ ), which results in an uncertainty of $0.0004(k=2)$ in the $x, y$ chromaticity when measuring a CIE Illuminant A source. The color scale is realized and maintained by the reference colorimeters. For measurement of sources other than Illuminant A, variable reference source models have been developed to avoid or reduce spectral mismatch errors. The model using Planckian radiation at a variable temperature has been introduced and tested. Using this model, the spectral mismatch errors for incandescent lamps are practically eliminated. Another model based on the primary colors of a display has also been tested and shown to be effective. Other models are being explored to measure LEDs and other sources. The transfer calibration to test colorimeters can be performed with real sources used in the variable reference source models or by using a spectrally tunable source to avoid spectral mismatch errors.

\section{References}

1. Eppeldauer, G. P. and Racz, M., Design and characterization of a photometer-colorimeter standard. Applied Optics, 2004. 43(13) p. 2621-2631.

2. Eppeldauer, G. P., Spectral response based calibration method of tristimulus colorimeters. J. Res. NIST, 1998. 103(6): p. 615-619.

3. Brown, S. W., Eppeldauer, G. P., and Lykke, K. R., NIST Facility for spectral irradiance and radiance responsivity calibrations with uniform sources. Metrologia, 2000. 37: p. 579-582.

4. Fryc, I., Brown, S. W., Eppeldauer, G. P., and Ohno, Y., A spectrally tunable solid-state source for radiometric, photometric, and colorimetric applications, Proc. SPIE, 2004. 5530, p. 150-159. 


\title{
DETECTOR-BASED CALIBRATION PROCEDURES FOR
}

\section{TRISTIMULUS COLORIMETER STANDARDS}

\author{
G. P. Eppeldauer, C. C. Miller, and Y. Ohno \\ National Institute of Standards and Technology \\ Gaithersburg, Maryland, USA
}

\begin{abstract}
Two reference and three working standard tristimulus colorimeters have been developed at the National Institute of Standards and Technology (NIST) to utilize the recently introduced detector-based colorimeter calibration method [1]. The tristimulus colorimeters are calibrated on three reference calibration facilities of NIST. The preliminary calibration procedures are described and the obtained results are evaluated. Future work to improve the uncertainty of detectorbased tristimulus color measurements is discussed.
\end{abstract}

Keywords: calibration, colorimetry, color temperature, spectral responsivity, tristimulus

\section{INTRODUCTION}

At present, routine calibrations of tristimulus colorimeters are performed against lamp standards. The expanded uncertainty using the NIST primary standard color temperature lamp standards is $8 \quad K \quad(k=2)$. This source-based calibration uncertainty can increase with the burning hours of the lamp standards. Both the long-term stability and the spectral distribution of the lamps can significantly change versus burning time. When the tristimulus colorimeter calibrations are performed against detector standards the uncertainty of the spectral responsivity calibrations of the colorimeter channels can dominate the color measurement uncertainty [2]. Also, long-term changes in the measurement uncertainty can be significantly decreased. The goal of this work is to utilize the advantages of the detector-based calibrations and to improve the present sourcebased color calibration uncertainty by at least a factor of two.

\section{NIST TRISTIMULUS COLORIMETERS}

First, a silicon trap-detector based reference tristimulus colorimeter was developed [2] with a temperature controlled filter-wheel for the NIST developed four filter combinations. The second reference tristimulus colorimeter, Model F100, was a similar design but the detector was a large area single-element silicon photodiode [2]. The picture of this colorimeter, with the front cover (where the aperture is mounted) removed, is shown in Fig. 1. Five equally designed and fabricated sets of filter combinations were purchased and installed in a temperature controlled copper filter-wheel. Since the blue filter combinations ( $X s$ and $Z$ ) had significant leakage in the near-IR, they have recently been replaced with improved-blocking filter packages. One filter set was installed in the second reference tristimulus colorimeter and three sets have been installed in three additionally fabricated working standard colorimeters of the same design.

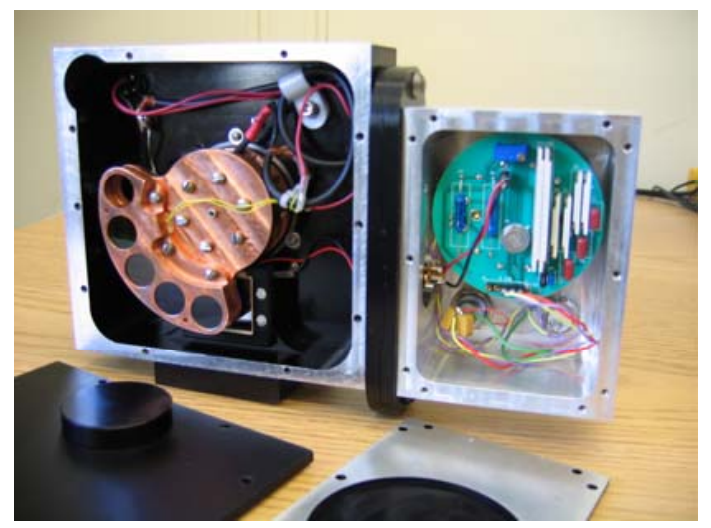

Figure 1. Picture of the prototype singleelement-Si based colorimeter 


\section{NIST CALIBRATION FACILITIES}

Both reference colorimeters were calibrated at the Spectral Irradiance and Radiance Responsivity Calibrations using Uniform Sources (SIRCUS) facility. The colorimeter channels were calibrated for spectral irradiance responsivity. The expanded responsivity uncertainty goal is $0.1 \%(k=2)$ or less. The monochromator-based Spectral Comparator Facility (SCF) was used to measure the channels of the second reference tristimulus colorimeter and the three working standard colorimeters for spectral power responsivity. These measurements were needed to check if the relative spectral responsivities of the corresponding channels of the four colorimeters agree. This spectral comparison is needed because the three working standard colorimeters will be calibrated against the second reference colorimeter in broad-band mode. The SCF measurements were made with an expanded relative uncertainty of $0.2 \%(k=2)$. The SCF measured the near-IR leakage of the filter packages to $1300 \mathrm{~nm}$.

The NIST Photometry Facility was used to measure the illuminance from lamp standards with the two reference tristimulus colorimeters.

\section{SPECTRAL RESPONSIVITIES}

After measuring the channel spectral responsivities on the SIRCUS and SCF, the results were evaluated.

In the channel-responsivities of the trapdetector based colorimeter, the rejection for near-infrared radiation was about four orders of magnitude and no fluorescence could be measured.

The spectral irradiance responsivity curves of the four channels of the second reference tristimuls colorimeter with the leaky blue filter combinations are shown in Fig. 2. The spectral irradiance responsivity measurements on the SIRCUS were made between $360 \mathrm{~nm}$ and $960 \mathrm{~nm}$. The SCF spectral power responsivity measurements were used to extend the responsivity curves to $1300 \mathrm{~nm}$, the sensitivity limit of the applied silicon detector. The logarithmic scale shows that the blue (Xs and Z) filter combinations have significant leakage in the nearIR range.
The wavelength shift of the monochromator was less than $0.1 \mathrm{~nm}$.

The relative spectral responsivities measured on the SIRCUS and SCF were compared. As an example, Fig. 3 shows the percent differences between the SIRCUS and SCF measured and then normalized spectral responses for the $Y$ channel. The left- $Y$ axis shows the normalized response at the peak wavelength of $555 \mathrm{~nm}$. The right- $Y$ axis shows the percent difference between the SIRCUS and SCF normalized responses.

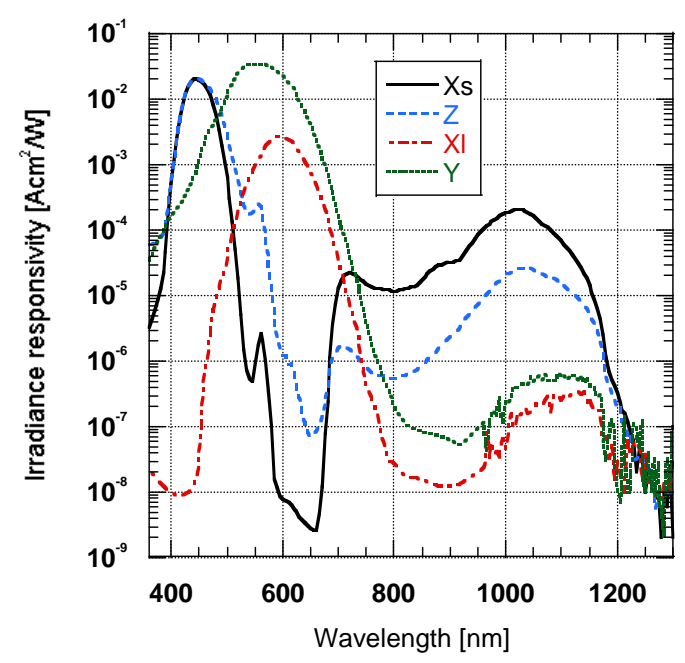

Figure 2. Spectral irradiance responsivities of the single-element-Si based colorimeter (F100) with the leaky blue filters.

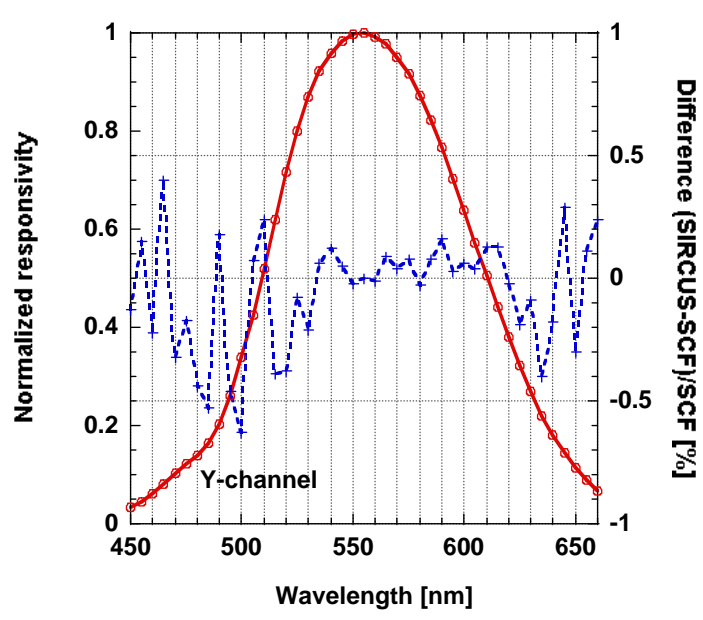

Figure 3. Percent differences between the SIRCUS and SCF measured and then normalized F100 spectral responses. 
The difference curve has structures. The differences around the peak are at the $0.1 \%$ level. However, at the sides of the curve, the structures are larger but their effect is lower. The structures originate from the SIRCUS calibrations and are caused by laser fluctuations and calibration protocol errors. Recent SIRCUS calibration procedures have been improved and results verified that spectral responsivity measurements can be performed with $0.05 \%(k=2)$ uncertainty.

The SIRCUS measured a $0.08 \%$ higher absolute responsivity value at the $555 \mathrm{~nm}$ peak of the Y-channel than the SCF.

The spectral responsivity of the $Y$-channel was re-measured at the SCF after the $Y$ filter package was flipped over (the front surface became the back surface) in the temperature controlled filter wheel. Figure 4 shows the normalized spectral responsivities before and after the flip on a log scale. An increased responsivity was measured in the blue between $380 \mathrm{~nm}$ and $450 \mathrm{~nm}$. The responsivity increase was caused by the fluorescence of the thin cut-on filter-layer. The change in the fluorescent signal, after reversing the filter package in the filterwheel, indicates that the exciting blue-light (producing the fluorescence) is attenuated differently at the two different orientations of the $Y$ filter package. An $f_{1}^{\prime}$ of $1.4 \%$ was calculated with the lower fluorescence and $1.7 \%$ with the higher fluorescence for the $Y$ channel.

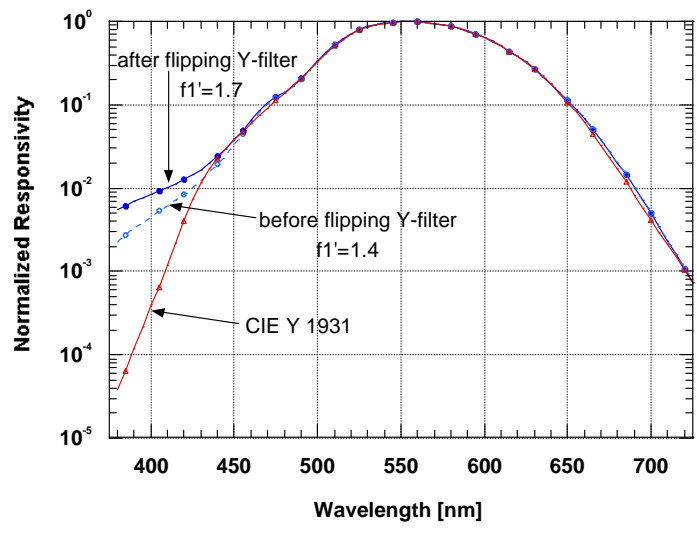

Figure 4. Fluorescence caused responsivity change in the F100 Y-channel before and after reversing the filter in its holder.

Figure 5 shows the fluorescence caused responsivity changes in the $\mathrm{Y}$-channel in a linear scale. The magnitude of the fluorescence caused contribution to the responsivity increase is not known. The increased responsivity at the original filter orientation, relative to the CIE Y 1931 function, can also be caused by the realized filter transmittance. The responsivity of the $\mathrm{Y}$-channel is about $0.4 \%$ higher (relative to the unity peak responsivity of the $Y$-channel) with the turned over filter compared to the original filter orientation at $410 \mathrm{~nm}$. At $400 \mathrm{~nm}$, at the original filter orientation, the responsivity is about $0.4 \%$ higher than the target ( $Y$ 1931) responsivity.

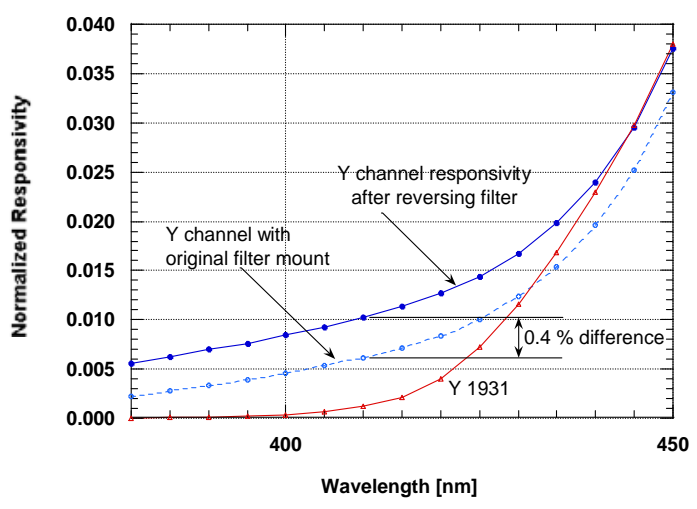

Figure 5. Fluorescence caused responsivity change in the F100 Y-channel on a linear scale at two different filter orientations.

Similar, but smaller, responsivity changes were measured in the $X s$ and $Z$ channels below $400 \mathrm{~nm}$. These changes were also caused by the fluorescence inside of the blue filter packages. The responsivity, after reversing the filter-package, changed from $0.1 \%$ to close to $1 \%$ (relative to the peakresponsivity at $445 \mathrm{~nm}$ ) at $390 \mathrm{~nm}$. After the flip, the $f_{1}{ }^{\prime}$ of the $Z$ channel decreased from $3.9 \%$ to $3.8 \%$. The $f_{1}{ }^{\prime}$ of the Xs channel, after the flip, increased from $2.9 \%$ to $3 \%$. The reason of the responsivity changes is again the different attenuation of the excitation light between the two (starting and reversed) filter positions. All filter packages were used at the orientations where the responsivity increase was smaller (lower fluorescence). The $f_{1}{ }^{\prime}$ of the $X_{l}$ channel was $4.3 \%$ and no fluorescence could be measured.

After eliminating the near-IR leakages in the blue filter combinations, the goal to achieve a $0.1 \%(k=2)$ expanded relative uncertainty 
for the channel spectral responsivity measurements is feasible.

\section{COLORIMETER CALIBRATIONS}

\subsection{Reference colorimeters}

The spectral irradiance responsivities of the channels of the F100 reference colorimeter (shown in Fig. 2) were determined against two silicon tunnel-trap detectors [3]. The picture of the calibration arrangement at the SIRCUS is shown in Fig. 6. The trapdetector reference standards were calibrated previously against the primarystandard electrical-substitution cryogenicradiometer in radiant power measurement mode. The aperture areas were determined at the NIST aperture measurement facility [4]. The aperture area times the power responsivity is equal to the irradiance responsivity of the trap detector. The two reference colorimeters were calibrated against the two trap detectors in irradiance measurement mode using the detector substitution method.

The (broad-band) calibration factor of a colorimeter channel can be calculated as the ratio of the CIE tristimulus value to the measured output signal (current) of the same (realized) channel [1]:

$$
\begin{aligned}
& k_{\mathrm{X} 1}=\frac{X_{1}}{I_{\mathrm{X} 1}}=\frac{K_{\mathrm{m}} \int_{\lambda} E(\lambda) \bar{x}_{1}(\lambda) d \lambda}{\int_{\lambda} E(\lambda) s_{\mathrm{X} 1}(\lambda) d \lambda} \\
& k_{\mathrm{X} 2}=\frac{X_{2}}{I_{\mathrm{X} 2}}=\frac{K_{\mathrm{m}} \int_{\lambda} E(\lambda) \bar{X}_{2}(\lambda) d \lambda}{\int_{\lambda} E(\lambda) s_{\mathrm{X} 2}(\lambda) d \lambda} \\
& k_{\mathrm{Y}}=\frac{Y}{I_{Y}}=\frac{K_{\mathrm{m}} \int_{\lambda} E(\lambda) V(\lambda) d \lambda}{\int_{\lambda} E(\lambda) s_{\mathrm{Y}}(\lambda) d \lambda} \\
& k_{\mathrm{Z}}=\frac{Z}{I_{\mathrm{Z}}}=\frac{K_{\mathrm{m}} \int_{\lambda} E(\lambda) \bar{Z}(\lambda) d \lambda}{\int_{\lambda} E(\lambda) s_{\mathrm{Z}}(\lambda) d \lambda}
\end{aligned}
$$

where $E(\lambda)$ is the relative spectral distribution of the reference source (which is not a standard in this detector-based calibration), $\bar{x}_{1}(\lambda), \bar{x}_{2}(\lambda), V(\lambda)$, and $\bar{z}(\lambda)$ are the CIE color matching functions, $K_{m}$ is the maximum spectral luminous efficacy, $683 \mathrm{Im} / \mathrm{W}$, $s_{X_{1}}(\lambda), s_{X 2}(\lambda), s_{Y}(\lambda)$, and $s_{Z}(\lambda)$ are the measured spectral responsivities of the realized channels (shown in Fig. 2), and $\lambda$ is the wavelength. The integrals were made from $360 \mathrm{~nm}$ to $1300 \mathrm{~nm}$ (involving the near-IR leakage) to correct for spectral mismatch and amplitude errors. As an example, the channel calibration factors calculated for the F100 reference colorimeter using $E(\lambda)=$ $2300 \mathrm{~K}, 2856 \mathrm{~K}$, and $3200 \mathrm{~K}$ Planckian reference distributions are shown in Table 1. The detector-based calibration factors in the Table were calculated with Planckian temperatures that are close to the color temperatures of the lamp-standard also measured by the F100 reference colorimeter.

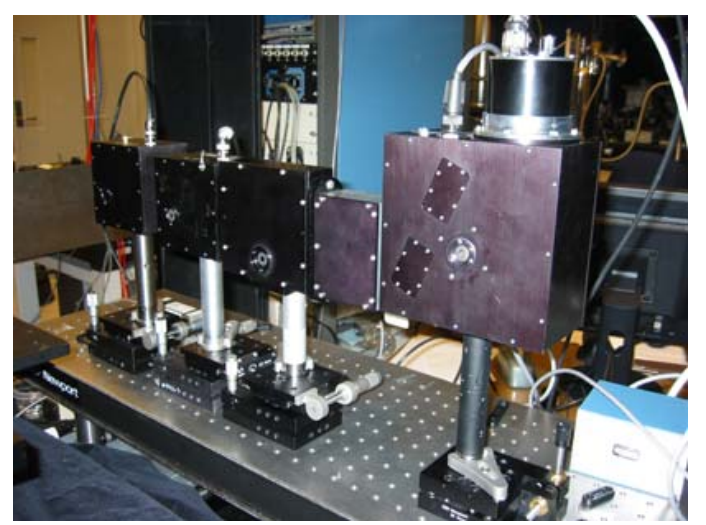

Figure 6. Arrangement of the two reference tristimulus colorimeters (right) and the two silicon trap-detectors (left) at the SIRCUS.

Table 1. Channel calibration factors of the F100 reference tristimulus colorimeter

\begin{tabular}{|c|c|c|c|c|}
\hline $\begin{array}{c}\text { Temp. } \\
{[\mathrm{K}]}\end{array}$ & $k_{\mathrm{X} 1}$ & $k_{\mathrm{X} 2}$ & $k_{\mathrm{Y}}$ & $k_{\mathrm{Z}}$ \\
\hline 2300 & $\begin{array}{c}27082 \\
6\end{array}$ & 5568 & 19225 & 54720 \\
\hline 2856 & $\begin{array}{c}27182 \\
9\end{array}$ & 9310 & 19247 & 59072 \\
\hline 3200 & $\begin{array}{c}27216 \\
3\end{array}$ & 10315 & 19252 & 59719 \\
\hline
\end{tabular}

Table 1 shows that $k_{x_{2}}$ of the $X_{S}$ blue channel, where the near-IR leakage is the highest, changed almost a factor of two for a $900 \mathrm{~K}$ temperature change. The change in the leaky $Z$ channel is close to $10 \%$ for the same temperature change. The change of $k_{\mathrm{X} 1}$ for the $\mathrm{X}_{1}$ channel is $0.5 \%$ and $k_{\mathrm{Y}}$ of the $Y$ channel is only $0.14 \%$ for the $900 \mathrm{~K}$ change of the lamp.

It is expected that after eliminating the nearIR leakage of the blue filter packages, the large changes in $k_{\mathrm{x} 2}$ and $k_{\mathrm{z}}$ will dramatically 
decrease and one set of calibration factors, calculated with the $2856 \mathrm{~K}$ reference source distribution, will be enough to make wide temperature-range measurements of tungsten lamps.

In order to compare the detector-based and source-based color temperature calibrations, the F100 colorimeter measured a color-temperature lamp-standard. The measurements were made inside of a lighttight photometer bench, with two baffles between the lamp standard and the F100 colorimeter. From the measured output signals of the four channels, using the above calibration factors, the tristimulus values were calculated for all four channels:

$$
\begin{aligned}
& X_{1}^{r}=k_{X 1} I_{X 1}^{r} \\
& X_{2}^{r}=k_{X 2} I_{X 2}^{r} \\
& Y^{r}=k_{Y} I_{Y}^{r} \\
& Z^{r}=k_{Z} I_{Z}^{r}
\end{aligned}
$$

where superscript $r$ indicates that the measurements were made with the F100 reference colorimeter.

From the tristimulus values the color temperatures are calculated using iterations. First, the $u^{\prime}, v^{\prime}$ chromaticity coordinates are calculated for a range a blackbody temperatures from $1000 \mathrm{~K}$ to $20000 \mathrm{~K}$. From the chromaticity coordinates of the test source, the distance is calculated to the blackbody curve (coordinates). The closest distance is chosen along with its neighbors. In a second step, the set of blackbody temperatures are extended to 20 points centered on the previous closest point and bounded by the neighbors. Again, the smallest distance is chosen along with its neighbors. This process is repeated four more times. The last step will calculate the correlated color temperature to within $0.01 \mathrm{~K}$ of the F100 measured chromaticity coordinate.

The color temperatures were also determined from spectral irradiance measurements (of the same lamp) at the Facility for Automated Spectral Calibrations (FASCAL). The expanded relative uncertainty of the FASCAL color-temperature calibrations at $2856 \mathrm{~K}$ is $2 \mathrm{~K}(k=2)$.

The color temperature values obtained with the source-based lamp and detector-based
F100 calibrations are shown in Table 2. The last column shows the differences at three different temperatures. The difference at $2300 \mathrm{~K}$ is higher than at higher lamp temperatures because the near-IR leakage of the blue filter-combinations plays a more significant role at the low temperature (2300 K) color measurement.

The color temperature uncertainty when propagated from the FASCAL calibrated lamp standard to the F100 on the photometer bench (at $2856 \mathrm{~K})$ increases to $8 \mathrm{~K}(k=2)$. The lamp was calibrated on the FASCAL at a distance of $0.5 \mathrm{~m}$. When it is used in the photometer bench with a $2 \mathrm{~m}$ separation from the F100 colorimeter, the spatial non-

Table 2. Color temperature measurements (in K) using detector-based (F100) and source-based (FASCAL) calibrations.

\begin{tabular}{|c|c|c|c|c|}
\hline $\begin{array}{c}\text { Source } \\
\text { Based }\end{array}$ & $\begin{array}{c}\text { F100 } \\
\text { ref }= \\
2300 \mathrm{~K}\end{array}$ & $\begin{array}{c}\text { F100 } \\
\text { ref }= \\
2856 \mathrm{~K}\end{array}$ & $\begin{array}{c}\text { F100 } \\
\text { ref }= \\
3200 \mathrm{~K}\end{array}$ & $\begin{array}{c}\text { F10 } \\
- \\
\text { FAS- } \\
\text { CAL }\end{array}$ \\
\hline 2292.9 & 2304.4 & & & 11.5 \\
\hline 2857.2 & & 2862.2 & & 5.0 \\
\hline 3198.4 & & & 3201.7 & 3.3 \\
\hline
\end{tabular}

uniformity of the irradiance (in the reference plane of the colorimeter) significantly changes versus lamp alignment. This spatial non-uniformity causes the large uncertainty increase in the source-based calibration of the F100 colorimeter.

The uncertainty goal for the detector-based reference colorimeter calibrations is $4 \mathrm{~K}$ $(k=2)$.

\subsection{Working standard colorimeters}

The three working standard colorimeters are not calibrated at the SIRCUS facility to save the long and expensive calibration time. Their channels will be measured for spectral power responsivity at the SCF and then compared to the channels of the reference colorimeter. All the filter combinations and the Si detectors were taken from the same batches. Before the SCF spectral responsivity measurements, it is important to check the wavelength accuracy of the monochromator to avoid responsivity errors when 
measuring the steep sides of the channel responsivity curves.

The working standard colorimeters will be calibrated against the reference colorimeter (of the same type) in broad-band mode using the illumination from a stable $2856 \mathrm{~K}$ lamp. The calibration factors can be transferred from the channels of the reference colorimeter to the equivalent channels of the working standard (test) colorimeters by measuring the signal-ratios. The ratios give the corrections to the calibration factors of the reference colorimeter to obtain the channel calibration factors for the working standard colorimeter. The working standard colorimeters are used as test colorimeters in this calibration transfer. Since a working standard colorimeter should measure the same tristimulus values for a CIE Illuminant $A$ as the reference colorimeter, it can be written for the $\mathrm{X}_{1}$ channel:

$$
\begin{gathered}
X_{1}{ }^{r}=X_{1}{ }^{t}=k_{x 1} I_{x 1}{ }^{r}=k_{x 1}{ }^{t} I_{X 1}{ }^{t} \\
k_{x 1}{ }^{t}=\frac{k_{x 1} I_{X 1}{ }^{r}}{I_{X 1}{ }^{t}}
\end{gathered}
$$

where superscript $t$ indicates testcolorimeter which is the working standard in this case. The other three calibration factors of the test-colorimeter can be derived in the same way. These calibration factors also include the spectral mismatch and channelamplitude corrections. However, this scale transfer will result in low color-temperature measurement uncertainty for different source distributions if the relative spectral responsivities of the corresponding channels are very similar to each other.

\section{CONCLUSIONS}

The above described reference colorimeters and calibration considerations, with the obtained preliminary measurement results indicate that tristimulus color calibrations of tungsten lamps on a detector-base can decrease the $8 \mathrm{~K}(\mathrm{k}=2)$ uncertainty of traditional source-based calibrations. The achievable small $f_{1}$ ' values are also promising to apply the detector-based calibration method for special test sources with spectral distributions other than tungsten. In order to achieve these goals, the infrared leakage of the blue filters must be dramatically decreased and the uncertainty of the meas- ured spectral responsivity data should be improved by about a factor of two.

\section{ACKNOWLEDGEMENTS}

The authors thank the US Air Force AFMETCAL for funding this project, and also Steve Brown, Thomas Larason, and Jeanne Houston for participating in the responsivity data evaluations.

\section{REFERENCES}

1. G. Eppeldauer, Spectral Response Based Calibration Method of Tristimulus Colorimeters, J. Res. Natl. Inst. Stand Technol. Vol. 103, No. 6. p. 615, 1998.

2. G. Eppeldauer and Y. Ohno, Development of the NIST Detector-based Color Temperature Scale, Proc. ISCC/CIE Expert Symposium '06 "75 Years of CIE Standard Colorimetric Observer", p. 5558, CIE Central Bureau, 27, Kegelgasse, A-1030, Vienna, Austria, 2006.

3. S. W. Brown, G. P. Eppeldauer, and K. R. Lykke, Facility for Spectral Irradiance and Radiance Responsivity Calibrations using Uniform Sources, Applied Optics, Vol. 45, No. 32, p. 8218-8237, 2006.

4. J. Fowler and M. Litorja, Geometric area measurements of circular apertures for radiometry at NIST, Metrologia, Vol. 40, No. 1, S9-S12, 2003.

\section{AUTHORS}

G. P. Eppeldauer, C. C. Miller, and Y. Ohno National Institute of Standards and Technology 100 Bureau Drive

Gaithersburg, Maryland 20899, USA

Phone: 301-975-2338, FAX: 301-869-5700,

Email: george.eppeldauer @nist.gov 


\title{
Directional error correction of radiometric and photometric standard detectors
}

\author{
Miklós Rácz \\ Hungarian Academy of Sciences Research Institute for Technical Physics and Materials Science \\ H-1525 Budapest-114, P.O.Box 49, Hungary \\ George Eppeldauer \\ National Institute of Standards and Technology, Optical Technology Division \\ Gaithersburg, MD 20899
}

\begin{abstract}
Changes in beam geometry during the calibration and subsequent testing of optical detector standards can cause errors which appear as uncertainties in the responsivities of the detectors. These uncertainties, caused by non-ideal detector input characteristics and changes in input beam geometry, can be decreased significantly if the detector response is always corrected to a reference beam geometry such as a collimated beam normally incident on the detector surface. An integral directional error can be determined for radiant power, luminous flux, irradiance and illuminance detectors by calculating the weighted integral of the product of the measured source radiance and the directional error over the solid angle produced by the light source. A conical directional error is introduced, and used to describe the case of circular and uniform radiant sources (e.g. exit port of an integrating sphere) and circularly symmetric detectors. Evaluation of the conical directional error and the directional response correction factor, the results of directional response measurements, and directional response correction factors for practical standard detectors (such as diffuser-input InGaAs and integrating-sphere-input silicon irradiance meters, silicon photodiode, and pyroelectric radiant power and irradiance measuring detectors) are presented.
\end{abstract}

Keywords: calibration, directional error, $\boldsymbol{f}_{2}$ error, conical directional error, standard detector

\section{Introduction}

The accuracy of primary standard radiometers has significantly improved in the last few years $[1,2,3]$. In order to benefit fully from these improvements, the accuracy of the derived radiometric scales needs to be improved. Greater accuracy in the scales means that some effects, formerly negligible, have become more important. One such effect is the directional response error of the detectors. At calibration the directional distribution of optical radiation (beam geometry) from a source may differ from that in subsequent tests of optical detector standards. These changes in beam geometry can be significant: here they are treated as equivalent to uncertainties in detector responsivity. Such uncertainties can be reduced by correcting the detector response to a reference input beam geometry. A collimated beam normally incident on the detectorsurface makes a practical choice for reference geometry. This paper introduces a conical directional error, $\boldsymbol{f}_{d}(\alpha)$, and a directional response correction factor, $\boldsymbol{C}_{\mathrm{d}}$, which can easily be derived from the directional error, $f_{2}(\varepsilon, \varphi)$, as defined by the International Commission on Illumination (CIE), for spatially uniform and circularly symmetric calibration sources. [4].

\section{Calculation of directional response correction factor}

The directional response correction factor $\boldsymbol{C}_{\boldsymbol{d}}$ of a radiometric detector is a quantity that corrects the detector response measured for the beam geometry $\boldsymbol{S}_{\boldsymbol{b}}$ to the collimated beam response $\boldsymbol{S}_{\boldsymbol{c}}$ :

$$
S_{c}=S_{b} / C_{d} \text {. }
$$

An integral directional error quantity $\boldsymbol{f}_{2}$ (defined in the CIE Publ. No 53) is given by:

$$
f_{2}=S_{b} / S_{c}-1 \text {. }
$$


It follows from Eqs. (1) and (2) that

$$
C_{d}=1+f_{2} .
$$

References [4] and [5] describe a method for determining the directional characteristics of irradiance, illuminance, and radiance meters by the directional error $f_{2}(\varepsilon, \varphi)$. For irradiance and illuminance meters, the CIE defined directional error is:

$$
f_{2}(\varepsilon, \varphi)=\frac{E_{\text {reading }}(\varepsilon, \varphi)}{E_{\text {reading }}\left(\varepsilon=0^{\circ}\right) \cdot \cos \varepsilon}-1,
$$

where $\boldsymbol{\varphi}$ is the azimuth angle (rotation around the detector axis), and $\boldsymbol{\varepsilon}$ is the angle of incidence. $\boldsymbol{E}_{\text {reading }}(\boldsymbol{\varepsilon} \varphi)$ and $\boldsymbol{E}_{\text {reading }}\left(\boldsymbol{\varepsilon}=\mathbf{0} \boldsymbol{9}\right.$ are the readings for an irradiance or illuminance, $\boldsymbol{E}$, arriving at the angles of incidence $\boldsymbol{\varepsilon}$ and $0^{\circ}$, respectively.

As the denominator shows, the response of an ideal irradiance or illuminance meter follows the cosine function. For radiant power and directional luminous flux meters, where ideally the response is independent of the direction, the directional error $f_{2}(\varepsilon, \varphi)$ is:

$$
f_{2}(\varepsilon, \varphi)=\frac{E_{\text {reading }}(\varepsilon, \varphi)}{E_{\text {reading }}\left(\varepsilon=0^{\circ}\right)}-1 .
$$

References [4] and [5] describe how to calculate the integral directional error $\boldsymbol{f}_{2}$ from the directional error $\boldsymbol{f}_{2}(\boldsymbol{\varepsilon} \varphi)$. The integral directional error $\boldsymbol{f}_{2}$ describes the response measurement error originating from the directional error of the detector. The CIE defined integral directional error $\boldsymbol{f}_{2}$ for irradiance and illuminance meters is:

$$
f_{2}=\int_{\Omega} f_{2}(\varepsilon, \varphi) \cdot L(\varepsilon, \varphi) \cdot \cos \varepsilon \cdot d \Omega / \int_{\Omega} L(\varepsilon, \varphi) \cdot \cos \varepsilon \cdot d \Omega,
$$

where $\boldsymbol{L}(\boldsymbol{\varepsilon} \varphi)$ is the source radiance, $\boldsymbol{d} \boldsymbol{\Omega}$ is the elementary solid angle, and $\boldsymbol{\Omega}$ is the total acceptance solid angle (field of view) of the detector.

For radiant power and directional luminous flux meters, where ideally the response is independent of the direction, the error $f_{2}$ is:

$$
f_{2}=\int_{\Omega} f_{2}(\varepsilon, \varphi) \cdot L(\varepsilon, \varphi) \cdot d \Omega / \int_{\Omega} L(\varepsilon, \varphi) \cdot d \Omega
$$

Using only angles $\varepsilon$ and $\varphi$, Eq (5a) can be written as:

$$
f_{2}=\int_{0}^{\pi / 2}\left[\int_{0}^{2 \pi} f_{2}(\varepsilon, \varphi) \cdot L(\varepsilon, \varphi) \cdot \cos \varepsilon \cdot 2 \pi \cdot \sin \varepsilon \cdot d \varphi\right] \cdot d \varepsilon / \int_{0}^{\pi / 2}\left[\int_{0}^{2 \pi} L(\varepsilon, \varphi) \cdot \cos \varepsilon \cdot 2 \pi \cdot \sin \varepsilon \cdot d \varphi\right] \cdot d \varepsilon .
$$

References [4] and [5] describe the cases where the irradiation comes from the $\boldsymbol{\Omega}=2 \pi$ hemisphere or the total $\Omega=4 \pi$ sphere. For detector calibrations, spatially uniform and circularly symmetrical calibration sources should be used where the source center is on the optical axis of the detector. For irradiance and illuminance meter calibrations, such radiation sources can be realized with integrating spheres. The radiance or luminance of the exit port of a high-quality integrating sphere can be considered spatially uniform. In this case $\boldsymbol{L}(\varepsilon, \varphi)=\boldsymbol{L}$, for $\varepsilon<\alpha$, and $\boldsymbol{L}(\boldsymbol{\varepsilon}, \varphi)=\mathbf{0}$ for $\boldsymbol{\varepsilon}>\boldsymbol{\alpha}$, where $\boldsymbol{\alpha}$ is the half viewing angle determined by the radius of the light source (the exit port of the integrating sphere) and the distance to the aperture center of the detector.

In case of spatially uniform and circularly symmetrical calibration sources, the integral directional error $\boldsymbol{f}_{2}$ depends on the viewing half angle $\alpha$ of the radiation (extended) source, that is, $\boldsymbol{f}_{2}=\boldsymbol{f}_{2}(\boldsymbol{\alpha})$. To avoid confusion with the CIE $\boldsymbol{f}_{2}(\boldsymbol{\varepsilon} \boldsymbol{\varphi})$ (and later $f_{2}(\varepsilon)$ ), we introduce the conical directional error $f_{d}(\alpha)$ instead of $f_{2}(\alpha)$.

The conical directional error $\boldsymbol{f}_{\boldsymbol{d}}(\boldsymbol{\alpha})$, using spatially uniform and circularly symmetrical sources, for irradiance and illuminance meters is: 


$$
f_{d}(\alpha)=\int_{0}^{\alpha}\left[\int_{0}^{2 \pi} f_{2}(\varepsilon, \varphi) \cdot \cos \varepsilon \cdot 2 \pi \cdot \sin \varepsilon \cdot d \varphi\right] \cdot d \varepsilon / \int_{0}^{\alpha}\left[\int_{0}^{2 \pi} \cos \varepsilon \cdot 2 \pi \cdot \sin \varepsilon \cdot d \varphi\right] \cdot d \varepsilon
$$

The calculation becomes simpler if the detector directional response is also circularly symmetrical (i.e. the response is independent of azimuth angle $\varphi$ ) resulting in $f_{2}(\varepsilon \varphi)=f_{2}(\varepsilon)$. In this case, the conical directional error for irradiance and illuminance meters is:

$$
f_{d}(\alpha)=\int_{0}^{\alpha} f_{2}(\varepsilon) \cdot \sin 2 \varepsilon d \varepsilon / \int_{0}^{\alpha} \sin 2 \varepsilon \cdot d \varepsilon
$$

Similar evaluation of Eq. (5b) will result in the conical directional error for radiant power and directional luminous flux meters:

$$
f_{d}(\alpha)=\int_{0}^{\alpha} f_{2}(\varepsilon) \cdot \sin \varepsilon d \varepsilon / \int_{0}^{\alpha} \sin \varepsilon \cdot d \varepsilon
$$

For radiant power and directional luminous flux meter calibrations, where the incident beam underfills the detector aperture, the uniform source can be realized by projecting a point source onto the detector entrance with a lens.

In accordance with Eq. (3), the conical directional error can be compensated for all kinds of radiometers by applying the directional response correction factor $\boldsymbol{C}_{\boldsymbol{d}}$ :

$$
C_{d}=1+f_{d}(\alpha)
$$

\section{Application of conical directional error and directional response correction factor}

At NIST, determination of detector directional response characteristics is done on a regular basis. Several of our measurements have been published by Eppeldauer et al. [6] and Lehman et al. [7].

For practical applications, the directional response correction factor $\boldsymbol{C}_{\boldsymbol{d}}$ is used to correct the detector response for the reference beam geometry, which is the axially incident parallel beam. Because the directional response correction factor $\boldsymbol{C}_{d}$ is close to unity, the conical directional error $\boldsymbol{f}_{d}(\alpha)$ gives better visual information.

The CIE $f_{2}(\varepsilon)$ directional errors were measured, the conical directional errors $f_{d}(\alpha)$, and the directional response correction factors $\boldsymbol{C}_{\boldsymbol{d}}$ were calculated for a number of detector standards. The results of four tested detectors are summarized in table . Here, directional error curves are shown as an example for a diffuser-input InGaAs irradiance meter only. This radiometer has an InGaAs photodiode with input optics consisting of a flashed opal glass diffuser, a precision aperture, and a field-of-view limiter. The characterization of the input optics and the directional response measurement results of this irradiance standard have been published by Eppeldauer et al. [6]. The detector directional response characteristics were measured with an incandescent lamp irradiation. The CIE directional error $\boldsymbol{f}_{\mathbf{2}}(\boldsymbol{\varepsilon})$ and the conical directional error $f_{d}(\alpha)$ of the detector can be seen in figure .

figure can be used to determine the directional response correction factor $\boldsymbol{C}_{\boldsymbol{d}}$. If the viewing half angle $\boldsymbol{\alpha}$ of the source from the detector center is known, the value of the conical directional error $f_{d}(\alpha)$ can be read from the right vertical axis. Thereafter, the directional response correction factor can be calculated using Eq. (9). The corrected detector response can be calculated from Eq. (1). 


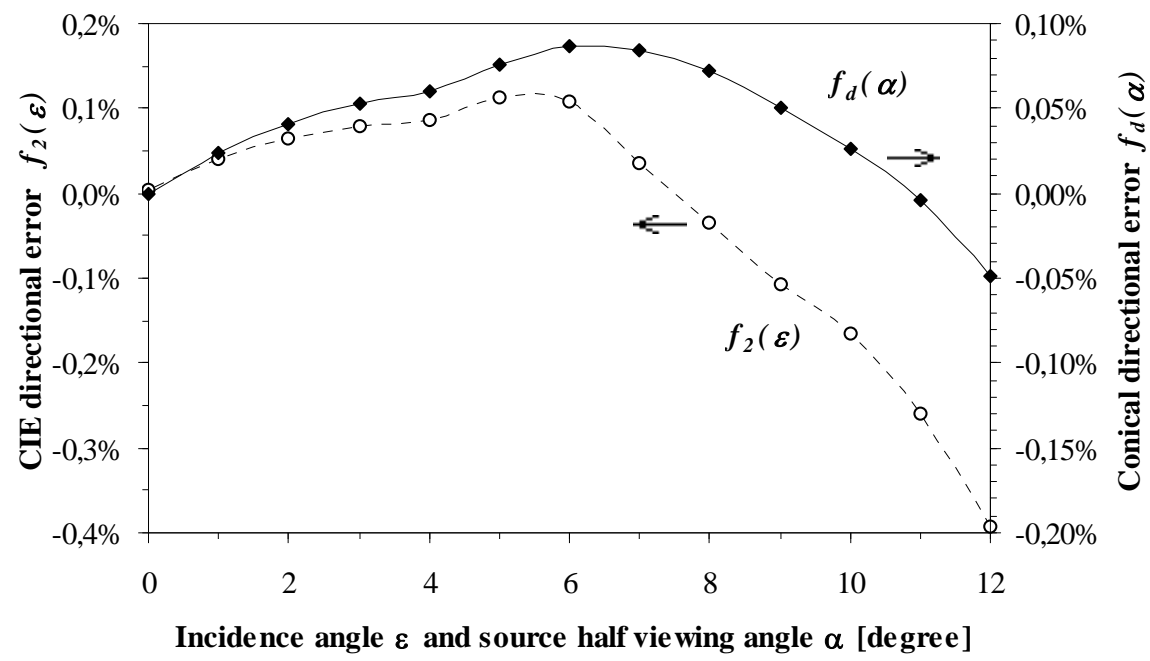

Figure 1. The directional error $\mathrm{f}_{2}(\varepsilon)$ (dotted line with open circles) and the conical directional error $\mathrm{f}_{\mathrm{d}}(\alpha)$ (solid line with black diamonds) of a diffuser input InGaAs irradiance meter. The uncertainties are less than $0.025 \%$ for $\mathrm{f}_{2}(\varepsilon)$ when $\varepsilon \leq 6^{\circ}$ and less than $0.023 \%$ for $\mathrm{f}_{\mathrm{d}}(\alpha)$ when $\alpha \leq 9^{\circ}(k=1)$.

Table 1. Conical directional errors $f_{d}(\alpha)$ and directional response correction factors $C_{d}$ of several irradiance and radiant power detector standards for f/9 and f/4 incidence beam geometry. The relative uncertainties of the error determinations are also given, $k=1$.

\begin{tabular}{|l|c|c|c|c|c|c|c|c|}
\hline \multirow{3}{*}{ Detector type } & \multicolumn{4}{|c|}{ Irradiance mode } & \multicolumn{3}{c|}{ Radiant flux (power) mode } \\
\cline { 2 - 9 } & $\mathrm{f} / 9$ beam, $\boldsymbol{\alpha}=3.18^{\circ}$ & \multicolumn{2}{|c|}{$\mathrm{f} / 4$ beam, $\boldsymbol{\alpha}=7.13^{\circ}$} & \multicolumn{2}{c|}{$\mathrm{f} / 9$ beam, $\boldsymbol{\alpha}=3.18^{\circ}$} & \multicolumn{2}{c|}{$\mathrm{f} / 4$ beam, $\boldsymbol{\alpha}=7.13^{\circ}$} \\
\cline { 2 - 9 } & $\boldsymbol{f}_{\boldsymbol{d}}$ & $\boldsymbol{C}_{\boldsymbol{d}}$ & $\boldsymbol{f}_{\boldsymbol{d}}$ & $\boldsymbol{C}_{\boldsymbol{d}}$ & $\boldsymbol{f}_{\boldsymbol{d}}$ & $\boldsymbol{C}_{\boldsymbol{d}}$ & $\boldsymbol{f}_{\boldsymbol{d}}$ & $\boldsymbol{C}_{\boldsymbol{d}}$ \\
\hline Sphere-input Si & $+0.033 \%$ & 1.00033 & $+0.166 \%$ & 1.00166 & & & & \\
(1) & $\pm 0.007 \%$ & & $\pm 0.005 \%$ & & & & & \\
\hline Diffuser-input & $+0.055 \%$ & 1.00055 & $+0.081 \%$ & 1.00081 & & & & \\
InGaAs & $\pm 0.010 \%$ & & $\pm 0.016 \%$ & & & & & \\
\hline Gold-black coated & $+0.036 \%$ & 1.00036 & $+0.022 \%$ & 1.00022 & $-0.003 \%$ & 0.99997 & $-0.006 \%$ & 0.99994 \\
pyroelectric (2) & $\pm 0.046 \%$ & & $\pm 0.033 \%$ & & $\pm 0.005 \%$ & & $\pm 0.004 \%$ & \\
\hline Si photodiode with & $+0.076 \%$ & 1.00076 & $+0.175 \%$ & 1.00175 & $-0.009 \%$ & 0.99991 & $+0.037 \%$ & 1.00037 \\
window (3) & $\pm 0.033 \%$ & & $\pm 0.026 \%$ & & $\pm 0.038 \%$ & & $\pm 0.026 \%$ & \\
\hline
\end{tabular}

In addition to the InGaAs radiometer, the test results of the following detectors are summarized in table :

(1) Integrating-sphere-input silicon irradiance meter. A Si photodiode was attached to the exit port of a $50 \mathrm{~mm}$ diameter integrating sphere. An early design of this irradiance meter has been published by Eppeldauer [8]. In the present version, a smaller (3.5 mm diameter) input aperture is used, and an additional light-tight cover is added to the housing. The directional characteristics were measured with an incandescent lamp.

(2) Pyroelectric radiant power and irradiance measuring detector. A gold black coated $\mathrm{LiNbO}_{3}$ pyroelectric detector, published by Lehman et al. [7], was further tested here. The detector has an $8 \mathrm{~mm}$ diameter aperture with $1 \mathrm{~mm}$ separation from the detector surface. The power-mode directional measurements were performed with a laser beam focused onto the detector. The irradiance-mode directional characteristics were measured with an incandescent lamp source.

(3) Silicon photodiode. The most frequently used transfer standards are silicon photodiodes for the near UV, visible, and near IR spectral ranges, as described by Larason et al. [9]. The directional characteristics were measured on a widely used standard photodiode. This device has a window in front of the circular, $1 \mathrm{~cm}^{2}$ area photodiode. The tests were made with incandescent lamp irradiation.

The results show that even for well-designed (optimized) radiometer standards, the conical directional error $f_{d}(\alpha)$ can 
exceed the $0.1 \%$ level $(\mathrm{k}=1)$. In order to achieve uncertainties below $0.1 \%$, application of the directional response correction factors can be necessary even for high-performance radiometer standards. In some cases, the uncertainties of our directional response measurements were too high to determine the directional response correction factors with the desired low uncertainty.

\section{Conclusions}

The conical directional error, $\boldsymbol{f}_{d}(\alpha)$, has been introduced and used to evaluate the effect of the CIE defined directional error, $f_{2}(\varepsilon \phi)$, for transfer standard radiometers. For a given incident beam geometry, $f_{d}(\alpha)$, can be determined as a weighted integral of the CIE $f_{2}(\varepsilon, \phi)$ directional error. A directional response correction factor, $\boldsymbol{C}_{d}$, has also been calculated. The uncertainty budget of detector response measurements can be decreased by applying this response correction. The CIE directional error can thus be replaced by the directional response correction factor determination, which has a smaller uncertainty. The suggested calculation can be applied to all irradiance, illuminance, power, and directional luminance flux meters. For the most frequently used beam geometries at the NIST, the conical directional error $f_{d}(\alpha)$ ranged between $+0.022 \%$ and $+0.175 \%$ for irradiance meters, and from $-0.003 \%$ to $+0.037 \%$ for radiant power meters. In order to simplify the calculation of $\boldsymbol{C}_{\boldsymbol{d}}$, circularly symmetric radiometers should be designed for standardization. A high-accuracy measurement setup is required to determine the directional response correction factor with a small enough uncertainty for the needs of standards-quality detectors. The described response correction method can be applied for medium-performance detector standards as well.

\section{Acknowledgements}

The authors thank Eric L. Shirley of the NIST Optical Technology Division and Jack J. Hsia of the NIST Office of International and Academic Affairs for their valuable advices, and Yuqin Zong of the NIST Optical Technology Division for helping in the directional response measurements.

\section{References}

1. Gentile T. R., Houston J. M., Hardis J. E., Cromer C. L., and Parr A. C., National Institute of Standards and Technology high-accuracy cryogenic radiometer, Applied Optics Vol. 35, No. 7, 1 March 1996. p. 1056-1068.

2. Goebel R., Pello R., Stock K. D. and Hofer H., Direct comparison of cryogenic radiometers from the BIPM and the PTB, Metrologia, 1997, 34, p. 257-259.

3. Köhler R, Goebel R and Pello R., Experimental procedures for the comparison of cryogenic radiometers at the highest accuracy, Metrologia, 1996, 33, p. 549-554.

4. Publication CIE 53-1982 Methods of Characterizing the Performance of Radiometers and Photometers.

5. Publication CIE 69-1987 Methods of Characterizing Illuminance Meters and Luminance Meters.

6. Eppeldauer G., Rácz M., and Larason T., Optical characterization of diffuser-input standard irradiance and illuminance meters, Optika’98, 14-17 Sep. 1998, Budapest, Hungary, SPIE 3573, p. 220-224, 1998.

7. Lehman J., Eppeldauer G., Aust J. A., and Rácz M., Domain-engineered pyroelectric radiometer, 1999, In press.

8. Eppeldauer G., Near Infrared Radiometer Standards, Optical Radiation Measurements, 8-9 Aug. 1996, Denver, Colorado, SPIE Vol. 2815, p. 42-54, 1996.

9. Larason T. C., Bruce S. S., and Parr A. C., Spectroradiometric Detector Measurements: Part I-Ultraviolet Detectors and Part II-Visible to Near-Infrared Detectors, NIST Special Publication 250-41. 


\title{
THE REALIZATION AND THE DISSEMINATION OF THE DETECTOR-BASED KELVIN
}

\author{
Howard W. Yoon, Charles E. Gibson, David W. Allen, Robert D. Saunders, Maritoni Litorja, \\ Steven W. Brown, George P. Eppeldauer, Keith R. Lykke \\ Optical Technology Division \\ National Institute of Standards and Technology \\ Gaithersburg, MD 20899 \\ USA
}

\begin{abstract}
In the International Temperature Scale of 1990 (ITS-90), temperatures above the freezing temperature of silver are determined with radiation thermometers calibrated using spectral radiance ratios to one of the Ag-, Au- or $\mathrm{Cu}$-freezing temperature blackbodies and the Planck radiance law. However, due to the use of spectral radiance ratios, the temperature uncertainties of the ITS-90 increase as the square of the temperature ratios, and recent acoustic-gas thermometry measurements have also shown that the underlying thermodynamic temperatures used in the radiance ratios in determining the Ag- and Au-fixed point temperatures could be in error. Since the establishment of ITS-90, much progress has been made in the development of radiation thermometers and blackbody sources. Cryogenic electrical-substitution radiometry is widely used in detector and radiometer calibrations, and stable, high-temperature metal-carbon eutectic blackbodies are under development. Radiation thermometers can be calibrated for absolute radiance responsivity, and blackbody temperatures determined from the amount of optical power without the use of any fixed points thus making possible direct dissemination of thermodynamic temperatures. We show that these temperatures can be measured with lower final uncertainties than the ITS-90 derived temperatures. We have shown that these "Absolute Pyrometers" can be used to determine the thermodynamic temperatures of the ITS-90 fixed points as well as also being used in bilateral comparisons of temperature scales. Many leading national measurement institutes are already utilizing detectorbased temperatures in establishing spectroradiometric source scales. We believe, that due to these developments, the international temperature scale should be revised so that a thermodynamic temperature scale can be directly disseminated.
\end{abstract}

\section{INTRODUCTION}

The development of an international temperature scale and the periodic adjustments of the scale are attempts to disseminate and best reproduce thermodynamic temperatures. Thermodynamic temperatures can be determined by primary thermometry using equations of state which relate temperatures with measurable quantities without any temperature-dependent parameters such as in pressure-volume and acoustic gas thermometry, noise thermometry and detector-based radiation thermometry [1]. Due to the difficulties in developing facilities and performing experiments for primary thermometry, various phase transitions of material have been assigned temperatures based upon available thermodynamic temperature measurements, and these fixed-points cells and blackbodies are in-turn used to calibrate secondary thermometers or sensors whose output changes in response to temperature. Almost all the national measurement institutes (NMI) rely upon a set of these fixed-points to generate the international temperature scale instead of performing their own primary thermometry, and thus the current International Temperature Scale of 1990 (ITS-90) with its system of fixed-points and defining instruments is an attempt at achieving closest agreement with the thermodynamic temperature scale [2].

Although there are no fundamental, inherent temperature limitations in the equations describing the relationship between measurables and thermodynamic temperatures in primary thermometry, in practice, experimental concerns limit these primary thermometers to a range of temperatures. Since 
the experimental upper temperature limit for the lowest uncertainty constant-volume gas thermometry and acoustic-gas thermometry is $<900 \mathrm{~K}$, these temperature limitations have impacted the higher temperatures uncertainties of the ITS-90 [3]. Due to the conflicting results of two constant-volume gas thermometry measurements, the mean of the two results were used as reference values at $743 \mathrm{~K}$ for radiation thermometry in determining the Al-, Ag- and Au-freezing temperatures used in the ITS90 [1]. The latest acoustic-gas thermometry measurements have been found to be consistent with the work of Edsinger and Schooley that would conclude that the current ITS-90 assignments for the each of the high-temperature fixed-points have inherent temperature biases [4]. There have also been recent efforts in noise-thermometry in measurements of the Ag- and Pd-freezing temperatures, but the uncertainties of the noise-thermometry determinations are comparable to those assigned to the ITS-90 values of these fixed points [5]. Even with new efforts in noise thermometry using super-conducting junctions to generate noise standards, the thermodynamic temperature uncertainties above the Agpoint are not expected to be greatly reduced below the present uncertainties [6].

Detector-based radiation thermometry can used for thermodynamic temperature determinations which are applicable over a wide range of temperatures from $60 \mathrm{~K}$ to $>3000 \mathrm{~K}$. With the development of cryogenic electrical substitution radiometers (ESR), accurate measurements of optical power by comparison to electrical power resulted in the determinations of the Stefan-Boltzmann constant from total radiation thermometry [7]. As the temperatures of blackbodies are increased, spectrally-selective radiation thermometers calibrated using ESR-based radiometry can be used to determine the thermodynamic temperatures with low uncertainties using Si-diode based radiation thermometers [8]. Recognizing that these detector-based radiation thermometers be used to obtain lower temperature uncertainties than ITS-90 based radiation thermometers, many leading national measurement institutes have developed calibration facilities for detector-based temperatures [9]. The needs for the lowest uncertainties in temperature $>900 \mathrm{~K}$ arise chiefly from the spectroradiometric community where sources are used to calibrate remote-sensing instruments.

With the development of high-temperature metal-carbon and metal carbide-carbon eutectics, these stable sources can be used as fixed points for redefinition of the ITS [10]. However, for the lowest thermodynamic uncertainties needed for the scale, detector-based radiation thermometry should be used to determine the transition temperatures of the eutectics since the any practical temperature scale should be in agreement with the thermodynamic scale.

Although detector-based radiation thermometry is increasingly being used in the international radiometric community, the international temperature community still utilizes the source-based ITS-90 derived temperatures, leading to a possible inconsistency in the disseminated radiometric and temperature scales from a common NMI. The situation is analogous to that in the photometry with the transition from the platinum-point based candela to the redefinition of the candela based upon optical power in 1979 [11]. If a general redefinition of the ITS-90 over all temperatures is not feasible or needed at the present time, then the ITS-90 above the Ag-point or perhaps above the Al-point should be redefined to reflect the maturity of detector-based radiation thermometry.

In this paper, we describe the facilities and the experimental procedures in calibrating detector-based radiation thermometer traceable to a cryogenic electrical substitution radiometer. The radiation thermometer, Absolute Pyrometer 1 (AP1), has been constructed at NIST to be temporally stable and is characterized for linearity, size-of-source effect (SSE) and detector-based radiance responsivity. The AP1 was used to determine the thermodynamic temperatures of the Ag- and Au-freezing temperatures and was also used for a bilateral comparison of temperature scales with the National Physical Laboratory (United Kingdom) using high-temperature metal-carbon eutectic blackbodies. With these measurements, we demonstrate that primary thermometers such as the AP1 can be used to directly disseminate thermodynamic temperatures with lower uncertainties that any ITS-90 based thermometer. We describe improvements to radiation thermometer designs to reduce the SSE to $<5 \times 10^{-5}$ with long-term stability of the responsivity from the use of graphite reference rods in the 
construction. We believe that these results along with further advances at other NMIs will result in the adoption of detector-based thermodynamic temperature scale above the Ag-freezing temperature with possible extension down to the Al-freezing temperature.

\section{DETECTOR-BASED RADIANCE RESPONSIVITY CALIBRATIONS}

Without the development of facilities to derive radiance and irradiance responsivity from electrical substitution radiometers, detector-based radiation thermometry would not be possible. Radiation thermometers can be constructed to measure irradiance or radiance, and simple filter radiometers with a precision aperture can be used to measure the irradiance of the source if the source also has a defining aperture. If the spatial uniformity of the filter radiometer is quantified, then power responsivity measurements along with the aperture area will provide the irradiance responsivity. However, typical radiation thermometers are constructed using lenses to define a target area to measure in radiance mode, and blackbody temperatures can be determined using the detector-based radiance responsivities and Planck's law by the use of

$$
i_{c}=\int S_{L} \cdot \varepsilon \cdot L(\lambda, T) d \lambda,
$$

where $i_{c}$ is the calculated photocurrent, $S_{L}$ is the radiance responsivity, and $L(\lambda, T)$ is the Planck radiance law, $\varepsilon$ is the emissivity of the blackbody. With the use Eq. 1, blackbody temperatures can be determined directly with no adjustable parameters in the measurement equation relating the radiance responsivity to the thermodynamic temperature. Since there is a unique relationship between the calculated current and the blackbody temperatures in Eq. 1, then integrations can be performed to find a match between the calculated and the measured photocurrents to determine blackbody temperatures.

At NIST, the radiance and irradiance responsivities of radiometers are calibrated using the NIST Spectral Irradiance and Radiance responsivity Calibrations using Uniform Sources (SIRCUS) facility [12]. Briefly, the 6-element trap detectors used in this facility are calibrated for power responsivity using the NIST High-Accuracy Cryogenic Radiometer (HACR) [13], and from the knowledge of the spatial uniformity and the precision aperture area of the trap detectors [14], the detector-based spectral irradiance responsivity is determined as shown in Fig. 1.

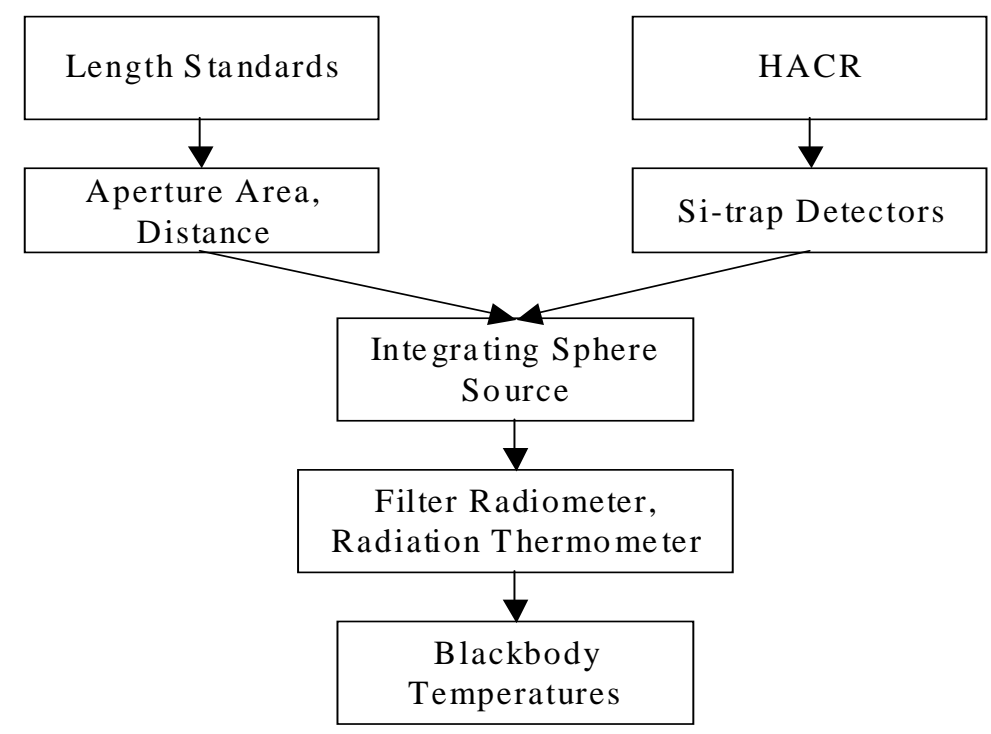

Figure 1. The realization chain for thermodynamic temperatures based upon detector-based radiation thermometry. 
With the irradiance responsivity of the Si-trap detectors calibrated from the power responsivity, the spectral irradiance of the laser-illuminated integrating sphere source (ISS) can be determined. Knowing the distance between the Si-trap detector and the precision aperture of the ISS determines the detector-based spectral radiance of the ISS. The above procedure is repeated with changing laser wavelengths, and thus the radiance or the irradiance responsivity of the filter radiometer or the radiation thermometer can be determined. Since the radiation thermometer is calibrated as a system, knowledge of the lens transmittances in not needed, and complex radiation thermometers with many optical elements can also be calibrated in the SIRCUS facility.

\section{THE DESIGN AND CHARACTERIZATION OF THE AP1}

With very few exceptions, the performances of commercial radiation thermometers are quite limited in the temporal stability, size-of-source and optical imaging so that new types of radiation thermometers need to be constructed. A schematic of the AP1 in front of the gold-point blackbody is shown in Fig. 2. The dimension of the AP1 from outside of the front plate to the back plate is $53 \mathrm{~cm}$. The optical elements are attached to three $50 \mathrm{~cm}$ long, $12.5 \mathrm{~mm}$ diameter invar rods for stability. A gradient-index singlet lens is chosen for the objective to minimize the possible scattered light from the lens interfaces and to improve the image quality. The objective distance is set at $50 \mathrm{~cm}$ using a fixed-

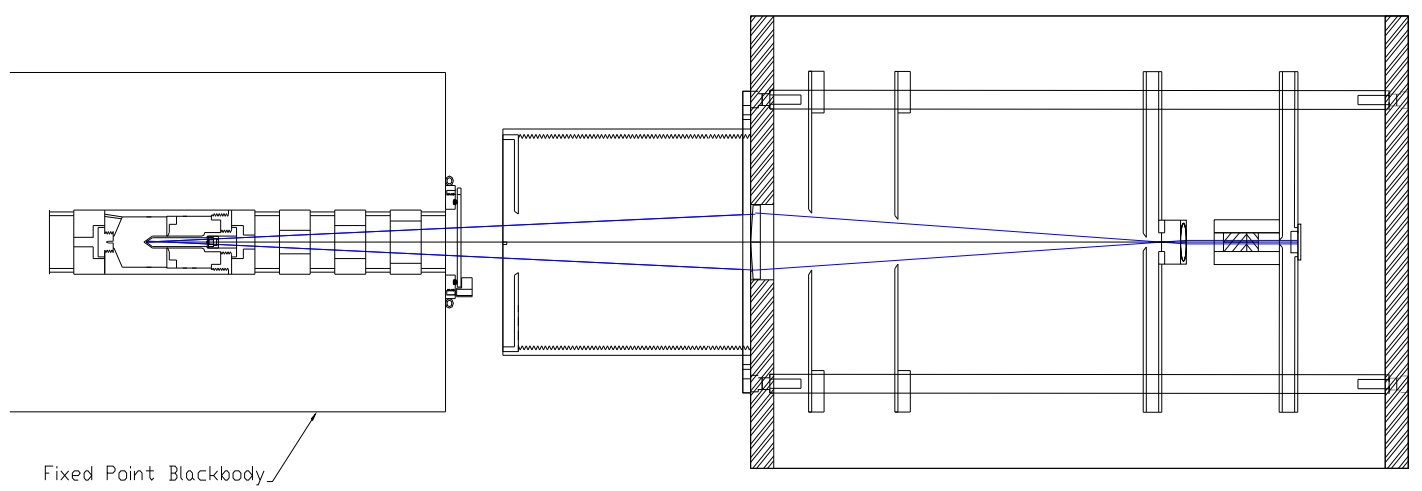

Figure 2. The schematic of the AP1 in front of the gold-point blackbody.

focus singlet lens distance of $33.3 \mathrm{~cm}$. A $0.5 \mathrm{~mm}$ diameter, blackened bi-metal pinhole is used as the field stop, which results in a nominal target size of $0.75 \mathrm{~mm}$ diameter. The size of the target area was chosen such that tungsten-strip lamps and fixed-point blackbodies could be compared to variabletemperature blackbodies with larger cavity apertures.

The re-imaging of the field stop is performed using an achromatic lens, which collimates the light. The collimated light passes through the interference filters and is incident on the $5.8 \mathrm{~mm}$ by $5.8 \mathrm{~mm}$ square Si photodiode. The spot size on the diode is estimated to be $5 \mathrm{~mm}$ diameter from ray-trace analysis. The spectral selection is performed using an ion-assisted deposited interference filter with center wavelength at $650 \mathrm{~nm}$. The spectral selection filter is temperature stabilized near room temperature, and the hermetically sealed Si photodiode has a 2-stage thermo-electric cooler for operation at $-15{ }^{\circ} \mathrm{C}$ to achieve a noise-equivalent power of $10 \mathrm{fW}$. The entire pyrometer can be purged with dry air or nitrogen to reduce effects due to humidity on the optical elements. 


\subsection{RADIANCE RESPONSIVITY CALIBRATION OF THE AP1}

The AP1 was calibrated for radiance responsivity using the NIST SIRCUS facility. The detectorbased radiance responsivity of the AP1 is shown in Fig. 3. The interference filter used in the AP1

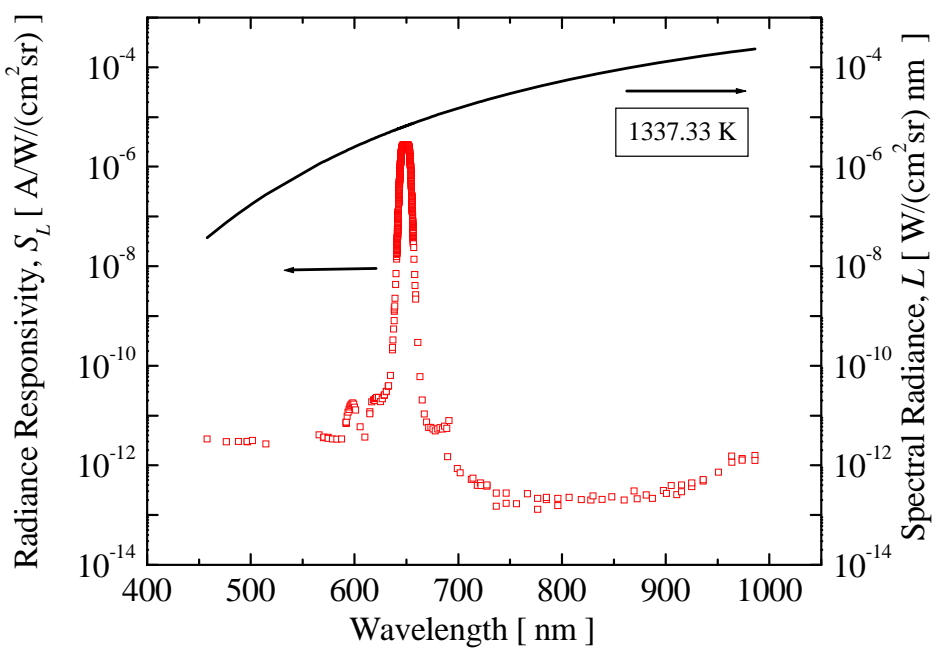

Figure 3: The radiance responsivity of the AP1 measured in the SIRCUS facility plotted with the Planck radiance at 1337.33 K. The center wavelength is $650 \mathrm{~nm}$ with a FWHM of $10 \mathrm{~nm}$. The out-of-band suppression of $<1 \times 10^{-7}$ is achieved in the wavelength region from $700 \mathrm{~nm}$ to $1000 \mathrm{~nm}$.

has $<1 \times 10^{-7}$ suppression in the out-of-band region from the peak value of the responsivity. The fullwidth at half-maximum (FWHM) of the radiance responsivity is $10 \mathrm{~nm}$. The calibration of the detector-based radiance responsivity is performed with the AP1 as a system and thus any other radiation thermometer can be calibrated similarly. The total expanded uncertainty of the radiance responsivity is $0.15 \%(k=2)$.

\subsection{DETERMINATION OF DETECTOR-BASED TEMPERATURES}

The calculated photocurrents from the use of Eq. 1 and the responsivity in Fig. 3 are plotted versus the respective blackbody temperatures in Fig. 4 and shows that the photocurrents range from $<10^{-10}$ A to

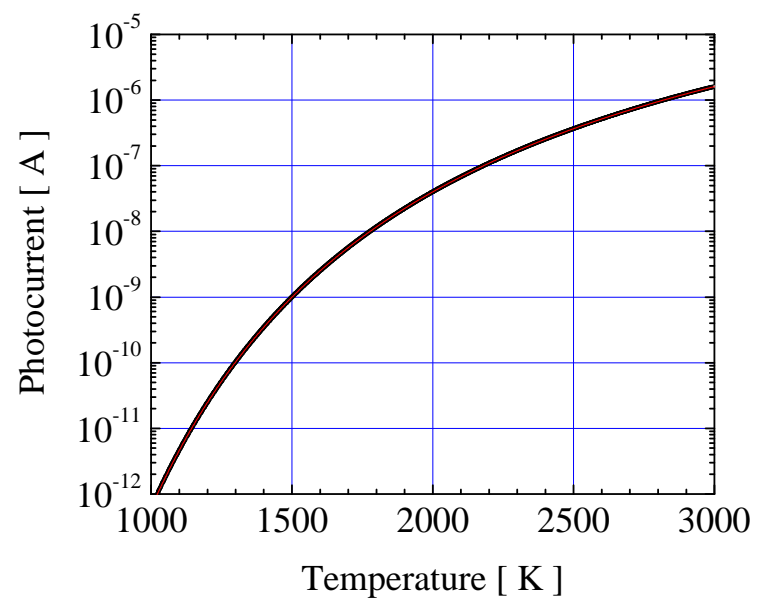

Figure 4. The calculated photocurrent from the use of Eq. 1 with emissivity of 1 . The temperature of the blackbody is determined using the relationship between the calculated photocurrent and the temperature. 
$>10^{-6} \mathrm{~A}$ from the Ag- and Au-point temperatures to $3000 \mathrm{~K}$. The range in photocurrent from the Agfreezing temperature to $>3000 \mathrm{~K}$ is in the linear photocurrent region for Si diodes if the radiation is not incident on the edges of the photodiode [15]. The photocurrent of $<10^{-10} \mathrm{~A}$ near the Ag-freezing temperatures indicates the need for low noise photodiodes and preamplifiers for these measurements.

\subsection{LINEARITY CHARACTERIZATION OF THE AP1}

Since the measurement of the radiance responsivity was performed in SIRCUS using gain 8, the normalization factors to the other gains are needed if the AP1 is used at different gain settings. Instead of the traditional determination of radiometer linearity using radiation sources, the preamplifier was measured separately with a programmable constant-current source and compared to the current-tovoltage conversions using the NIST Precision Preamplifier (NPP) as shown in Table 1. The NPP is a current meter whose the nominal decade values of the feedback resistors have a tolerance of $0.01 \%$. The temperature coefficients of the resistances are $10 \times 10^{-6} /{ }^{\circ} \mathrm{C}$. The AP1 preamplifier was calibrated against the NPP using an input-current on/off switch to eliminate the output offset voltages of the test and standard preamplifiers. As the noise and ripple pickup was minimized in the preamplifier standard, the test preamplifier could be calibrated with $0.022 \%(k=1)$ uncertainty even at gain 10 .

Table 1: AP1 preamplifier gains determined by reference to NIST Precision Preamplifier with $0.01 \%$ precision resistors used as feedback resistors.

\begin{tabular}{|c|c|c|c|c|}
\hline Gain & Gain factors [V/A] & Uncertainty $(\boldsymbol{k}=\mathbf{2})$ [\%] & Ratios & Normalization factors to gain10 $^{\mathbf{8}}$ \\
\hline 6 & $1.00010 \mathrm{E}+06$ & 0.02 & $10^{8} / 10^{6}=$ & $9.95920 \mathrm{E}+01$ \\
\hline 7 & $1.00008 \mathrm{E}+07$ & 0.014 & $10^{8} / 10^{7}=$ & $9.95940 \mathrm{E}+00$ \\
\hline 8 & $9.96020 \mathrm{E}+07$ & 0.014 & $10^{8} / 10^{8}=$ & $1.00000 \mathrm{E}+00$ \\
\hline 9 & $9.94820 \mathrm{E}+08$ & 0.022 & $10^{8} / 10^{9}=$ & $1.00121 \mathrm{E}-01$ \\
\hline 10 & $9.94330 \mathrm{E}+09$ & 0.022 & $10^{8} / 10^{10}=$ & $1.00170 \mathrm{E}-02$ \\
\hline
\end{tabular}

\section{DETERMINATION OF THE AG- AND AU-FREEZING TEMPERATURES}

One of the most critical tests of the detector-based radiation thermometer is the comparison of the thermodynamic temperatures of the Ag- and Au-freezing temperatures to the ITS-90 [16]. If the detector-based measurements are not within the combined uncertainties of the experiment then some unknown systematic uncertainty component could be improperly assigned. The AP1 was used to compare the thermodynamic Ag- and Au-freezing temperatures to the ITS-90 assigned values [17]. A summary of the measurements is listed in Table 2.

Table 2. Summary of the Ag- and Au-freezing temperature determinations with the ITS-90 assignments.

\begin{tabular}{|c|c|c|c|c|c|}
\hline $\begin{array}{c}\text { Freezing } \\
\text { Material }\end{array}$ & $\mathrm{T}(\mathrm{AP} 1)[\mathrm{K}]$ & $\begin{array}{c}\text { Uncertainty T } \\
(\mathrm{AP} 1)[\mathrm{K}](k=2)\end{array}$ & $\mathrm{T}_{90}[\mathrm{~K}]$ & $\begin{array}{c}\text { Uncertainty T } \\
(\mathrm{ITS}-90)[\mathrm{K}] \\
(k=2)\end{array}$ & $\mathrm{T}^{-\mathrm{T}_{90}[\mathrm{~K}]}$ \\
\hline $\mathrm{Ag}$ & 1234.956 & 0.106 & 1234.93 & 0.080 & 0.026 \\
\hline $\mathrm{Au}$ & 1337.344 & 0.121 & 1337.33 & 0.100 & 0.014 \\
\hline
\end{tabular}

From Table 2, the dominant uncertainty component is from the radiance responsivity uncertainty of $0.15 \%(k=2)$ which corresponds to an expanded temperature uncertainty of $0.121 \mathrm{~K}$ and $0.106 \mathrm{~K}$ at the Ag- and Au-freezing temperatures, respectively. Since the center wavelength of the AP1 is in the wavelength and temperature region where the Wien approximation is applicable, the uncertainty of the temperature from the uncertainty of radiance is found from the derivative of the Wien approximation, and the differences, $\mathrm{T}-\mathrm{T}_{90}$, are found to be within the combined uncertainties. 


\section{COMPARISON OF NPL ITS-90 AND NIST THERMODYNAMIC TEMPERATURES}

The NIST thermodynamic temperature scale based on the AP1 was also used for bi-lateral comparison of temperature scales with the National Physical Laboratory (NPL), United Kingdom, using a set of metal-carbon eutectics [18]. The temperatures of the eutectics were assigned at the NPL using ITS-90 temperatures and then compared by transporting the crucibles to NIST for measurements with the AP1. The results are shown Fig. 5. The solid lines are the combined uncertainties of the measurements and the dotted lines are the uncertainties of the AP1 measurements. The differences are

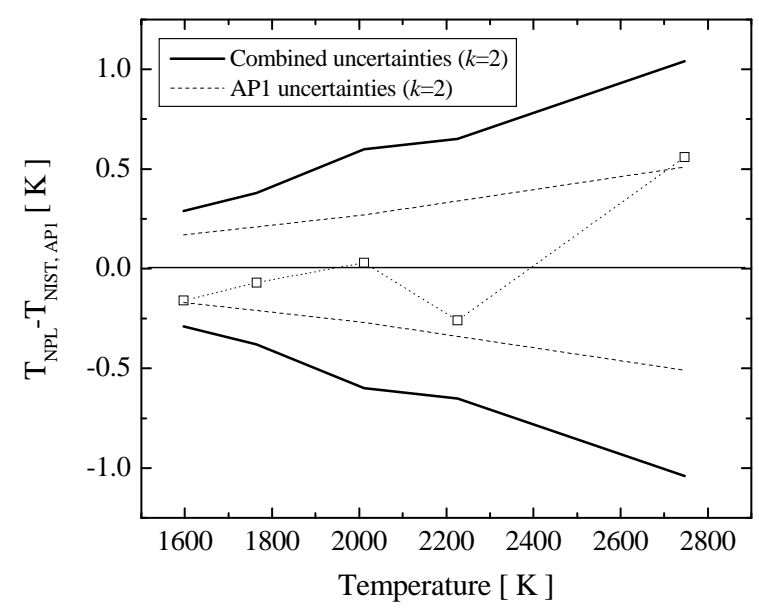

Figure 5. The differences between the NPL (ITS-90) assigned temperatures and the NIST thermodynamic temperatures of the metal-carbon eutectics. The solid line is the combined temperature uncertainties and the dotted line denotes only the AP1 uncertainties.

within the combined uncertainties of the comparison and within the uncertainties of the detector-based temperatures as well. The uncertainties of the NIST detector-based temperature scale is about x2 smaller than the ITS-90 based scale of NPL.

\section{NEW DESIGNS FOR RADIATION THERMOMETERS}

Our experience with the AP1 and further analysis of the contributions to the SSE in radiation thermometers have lead to new designs for future NIST radiation thermometers. These radiometers will be used for detector-based radiation thermometry and for ratio radiation thermometry to obtain the lowest uncertainties at temperatures to the Sn-point. A design to be used for detector-based radiation thermometry from $350 \mathrm{~nm}$ to $2500 \mathrm{~nm}$ utilizing different detectors and lenses is shown in Fig. 6. The outer case is constructed with $25 \mathrm{~cm}$ diameter anodized aluminum, and the large outer case is for suppression of scatter from internal reflections. The internal walls are covered with low-reflective black appliqué, and the field stop is tilted to avoid direct reflections back onto the objective lens. For temporal stability, the optical elements are attached to graphite reference rods which have low coefficient of thermal expansion. If the mechanical stability of the graphite rods are not deemed adequate, then the reference rods can be exchanged with those constructed from invar. The radiometer is also designed for removal of the objective lens from the outside for easy cleaning and replacement if the objective lens becomes damaged. Our goal for the uncertainty due to long-term stability of the radiance responsivity is $0.01 \%$ or $100 \mathrm{ppm}$.

The optical design of the radiometer has been influenced by our studies of the contributions to the SSE [19]. The main source of the SSE has been determined to be the scatter from the objective lens. The scatter can be reduced by the placement of the Lyot stop or the aperture stop behind the field stop 
such that the image of the objective lens is formed on the Lyot stop by the collimating lens. The optical

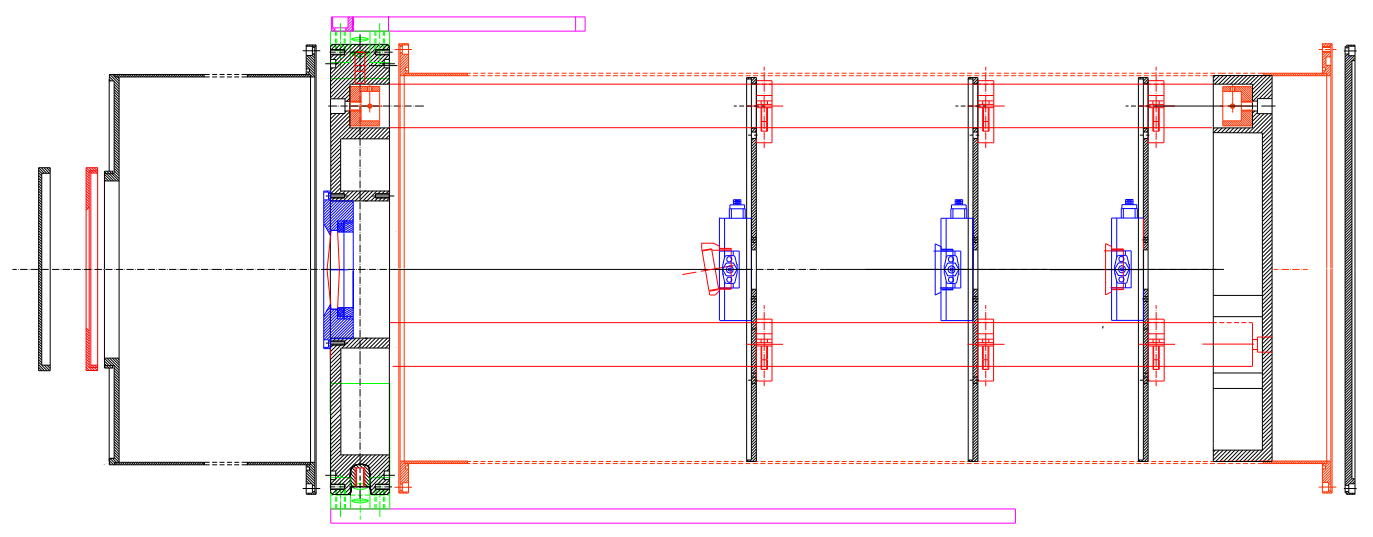

Figure 6. A side-view schematic of the graphite-rod referenced radiation thermometer. The outer diameter of the radiation thermometer is $25 \mathrm{~cm}$.

imaging and chromatic aberrations have been assessed for various different types of lenses using a commercial optical modeling software. We have found that for radiation thermometry, the simplest objective lens design leads to the lowest SSE as shown in Fig. 7.

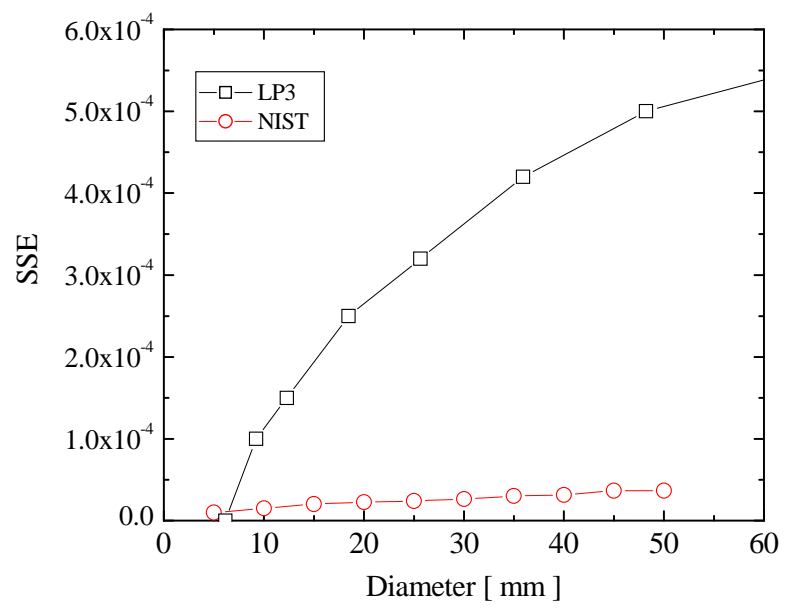

Figure 7. A comparison of the SSE between the NIST radiation thermometer design and the LP3.

In Figure 7, the optical design utilized in the NIST radiation thermometers is compared against the SSE from the Linear Pyrometer 3 (LP3)*. The simpler objective lens can lead to $>10$ reduction in the SSE. Custom lens designs are being assessed to obtain both optical performance as well as low surface scatter. 


\section{THE DEVELOPMENT OF A FUTURE TEMPERATURE SCALE}

One of the dominant factors in the increase in the ITS-90 temperature uncertainties above the Agfreezing point is the lack of higher-temperature fixed points above $1400 \mathrm{~K}$. Recent developments in high-temperature metal-carbon and metal-carbide-carbon eutectic blackbodies could lead to lower temperature uncertainties if the eutectic blackbodies are determined to be suitable for use as high temperature fixed-points [20]. Spectral radiances of the new metal- carbon and metal carbide-carbon eutectic blackbodies are shown along with the Au- and the Ag-fixed points in Fig. 8. For any future temperature scale to be closely in agreement with the thermodynamic scale, the temperatures of the high temperature eutectic blackbodies must be determined using absolute detector-based techniques. If radiance ratios from the Au-point temperature are used to determine the temperatures of the eutectics then the uncertainties would be unacceptably large, since the uncertainties are found by using

$$
u\left(T_{\mathrm{BB}}\right)=\frac{u\left(T_{\mathrm{REF}}\right)}{T_{\mathrm{REF}}^{2}} T_{\mathrm{BB}}^{2},
$$

with $T_{\mathrm{REF}}$ denoting the fixed point blackbody, and $T_{\mathrm{BB}}$ the unknown temperature of the eutectic-point blackbody.

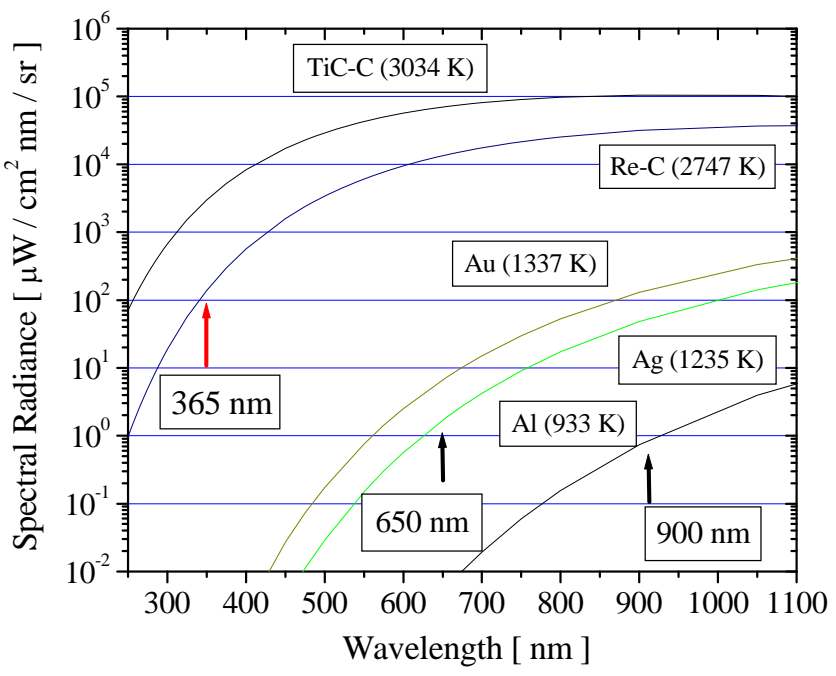

Figure 8. The spectral radiances of the blackbodies at the respective temperatures. The measurements of the Au- and the Ag-freezing points at $650 \mathrm{~nm}$ are compared with the planned measurements of the eutectic points at shorter wavelengths.

However, there is still a role for ratio radiometry in the new temperature scale. If the temperature of the reference fixed-point blackbody described in Eq. 2 is greater than the temperatures of the blackbodies under measurement, then the temperature uncertainties of the lower temperature blackbodies become smaller by the ratio of the squares of the respective temperatures. A possible future temperature scale could be based upon an absolute temperature determination of several hightemperature metal-carbon eutectic blackbodies $\sim 3000 \mathrm{~K}$ with the temperatures of the lowertemperature fixed point determined using radiance ratios.

Table 5 shows the temperature uncertainties both using absolute detector-based techniques and radiance ratios to the TiC-C blackbody, and the uncertainties of the detector-based temperature determinations can be estimated from the derivative of the Wien approximation to Planck's radiance law, 


$$
\frac{\Delta L}{L}=\frac{\mathrm{C}_{2}}{\lambda} \frac{\Delta T}{T^{2}},
$$

which shows the relationship between the uncertainty in radiance, $L$, to the uncertainty in blackbody temperature, $T$, where $\mathrm{c}_{2}$ is the second Planck's constant, and $\lambda$ is the wavelength. If the temperatures of the respective blackbodies are measured at the wavelengths shown in Col. 3, then the radiance uncertainties shown in Col. 4 lead to the uncertainties in Col. 5 as found using Eq. 3. However, if the temperatures of the lower temperature fixed points are determined using radiance ratios to the TiC-C, then the temperature uncertainties decrease as in Eq. 2 thus leading to lower uncertainties as shown in Col. 6. For the lowest uncertainties in the temperature scale, both types of temperature determinations are needed to verify the assigned uncertainties in addition to the ratio radiometry based on the lowertemperature acoustic-gas thermometry.

Table 5. A comparison of the temperature uncertainties found using absolute detector-based techniques and radiance ratios.

\begin{tabular}{|c|c|c|c|c|c|}
\hline & & Ravelength & $\begin{array}{c}\text { Radiance } \\
\text { Uncertainty } \\
(\mathbf{\%}) \\
(\boldsymbol{k}=\mathbf{2})\end{array}$ & $\begin{array}{c}\text { Detector-based } \\
\text { Temperature } \\
\text { Uncertainty (K) } \\
(\boldsymbol{k}=\mathbf{2})\end{array}$ & $\begin{array}{c}\text { Ratio to TiC-C } \\
\text { Ratio Pyrometry } \\
(\mathbf{K}) \\
(\boldsymbol{k}=\mathbf{2})\end{array}$ \\
\hline $\mathrm{TiC}-\mathrm{C}$ & 3034 & 365 & 0.1 & 0.234 & -- \\
\hline $\mathrm{Re}-\mathrm{C}$ & 2748 & 400 & 0.1 & 0.210 & 0.172 \\
\hline $\mathrm{Cu}$ & 1357.77 & 650 & 0.1 & 0.083 & 0.042 \\
\hline $\mathrm{Au}$ & 1337.33 & 650 & 0.1 & 0.081 & 0.041 \\
\hline $\mathrm{Ag}$ & 1234.93 & 800 & 0.1 & 0.069 & 0.035 \\
\hline $\mathrm{Al}$ & 933.473 & 900 & 0.1 & 0.055 & 0.020 \\
\hline $\mathrm{Zn}$ & 692.677 & 1250 & 0.3 & 0.125 & 0.011 \\
\hline
\end{tabular}

\section{DISCUSSION}

With the improvements in radiation thermometer designs for long-term stability and low SSE, the total radiance responsivity uncertainty in detector-based thermometry is expected to decrease to $0.1 \%$ $(k=2)$ in the wavelength region from $350 \mathrm{~nm}$ to $1000 \mathrm{~nm}$. Although, even with the current design of the AP1, the detector-based determinations of the Ag- and Au-freezing temperature are in agreement with the combined uncertainties, further reduced uncertainties will lead to the thermodynamic uncertainties as shown in Table 6. The present thermodynamic uncertainties in the ITS-90 assigned temperatures are listed. Although the uncertainties appear comparable, the limiting uncertainties in noise-thermometry are due to random uncertainties, while the dominant uncertainties in detector-based thermometry are due to systematic uncertainties, indicating that even lower total uncertainties are achievable with detector-based thermometry.

Table 6. A comparison of the thermodynamic temperature uncertainties obtained using noise and detector-based thermometry with the current uncertainties in the ITS-90 assignments. The detector-based thermometry is determined using $0.1 \%(k=2)$ uncertainty in the radiance responsivity at $650 \mathrm{~nm}$.

\begin{tabular}{|c|c|c|c|c|}
\hline Metal & $\mathbf{T}_{\mathbf{9 0}}[\mathbf{K}]$ & $\begin{array}{c}\mathbf{T}_{\mathbf{9 0}} \text {, Thermodynamic } \\
\text { temperature uncertainty } \\
{[\mathbf{m K}](\boldsymbol{k}=\mathbf{2})}\end{array}$ & $\begin{array}{c}\text { Noise thermometry } \\
\text { uncertainty } \\
{[\mathbf{m K}](\boldsymbol{k}=\mathbf{2})}\end{array}$ & $\begin{array}{c}\text { Detector-based } \\
\text { thermometry } \\
\text { uncertainties [mK] } \\
(\boldsymbol{k}=\mathbf{2})\end{array}$ \\
\hline $\mathrm{Al}$ & 933.473 & 50 & -- & 55 \\
\hline $\mathrm{Ag}$ & 1234.93 & 80 & 80 & 68 \\
\hline $\mathrm{Au}$ & 1337.33 & 100 & -- & 81 \\
\hline $\mathrm{Cu}$ & 1357.77 & 120 & 126 & 83 \\
\hline $\mathrm{Pd}$ & 1827.95 & 200 & 212 & 151 \\
\hline
\end{tabular}


We have shown that detector-based thermometry can achieve comparable uncertainties to the ITS-90 uncertainties near the Ag- and Au-freezing temperatures and can attain lower uncertainties than any ITS-90 based radiation thermometry at higher temperatures. The promise of lower uncertainties and the requirements for determining the metal-carbon eutectic transitions with the lowest uncertainties have led to efforts at NPL, PTB, NMIJ/BIPM, CSIRO and HUT, among others, to set up similar detector-based radiance responsivity calibration facilities, and with such facilities, direct disseminations of thermodynamic temperatures are possible with either calibrated radiation thermometers or secondary transfer standard sources.

We believe that these developments will lead to a reassessment of the ITS-90 and the future international temperature scale. Since it is possible, with detector-based radiation thermometry, to directly disseminate thermodynamic temperatures, the present $\mathrm{Ag}-\mathrm{Au}-\mathrm{Cu}$ - and even the $\mathrm{Al}$-freezing points will not remain as fixed points but will be allowed to vary within the uncertainty of the thermodynamic temperature determinations. Remaining within the framework of ITS-90 could result in higher temperature uncertainties than is possible with a detector-based scale, and NIST has already recognized the restrictions that a fixed-point scale places upon the temperature uncertainties. The current situation is analogous to the time-period when the IPTS-68 was utilized prior to ITS-90. Although the deviations from the thermodynamic temperature scale of the IPTS-68 was recognized, restrictions of a fixed-point based scale meant that the discrepancies could not be resolved without new temperatures assigned to the fixed points. Recognizing these restrictions, NIST has been detector-based since 1990 with a separate, independent, thermodynamic temperature determination of the Au-freezing temperature [21], and with the new generation of radiation thermometers, will soon be completely detector-based. If the ITS-90 scale is desired, then correction factors can be applied to the NIST detector-based scale for international comparisons and to comply with mutual recognition arrangements.

\section{CONCLUSION}

We show that much progress has been made toward an implementation of a detector-based thermodynamic temperature scale. The impetus for such progress has arisen from the spectroradiometric community and the need for the lowest-uncertainty temperature measurements of the new metal-carbon eutectic blackbodies. These detector-based primary radiation thermometers have been used to determine the Ag- and Au-freezing temperatures and even for bilateral comparison of temperature scales. With continuing efforts at many different NMIs, direct dissemination of thermodynamic temperatures will be possible in the near future. We believe that a reassessment of the ITS-90 is needed to reflect the developments in detector-based radiation thermometry.

\section{REFERENCES}

* $\quad$ Certain commercial equipment, instruments, or materials are identified in this paper to foster understanding. Such identification does not imply recommendation or endorsement by the National Institute of Standards and Technology, nor does it imply that the material or equipment are necessarily the best available for the purpose.

[1] Quinn T. J., Temperature, 2 nd ed., London, Academic Press Ltd., 1990.

[2] Preston-Thomas H., “The International Temperature Scale of 1990 (ITS-90)”, Metrologia 27, 1990, pp. 3-10.

[3] Ref [1] pp. 56-57.

[4] Strouse G. F., Defibaugh D. R., Moldover M. R., Ripple D. C., ”Progress in primary acoustic thermometry at NIST: 273 K to 505 K”, TMCSI, Vol. 7, Chicago, edited by Ripple D. C., 2003, 
pp. 31-36.

[5] Edler F., Kühne M., Tegeler E., "Noise Thermometry above $960{ }^{\circ} \mathrm{C}$ ”, Proceedings of TEMPMEKO 1999, Delft, edited by Dubbeldam J. F., de Groot, M. J., 1999, pp. 394 -399.

[6] Nam S. W., Benz S. P., Martinis J. M., Dresselhaus P., Tew W. L., White D. R., ”A Ratiometric Method for Johnson Noise Thermometry Using a Quantized Voltage Noise Source”, TMCSI, Vol. 7, Chicago, edited by Ripple D. C., 2003, pp. 37 -42.

[7] Quinn T. J., Martin, J. E., ”A radiometric determination of the Stefan-Boltzmann constant and thermodynamic temperatures between $-40{ }^{\circ} \mathrm{C}$ and $+100{ }^{\circ} \mathrm{C}$ ”, Philosophical Transactions of the Royal Society of London A 316, 1985, pp. 85 -189.

[8] Allen D. W., Saunders R. D., Johnson B. C., Gibson C. E., Yoon H. W., “The Development and Characterization of an Absolute Pyrometer Calibrated for Radiance Responsivity”, TMCSI, Vol. 7, Chicago, edited by Ripple D. C., 2003, pp. $577-582$

[9] Fox N. P., Metrologia 32, 1995/1996, pp. 535 -543.; Taubert D. R., Hartmann J., Hollandt J., Fischer J., "Investigation of the Accuracy of the ITS-90 with Reference to Thermodynamic Temperature in the Range from $400{ }^{\circ} \mathrm{C}$ up to $600^{\circ} \mathrm{C} ”$, TMCSI, Vol. 7, Chicago, edited by Ripple D. C., 2003, pp. 7 -12.

[10] Yamada Y., Sakate H., Sakuma F., Ono A., “A Possibility of Practical High Temperature Fixed Points above the Copper Point, Proceedings of TEMPMEKO1999, Delft, edited by Dubbeldam J. F., de Groot, M. J., 1999, pp. $535-540$.

[11] Blevin W. R., and Steiner B., "Redefinition of the Candela and Lumen”, Metrologia 11, 1975, pp. 97104.

[12] Brown S. W., Eppeldauer G. P., Lykke K. R., "NIST Facility for Spectral Irradiance and Radiance Responsivity Calibrations with Uniform Sources”, Metrologia 37, 2000, pp. 579-582.

[13] Gentile T. R., Houston J. M., Cromer C. L., "National Institute of Standards and Technology Highaccuracy Cryogenic Radiometer”, Appl. Opt. 35, 1996, pp. 4392-4403.

[14] Fowler J. and Litorja M., "Geometric Area Measurements of Circular Apertures for Radiometry at NIST”, Metrologia 40, 2003, pp. S9-S12.

[15] Battuello M., Bloembergen P., Girard F., Ricolfi, “A Comparison of Two Methods for Measuring the Nonlinearity of Infrared Radiation Thermometers”, Temperature: Its Measurement and Control in Science and Industry, Vol. 7, edited by D. C. Ripple, 2003, pp. 613-618.

[16] Yoon H. W., Allen D. W., Gibson C. E., Litorja M., Saunders R. D., Brown S. W., Eppeldauer G. P., Lykke K. R., "Temperature Determination of the Ag- and Au-freezing points using a Detector-based Radiation Thermometer”, submitted to the Proceedings of Tempmeko 2004, Croatia, 2004.

[17] Ref. [1] p. 59.

[18] Machin G., Gibson C. E., Low D., Allen D.W., Yoon H.W., “A Comparison of ITS-90 and Detectorbased Scales between NPL and NIST Using Metal-carbon Eutectics ”, submitted to the Proceedings of Tempmeko 2004, Croatia, 2004.

[19] Yoon H.W., Allen D.W. Saunders R.D., "Methods to reduce the size-of-source effect in radiation thermometers", submitted to the Proceedings of Tempmeko 2004, Croatia, 2004.

[21] Bloembergen P., Yamada Y., Yamamoto N., Hartmann J., "Realizing the High-temperature Part of a Future ITS with the Aid of Eutectic Metal-Carbon Fixed Points”, Temperature: Its Measurement and Control in Science and Industry, Vol. 7, edited by D. C. Ripple, 2003, pp. 291-296.

[21] Mielenz K. D., Saunders R. D., Shumaker J. B., "Spectroradiometric Determination of the Freezing Temperature of Gold”, J. Res. Natl. Inst. Stand. Technol. 95, 1990, pp. 45-67.

Address of the Principal Author: Howard W. Yoon, Optical Technology Division, NIST, 100 Bureau Drive Gaithersburg, MD 20899-8441, USA, tel. (301) 610-0094, fax (301) 869-5700, e-mail: hyoon@nist.gov 


\title{
Thermodynamic-temperature determinations of the Ag and $\mathrm{Au}$ freezing temperatures using a detector-based radiation thermometer
}

\author{
Howard W. Yoon, David W. Allen, Charles E. Gibson, Maritoni Litorja, Robert D. Saunders, \\ Steven W. Brown, George P. Eppeldauer, and Keith R. Lykke
}

\begin{abstract}
The development of a radiation thermometer calibrated for spectral radiance responsivity using cryogenic, electrical-substitution radiometry to determine the thermodynamic temperatures of the Ag- and $\mathrm{Au}$ freezing temperatures is described. The absolute spectral radiance responsivity of the radiation thermometer is measured in the NIST Spectral Irradiance and Radiance Responsivity Calibrations using Uniform Sources (SIRCUS) facility with a total uncertainty of $0.15 \%(k=2)$ and is traceable to the electrical watt, and thus the thermodynamic temperature of any blackbody can be determined by using Planck radiation law and the measured optical power. The thermodynamic temperatures of the Ag- and Au-freezing temperatures are determined to be $1234.956 \mathrm{~K}( \pm 0.110 \mathrm{~K})(k=2)$ and $1337.344 \mathrm{~K}( \pm 0.129 \mathrm{~K})(k=2)$ differing from the International Temperature Scale of 1990 (ITS-90) assignments by $26 \mathrm{mK}$ and $14 \mathrm{mK}$, respectively, within the stated uncertainties. The temperatures were systematically corrected for the size-of-source effect, the nonlinearity of the preamplifier and the emissivity of the blackbody. The ultimate goal of these thermodynamic temperature measurements is to disseminate temperature scales with lower uncertainties than those of the ITS-90. These results indicate that direct disseminations of thermodynamic temperature scales are possible. (C) 2007 Optical Society of America
\end{abstract}

OCIS codes: $120.6780,120.3940$.

\section{Introduction}

The measurements of temperature can be classified into primary and secondary thermometry. ${ }^{1}$ Primary thermometry is defined as determining thermodynamic temperatures using equations of state and physical constants without any adjustable temperature-dependent parameters; some examples are constant-volume gas thermometry, ${ }^{2}$ speed-of-sound measurements in gases,$^{3}$ or noise thermometry. ${ }^{4}$ Primary measurements based on the measurements using gases, however, are limited in temperature range owing to the deviation of the gas from ideal behavior and other experimental limitations, ${ }^{5}$ and noise thermometry is limited by the need for long measurement times required for sufficient statistical precision. ${ }^{6}$

The authors are with the Optical Technology Division, National Institute of Standards and Technology, Gaithersburg, Maryland 20899-8441, USA. H. Yoon's e-mail address is hyoon@nist.gov.

Received 14 November 2006; accepted 18 December 2006; posted 17 January 2007 (Doc. ID 77061); published 1 May 2007.

0003-6935/07/152870-11\$15.00/0

(C) 2007 Optical Society of America
Since primary thermometers are not easily transportable and are difficult to use, secondary thermometers are used as transfer devices. Because of the difficulties in maintaining primary thermometers for routine calibrations, the International Temperature Scale of 1990 (ITS-90) assigns fixed values for the phase-transition temperatures of various pure materials based upon the best thermodynamic temperature determinations available at the time of specifying the scale. ${ }^{7}$ Secondary interpolating thermometers are based upon these fixed points, and the assigned temperatures are ultimately traceable to primary thermometers through the fixed points. Some examples of secondary thermometers are standard platinum resistance thermometers and thermocouples.

Another type of primary thermometer is the total and the spectral radiometer, calibrated using electrical substitution radiometry and used in conjunction with a blackbody. Such total radiometers in the past have determined the Stefan-Boltzmann constant using the wavelength integrated Planck's radiance law to a relative combined uncertainty of $0.013 \% .{ }^{8}$ If only a narrow spectral region is selected by the use of spectral filters, then the spectral radiometer can be 
used to determine the temperatures of blackbodies with the use of Planck's radiation law within the spectrally selected region.

In the past, detector-based radiance temperature measurements have been performed using filter radiometers calibrated for spectral irradiance responsivity. ${ }^{9}$ These filter radiometers with precision apertures are typically used in nonimaging applications to measure the temperatures of blackbodies, but such optical flux techniques are difficult to use to measure the fixed-point blackbodies with subcentimeter diameter cavity openings. The nonimaging radiometers are used to measure spectral irradiance using double-aperture flux transfer techniques, and the small, $1 \mathrm{~cm}$ internal apertures of fixed-point blackbodies make it difficult to define a source aperture. Such techniques can also result in large uncertainties in the diffraction corrections needed from the use of the small defining apertures. However, small aperture blackbodies can be measured by using imaging radiometers in which the field stop defines the area of measurement. In the past, absolute imaging radiometers or pyrometers have been constructed from filter radiometers by using a lens with a measured transmittance, ${ }^{10}$ but similar component-based calibrations cannot be used with low final uncertainties if a number of optical elements such as lenses and filters are introduced into the optical path.

The ultimate goal of detector-based radiance temperatures is the direct dissemination of thermodynamic temperatures without the use of physical artifacts such as fixed-point blackbodies. Such a transition occurred for the definition of the candela in 1979 when it was changed from being derived from the Pt-freezing point to that of optical power, ${ }^{11}$ and the uncertainties in the realization of the candela can be improved with the development of new detectors and procedures.

In this work, measurements of the thermodynamic temperatures of the $\mathrm{Ag}$ - and $\mathrm{Au}$-freezing points using the NIST Absolute Pyrometer (AP1) are described in which the radiation thermometer is calibrated at the system level. The AP1 is designed and constructed at the NIST and is calibrated for radiance responsivity as a system using a cryogenic, electrical-substitution radiometer-based method. The characterization of the AP1 for size-of-source effect and linearity are de- scribed, and the measured temperatures are corrected for these systematic effects. The thermodynamic temperatures are compared to the previous temperature measurements and found to be in agreement within the combined uncertainties. This work demonstrates that the thermodynamic temperatures can be directly disseminated in this range using detector-based calibrations with comparable or lower uncertainties than that of the ITS-90.

\section{Experimental Section}

\section{A. Description of the Absolute Pyrometer}

A schematic of the AP1 in front of the gold-point blackbody is shown in Fig. 1. The dimension of the AP1 from outside of the front plate to the back plate is $53 \mathrm{~cm}$. The optical elements are attached to three, $50 \mathrm{~cm}$ long, $12.5 \mathrm{~mm}$ diameter Invar rods for structural stability with a low-temperature coefficient of expansion. The radiometer uses an on-axis design with a plano-convex singlet lens (focal length $=20 \mathrm{~cm}$ ) for the front objective with the aperture stop (AS) in front of the lens. For the objective lens, a gradientindex singlet lens was chosen to minimize the possible scattered light from the lens interfaces and to improve the image quality. The $50 \mathrm{~mm}$ diameter objective lens is set at a fixed object distance of $50.0 \mathrm{~cm}$ with the field stop (FS) at an image distance of $33.3 \mathrm{~cm}$. A $0.5 \mathrm{~mm}$ diameter blackened bimetal aperture is used as the field stop, which results in a nominal target size of $0.75 \mathrm{~mm}$ diameter. The actual field of view was measured by raster scanning a source with a small $10 \mu \mathrm{m}$ pinhole aperture. The size of the target area was chosen such that tungsten-strip lamps and fixed-point blackbodies could be compared to variable-temperature blackbodies with larger cavity opening apertures.

The reimaging of the field stop is performed using a gradient-index achromatic lens (RL), which collimates the light. The light passing through the interference filters (IF) is collimated, and no further lenses are used between the filters and the $5.8 \mathrm{~mm}$ by $5.8 \mathrm{~mm}$ square Si photodiode (PD) with the diode suppression of the near-infrared power responsivity. The spot size on the diode is estimated to be $5 \mathrm{~mm}$ diameter from the ray-trace analysis. The spectral selection is performed using ion-assisted-deposited

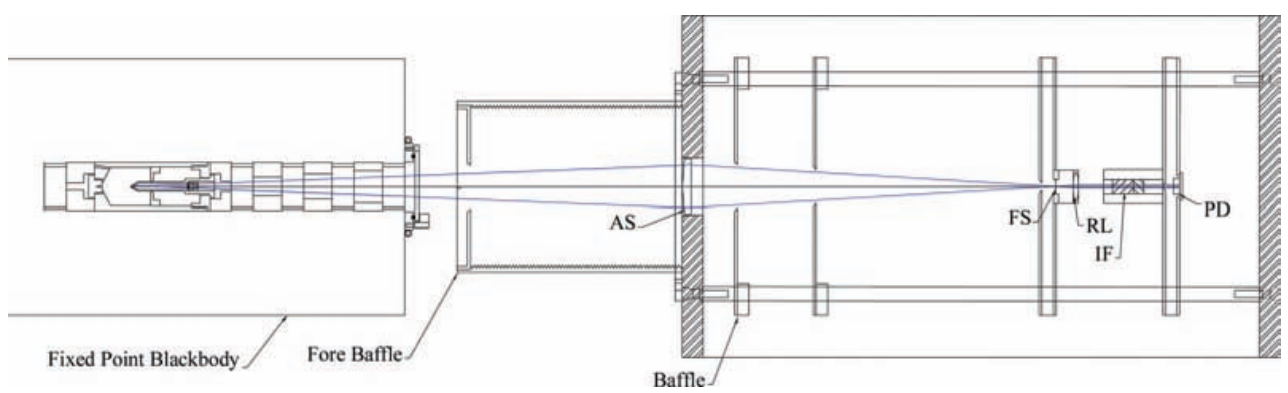

Fig. 1. (Color online) Schematic of the AP1 placed in front of the gold-point blackbody. The aperture stop (AS), field stop (FS), relay lens $(\mathrm{RL})$, interference filter (IF), and the photodiode (PD) are shown. 
interference filters ${ }^{12}$ with the center wavelengths at $650 \mathrm{~nm}$ for at least $10^{-7}$ suppression of the responsivity in the out-of-band region to $1100 \mathrm{~nm}$. The spectral selection filter is temperature stabilized using a thermoelectric (TE) cooler near room temperature, and the hermetically sealed Si photodiode has a twostage TE cooler for operation at $-15{ }^{\circ} \mathrm{C}$ to achieve a noise-equivalent power (NEP) of $<10 \mathrm{fW}$. The AP1 can be purged with dry air or nitrogen to reduce effects attributable to humidity on the interference filters.

\section{B. Radiance Responsivity Calibration of the Absolute Pyrometer}

The AP1 was calibrated for absolute radiance responsivity using the NIST Spectral Irradiance and Radiance Responsivity Calibrations using Uniform Sources (SIRCUS) facility ${ }^{13}$ as illustrated in the sequence of measurements in Figs. 2(a)-2(d). The optical power of the power-stabilized and collimated laser source is measured using a cryogenic electrical substitution radiometer, the NIST Primary Optical Power Radiometer (POWR) in Fig. 2(a). Since the POWR is difficult to use on a daily basis, the power responsivity is transferred to six-element Si-trap detectors in Fig. 2(b). From the knowledge of the spatial uniformity and the area of the precision defining aperture on the trap detectors, the detector-based spectral irradiance responsivity is determined. The laser is input into an integrating sphere to obtain a spatially uniform and Lambertian source in Fig. 2(c), and the spectral irradiance of the source is determined using the Si-trap detector. The spatial uniformity at the exit aperture of the integrating sphere is mapped to determine the uncertainties attributable to the nonuniformity of the sphere. The power-stabilized laser is transmitted through a fiber, which is agitated in a water-filled ultrasonic bath. The rapid vibrations in the ultrasonic bath remove the laser speckle in the sphere output since the integration times of the detectors are much longer than the time duration of the speckle pattern. A small $38.1 \mathrm{~mm}$ diameter integrating sphere is used to increase the throughput of the sphere, and the $5 \mathrm{~mm}$ diameter exit port of the integrating sphere source is chosen to approximate the $6 \mathrm{~mm}$ diameter opening of the fixed-point blackbody cavities. With precision apertures on both the output port of the integrating sphere and the entrance of the Si-trap detector combined with the knowledge of the distance separating the sphere and the trap detector, the monochromatic spectral radiance of the integrating sphere can be assigned from the detector-calibrated irradiance responsivity of the trap detector. The aperture area and the distance measurement device are traceable to length standards. The AP1 is then calibrated for spectral radiance responsivity by substitution with the trap detector as shown in Fig. 2(d). Once the monochromatic, spectral radiance of the integrating-sphere source is known, any radiometer or radiation thermometer can be calibrated at the system level. The procedure is repeated at other wavelengths until the full spectrum of the radiance responsivity is obtained.

The detector-based radiance responsivity of the AP1 is shown along with the spectral radiance of the gold-point blackbody in Fig. 3. The interference filter used in the AP1 shows $>1 \times 10^{-7}$ suppression in the out-of-band region relative to the peak value of the responsivity. The FWHM of the radiance responsivity is $10 \mathrm{~nm}$ with the centroid wavelength of $649.115 \mathrm{~nm}$. The total expanded uncertainty of the radiance responsivity at a single wavelength is $0.15 \%(k=2)$.

$\pm$

(a)

(b)

(c)

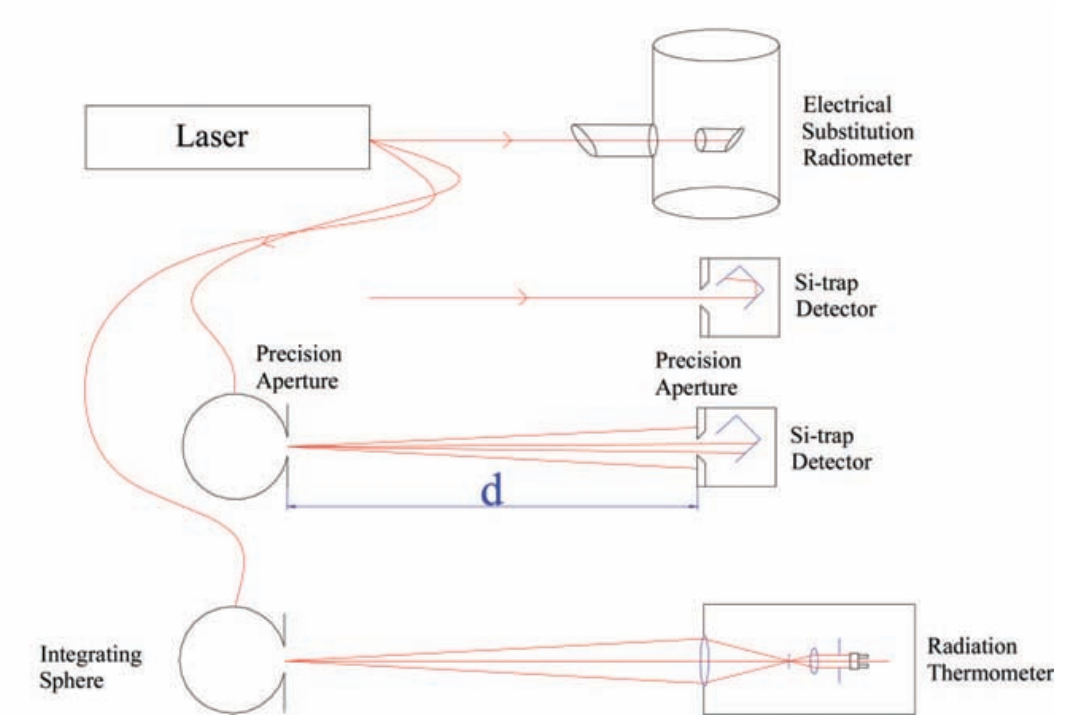

Fig. 2. (Color online) Procedure for detector-based spectral radiance responsivity calibrations with the use of the electrical substitution radiometer. (a) Measurement of the spectral power of the laser with the cryogenic electrical substitution radiometer; (b) determination of the spectral power responsivity of the Si-trap detector; (c) measurement of the spectral irradiance of the integrating sphere source; (d) determination of the radiance responsivity of the radiation thermometer as a system. 


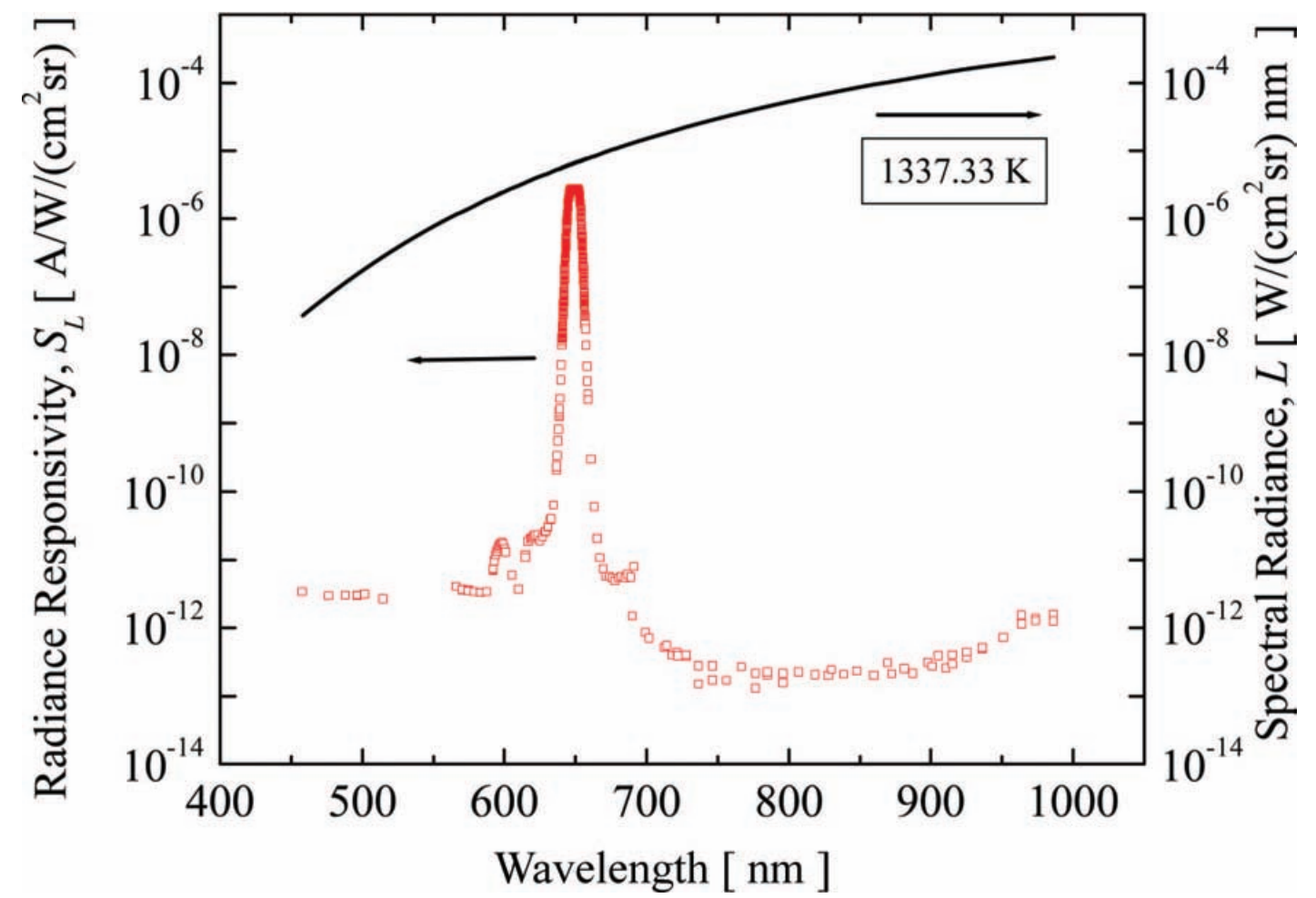

Fig. 3. (Color online) Radiance responsivity of the AP1 measured in the SIRCUS facility plotted with the Planck radiance at $1337.33 \mathrm{~K}$. The center wavelength is $650 \mathrm{~nm}$ with a FWHM of $10 \mathrm{~nm}$. The out-of-band suppression of $<1 \times 10^{-7}$ is achieved in the wavelength region from 700 to $1000 \mathrm{~nm}$.

The wavelength spacing between calibrations was decreased near the peak of the spectral responsivity since interference effects from interreflections can lead to oscillations in the spectral responsivity, which are wavelength dependent, as seen in Fig. 4. The sources of the interference effects are from the remaining coherence of the laser through the integrat-

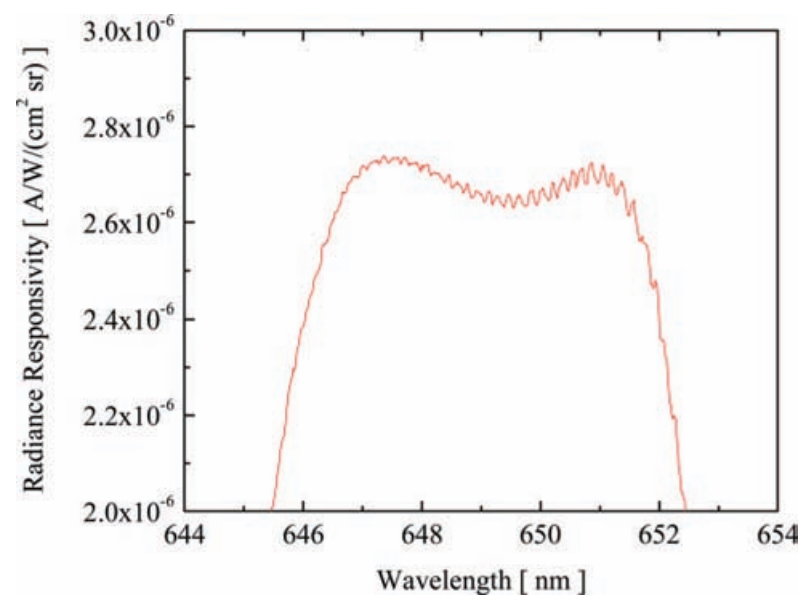

Fig. 4. (Color online) Spectral radiance responsivity near the peak of the responsivity function showing the interference fringes. The fringes arise from the interference of the radiation with the planar reflective surfaces in the AP1. The presences of the fringes require measurements at sufficient wavelength intervals to resolve them. ing sphere source combined with the interreflections between the optical elements in the radiation thermometer. Although an effort was made to reduce the interference effects by designing the optical filter with a $5^{\circ}$ tilt of one of the surfaces from the optical axis, the interference effects are evident as $<1 \%$ peak oscillations of the responsivity. Since the radiation thermometer is used to measure the radiance from a blackbody, which is an incoherent source, the convolved radiance will not exhibit the interference effects, and the interference effects in the radiance responsivity will depend on the spatial coherence of the incident radiation. The increase in the uncertainties attributable to the interference effects can be minimized by performing measurements at closely spaced wavelength intervals.

The thermodynamic temperatures of blackbodies are found from the radiance responsivity and Planck's law by the use of

$$
i_{c}=\int S_{L} L(\lambda, T) \mathrm{d} \lambda
$$

where $i_{c}$ is the calculated photocurrent, $S_{L}$ is the absolute radiance responsivity, and $L(\lambda, T)$ is the spectral radiance given by Planck radiance law. Planck radiance law is

$$
L(\lambda, T)=\frac{\mathrm{c}_{1 \mathrm{~L}}}{n^{2} \lambda^{5}} \frac{1}{\exp \left[\mathrm{c}_{2} /(n \lambda T)\right]-1}
$$




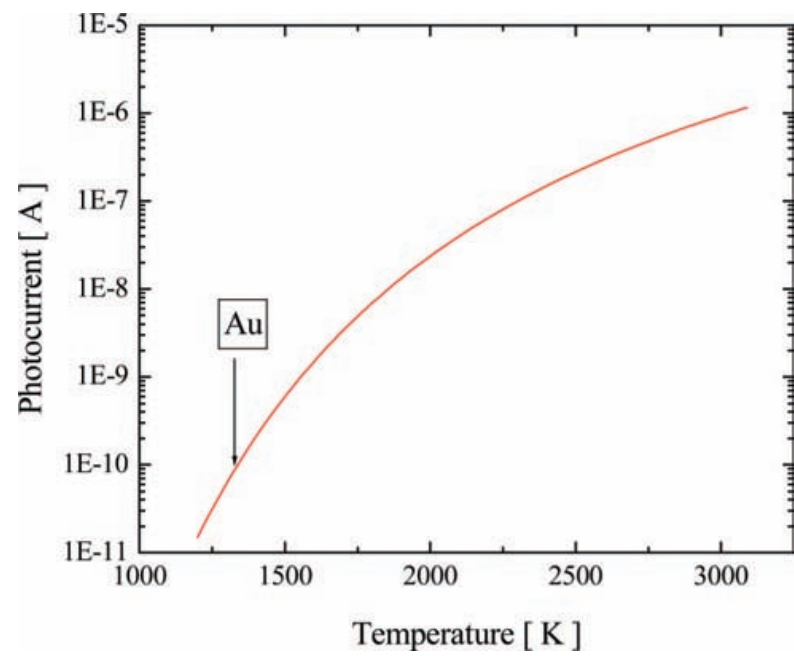

Fig. 5. (Color online) Calculated photocurrent as a function of the blackbody temperature. The calculation is performed with no adjustable parameters.

where $c_{1 L}$ and $c_{2}$ are the first and second radiation constants, $T$ is the thermodynamic temperature, $\lambda$ is the wavelength of the radiation in air, and $n$ is the refractive index. For these calculations, the CODATA values $^{14}$ for the radiation constants and the refractive index of air of $n=1.00029$ were utilized.

There are no adjustable parameters in the measurement equation relating the radiance responsivity of the AP1 to the thermodynamic temperature, and Eq. (2) can be used to generate a curve of photocurrent to blackbody temperatures. The one-to-one relationship is shown in Fig. 5. The photodiode used in the AP1 is operated in the photovoltaic mode without any bias voltages, and this type of photodiode in the photovoltaic mode has been shown to be linear over a wide dynamic range. ${ }^{15}$ The range of calculated photocurrents of the AP1 are from $1 \times 10^{-11} \mathrm{~A}$ at the Ag point to $1 \times 10^{-6} \mathrm{~A}$ near $3000 \mathrm{~K}$, which is in the linear regime for photodiodes. The plot in Fig. 5 also indicates the need for low-noise current-to-voltage amplifiers to measure the low photocurrents near the Ag- and Aufreezing temperatures. Other corrections such as the size-of-source effect, the nonlinearity of the preamplifier, and the emissivity of the blackbody will be applied as corrections to the measured photocurrents before comparison to the calculated photocurrents in Fig. 5. The relationship between photocurrent and blackbody temperatures can be fit with an interpolation func$\operatorname{tion}^{16}$ to facilitate conversions from signals to temperatures.

\section{Instrumental Characterizations of the Absolute Pyrometer}

\section{A. Gain Linearity}

To reduce the uncertainties in the linearity corrections, the same preamplifier was used for both AP1 calibrations and the temperature determinations. The SIRCUS calibrations were performed at a gain of
Table 1. AP1 Preamplifier Gain Corrections Measured 11 June 2002

\begin{tabular}{rcccc}
\hline & & $\begin{array}{c}\text { Uncertainty } \\
(k=2) \\
{[\%]}\end{array}$ & Ratios & $\begin{array}{c}\text { Normalization } \\
\text { Factors to Gain } \\
10^{8}\end{array}$ \\
\hline 6 & $1.00010 \times 10^{6}$ & 0.02 & $10^{8} / 10^{6}=$ & $9.9592 \times 10^{1}$ \\
7 & $1.00008 \times 10^{7}$ & 0.014 & $10^{8} / 10^{7}=$ & 9.9594 \\
8 & $9.96020 \times 10^{7}$ & 0.014 & $10^{8} / 10^{8}=$ & 1.0000 \\
9 & $9.94820 \times 10^{8}$ & 0.022 & $10^{8} / 10^{9}=$ & $1.0012 \times 10^{-1}$ \\
10 & $9.94330 \times 10^{9}$ & 0.022 & $10^{8} / 10^{10}=$ & $1.0017 \times 10^{-2}$ \\
\hline
\end{tabular}

$10^{8} \mathrm{~V} / \mathrm{A}$. If the AP1 is used with any other gain setting on the preamplifier, then the gain normalization factors to the $10^{8} \mathrm{~V} / \mathrm{A}$ gain are needed to compare the measurements at the different gains. These gain factors were measured at the beginning of the study and the values used through the measurements. Since the gain factor nonlinearities primarily arise from the deviation of the feedback resistance values from their precision stated values, the resistances values are not expected to change during the operational lifetime of the radiation thermometer. The gain factors for the AP1 preamplifier were determined by comparison to the NIST Precision Preamplifier (NPP) with $0.01 \%$ precision resistors used as feedback resistors, and the gain correction factors are listed in Table 1. These gain factors can also be obtained by the use of a stable radiation source such as a light-emitting diode whose intensity can be varied by neutral density filters. A programmable-current source was used to provide constant current for the ratio measurements between the AP1 preamplifier and the NPP. The linearity of the NPP and the AP1 preamplifier depends upon the accuracy of the electrical resistance measurements as compared to the optical linearity characterizations used for other radiation thermometers. Since the AP1 is used at gain $10^{10} \mathrm{~V} / \mathrm{A}$ to $10^{8} \mathrm{~V} / \mathrm{A}$, the normalization factors are found by using ratios of gains and are found to be $1.00170 \times 10^{-2}[ \pm 0.022 \%(k=2)]$. The linearity of the Si diode in the AP1 was not measured separately since the Si diode has been demonstrated to have linear response over a 14-decade ratio of photocurrents. ${ }^{15}$

\section{B. Size-of-Source Effect Characterization}

Since the integrating sphere source used for the detector-based spectral radiance responsivity calibration and the Ag- and Au-freezing temperature blackbody have different spatial distributions, systematic corrections are needed. The size-of-source effect was characterized by using two different techniques. First, the point-spread function of the AP1 was measured using a $10 \mu \mathrm{m}$ diameter pinhole aperture, which was illuminated by a laser diode centered at $650 \mathrm{~nm}$. A microscope objective was used to collect the collimated radiation from the laser diode and made incident on the pinhole. The $10 \mu \mathrm{m}$ source was translated on the $x$ and $y$ axis stages in $100 \mu \mathrm{m}$ steps at the object plane of the AP1, and the point-spread function determined, as shown in Fig. 6. The FWHM was 


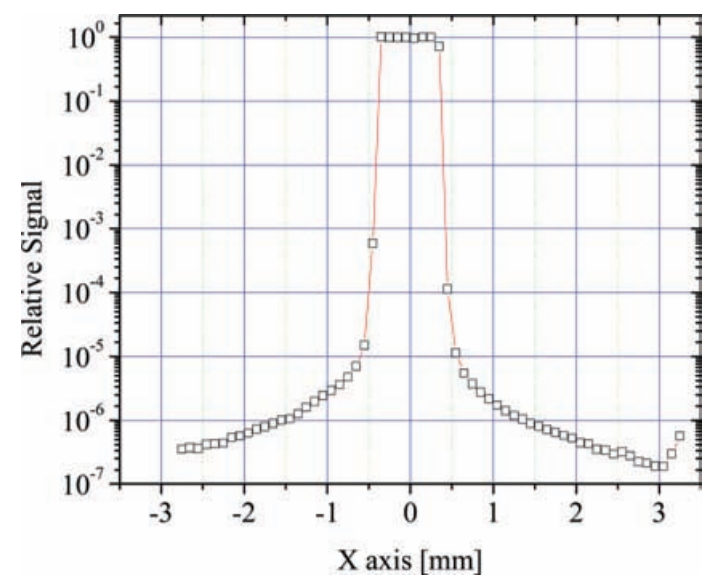

Fig. 6. (Color online) Spatial responsivity of the AP1 measured using an illuminated $10 \mu \mathrm{m}$ pinhole scanned across the object plane.

determined to be $0.78 \mathrm{~mm}( \pm 0.01 \mathrm{~mm})$ with a difference between $10 \%$ and $90 \%$ of the peak value of $0.11 \mathrm{~mm}( \pm 0.01 \mathrm{~mm})$. The measured spot size is in agreement with the expected spot size based on geometric optics.

Since the point-spread function determines only the optical imaging quality of the AP1, a large-area, radiance source was used to determine the size-ofsource effect (SSE). The AP1 was characterized for SSE using a LED illuminated radiance source with a $5 \mathrm{~mm}$ diameter conical, central obscuration using the so-called indirect technique. The outer diameter of the source was changed with a variable-diameter iris. The SSE is plotted in Fig. 7 using

$$
\sigma\left(d, d_{o}\right)=\frac{\mathrm{v}(L, d)-\mathrm{v}\left(L, d_{o}\right)}{\mathrm{v}(L)}
$$

where $d$ is the diameter of the uniform radiance source, $d_{o}$ is the diameter of the central obscuration, and $L$ is the radiance of the source. The signal measured while viewing the unobstructed radiance source

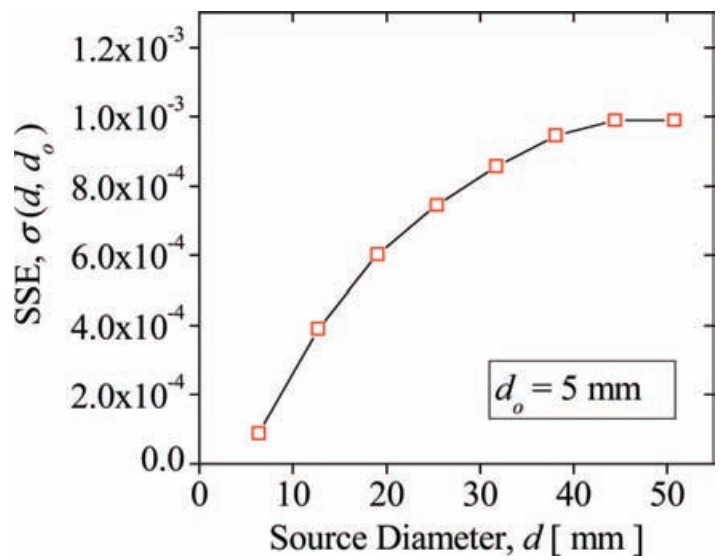

Fig. 7. (Color online) SSE measured for the AP1 using an LED radiance source with a $5 \mathrm{~mm}$ diameter central obscuration.

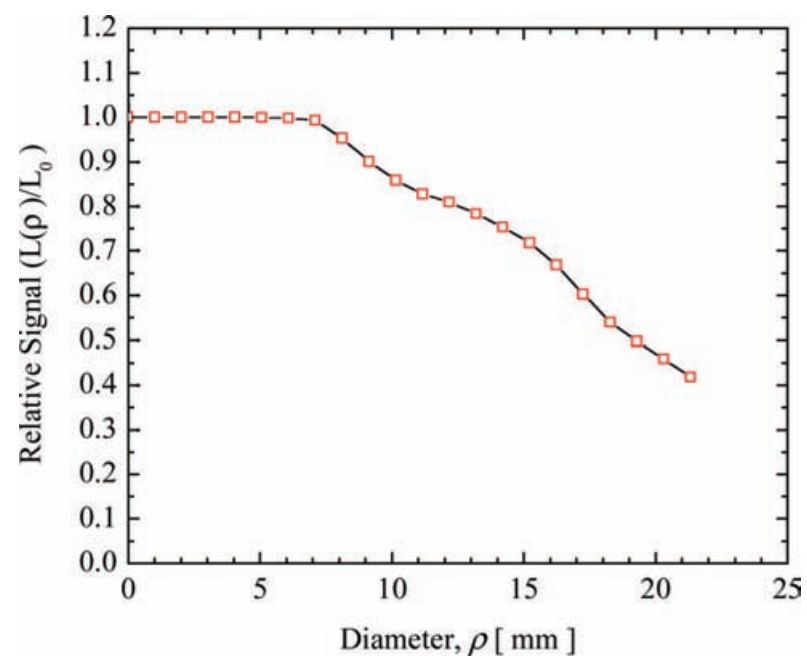

Fig. 8. (Color online) Spatial scans of the Au-freezing temperature blackbody during the freeze for use in SSE corrections.

is $\mathrm{v}(L)$, and the signal measured while viewing the central obscuration with the diameter of the radiance source, $d$, is $\mathrm{v}(L, d)$. The signal measured with the diameter of the variable aperture at the diameter of the central obscuration, $d_{o}$, is $\mathrm{v}\left(L, d_{o}\right)$.

With knowledge of the SSE using a uniform source, the SSE must be corrected for the nonuniform blackbody source by spatial scans during the freeze. The spatial scans of the Au-freezing temperature blackbody is shown in Fig. 8. The scans were performed with the AP1 on a motorized stage while the Au-point blackbody was in the freeze plateau. Since the electrical heating is reduced during the freeze, there is contrast observed between the central cavity and the surrounding graphite crucible seen as a reduction in the signal past $7 \mathrm{~mm}$.

The SSE corrections for the Ag and Au freezes were determined using the spatial distribution of the radiance from Fig. 8 measured by scanning the AP1 convolved with the SSE measurements shown in Fig. 7 along with Eq. (3) according to

$$
\sigma^{\prime}\left(r, r_{o}\right)=\sum_{i} \frac{L(\rho)}{L_{o}}\left(\frac{\Delta \sigma_{i}\left(\rho, r_{o}\right)}{\Delta \rho}\right) \Delta \rho,
$$

where a modified SSE, $\sigma^{\prime}$, is found from the product of the differential increase in the SSE, $\Delta \sigma_{i}$, and the measured relative radiance distribution as a function of the radius, $L(\rho)$. Since the $\mathrm{Ag}$ and $\mathrm{Au}$ furnaces utilize the same designs and components, the spatial scans of the Au-point furnace was also used to correct the Ag-point furnace.

\section{Blackbody Design}

The physical diagram of the blackbody and the furnace is shown in Fig. 9. The graphite crucible is held in an alumina tube with an argon purge at the rear. The alumina tube is placed in a Na-heatpipe liner constructed from Inconel 601 for temperature uniformity and is sealed except for the front opening. The 


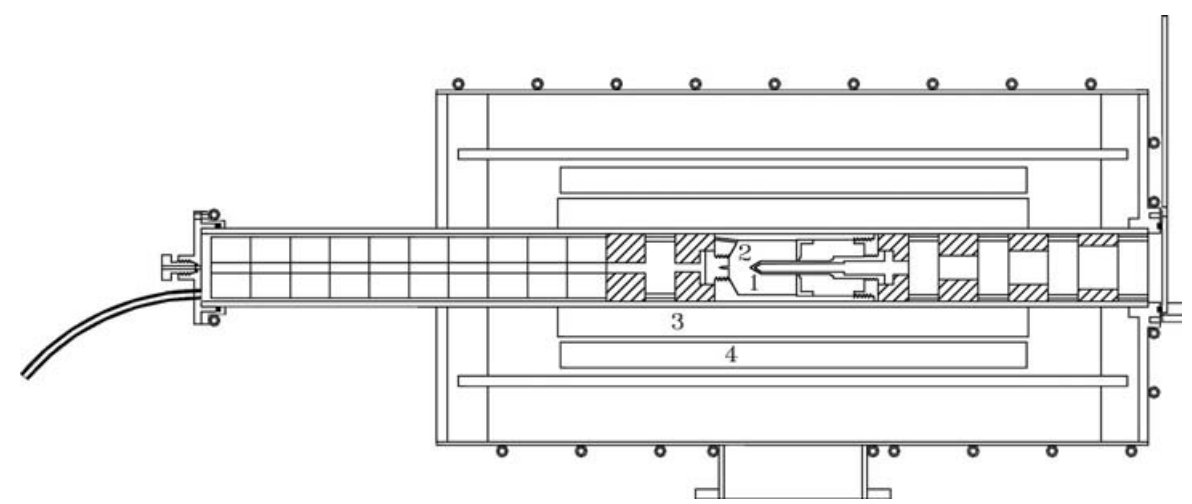

Fig. 9. Schematic of the NIST furnace showing the (1) graphite cavity and the (2) crucible, which is surrounded by the (3) cylindrical heat pipe, which is heated by the (4) semicylindrical heater elements. The alumina tube separates the crucible from the Inconel liner, and the argon purge enters from the rear and exits at the front opening of the furnace to reduce oxidation of the graphite elements.

heatpipe is heated using two cylindrical heaters that surround the heatpipe, and the alumina tube separates the crucible from the heatpipe to avoid possible contamination of the pure metal in the crucible from the Inconel heatpipe liner. The remaining space in the furnace is filled with fiberglass-insulation material, and the outer jacket is water cooled. Both the Agand Au-freezing points utilize the same furnace and crucible designs. The temperature uniformity of the crucible is increased with the use of graphite spacer rings, which restrict the field of view to $f / 6$. The temperature of the crucible is monitored for electrical heating with a thermocouple encased in a fused-silica holder in contact with the rear of the crucible. The furnace is operated as a two-level power control with different constant-current levels to either initiate the melt from the solid or to initiate the freeze from the melt.

A detailed view of the crucible is shown in Fig. 10. The crucible is constructed of graphite and filled with $1.3 \mathrm{~kg}$ of high-purity (99.999\% mass fraction) gold. Although the opening of the blackbody can be made smaller than a $6 \mathrm{~mm}$ diameter with graphite apertures, none were used during these measurements. The emissivity of the blackbody was determined using Monte Carlo calculations ${ }^{17}$ based upon the geometry shown in Fig. 10, and the calculated emissivities are listed in Table 2 for graphite emissivity of 0.86 (col. 2) and 0.80 (col. 3), showing the relative insen-

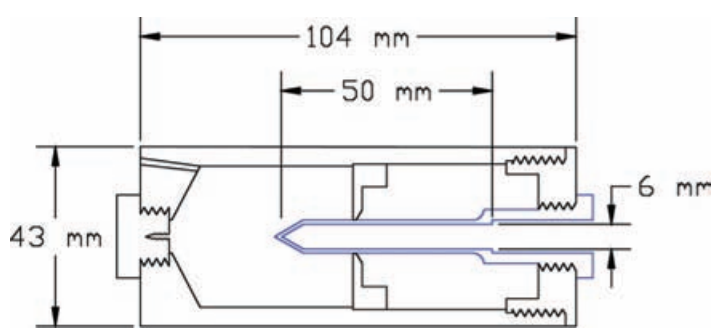

Fig. 10. (Color online) Design of the Ag- and Au-freezing temperature crucibles. The graphite crucible is operated inside a furnace with Na-heatpipe liner for temperature uniformity. The conical cavity has an apex angle of $57^{\circ}$. sitivity of the emissivity calculation with initial conditions. The graphite surface is expected to increase in emissivity with increasing operational hours owing to surface roughening, and thus the higher graphite emissivity value was used for this study. A uniform temperature distribution was assumed for the calculations with the emissivity of graphite of 0.86 at room temperature. ${ }^{18}$ These calculated emissivities are in agreement with experimental measurements $^{19}$ and with other calculations. ${ }^{20}$

\section{Summary of Systematic Corrections to Absolute Pyrometer}

The correction factors listing the dominant systematic corrections to the AP1 signals are listed in Table 3 . The AP1 signals were corrected prior to the application of Eq. (1) by using

$$
i=G \frac{\sigma}{\varepsilon} i_{m}
$$

where $i_{m}$ is the measured photocurrent, $i$ is the corrected photocurrent, $G$ is the gain correction, $\varepsilon$ is the emissivity, and $\sigma$ is the SSE correction. The corrected photocurrent can then be directly compared to the calculated photocurrents in Fig. 5. The gain correction factor is the normalization factor of gain $10^{10}$ to gain $10^{8}$ as shown in Table 1 since the responsivity calibrations were performed at gain $10^{8}$ while the $\mathrm{Ag}$ and $\mathrm{Au}$-freezing temperatures were measured at gain $10^{10}$. The SSE correction will have the effect of reduc-

\begin{tabular}{|c|c|c|}
\hline $\begin{array}{c}\text { Aperture } \\
\text { Diameter } \\
{[\mathrm{mm}]}\end{array}$ & $\begin{array}{c}\text { Emissivity with } \\
\text { Graphite Emissivity } \\
\varepsilon=0.86\end{array}$ & $\begin{array}{c}\text { Emissivity with } \\
\text { Graphite Emissivity } \\
\qquad \varepsilon=0.80\end{array}$ \\
\hline 6.0 & 0.99964 & 0.99943 \\
\hline 2.0 & 0.99996 & 0.99993 \\
\hline 1.5 & 0.99998 & 0.99996 \\
\hline 1.0 & 0.99999 & 0.99998 \\
\hline
\end{tabular}


Table 3. Systematic Correction Factors to the AP1 Signal

\begin{tabular}{llll}
\hline \multicolumn{1}{c}{ Type of Correction } & Gain, $G$ & Emissivity, $\varepsilon$ & SSE, $\sigma$ \\
\hline Signal correction factor & 1.0017 & 0.99964 & 0.99963 \\
Uncertainty $(k=2)$ & $0.022 \%$ & $0.02 \%$ & $0.02 \%$ \\
\hline
\end{tabular}

ing the measured signal since the responsivity calibration was used with a $5 \mathrm{~mm}$ diameter opening of the integrating sphere source with no other source of radiation outside the opening. The emissivity of blackbodies will also reduce the radiance, and thus compensation can be made using Eq. (5).

\section{Measurements of the $\mathrm{Ag}$ and Au Melts and Freezes}

The Au-freezing temperatures were measured over a 2 week period from 15 May 2003 to 28 May 2003. Since the blackbody power supply can be operated under computer control, measurements were made over a $24 \mathrm{~h}$ period for each of the runs listed in Tables 4 and 5 . A representative Au melt and freeze temperature measurement is shown in Fig. 11. Here the operation of the furnace is briefly described. Since the heatpipe furnace liner provides temperature uniformity over the length of the blackbody cavity, the furnace is operated with two-level constant-current control of the furnace power supply for inducing either the melting or the freezing transition. The temperature of the blackbody is held initially at approximately $15 \mathrm{~K}$ below the melting temperature, and then the melting transition is induced by ramping the current to approximately $9 \mathrm{~A}$ and then holding the current at that level. The time response of the furnace is much slower than the change in the current due to the thermal capacity of the furnace. The temperature rises up to the melting temperature and then is made constant until all the metal is melted. If there are temperature gradients in the furnace or impurities in the pure metal, then the rapid temperature rise above the melting temperature is expected to be rounded or less sharp. After the metal has completely melted with the temperature of the molten metal at approximately $20 \mathrm{~K}$ above the transition temperature, the current is lowered to approximately $5 \mathrm{~A}$, inducing the freeze. The temperature of the molten metal decreases until the supercool, and the freezing transition occurs at slightly higher temperatures from the supercool. The time duration of the freeze plateau is typically $20 \mathrm{~min}$, and the duration of the freeze plateau depends on the amount of the metal, the furnace parameters, and the power supply setting, among other parameters.

Table 4. Summary of the Au-Freezing Temperature Measurements

\begin{tabular}{cccr}
\hline & & Temperature & \multicolumn{1}{c}{$\begin{array}{c}T-T_{90} \\
{[\mathrm{~K}]}\end{array}$} \\
\hline 15 Mate & No. of Freezes & 1337.372 & 0.042 \\
23 May 2003 & 12 & 1337.329 & -0.001 \\
28 May 2003 & 12 & 1337.332 & 0.002 \\
& Average & 1337.344 & 0.014 \\
\hline
\end{tabular}

Table 5. Summary of the Ag-Freezing Temperature Measurements

\begin{tabular}{cccr}
\hline & Temperature & \multicolumn{1}{c}{$T-T_{90}$} \\
Date & No. of Freezes & {$[\mathrm{K}]$} & \multicolumn{1}{c}{\begin{tabular}{c} 
[K] \\
\hline 3 June 2003
\end{tabular} 9 June 2003 } \\
& 8 & 1235.010 & 0.080 \\
& Average & 1234.901 & -0.029 \\
& 1234.956 & 0.026 \\
\hline
\end{tabular}

A more detailed view of the melts and freezes with an expanded temperature scale is shown in Fig. 12. The melts and freezes are plotted with the thermodynamic temperature scale from the AP1, and a line at the ITS-90 temperature shows the agreement between the detector-based scale and the ITS-90. Slight temperature differences of $20 \mathrm{mK}$ are observed between the melting and the freezing temperatures. We believe that these differences are attributable to the temperature gradients in the furnace since the differences between the melting and freezing temperatures became $<2 \mathrm{mK}$ with a new heatpipe liner and heating elements. The gradual increase in the freezing temperatures is attributable to the drift in the dark current in the preamplifier of the AP1 and is not caused by changes in the Au-freezing temperature. The dark current correction to the signal was measured only at the beginning of the cycles, and the same correction was applied to the following melt and freeze cycles. At the high gains of $10^{10} \mathrm{~V} / \mathrm{A}$ used for these measurements, the circuit in the transimpedance amplifier could be susceptible to room-temperature drift or humidity changes, and dark-current corrections for each melt and freeze transition led to a reduction in the temperature drift of less than $10 \mathrm{mK}$ in later studies. For each freeze, as shown in Fig. 13, a mean temperature was determined with the uncertainties attributable to the averaging process estimated to be less than $5 \mathrm{mK}$. The noise-equivalent temperature at the Aufreezing temperature is less than $3 \mathrm{mK}$ leading to a noise-equivalent power of $<4 \mathrm{fW}$. The summaries of

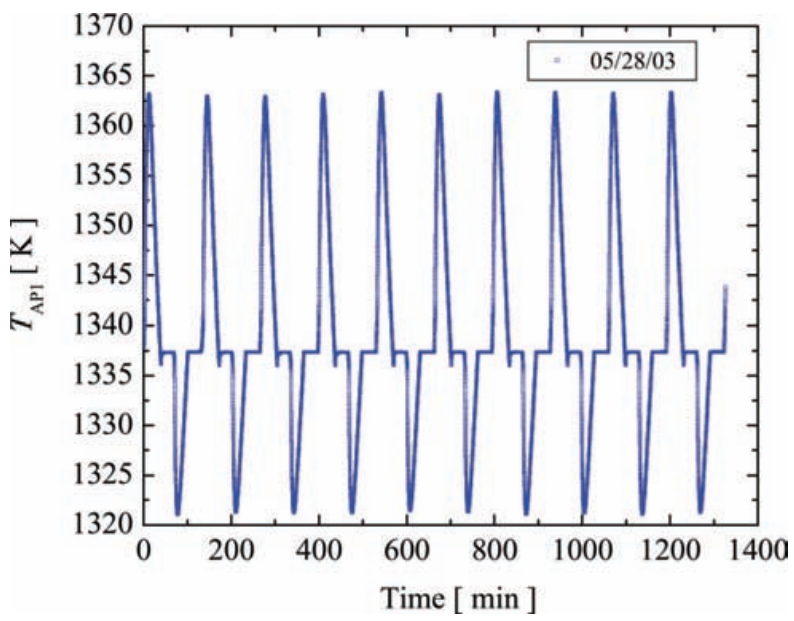

Fig. 11. (Color online) Repeat temperature cycle of melts and freezes of the Au-point blackbody measured with the AP1 showing the melt, slight supercool, and the freeze. 


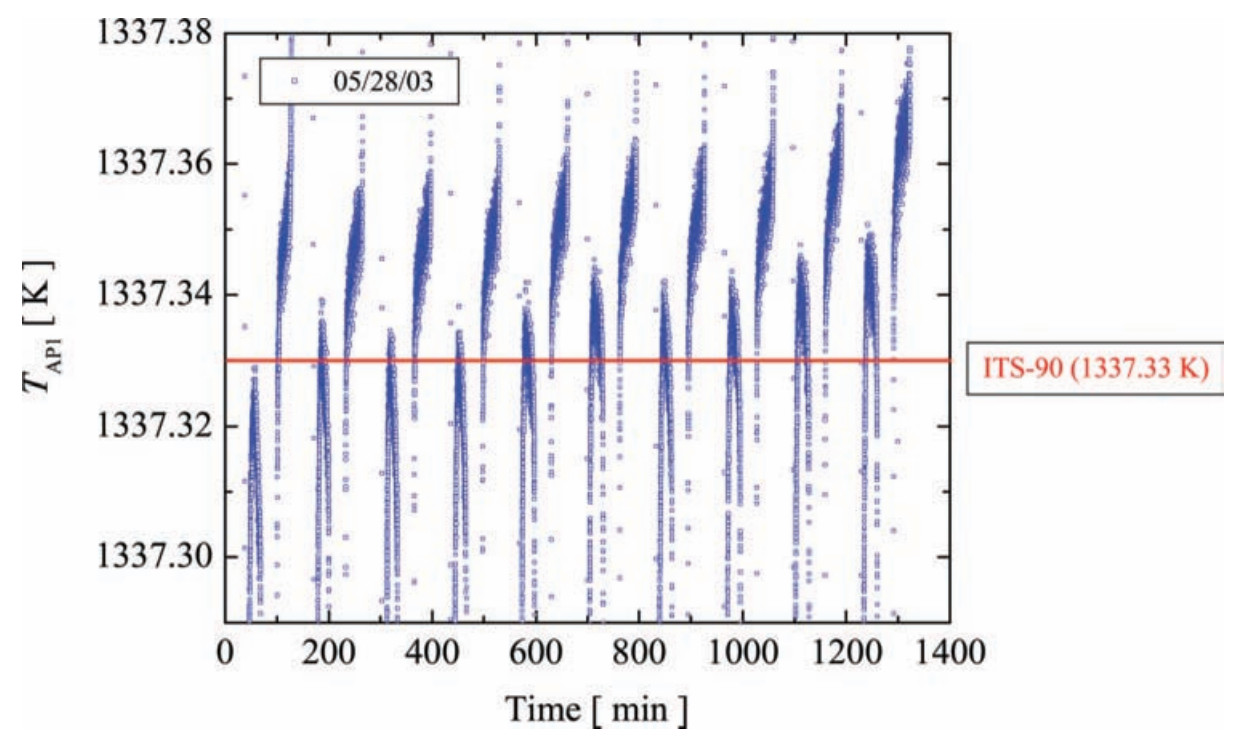

Fig. 12. (Color online) Plot of the melts and freezes of the Au point on an expanded temperature scale. The slight drift in the temperatures is attributable to the changes in the dark signal of the AP1.

the all the Ag- and Au-freezing temperature measurements are shown in Figs. 14 and 15, respectively, along with the ITS-90 temperatures and associated uncertainties.

The discussion of the uncertainties of the measurements must start from the initial uncertainties arising from the initial realization to the cryogenic electrical substitution radiometer in the NIST SIRCUS facility, and the component uncertainties are listed in Table 6. The component uncertainties arise from the individual transfers and steps as shown in Fig. 2. The two largest components, lines 4 and 7 , are attributable to the spatial uniformity and the uniformity of the angular output of the integrating sphere and the long-term stability of the trap detectors. Since the windows in the multielement diode trap detectors are removed to above the multiple reflections between the windows and the diode surfaces, the power responsivities of the diodes can

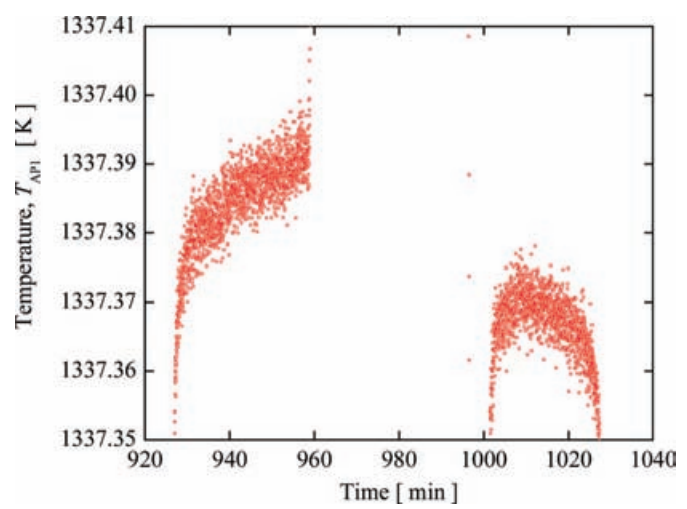

Fig. 13. (Color online) Representative Au melt and freeze temperatures measured using the AP1. The slight positive slope in the melt and the negative slope in the freeze is likely attributable to the size-of-source effect of the AP1. change owing to contamination or surface damage. The final total uncertainty leads to the uncertainties in Table 7. Additional information on the uncertainties in the detector-based realizations can be found in Ref. 13.

The individual uncertainties are listed in Table 7 as a percentage of the signals and then converted to temperature uncertainties. The dominant contribution to the expanded temperature uncertainties is the uncertainty in the radiance responsivity of $0.15 \%(k=2)$. Since the center wavelength of the AP1 is in the wavelength and temperature region where the Wien approximation is applicable, the uncertainty of the temperature from the uncertainty of radiance is found from the derivative of the Wien approximation

$$
\frac{\mathrm{d} L}{L}=\frac{\mathrm{c}_{2}}{\lambda} \frac{\mathrm{d} T}{T^{2}},
$$

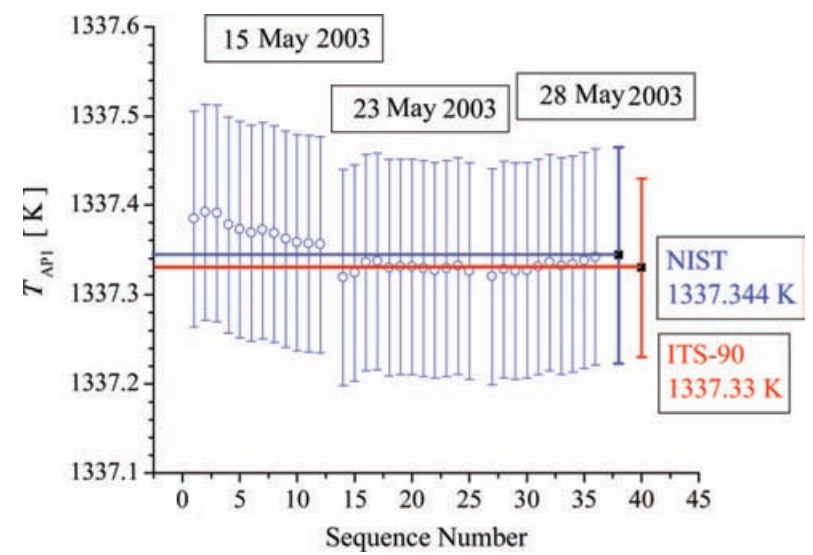

Fig. 14. (Color online) Summary of the Au-point measurements with the AP1 with the line indicating the ITS-90 assigned temperature. 


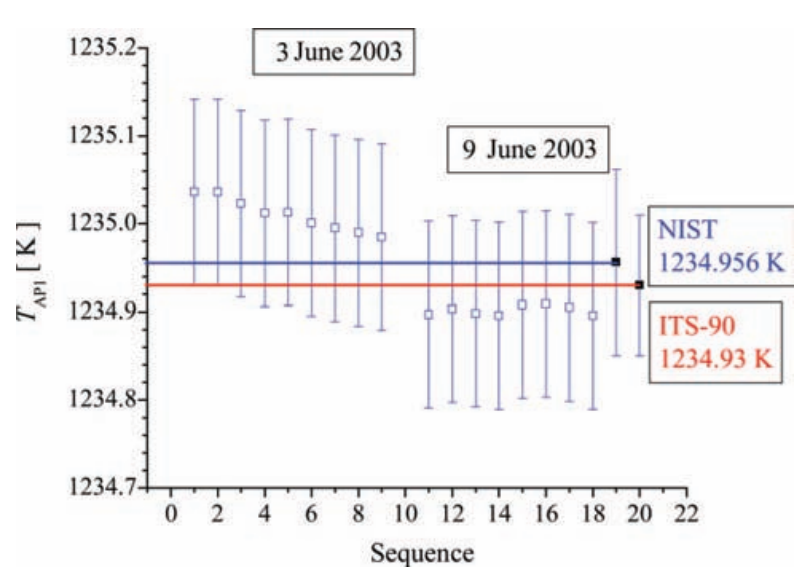

Fig. 15. (Color online) Summary of AP1 measurements of the Ag point along with the ITS-90 assigned temperature.

where $L$ is the radiance, $\lambda$ is the centroid wavelength, $T$ is the temperature, and $c_{2}$ is the second radiation constant.

\section{Discussion}

Our detector-based Ag- and Au-freezing temperatures are in agreement with the measurements of Fox et al. ${ }^{21}$ who also used a detector-based filter radiometer to measure the $\mathrm{Ag}$ - and $\mathrm{Au}$-freezing temperatures to be $1235.009 \mathrm{~K}( \pm 0.088 \mathrm{~K})$ and $1337.330 \mathrm{~K}( \pm 0.098 \mathrm{~K})$ respectively. The differences between our temperature determinations and the earlier results by Fox et $a l .{ }^{21}$ are within the combined uncertainties. The Au-freezing temperature is also in agreement with the detector-based measurements of Mielenz et al. ${ }^{22}$

Our results are also in agreement with the ITS-90 temperatures. The ITS-90 temperature assignments of the respective freezing temperatures are primarily from the ratio pyrometry work of Jung and Fischer ${ }^{23}$ with radiance ratios taken from a lower temperature blackbody at $730 \mathrm{~K}$. The thermodynamic temperature of the blackbody was assigned from two conflicting constant-volume gas thermometry measurements ${ }^{24}$ with differences of $30 \mathrm{mK}$ at $730 \mathrm{~K}$. Since the difference could not be resolved at the assignments of the ITS-90 fixed points, a decision was made to compro-

Table 6. Total Uncertainty of the Spectral Radiance Responsivity Calibrations in the NIST SIRCUS Facility Broken Down into the Component Uncertainties

SIRCUS Uncertainty Components $(\mathrm{k}=1) \quad$ Type

$\begin{array}{llcl}1 & \text { Trap }^{a} \text { responsivity } & \text { B } & 0.025 \% \\ 2 & \text { Aperture areas } & \text { B } & 0.004 \% \\ 3 & \text { Distance } & \text { B } & 0.010 \% \\ 4 & \text { Sphere spatial and angular uniformity } & \text { B } & 0.050 \% \\ 5 & \text { Amplifier gain } & \text { A } & 0.010 \% \\ 6 & \text { Temperature coefficient of trap } & \text { B } & 0.003 \% \\ 7 & \text { Temporal stability of trap } & \text { B } & 0.050 \% \\ & \text { Combined standard uncertainty }(\mathrm{k}=1) & & 0.076 \%\end{array}$

${ }^{a}$ Trap refers to the Si-diode trap detector used for the realization.
Table 7. Uncertainty Components for the Ag- and Au-Freezing Temperature Measurements

\begin{tabular}{llll}
\hline \multicolumn{5}{c}{ Signal Uncertainty Component } \\
{$[\%](k=2)$} & $\mathrm{Ag}$ & $\mathrm{Au}$ \\
\hline 1 & Spectral radiance responsivity & 0.15 & 0.15 \\
2 & Temporal stability of & 0.03 & 0.03 \\
$\quad \quad$ responsivity & & \\
3 & Plateau identification & 0.01 & 0.01 \\
4 & Emissivity & 0.02 & 0.02 \\
5 & Nonlinearity & 0.022 & 0.022 \\
6 & Dark current drift & 0.03 & 0.03 \\
7 & Size-of-source effect & 0.02 & 0.02 \\
& $\quad$ Total uncertainty in signal & 0.16 & 0.16 \\
& Temperature uncertainty & 0.110 & 0.129 \\
& {$[\mathrm{~K}](k=2)$} & & \\
\hline
\end{tabular}

mise between the two results and agree upon a compromise of $15 \mathrm{mK}$ at $730 \mathrm{~K}$, or half the value of the $30 \mathrm{mK}$ difference. Since the uncertainties in temperature increase as

$$
\Delta T_{a}=\frac{T_{a}^{2}}{T_{b}^{2}} \Delta T_{b}
$$

where $\Delta T_{a}$ and $\Delta T_{b}$ are the uncertainties of the blackbody at temperatures $T_{a}$ and $T_{b}$, the resulting thermodynamic temperature offset at $1337 \mathrm{~K}$ resulting from ratio pyrometry is expected to be $50 \mathrm{mK}$.

In addition to the thermodynamic temperature uncertainties, the uncertainties in realizing a temperature scale using fixed points include uncertainties due to material purity, emissivity of the cavity, furnace-temperature uniformity, and furnace operation. Even if the fixed point is filled initially with ultrapure (99.999\%) metal, the metal in the blackbody can become contaminated due to operations at high temperatures, and any contamination will suppress the freezing temperature. To have reproducible and stable freeze plateaus, the furnace should have a uniform temperature distribution. The electrical power heating of the furnace also has to be optimized to achieve reproducible and flat freeze plateaus. The dependence of the freezing temperature on the impurities and the operational parameters can lead to realization uncertainties of $50 \mathrm{mK}(k=2),{ }^{24}$ and temperature scale comparisons can require side-by-side operation of the respective fixed points to achieve lower temperature uncertainties.

The transition to detector-based radiance temperatures would lead to better agreement with thermodynamic temperatures and lead to direct dissemination of thermodynamic temperatures. This transition would be in accordance with the movement to having defined quantities or fixed values for the fundamental constants as the basis for the SI units instead of fixed artifacts as has already occurred for the candela in 1979. The dissemination of detector-based temperatures would also result in having a common basis, the electrical watt and length units, for photometric, ra- 
diometric, and temperature scales possibly leading to a resolution of any inconsistencies in the national scales. The uncertainties in the SIRCUS-based radiance responsivity calibrations are expected to be near $0.05 \%(k=2)$ in the near future with more frequent cryogenic radiometer realizations and finer wavelength intervals in the realized wavelengths. The resulting temperature uncertainty at the Au-freezing temperatures from such improvements is expected to result in $\sim 50 \mathrm{mK}(k=2)$ uncertainties.

\section{Conclusions}

The thermodynamic temperatures of the $\mathrm{Ag}$ - and Au-freezing temperatures are determined to be $1234.956 \mathrm{~K}[ \pm 0.110 \mathrm{~K}(k=2)]$ and $1337.344 \mathrm{~K}$ $[ \pm 0.129 \mathrm{~K}(k=2)]$ differing from the ITS-90 assigned values of $1234.93 \mathrm{~K}[ \pm 80 \mathrm{mK}(k=2)]$ and $1337.33 \mathrm{~K}[ \pm 90 \mathrm{mK}(k=2)]$ by $26 \mathrm{mK}$ and $14 \mathrm{mK}$, respectively. The temperatures are in agreement with the thermodynamic temperature uncertainties of the ITS-90 assigned values. The temperatures were systematically corrected for the size-of-source effect, the linearity of the preamplifier, and the emissivity of the blackbody. The ultimate goal of these thermodynamic temperature measurements is to disseminate temperature scales with lower uncertainties than those of the ITS-90. These results indicate that direct disseminations of thermodynamic temperature scales are possible.

Certain commercial equipment, instruments, or materials are identified in this paper to foster understanding. Such identification does not imply recommendation or endorsement by the National Institute of Standards and Technology nor does it imply that the material or equipment are necessarily the best available for the purpose.

\section{References}

1. T. J. Quinn, Temperature (Academic, 1990), pp. 20-22.

2. L. A. Guildner and R. E. Edsinger, "Deviation of the international practical temperatures from thermodynamic temperatures in the temperature range from $273.16 \mathrm{~K}$ to $730 \mathrm{~K}$," J. Res. Natl. Bur. Stand. 80A, 703-738 (1976).

3. M. R. Moldover, S. J. Boyes, C. W. Meyer, and A. R. H. Goodwin, "Thermodynamic temperatures of the triplepoints of mercury and Gallium in the interal 217 K to 303 K," J. Res. Natl. Inst. Stand. Technol. 104, 11-46 (1999).

4. L. Crovini and A. Actis, "Noise thermometry in the range $630{ }^{\circ} \mathrm{C}$ to $962{ }^{\circ} \mathrm{C}$," Metrologia 14, 69-78 (1978).

5. R. E. Edsinger and J. F. Schooley, "Differences between thermodynamic temperature and $\mathrm{t}$ (IPTS-68) in the range $230{ }^{\circ} \mathrm{C}$ to 660 C," Metrologia 26, 95-106 (1989).

6. D. R. White, R. Galleano, A. Actis, H. Brixy, M. De Groot, J. Dubbeldam, A. L. Reesink, F. Edler, H. Sakurai, R. L. Shepard, and J. C. Gallop, "The status of Johnson noise thermometry," Metrologia 33, 325-335 (1996).

7. Supplementary Information for the International Tempera- ture Scale of 1990, Sèvres, Bureau International des Poids et Mesures, 1990.

8. T. J. Quinn and J. E. Martin, "A radiometric determination of the Stefan-Boltzmann constant and thermodynamic temperatures between $-40{ }^{\circ} \mathrm{C}$ and $+100{ }^{\circ} \mathrm{C}$," Philos. Trans. R. Soc. 316, 85-189 (1985).

9. H. W. Yoon and C. E. Gibson, in Proceedings of TEMPMEKO'99, J. F. Dubbledam and M. J. de Groot, eds., Vol. II (IMEKO/Nmi Van Swinden Laboratorium, Delft, 1999, pp. 737-742.

10. N. P. Fox, J. E. Martin, and D. H. Nettleton, "Radiometric aspects of an experiment to determine the melting/freezing temperature of gold," Metrologia 28, 221-227 (1991).

11. W. R. Blevin and B. Steiner, "Redefinition of the candela and lumen," Metrologia 11, 97-104 (1975).

12. P. J. Martin, H. A. Macleod, R. P. Netterfield, C. G. Pacey, and W. G. Sainty, "Ion-beam-assisted deposition of thin films," Appl. Opt. 22, 178-184 (1983).

13. S. W. Brown, G. P. Eppeldauer, and K. R. Lykke, "Facility for spectral irradiance and radiance responsivity calibrations using uniform sources,” Appl. Opt. 45, 8218-8237 (2006).

14. P. J. Mohr and B. N. Taylor, "CODATA recommended values of the fundamental physical constants: 2002," Rev. Mod. Phys. 77, 1-108 (2005).

15. G. P. Eppeldauer and J. E. Hardis, "Fourteen-decade photocurrent measurements with large-area silicon photodiodes at room temperature," Appl. Opt. 30, 3091-3099 (1991).

16. F. Sakuma and S. Hattori, "Establishing a practical temperature standard by using a narrow-band radiation thermometer with a silicon detector," in Temperature: Its Measurement and Control in Science and Industry, J. F. Schooley, ed. (AIP, 1982), Vol. 5, pp. 421-427.

17. V. I. Sapritsky and A. V. Prokhorov, "Spectral effective emissivities of nonisothermal cavities calculated by the Monte Carlo method," Appl. Opt. 34, 5645-5652 (1995).

18. G. Bauer and K. Bischoff, "Evaluation of the emissivity of a cavity source by reflection measurements," Appl. Opt. 10, 2639-2643 (1971).

19. C. K. Ma, "Method for the measurement of the effective emissivity of a cavity," in Proceedings of TEMPMEKO'04, D. Zvizdic, ed. (Laboratory for Process Measurement, Zagreb, 2005), pp. 575-580.

20. F. Sakuma and L. Ma, "Evaluation of the fixed-point cavity emissivity at NMIJ," in Proceedings of TEMPMEKO'04, D. Zvizdic, ed. (Laboratory for Process Measurement, Zagreb, 2005), pp. 563-568.

21. N. P. Fox, J. E. Martin, and D. H. Nettleton, “Absolute spectral radiometric determination of the thermodynamic temperatures of the melting/freezing points of gold, silver, and aluminum," Metrologia 28, 357-374 (1991).

22. K. D. Mielenz, R. D. Saunders, and J. B. Shumaker, "Spectroradiometric determination of the freezing temperature of gold,” J. Res. Natl. Inst. Stand. Technol. 95, 49-67 (1990).

23. R. L. Rusby, R. P. Hudson, M. Durieux, J. F. Schooley, P. P. M. Steur, and C. A. Swenson, "Thermodynamic basis of the ITS90," Metrologia 28, 9-18 (1991).

24. J. Fischer, M. Battuello, M. Sadli, M. Ballico, S. N. Park, P. Saunders, Y. Zundong, B. C. Johnson, E. Van der Ham, F. Sakuma, G. Machin, N. Fox, W. Li, S. Ugur, and M. Matveyev, "Uncertainty budgets for realization of ITS-90 by radiation thermometry," in Temperature: Its Measurement and Control in Science and Industry, D. C. Ripple, ed. (AIP, 2003), Vol. 7, pp. 631-638. 


\title{
Uncertainty Analysis and Maintenance of the NIST Detector- based Temperature Scale
}

\author{
H. W. Yoon, C. E. Gibson, G. P. Eppeldauer, A. W. Smith, \\ S. W. Brown, K. R. Lykke \\ (Optical Technology Division \\ National Institute of Standards and Technology \\ Gaithersburg, MD 20899 USA)
}

\begin{abstract}
Thermodynamic temperatures can be directly determined using the Planck radiance law and detector-based radiation thermometers. Although the final uncertainties in the detector-based radiation thermometry can be lower than in the ITS-90 based temperatures, detailed uncertainty analyses for each step of the detector-based realization are needed to validate these low uncertainties. We list the measurement steps in the detector-based temperatures and also describe the characterizations in determining the measurement uncertainties for each of the steps. We further describe measures to maintain the low uncertainties obtained from the radiometric calibrations.
\end{abstract}

Key words: Thermodynamic temperature; Temperature measurement; Radiation Thermometry; Uncertainty Analysis

\section{Introduction}

A fundamental goal in the establishment of the Système International d'Unités (SI) is to base the SI units on constants which are invariant in space and time. The movement to define the SI units based upon fundamental physical constants and, in particular, to adopt a fixed Boltzmann constant as the basis of the unit of temperature, the kelvin, is in pursuit of this goal [1]. With the redefinition of the kelvin, work is underway in the Consultative Committee on Thermometry (CCT) to give guidance on the practical realization of the kelvin or the mise en practique (MeP). The new MeP will include descriptions and references to all primary thermometry techniques including, among others, constant-volume gas thermometry, acoustic thermometry, noise thermometry, dielectric-

Received: 2008-00-00

Corresponding e-mail address: hyoon@nist.gov constant gas thermometry, and detector-based radiation thermometry [2]. The MeP will also list the uncertainties associated with each of these primary thermometry techniques. The common value linking these different methods is the fixed Boltzmann constant which also fixes the kelvin temperature scale.

Presently, the International Temperature Scale of 1990 (ITS-90) defines the kelvin scale and is used successfully in most applications. However, the greatest weakness of the ITS-90 is recognized to be at temperatures above the Ag freezing temperatures where temperatures are defined by the use of Planck radiance ratios from either the Ag-, $\mathrm{Au}$ - or the $\mathrm{Cu}-$ freezing temperature blackbodies [3]. This weakness is due to the use of extrapolations to higher temperatures from the defining fixed point using radiation thermometers which can have many systematic biases which, in turn, can lead to large temperature differ- 
ences. Detector-based radiation thermometry is primary thermometry which can lead to lower uncertainties than in ITS-90 based temperatures, but the uncertainty components need to be characterized and measured.

In this work, we describe the uncertainty components in the detector-based radiation thermometry where the radiance responsivity of the radiation thermometer is measured using a tunable laser-irradiated, integrating sphere source. The uncertainty analysis is separated into those related to the calibrations of the radiance responsivity and the characterizations of the radiation thermometer. Both spatial and angular uniformity characterizations of the integrating sphere used in the realization are described. The interference fringes observed at the peak of the radiance responsivity is analyzed to determine the thickness of the layer causing the interference fringes. This listing of the uncertainty components will assist other laboratories attempting to develop similar primary thermometry capabilities.

\section{Detector-based Radiance Responsivity Calibrations}

The spectral radiance responsivity calibrations are performed in the NIST Spectral Irradiance and Radiance responsivity Calibrations using Uniform Sources (SIRCUS) facility [4]. In Tab. 1, the uncertainty

Tab. 1 The total uncertainty of the radiance responsivity calibrations in the NIST SIRCUS facility broken down into the component uncertainties. Trap refers to the Si-diode trap detector used for the realization.

\begin{tabular}{|c|l|c|c|}
\hline & $\begin{array}{l}\text { SIRCUS Uncer- } \\
\text { tainty Components } \\
(\boldsymbol{k}=\mathbf{1})\end{array}$ & Type & [ \% ] \\
\hline 1 & Trap responsivity & A & 0.025 \\
\hline 2 & Aperture Area & A & 0.004 \\
\hline 3 & Distance & B & 0.01 \\
\hline 4 & $\begin{array}{l}\text { Sphere Spatial and } \\
\text { Angular Uniformity }\end{array}$ & B & 0.025 \\
\hline 5 & Amplifier gain & A & 0.005 \\
\hline 6 & $\begin{array}{l}\text { Temperature coeffi- } \\
\text { cient of Trap }\end{array}$ & B & 0.002 \\
\hline 7 & $\begin{array}{l}\text { Temporal Stability of } \\
\text { Trap }\end{array}$ & B & 0.013 \\
\hline & $\begin{array}{l}\text { Combined Standard } \\
\text { Uncertainty }(k=1)\end{array}$ & & 0.040 \\
\hline & $\begin{array}{l}\text { Expanded Uncer- } \\
\text { tainty }(\boldsymbol{k}=2)\end{array}$ & & 0.080 \\
\hline
\end{tabular}

components of the detector-based radiance responsivity calibrations are listed. Although these uncertainties are discussed in Ref. 4, additional measurements to support the uncertainties have been further performed since the initial publication about the SIRCUS facility.

\subsection{Si-trap detector responsivity}

At NIST, the detector-based radiation thermometry begins with the calibration of detectors using the Primary Optical Watt Radiometer (POWR) [5] which is a cryogenic electrical substitution radiometer. Cryogenic radiometers have been compared against each other using Si-trap detectors and have been found to be in agreement to within $0.012 \%(\mathrm{k}=1)$. In the wavelength region from $400 \mathrm{~nm}$ to $920 \mathrm{~nm}$, the Sitrap detector is calibrated against the POWR in the NIST SIRCUS facility for spectral power responsivity at many different laser wavelengths as shown in Fig. 1. Since there is some scatter in the measurements, multiple measurements at various wavelengths are fitted using a modified Gaussian-Lorentzian fit and the residuals from this fit are shown in Fig. 1. Although the physical model of the quantum efficiencies is typically used to fit the spectral power responsivity of the trap detectors [6], such fitting is not needed if many laser wavelengths are used for the calibrations. The residuals of the measurements from the fitted functions are distributed evenly about the zero crossing.

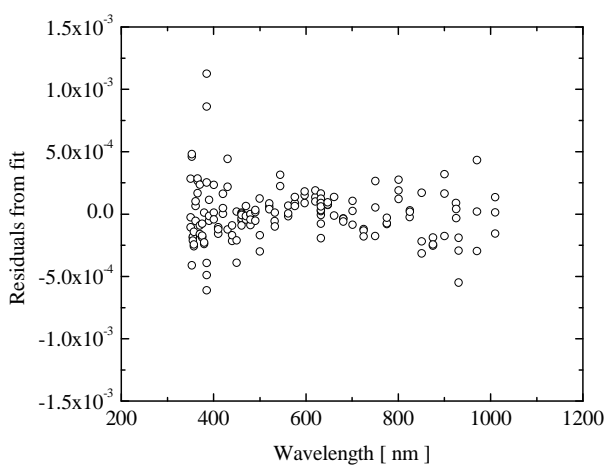

Fig. 1 The residuals of the fitted spectral power responsivity of the trap detector from the individual measurements 


\subsection{Aperture area uncertainties}

The uncertainties in row 2 of Tab. 1 are due to the aperture areas on both the trap detector and the integrating sphere sources (ISS), and the uncertainties in row 3 are due to the uncertainty in the separation distance between the precision aperture on the ISS and the trap detector. An international key comparison of aperture areas was recently completed to determine the whether the measurements at the different national measurement institutes (NMI) were within their stated uncertainties [7]. The comparison showed that the uncertainties of an individual laboratory for certain type of apertures could be at the value cited in Tab. 1 but the differences between the NMIs could be larger than the uncertainties cited by the NMIs.

\subsection{Distance measurement uncertainties}

The distance between the ISS and the trap detectors are determined through a modified $1 / \mathrm{r}^{2}$ fit,

$$
\frac{S_{o}}{\left(r-r_{o}\right)^{2}}
$$

where both the amplitude, $S_{o}$, and the offset distance, $r_{0}$, are fitted parameters using and the uncertainties are determined from the residuals of the fit. Furthermore, presence of scattered radiation from improper baffling can be determined from the residuals of the $1 / r^{2}$ fit .

\subsection{Amplifier gain uncertainties}

The uncertainties in the amplifier gains are attributed to the uncertainties in the current-to-voltage conversion of the transimpedance amplifiers. The current measurements are performed with custom-designed preamplifiers with gains determined by high-precision, metal-film, feedback resistors in the operational amplifier circuit. The preamplifier gains are determined from electrical resistance calibrations of the feedback resistors. Care should be taken that the current-tovoltage conversion is linear since the parallel connections of the Si diodes in the trap detector results in a shunt resistance of the trap detector being lower than the shunt resistance of any single diode element.

\subsection{Si-trap detector temperature dependence and long-term stability}

The next two rows in Table 1 are due to the temperature dependence of the trap detector responsivity and the long-term stability of the trap detector. Since the trap detector is constructed from three or more $\mathrm{Si}$ diodes which could have a temperature dependence, change in the spectral power responsivity could result from the temperature difference between the calibration against the cryogenic radiometer and the subsequent use of the trap detector. Such temperature dependence is expected to be greater near the band-gap energy of the Si diode.

Since the trap detectors are only calibrated on an annual or biennial basis, the responsivity could change over time. The trap detectors are constructed using Si diodes without any windows, and any change in the reflectance of Si diodes due to oxide accumulation would be compensated by the increase in the photocurrent of the other Si diodes. Although, the trap detectors are stored in dust-free containers when not in use, any particulate contamination would lead to a change in the responsivity. Such contamination is evident in the change in the spatial uniformity of the trap detector or changes in the power responsivity when compared to the other sets of trap detectors. The results of the annual calibrations of the working standard trap detectors $\mathrm{T}-01$ and $\mathrm{T}-02$ are shown in Table 2. The power responsivities are determined by comparison against the cryogenic radiometer and the

Tab. 2 The external quantum efficiencies of trap detectors T01 and T-02 with repeat calibrations in 2005, 2006 and 2007.

\begin{tabular}{|c|c|c|c|c|}
\hline & & $\begin{array}{c}\text { T-01 Exter- } \\
\text { nal Quantum } \\
\text { Efficiency } \\
\text { Year }\end{array}$ & $\begin{array}{c}\text { T-02 Ex- } \\
\text { ternal } \\
\text { Quantum } \\
\text { Efficiency } \\
\text { (EQE) }\end{array}$ & $\begin{array}{c}\text { Ratio } \\
\text { (T-1 } \\
\text { EQE/T- } \\
02 \text { EQE) }\end{array}$ \\
\hline 2005 & 649.906 & 0.99573 & 0.99772 & 0.99801 \\
\hline 2005 & 649.9 & 0.99583 & 0.99778 & 0.99804 \\
\hline & & & & \\
\hline 2006 & 647.1600 & 0.99543 & 0.99766 & 0.99777 \\
\hline 2006 & 647.1400 & 0.99545 & 0.99766 & 0.99778 \\
\hline 2006 & 647.1200 & 0.99545 & 0.99768 & 0.99777 \\
\hline & & & & \\
\hline 2007 & 653.81 & 0.99566 & 0.99783 & 0.99783 \\
\hline 2007 & 653.84 & 0.99564 & 0.99785 & 0.99779 \\
\hline
\end{tabular}

external-quantum efficiencies (EQE) are determined. 
The slight differences in the wavelengths of the measurements and the resulting differences in the EQE are not significant, and if the trap detector has remained temporally stable, then the EQE's are expected to repeat within the assigned uncertainties. One way to determine if the trap detectors have remained stable is to plot the ratios of the EQE's of the trap detectors over time as shown in Fig. 2. The mean value of the ratios is indicated by the middle line and the expanded uncertainties assigned to the temporal stability of $0.026 \%$ is shown by the bracketing dotted lines. The data indicates that the temporal stabilities of the trap detectors are adequately accounted by the assigned uncertainties.

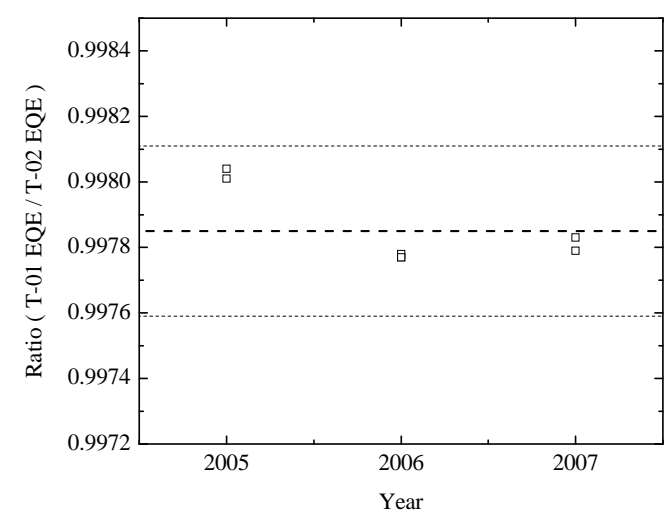

Fig. 2 The ratios of the EQE's of the trap detectors T-01 and T-02 over time shown with the mean and the assigned expanded uncertainties of $0.026 \%$ indicated by dotted lines.

\section{Spatial Uniformity of the Integrating Sphere Source}

One of the most important characterization steps in the detector-based radiance responsivity is the measurements of the spatial and angular uniformity of the ISS. When the radiation thermometer is calibrated for radiance responsivity, the ISS acts as a monochromatic substitute for a blackbody, and the radiance output of the ISS, in turn, must be as uniform and lambertian as a Planckian source. It is difficult to have the ISS achieve the spatial and angular uniformity of a blackbody, since unlike a blackbody, the radiation enters from one or few ports and is reflected. The high emissivity of the blackbody is achieved by having every surface inside the blackbody emitting at a uniform temperature.

The spatial uniformity of the ISS can be measured with a radiometer which can be limited to a small field of view. The spatial uniformities of different diameter integrating sphere sources with $5 \mathrm{~mm}$ diameter openings measured with a radiometer limited to $1 \mathrm{~mm}$ spot size at f/12 geometry are shown. Fig. 3 shows the spatial uniformity of the $25 \mathrm{~mm}$ diameter sphere with the $5 \mathrm{~mm}$ diameter opening. All the ISS used in this study are internally coated with sintered polytetrafluoroethylene (PTFE) for the highest reflectance over a wide range of wavelengths. The laser is introduced into the ISS with a fiber input at $90^{\circ}$ at the side of the sphere. Due to the small size of the sphere, the bright illumination of the initial bounce from the laser can be seen as a spatial non-uniformity of $0.1 \%$ toward the upper right of the figure.

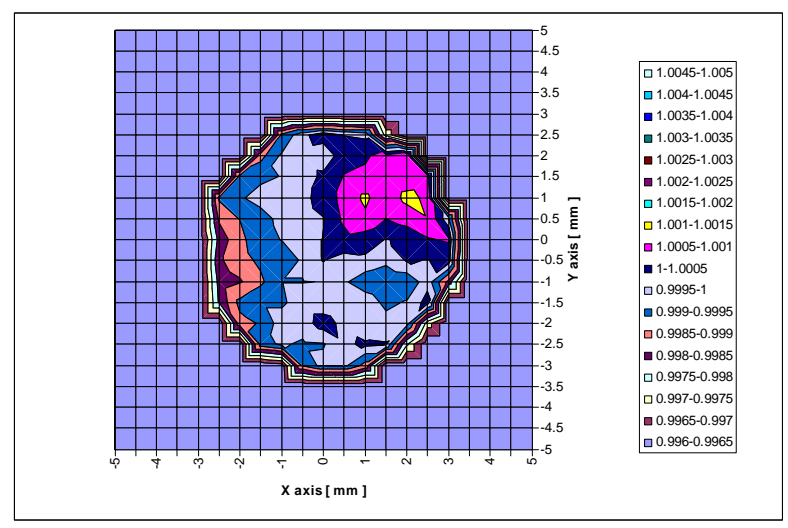

Fig. 3 The spatial uniformity of the $25 \mathrm{~mm}$ diameter PTFE sphere with a $5 \mathrm{~mm}$ diameter opening. The insufficient mixing of the radiation can be observed from the non-uniformity of $0.1 \%$ toward the upper right side.

Two other PTFE-lined ISS with $38 \mathrm{~mm}$ and $300 \mathrm{~mm}$ diameters have also been measured for spatial uniformity as shown in Figs. 4 and 5 with the same intensity scales as in Fig. 3. As expected, the spatial uniformity is found to increase with the diameter of the sphere. The throughput of the sphere decreases with increasing diameter so that the incident laser power must be increased to obtain the same irradiances or radiances as with the smaller diameter spheres. 


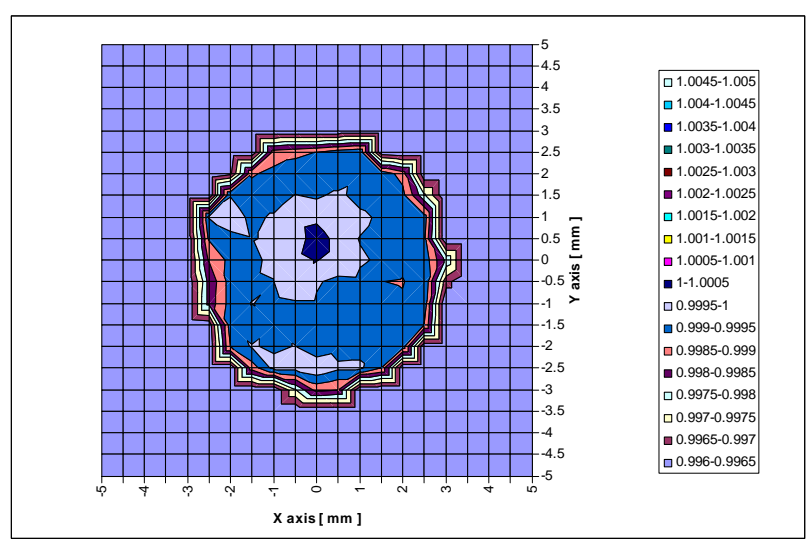

Fig. 4 The spatial uniformity of the $38 \mathrm{~mm}$ diameter PTFE sphere with a $5 \mathrm{~mm}$ diameter opening. A center spot introduces a nonuniformity of $0.05 \%$.

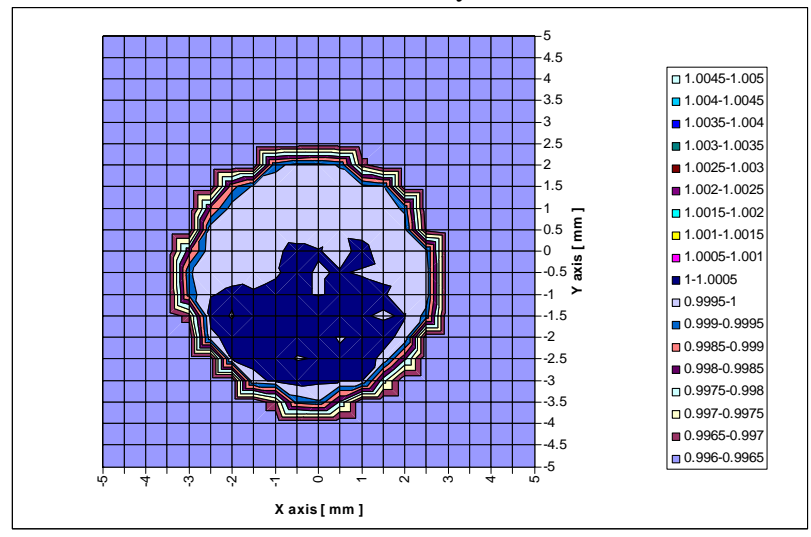

Fig. 5 The spatial uniformity of the $300 \mathrm{~mm}$ diameter PTFE sphere with a $5 \mathrm{~mm}$ diameter opening. The large sphere with a small opening results in uniformity of $0.025 \%$.

\section{Angular Uniformity of the Integrating Sphere Source}

The angular uniformity of the ISS can be measured with a trap detector with a characterized angular responsivity. If the internal diodes which form the trap configuration are not well aligned, then the angular responsivity of the trap detector will not be symmetric and be independent of the angular direction. Once the trap detector has been characterized, the ISS can be measured for angular output. The optical layout of the radiometric measurements is shown in Fig. 6 . The integrating-sphere source is shown at left and the precision aperture of the trap detector is shown on the right. If the two apertures are translated in a perpendicular plane then the closest distance between the two apertures is indicated by the distance $\mathrm{d}$, and the distance between the apertures when the trap detector is out of the plane is given by the distance $r$. For the optical configuration shown in Fig. 6, the relationship between $r$ and $d$ is given by $d=r \cos \theta$, where $\theta$ is the angle between the centers of the ISS and the trap detector. In addition to the change in the distance between the ISS and the trap detector, the projected areas of both the source and the detector apertures decrease by the $\cos \theta$, leading to a relationship between the irradiance at $r$ as compared to the irradiance at position d,

$$
E(r)=E(d) \cos ^{4} \theta
$$

Thus, a plot of the normalized signal versus angle can

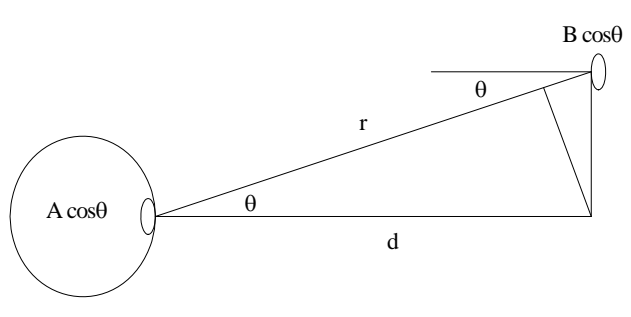

Fig. 6 The optical schematic for determining the angular uniformity of the irradiance at the plane of the trap detector.

be fitted with Eq. 2 to determine any deviations from lambertian output of the ISS. The irradiance ratios are plotted in Fig. 7 and shown to be in agreement with Eq. 2 within \pm 0.05 radian.

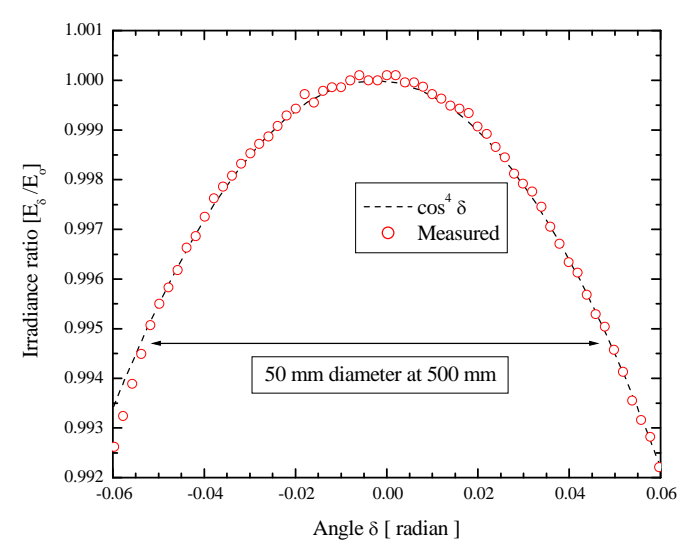

Fig. 7 The irradiance ratios to the center measured by scanning the trap detector. Although the detector was scanned in the $\mathrm{x}$ and $\mathrm{y}$ planes, a one dimensional plot is shown to better demonstrate the fit to $\cos ^{4} \theta$ dependence. 


\section{Radiation thermometer uncertainties}

The uncertainties for the use of the radiation thermometer in measuring the temperature of fixed-point blackbodies are shown in Tab. 3. The uncertainty of the spectral radiance responsivity is transferred from Tab. 1, but additional uncertainties arise in the specific use of the radiation thermometer. One of the largest uncertainty components is due to the temporal stability of the responsivity arising from the change in the radiation thermometer throughput. The change in the throughput could be due to many factors such as the changes from the filter transmittance, the detector responsivity, contamination of the optics or structural changes of the radiation thermometer related to changes in the ambient temperature, among others. Although care is taken to design the radiation thermometer to minimize such unpredicted changes, it is difficult to eliminate all sources of temporal changes.

Tab. 3 Uncertainty components for a fixed-point blackbody temperature determination.

\begin{tabular}{|c|c|c|}
\hline & Uncertainty Component & [ \% ] \\
\hline 1 & Spectral Radiance Responsivity & 0.040 \\
\hline 2 & Temporal Stability of Responsivity & 0.050 \\
\hline 3 & Plateau Identification & 0.005 \\
\hline 4 & Emissivity & 0.010 \\
\hline 5 & Preamplifier Gain & 0.025 \\
\hline 6 & Dark current drift & 0.015 \\
\hline 7 & Size-of-source effect & 0.010 \\
\hline & Total Uncertainty in Signal & 0.072 \\
\hline & Expanded Uncertainty $(\boldsymbol{k}=\mathbf{2})$ & 0.144 \\
\hline & & \\
\hline & Temperature Uncertainty [ K ] $(\boldsymbol{k}=\mathbf{2})$ & $\mathbf{0 . 1 1 6}$ \\
\hline
\end{tabular}

The low uncertainty of the wavelength and dynamic range of the responsivity calibrations are made possible by the use of laser-based responsivity calibrations. However, due to the narrow linewidth of the lasers, interference fringes can be present in the responsivity calibrations. The radiance responsivity of the NIST Absolute Pyrometer 1 (AP1) is shown in Fig. 8. The interference fringes are observed at the peak of the responsivity, and the additional modulation can be

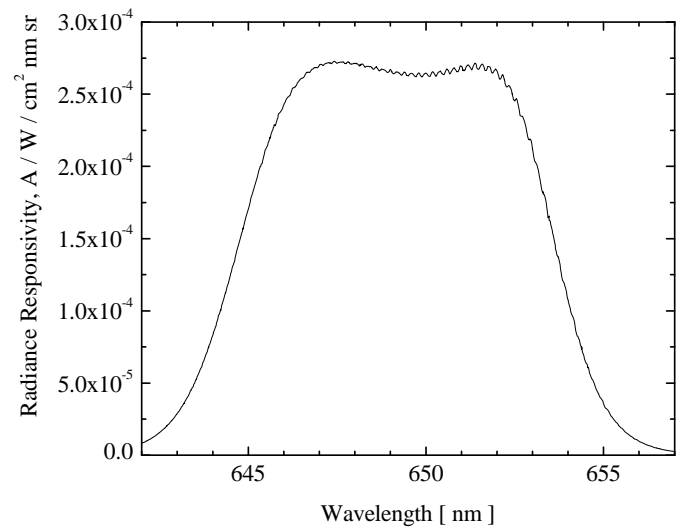

Fig. 8 The radiance responsivity of the NIST Absolute Pyrometer 1 (AP1) showing the interference fringes at the peak.

observed from the changes in the amplitude of the interference fringes. The thickness of the material responsible for the interference fringes can be determined from

$$
\mathrm{m} \lambda=2 \mathrm{nd} \cos \varphi
$$

where $m$ is an integer, $\lambda$ is the wavelength of the radiation, $\mathrm{n}$ is the refractive index, $\mathrm{d}$ is the thickness of the material, and $\varphi$ is the incident angle of the radiation. For determining the cause of the interference fringes, an expanded plot of the peak responsivity is shown in Fig. 9. In Fig. 9, the interference fringes are found to be temporally stable with repeated calibrations from 2003 to 2006. Since the wavelength interval between the fringes can be measured from Fig. 9, the thickness of the material can be found using

$$
\mathrm{d}=\frac{1}{2 \mathrm{n} \Delta \chi},
$$

where

$$
\Delta \chi=\frac{1}{\lambda_{m}}-\frac{1}{\lambda_{m+1}} .
$$

If the refractive index is assumed to be 1.55 then the thickness of the material which results in the interference fringes seen in Fig. 9 is estimated to be $0.67 \mathrm{~mm}$. Although the interferences could arise from several different sources, the fringes in the AP1 are determined to be from an element in the interference filter centered at $649.5 \mathrm{~nm}$. At first, the protective window 
on the thermo-electrically cooled diode was postulated to cause the interference fringes. Both roughening the window surface and increasing the thickness of the window by gluing an additional glass window did not affect the fringes. However, when the Lyot stop diameter was increased, the amplitudes of the fringes were reduced, and the amplitudes increased with a decrease in the Lyot stop diameter. Since the Lyot stop is placed directly in front of the interference filter, one explanation for this behavior is that a layer within the filter is the cause of the fringes. The total thickness of the interference filter is about $11 \mathrm{~mm}$ so that the front and back surfaces of the filter are not the source of the fringes as estimated from Eq. 4. If this layer inside the filter has interfaces which are not parallel over the entire area of the filter, then the radiation is expected to have a greater degree of destructive interference when the radiation is transmitted over a larger surface of the filter.

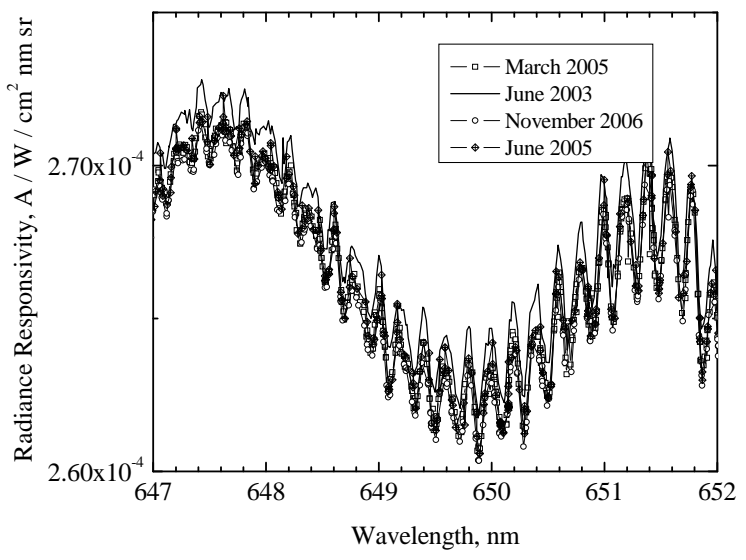

Fig. 9 An expanded plot of radiance responsivity showing the changes with each calibration and the persistence of the interference fringes.

The presence of the fringes are not problematic if the radiance responsivity calibrations can adequately measure the peaks and valleys of the fringes, but the number of calibrations at the different wavelengths could be extremely time consuming. Furthermore, if the amplitude of the fringes were much larger than the $<2 \%$ observed, then the sharp radiance responsivity changes with wavelength would require lower wavelength uncertainties. Any wavelength fluctuations of the source would be magnified by the amplitude changes of the responsivity function resulting in large uncertainties.

\section{Blackbody uncertainties}

For fixed-point blackbodies the uncertainty due to the plateau identification is low due to the long time duration of the freeze. Since the cavity is also surrounded by a constant temperature material undergoing the phase transition, the emissivity of the cavity is high and can be determined with low uncertainties. The uncertainties related to the use of fixed point blackbodies are well understood [8].

\section{Systematic uncertainties of the radiation thermometer}

Since the preamplifier gain during the calibration of the radiance responsivity and the use of radiation thermometer can be different, uncertainties arise when the RT is used to measure blackbody temperatures which can vary widely in radiance. The size-ofsource effect can be controlled to low levels by using clean, low-scatter optics and a Lyot stop [9]. The additional sources of uncertainties are discussed in a prior publication [10]. The total uncertainties at the bottom of Tab. 3 are then converted to temperature uncertainties using the derivative of the Wien approximation as discussed in Ref. 10.

\section{Conclusions}

The uncertainties in the detector-based radiation thermometry have been discussed and the experimental results for determining those uncertainties have been shown. The critical characterizations related to the fitting of the quantum efficiencies of the trap detectors and their long-term stabilities are shown. The spatial and angular uniformity of the integrating sphere sources are shown and found to be dependent on the ratio of the opening of the sphere to its internal surface area. The interference fringes observed in the radiance responsivities are described and minimized by increasing the radiated area on the spectrally selective filter. These characterizations are needed if the direct realizations of thermodynamic temperatures are to achieve lower uncertainties than the ITS-90 based temperatures. 


\section{REFERENCES}

1. I. M. Mills, P. J. Mohr, T. J. Quinn, B. N. Taylor, and E. R. Williams. Metrologia. 2006, 43 (3): 227-246.

2. B. Fellmuth, Ch. Gaiser, and J. Fischer. Meas. Sci. Technol. 2006. 17 (10): R145-R159.

3. Supplementary Information for the International Temperature Scale of 1990, H. Preston-Thomas, P. Bloembergen, T. J. Quinn, Sèvres, Bureau International des Poids et Mesures, 1990.

4. S. W. Brown, G. P. Eppeldauer, K. R. Lykke. Applied Optics. 2006, 45(32): 8218-8237.
5. J. M. Houston, J. P. Rice. Metrologia. 2006. 43(2): S31S35.

6. T. R. Gentile, J. M. Houston, and C. L. Cromer. Applied Optics. 1996, 35(22): 4392-4403.

7. M. Litorja et al. Metrologia. 2007, 44: 02002.

8. P. Saunders et al. International Journal of Thermophysics. 2008, 29:1066-1083.

9. H. W. Yoon, D. W. Allen, and R. D. Saunders. Metrologia. 2005, 42(2): 89-96.

10. H. W. Yoon, D. W. Allen, C. E. Gibson, M. Litorja, R. D. Saunders, S. W. Brown, G. P. Eppeldauer, K. R. Lykke, Applied Optics. 2007, 46(15): 2870-2880. 


\title{
SSE- and noise-optimized InGaAs radiation thermometer
}

\author{
H W Yoon , C E Gibson, V Khromchenko, G P Eppeldauer \\ Optical Technology Division \\ National Institute of Standards and Technology, Gaithersburg, USA
}

\begin{abstract}
For the measurements of radiance temperatures in the range from $150{ }^{\circ} \mathrm{C}$ to $1000{ }^{\circ} \mathrm{C}$, low uncertainties in the temperature measurements can be achieved by using near-infrared InGaAs radiation thermometers. We describe the design and construction of the NIST near-infrared radiation thermometer (NIRT) which is optimized for low size-of-source effect and noiseequivalent temperatures. The NIRT utilizes a $50 \mathrm{~mm}$ diameter achromatic objective lens with low scatter which images a $4.5 \mathrm{~mm}$ diameter spot at a distance of $50 \mathrm{~cm}$ away from the objective in an on-axis design. A Lyot stop is implemented in the design with the aperture stop placed after the field stop resulting in a collection $\mathrm{f} / \#=12$. A $3 \mathrm{~mm}$ diameter InGaAs detector is cooled to $85^{\circ} \mathrm{C}$ using a 4-stage thermoelectric cooler to obtain high-shunt resistance for linear, low-noise operation at high trans-impedance amplifier gains. For thermal and structural stability, the optical components are placed on four $15 \mathrm{~mm}$ diameter graphite-epoxy rods making the optical throughput stable. Optical ray tracing using a commercial program was used to determine the Strehl ratio and other imaging parameters. We discuss a possible approach for detector-based temperature scale in this range which could result in $10 \mathrm{mK}(k=2)$ thermodynamic temperature uncertainties at the In-point.
\end{abstract}

KEY WORDS: radiance temperature, ITS-90, thermodynamic temperature, size-of-source effect, near-infrared radiation thermometer, noise-equivalent temperature difference 


\section{INTRODUCTION}

Accurate determinations of radiance temperatures from $150{ }^{\circ} \mathrm{C}$ to $1000{ }^{\circ} \mathrm{C}$ are important for calibration of radiation thermometers which are used in turn for process control or used to transfer the calibration to other radiation thermometers. In this temperature range, one can establish a scale on radiation thermometers from the International Temperature Scale of 1990 (ITS-90) using the ITS-90 fixed-point blackbodies (FPBBs) from the In-point to the Ag-point and applying an interpolation functional fit or by the use of a variable-temperature blackbody (VTBB) whose temperatures are determined using contact platinum resistance thermometers (PRT) [1]. In both approaches, the FPBB or the VTBB emissivity has to be measured or modeled so that the lowest uncertainties in the transfer to the radiation thermometer can be achieved. International comparisons of scales in this temperature range has revealed that the lowest uncertainties in radiance temperatures can be achieved using the fixed-point interpolation technique instead of using a variable-temperature blackbody [2]. The lower uncertainties by utilizing the fixed-points are possible since the temperature is well known with high spatial uniformity of the cavity. Variable-temperature blackbodies are expected to have greater issues with achieving uniform temperature distributions within the cavity.

Although the method of calibrations using fixed-points is well established, commercial radiation thermometers which are temporally stable and able to view the small cavity openings of the FPBBs are not readily available. Furthermore, custom InGaAs radiation thermometers constructed by the national measurement institutes have conflicting designs $[3,4]$. Although reduction of size-of-source effect is a critical design feature due to the comparison of small-area 
fixed point blackbody cavities to large variable-temperature blackbody openings, implementation of the Lyot stop has been lacking.

In this paper, we describe a generalized design for an InGaAs-based radiation thermometer (RT) capable of measurements from the In-point to the Ag-point. The radiation thermometer also incorporates a Lyot stop while utilizing a low-scatter objective lens. The construction utilizes carbon-epoxy rods for structural stability with temporal stability of the responsivity increased by the use of room temperature-stabilized filter and cooled detector. The detector is contained in a hermetically sealed package to decrease the detector temperature in order to increase the shunt resistance for low-noise operations. It is hoped that instrumental designs discussed in this paper can be adopted in future radiation thermometer designs to improve the performance of transfer standard radiation thermometers.

\section{RADIATION THERMOMETER CHARACTERIZATION}

\subsection{Physical Design}

The schematic of the radiation thermometer is shown in Fig. 1. The internal elements are mounted on four 15 mm diameter graphite-epoxy rods for structural stability. The graphiteepoxy rods have a coefficient of thermal expansion (CTE) of $-0.5 \times 10^{-6} \mathrm{~mm} / \mathrm{mm}$ which is smaller than that of fused silica [5]. The rods are attached to the front plate holding the objective lens and the various separation between the optical elements can be smoothly adjusted by sliding the through rods. The angled field stop is constructed from polished stainless steel with a beveled $3 \mathrm{~mm}$ diameter hole. The field stop angled to reduce the back reflection of the image onto the objective lens thus reducing the SSE. The specular reflection from the field stop is directed to a flat mirror for viewing through the telescope objective. The radiation through the field stop is 
collimated by the $70 \mathrm{~mm}$ focal length achromat and the plane of the objective lens is focussed onto the Lyot stop. The presence of the Lyot stop is critical in reducing the SSE.

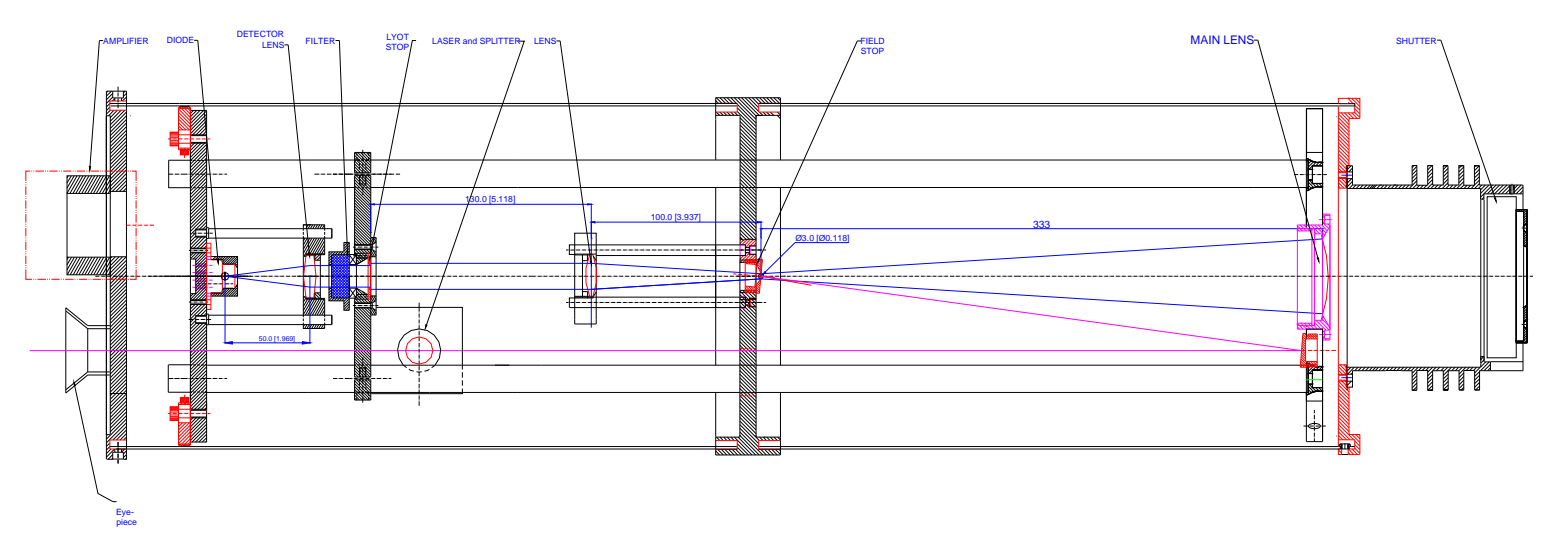

Figure 1. The schematic of the InGaAs-based radiation thermometer. The outer diameter of the main body is $20.3 \mathrm{~cm}$ with a total length of $75 \mathrm{~cm}$ excluding the front baffle.

The room-temperature stabilized interference filter is constructed using hard, ion-assisted deposited layers at a center wavelength of $1550 \mathrm{~nm}$. The full-width at half-maximum bandpass of the filter is $100 \mathrm{~nm}$. Due to the 4-stage thermoelectric cooler which limits the maximum size of the regular InGaAs detector, a $3 \mathrm{~mm}$ diameter detector is used. The detector lens focusses the $3 \mathrm{~mm}$ diameter object at the field stop onto a $1.5 \mathrm{~mm}$ diameter region on the detector, leading to an underfilled detector.

The outer case of the radiation thermometer is floating from the optical elements which are attached to the graphite rods and is only fixed at the middle supporting plate. The case is constructed using $1.83 \mathrm{~mm}$ thick anodized aluminum tubing. The use of the al tube facilitates rapid thermal equilibrium between the laboratory temperature and the internal temperatures. The 
heat-sink plate for the InGaAs detector is additional thermally connected to the external tubing with copper-braided contacts which work to dissipate the heat generated from the TE cooler. We are able to achieve $-85^{\circ} \mathrm{C}$ with only passive cooling without the use of fans. Additional information on the construction of the radiation thermometer is given in Table 1.

Table 1. The design parameters of the InGaAs radiation thermometer (RT).

\begin{tabular}{|c|c|}
\hline Object Distance & $50 \mathrm{~cm}$ \\
\hline Image Distance & $33.3 \mathrm{~cm}$ \\
\hline Objective Focal Length & $20 \mathrm{~cm}$ \\
\hline Field Stop Diameter & $3.0 \mathrm{~mm}$ \\
\hline Target Diameter & $4.5 \mathrm{~mm}$ \\
\hline $\mathrm{f} / \#$ & 12 \\
\hline Lyot Stop Diameter & $8 \mathrm{~mm}$ \\
\hline Detector Type & $\mathrm{InGaAs}$ \\
\hline Detector Diameter & $3 \mathrm{~mm}$ \\
\hline Detector Temperature & $-85^{\circ} \mathrm{C}$ \\
\hline Filter Center Wavelength & $1550 \mathrm{~nm}$ \\
\hline Filter FWHM bandpass & $100 \mathrm{~nm}$ \\
\hline Filter Temperature & $22^{\circ} \mathrm{C}$ \\
\hline Collimator Lens Focal Length & $70 \mathrm{~mm}$ \\
\hline Detector Lens Focal Length & $35 \mathrm{~mm}$ \\
\hline Image Diameter on Detector & $1.5 \mathrm{~mm}$ \\
\hline RT Diameter & $20.3 \mathrm{~cm}$ \\
\hline RT Length & $76.2 \mathrm{~cm}$ \\
\hline
\end{tabular}

\subsection{Detector optimization}

The most important aspect of radiation thermometer design is the selection of the detector to be used in the radiation thermometer. Due to the linearity and the wavelength region of the spectral power responsivity, regular InGaAs diode was chosen. Since the small target diameter and the $\mathrm{f} / \#$ limits the throughput of the RT, the preamplifier should have a dynamic range from $10^{10} \mathrm{~V} / \mathrm{A}$ to $10^{5} \mathrm{~V} / \mathrm{A}$ for measurements of blackbodies from the In-point to the Ag-point. In a transimpedance amplifier circuit, the shunt resistance of the input diode should have a value greater or 
equal to the feedback resistance for linear, low noise amplification of the current. The measurement of the shunt resistances of both $1 \mathrm{~mm}$ diameter and $3 \mathrm{~mm}$ diameter InGaAs diodes in Fig. 2 shows that the shunt resistances have a semi-logarithmic relationship to temperature.

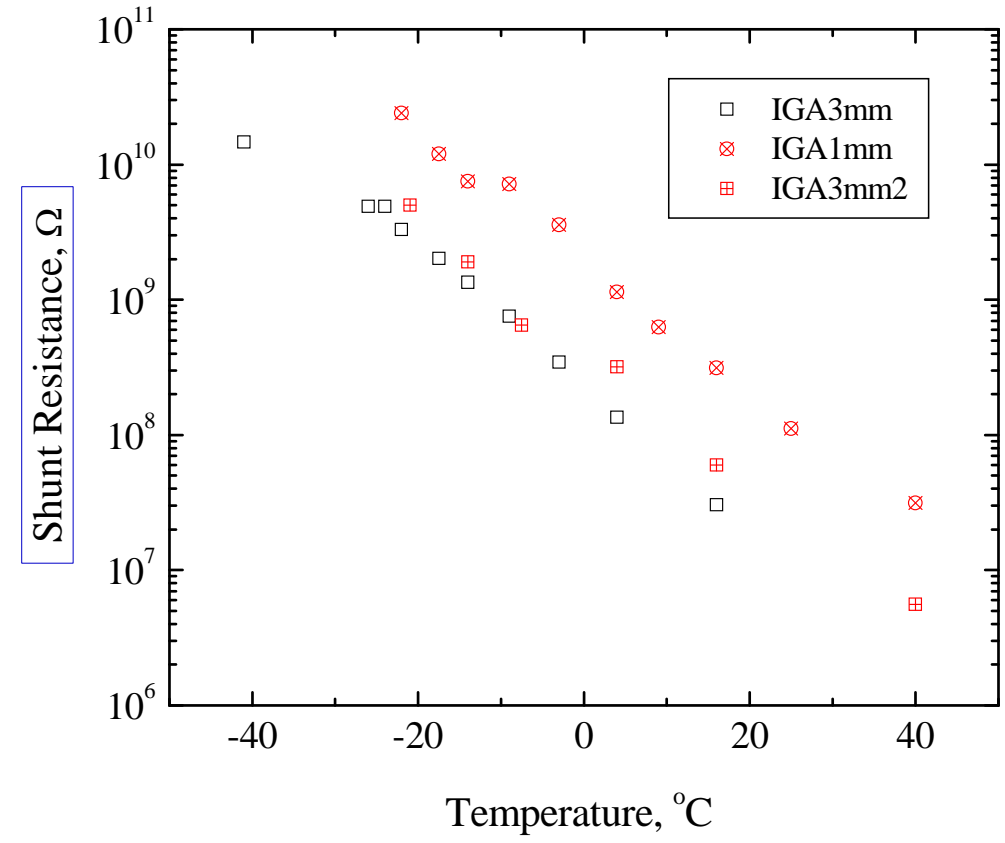

Fig. 2. The temperature-dependent shunt resistances of $1 \mathrm{~mm}$ and $3 \mathrm{~mm}$ diameter InGaAs diodes. The shunt resistance of the diode should be comparable to the feedback resistance of the preamplifier circuit for low noise, linear operation.

The shunt resistances also increase as linear function of the detector area as expected for a defects with a uniform surface density. The Fig. 2 shows that for $1 \mathrm{~mm}$ diameter diodes, shunt resistances of $>10^{10} \Omega$ are achieved at detector temperatures of $-20{ }^{\circ} \mathrm{C}$ which is possible with 2stage TE cooling. For $3 \mathrm{~mm}$ diameter diodes, detector temperatures of at least $-40{ }^{\circ} \mathrm{C}$ are needed to achieve $>10^{10} \Omega$ shunt resistance. Unless the diode material quality is improved, larger diode are expected to require greater cooling to obtain the same shunt resistances. 


\subsection{Temperature-dependent detector performance}

The importance of the ability to cool the detector to below ambient temperature is shown by

Fig. 3. The equivalent dark current (calculated from the output offset voltage in dark) and its standard deviation at a preamplifier gain of $10^{10} \mathrm{~V} / \mathrm{A}$ are shown. The standard deviations are measured using 10 measurements at number of power-line cycles (NPLC) of 10 or $0.13 \mathrm{~Hz}$. The equivalent dark current is only weakly dependent on the detector temperature with a decrease from $3 \mathrm{pA}$ to about $1 \mathrm{pA}$ as the detector is cooled from room temperature to $-70{ }^{\circ} \mathrm{C}$. The change in the dark current with decreasing temperature indicates that the stabilizing the detector temperature is important in avoiding dark-current drift, especially at the In point where the net photocurrent is only about $23 \mathrm{pA}$. With cooling of the detector, the standard deviation of the equivalent dark current changes much more, from about 100 fA to 2.4 fA.

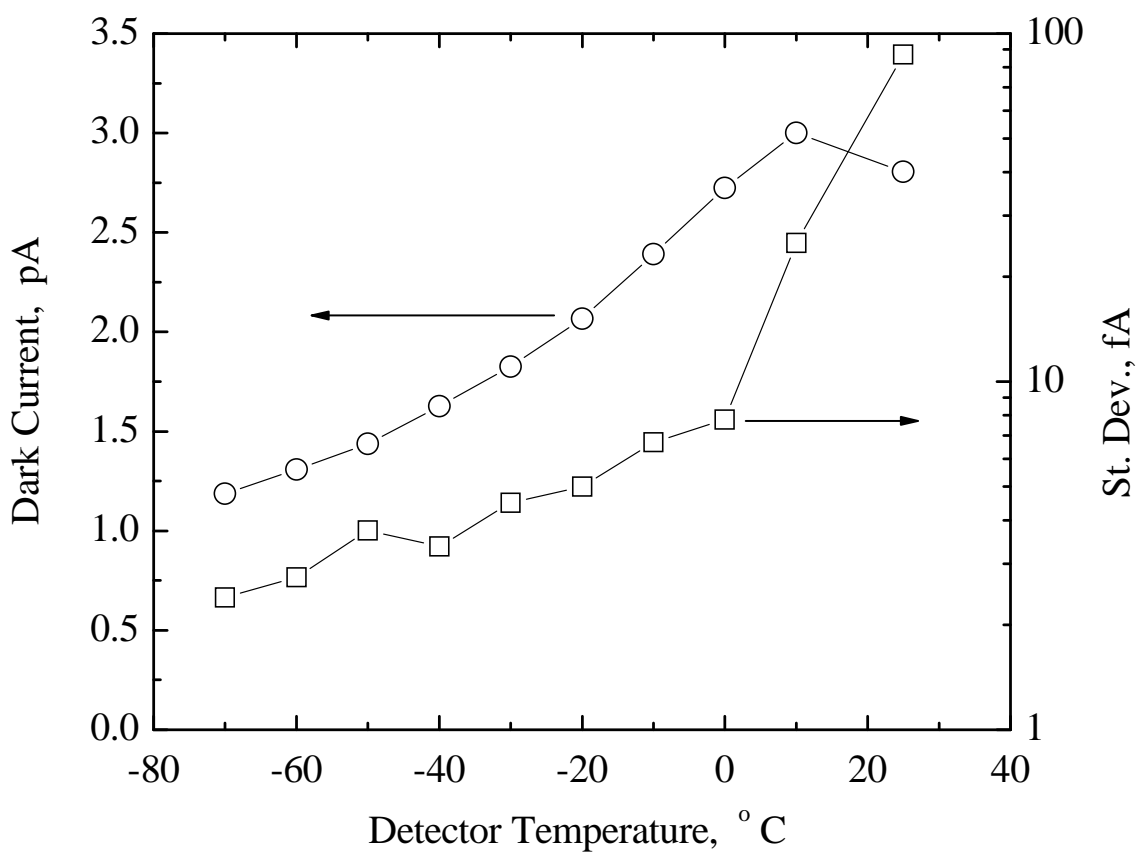

Fig. 3. Equivalent dark current and its standard deviation versus temperature. 
Although the detector was cooled to lower temperatures than $-70^{\circ} \mathrm{C}$, the heat generated from the TE coolers could not be readily dissipated which lead to self heating of the detector. We observed a slow drift of the equivalent dark current when the temperature of the detector was set to lower temperatures which precluded stable measurements of standard deviations of the equivalent dark current. These measurements indicate that close to Si-type performance of few fA noise-equivalent current [6] is possible with 4-stage TE cooling. This low-noise performance is especially important at the In-point where any decrease in the noise-equivalent power directly leads to a decrease in the noise-equivalent temperatures.

\subsection{Size-of-source effect}

Since the radiation thermometer is designed for transfer of the ITS-90 temperatures to other blackbodies, the suppression of the SSE in comparing small fixed-point cavities to larger area variable temperature blackbody cavities is critical. In the design of a radiation thermometer to have small SSE, it is important to use a low-scatter objective lens in addition to the incorporation of a Lyot stop [7]. Although SSE can be changed by removing the objective lens, such drastic steps will result in decrease of the entrance pupil leading to extremely low throughput and inability to measure at low temperatures due to the high noise. Furthermore, the introduction of any element which can lead to further scatter into the field stop should be discouraged such as protective windows or viewing beamsplitters which are directly in the optical path of the radiation. The SSE measured using a $10 \mathrm{~mm}$ diameter central obscuration is shown in Fig. 4. The measurements are performed using a high-power $1 \mathrm{~W}$ LED source as described previously [8]. The LED source has sufficient output in the near infrared wavelength region for these measurements. The low SSE measured at $50 \mathrm{~mm}$ source diameter indicates that our design is effective at suppressing internal scatter. The comparison of the SSE in terms of temperature 
corrections are shown in Table 2 with SSE of $7 \times 10^{-5}$ and $1 \times 10^{-3}$ converted to their temperature corrections at the respective fixed points. If the SSE can be reduced to $7 \times 10^{-5}$ then the corrections can be included as terms in the total uncertainty budget, while larger SSE require systematic correction terms as shown in Table 2.

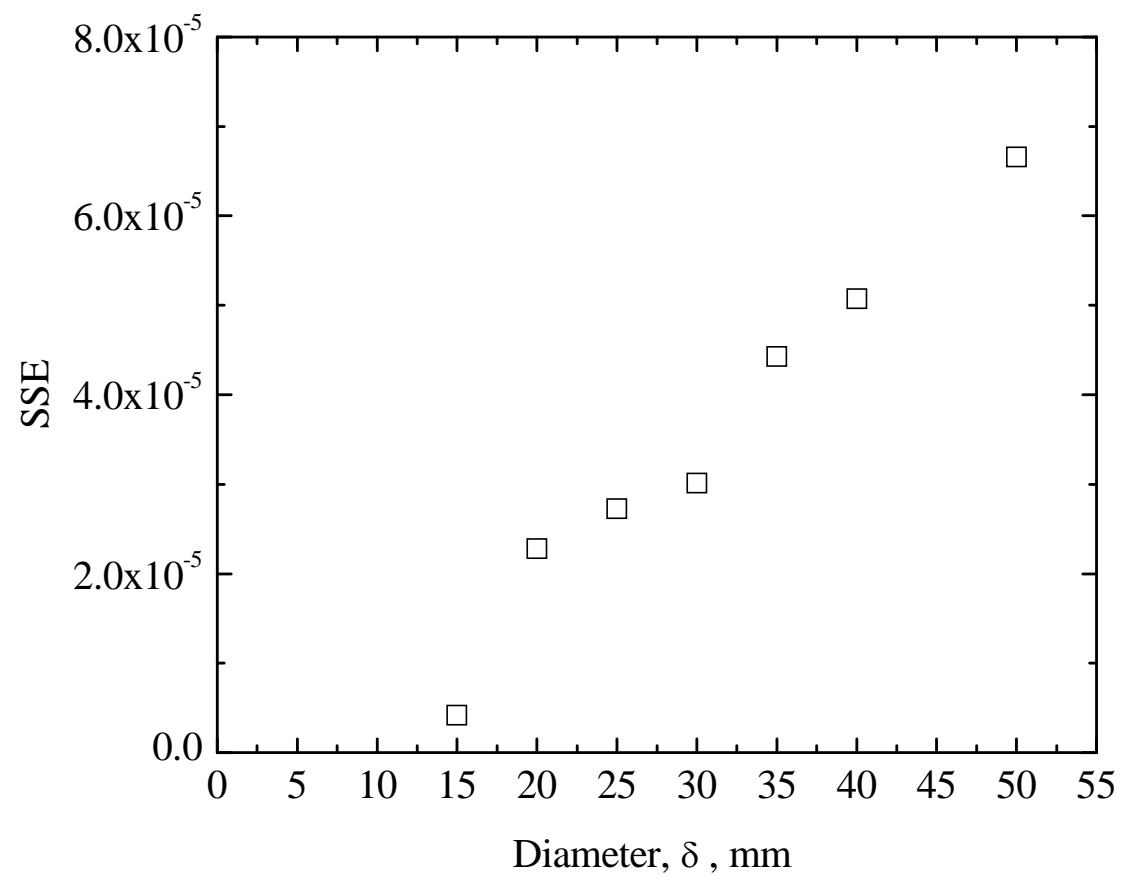

Fig. 4. The SSE measured using a central obscuration of $10 \mathrm{~mm}$ diameter with the outer diameter opened up to $50 \mathrm{~mm}$. The use of low scatter objective with a tilted field stop along with a Lyot stop results in low SSE. 
Table 2. The SSE $=7 \times 10^{-5}$ and $1 \times 10^{-3}$ converted to temperature corrections. The smaller SSE corrections can be folded into the uncertainty budget while the larger SSE requires a correction factor.

\begin{tabular}{|c|c|c|c|}
\hline Fixed Point & Temperature [ ${ }^{\circ} \mathbf{C}$ ] & $\begin{array}{c}\text { SSE }=\mathbf{7 x 1 0} \\
{[\mathbf{~ K ~ ] ~}}\end{array}$ & $\begin{array}{c}\text { SSE }=\mathbf{1 x 1 0}^{-3} \\
{[\mathbf{~ K ~ ] ~}}\end{array}$ \\
\hline In & 156.5985 & 0.001 & 0.020 \\
\hline Sn & 231.928 & 0.002 & 0.027 \\
\hline $\mathrm{Zn}$ & 419.527 & 0.004 & 0.052 \\
\hline $\mathrm{Al}$ & 660.323 & 0.007 & 0.094 \\
\hline $\mathrm{Ag}$ & 961.78 & 0.011 & 0.164 \\
\hline
\end{tabular}

\subsection{Optical Performance}

The InGaAs radiation thermometer utilizes a commercial $50 \mathrm{~mm}$ diameter achromat with a focal length of $200 \mathrm{~mm}$ with nearly 1:1 finite-conjugate imaging. Since commercial achromatic lenses are designed for infinite-conjugate imaging, the imaging performance suffers as shown by the Strehl ratio in Table 3. The Strehl ratio for a perfect point source imaging will be 1.0, and the ratio will approach this value as the imaging performance improves. The Strehl ratio increases as the wavelength increases which indicates that imaging performance is better at $1550 \mathrm{~nm}$ than at $500 \mathrm{~nm}$. Furthermore, achromatic lenses will generally have two wavelengths were the images distances will be equal for a set object distance with the chromatic focal shift depending on the lens material and thickness. Since the radiation thermometer is designed to transfer a temperature scale from one blackbody to another without any desired spatial structure within the cavity, the need for imaging performance is much reduced. Although a single achromat will have much worse imaging performance than that of multi-element photographic-type lenses, the single, cemented achromat will have reduced scatter and consequently much lower SSE than that of multi-element lenses. All these optical performance metrics can be modeled using a commercial 
modeling program. Table 3 shows that although the alignment of the radiation thermometer can be performed in the visible wavelength, the object distances to have the same image distance at $1550 \mathrm{~nm}$ and at $500 \mathrm{~nm}$ differ by $25 \mathrm{~mm}$. Since the internal focus is difficult to adjust, the object distance can be offset by this difference due to chromatic aberrations.

Table 3. The optical parameters of the $200 \mathrm{~mm}$ focal length achromatic objective lens. Moving the object distance for $1550 \mathrm{~nm}$ results comparable image distance to the $500 \mathrm{~nm}$ wavelength. The Strehl ratio also improves at $1550 \mathrm{~nm}$ as compared to $500 \mathrm{~nm}$ indicating better imaging performance.

\begin{tabular}{|c|c|c|c|}
\hline Wavelength [ nm ] & $\begin{array}{c}\text { Object Distance } \\
{[\mathbf{~ m m}]}\end{array}$ & $\begin{array}{c}\text { Image Distance } \\
{[\mathbf{~ m m}]}\end{array}$ & $\begin{array}{c}\text { Strehl } \\
\text { Ratio }\end{array}$ \\
\hline 500 & 500 & 323.91 & 0.0738 \\
\hline 1550 & 500 & 334.49 & 0.213 \\
\hline 1550 & 525 & 324 & 0.238 \\
\hline
\end{tabular}

\subsection{Preamplifier Gain Corrections}

Since the InGaAs radiation thermometer should have a linear signal versus radiance of the blackbody, the preamplifier gain settings need to be normalized against each other. In this radiation thermometer, precision, metal-film-on-ceramic resistors are used in the trans-impedance amplifier. The resistors are chosen to have low temperature variation of resistance. The preamplifier was attached to a Si detector with high shunt resistance and placed in front of a broad-band white LED source. The $1 \mathrm{~W}$, high-power LED source was chosen for its temporally stable output so that the gain ratios can be determined with low uncertainty. The optical power of the LED source incident on the detector was varied by using a combination of diffusers, variable 
source apertures or by changing the distance between the source and the detector. The LED source, for the most stable operations, was left on for about 24 hours to reach a thermal equilibrium. The ratios of gain factors shown in Table 4 indicate that at the lower gain settings, the ratios between two gains different by 10 are at this value within the uncertainties of the measurements and the resistances. The greatest deviation is present at gain factors of $10^{10} \mathrm{~V} / \mathrm{A}$ and $10^{9} \mathrm{~V} / \mathrm{A}$ where the resistors used in the preamplifer deviate more from their assigned values.

Table 4. The gain ratios of the preamplifier measured using a $1 \mathrm{~W}$ white LED source with a Si detector.

\begin{tabular}{|c|c|c|}
\hline $\begin{array}{c}\text { Gains } \\
\text { Compared }\end{array}$ & Gain Ratios & $\begin{array}{c}\text { Measurement } \\
\text { Uncertainty } \\
(\boldsymbol{k}=\mathbf{2})\end{array}$ \\
\hline $10^{6} / 10^{5}$ & 9.998 & 0.002 \\
\hline $10^{7} / 10^{6}$ & 10.001 & 0.002 \\
\hline $10^{8} / 10^{7}$ & 10.000 & 0.002 \\
\hline $10^{8} / 10^{9}$ & 10.000 & 0.002 \\
\hline $10^{9} / 10^{10}$ & 9.957 & 0.002 \\
\hline
\end{tabular}

\section{BLACKBODY MEASUREMENTS}

The InGaAs radiation thermometer was placed in front of a variable-temperature blackbody (VTBB) which was set at the respective ITS-90 fixed-point temperatures. The measurements on actual fixed-points could not be performed in time for this submission. These measurements are used to determine the gain settings of the preamplifier, the throughput of the radiation thermometer and to measure the noise-equivalent temperature difference at the fixed-point temperatures. Work is planned to measure the freezing-points directly using the InGaAs radiation thermometer. The measured NETD could be affected by the temporal stability of the 
VTBB, and our measurements shown in Table 5 give the upper value of this parameter. The photocurrent at the In-point is comparable to InGaAs radiation thermometers developed at other national measurement institutes indicating that the throughputs are comparable. The lower NETD exhibited by the NIST design is due to the optimization of the preamplifier and detector, especially by 4-stage cooling of the detector and the resultant decrease in the noise-equivalent photocurrent seen in Fig. 5. Once the NIST InGaAs radiation thermometer is calibrated using the ITS-90 fixed points, a modified 3-parameter Planck equation will be used for the interpolation.

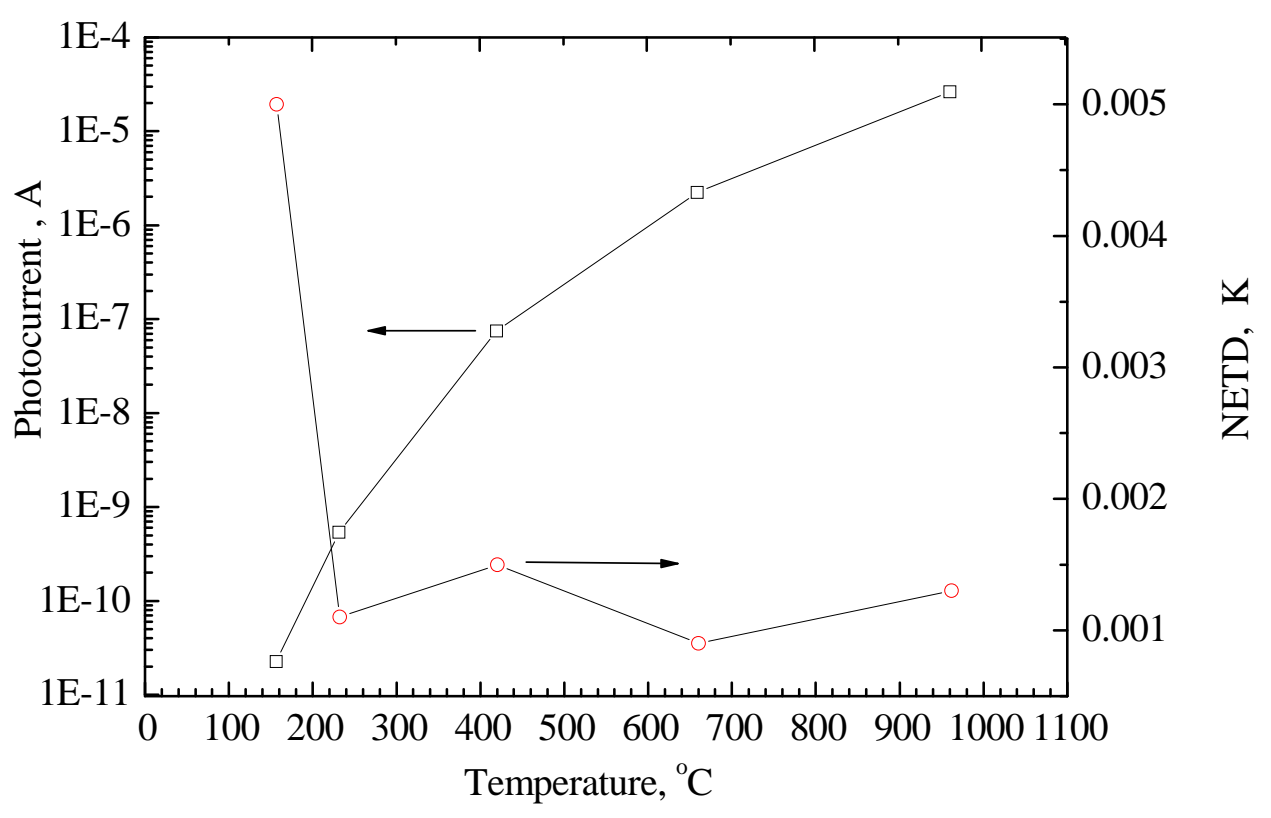

Fig. 5. The measured photocurrents of the InGaAs radiation thermometer at the various fixed point temperatures. For convenience, the measurements were performed using a variabletemperature blackbody set to the ITS-90 temperatures. The NETD was determined from the standard deviation of the measurements. 
Table 5. The gains and the photocurrents at the fixed-point temperatures measured using a variable-temperature blackbody set at the respective temperatures.

\begin{tabular}{|c|c|c|c|c|}
\hline Fixed Point & Temperature $\left[{ }^{\circ} \mathbf{C}\right.$ ] & Gain [ V/A ] & Signal [ A ] & NETD [ ${ }^{\circ} \mathbf{C}$ ] \\
\hline In & 156.5985 & $10^{10}$ & $2.26251 \mathrm{E}-11$ & 0.0050 \\
\hline $\mathrm{Sn}$ & 231.928 & $10^{9}$ & $5.36748 \mathrm{E}-10$ & 0.0011 \\
\hline $\mathrm{Zn}$ & 419.527 & $10^{7}$ & $7.45110 \mathrm{E}-08$ & 0.0015 \\
\hline $\mathrm{Al}$ & 660.323 & $10^{6}$ & $2.23358 \mathrm{E}-06$ & 0.0009 \\
\hline $\mathrm{Ag}$ & 961.78 & $10^{5}$ & $2.63766 \mathrm{E}-05$ & 0.0013 \\
\hline
\end{tabular}

We have also tried to use alternating-current (AC) mode measurements using a $10.1 \mathrm{~Hz}$ tuningfork chopper placed directly in front of the field stop with a lock-in detection system. With the AC mode, lower blackbody temperatures, to $80^{\circ} \mathrm{C}$, could be measured, although with the added complexities of using the lock-in amplifier. Further work is pursued to make low uncertainty AC mode measurements.

\section{DISCUSSION AND FUTURE WORK}

Thus far, radiation thermometers with InGaAs detectors have only been used to transfer the source-based ITS-90 scale to other sources or blackbodies. Due to the developments of laserbased calibration facilities and low-noise and high linearities of InGaAs detectors, the possibility of detector-based thermodynamic temperature determinations are being explored. The detectorbased calibration of the InGaAs radiation thermometer would proceed in a similar way as with Sidiode based radiation thermometer [9]. A transfer radiometer would need to be calibrated for spectral power responsivity by comparison to the cryogenic electrical-substitution radiometer using infrared lasers, and thus the optical power scale would be derived from the electrical power. The transfer radiometer would need a precision aperture and known spatial uniformity of responsivity so that the power responsivity can be converted to spectral irradiance responsivity. 
The conversion to irradiance responsivity can be performed with detectors in a trap configuration or with detectors which are attached to integrating sphere inputs. With a laser-irradiated sphere along with the calibrated transfer radiometer, the InGaAs radiation thermometer can be calibrated for absolute detector-based radiance responsivity. Since Spectralon and other sintered or packed polytetrafluoroethylene (PTFE) materials have a high reflectance at $1.55 \mu \mathrm{m}$, integrating spheres have high throughput and spatial uniformity. It is anticipated that such a detector-based radiation thermometer could be calibrated to $0.05 \%(k=2)$ [10]. The resulting thermodynamic temperature uncertainties are shown in Table 6. Although these uncertainties are much larger than those obtained using gas thermometry, the uncertainties are lower than those achieved by noise-thermometry.

Table 6. The thermodynamic temperature uncertainties for total uncertainty of $0.05 \%(k=2)$ at the respective fixed-point temperatures.

\begin{tabular}{|c|c|c|}
\hline Fixed Point & Temperature [ ${ }^{\circ} \mathbf{C}$ ] & $\begin{array}{c}\text { Total Uncertainty }=\mathbf{0 . 0 5 \%} \\
(\boldsymbol{k}=\mathbf{2})[\mathbf{K}]\end{array}$ \\
\hline In & 156.5985 & 0.0099 \\
\hline Sn & 231.928 & 0.0137 \\
\hline Zn & 419.527 & 0.0258 \\
\hline Al & 660.323 & 0.0469 \\
\hline Ag & 961.78 & 0.0821 \\
\hline
\end{tabular}

\section{CONCLUSIONS}

We described a noise-optimized InGaAs radiation thermometer operating at $1550 \mathrm{~nm}$ which is capable of $<5 \mathrm{mK} \mathrm{NETD} \mathrm{at} 157^{\circ} \mathrm{C}$. The SSE has also been reduced to $<7 \times 10^{-5}$ at $50 \mathrm{~mm}$ which leads to low uncertainties when comparing fixed-point blackbodies to other larger-area 
variable-temperature blackbodies. These results are due to the implementation of 4-stage cooling of the diode resulting in high shunt resistances along with a Lyot stop which acts as an aperture stop. A stable chassis design is shown using graphite-epoxy rods for long-term stability of radiance responsivity. The preamplifier gain ratios are measured for use over the 5-decade dynamic range required of the radiation thermometer as the blackbody temperatures range from $150{ }^{\circ} \mathrm{C}$ to $1000{ }^{\circ} \mathrm{C}$. In addition to the use of this radiation thermometer as an ITS-90 transfer radiation thermometer, we outline a possible approach to directly measuring thermodynamic temperature using an absolute detector-based radiance responsivity calibration.

\section{REFERENCES}

${ }^{1}$ T. Ricolfi, M. Battuello, F. Girard, G. Machin, H. McEvoy, S. Ugur, A. Diril, Meas. Sci. Technol. 13, $2090(2002)$

${ }^{2}$ F.Girard, T. Ricolfi, in Proceedings TEMPMEKO 2004, 9th International Symposium on Temperature and Thermal Measurements in Industry and Science, ed. by D. Zvizdi'c (FSB/LPM, Zagreb, Croatia, 2005), pp. 827-832

${ }^{3}$ T. Ricolfi, F. Girard, in Proceedings of TEMPMEKO '99, 7th International Symposium on Temperature and Thermal Measurements in Industry and Science, ed. by J.F. Dubbeldam, M.J. de Groot (Edauw Johannissen bv, Delft, 1999), pp. 593-598

${ }^{4}$ B. Gutschwager, S. Schiller, J. Hartmann, J. Hollandt, in Proceedings TEMPMEKO 2004, 9th International Symposium on Temperature and Thermal Measurements in Industry and Science, ed. by D. Zvizdi'c (FSB/LPM, Zagreb, Croatia, 2005), pp. 605-610

${ }^{5}$ G. Romeo, G. Frulla, J. Compos. Mater. 29, 751 (1995)

${ }^{6}$ G.P. Eppeldauer, J. Res. Natl. Bur. Stand. (U.S.) 105, 29 (2000)

${ }^{7}$ H.W. Yoon, D.W. Allen, R.D. Saunders, Metrologia 42, 89 (2005)

${ }^{8}$ D.W. Allen, G. Dezsi, H.W. Yoon, in Proceedings TEMPMEKO 2004, 9th International Symposium on Temperature and Thermal Measurements in Industry and Science, ed. by D. Zvizdi'c (FSB/LPM, Zagreb, Croatia, 2005), pp. 817-822

${ }^{9}$ H.W. Yoon, D.W. Allen, C.E. Gibson, M. Litorja, R.D. Saunders, S.W. Brown, G.P. Eppeldauer, K.R. Lykke, Appl. Optics 46, 2870 (2007)

${ }^{10}$ S.W. Brown, G.P. Eppeldauer, K.R. Lykke, Appl. Optics 45, 8218 (2006) 


\title{
Linearity of InGaAs photodiodes
}

\author{
H W Yoon, J J Butler, T C Larason and G P Eppeldauer
}

Optical Technology Division, NIST, Gaithersburg, MD 20899-8441, USA

Published 7 February 2003

Online at stacks.iop.org/Met/40/S154

\begin{abstract}
In radiometry or pyrometry, radiometers are often used to assign the spectral radiance or radiance temperatures of sources using ratios of signals which can differ by several decades. For performing ratios between such sources with low uncertainties, the linearity of the detectors used in the transfer radiometers needs to be characterized. The linearity of InGaAs photodiodes has been studied using the flux-addition method using a broadband infrared source with a visible-blocking filter. Using this technique, $18 \mathrm{InGaAs}$ photodiodes from four different vendors were studied without spectral filtering in a broad wavelength region from $900 \mathrm{~nm}$ to $1700 \mathrm{~nm}$ with the diodes underfilled by the incident flux. The linearity of InGaAs photodiodes was determined within the range of photocurrents from $10^{-8} \mathrm{~A}$ to $10^{-4} \mathrm{~A}$. All the InGaAs photodiodes demonstrated linearity from $10^{-7} \mathrm{~A}$ to $10^{-4} \mathrm{~A}$ within the expanded uncertainties of $0.08 \%(k=2)$. The uncertainty in the linearity measurement below $10^{-8} \mathrm{~A}$ is increased due to the increased noise in the photocurrent arising from the feedback resistance of the transimpedance amplifier being greater than the shunt resistance of the photodiode.
\end{abstract}

\section{Introduction}

InGaAs photodiodes are critical components in optical networks, and as the manufacturing process for InGaAs improves, they are being increasingly used as detectors in thermal imaging, pyrometry [1] and spectroradiometry [2]. Previously, the electronic characteristics of InGaAs and Ge photodiodes using current preamplifiers were compared, and the InGaAs photodiodes were found to have much a smaller temperature dependence and lower noise floor than Ge photodiodes [3]. Although InGaAs photodiodes are widely used as receivers in optical networks, they have not been extensively evaluated for use in spectroradiometry. Since the detector could be used to assign the spectral radiances of vastly different sources using radiance ratios or signal ratios from one source to another, the linearity of the detectors must be quantified for use in spectroradiometry. In addition, these particular photodiodes have been used as working standards in the key comparison K2.a of the Consultative Committee on Photometry and Radiometry for the intercomparison of spectral power responsivity from $900 \mathrm{~nm}$ to $1600 \mathrm{~nm}$ [4]. Since each laboratory participating in the intercomparison could use different optical power levels to assign the spectral responsivity, the determination of the linearity of these InGaAs photodiodes is critical. To our knowledge, there has not been a study of InGaAs photodiode linearity although the linearity of these detectors is an important issue in many applications. The different InGaAs diodes studied and their electrical characterizations are listed in table 1 .

The linearity of detectors can be measured with several different techniques. A common technique is to insert a filter into the optical path at different optical power levels. The nonlinearity is measured as a change in the filter-in to the filter-out signal ratios. Another well-known method is the beam-flux addition using double apertures. In this study, we use the combination of these two methods with the Beamconjoiner III apparatus developed at the National Institute of Standards and Technology [5].

Although the linearity of photodiodes can also be dependent on the wavelength of the measurements, such wavelength dependences were not investigated during this study. The InGaAs photodiode was illuminated using a broadband source, an FEL lamp, and only a single visible blocking filter was placed in front of the photodiode. All the photodiodes studied had active areas of $5 \mathrm{~mm}$ diameter, and the incident flux was made to underfill the diode. Wavelengthdependent non-linearities are possible, especially near the band-gap wavelength of the InGaAs around $1.7 \mu \mathrm{m}$.

The linearity of InGaAs photodiodes was determined to an expanded uncertainty of $0.08 \%(k=2)$ within the range of photocurrents from $10^{-8} \mathrm{~A}$ to $10^{-4} \mathrm{~A}$. Below $10^{-8} \mathrm{~A}$, the uncertainty in the linearity measurement is larger due to the increased noise in the photocurrent arising from the feedback resistance of the transimpedance amplifier being greater than 
Table 1. The shunt resistances and the shunt capacitances of all the tested photodiodes are shown. The shunt capacitance of the NIST 14 photodiode could not be measured.

\begin{tabular}{|c|c|c|c|c|}
\hline Detector & Manufacturer and model & $\begin{array}{l}\text { Serial } \\
\text { number }\end{array}$ & $\begin{array}{l}\text { Shunt } \\
\text { resistance/ } \\
\mathrm{M} \Omega\end{array}$ & $\begin{array}{l}\text { Shunt } \\
\text { capacitance/ } \\
\mathrm{nF}\end{array}$ \\
\hline NIST 01 & Fermionics & 123 & 17.50 & 1.41 \\
\hline NIST 02 & Fermionics & 124 & 12.80 & 1.43 \\
\hline NIST 03 & Fermionics & 125 & 15.40 & 1.42 \\
\hline NIST 04 & GPD GAP5000, D/C 9812 & 1 & 1.78 & 1.33 \\
\hline NIST 05 & GPD GAP5000, D/C 9812 & 2 & 2 & 1.1 \\
\hline NIST 06 & GPD GAP5000, D/C 9812 & 3 & 1.9 & 1.14 \\
\hline NIST 07 & GPD GAP5000, D/C 9812 & 4 & 1.8 & 1.2 \\
\hline NIST 08 & EG\&G Optoelec. C30723G & 6 & 3.75 & 0.826 \\
\hline NIST 09 & EG\&G Optoelec. C30723G & 9 & 5.00 & 1.50 \\
\hline NIST 10 & EG\&G Optoelec. C30723G & 11 & 5.71 & 1.58 \\
\hline NIST 11 & EG\&G Optoelec. C30723G & 13 & 4.44 & 1.67 \\
\hline NIST 12 & TDC P/N: 35PD5M-TO & 2 & 5 & 1.8 \\
\hline NIST 13 & TDC P/N: 35PD5M-TO & 3 & 2.5 & 1.87 \\
\hline NIST 14 & TDC P/N: 35PD5M-TO & 4 & 0.00125 & - \\
\hline NIST 15 & TDC P/N: 35PD5M-TO & 5 & 5 & 1.85 \\
\hline NIST 16 & GPD GAP5000, D/C 9849 & 11 & 1.80 & 1.27 \\
\hline NIST 17 & GPD GAP5000, D/C 9849 & 12 & 0.58 & 1.37 \\
\hline NIST 18 & GPD GAP5000, D/C 9841 & 13 & 1.40 & 1.44 \\
\hline
\end{tabular}

the shunt resistance of the photodiode. The shunt resistances and the junction capacitances of the photodiodes have also been measured, and the increased noise at the low power levels is correlated with the differences in the respective shunt resistances.

\section{Experimental method}

The linearity of the InGaAs photodiodes was determined using the Beamconjoiner III apparatus. Our method utilizes a visible-blocking filter (Hoya R72) ${ }^{1}$ and reflective neutral density filters to provide 40 different flux values, resulting in 120 different combinations to attenuate the light source, a current-stabilized FEL lamp. The incident light on the InGaAs diodes is broadband infrared with the visible range filtered. The neutral density filters were constructed using metallized quartz substrates for transmission from $0.2 \mu \mathrm{m}$ to $2.5 \mu \mathrm{m}$. The optical diagram of the Beamconjoiner III is shown in figure 1 . The incident flux is introduced into the opening of the lighttight box and is split into two directions by the beamsplitter 1 . Each of the filter wheels 1 and 2 has four different reflective neutral density filters with circular openings of $4 \mathrm{~cm}$ diameter, and the filter wheel 3 has five filters. Combined with the blocked value for each of the filter wheels, a total of $(5 \times 5 \times 6)$ 150 different combinations can be formed. The filter wheel 3 acts to reduce the total level of the combined flux, and the dynamic range in the optical flux of the highest level to the lowest level is about $500: 1$. The rotation of the filter wheels is controlled using three separate stepper motors, and the rotational positions of the filter wheels are verified with a check of the home position optical switch each time the stepper motor is rotated. The change in the optical flux is performed in the collimated beam before the imaging of the flux onto the detector by the spherical mirror.

\footnotetext{
1 Certain commercial equipment, instruments or materials are identified in this paper to foster understanding. Such identification does not imply recommendation or endorsement by the National Institute of Standards and Technology, nor does it imply that the material or equipment are necessarily the best available for the purpose.
}

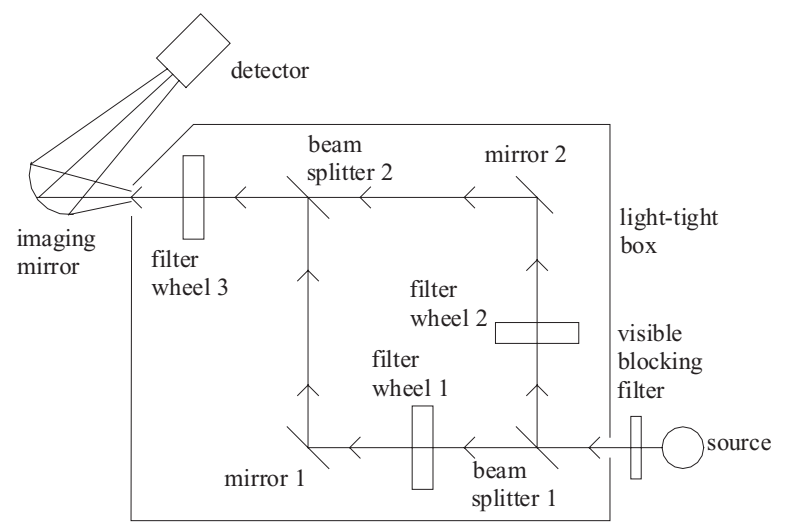

Figure 1. Optical diagram of the Beamconjoiner III apparatus. The source is split into two directions using the beamsplitter 1 and recombined at the detector using beamsplitter 2 . The combination of the three filter wheels can produce 120 different signal levels using 40 different flux levels. The optical flux is changed in the collimated beam before imaging onto the detector.

In the measurement protocol, each of the filter wheel positions is assigned a distinct value $i, k, j$ corresponding to the positions of the filter wheel 1,2,3, respectively. Each of the 120 different filter combinations is in turn also assigned a distinct number, and the sequence of filter wheel positions for each run is determined using a random number generator prior to each run. Every group of four measurements is followed by a dark measurement, resulting in 30 separate dark measurements for each run. Since each dark signal has a time stamp, any change in the dark signal can be monitored. None of the InGaAs photodiodes discussed in this study showed any evidence of dark signal drift.

Since each run could take up to $1.5 \mathrm{~h}$ or longer depending upon the signal averaging needed for optimal signal-to-noise ratios, the source flux has to be temporally stable. The lamp was stabilized by measuring the voltage drop across a calibrated resistor to determine the current, and a closed-loop 
current stabilization was used, which adjusted the current every $5 \mathrm{~s}$ to the desired value.

Although the power responsivity of the InGaAs photodiodes is temperature sensitive, the photodiodes used in the CCPR K2.a were not operated with temperature stabilization. The temperature was monitored using a thermistor held in contact with the back of the photodiode, and each photodiode was allowed sufficient time to come to temperature equilibrium with the ambient laboratory environment. Temperature changes of the photodiode of few degrees resulted in noisy signal/flux values since the doublebeam flux method requires both the source and the detector to be stable during the 120 different measurements.

\section{Linearity analysis}

Although 150 different signals are possible, only 120 signals are measurements of fluxes from the source. Each signal can be written as a response to the incident flux, or conversely, each flux results in signal as a function of the flux,

$$
\begin{aligned}
& \phi(i, j, k)=\phi(i, j)+\phi(j, k) \\
& \quad=r_{0}+s(i, j, k)+r_{2} s^{2}(i, j, k)+\cdots+r_{n} s^{n}(i, j, k)
\end{aligned}
$$

where $\phi(i, j, k)$ denote the flux, $s(i, j, k)$ is the measured signal at the detector, and $r_{0}, r_{2}, \ldots, r_{n}$ are the coefficients which relate the signal to the flux. The total flux at the detector is a sum of the individual fluxes, $\phi(i, j)$ and $\phi(j, k)$, and flux addition is inherently linear. Since each flux can be an independent flux or sums of independent fluxes, and although 120 signals are measured along with 30 dark signals, only 40 different fluxes exist with 20 fluxes from each of the two paths. Thus equation (1) becomes an over-determined equation with 40 unknown fluxes and one or many unknown coefficients, $r_{0}$, $r_{2}, \ldots, r_{n}$, but with 120 equations. The unknown fluxes are found by setting up a system of linear equations and performing a least-squares fit of the signals for the 40 unknown fluxes and the coefficients.

Due to the complexity of the matrix inversion and the possibility of errors in the convergence, the analysis program was tested using artificial data sets which simulated the experimental results from the Beamconjoiner. Different constant values were used in the simulations, and second-order correction factors were also simulated. The analysis program was able to correctly converge on all of the coefficients in the artificial data sets.

The determination of linearity is measured using the residuals of the fluxes found using the fit to the measured signal corrected by the appropriate coefficients. Initially, only the constant correction, $r_{0}$, is applied and the residuals of the fit,

$$
\phi(i, j, k)-\left[r_{0}+s(i, j, k)\right]
$$

are plotted against the signals and examined for indications of the need for higher order corrections. The linearity of the signals is determined from a plot of the ratio of the measured signal to the calculated flux,

$$
\frac{s(i, j, k)}{\phi(i, j, k)}
$$

and the ratios are fitted using a linear function to determine the uncertainty component of the total signal due to non-linearity.
Since flux is inherently linear, and the signals could be nonlinear, any non-linearity of the signal will be evident from the curvature of the ratios.

\section{Discussion}

A plot of the ratios of the measured signals to the fluxes versus the signals for the NIST 01 photodiode is shown in figure 2. The plot shows signal/flux ratios from three separate runs, each consisting of 120 signal measurements. The measurements were performed using an additional neutral density filter between the filter wheel 3 and the detector to reduce the overall signal such that the wide dynamic range could be spanned from $10^{-9} \mathrm{~A}$ to $10^{-4} \mathrm{~A}$. The symmetry of the residuals above and below the $y=0$ axis in figure 2 indicates the goodness of fit, since any non-linearity would be manifest in deviations from a symmetric distribution. From the plot, the detector is determined to be linear from $1 \times 10^{-8} \mathrm{~A}$ to $1 \times 10^{-4} \mathrm{~A}$ in photocurrent with an uncertainty of $0.08 \%$ $(k=2)$. The uncertainty is determined from the goodness of a linear fit to the values in the same range.

All the InGaAs photodiodes examined showed linear behaviour with an uncertainty of $0.08 \%$ between $5 \times 10^{-8} \mathrm{~A}$ and $1 \times 10^{-4} \mathrm{~A}$. Some of the photodiodes also exhibited increased noise below $5 \times 10^{-8} \mathrm{~A}$ with decreasing photocurrent. A plot of the signal/flux is shown for the detector NIST 18 in figure 3 and shows that the signal to flux ratios below $5 \times 10^{-8} \mathrm{~A}$ become noisy as compared to the same measurements of NIST 01 . The reasons for the increase in the signal noise will be explored in the noise analysis section of this paper. For example, the Beamconjoiner was used to measure the linearity of silicon photodiodes from $10^{-11} \mathrm{~A}$ to $10^{-3} \mathrm{~A}$ to within $0.054 \%(k=2)$, since $\mathrm{Si}$ diodes have typical shunt resistances of $1 \mathrm{G} \Omega$ [5].

In conjunction with the linearity testing, all the InGaAs photodiodes were also measured for shunt resistance and shunt capacitance. Table 1 shows the measured shunt resistances and the shunt capacitances of the individual InGaAs photodiodes. With the exception of two diodes, NIST 08 and NIST 14, all

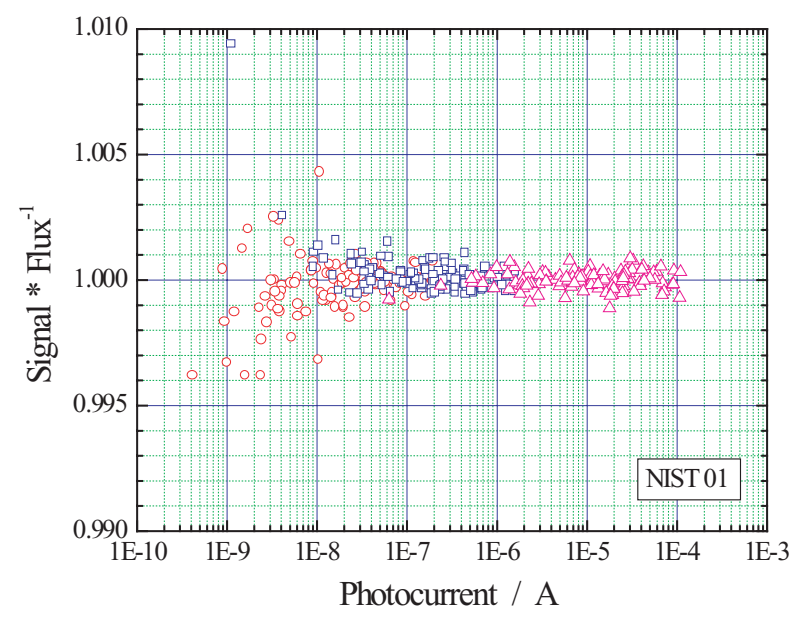

Figure 2. The ratios of the signal divided by the calculated flux plotted versus the signal for the NIST 01 photodiode (17.5 $\mathrm{M} \Omega$ shunt resistance). A total of three runs are plotted with each run consisting of 120 different signal levels. The ratios can be measured with $<0.1 \%$ uncertainty for photocurrents below $1 \times 10^{-8} \mathrm{~A}$. 


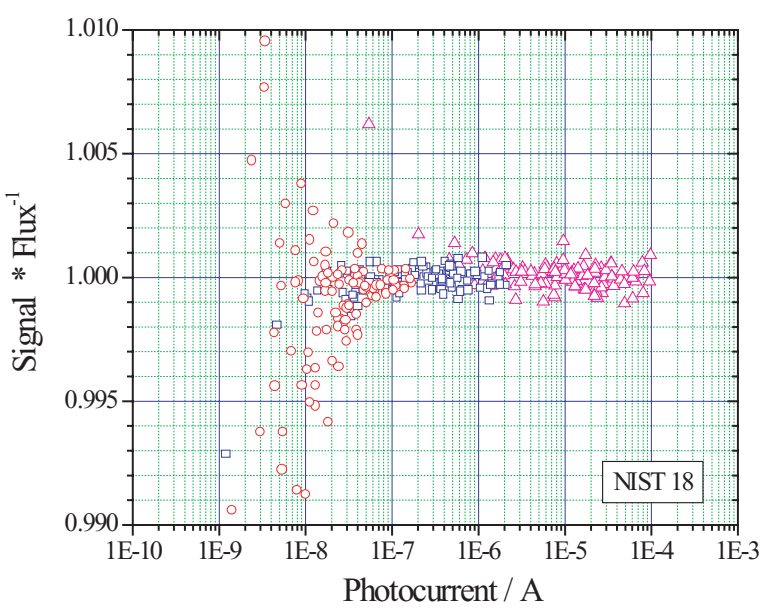

Figure 3. The ratios of the signal divided by the calculated flux plotted versus the signal for the NIST 18 photodiode $(1.40 \mathrm{M} \Omega$ shunt resistance). The ratios can be measured within the $<0.1 \%$ uncertainty for photocurrents below $5 \times 10^{-8} \mathrm{~A}$, and the noise increases rapidly at lower photocurrents.

the diodes had similar shunt capacitances ranging from $1.10 \mathrm{nF}$ to $1.87 \mathrm{nF}$. The shunt resistances varied from $0.583 \mathrm{M} \Omega$ (NIST 17) to $17.5 \mathrm{M} \Omega$ (NIST 01).

\section{Noise analysis}

According to our previous measurements [3], there are two major noise components at the output of an InGaAs photodiode current meter- $1 / f$ voltage noise and the resistor noise. The $1 / f$ voltage noise arises from the operational amplifier in the current-to-voltage converter. The other major noise component is the resistor noise that is determined by the parallel connection of the detector shunt resistance and the feedback resistance.

Figure 4 shows the two major noise components at the output of the InGaAs photocurrent meter plotted as a function of the shunt resistance. The resistor noise was calculated with two different electrical bandwidths and the output $1 / f$ noise was calculated with two different feedback resistors. In the first example; the resistor noise was calculated with $0.3 \mathrm{~Hz}$ bandwidth (lowest, dotted curve) and a feedback resistor of $R_{f}=100 \mathrm{M} \Omega$. The output $1 / f$ noise, shown with the shorter dashed curve, is independent of the bandwidth selection [6]. The $0.3 \mathrm{~Hz}$ bandwidth originates from the $1.7 \mathrm{~s}$ integration time (duration of 100 power line cycles) of the DVM connected to the output of the photocurrent meter (current-to-voltage converter). We used $0.35 \mu \mathrm{V} 1 / f$ noise in the present calculations. This is a typical value from our earlier measurements on OPA128 and OPA111 operational amplifiers [6]. In the second example, the $1 / f$ output noise was calculated with a feedback resistor of $1 \mathrm{G} \Omega$ (longer dashed curve on the top). This noise was an order of magnitude larger than that with $100 \mathrm{M} \Omega$. The resistor noise in this case was calculated with a bandwidth of $1 \mathrm{~Hz}$ (solid line). The shunt effect of the $1 \mathrm{G} \Omega$ feedback resistor for the maximum $200 \mathrm{M} \Omega$ shunt resistance was neglected.

Since the resistor noise increases and the voltage amplification decreases with increasing shunt resistance, the

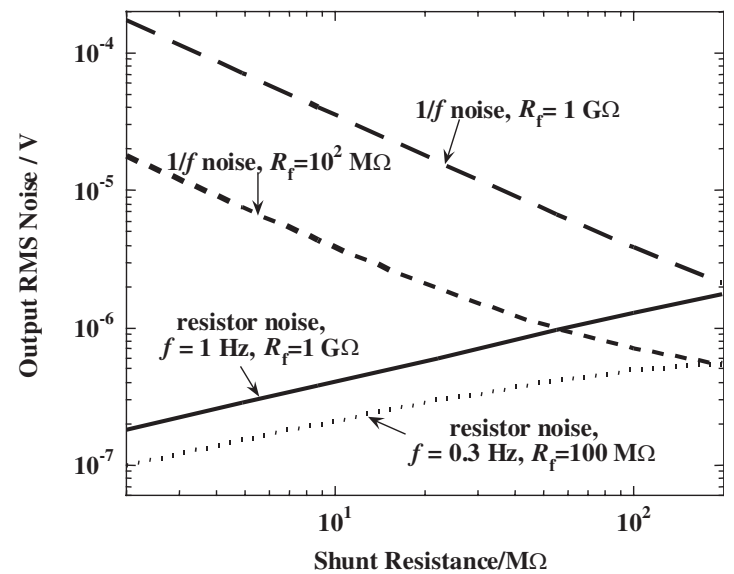

Figure 4. Output root-mean-squared (RMS) noise components of the InGaAs photocurrent meter for the $10^{8} \mathrm{~V} \mathrm{~A}^{-1}$ and $10^{9} \mathrm{~V} \mathrm{~A}^{-1}$ gains versus photodiode shunt resistance.

output noise could be optimized. In both examples, the two output noise components could be equalized at a shunt resistance of $200 \mathrm{M} \Omega$. The shunt resistance increases when the photodiode is cooled. Our earlier experiments showed that a $2 \mathrm{M} \Omega$ shunt resistance at $25^{\circ} \mathrm{C}$ could be increased to $55 \mathrm{M} \Omega$ at a controlled temperature of $-30^{\circ} \mathrm{C}$ [3]. Accordingly, an InGaAs photodiode with $7.3 \mathrm{M} \Omega$ shunt resistance at $25^{\circ} \mathrm{C}$ could produce $200 \mathrm{M} \Omega$ shunt resistance at $-30^{\circ} \mathrm{C}$. A $300 \mathrm{M} \Omega$ shunt resistance will equalize the two major output noise components for a $1 \mathrm{G} \Omega$ feedback resistor and a bandwidth of $0.3 \mathrm{~Hz}$. To perform the optimization in this third example, an InGaAs photodiode with a shunt resistance of about $11 \mathrm{M} \Omega$ at $25^{\circ} \mathrm{C}$ should be selected. The achievable (equivalent) photocurrent noise at $-30^{\circ} \mathrm{C}$ will be about $1 \mathrm{fA}(1 \mu \mathrm{V} / 1 \mathrm{G} \Omega)$.

In our present linearity measurements, the feedback resistors could be changed from $10^{4} \Omega$ to $10^{9} \Omega$. At the $100 \mathrm{M} \Omega$ and $1 \mathrm{G} \Omega$ gain selections, the resistor noise was dominated by the photodiode shunt resistance. The shunt resistance at room temperature was smaller than $20 \mathrm{M} \Omega$ for all measured photodiodes. At the low gain selections, where the feedback resistor was smaller than the shunt resistance, the resultant resistance was dominated by the feedback resistor. At these gain selections, the voltage amplification for the $1 / f$ input voltage noise was close to unity. Accordingly, resistor noise and output $1 / f$ noise were similar for the shunt resistance interval (about one decade) shown in table 1. For detectors with small shunt resistance, the voltage amplification at high feedback resistor selections can be very large, resulting in a dominating $1 / f$ noise component in the output signal. This is why the noise floor of the NIST 18 photodiode was an order of magnitude higher than that of the NIST 01.

\section{Conclusion}

The linearity and signal dynamic range of $18 \mathrm{InGaAs}$ photodiodes have been studied. The major noise components of the InGaAs photocurrent meters were analysed versus shunt resistance, signal gain and bandwidth. A limit sensitivity of $1 \mathrm{fA}$ can be achieved at $10^{9} \mathrm{~V} \mathrm{~A}^{-1}$ gain if the amplified $1 / f$ noise and the resistor noise are equalized at the output of the current meter. For the tested room temperature photodiodes, 
linearity could be measured from $10^{-8} \mathrm{~A}$ to $10^{-4} \mathrm{~A}$ with an expanded uncertainty of $0.08 \%(k=2)$. When the shunt resistance was increased by cooling one of the photodiodes, the linearity measurement range could be extended a decade due to the increase of the shunt resistance.

\section{References}

[1] Gutschwager B and Fischer J 1999 An InGaAs radiation thermometer with an accurate reference function as transfer standard Proc. TEMPMEKO '99 (Delft, Netherlands) ed J F Dubbledam and M J deGroot
[2] Yoon H W, Proctor J E and Gibson C E 2003 FASCAL 2: A new NIST facility for the calibration of the spectral irradiance of sources Metrologia 40 S30-4

[3] Eppeldauer G 1997 Electronic characteristics of Ge and InGaAs radiometers Proc. SPIE 3061 833-8

[4] Larason T C, Brown S W and Lykke K R 2002 InGaAs photodiodes used in a CCPR near-infrared key comparison Proc. NEWRAD02 (Gaithersburg, MD)

[5] Thompson A and Chen H M 1994 Beamcon III, a linearity measurement instrument for optical detectors J. Res. Natl. Inst. Stand. Technol. 99 751-5

[6] Eppeldauer G and Hardis J E 1991 Fourteen-decade photocurrent measurements with large-area silicon photodiodes at room temperature Appl. Opt. 30 3091-9 


\title{
Short-wave infrared radiometers design and characterizations
}

\author{
George P. Eppeldauer and Howard W. Yoon \\ National Institute of Standards and Technology \\ Gaithersburg, MD USA 20899
}

\begin{abstract}
Short-wave infrared (SW-IR) radiometers have been developed to extend the National Institute of Standards and Technology (NIST) reference responsivity scales from the silicon wavelength range to $2500 \mathrm{~nm}$. In addition to spectral power responsivity measurements, where $5 \mathrm{~mm}$ diameter extended-InGaAs (EIGA) detectors are underfilled by the incident radiation, irradiance responsivity calibrations are needed. Irradiance measuring radiometers are used as reference detectors to calibrate field radiometers in both irradiance and radiance measurement modes. In irradiance mode, smaller detectors with high shunt resistance, such as $1 \mathrm{~mm}$ diameter short-wave HgCdTe and EIGA detectors are used. Mechanical, optical, thermal, and electronic design considerations of SW-IR radiometers are discussed. Noise equivalent currents (NEC) were measured to evaluate noise equivalent power (NEP) and D*.
\end{abstract}

Keywords: detectivity, extended-InGaAs, infrared, infrared detectors, noise equivalent power, short-wave infrared, shunt resistance, spectral responsivity, thermoelectric cooling

\section{INTRODUCTION}

While Si, Ge, and InGaAs detector applications, characterizations, and calibrations have been described in detail [1, 2], understanding of radiometer design issues, characterizations and calibrations for short-wave infrared (SW-IR) detectors is still lacking. Photovoltaic extended-InGaAs (EIGA) and short-wave HgCdTe (PV-MCT) detectors are commercially available with high detector shunt resistance [3]. These detectors do not have large ambient-background-produced signals because their upper wavelength cut-off is low. Consequently, higher radiometer responsivity and lower radiometer output noise can be achieved, which is an important requirement for the SW-IR range where the radiant flux from ambient temperature targets is lower than at longer wavelengths [3]. Using small-area detectors the shunt resistance can be further increased, which is important to make radiometers with decreased output noise, linear operation, and low photocurrent-to-voltage conversion uncertainty [1]. The main goal in designing SW-IR radiometers is to increase the detectivity $\left(\mathrm{D}^{*}\right)$ close to the background-noise-produced theoretical limit. These improved $\mathrm{D}^{*} \mathrm{SW}$-IR radiometers can be used for ambient temperature measurements [4]. It is anticipated that NIST, in the near future, will begin to issue detector calibration services in the SW-IR wavelength region to $2.5 \mu \mathrm{m}$ utilizing these standards.

The design of high-D* SW-IR radiometers needs special considerations. Cryogenic cooling is not needed for these detectors, but 4-stage thermoelectric (TE) coolers must be used to operate the detectors at temperatures close to -80 ${ }^{\circ} \mathrm{C}$ in order to obtain high shunt resistance. Delivering the dissipated heat away from the TE coolers is an additional problem compared to traditional temperature control of Si, Ge, and InGaAs detectors.

Applying SW-IR detectors in a new radiometer design, the NIST spectral power responsivity scale can be extended to $2.5 \mu \mathrm{m}$. Also, in addition to spectral radiant power responsivity, spectral irradiance and radiance responsivities can be measured by changing the input optics of these radiometers. Characterization of SW-IR radiometers for measuring the different radiometric quantities is necessary to keep the measurement uncertainty low. SW-IR transfer standard radiometers have also been developed within this project to convert power responsivity into irradiance responsivity. These irradiance mode radiometers will be used as reference standards to calibrate both irradiance and radiance

Infrared Technology and Applications XXXIII, edited by Bjørn F. Andresen, Gabor F. Fulop, Paul R. Norton, Proc. of SPIE Vol. 6542, 654200, (2007) · 0277-786X/07/\$18 · doi: 10.1117/12.720548 
measuring SW-IR radiometers. The calibrated SW-IR radiometers are used as working standards to disseminate the pyroelectric radiometer realized NIST infrared responsivity scales in the $1 \mu \mathrm{m}$ to $2.5 \mu \mathrm{m}$ wavelength range [5, 6].

\section{SW-IR DETECTORS}

Photovoltaic EIGA detectors are available with $1.9 \mu \mathrm{m}, 2.2 \mu \mathrm{m}, 2.4 \mu \mathrm{m}$, and $2.6 \mu \mathrm{m}$ (50 \%) wavelength cut-offs. The wavelength cut-off of PV-MCT detectors can be tuned by changing the material composition [3]. The PV-MCT detectors, tested in this work, have a $2.8 \mu \mathrm{m}$ wavelength cut-off. While the diameter of the EIGA detectors can be as large as $5 \mathrm{~mm}$, PV-MCT detectors are usually not larger than $2 \mathrm{~mm}$.

The custom-made detector can for a $5 \mathrm{~mm}$ diameter EIGA detector is shown in Fig. 1. The sapphire window that has a $4^{\circ}$ wedge angle is positioned above the detector-chip tilted by $4^{\circ}$ (both are shown within circle C). The non-parallel surfaces serve to eliminate interference effects in the detector response. In case of traditional detector cans (such as Model 3CN), the side walls can absorb heat from the thermal energy rejected by the TE cooler. To avoid this problem, a cold field-of-view limiter (e.g. with $30^{\circ}$ acceptance angle) can be applied to 3CN cans to keep the background signal low. The main advantage of the shown custom-made detector can is that the front and side surfaces around the detector will not warm up because of the heat conducted from the hot side of the 4-stage TE cooler and there will be no excess background radiation for the detector.

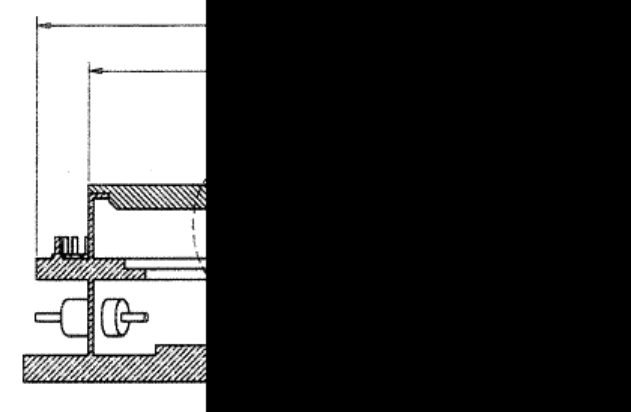

Fig. 1. SW-IR detector can with a wedged window and a tilted $5 \mathrm{~mm}$ diameter detector.

Shunt resistance is one of the most important detector characteristics [1, 3]. High shunt resistance is required to obtain low noise at a radiometer output, high signal-gain in the photocurrent meter, and high stability and repeatability in signal measurements. In order to achieve a high shunt resistance, the detectors have to be operated at a low and stable temperature. Figure 2 shows that the shunt resistance changes exponentially with the detector temperature (the Y scales are logarithmic and the $\mathrm{X}$ scale is linear). While the shunt resistance of a $1 \mathrm{~mm}$ diameter regular InGaAs (IGA) detector, that has a $1.7 \mu \mathrm{m}$ wavelength cut-off, can reach $10 \mathrm{G} \Omega$ at a temperature close to $-20{ }^{\circ} \mathrm{C}, 1 \mathrm{~mm}$ diameter SW-IR detectors (EIGA and PV-MCT) need cooling to about $-80{ }^{\circ} \mathrm{C}$ to obtain a shunt resistance close to $10 \mathrm{M} \Omega$. The IGA detector needs only two-stage thermoelectric (TE) cooling to obtain that high shunt resistance. For SW-IR detectors four-stage TE coolers must be used to achieve about $-80{ }^{\circ} \mathrm{C}$ detector temperature. While 3 mm diameter EIGA detectors can have shunt resistances between $1 \mathrm{M} \Omega$ and $3 \mathrm{M} \Omega$ at $-70{ }^{\circ} \mathrm{C}$, shunt resistances only between $70 \mathrm{k} \Omega$ and $90 \mathrm{k} \Omega$ were measured on $5 \mathrm{~mm}$ diameter EIGA detectors at this same temperature. Material quality problems were the reasons of these lower-than-expected shunt resistances. 


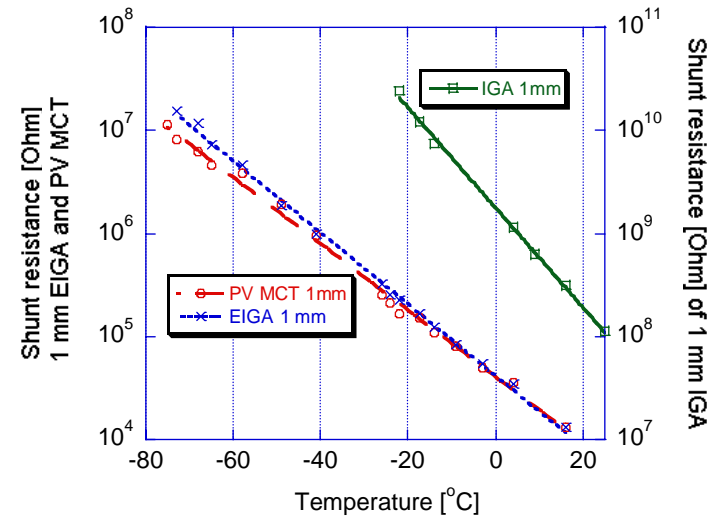

Fig. 2. Shunt resistance versus temperature of regular IGA and SW-IR (EIGA and PV-MCT) photodiodes.

\section{SW-IR RADIOMETER DESIGN}

The radiometer measuring-head design for SW-IR detectors needs several important considerations. The measuring head includes input optics, a heat removing attachment for the TE cooler, temperature control for the detector (using the TE cooler and the temperature sensor), and a photocurrent-to-voltage converter.

\subsection{Measuring head}

The cross section of an irradiance mode measuring head is shown in Fig. 3 to illustrate the input optics and the side fan for cooling. An aperture is usually located at the front. There is an IR diffuser between the aperture and the $3 \mathrm{~mm}$ diameter EIGA detector to maintain the angular responsivity close to the theoretical cosine function of irradiance measurements [7]. While the aperture is underfilled by the incident radiation (beam) in power mode measurements, it is overfilled in irradiance measurement mode. In this measuring head design, the 4-stage TE cooled detector is inside of a traditional (Model 3CN) can which is mounted on a thick copper plate. The plate conducts the heat from the hot side of the TE cooler to the heat sink, which is part of the cylindrical Aluminum housing. The fan attached to the side of the housing produces forced air cooling for the heat sink. The thermistor pins and the pins of the 4-stage TE cooler are connected from the bottom of the detector can to a multiple-pin connector on the side of the housing. An external temperature controller that regulates the detector temperature to a low (about $-85^{\circ} \mathrm{C}$ ) and constant value is attached to this connector. The detector leads are connected to the inputs of a photocurrent-to-voltage converter through a 4-pin connector located at the right side of the shown housing. The converter is inside of another cylindrical housing (not shown here) which can be attached to the bottom (on the right) of the shown housing and has the same outside diameter.

Another measuring head design is shown in Fig. 4 using the detector-can of Fig. 1. In this head design, the heat removal is performed with water cooling. The water coolant that delivers the heat away from the 4-stage TE cooler of the $5 \mathrm{~mm}$ diameter detector is circulated with a heat exchanger (radiator). A selectable-speed fan is built into the heat exchanger that removes the heat from the water coolant. The temperature stabilization is done by an external temperature controller. The side view of the measuring head is shown on the left side and the cross section is shown on the right side of the figure. The different heat dumping solutions shown on Figs. 3 and 4 can work equally well. As a result of the efficient heat removal, the external temperature controller can regulate the detector temperature to $<0.01{ }^{\circ} \mathrm{C}$.

Proc. of SPIE Vol. 6542 654200-3 


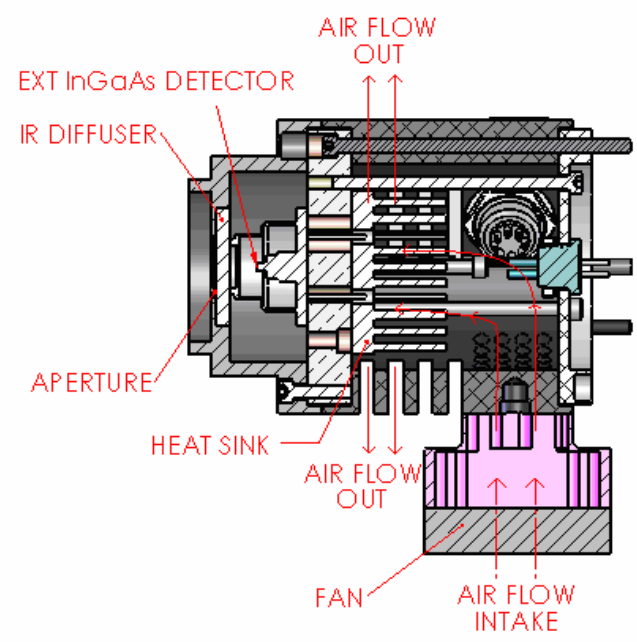

Fig. 3. Radiometer head for a 3 mm EIGA detector using fan produced air flow for heat removal.
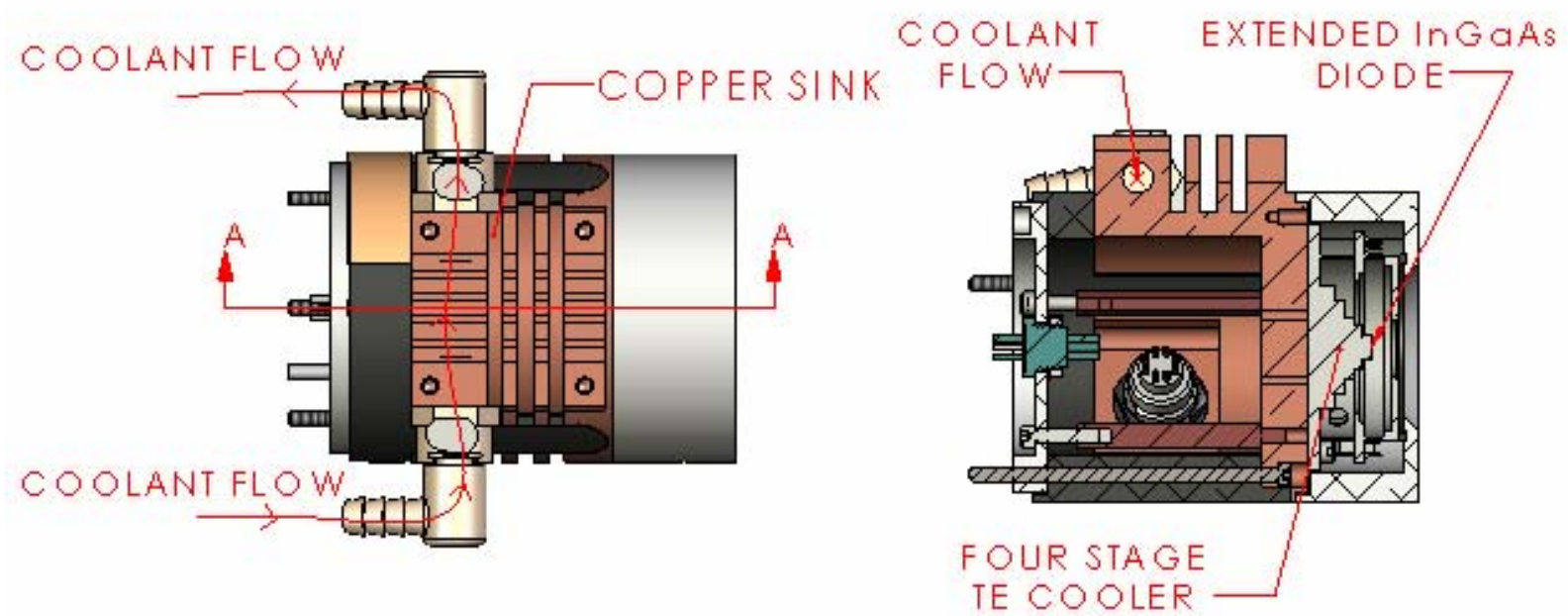

SECTION A-A

Fig. 4. Side view and cross-section of a radiometer head built for a $5 \mathrm{~mm}$ EIGA detector. The detector can shown in Fig. 1 is used and water-coolant is circulated for heat removal.

\subsection{Electronic design considerations}

The photocurrent meter characteristics are matched to the electrical characteristics of the SW-IR detector. The shunt resistance of SW-IR detectors (as discussed above) is significantly lower than that of Si and cooled regular InGaAs photodiodes. In case of high shunt resistance (such as with Si and cooled regular InGaAs detectors) and high signal gain (feedback resistor of the current-to-voltage converter) selections, the output noise of a radiometer (measuring head) is dominated by the resistor noise [1]. The equivalent source-resistance of a photodiode photocurrent meter can be calculated as a parallel connected shunt resistance and feedback resistance. For instance, when the shunt resistance is 
$3 \mathrm{M} \Omega$ and the feedback resistance is higher, the $3 \mathrm{M} \Omega$ shunt resistance will shunt the feedback resistance and will dominate the resultant source-resistance. This means that the resistor noise will remain small and constant for feedback resistors higher than the $3 \mathrm{M} \Omega$ shunt resistance. In this case, the resistor noise will not be the dominating noise component of this radiometer. The dominating output noise, in contrast to other IR (such as InSb) detector applications, will be the amplified input voltage noise of the operational amplifier of the current-to-voltage converter. This input noise has both white noise and 1/f noise components. In contrast to Si photodiode current meters, where low inputcurrent operational amplifiers are used (for the $2 \mathrm{G} \Omega$ to $5 \mathrm{G} \Omega$ optimum shunt resistances), low input voltage noise operational amplifiers are to be selected for SW-IR detectors. The amplification for the input voltage noise is shunt resistance dependent [1]. The higher the shunt resistance the lower the voltage amplification (for feedback resistors higher than the shunt resistance). Accordingly, increasing shunt resistance will decrease the dominant output voltage noise component for the radiometer output. At the same time (using the same feedback resistor), the background current noise will not change at the output. The goal is to decrease the output voltage noise, by increasing the shunt resistance, until the background current noise will be the dominating noise component. In this case, there will be a reasonable limit for increasing the detector shunt resistance. AC mode Si radiometer noise-performance will not be achieved with SW-

IR radiometers even if the shunt resistances were increased to G $\Omega$ values and a cold field-of-view limiter was applied for the EIGA detector. In case of an optimized SW-IR radiometer design, the noise performance will be limited by the background noise, which is small in the SW-IR wavelength range but never as small as the noise-equivalent-power (NEP) of noise-optimized AC mode Si radiometers [1]. Test measurements can determine the reasonable limit for the shunt resistance increase.

In case of SW-IR detectors, the background current is orders of magnitude smaller than with InSb detectors. However, it still can be comparable to the photocurrent of the optical signal to be measured. High sensitivity can be achieved if the useful signal (to be measured) is chopped and is measured by a lock-in amplifier. In this case, the DC background signal and its changes will not be measured because it will not have any frequency components that the chopping frequency synchronized lock-in amplifier could measure. Because of the low DC background current, the feedback resistor can be two orders of magnitude higher than with background optimized InSb detectors. Accordingly, the responsivity of SWIR radiometers can be much higher than InSb radiometers. The highest value for the feedback resistor in the current-tovoltage converter is limited by the required upper roll-off frequency of the radiometer. The upper roll-off is determined by the time constant of the feedback impedance (the product of the feedback resistance and the feedback capacitance). It is important to keep the parallel stray (feedback) capacitance low if high roll-off frequencies are needed at high signalgains. In an optimum design, the chopping frequency is about one order of magnitude lower than the upper roll-off frequency, to obtain an operating point on the flat (constant) signal-gain versus frequency function of the photodiode current-meter. Accordingly, the chopping frequency should be high enough to be near the elbow of the $1 / \mathrm{f}$ noise spectrum of the current-to-voltage converter and small enough to avoid problems when optimizing the feedback capacitance during frequency compensations. Partial frequency compensations are needed in AC measurement modes to optimize the three fundamental gains of photodiode current meters. These gains are the signal-gain (discussed above), the closed-loop voltage gain (that gives the amplification for the input voltage noise), and the loop gain (product of the operational amplifier open-loop gain and the feedback attenuation produced by the feedback resistor and the detector shunt resistance) that determines the uncertainty of the current-to-voltage conversion [1].

\section{CHARACTERIZATIONS}

In order to verify the above design considerations, test radiometers have been made and their performance characteristics were measured. First, tests were made to evaluate the minimum signal (photocurrent) that can be measured in DC mode with a reasonably low uncertainty. This information is needed for high signal (such as laserbased source) measurements which is typical in responsivity scale transfers. However, the main goal of the performed tests was to determine the noise equivalent current (NEC), the noise-equivalent power (NEP), and the D* values of the newly developed SW-IR radiometers.

The DC tests were made at detector temperatures of $-85{ }^{\circ} \mathrm{C}$. The background current measured on a 3 mm EIGA detector was $17 \mathrm{nA}$. The background current of a $1 \mathrm{~mm}$ EIGA detector, equipped with a cold $30^{\circ}$ field-of-view limiter, was only $0.6 \mathrm{nA}$. These small DC currents can be easily compensated in the current-to-voltage converter. The DC mode NEC will depend on the fluctuations of the background current if the temperature of the detector is kept constant. The

Proc. of SPIE Vol. 6542 654200-5 
$3 \mathrm{~mm}$ detector, which has a shunt resistance of $3 \mathrm{M} \Omega$, was measured at a signal-gain of $10^{7} \mathrm{~V} / \mathrm{A}$. A $40 \mu \mathrm{V}$ standard deviation was obtained from 20 measurements. Each measurement was taken using an integrating digital voltmeter with a measurement integration time of 100 power line cycles. At this $0.3 \mathrm{~Hz}$ bandwidth [1], a DC mode NEC= 4 pA can be calculated from the ratio of the measured $40 \mu \mathrm{V}$ output noise to the $10^{7} \mathrm{~V} / \mathrm{A}$ signal-gain. The minimum signal will be 4 $\mathrm{nA}$ to receive a signal-to-noise ratio of 1000 for performing a $0.1 \%$ measurement uncertainty. The DC mode NEC of the $1 \mathrm{~mm}$ EIGA detector has not been tested but it is estimated to be significantly lower because of the higher shunt resistance (15.3 $\mathrm{M} \Omega$ ) and the 30 times lower background current.

In AC measurement mode, the output total noise voltage of the EIGA radiometers was measured at different signalgains. The output noise voltage was measured with a lock-in amplifier attached to the output of the current-to-voltage converter. The chopping frequency was $7.5 \mathrm{~Hz}$ and the integrating time constant of the lock-in amplifier was $1 \mathrm{~s}$. The measured voltage noise changed from $0.1 \mu \mathrm{V}$ at gain $10^{6} \mathrm{~V} / \mathrm{A}$ to $20 \mu \mathrm{V}$ at gain $10^{9} \mathrm{~V} / \mathrm{A}$. Figure 5 shows the measured output voltage noise on the left-Y axis. The right-Y axis shows the NEC which was calculated as the ratio of the output voltage noise to the selected feedback resistor. A constant NEC=20 fA was obtained for the high signal gains from $10^{7} \mathrm{~V} / \mathrm{A}$ to $10^{9} \mathrm{~V} / \mathrm{A}$. The NEC of the $1 \mathrm{~mm}$ EIGA detector was tested at a signal-gain of $10^{8} \mathrm{~V} / \mathrm{A}$, a chopping frequency of $9 \mathrm{~Hz}$, and an electrical bandwidth of $0.16 \mathrm{~Hz}$. This detector has five times larger (15.3 M $\Omega$ ) shunt resistance and about 30 times lower background current. The obtained NEC was $7 \mathrm{fA}$.

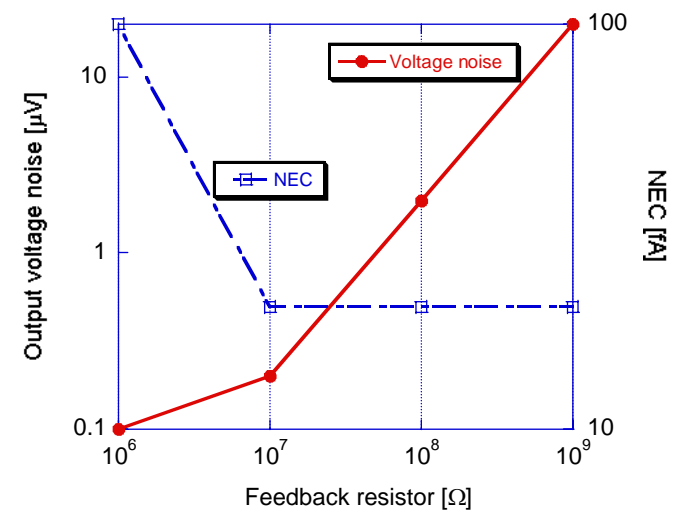

Fig. 5. The output voltage noise and the noise equivalent current (NEC) of a

$3 \mathrm{~mm}$ EIGA radiometer with a shunt resistance of $3 \mathrm{M} \Omega$.

The NEP of a SW-IR radiometer can be calculated from its NEC:

$$
\mathrm{NEP}=\frac{\mathrm{NEC}}{R}
$$

where $R$ is the peak responsivity of the detector in A/W. Using a typical $1.3 \mathrm{~A} / \mathrm{W}$ peak responsivity (manufacturer's data), an $\mathrm{NEP}=15.4 \mathrm{fW}$ can be calculated for the above discussed $3 \mathrm{~mm}$ EIGA radiometer. An NEP=5.4 fW was calculated for the radiometer using the $1 \mathrm{~mm}$ EIGA detector with the cold FOV limiter. This result is very close to the $3 \mathrm{fW}$ DC-mode NEP of well-designed silicon radiometers [8].

The detectivity $\mathrm{D}^{*}$ of a SW-IR radiometer (with detector area A) can be calculated as:

$$
D^{*}=\frac{\sqrt{\mathrm{A}}}{\mathrm{NEP}}
$$


The wavelength dependence of the $\mathrm{D}^{*}$ of several high quality AC measurement-mode radiometers, such as photovoltaic Si and InGaAs, EIGA, PV-MCT and InSb are shown in Fig. 6. The background-limited power (BLIP) curve, that gives the theoretical limit for the $\mathrm{D}^{*}$ was calculated with an f/2 detector field-of-view (FOV) for a $295 \mathrm{~K}$ background radiation. The noise due to the ambient background at temperature, $T$, is given by the mean square deviation in the total rate of photon arrival per unit area and per unit interval given by

$$
(\Delta N)^{2}=\frac{2 \pi v^{2}}{\mathrm{c}^{2}} \frac{e^{h v / k T}}{\left(e^{h v / k T}-1\right)^{2}},
$$

where $\mathrm{c}$ is the speed of light, $v$ is the frequency, $h$ is the Planck constant and $k$ is the Boltzmann constant. The BLIP is then given by

$$
\mathrm{D}_{\mathrm{BLIP}}^{*}(T, \lambda)=\frac{\mathrm{c} \eta}{2 \pi^{1 / 2} h v}\left[\int_{0}^{\infty} \eta(v) \frac{v^{2} e^{h v / k T}}{\left(e^{h v / k T}-1\right)^{2}} d v\right]^{-1 / 2}
$$

where $\eta$ is the quantum efficiency of the detector [9]. The graph shows that the D* of a room temperature (RT) E-IGA radiometer was increased by almost three orders of magnitude when the detector was cooled to $-85^{\circ} \mathrm{C}$. As a result of the above discussed design considerations, the obtained $D^{*}$ value of $3 \times 10^{13} \mathrm{~cm} \mathrm{~Hz}^{1 / 2} / \mathrm{W}$ is very close to the given background noise theoretical limit. The PV-MCT radiometer, that has a $2.8 \mu \mathrm{m}$ cut-off, is also very close to the BLIP curve. The $\mathrm{D}^{*}$ of an InSb radiometer (with optimized background rejection) is about two orders of magnitude lower. The AC mode Si and InGaAs radiometers have very high $\mathrm{D}^{*}$ values but they are not suited for detection of IR radiation since there is insufficient signal at these wavelengths (the BLIP is $>10^{18} \mathrm{~cm} \mathrm{~Hz}^{1 / 2} / \mathrm{W}$ ). We note that the detectivities of the TE-cooled SW-IR detectors greatly exceed that of the liquid nitrogen-cooled InSb detectors.

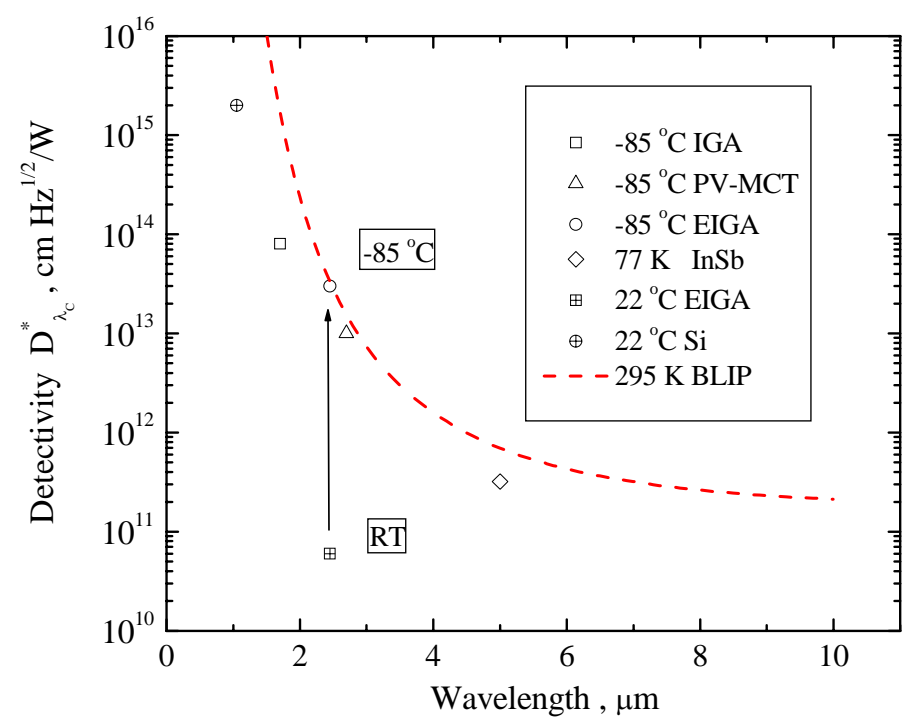

Fig. 6. D* of AC mode Si, InGaAs, EIGA, PV-MCT and InSb radiometers with the background-limited power curve at $295 \mathrm{~K}$ calculated with an f/2 detector FOV and electrical bandwidth of $0.16 \mathrm{~Hz}$. 


\section{CALIBRATION TRANSFER}

The spectral responsivity calibration of the above discussed SW-IR radiometers will be derived from the NIST electrical-substitution cryogenic radiometer (ESR). The ESR measures the total radiant power in the incident laser beam with a relative expanded uncertainty of $0.02 \%(k=2)$. In order to propagate the radiant power responsivity scale with a minimal increase in the uncertainty, transfer standard SW-IR radiometers have been developed. These radiometers are expected to measure the total beam power with uncertainties of about $0.1 \%(k=2)$. The spatial non-uniformity of response of EIGA detectors (as measured by the manufacturer) is between $0.5 \%$ and $1 \%$. This non-uniformity is too high to propagate the responsivity scale with the expected measurement uncertainty. In order to improve the spatial nonuniformity of response, two of the EIGA radiometers (with $3 \mathrm{~mm}$ diameter detectors) were modified. Spectralon ${ }^{\#}$ coated integrating spheres of $38 \mathrm{~mm}$ diameter with $5 \mathrm{~mm}$ diameter input apertures were attached to the front of the radiometers. This attachment makes it possible to convert an EIGA radiometer from power measurement mode into irradiance measurement mode. The irradiance responsivity is equal to the product of the power responsivity and the aperture area. The picture of a modified EIGA radiometer head is shown in Fig. 7. The picture shows not only the entrance aperture in the sphere, but also the side connector for the temperature controller and the tube-connector on the heat sink for circulating the water-coolant. The uniformity was tested by scanning a $1 \mathrm{~mm}$ diameter beam over the $8 \mathrm{~mm}$ diameter sphere entrance port (with the $5 \mathrm{~mm}$ aperture removed). The precision aperture was removed during this test. The measurement results are shown in Fig. 8. The input sphere improved the spatial non-uniformity of the EIGA radiometer by about a factor of five. This improvement is needed to perform $0.1 \%(k=2)$ responsivity uncertainty in power measurement mode even if different beam positions and different beam diameters are used. Using these optimized transfer standard radiometers, a factor of three uncertainty decrease can be expected compared to the present near-IR detector spectral responsivity calibrations [2]. Also, these radiometers make it possible to extend the NIST spectral responsivity calibrations to $2.5 \mu \mathrm{m}$ with close to $0.1 \%(k=2)$ responsivity uncertainties in both radiant power and irradiance measurement modes.

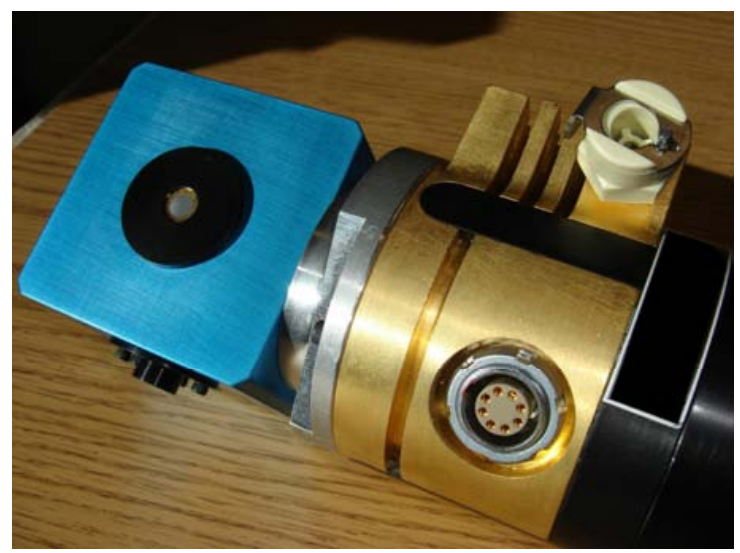

Fig. 7. Transfer standard EIGA radiometer head with an aperture equipped integrating sphere. 


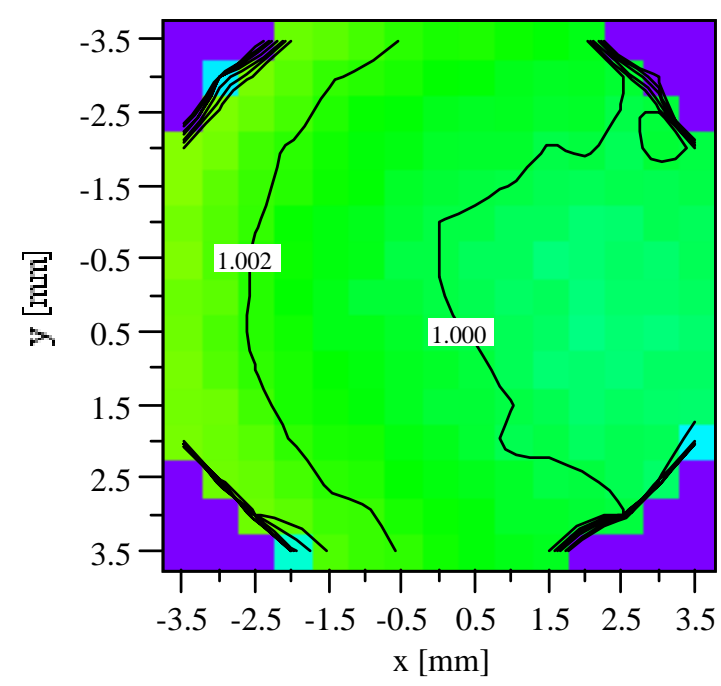

Fig. 8. Spatial non-uniformity of response of a sphere-input radiometer at $1250 \mathrm{~nm}$.

\section{CONCLUSIONS}

Radiant power and irradiance measuring transfer- and working-standard SW-IR radiometers have been developed to extend the NIST spectral responsivity scale to $2.5 \mu \mathrm{m}$ with close to $0.1 \%(k=2)$ responsivity uncertainty. Instead of cryogenic cooling, 4-stage thermoelectric coolers were used to stabilize the temperature of the applied $5 \mathrm{~mm}, 3 \mathrm{~mm}$, and $1 \mathrm{~mm}$ diameter detectors at $-85^{\circ} \mathrm{C}$. The dissipated heat from the TE coolers was removed using heat sinks constructed with either air flow or circulated water cooling. As a result of an additional analog control loop, the temperature of the applied SW-IR detectors could be stabilized at temperatures as low as $-85{ }^{\circ} \mathrm{C}$. The radiometers were characterized in both DC and AC measurement modes. In DC mode, at a bandwidth of $0.3 \mathrm{~Hz}$, an NEC of 4 pA was measured with a $3 \mathrm{~mm}$ diameter EIGA detector. In AC mode, an NEC=7 fA was measured with a $1 \mathrm{~mm}$ diameter EIGA detector (using a cold FOV limiter) at a bandwidth of $0.16 \mathrm{~Hz}$. This corresponds to an AC-mode NEP $=5.4 \mathrm{fW}$ which is close to the 3 fW DC-mode NEP of silicon photodiodes.

\section{ACKNOWLEDGEMENTS}

This project was funded by the U.S. Air Force Primary Standards Laboratory in the Calibration Coordination Group. The authors thank C. R. Lustenberger of Gamma Scientific, Inc. for participating in the design of the radiometers and T. C. Larason of NIST for measuring the spatial uniformity of response of the sphere radiometers.

\# Certain commercial equipment, instruments, or materials are identified in this paper to foster understanding. Such identification does not imply recommendation or endorsement by the National Institute of Standards and Technology, nor does it imply that the material or equipment are necessarily the best available for the purpose.

\section{REFERENCES}

1. G. P. Eppeldauer, NIST Technical Note 1438, Optical Radiation Measurement with Selected Detectors and Matched Electronic Circuits Between 200 nm and $20 \mu \mathrm{m}$, U.S. Government Printing Office, Washington, DC, $20402,2001$.

2. T. C. Larason, S. S. Bruce, and A. C. Parr, Spectroradiometric Detector Measurements: Part II - Visible to NearInfrared Detectors, Special Publication 250-41, U.S. Department of Commerce, February, 1998. 
3. H. W. Yoon, M. C. Dopkiss, and G. P. Eppeldauer, Performance comparisons of InGaAs, extended InGaAs and short-wave HgCdTe detectors between $1 \mu \mathrm{m}$ and $2.5 \mu \mathrm{m}$. SPIE, Vol. 6297, p. 629703-1 to 629703-10, 2006.

4. G. P. Eppeldauer and H. W. Yoon, AC-Mode SW-IR Radiation Thermometers for Measurement of Ambient Temperatures, Tempmeko 2007, to be published.

5. G. P. Eppeldauer, J. Zeng, and L. M. Hanssen, Development and calibration of pyroelectric radiometer standards at NIST, SPIE Proc. Vol. 6201, pp. 620119-1 to 620119-12, 2006.

6. G. P. Eppeldauer, M. Racz, and L.M. Hanssen, Spectral responsivity determination of a transfer-standard pyroelectric radiometer, SPIE Infrared Spaceborne Remote Sensing X., Symposium Proc. Volume 4818, p. 118-126, Seattle, Washington, July 10-11, 2002.

7. G. Eppeldauer, M. Racz, and T. Larason, Optical Characterization of Diffuser-input Standard Irradiance Meters, Optika’98, 5th Congress on Modern Optics, 14-17 September, Budapest, Hungary. SPIE Proc. Vol. 3573, p. 220-224, 1998.

8. G. Eppeldauer, Noise-optimized silicon radiometers, J. Res. Natl. Inst. Stand. Technol. Vol. 105, No. 209, p. 209219, 2000.

9. P. R. Norton, in “Photodetectors,” Handbook of Optics, ed. By M. Bass, 1995 (McGraw-Hill, New York) P. 15.11 to 15.13 . 


\title{
Measurement of thermal radiation using regular glass optics and short-wave infrared detectors
}

\author{
H. W. Yoon ${ }^{1 *}$, G. P. Eppeldauer ${ }^{1}$ \\ ${ }^{1}$ Optical Technology Division \\ National Institute of Standards and Technology \\ 100 Bureau Drive \\ Gaithersburg, MD 20899, USA \\ *Corresponding author: hyoon@nist.gov
}

\begin{abstract}
The measurement of thermal radiation from ambienttemperature objects using short-wave infrared detectors and regular glass optics is described. The detectors are chosen to operate in the $2.0 \mu \mathrm{m}$ to $2.5 \mu \mathrm{m}$ atmospheric window. Selection of detectors with high shunt resistance along with the 4-stage thermo-electric cooling of the detectors to $-85{ }^{\circ} \mathrm{C}$ results in detectivity, $\mathrm{D}^{*}$, of $4 \times 10^{13} \mathrm{~cm} \mathrm{~Hz}^{1 / 2} / \mathrm{W}$ which is near the back-ground limited performance at $295 \mathrm{~K}$. Furthermore, the use of the regular-glass commercial optics to collect the thermal radiation results in diffraction-limited imaging. The use of a radiation thermometer constructed with these elements for the measurement of a blackbody from $20{ }^{\circ} \mathrm{C}$ to $50{ }^{\circ} \mathrm{C}$ results in noise-equivalent temperature difference (NETD) of $<3 \mathrm{mK}$ at $50^{\circ} \mathrm{C}$. The operation at shorter wavelengths than traditional thermal imagers also leads to lower sensitivity to the emissivity of the object in determining the temperature of the object. These elements are used to construct a calibrator for an infrared collimator, and such a system demonstrates noise-equivalent irradiances of $<5 \mathrm{fW} / \mathrm{cm}^{2}$. These results indicate that thermal imagers using short-wave infrared sensors could be constructed utilizing commercial glass optics with possible better imaging performance and lower NETD than existing, cryogenically-cooled thermal imagers.
\end{abstract}

(C) 2007 Optical Society of America

OCIS codes: (040.3060) Infrared Detectors; (120.4640) Optical Instrumentation

\section{References and links}

1. A. Rogalski and K. Chrzanowski, Infrared devices and technique, Opto-Electronics Review 10(2): 111(2002).

2. D.G. Crowe, P.R. Norton, T. Limperis and J. Mudar, "Detectors," in Electro-Optical Components, W. D.

Rogatto ed., Infrared Information Analysis Center, Michigan, 1993.

3. Schott glass designations.

4. H.W. Yoon, D.W. Allen, and R.D. Saunders, "Methods to reduce the size-of-source effect in radiometers,” Metrologia 42, 89-96 (2005).

\# Certain commercial equipment, instruments, or materials are identified in this paper to foster understanding. Such identification does not imply recommendation or endorsement by the National Institute of Standards and Technology, nor does it imply that the material or equipment are necessarily the best available for the purpose. 


\section{Introduction}

The needs for measurement of thermal radiation from ambient-temperature and highertemperature objects are vast and wide ranging [1]. These needs arise from requirements of surveillance and non-destructive testing to early and accurate detection of hot objects at far distances for defense and other applications. At present, these needs are met by the use of cryogenically-cooled quantum detector materials such as InSb or HgCdTe or by the use of thermal detectors such as thermopiles, pyroelectrics or bolometers [2]. Although the quantum detectors have sufficient sensitivities to be able to measure thermal radiation with low noiseequivalent temperature difference (NETD), the need for cryogens or cryo-coolers and the accompanying vacuum jacket for low-noise operations limit their versatility in many applications. Furthermore, the thermal cycling and eventual ice build-up inside the cryostat lead to poor long-term stability of the responsivity and require routine maintenance of the system. Instruments utilizing thermal detectors suffer from low signal-to-noise ratios resulting from the low-power responsivities of the thermal detectors which lead to high NETD. Also, the use of thermal detectors can result in drift of the measured signal from any change in the surrounding ambient temperatures. For collection optics, these traditional detectors also require the use of either reflective mirrors or refractive lenses which transmit in the midinfrared and long-infrared wavelength such as those constructed using $\mathrm{Si}$, Ge or ZnSe.

In the past, the use of short-wave infrared (SWIR) wavelength region for thermal imaging has been limited due to the poor signal-to-noise performances of the existing detectors. The most commonly utilized detector in this wavelength region is the photoconductive $\mathrm{PbS}$ which requires operation with dry ice at $193 \mathrm{~K}$ for optimum operation. However, photoconductive $\mathrm{PbS}$ was found to be limited by its high noise-equivalent power, poor long-term stability of response and possible signal nonlinearity. Other SWIR detectors such as photoconductive HgCdTe (MCT), PbSe and InAs detectors have also been used but with limited success.

Due to the rapid improvements in InGaAs material fabrication driven by the optical fiber communications, the InGaAs photodiodes are the preferred detectors in the $900 \mathrm{~nm}$ to $1700 \mathrm{~nm}$ range. With modification of the fabrication process, InGaAs can be made to be sensitive to infrared radiation from $900 \mathrm{~nm}$ to $2500 \mathrm{~nm}$, with the long-wavelength bandgap occurring at around $2500 \mathrm{~nm}$. However, the extension of the responsivity to longer wavelengths results in the ex-InGaAs diodes having lower shunt resistances than the regular InGaAs photodiodes. The shunt resistances of the ex-InGaAs diodes can be increased by cooling the diode with 1-stage to 4-stage thermo-electric (TE) coolers. Similarly, photovoltaic (pv) MCT photodiodes can also be used. The MCT photodiodes can be fabricated for sensitivity from $2.0 \mu \mathrm{m}$ to $2.5 \mu \mathrm{m}$ with long-wavelength cutoffs near $2.5 \mu \mathrm{m}$, distinct from the usual $10 \mu \mathrm{m}$ to $12 \mu \mathrm{m}$ operation of these devices. The shunt resistances of the SWIR MCT detectors can also be increased by TE cooling, and such detectors can be substituted for ex-InGaAs.

In this work, the use of TE cooled, extended InGaAs and short-wave, pv MCT diodes for the measurement of thermal radiation from ambient-temperature objects is described. The work is motivated by the examination of the thermal emission and the radiance ratios between the object and the background radiation. The SWIR radiometers can be designed to operate in a clear, atmospheric window between $2.0 \mu \mathrm{m}$ and $2.5 \mu \mathrm{m}$. In this wavelength region, in contrast to other IR bands, off-the shelf, glass optics can be utilized for the collection of the emitted radiation. Optical modeling using glass lenses indicates that diffraction-limited performance is achieved. As examples, we demonstrate, using a TE-cooled ex-InGaAs radiation thermometer and a radiometer for measuring collimated sources, that such radiometers can achieve significantly better irradiance and radiance sensitivity than radiometers utilizing cryogenically-cooled InSb. 


\section{Planck Radiance Analysis}

The choice of the optimum wavelengths for the detection of Planck radiation depends upon many factors. Although the obvious choice of the wavelength would be at the peak of the Planck radiance of the emitting body, other considerations can mitigate the signal advantages from measuring at the peak of the spectral distribution. Often, object of interest will be in the presence of ambient background radiation which is also detected by the sensors leading to a large contaminating, background signal. The background radiation could come from objects in the field-of-view of the radiometer or from the thermal emission from body of the radiometer. Much of the unwanted background signal can be eliminated by the use of lock-in detection and the placement of the modulator. However, outside of the laboratory environment, the optimum modulation, which is directly at the source, is not feasible.

In many applications, the detection of thermal radiation from an object which is hotter than the ambient background is desired. In such cases, the advantages of using the shortest wavelength while having sufficient signals from the target area can be seen from Fig. 1. The

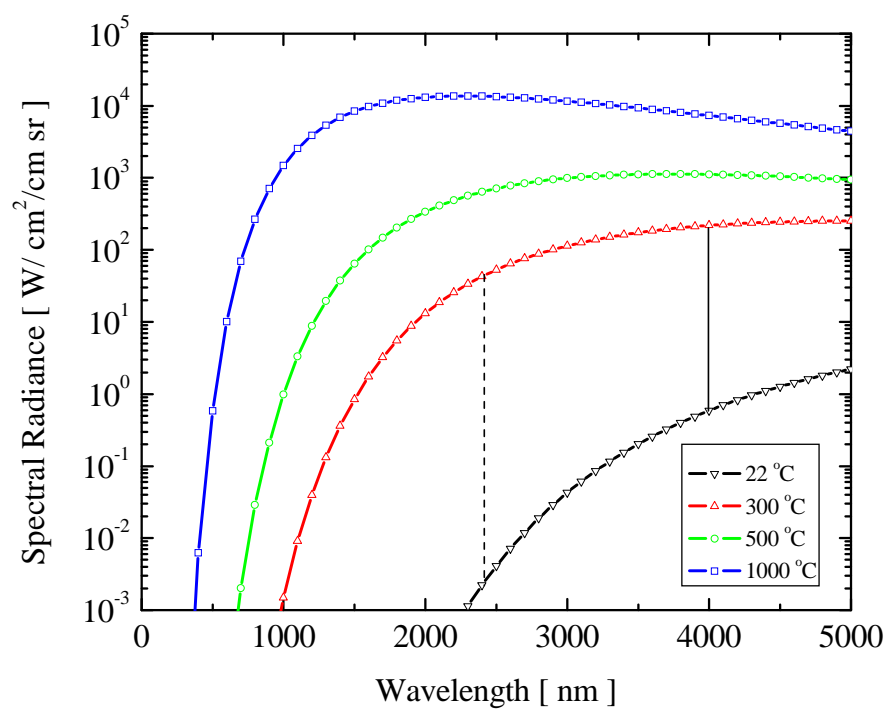

Figure 1. The spectral radiances from the use of the Planck radiance law for blackbodies at the respective temperatures. In the spectral region from $2.0 \mu \mathrm{m}$ to $2.5 \mu \mathrm{m}$, a blackbody at $22{ }^{\circ} \mathrm{C}$ and a blackbody at $300{ }^{\circ} \mathrm{C}$ will differ by a factor $>19,000$ while at a center wavelength of $4.0 \mu \mathrm{m}$, such ratio is $\sim 370$.

Planck radiances for the temperatures of interest are plotted, and for comparisons, the radiance differences between the ambient background at $22{ }^{\circ} \mathrm{C}$ and the target at $300{ }^{\circ} \mathrm{C}$ at $2.4 \mu \mathrm{m}$ and at $4.0 \mu \mathrm{m}$ can be compared. At $4.0 \mu \mathrm{m}$, the spectral radiance ratio from the roomtemperature $22{ }^{\circ} \mathrm{C}$ background and a blackbody at $300{ }^{\circ} \mathrm{C}$ is

$$
\frac{L_{4.0 \mu m}\left(300^{\circ} \mathrm{C}\right)}{L_{4.0 \mu \mathrm{m}}\left(22^{\circ} \mathrm{C}\right)} \cong 370 \text {. }
$$

However, at a center wavelength of $2.4 \mu \mathrm{m}$, the Planck radiance ratio is much larger at 


$$
\frac{L_{2.4 \mu m}\left(300^{\circ} C\right)}{L_{2.4 \mu m}\left(22^{\circ} C\right)} \cong 1.9 \times 10^{4} \text {. }
$$

The radiance from the $22{ }^{\circ} \mathrm{C}$ ambient at $2.4 \mu \mathrm{m}$ is lower than at $4.0 \mu \mathrm{m}$ by a factor of 260 which will result in lower interfering radiances from the background at the shorter wavelengths. This simple analysis indicates that if sufficiently sensitive detectors with low noise and high responsivity can be developed, then such detectors will be less influenced by the background radiation while still being able to detect the radiation source. It is critical that the band-gap of the detectors cut-off is at or near $2.5 \mu \mathrm{m}$ so that the sensor is less sensitive to the ambient background radiation. The Planck radiances also indicate that the dynamic range of the signals (the useful-signal and background-signal ratios) for a source at $300{ }^{\circ} \mathrm{C}$ and at $1000{ }^{\circ} \mathrm{C}$ will be greater at $2.25 \mu \mathrm{m}$ than at $4.0 \mu \mathrm{m}$, thus leading to greater contrast ratios between the objects.

\section{SWIR Detectivity or $\mathbf{D}^{*}$}

The relative performances of detectors can be compared independently from the detector element sizes using the criterion of $\mathrm{D}^{*}$ or detectivity (sometimes called Jones) with units of $\mathrm{cm} \mathrm{Hz} / \mathrm{W}$. The $\mathrm{D}^{*}$ of the detector can be increased by decreasing the noise-equivalent power (NEP). A decrease in the NEP can be achieved by increasing the shunt resistance, $R_{S}$, of the detector which will decrease the noise at the output of the photodiode trans-impedance amplifier. The $\mathrm{R}_{\mathrm{S}}$ can be increased by several techniques. Since the shunt resistance is typically due to the areal density of the electrical imperfections of the detector, it depends on the surface area of the detector. A detector with a smaller surface area will have higher shunt resistance resulting in a lower NEP and a higher $\mathrm{D}^{*}$.

The shunt resistances of semiconductor photodiodes also depend on the temperature of the diode. The shunt resistances of $3 \mathrm{~mm}$ and $1 \mathrm{~mm}$ diameter ex-InGaAs and short-wave MCT (2.8 $\mu \mathrm{m}$ bandgap) detectors were measured as a function of diode temperature. The temperatures were varied using the built-in 4-stage thermoelectric coolers with thermistor feedback controls. The shunt resistances shown in Fig. 2 were determined from the inverse 


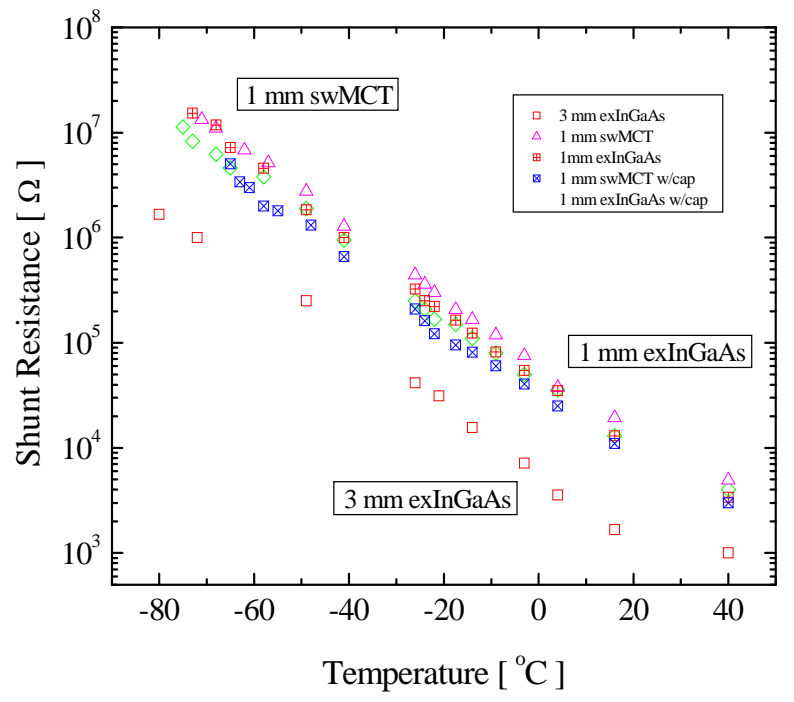

Figure 2. The temperature dependence of shunt resistance for $1 \mathrm{~mm}$ and $3 \mathrm{~mm}$ diameter InGaAs, extended InGaAs and $1 \mathrm{~mm}$ diameter swMCT detectors. Shunt resistance increase of 1000 over the room temperature values are easily achieved using TE cooling. The notation "w/cap" refers to the addition of a TE-cooled $30^{\circ}$ field-of-view limiting baffle incorporated onto the front of the diode in the detector housing.

slope of the current-voltage (I-V) curves. All the detectors are found to have a semilogarithmic dependence on diode temperature. These results indicate that the shunt impedances for the ex-InGaAs detector and the sw-MCT can be increased by a factor $>1000$, resulting in $3 \times 10^{6} \Omega$ with the $3 \mathrm{~mm}$ diameter detector cooled to $-85{ }^{\circ} \mathrm{C}(188 \mathrm{~K})$. The $3 \mathrm{~mm}$ diameter diode has a lower shunt resistance than the $1 \mathrm{~mm}$ diameter diode which is inversely proportional to the area of the diode. This indicates that the defects which limit the shunt resistance are uniformly distributed across the diode area.

The $\mathrm{D}^{*}$ or the detectivity of various detectors at the peak wavelength of their spectral responsivities are shown in Fig. 3 along with the back-ground-limited performance (BLIP) at 


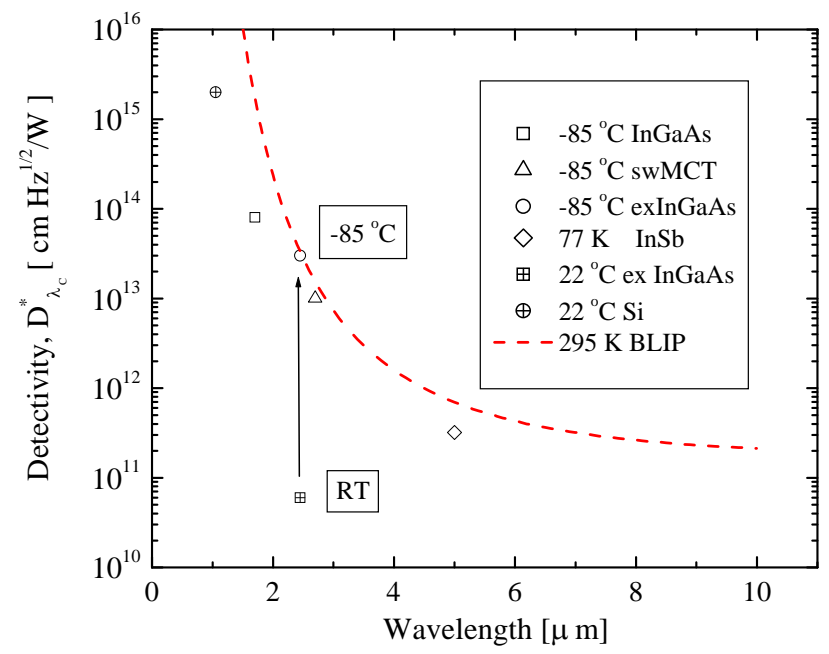

Figure 3. The background-limited power (BLIP) restricted to F/2 field-of-view for $295 \mathrm{~K}$ background with the detectivity of NIR, SWIR and MIR sensors plotted versus their bandgap wavelengths. The detectivities plotted are for electrical bandwidth of $0.16 \mathrm{~Hz}$. The detectivities of the uncooled exInGaAs at room temperature (RT) does not meet the BLIP curve while the 4-staged TE cooled detectors lie near the BLIP curve.

$295 \mathrm{~K}$. The BLIP limits the performances of detectors operating in typical laboratory environments since the fluctuations in the arrival rate of photons from the ambient background will limit the noise floor. One can observe that much higher detectivities, without being limited by the BLIP, are possible as the detection wavelengths are made shorter than the peak of the $295 \mathrm{~K}$ Planck radiances at $10 \mu \mathrm{m}$. With cooling of the ex-InGaAs diode, the measured $\mathrm{D}^{*}$ is $>3 \times 10^{13} \mathrm{~cm} \mathrm{~Hz}^{1 / 2} / \mathrm{W}$, which is almost a factor of 100 greater than that of liquid-nitrogen cooled InSb detector at $\sim 5 \times 10^{11} \mathrm{~cm} \mathrm{~Hz}^{1 / 2} / \mathrm{W}$. These detectivities at long wavelengths would be unusable in ambient environments because of the limitations of the BLIP, but at the shorter wavelengths, the reduction in the BLIP allows even higher D*.

\section{Spectral Responsivity and Atmospheric Transmission}

The advantages of higher detectivities for these detectors cannot be utilized if the spectral responsivities of the detectors are in regions of atmospheric absorption bands. The spectral irradiance responsivity of a 2-stage cooled ex-InGaAs photodiode is shown in Fig. 4. The 


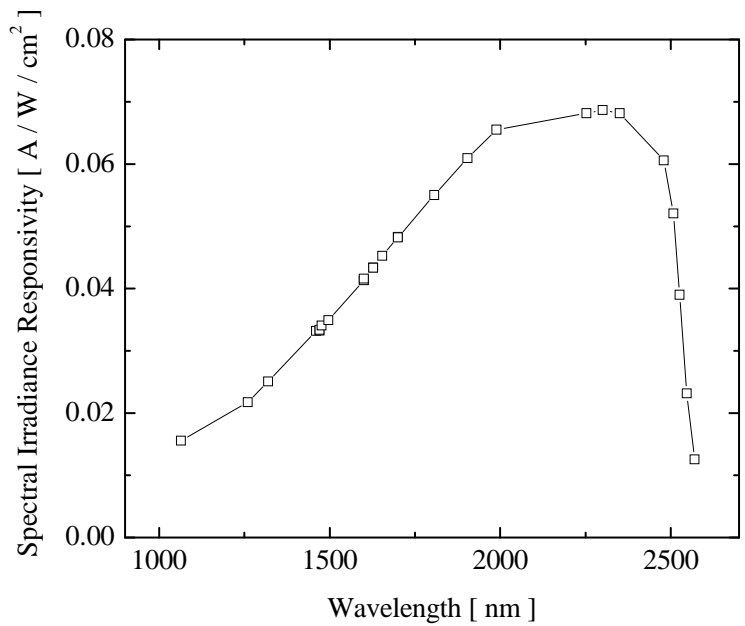

Figure 4. The NIST-measured spectral irradiance responsivity of an ex-InGaAs detector demonstrating the peak of the responsivity lies in the $2.0 \mu \mathrm{m}$ to $2.5 \mu \mathrm{m}$ region.

peak of the responsivity can be tuned by selecting the material composition such that the band-gap energy of InGaAs or HgCdTe diodes can be made to occur at a range of wavelengths from $1.7 \mu \mathrm{m}$ to $2.6 \mu \mathrm{m}$. Since these are direct band-gap materials, the quantum efficiency of the photon-to-electron conversion is also high.

In Fig. 5, the atmospheric transmission as a function of wavelength is plotted showing

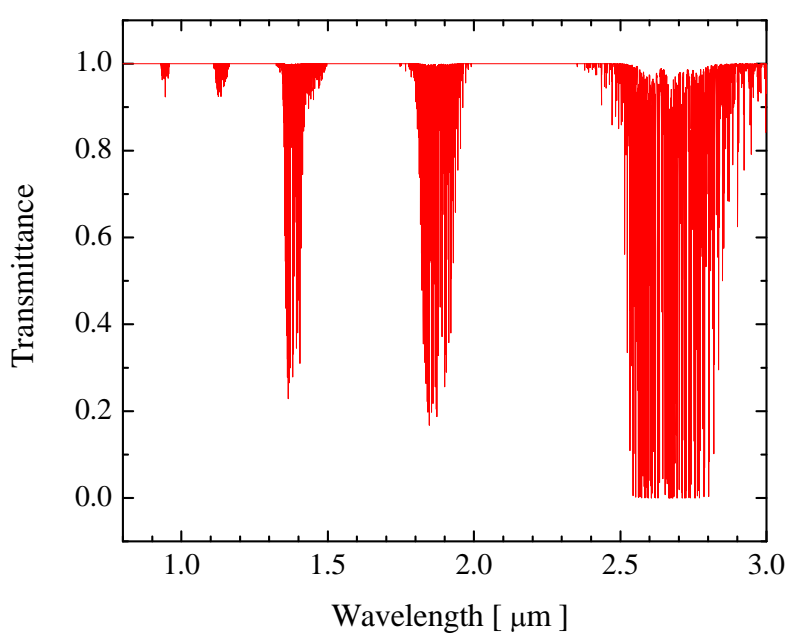

Figure 5. The transmittance at sea level for a $1 \mathrm{~m}$ path length of atmosphere showing the window between $2.0 \mu \mathrm{m}$ to $2.5 \mu \mathrm{m}$.

that such spectrally selected radiometers can operate in an atmospherically clear transmission region. To avoid fluctuations due to atmospheric changes in the transmission or absorption, ex-InGaAs radiometers can be constructed with cut-on filters to further restrict the spectral region from $2.0 \mu \mathrm{m}$ to $2.5 \mu \mathrm{m}$. 


\section{Detector Signals from Ambient Objects}

The results of initial feasibilities studies of using SWIR detectors for measurements of ambient temperature objects are shown in Table 1 . A 3 mm diameter ex-InGaAs detector was

Table 1. The signals from various objects at the estimated temperatures filling the field-of-view with the $3 \mathrm{~mm}$ diameter, 4-stage TE cooled $\left(-85^{\circ} \mathrm{C}\right)$ ex-InGaAs photodiode with a preamplifier gain of $10^{7} \mathrm{~V} / \mathrm{A}$. These signals clearly demonstrate that there is sufficient signal for thermal imaging. The differences in the spectral emissivity of the room temperature objects account for the different signals.

\begin{tabular}{ccc}
\hline Material & Signal [ V ] & St. Dev. [ V ] \\
$\mathrm{LN}_{2}$ dewar $(77 \mathrm{~K})$ & 0.026 & $2.5 \times 10^{-5}$ \\
Al foil $(295 \mathrm{~K})$ & 0.0912 & $2.5 \times 10^{-6}$ \\
Anodized Al ( 295 K ) & 0.115 & $2.5 \times 10^{-6}$ \\
Black polymer ( 295 K ) & 0.137 & $2.5 \times 10^{-6}$ \\
Human hand ( $307 \mathrm{~K})$ & 0.226 & $2.5 \times 10^{-6}$ \\
\hline
\end{tabular}

TE cooled to $-85^{\circ} \mathrm{C}$ and the photocurrents were converted to voltages using a current-tovoltage amplifier at a gain of $10^{7} \mathrm{~V} / \mathrm{A}$. Various objects with differing temperatures and emissivities were placed to fill the field-of-view of the detector. Although the detector was placed over the mouth of a liquid-nitrogen $\left(\mathrm{LN}_{2}\right)$ dewar to reduce the ambient background signal, the field-of-view could not be completely filled with a $77 \mathrm{~K}$ environment. The signals from the various objects are listed in col. 2 of Table 1. The lower temperature background of the $\mathrm{LN}_{2}$ dewar results in lower signals, and the low emissivity of the aluminum foil also leads to low signals. The difference in the signals with the changing temperature and the emissivity of the objects at room temperature and human body temperatures can be observed. These results clearly indicate that SWIR detectors have the sensitivity to measure objects at ambient temperatures.

\section{Use of Traditional Glass Refractive Optics}

One of the greatest advantages of operating in this atmospheric window is that unlike the traditional mid- or thermal-infrared regions where the choices of refractive lens materials are limited, many optical glasses transmit to $2.5 \mu \mathrm{m}$. The transmittances of $10 \mathrm{~mm}$ thick BK7 and SF5 glasses [3], which are used in typical achromat lens combinations, are shown in Fig. 6. These off-the- 


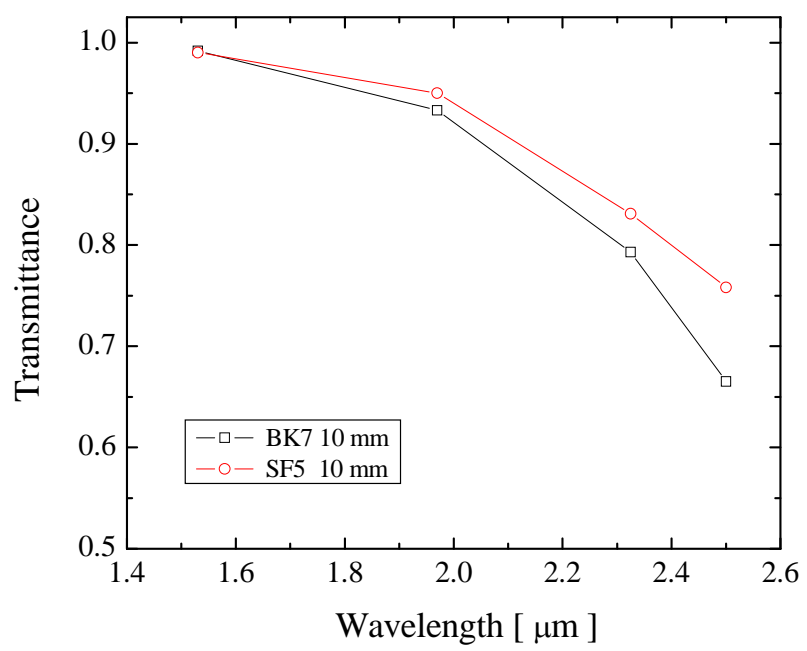

Figure 6. The transmittances of $10 \mathrm{~mm}$ thick BK7 (crown) glass and SF5 (flint) glass elements which are commonly used to form achromat lenses. Other optical glasses have generally similar transmittances.

shelf achromats are used to collect the SWIR radiation. Since these glasses also transmit in the visible radiation, the optical alignment can be performed using visible radiation and then refocused to optimize the thermal infrared signal. Such lenses could be easily optimized for diffraction-limited optical performance as shown later by optical modeling using commercial software. In addition, if the detectors are designed so that the back substrate is thinned, then the spectral responsivities can be made to range from the visible to $2.5 \mu \mathrm{m}$. For such a detector, these optical glass lenses can be refocused to use a single lens system for visible to thermal imaging.

\section{Imaging Performance of Traditional Glass Optics in the SWIR}

Optical performances of lenses used in the visible wavelength region can be assessed for the SWIR by using an optical modeling software. In Table 2, typical achromat specifications are

Table 2. The specifications of the $200 \mathrm{~mm}$ focal length achromatic lens used for the optical modeling.

\begin{tabular}{cccc}
\hline $\begin{array}{c}\text { Radius of Curvature } \\
{[\mathrm{mm}]}\end{array}$ & Thickness [ mm ] & $\begin{array}{c}\text { Radius of } \\
\text { Entrance Aperture } \\
{[\mathrm{mm}]}\end{array}$ & Material \\
\hline 121.342 & 8.0 & 25.4 & BK7 \\
\hline-91.445 & 4.0 & 25.4 & SF5 \\
\hline-271.562 & -- & 25.4 & -- \\
\hline
\end{tabular}

shown with the surface curvatures and thicknesses along with the glass types. The achromat specifications are for an off-the-shelf achromat which has been optimized for infinite conjugate in the visible wavelength region. Typically, if the optics has been optimized at a shorter wavelengths, the performance at longer wavelengths will be better than at the optimized wavelengths. The specifications in Table 2 for a $200 \mathrm{~mm}$ focal length lens are used to model the optical performance shown in Table 3 at $2.45 \mu \mathrm{m}$. Table 2 shows the root-mean- 
Table 3. The optical performance of the achromatic lens at infinite conjugate at a wavelength of $2.45 \mu \mathrm{m}$. The performance is diffraction limited as indicated by a smaller geometric, root-mean-squared (RMS) radius than the diffraction limit radius.

\begin{tabular}{cccc}
\hline Beam Entrance & Geometric RMS & Diffraction Limit & Strehl Ratio \\
Radius [ mm ] & Radius [ mm ] & Radius [ mm ] & 0.9883 \\
24.0 & 0.002123 & 0.013145 & 0.5 \\
\hline
\end{tabular}

squared radius as compared to the diffraction-limited radius indicating that the lens is diffraction limited with close to optimum Strehl ratio of 1.0 for an infinite conjugate operation at $2.45 \mu \mathrm{m}$. Although the optical performance will suffer if infinite conjugate optimized lenses are used in finite conjugate (1:1) imaging, custom lenses with computer-optimized curvatures and thicknesses can be fabricated if better performance is desired.

\section{SWIR detector performance in radiation thermometers}

To compare the performances of SWIR detectors against traditional cryogenically cooled detectors, the SWIR detectors were constructed into experimental radiation thermometers. Since optical glasses transmit at these wavelengths, regular achromats were used as objective lenses in the prototype shown in Fig. 7. A variable-temperature blackbody set to room

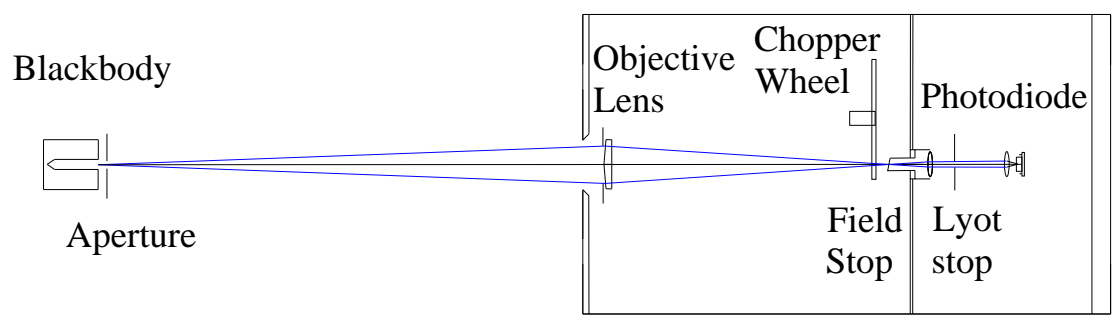

Figure 7. The SWIR detector was constructed into a radiation thermometer configuration to determine the noiseequivalent temperature difference as a function of the blackbody temperature. The distance between the objective lens and the blackbody aperture was $500 \mathrm{~mm}$ with a $50 \mathrm{~mm}$ diameter objective resulting in roughly a f/10 collection geometry. A $6 \mathrm{~mm}$ diameter target was focused at the blackbody opening.

temperatures and higher was used as the source of radiation. A $6 \mathrm{~mm}$ diameter target was imaged by the radiation thermometer at a distance of $50 \mathrm{~cm}$ between the source aperture and front of the objective lens. The radiation was imaged onto a field stop with a chopper wheel placed close to the field stop. The radiation was collimated and focused onto the $3 \mathrm{~mm}$ diameter detector with achromats. The ex-InGaAs detector was cooled to $-85{ }^{\circ} \mathrm{C}$ with thermoelectric cooling. The Lyot stop [4] serves as the aperture stop behind the field stop. Due to the low scatter objective, the Lyot stop and the internal field-of-view limiter, this radiation thermometer design has out-of-field rejection which enables internal chopping. A novel chopper using a mirrored surface was used to have the TE-cooled diode view itself using the self-staring or "Narcissus" effect. The reference for the chopped radiation then becomes the radiation from the temperature-stabilized detector leading to long-term stability of the chopped signal. 
The results of the measurements with the blackbody at $50{ }^{\circ} \mathrm{C}$ can be seen in Fig. 8 . A

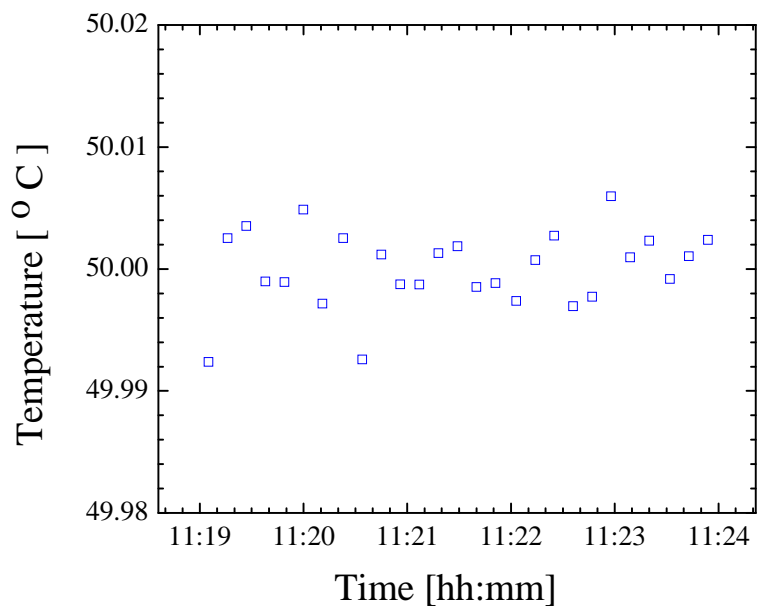

Figure 8. The stability of the chopped radiation thermometer measuring the radiance temperature of the $50{ }^{\circ} \mathrm{C}$ blackbody. The blackbody was constructed with a spherical geometry and a control loop was used to stabilize the blackbody temperature.

noise-equivalent temperature difference (NETD) of $<3 \mathrm{mK}$ can be observed from the standard deviation of the temperature measurements taken with an electrical bandwidth of $0.16 \mathrm{~Hz}$. A longer time sequence of temperatures showed changes due to the fluctuations from the heater controller of the blackbody which are not shown in the short time sequence in Fig. 8.

The NETD plotted as a function of the blackbody temperature is shown in Fig. 9. The

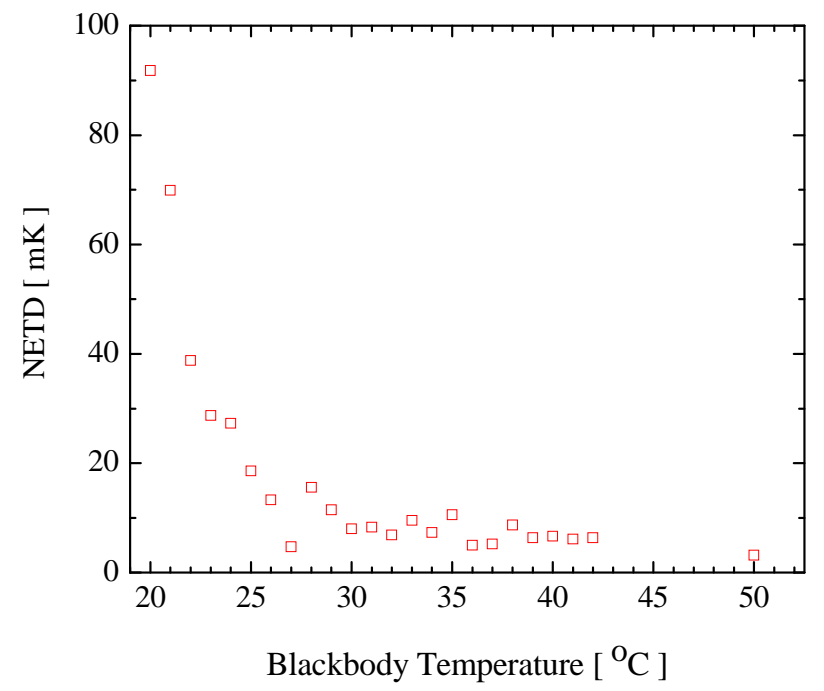

Figure 9. The noise-equivalent temperature difference (NETD), at an electrical bandwidth of $0.16 \mathrm{~Hz}$, obtained from the standard deviation of the radiation thermometer measurements at the respective blackbody temperatures. The 
increase in the NETD from the low signals as the blackbody temperature approaches the laboratory ambient temperatures is observed. The NETD at human body temperature of $36^{\circ} \mathrm{C}$ is below $10 \mathrm{mK}$.

NETD increases rapidly as the target temperature becomes comparable to the chopper-wheel temperature, but at the human body temperature of $36{ }^{\circ} \mathrm{C}$, the NETD remains $<10 \mathrm{mK}$. These results demonstrate that the NETD is comparable to that of InSb detectors for blackbody temperatures $>25^{\circ} \mathrm{C}$ without the need for cryogenic cooling.

\section{Noise-equivalent Irradiance}

To test the limits of detection for irradiance, a blackbody at $300{ }^{\circ} \mathrm{C}$ was placed at the entrance to the $1.24 \mathrm{~m}$ focal length collimator as shown in Fig. 10. An aperture wheel was placed in

\section{$1.24 \mathrm{~m}$ fl Collimator}

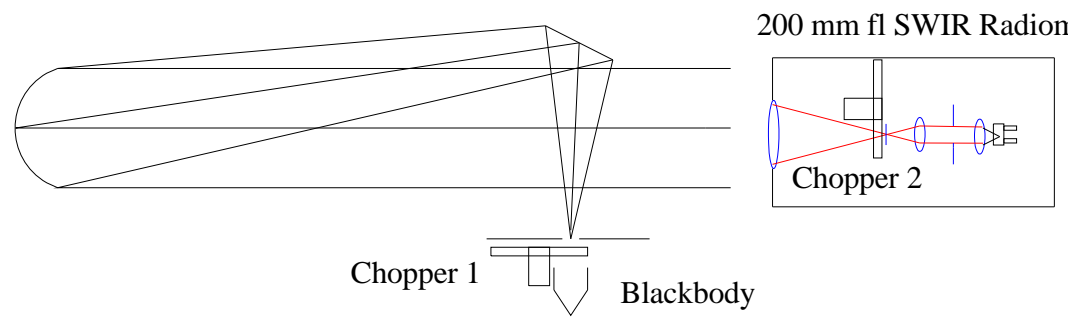

Figure 10. The schematic of the SWIR radiometer used to measure the collimated output from an infrared collimator. The use of the two chopper positions allows comparison of "upstream" chopping at the source to "down-stream" chopping inside the radiometer.

front of the blackbody, and the chopper wheel (Chopper 1) was placed between the blackbody and the aperture wheel. From the geometry of the collimator, the calculated irradiance at the $40 \mathrm{~mm}$ diameter entrance pupil of the radiometer can be determined. Another alternatively used chopper wheel (Chopper 2) was placed inside the radiometer for “down-stream" chopping at the radiometer. The measurements of the noise-equivalent irradiance (NEI) with the setup in Fig. 11 are shown in Table 4.

Table 4. The noise-equivalent irradiances found using the SWIR radiometers from the measurements of the collimator shown in Fig. 10 with the blackbody source with the respective source diameters at $300{ }^{\circ} \mathrm{C}$.

\begin{tabular}{ccccc}
\hline $\begin{array}{c}\text { Diameter } \\
{[\text { um ] }}\end{array}$ & $\begin{array}{c}\text { Measured } \\
\text { Signal [ V ] }\end{array}$ & $\begin{array}{c}\text { Standard } \\
\text { Deviation } \\
{[\mathrm{V}]}\end{array}$ & $\begin{array}{c}\text { Standard } \\
\text { Deviation/Signal }\end{array}$ & $\begin{array}{c}\text { Noise equivalent } \\
\text { irradiance [ W/ } / \mathrm{cm}^{2} \text { ] }\end{array}$ \\
250.0 & $9.54 \times 10^{-3}$ & $\begin{array}{c}1.50 \times 10^{-6} \\
150.0\end{array}$ & $1.57 \times 10^{-4}$ & $1.00 \times 10^{-14}$ \\
50.0 & $3.62 \times 10^{-3}$ & $6.40 \times 10^{-7}$ & $1.77 \times 10^{-4}$ & $4.06 \times 10^{-15}$ \\
\hline
\end{tabular}




\section{Applications of SWIR Radiometers}

One of the advantages of using the SWIR radiometers is the transparency of glass in buildings, automobiles and other equipment at these wavelengths. If SWIR thermal imagers with low NETD could be developed at these wavelengths, then it would be possible to perform thermal imaging for human detection through the automotive glass and building windows. Firefighters and emergency personnel could determine the presence human beings without having to break the window and risk acceleration of smoldering fires. Such thermal imaging is not possible for the currently used long-wavelength infrared detectors. In addition, thermal imagers are used for diagnostics of electrical power switches where the operator must be protected from potentially lethal voltages with a protective cover. Often, these covers require special infrared transmitting glasses for thermal imaging in order to determine which switches are overheating and about to fail. Regular window glass covers could be used in these applications. There are also a large number of military applications, especially in target discrimination at long distances where the traditional InSb and MCT detectors are not sensitive enough for accurate sensing.

\section{Conclusions}

We demonstrate that SWIR detectors with a $2.5 \mu \mathrm{m}$ wavelength cut-off can be used as new generation of sensors for IR radiometers and radiation thermometers. Although the cut-on at $2.0 \mu \mathrm{m}$ can be accomplished with a filter, the cut-off at around $2.5 \mu \mathrm{m}$ must be intrinsic to the detector material from the fabrication process to avoid large background currents. These detectors can be used in a spectral wavelength region where the atmosphere has a clear window from $2.0 \mu \mathrm{m}$ to $2.5 \mu \mathrm{m}$, leading to the use of refractive-glass optics without central obscurations. Furthermore, equivalent or better infrared detection capability than the traditionally used detectors can be achieved in a compact, low-maintenance design with the capabilities of measuring human body temperatures. 
\# Certain commercial equipment, instruments, or materials are identified in this paper to foster understanding. Such identification does not imply recommendation or endorsement by the National Institute of Standards and Technology, nor does it imply that the material or equipment are necessarily the best available for the purpose.

\section{References}

1. A. Rogalski and K. Chrzanowski, Infrared devices and technique, Opto-Electronics Review 10(2): 111(2002).

2. D.G. Crowe, P.R. Norton, T. Limperis and J. Mudar, “Detectors," in Electro-Optical Components, W. D. Rogatto ed., The Infrared and Electro-Optical Systems Handbook, Infrared Information Analysis Center, Michigan, 1993.

3. Schott glass designations.

4. H.W. Yoon, D.W. Allen, and R.D. Saunders, "Methods to reduce the size-of-source effect in radiometers," Metrologia 42, 89-96 (2005). 


\title{
AC-Mode Short-Wavelength IR Radiation Thermometers for Measurement of Ambient Temperatures
}

\author{
G. P. Eppeldauer and H. W. Yoon \\ Optical Technology Division, National Institute of Standards and Technology, \\ Gaithersburg, Maryland 20899, USA
}

\begin{abstract}
Recent improvements in the fabrication of short-wave infrared (SW-IR) quantum detectors have opened a new era in radiation thermometry. Ambient and higher temperatures can be measured with low uncertainties using thermoelectrically (TE) cooled extended-InGaAs (E-IGA) and short-wave photovoltaic-HgCdTe (SW-MCT) detectors. Since these detectors have low cut-off wavelengths $(2.5 \mu \mathrm{m}$ and $2.8 \mu \mathrm{m}$, respectively), they do not respond past cut-off and are less sensitive to the background infrared radiation, resulting in orders of magnitude lower background noise than traditionally used broad-band infrared detectors such as cryogenically cooled quantum detectors or thermal detectors. At the same time, the cut-off is far enough in the infrared to obtain a large enough signal from the source of interest. Because of the low detector cut-off wavelength, traditional glass-based optics can be used in the radiation thermometers. A chopper produced alternating-current (AC) signal was used to measure low temperatures by separating the AC signal from the background-radiation-produced direct-current (DC) signal and its fluctuations. Design considerations and characteristics of a newly developed SW-IR radiation thermometer are discussed. A noise-equivalent temperature difference (NETD) of $<3 \mathrm{mK}$ for a $50{ }^{\circ} \mathrm{C}$ blackbody was measured. At the human body temperature of $36{ }^{\circ} \mathrm{C}$, the obtained NETD of $\sim 10 \mathrm{mK}$ indicates that these detectors can be used in non-contact temperature measurements to replace thermopile- or pyroelectric-based radiation thermometers.
\end{abstract}

KEY WORDS: ambient temperatures; infrared; input optics; NETD; NEP; noise; optical radiation; radiance; radiation thermometer; responsivity; non-contact body temperature

\section{INTRODUCTION}

Traditional infrared detectors, such as cryogenically cooled quantum detectors and thermal detectors, operate in background-limited mode because of their long cut-off wavelengths. In this mode, the background radiation is orders of magnitude larger than the target radiation. In addition, the high background-radiation-produced DC signal is not stable and has a large noise component. The signal to be measured is usually AC to separate it from the DC background signal. However, the large noise component of the DC background signal will dominate the output noise of the radiometer unless the background signal is significantly reduced. 
Furthermore, ambient-temperature thermal detectors (e.g. thermopiles or pyroelectric detectors) have low responsivities and, thus, high noise-equivalent powers (NEP). They need large signals (sufficient thermal radiation within the detector system bandpass) to obtain reasonable signal-to-noise ratios at the output of the radiometers. In many applications, large thermal radiation to be measured is not available, and the signal (photocurrent) gain in the radiometers cannot be increased to improve the poor signal-tonoise ratios. Both of the above limitations are apparent in current designs of radiation thermometers for measuring human body temperatures. They are prone to drift from changes in the immediate thermal environment around the detector and also are not capable of transferring a temperature scale to within $\pm 0.2{ }^{\circ} \mathrm{C}$ from $32{ }^{\circ} \mathrm{C}$ to $45^{\circ} \mathrm{C}$ [1].

We describe the development of a short-wave infrared (SW-IR) radiation thermometer that can be used as a transfer standard to measure low-temperature radiometric sources with low uncertainty and high stability. The design issues and the characteristics of this thermometer are discussed here, and we show that cryogenic, quantum detector-based radiation thermometer performance can be achieved with thermo-electrically cooled SW-IR radiation thermometers.

\section{NETD AND NEP OF RADIATION THERMOMETERS}

The noise-equivalent temperature difference (NETD) and the NEP of a radiation thermometer are noise figures-of-merit that are closely related. The NETD shows the sensitivity (temperature resolution) of a thermometer while the NEP is used to optimize the detectivity of a radiation thermometer.

The NETD is determined when the thermometer measures the radiation from a stable source. In this case, the standard deviation (STD) of the measured photocurrent values is divided by the mean of the values to obtain the NETD. The noise equivalent signal fluctuation $\Delta S / S$ can come from both the emitted radiation and the noise in the output signal of the thermometer. Using the derivative of the Wien approximation,

$$
\frac{\Delta S}{S}=\frac{\Delta L}{L}=\frac{c_{2}}{\lambda} \frac{\Delta T}{T^{2}}
$$

where $L$ is the source radiance, $c_{2}=1.4387752 \times 10^{-2} \mathrm{~m} \cdot \mathrm{K}, T$ is the temperature of the source, and $\lambda$ is the wavelength where the detector is responsive to the broad-band source radiation (such as a blackbody), the NETD can be equated with the calculated $\Delta T$.

In contrast to the above suggested NETD measurements, the NEP is measured at zero (dark or blocked) signal, or close to zero signal. The NEP is the ratio of the output noise $N$ to the responsivity $R$ of the radiation thermometer, usually at an electrical bandwidth of $1 \mathrm{~Hz}$,

$$
N E P=\frac{N(\text { output })}{R} .
$$

The output noise can be obtained as the standard deviation (STD) of the thermometer photocurrent (output voltage per photocurrent-to-voltage gain). The unit of NEP is $\mathrm{W} \cdot \mathrm{Hz}^{-}$ $1 / 2$. The detectivity $D^{*}$ of the thermometer (comprised of the radiation detector and the preamplifier) can be calculated from the NEP, 


$$
D^{\star}=\frac{\sqrt{A}}{N E P},
$$

where $A$ is the active area of the detector. The unit of detectivity is $\mathrm{cm} \cdot \mathrm{Hz}^{1 / 2} \cdot \mathrm{W}^{-1}$.

In traditional broad-band detector applications, where the background-produced photocurrent is high, the photocurrent-to-voltage gain (a component of $R$ ) cannot be increased to high values without saturating the photocurrent-to-voltage converter, but the low $R$ will result in a high NEP. The lower background signal of SW-IR detectors makes it possible to increase the photocurrent-to-voltage gain in the preamplifier of the radiometer.

The NETD of ambient thermal detectors is higher than $0.1 \mathrm{~K}$, which makes them difficult to use for low-uncertainty measurements in many applications, such as for ear thermometers [1]. The responsivity of such detectors, i.e. thermopiles and pyroelectric detectors, is low. For example, the power responsivity of a pyroelectric detector is 6 orders of magnitude lower than that of InGaAs (including extended-InGaAs) detectors, resulting in 6 orders of magnitude higher NEP.

Detectors with high responsivity for the SW-IR and low responsivity (high rejection) for the long-wave infrared (LW-IR) should be used to achieve low NEP and NETD in radiation thermometers. In order to obtain maximum responsivity, the feedback resistance of the photocurrent-to-voltage converter should be equal to the shunt resistance of the detector. Also, the output noise, $N$, should be minimized.

\section{DECREASE OF THERMOMETER OUTPUT NOISE}

The output noise of a thermometer can be decreased in a few ways. The dominating noise components are the amplified $1 / f$ noise of the current measuring preamplifier, the resistor (Johnson) noise (which is obtained from the parallel combination of the detector shunt resistance and the feedback resistance of the current meter), and the remaining background noise [2]. These three noise components must be minimized to achieve low values of NEP. This is in contrast to the dominantly background-noise-limited operational mode of traditional mid-IR and long-IR thermometers.

The $1 / f$ noise can be significantly decreased using AC signal techniques. In this case, the signal frequency is much higher than in DC mode measurements. The signal frequency should be selected near the elbow of the $1 / f$ noise spectrum of the current-tovoltage converter (preamplifier). A high photodiode shunt resistance can keep the closedloop voltage gain of the photocurrent-to-voltage converter low [3], resulting in low noise at the output of the radiometer. The noise of the remaining background signal can be further decreased using a metal detector case with a built-in thermoelectric (TE) cooler and a cold field-of-view (FOV) limiter for the detector. The side of the metal can, viewed by the detector, should remain at room temperature to avoid radiating excess heat onto the detector. The achievable signal-to-noise ratio is determined by the performance of the first-stage amplifier (preamplifier), which includes the detector and the current-tovoltage converter. As the noise is electrical bandwidth dependent, it can be further reduced if the AC signal of the radiometer (thermometer) output is measured with a lockin amplifier that has a large time-constant low-pass filter. 


\section{PHOTOCURRENT MEASUREMENT}

In order to describe the effects of detector and feedback impedances and noise sources in a photodiode current meter, the equivalent circuit must be discussed. As shown in Fig. 1, a photodiode $\mathrm{P}$ can be substituted by an ideal current source (double circle) and parallelconnected shunt resistance $R_{\mathrm{S}}$ and junction capacitance $C_{\mathrm{J}}$. The photocurrent $I_{\mathrm{P}}$ from photodiode $\mathrm{P}$ is converted into a voltage $V$ at the output of the operational amplifier (OA). $R$ is the feedback resistance and $C$ is the feedback capacitance of the OA. $I_{\text {IN }}$ is the input current noise and $V_{\mathrm{VN}}$ the input voltage noise of the OA. The resistor noise that originates from the parallel connection of $R$ and $R_{\mathrm{S}}$ is not illustrated here to keep the equivalent circuit simple. The resistor noise will be superimposed on $V$ without any amplification.

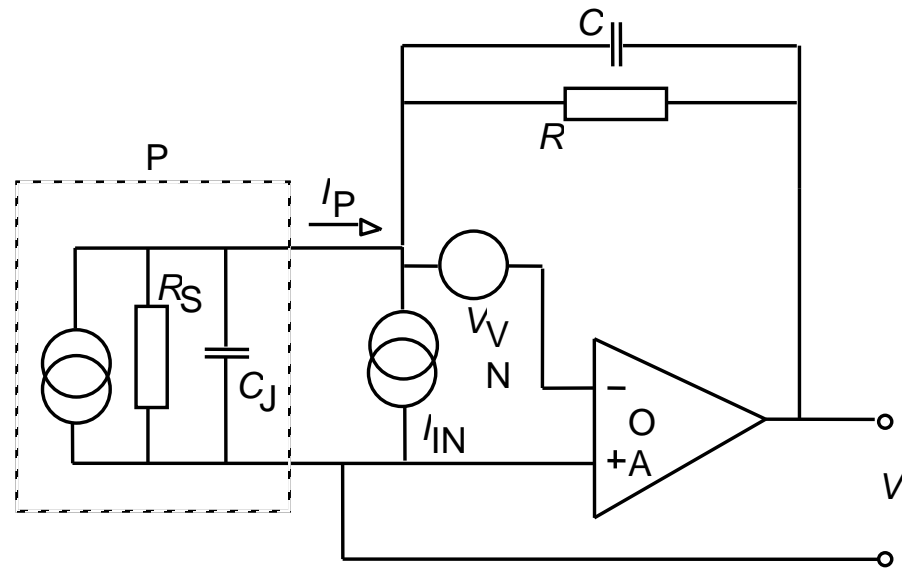

Fig. 1. Equivalent circuit of a photodiode current meter. The photodiode $\mathrm{P}$ is denoted by the dotted line.

The signal gain (current-to-voltage conversion) is the following:

$$
\frac{V}{I_{\mathrm{P}}}=R\left(\frac{1}{1+j \omega R C}\right)\left(\frac{1}{1+G^{-1}}\right)
$$

where $R C$ is the time constant produced by the feedback components. It determines the upper frequency roll-off of the signal. $G$ is the loop gain and it must be large at the signal (i.e. the chopping) frequency to keep the uncertainty of the current-to-voltage gain low. The loop gain (the product of the OA open-loop gain $A$ and the feedback attenuation $\beta$ ) is

$$
G=A \beta
$$

where $\beta=R_{\mathrm{S}} /\left(R_{\mathrm{S}}+R\right)$ at low frequency, i.e. as with DC measurements. High loop gain can be obtained if $\beta$ is close to unity (its maximum value). To achieve this condition, the shunt impedance, $Z_{S}$ ( $\equiv R_{S} / 1+j \omega R_{S} C_{S}$ ), must be high at the signal frequency. $C_{S}$ is the shunt capacitance of the photodiode.

The closed-loop voltage gain that determines the amplification of the input noise, offset, and drift for the output of the current-to-voltage converter is the reciprocal of $\beta$ : 


$$
A_{V}=\frac{R_{S}+R}{R_{S}}
$$

Again, $A_{\mathrm{V}}$ can be kept small (close to unity) if the shunt resistance is high relative to the feedback resistance. As with the signal-gain above, both $G$ and $A_{\mathrm{V}}$ are frequency dependent [4].

When the shunt resistance is high, the feedback resistor of the current-to-voltage amplifier (that determines the signal gain) can be high as well.

The input impedance of the above photocurrent meter can be calculated as the parallel connection of the down-transformed feedback resistor and the up-transformed feedback capacitor of the OA:

$$
\left(Z_{1}\right)^{-1}=\left(\frac{R}{A}\right)^{-1}+\left(\frac{1}{j \omega A C}\right)^{-1}
$$

Linear signal-gain (response) can be achieved if the short-circuit photocurrent of the photodiode is measured. In this case, the photodiode shunt impedance must be much larger than the input impedance of the current meter:

$$
Z_{1}<<Z_{P}
$$

The preamplifier should not produce any distortion in the wave-shape of the chopped optical signal. This requirement can be achieved if the upper roll-off frequency of the pre-amplifier is selected high enough:

$$
f=\frac{1}{2 \pi R C}
$$

where $R C$ is the time constant from Eq. 4. In practice, the time constant is tuned by changing $C$. In addition to affecting the frequency dependence of the signal gain, changing $C$ will modify the frequency dependence of $G$ and $A_{\mathrm{V}}$ as well. Therefore, these three gains are to be optimized together for the signal (chopping) frequency at all signalgain selections [3].

\section{SECOND-STAGE LOCK-IN AMPLIFIER}

A large signal-to-noise ratio, as the result of the above discussed first-stage (detectorcurrent meter) optimization, can be further increased using a second-stage lock-in amplifier. The lock-in amplifier makes a phase-sensitive rectification of its AC input signal and filters out those signal components at frequencies other than the chopping frequency (of the optical signal to be measured). The filter of the lock-in amplifier limits the electrical bandwidth around the signal (chopping) frequency. Using a sine-wave measuring lock-in amplifier, only the fundamental frequency component of the AC input signal will be measured. The theoretical reading (conversion factor) of a sine-wave measuring lock-in amplifier is:

$$
S_{1}=\frac{H}{\sqrt{2}} \frac{4}{\pi}=0.9003 H
$$

where $H$ is half of the peak-to-peak amplitude of the square wave signal (which is equal to zero when the chopper blocks the radiation). This conversion factor is not always 
accurate. It can be calibrated against a digital voltmeter (DVM) in DC mode if the input signal has a large signal-to-noise ratio. If the chopper is stopped, the DVM reading will be $S_{2}=2 H$. The real correction factor will be the ratio of the lock-in amplifier reading to the DVM reading.

In many cases, the frequency-dependent responsivity of a detector-preamplifier standard is measured during its calibration and the DC responsivity is reported. When this detector-preamplifier standard is used in an application where the chopper and the lock-in amplifiers are different, the frequency-dependent responsivity is measured again with these different instruments and the DC responsivity is extended to the signal frequency of this application using the measured curve. Using the theoretical conversion factor $S_{1}$, the DC responsivity is $2 / 0.9003=2.22$ times higher than the value measured by the lock-in amplifier.

\section{DESIGN OF SENSITIVE RADIATION THERMOMETERS}

The wavelength interval for a sensitive radiation thermometer should be properly selected to obtain high ratios of signal from the target to that of the background. Figure 2 shows different blackbody radiance curves for target temperatures ranging from $-63{ }^{\circ} \mathrm{C}$ to $850{ }^{\circ} \mathrm{C}$. The graph shows that at long wavelengths the target-signal to background-signal (contrast) ratios are much smaller than in the atmospheric window between $2 \mu \mathrm{m}$ and $2.5 \mu \mathrm{m}$ where the SWIR detectors operate. The vertical lines show orders of magnitude increase in the contrast ratio when the thermometer measures at $2.25 \mu \mathrm{m}$ instead of $4 \mu \mathrm{m}$.

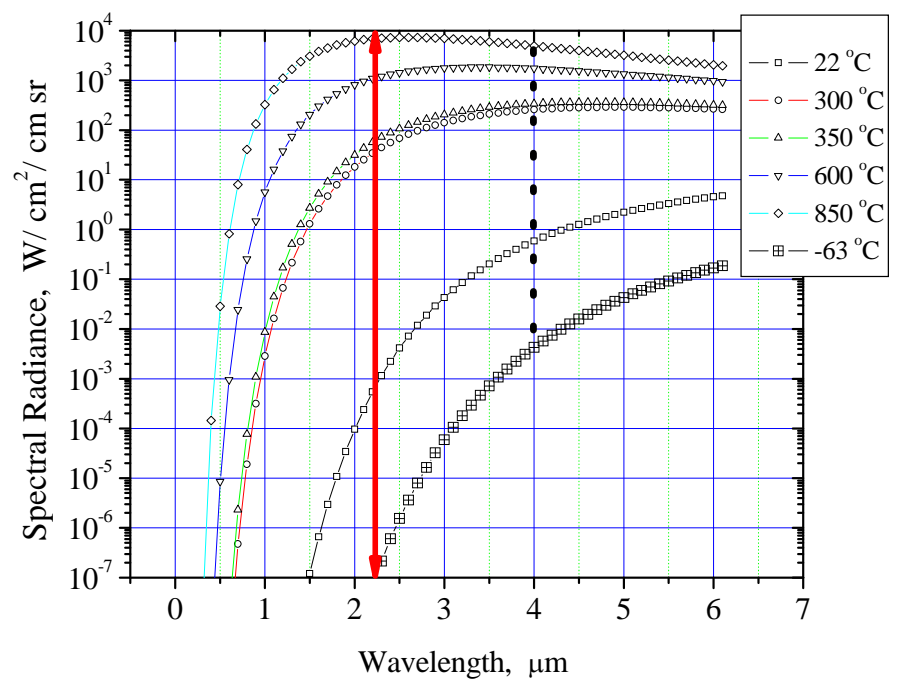

Fig. 2. Planck radiance curves between $-63^{\circ} \mathrm{C}$ and $850{ }^{\circ} \mathrm{C}$. The target-signal to background-signal ratios are shown with vertical lines at $2.25 \mu \mathrm{m}$ and $4 \mu \mathrm{m}$.

Figure 2 also shows that the target signal at $2.25 \mu \mathrm{m}$ is about an order of magnitude smaller than at $4 \mu \mathrm{m}$ or longer. Therefore, detectors with high $\mathrm{D}^{*}$ should be selected for the atmospheric window between $2 \mu \mathrm{m}$ and $2.5 \mu \mathrm{m}$. Figure 3 shows the 
spectral power responsivity of an extended-InGaAs (E-IGA) detector. It can be seen that the responsivity is high in the $2 \mu \mathrm{m}$ to $2.5 \mu \mathrm{m}$ wavelength range.

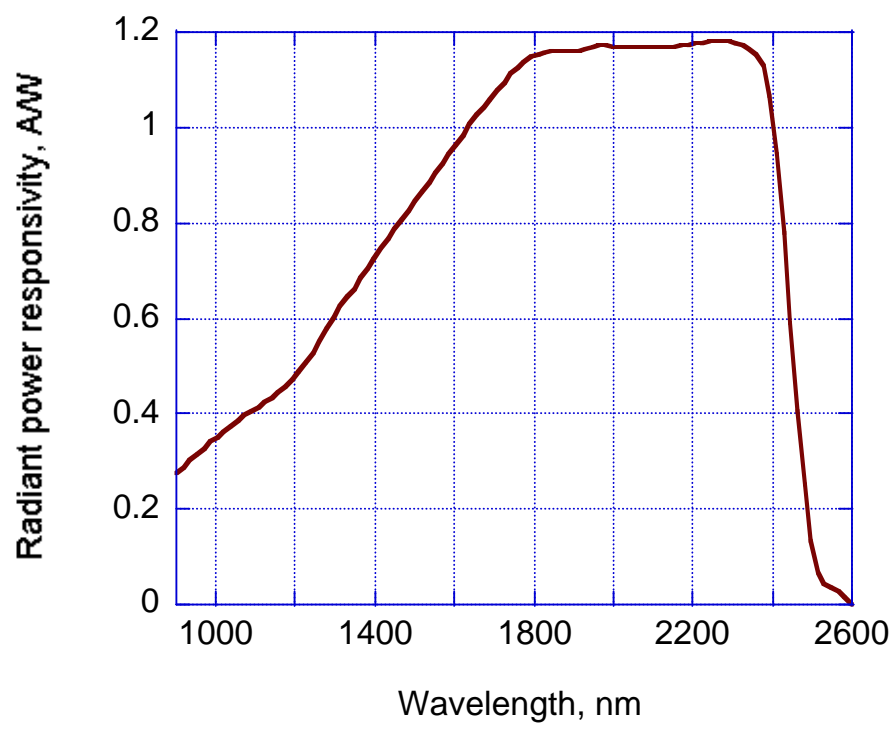

Fig. 3. Spectral power responsivity of an extended-InGaAs detector.

Figure 4 shows the wavelength dependence of $\mathrm{D}^{*}$ of several high radiometric quality detector-preamplifier units (radiometers), such as Si and InGaAs (near-IR) radiometers and different infrared radiometers, including SW-IR such as E-IGA and SWHgCdTe (SW-MCT), and mid-wave infrared (mid-IR) InSb.

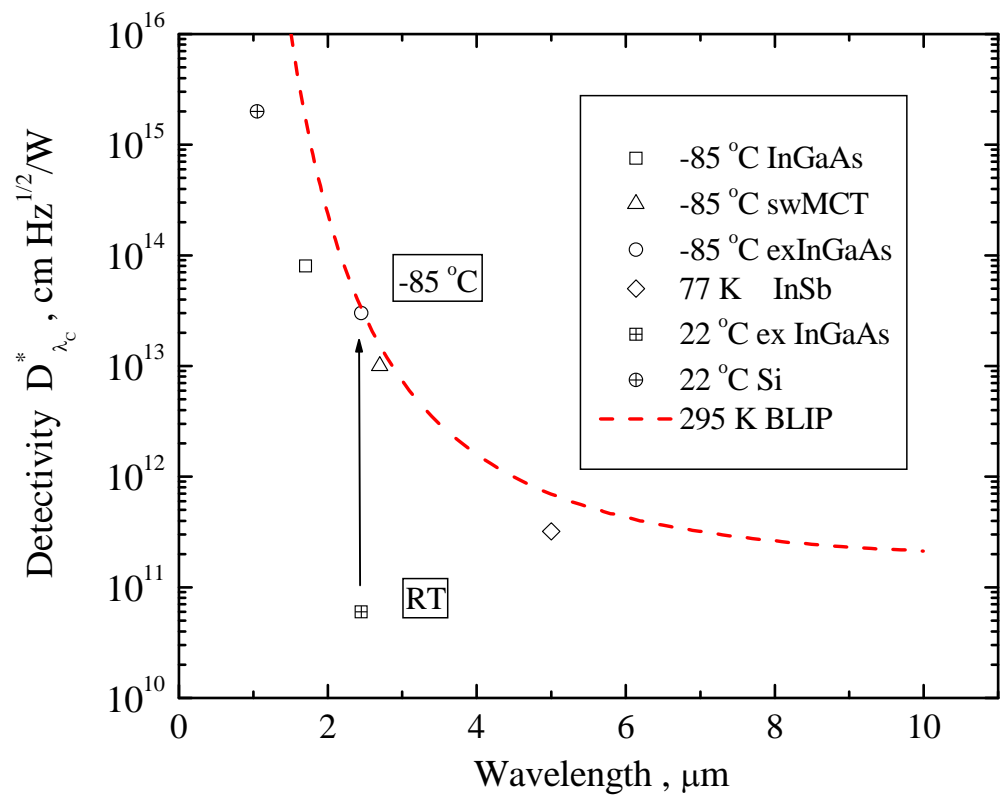

Fig. 4. D* of silicon, near-IR, SW-IR, and mid-IR radiometers with the backgroundlimited power curve at $295 \mathrm{~K}$ calculated with an $\mathrm{f} / 2$ detector FOV and an electrical bandwidth of $0.16 \mathrm{~Hz}$. 
The background-limited power (BLIP) curve, which gives the theoretical limit for the $D^{*}$ [5], was calculated with an $f / 2$ detector field-of-view (FOV) for a $295 \mathrm{~K}$ background radiation. The graph shows that the $\mathrm{D}^{*}$ of a room-temperature E-IGA radiometer was increased by almost three orders of magnitude when the detector was cooled down to $-85^{\circ} \mathrm{C}$. As a result of equalizing the three dominant noise components, the obtained $\mathrm{D}^{*}$ value of $3 \times 10^{13} \mathrm{~cm} \cdot \mathrm{Hz}^{1 / 2} \cdot \mathrm{W}^{-1}$ is very close to the background noise theoretical limit. The SW-MCT radiometer, with a $2.8 \mu \mathrm{m}$ cut-off, is also very close to the BLIP curve. The $\mathrm{D}^{*}$ of an InSb radiometer (with optimized background rejection) is about two orders of magnitude lower. The Si and InGaAs radiometers have very high $\mathrm{D}^{*}$ values but they are not suited for detection of objects at ambient temperatures since there is insufficient signal at these wavelengths (the BLIP is $>10^{18} \mathrm{~cm} \cdot \mathrm{Hz}^{1 / 2} \cdot \mathrm{W}^{-1}$ ).

Thermometers with high $\mathrm{D}^{*}$ (high responsivity and low noise) can be developed if detectors with high shunt resistance are used. The shunt resistance of a $1 \mathrm{~mm}$ diameter SW-IR detector can be increased to about $10 \mathrm{M} \Omega$ from $10 \mathrm{k} \Omega$ (at room temperature) when it is cooled to about $-70{ }^{\circ} \mathrm{C}$ or lower [6]. Instead of cryogenic cooling, which is traditionally used for InSb detectors, four-stage thermo-electric (TE) coolers can be used.

\section{SW-IR RADIATION THERMOMETER CONSTRUCTION}

Using the above design considerations, an experimental radiation thermometer was constructed to determine the NETD as a function of blackbody temperature. Since optical glasses transmit at these wavelengths, regular achromats were used as objective lenses in the prototype shown in Fig. 5. A variable-temperature blackbody set to room

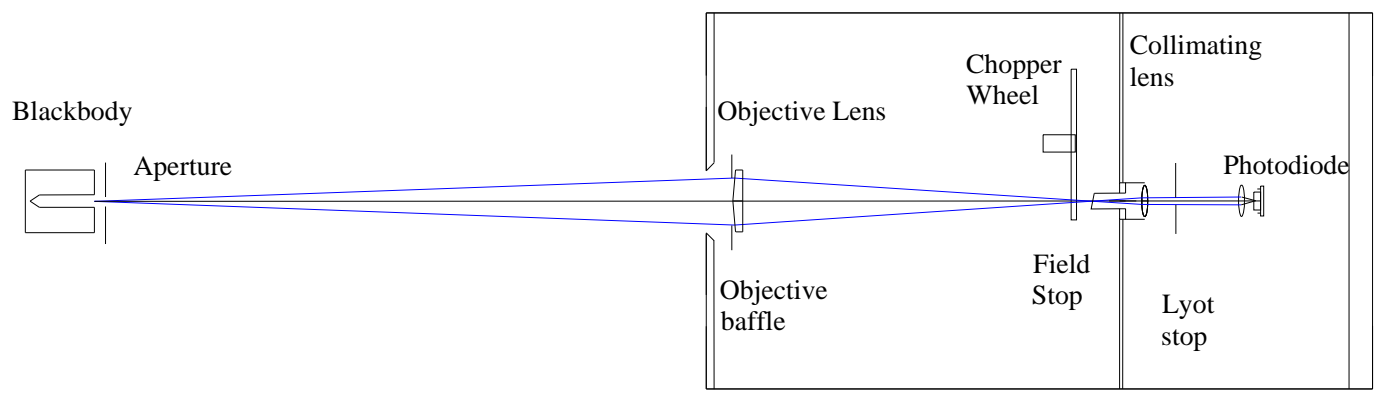

Fig. 5. SW-IR radiation thermometer construction.

temperatures and higher was used as the source of radiation. A $6 \mathrm{~mm}$ diameter target was imaged by the radiation thermometer at a distance of $50 \mathrm{~cm}$ between the source aperture and the front of the objective lens. The radiation was imaged onto a field stop with a chopper wheel placed close to the field stop. The radiation was collimated and focused onto a $1 \mathrm{~mm}$ diameter detector with achromats. The detector was underfilled by the image. The extended-InGaAs detector was cooled to $-85{ }^{\circ} \mathrm{C}$ with $\mathrm{TE}$ cooling. The detector was attached to a short-circuit photocurrent meter built with a low-noise operational amplifier. The Lyot stop [7] serves as the aperture stop behind the field stop. 
Due to the low-scatter objective and the Lyot stop, the thermometer has excellent out-offield rejection that enables internal chopping.

\section{NEP AND NETD MEASUREMENT RESULTS}

The output total noise voltage was measured versus signal-gain selections for a $3 \mathrm{~mm}$ (3 $\mathrm{M} \Omega$ shunt resistance) extended-InGaAs photodiode current-meter. The temperature of the detector was controlled to $-85{ }^{\circ} \mathrm{C}$ using a 4-stage TE cooler. The noise equivalent current (NEC) is equal to the measured total noise voltage divided by the current-tovoltage gain (the value of the feedback resistor). The total noise voltage was measured by a lock-in amplifier attached to the output of the photocurrent meter. The chopping frequency was $7.5 \mathrm{~Hz}$ and the integrating time constant of the lock-in amplifier was $1 \mathrm{~s}$. The signal source was a $314{ }^{\circ} \mathrm{C}$ blackbody located about $50 \mathrm{~cm}$ from the radiometer. The NEP was calculated as the ratio of the NEC to the detector (peak) power responsivity of $1.2 \mathrm{~A} / \mathrm{W}$ (at $2 \mu \mathrm{m}$ ). The NEC and NEP results are shown on the two Y-axes of Fig. 6.

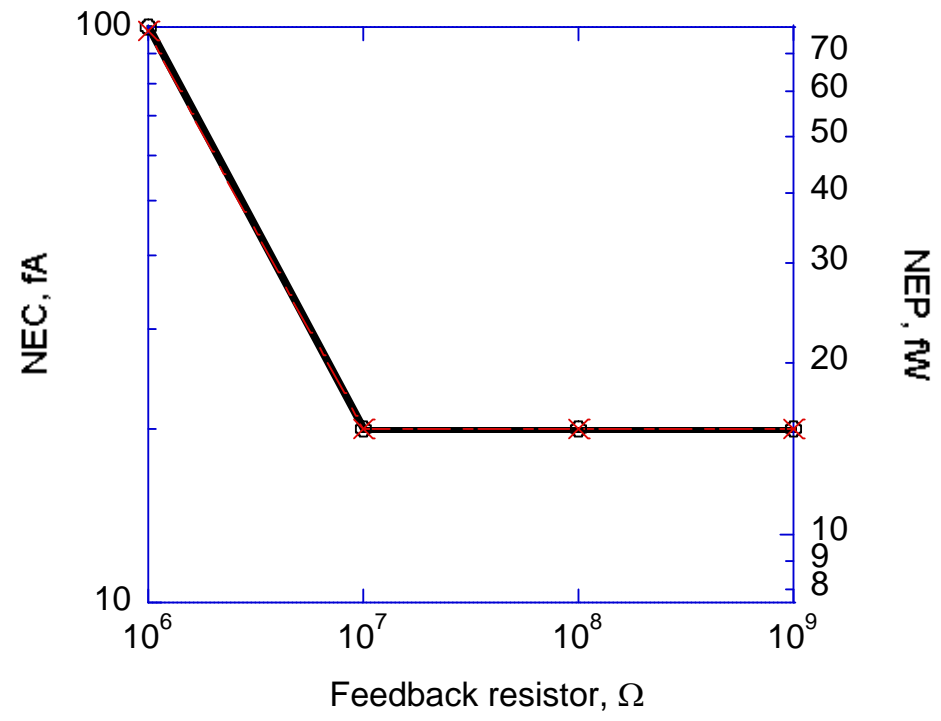

Fig. 6. NEP (right-Y) and NEC (left-Y) of a $3 \mathrm{M} \Omega$ shunt resistance E-IGA photodiode current meter at a lock-in amplifier integrating time constant of $1 \mathrm{~s}$.

The measured data points are connected with straight lines for better illustration. The graph shows that a NEP=15 fW was obtained with the $1 \mathrm{~s}$ integration time constant. The NEP of this SWIR detector is more than six orders of magnitude lower than the 50-100 $\mathrm{nW} \cdot \mathrm{Hz}^{-1 / 2} \mathrm{NEP}$ of pyroelectric detectors. The $15 \mathrm{fW}$ (obtained with $1 \mathrm{~s}$ time constant) NEP (and the related output signal-to-noise ratio) could not be improved at the $10^{8} \mathrm{~V} \cdot \mathrm{A}^{-1}$ and $10^{9} \mathrm{~V} \cdot \mathrm{A}^{-1}$ signal-gains relative to the $10^{7} \mathrm{~V} \cdot \mathrm{A}^{-1}$ selection where the shunt resistance is roughly equal to the feedback resistance. At gain selections of $10^{7} \mathrm{~V} \cdot \mathrm{A}^{-1}$ and higher, the constant $3 \mathrm{M} \Omega$ shunt resistance dominates the resistor noise. The $10^{8} \mathrm{~V} \cdot \mathrm{A}^{-1}$ and higher signal gains should be utilized only with higher than $3 \mathrm{M} \Omega$ shunt resistance detectors 
because the loop gain decreases and there is no further signal-to-noise advantage. Higher shunt resistances of $10 \mathrm{M} \Omega$ - $15 \mathrm{M} \Omega$ were measured for $1 \mathrm{~mm}$ diameter E-IGA and SWMCT detectors. In these cases, the NEP can be further decreased.

At a blackbody temperature of $50{ }^{\circ} \mathrm{C}$, a NETD of $<3 \mathrm{mK}$ was obtained from the standard deviation of the temperature measurements taken with an electrical bandwidth of $0.16 \mathrm{~Hz}$.

The NETD plotted as a function of the blackbody temperature is shown in Fig. 7. The NETD increases rapidly as the target temperature becomes comparable to the chopper-wheel temperature, but at the human body temperature of $36{ }^{\circ} \mathrm{C}$, the NETD is $10 \mathrm{mK}$. These results demonstrate that the NETD is comparable to that of InSb detectors for blackbody temperatures $>25^{\circ} \mathrm{C}$ without the need for cryogenic cooling.

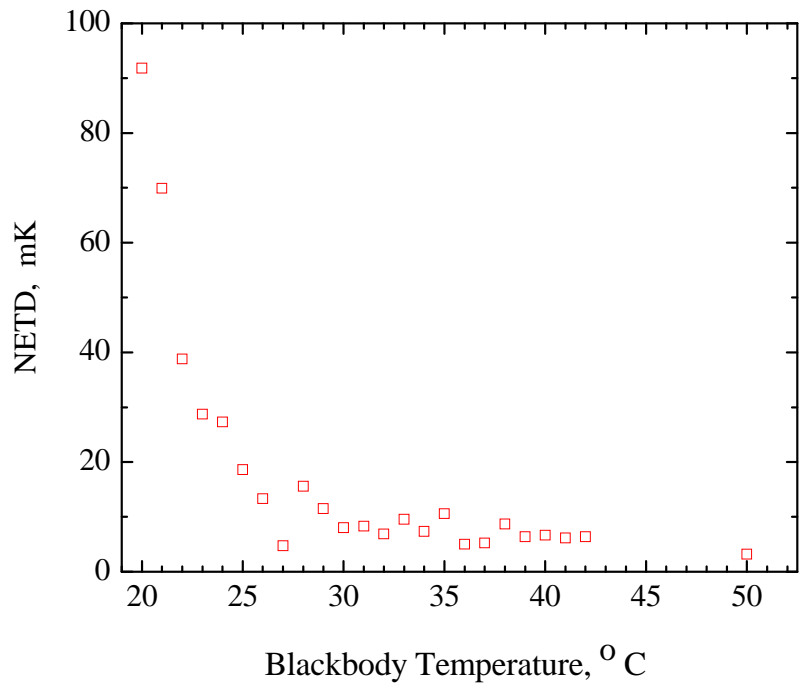

Fig. 7. NETD of the experimental radiation thermometer versus target temperature.

\section{CONCLUSIONS}

Thermoelectrically-cooled SW-IR detectors attached to noise-optimized photocurrent meters can be incorporated into radiation thermometers utilizing traditional achromats to measure objects at human-body temperatures. The SW-IR thermometers operate in the $2 \mu \mathrm{m}$ to $2.5 \mu \mathrm{m}$ atmospheric window where the target signal is lower than in the mid-IR. However, the target-signal to background-signal ratio is orders of magnitude higher than in the mid-IR or long-IR ranges. The NEP of the SW-IR thermometers is more than six orders of magnitude lower than that of pyroelectric thermometers. An optically- and electronically-optimized SW-IR radiation thermometer was designed, built, and tested. Regular glass input optics are used and the incident radiation is chopped. The achieved NEP was $15 \mathrm{fW}$ (with $1 \mathrm{~s}$ integrating time constant) and the NETD increased from $3 \mathrm{mK}$ at $50{ }^{\circ} \mathrm{C}$ to $10 \mathrm{mK}$ at $36{ }^{\circ} \mathrm{C}$. While standards-quality radiation thermometers with high sensitivity are not presently available, the described SW-IR thermometer can measure ambient temperature sources, such as water-bath or tin-point blackbodies, with very low 
uncertainties. With this suggested radiation thermometer standard, the temperature of the human body can be measured with a NETD of $10 \mathrm{mK}$, which is about an order of magnitude improvement over existing ear-thermometers. These results indicate that transfer standard radiation thermometers for measurements of human-body temperatures can be constructed without utilizing cryogenic or thermal detectors.

\section{REFERENCES}

1. I. Pusnik, E.van der Ham, J. Drovsek, Physiol. Meas. 25, 699 (2004)

2. $\quad$ G.P. Eppeldauer, A.L. Migdall, L.M. Hanssen, Metrologia 35, 485 (1998)

3. G.P. Eppeldauer, Optical radiation measurement with selected detectors and matched electronic circuits between $200 \mathrm{~nm}$ and $20 \mu \mathrm{m}$. NIST Technical Note, 2001, 1438 (U.S. Government Printing Office).

4. $\quad$ G.P. Eppeldauer, J. Res. NIST, 103, 153 (1998)

5. A. Rogalski, K. Chrzanowski, Opto-Electronics Review 10, 111 (2002)

6. H.W. Yoon, M.C. Dopkiss, G.P. Eppeldauer, Performance comparisons of InGaAs, extended InGaAs and short-wave HgCdTe detectors between 1 and 2.5 micrometers. SPIE Proceedings, 2006. 6297: p. 629703-1 to 629703-10.

7. H.W. Yoon, D.W. Allen, R.D. Saunders, Metrologia 42, 89 (2005) 


\title{
System-level calibration of a transfer radiometer used to validate EOS radiance scales
}

\author{
B. C. JOHNSON*, S. W. BROWN, G. P. EPPELDAUER and \\ K. R. LYKKE
}

National Institute of Standards and Technology, Optical Technology Division, Gaithersburg, MD 20899, USA

\begin{abstract}
A number of transfer radiometers have been developed by the National Institute of Standards and Technology (NIST) with the support and funding of the National Aeronautics and Space Administration's (NASA's) satellite sensor calibration programmes. The transfer radiometers travel to NASA satellite builder and calibration facilities and validate the radiance scale of calibration sources at those facilities. One of these instruments, the Earth Observing Systems (EOS) Visible Transfer Radiometer (VXR), was calibrated for spectral responsivity in different facilities at NIST using two types of integrating sphere sources (ISSs): an incandescent lamp-illuminated ISS and a laser-illuminated ISS. The laserilluminated ISS results in reduced uncertainties and a greater dynamic range in radiance responsivity. We will discuss the calibration procedures using the two types of source, compare results of calibrations of two of the VXR filter channels in the two NIST facilities, and suggest additional irradiance and radiance responsivity calibrations that are now possible using the new laser-illuminated facility.
\end{abstract}

\section{Introduction}

The National Institute of Standards and Technology (NIST) has undertaken a role to support the Earth Observing System (EOS) calibration programme of the National Aeronautics and Space Administration (NASA). The EOS programme is a major component of NASA's Earth Sciences Enterprise, which is, in turn, a significant component of the US Global Change Research Program. The EOS programme utilizes space-based and ground-based measurement systems to provide the scientific basis for understanding global change. The unprecedented accuracies and long-term stability of the measurements required place new and stringent demands upon the metrology systems that underpin the effort. One of the requirements of the EOS calibration programme is to document the relationship between radiometric quantities as realized by NIST with those realized by EOS participants, who often use NIST-traceable radiometric standards (Butler and Barnes 1998).

To accomplish this task, NIST has developed a number of portable artefacts for establishing the shortest possible measurement chains to the EOS community. These include transfer radiometers for the visible (Johnson et al. 1998), the short-wave

*e-mail: cjohnson@nist. gov.

Paper presented at the Conference on Characterization and Radiometric Calibration for Remote Sensing held at Utah State University, Logan, 9-11 November 1999.

International Journal of Remote Sensing

ISSN 0143-1161 print/ISSN 1366-5901 online (C) 2003 Taylor \& Francis Ltd

http://www.tandf.co.uk/journals DOI: $10.1080 / 01431160110113205$ 
infrared (SWIR; Brown, et al. 1998), and the thermal infrared (Rice and Johnson 1998, Rice et al. 2002) wavelength regions as well as a portable radiance source (Brown and Johnson 1999, 2002). The transfer artefacts travel to NASA instrument builder and calibration facilities to verify the radiance of sources used to calibrate the responsivity of air-and space-borne sensors. They therefore serve as a direct link from the instrument builder and NASA calibration facilities to the national radiometric scales maintained at NIST.

This strategy has been successfully implemented at a number of facilities and locations (Sakuma et al. 1996, Johnson et al. 1997, Butler et al. 1999). The comparisons in Japan in 1995 for the support of the Ocean Color and Temperature Scanner (OCTS) and the visible radiometer for the Advanced Spaceborne Thermal Emission and Reflection Radiometer (ASTER) are of particular interest because they related radiometric scales from two national metrological institutes. For these comparisons, the agreement between the results with the transfer radiometers and the values determined by the manufacturer (NEC Corporation) was within $\pm 4 \%$ (Sakuma et al. 1996, Johnson et al. 1997).

A more involved approach was used with the Sea-viewing Wide Field-of-view Sensor (SeaWiFS) in 1997 (Johnson et al. 1999). In addition to the radiometric calibration performed by the instrument manufacturer, a pre-flight radiometric calibration was done at the spacecraft integrator's facility using an integrating sphere source that was calibrated at NIST (Early and Johnson 1997). The radiance of this source at the time of the SeaWiFS pre-flight calibration was determined by using the SeaWiFS transfer radiometer (SXR) (Johnson et al. 1998) to relate the spectral radiance of the source from the NIST calibration (in April 1995) to the time of the SeaWiFS measurements (in spring 1997). Including sphere degradation effects, radiance non-uniformity and other sources of additional uncertainty, the SeaWiFS radiance responsivity was determined with standard uncertainties of $1.2-3.3 \%$, depending on the instrument channel (Johnson et al. 1999).

On-orbit solar and ocean colour measurements confirmed the SeaWiFS prelaunch calibration strategy. Solar-based transfer-to-orbit experiments agreed on-orbit with predicted ground-based values to within 2.5\% (Barnes and McClain 1999). Similarly, vicarious calibrations of SeaWiFS using an ocean buoy near Hawaii under the auspices of the Marine Optical Buoy (MOBY) programme (Clark et al. 1997, Herring 1997) to measure the spectral radiance of the ocean has provided corrections of $3.2 \%$ or less to the laboratory-derived calibration coefficients (Barnes and McClain 2000). The good agreement between the pre-launch calibration and on-orbit measurements is a consequence of the thorough calibration approach used by the SeaWiFS and the MOBY programmes, in particular the direct traceability to NIST established by the transfer radiometers for the satellite sensor calibration and during field calibrations of radiometers used at the MOBY site (Cromer and Johnson 1996).

Owing to the increasing constraints in the uncertainties of on-orbit radiometric measurements by satellite sensors, it is desirable to calibrate the transfer radiometers with the lowest possible uncertainty. For example, the Moderate Resolution Imaging Spectroradiometer (MODIS) requires on-orbit radiometric uncertainties* of 5\% or less in radiance and $2 \%$ or less in reflectance over the spectral range $412-2130 \mathrm{~nm}$

*The coverage probability is not stated explicity but corresponds to an estimated standard deviation. 
(Guenther et al. 1996). For an on-orbit satellite sensor standard uncertainty in radiance responsivity of $5 \%$, we would like to validate the calibration source radiance in the laboratory with standard uncertainties of $1-2 \%$. Current uncertainties in the spectral responsivity of transfer radiometers in the visible and near-infrared (VNIR) approach those target levels (Butler et al. 2000), while they are between 2\% and 4\% in the SWIR (Yoon et al. 1998), so reduction in the uncertainties of the SWIR radiometers is important to achieve the laboratory calibration goals.

To understand better the origin of our calibration uncertainties, and in an attempt to reduce those uncertainties, we calibrated the EOS Visible Transfer Radiometer (VXR) using two approaches relying on very different types of integrating sphere sources (ISS): a typical incandescent lamp-illuminated ISS and a laser-illuminated ISS. The lamp-illuminated source was calibrated for spectral radiance on the NIST Facility for Automated Spectral Radiance Calibrations (FASCAL) (Walker et al. 1987); the radiance scale in this facility is derived from a variable temperature black body that is traceable to a gold-point black body. This method was the only one available at NIST until recently.

The new laser-illuminated ISS takes advantage of the recent technological advances in the development of laser sources. Sources are now available to cover the spectral range from the ultraviolet (below $0.2 \mu \mathrm{m})$ to the infrared $(20 \mu \mathrm{m})$. We have taken advantage of these laser sources in a new facility termed the Spectral Irradiance and Radiance response Calibrations with a Uniform Source (SIRCUS) (Lykke et al. 1998, Brown et al. 2000, Eppeldauer et al. 2000). In this facility, the output from a variety of lasers is directed into an integrating sphere. The integrating sphere serves as a uniform, monochromatic, Lambertian source for both irradiance and radiance response calibrations. Calibrations in the SIRCUS facility are based on the direct substitution method using detectors whose responsivity is traceable to the NIST High Accuracy Cryogenic Radiometer (HACR) (Gentile et al. 1996a, b).

A schematic diagram of the VXR is shown in figure 1. An objective lens forms an intermediate image at the field stop. Wedged mirrors reflect the field stop onto the six different filter channels. Each VXR channel consists of a narrow-band interference filter and a silicon photodiode; channel band-moment wavelengths and

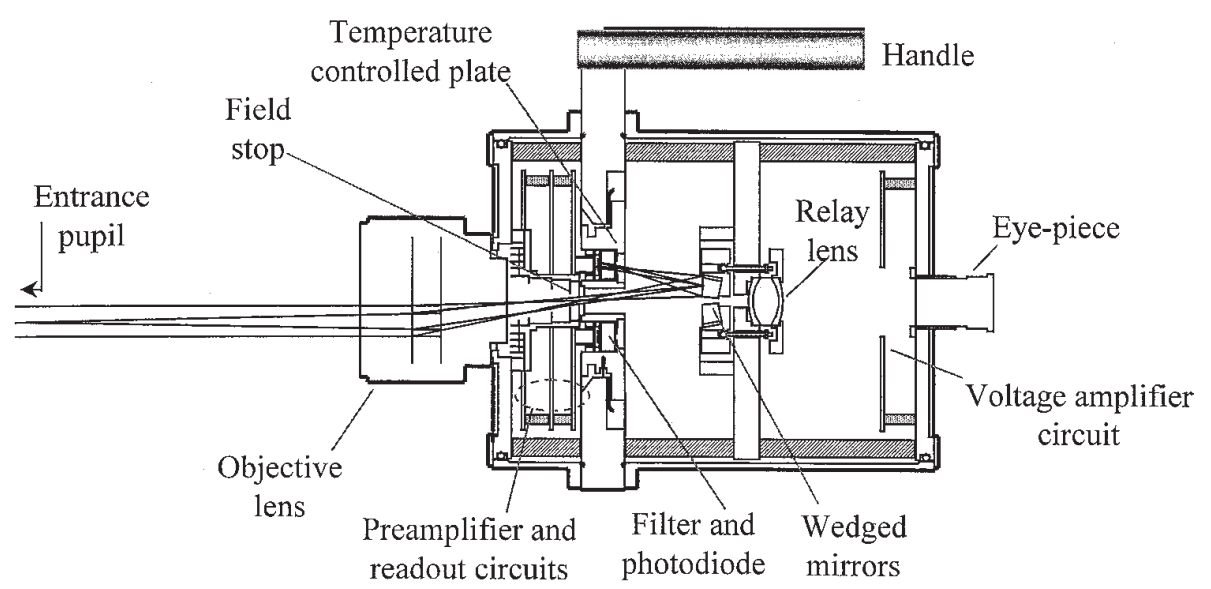

Figure 1. Schematic diagram of the VXR, with the critical components labelled. The side view of the optical system is shown using marginal and chief rays for a single channel. 
full-width-square (FWS) bandwidths are given in table 1. (The band-moment wavelengths are a weighted average using the relative spectral responsivity of the channel for the weight.) The interference filters, photodiodes, and the precision aperture that functions as the field stop are all mounted on a temperature-controlled plate. During operation, they are maintained at $26^{\circ} \mathrm{C}$. A relay lens images the field stop onto an eyepiece used to align the VXR to its target.

We will describe the calibration strategies using two ISSs and compare calibrations of two VXR filter channels using the different approaches. We will then discuss future applications of the laser-illuminated ISS.

\section{Calibration using a lamp-illuminated ISS}

The measurement of a broadband source for one channel of the VXR is described by a simplified measurement equation:

$$
S=A \Omega G \int \tau(\lambda) R_{\mathrm{f}}(\lambda) L_{\lambda}(\lambda) d \lambda
$$

where $S$ is the output signal (V), the product $A \Omega$ is the throughput $\left(\mathrm{m}^{2} \mathrm{sr}\right), G$ is the (fixed) gain of the transimpedance pre-amplifier $\left(\mathrm{V} \mathrm{A}^{-1}\right), \tau(\lambda)$ is the spectral transmittance of the optical system (dimensionless), $R_{\mathrm{f}}(\lambda)$ is the spectral flux (subscript $\mathrm{f}$ for flux) responsivity of the silicon photodiode $\left(\mathrm{A} \mathrm{W}^{-1}\right), L_{\lambda}(\lambda)$ is the spectral radiance of the ISS ( $\mathrm{W} \mathrm{m}^{-2} \mathrm{sr}^{-1} \mathrm{~nm}^{-1}$ ), and $\lambda$ is the wavelength (nm). The variables $A \Omega, G$, $\tau(\lambda)$, and $R_{\mathrm{f}}(\lambda)$ depend on the VXR channel under consideration. The output $S$ is then the integrated response for the channel.

Let the function $f(\lambda)=\tau(\lambda) R_{\mathrm{f}}(\lambda)$ represent the spectral portion of the VXR response. Also define the relative spectral responsivity $r\left(\lambda, \lambda_{j}\right)=f(\lambda) / f\left(\lambda_{j}\right)$, where the reference wavelength $\lambda_{j}$ is arbitrary but usually near the value that results in the maximum value of $f(\lambda)$. The equation (1) can be written as

$$
S=\left(A \Omega G f\left(\lambda_{j}\right) \int r\left(\lambda, \lambda_{j}\right) d \lambda\right) \frac{\int r\left(\lambda, \lambda_{j}\right) L_{\lambda}(\lambda) d \lambda}{\int r\left(\lambda, \lambda_{j}\right) d \lambda}
$$

The factor inside the parentheses on the right-hand side of equation (2) is the radiance responsivity (or calibration coefficient) for one channel in the VXR, and it is independent of the choice of the normalization wavelength $\lambda_{j}$. The ratio of the integrals is also independent of $\lambda_{j}$. Equation (2) and measurements of an ISS with known spectral radiance $L_{\lambda}(\lambda)$ are used to determine the calibration coefficient $D_{\mathrm{b}}$ ( $\mathrm{V} \mu \mathrm{W}^{-} \mathrm{cm}^{2} \mathrm{sr} \mathrm{nm}$ ). The subscript $\mathrm{b}$ stands for broadband.

$$
D_{\mathrm{b}}=\frac{S}{\left(\int r(\lambda) L_{\lambda}(\lambda) d \lambda\right) /\left(\int r(\lambda) d \lambda\right)^{-1}}
$$

Table 1. Channel, band-moment wavelength and full-width square bandpass of the VXR filter channels.

\begin{tabular}{lcc}
\hline Channel & $\begin{array}{c}\text { Wavelength } \\
(\mathrm{nm})\end{array}$ & $\begin{array}{c}\text { Bandpass } \\
(\mathrm{nm})\end{array}$ \\
\hline 1 & 411.77 & 10.76 \\
2 & 441.01 & 10.50 \\
3 & 548.40 & 10.21 \\
4 & 661.40 & 9.47 \\
5 & 775.47 & 11.11 \\
6 & 869.93 & 13.35 \\
\hline
\end{tabular}


Because the explicit dependence on the normalization wavelength is not necessary, $r\left(\lambda, \lambda_{j}\right)$ is written as $r(\lambda)$. Equation (3) also applies when the VXR is used to assess the accuracy of sources calibrated independently by the EOS participants. The comparison is between the actual signals obtained with the VXR to those predicted using equation (3) and the independent determination of the spectral radiance.

From equation (3), it is clear that three types of measurements are required to characterize and calibrate the VXR using the lamp-illuminated ISS: (1) determination of the spectral radiance $L_{\lambda}(\lambda)$ of the ISS; (2) determination of the relative spectral responsivity $r(\lambda)$ of the VXR channels; and (3) determination of the net VXR signal $S$ corresponding to measurements of the ISS when it is operated to produce the same radiance as during its calibration. These three steps are illustrated in figure 2 . In general, the ISS need only be calibrated at a single radiance level, but implementation of the basic scheme at multiple, independent levels provides a measure of the reproducibility of the procedure.

\subsection{ISS calibration on FASCAL}

The VXR has been calibrated using a number of lamp-illuminated ISSs, most recently the NIST Portable Radiance Source (NPR) source (Brown and Johnson 1999, 2001). The VXR measured the source a total of 10 times at NIST from January 1999 through September 1999. Monitor photodiodes mounted in the sphere were used to verify the stability of the ISS radiance over this time period (Brown and Johnson 2002).

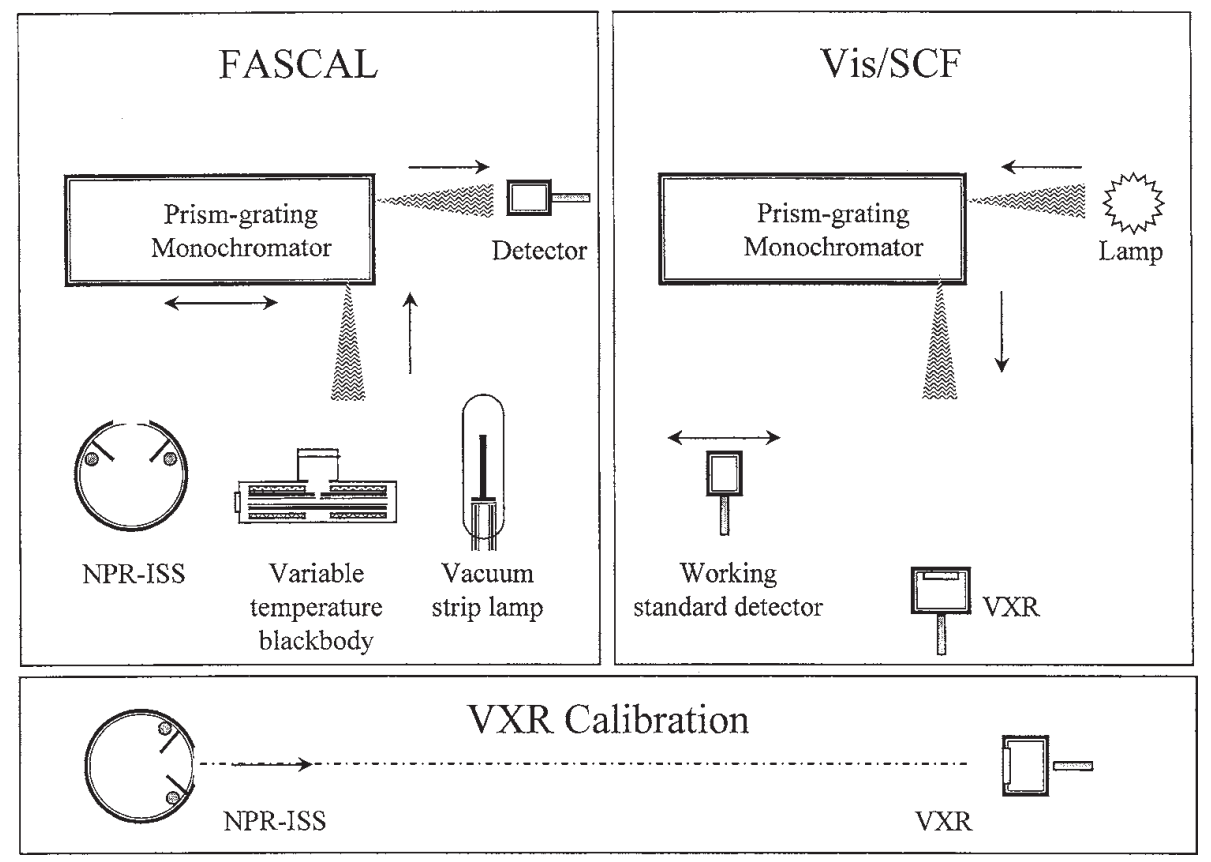

Figure 2. Calibration and characterization of the VXR using the Vis/SCF, FASCAL and the NPR ISS. The propagation of the radiant flux is indicated by the direction of the lines with single arrows; lines with double arrows indicate linear translation for the purpose of source or detector substitution. 
The NPR was calibrated for spectral radiance over the wavelength range $300-900 \mathrm{~nm}$ on FASCAL in May 1999. The primary radiometric standard in FASCAL is a fixed-point black body at the freezing temperature of gold. The spectroradiometrically determined temperature $(1337.3 \pm 0.1) \mathrm{K}(k=1)$, is in agreement with the value defined on the International Temperature Scale of 1990 (Mielenz et al. 1990). The temperature of the gold-point black body is too low to produce any measurable flux below about $500 \mathrm{~nm}$, so a higher temperature black body is used to calibrate the ISS. A number of steps are required to calibrate the higher temperature black body. First, a spectroradiometer, consisting of a prism-grating monochromator and a photomultiplier, is used to match the spectral radiance of the gold-point black body to that from a vacuum tungsten-strip lamp at $654.6 \mathrm{~nm}$. Next, the tungsten-strip lamp is used to calibrate a higher temperature, commercial, vacuum tungsten-strip lamp so that its radiance corresponds to a black body at a temperature of about $1530 \mathrm{~K}$. This lamp holds the scale, and is used to determine the temperature of a high emittance, variable temperature black body source, again at $654.6 \mathrm{~nm}$. Once the temperature of the black body is known, its spectral radiance at any wavelength can be calculated using Planck's equation.

The spectroradiometer is used to transfer the radiance scale from the variable temperature black body to the ISS. The typical measurement interval for an ISS to be used with a VNIR instrument such as the VXR is $350-900 \mathrm{~nm}$ with a step size of $10 \mathrm{~nm}$ in the ultraviolet, $50 \mathrm{~nm}$ in the visible and $100 \mathrm{~nm}$ in the SWIR. The NPR spectral radiance and the FASCAL uncertainty of the spectral radiance calibration are shown in figure 3.

\subsection{Relative spectral responsivity measurements}

The relative spectral responsivity for the VXR was determined by adaptations of the NIST Visible/Spectral Flux Comparator (Vis/SCF) (Larason et al. 1998) for

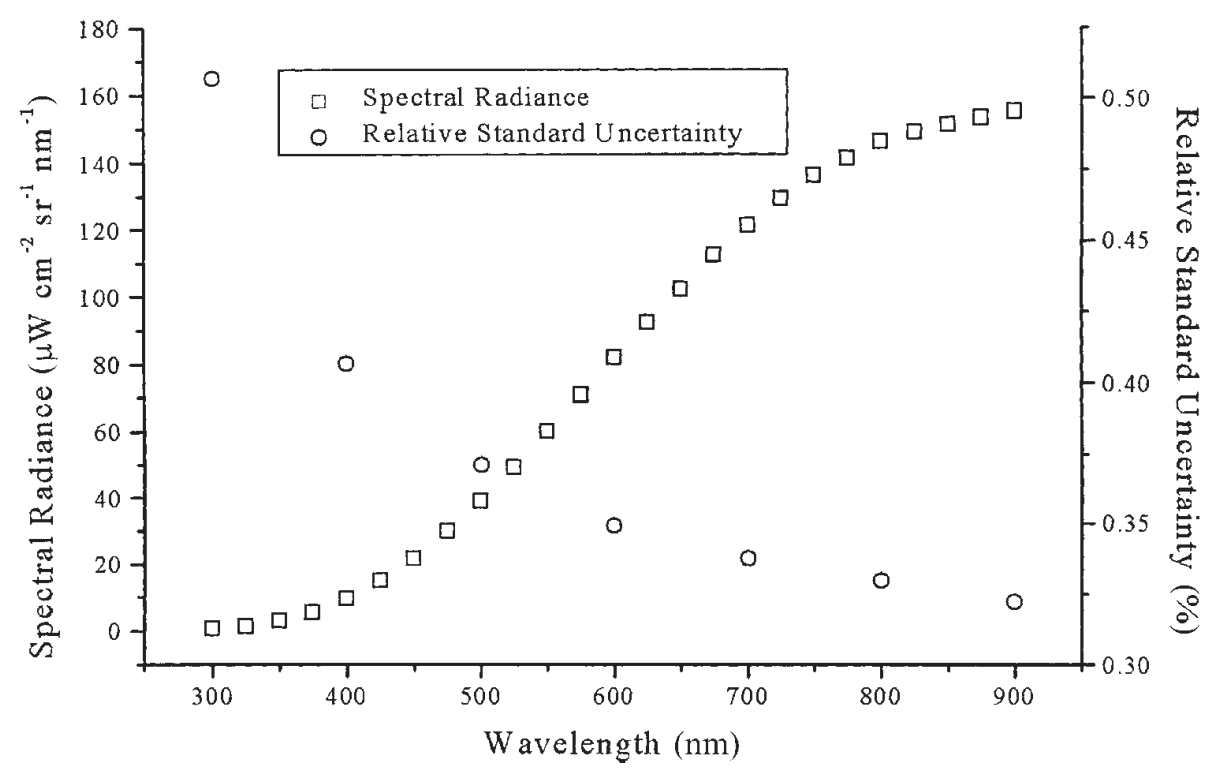

Figure 3. The spectral radiance of the NPR ISS with four lamps in operation. The values and their relative standard uncertainties, which are identified in the legend, were determined using FASCAL. 
operation in a relative mode. The Vis/SCF consists of a prism-grating monochromator illuminated by a lamp, a monitor detector, a shutter and a set of working standard detectors (silicon and germanium photodiodes). These detectors are calibrated using transfer standards (silicon photodiode trap detectors) that are calibrated against the HACR.

The relative spectral responsivity of the VXR was determined using the same procedures as described for the SXR (Johnson et al. 1998). As with the SXR, the low flux levels meant that the instrumental bandwidth could not be smaller than about $1 \mathrm{~nm}$, which is finite compared to the $10 \mathrm{~nm}$ bandwidth of the VXR channels. Because the output of the Vis/SCF did not fill the entrance pupil of the VXR, several steps were required. First, the VXR was aligned on the optical axis of the exit beam of the Vis/SCF and focused to image the intermediate image of the exit slit of the monochromator. Because the output of the monochromator is $f / 8$ by $f / 16$, this underfilled the VXR's field of view. A translation stage could be positioned between the VXR and the monochromator such that the intermediate image of the exit slit coincided with the working standard detector. A bandwidth of $1 \mathrm{~nm}$ and a step size of $0.5 \mathrm{~nm}$ was used near the centre of each channel, and a bandwidth of $4 \mathrm{~nm}$ and a step size of $3-5 \mathrm{~nm}$ was used in the wings. The monitor detector was used to reduce the variability from source fluctuations.

A second scan across the central portion of each channel was performed under conditions that filled the VXR field of view, but with poor measurement resolution due to the reduced flux. A diffuse reflectance standard was placed perpendicular to the optical axis at a distance that gave a spot size large enough to fill the VXR entrance window. The VXR was aligned to measure the radiance reflected from the diffuser. The field of view was then filled, resulting in a small wavelength shift compared to the on-axis measurements. The data acquired with the diffuser were used to correct the on-axis data for this small shift. The largest identifiable uncertainty components in the determination of the relative spectral responsivity result from the responsivity of the working standard and the effect of the $0.1 \mathrm{~nm}$ uncertainty in the $\mathrm{Vis} / \mathrm{SCF}$ wavelength calibration. The magnitude of these effects depends on the wavelength (VXR channel). A likely, but difficult to quantify, additional component of uncertainty is that associated with the procedure itself, including the finite bandwidth of the source, corrections for the field of view effects, and matching the in- and out-of-band scans.

\subsection{VXR calibration}

Given the FASCAL values for $L_{\lambda}(\lambda)$ of the ISS at one set of discrete wavelengths, and the function $r(\lambda)$ from the Vis/SCF data, the integrals in equation (1) were determined numerically using the alternative extended Simpson's rule. The wavelength step corresponded to the $r(\lambda)$ data. A commercial cubic spline routine was used to determine the values of $L_{\lambda}(\lambda)$ at these wavelengths, with the input values just encompassing the $r(\lambda)$ dataset. For example, $r(\lambda)$ for channel 1 of the VXR was measured from $380-600 \mathrm{~nm}$ and the $L_{\lambda}(\lambda)$ values for one calibration of the ISS on FASCAL at 360, 380, 400,450, 500, 550 and $600 \mathrm{~nm}$ were input to the cubic spline routine.

The relative combined standard uncertainty of the individual determinations of $D_{\mathrm{b}}$ using the NPR and the relative responsivity measurements on the Vis/SCF are given in table 2 for VXR channels 1 and 5 . The $0.1 \mathrm{~nm}$ uncertainty in the wavelength calibration of the monochromators for the $r(\lambda)$ and $L_{\lambda}(\lambda)$ measurements affects the 
Table 2. Relative combined standard uncertainty of the individual determinations of $D_{\mathrm{b}}$ using the NPR and relative responsivity measurements on the Vis/SCF.

\begin{tabular}{|c|c|c|c|c|}
\hline Uncertainty origin & $\begin{array}{c}\text { Channel } 1 \\
\text { type A } \\
(\%)\end{array}$ & $\begin{array}{c}\text { Channel } 1 \\
\text { type B } \\
(\%)\end{array}$ & $\begin{array}{c}\text { Channel } 5 \\
\text { type A } \\
(\%)\end{array}$ & $\begin{array}{c}\text { Channel } 5 \\
\text { type B } \\
(\%)\end{array}$ \\
\hline \multicolumn{5}{|l|}{ Effects that are always present } \\
\hline VXR alignment & & 0.10 & & 0.10 \\
\hline VXR linearity & & 0.10 & & 0.10 \\
\hline Vis/SCF, spectral flux scale* & & 0.11 & & 0.09 \\
\hline Vis/SCF, measurement uncertainty & $<0.002$ & & $<0.0002$ & \\
\hline $\mathrm{Vis} / \mathrm{SCF}$, overall technique & & 0.21 & & 0.10 \\
\hline Vis $/$ SCF and FASCAL $\lambda$ calibration & & 0.32 & & 0.02 \\
\hline \multicolumn{5}{|l|}{ Effects that depend on the ISS } \\
\hline Lamp current & 0.40 & & 0.21 & \\
\hline Radiance uniformity in exit aperture & & 0.15 & & 0.15 \\
\hline Short-term drift & & 0.10 & & 0.03 \\
\hline FASCAL calibration & & 0.40 & & 0.33 \\
\hline Numerical analysis & & 0.10 & & 0.10 \\
\hline \multicolumn{5}{|l|}{ Effects that depend on each measurement } \\
\hline VXR measurement uncertainty & $<0.1$ & & $<0.06$ & \\
\hline DVM accuracy & & $<0.01$ & & $<0.01$ \\
\hline VXR amplifier gain & & 0.02 & & 0.02 \\
\hline Relative combined standard uncertainty & 0.7 & & & 48 \\
\hline
\end{tabular}

*Larason et al. (1998).

determination of the band-weighted radiance by an amount that is proportional to the relative change in the spectral radiance with wavelength (Early and Thompson 1996). The effect of the current resolution of the lamp power supply is proportional to inverse wavelength. At $654.6 \mathrm{~nm}$, we used the scaling law $\left[L_{\lambda}(\lambda)+\Delta L_{\lambda}(\lambda)\right] / L_{\lambda}(\lambda)=$ $[(I+\Delta I) / I]^{6.24}$ (Walker and Thompson 1994) to estimate this uncertainty component. The FASCAL calibration of the NPR also results in an uncertainty that is wavelength-dependent. Not given in table 2 is the long-term drift of the NPR over the nine-month measurement interval. This component cannot be estimated from the FASCAL data because only one calibration has been performed to date. However, the output of the NPR internal monitor photodiodes was stable from April 1999 to September 1999 (Brown and Johnson 2002). Also, the standard deviation of the VXR measurements over this interval is less than $0.2 \%$. Because this is less than the uncertainty of the FASCAL spectral radiance calibration, it is satisfactory to model the sphere as a constant source of radiance during the measurement interval.

\section{Calibration using a laser-illuminated ISS}

We use a number of tunable and discrete laser sources to calibrate the VXR on SIRCUS over the spectral range 400-1000 $\mathrm{nm}$. Continuous wave dye lasers and a Ti:sapphire laser provide tunable coverage from $550-1000 \mathrm{~nm}$, while frequency doubling these lasers gives a tunable source over the spectral range $275-500 \mathrm{~nm}$. The spectral range $500-550 \mathrm{~nm}$ is currently covered using discrete emission lines from various lasers, which affects the calibration of VXR channel 3. We expect to have continuous coverage over this spectral range using a dye laser pumped by an argon ion laser in the near future. The output from the various lasers is sent through 
an intensity stabilizer prior to entering the ISS. The intensity stabilizer reduces the relative standard deviation of the power in the laser beam to less than $0.05 \%$, and enables variable control over the power in the beam sent into the integrating sphere.

We used a $20 \mathrm{~cm}$ diameter Spectralon ${ }^{(i)}$ (a product of Labsphere, Inc.*) integrating sphere with a $5.1 \mathrm{~cm}$ diameter exit port to calibrate the VXR. The source is of sufficiently extended area that it overfills the VXR entrance pupil. Typical power levels of radiation introduced into the ISS range from a few milliwatts to a few hundred milliwatts, depending on the laser used, giving a source radiance varying from $2 \times 10^{-5} \mathrm{~W} \mathrm{~mm}^{-2} \mathrm{sr}^{-1}$ to $1 \times 10^{-7} \mathrm{~W} \mathrm{~mm}^{-2} \mathrm{sr}^{-1}$.

\subsection{Radiance responsivity calibrations on SIRCUS}

The measurement of an extremely narrow-band source for one channel of the VXR is also given by equation (1). The laser-illuminated source is so narrow, with a bandwidth of about $2.5 \times 10^{-5} \mathrm{~nm}$, that the source spectral radiance is adequately described by a delta function and equation (1) becomes

$$
S(\lambda)=A \Omega G \tau(\lambda) R_{\mathrm{f}}(\lambda) L(\lambda)
$$

where $L(\lambda)$ is the radiance ( $\mathrm{W} \mathrm{m}^{-2} \mathrm{sr}^{-1}$ ) of the laser-illuminated ISS at wavelength $\lambda$. At each $\lambda$, the radiance responsivity $R(\lambda)$ is determined:

$$
\frac{S(\lambda)}{L(\lambda)}=A \Omega G \tau(\lambda) R_{\mathrm{f}}(\lambda) \equiv R(\lambda)
$$

The responsivity for each VXR channel is found from integration over all wavelengths where the radiance responsivity is non-negligible:

$$
D_{\mathrm{d}}=\int R(\lambda) d \lambda
$$

Here $D_{\mathrm{d}}$ is the laser-illuminated calibration coefficient. For the EOS intercomparisons, an equation of the form of equation (3) is used, with $D_{\mathrm{b}}$ replaced by $D_{\mathrm{d}}$ and $r(\lambda)$ replaced by $R(\lambda)$.

From equation (5), it is clear that two types of measurements are required to characterize and calibrate the VXR using the laser-illuminated ISS: (1) determination of the radiance $L(\lambda)$ of the ISS; and (2) determination of the net VXR signal $S$ corresponding to measurements of the laser-illuminated ISS at the same time $L(\lambda)$ is determined. In comparison to the lamp-illuminated determination of the VXR calibration coefficients, the SIRCUS method is more direct, with a shortened measurement chain to the HACR.

The calibration strategy is shown in figure 4. Test sensors and a reference detector are located on a translation stage at a fixed (and measured) distance from the sphere exit port. The test sensors are calibrated against the reference detector using the substitution method. A monitor photodiode mounted in a small port on the side wall of the ISS corrects for small source intensity fluctuations during a calibration. A computer controls and monitors the laser power and wavelength over much of the spectral range, controls the positions of the detectors and sources, and records test and reference detector signals.

*Certain commercial equipment, instruments or materials are identified in this technical memorandum to foster understanding. Such identification does not imply recommendation or endorsement by NIST, nor does it imply that the materials or equipment used are necessarily the best available for the purpose. 


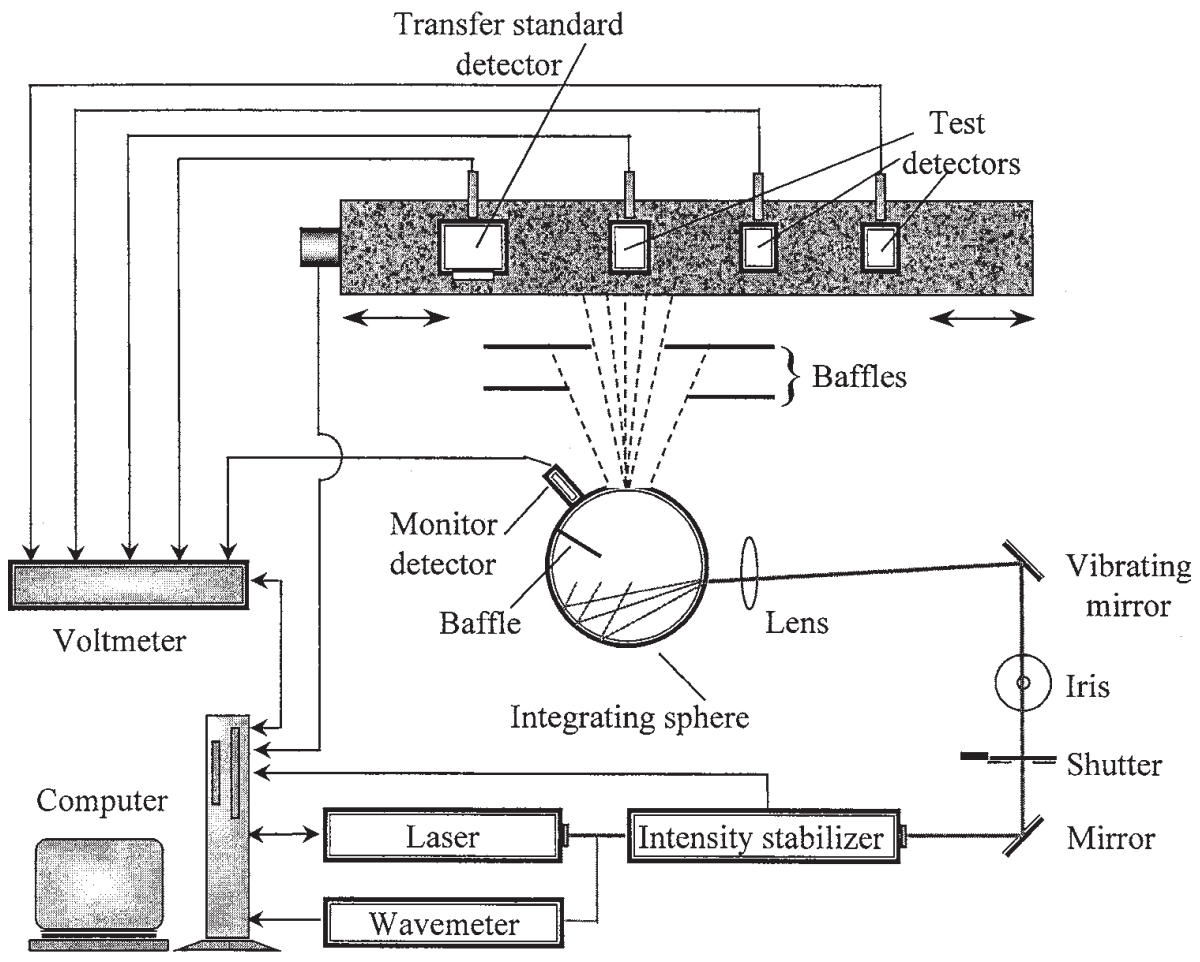

Figure 4. Experimental system for the determination of the radiance responsivity of the VXR using SIRCUS. The propagation of the radiant flux is indicated by the direction of the lines with single arrows; lines with double arrows indicate linear translation for the purpose of detector substitution. The vibrating mirror reduces the non-uniformities of the radiance in the exit port of the sphere that arise from speckle.

We use a silicon trap detector equipped with a precision aperture as our reference detector for calibrations over the wavelength range $406-920 \mathrm{~nm}$. An aperture of known area is located at the sphere exit port for radiance calibrations using the trap detector as an irradiance standard. Before the trap detector measures the sphere radiance, its responsivity must be determined. The optical power responsivity of the trap detector is derived from measurements on HACR. HACR is an electrical substitution radiometer, relating optical power (flux) to the electrical watt. It serves as the primary US national standard for optical power measurements. The irradiance responsivity of the trap detector is the product of its power responsivity and the aperture area. The source radiance is calculated from the irradiance on the trap detector using the distance between the trap detector aperture and the source aperture and the area of the source aperture.

During a calibration, a laser is tuned to the wavelength of interest. The trap detector is then placed in front of the sphere exit port, typically at a distance of approximately $1 \mathrm{~m}$, and measures the irradiance of the sphere. It is moved out of the way, and replaced by the radiance detector under test. The test detector measures the radiance within the exit port and the radiance responsivity $R(\lambda)$ is calculated by dividing the test detector output signal by the sphere radiance (see equation (5)). 
Any changes in the sphere radiance between the reference detector measurements and the test detector measurements are accounted for by referencing both signals to the output of the monitor photodiode. For a full spectral calibration, the wavelength is changed, and the calibration sequence repeated.

\subsection{Uncertainty budget for $V X R$ radiance response calibration}

Each component in the calibration contributes to the overall uncertainty in radiance responsivity calibrations. Additional uncertainties arise from the repeatability and reproducibility of the measurements, and include thermal effects as well as any small detector ageing effects. Table 3 lists all known contributions to the uncertainty in radiance responsivity calibrations on SIRCUS. While the uncertainty in the transfer to test detector will vary slightly from detector to detector, the current combined standard uncertainty in radiance responsivity for a typical test detector is $0.25 \%$.

\section{Calibration of the VXR}

In figure 5, the spectral responsivity determinations from the two techniquesusing the Vis/SCF and SIRCUS - are shown for two channels of the VXR. The channel at $412 \mathrm{~nm}$ was selected because this value of $D_{\mathrm{b}}$ is the most uncertain due to the wavelength-dependent components of uncertainty described previously (table 2). Also, experience with two other ISSs at NIST had revealed temporal drifts of the sphere radiance that resulted in larger than desired combined uncertainties. Hence SIRCUS could be expected to make the largest impact on the calibration of the VXR at $412 \mathrm{~nm}$. The channel at $775 \mathrm{~nm}$ was selected because the ISS-based uncertainties are lower and because application of VXR measurements for EOS programmes is often of interest for a broad wavelength interval.

For comparison purposes, the data were normalized using the maximum values in each dataset (figure 5). The Vis/SCF data were acquired in November and December 1996 and the SIRCUS measurements were performed in June 1999. At $412 \mathrm{~nm}$ (shown in figure 5(a)), there is a wavelength shift of about $0.33 \mathrm{~nm}$, with the

Table 3. Relative combined standard uncertainty of radiance responsivity calibrations on SIRCUS.

\begin{tabular}{lcc}
\hline $\begin{array}{l}\text { Uncertainty origin } \\
(\%)\end{array}$ & $\begin{array}{c}\text { Type B } \\
(\%)\end{array}$ \\
\hline $\begin{array}{l}\text { Source radiance } \\
\text { Trap detector spectral power responsivity }\end{array}$ & & 0.15 \\
$\quad$ Trap detector aperture area* & & 0.01 \\
$\quad$ Source-to-trap detector distance (squared) & 0.05 & \\
$\quad$ Trap detector repeatability & 0.04 & 0.05 \\
$\quad$ Source aperture area & & 0.04 \\
$\quad$ Amplifier gain & & 0.1 \\
Transfer to test detector & 0.05 & \\
$\quad$ Source spatial radiance non-uniformity & & \\
$\quad$ Test detector repeatability & & 0.15 \\
$\quad$ Test detector size-of-source effect & & 0.04 \\
$\quad$ Amplifier gain & & \\
Relative combined standard uncertainty & & \\
\hline
\end{tabular}

* Fowler et al. (1998). 

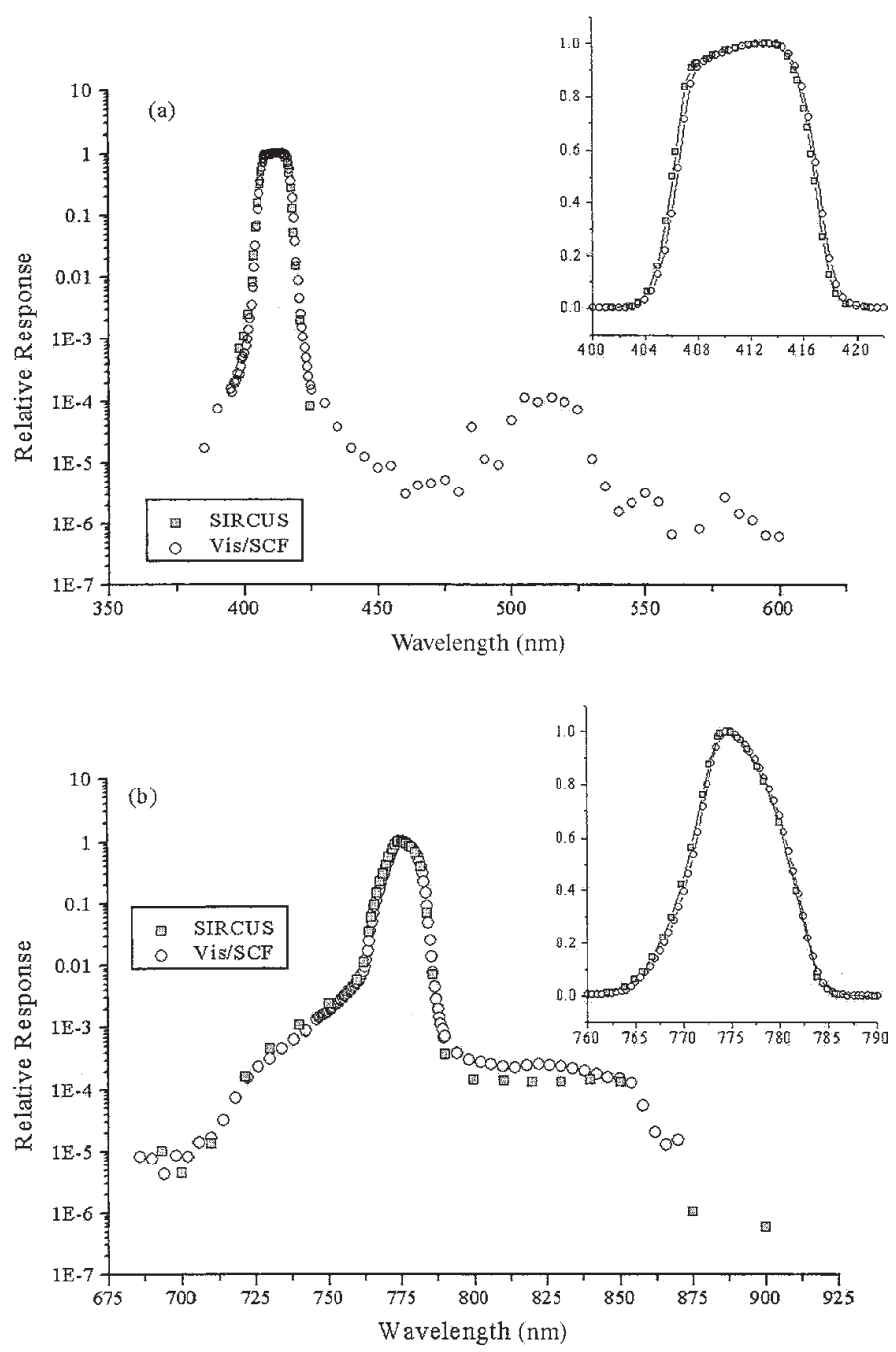

Figure 5. Comparison of the spectral shapes of the relative responsivity determined on the Vis/SCF in 1996 and the normalized radiance responsivity determined using SIRCUS in 1999 for the (a) $412 \mathrm{~nm}$ and (b) $775 \mathrm{~nm}$ channel.

SIRCUS results at the shorter wavelength. There are also small differences in the shape of the responsivity functions in the region $408-415 \mathrm{~nm}$. The SIRCUS measurements were made only over the central portion of the response, but the normalized data can be compared from unity to about $10^{-4}$ of the maximum value. The effect of the missing out-of-band response in the SIRCUS results can be estimated from the Vis/SCF data, where the integrated response from $450-600 \mathrm{~nm}$ is $0.05 \%$ of the total integrated response. We estimate the effect for measurements of a broadband source to be $0.1 \%$ or less.

At $775 \mathrm{~nm}$, there is a wavelength shift of about $0.35 \mathrm{~nm}$, again with the SIRCUS results at the shorter wavelength. The Vis/SCF results are larger than those of 
SIRCUS from $790-850 \mathrm{~nm}$ by about $50 \%$ on average, but the effect on the integrated response is only $0.05 \%$. It is clear from the SIRCUS measurements beyond $850 \mathrm{~nm}$ that this laser-illuminated determination is able to achieve accurate values at $10^{-6}$ of the peak. There was evidence in the Vis $/ \mathrm{SCF}$ data for the SXR at $775 \mathrm{~nm}$ of a decrease in responsivity beyond $850 \mathrm{~nm}$ (Johnson et al. 1998), but with signal-tonoise ratios of less than unity, interpretation was difficult. Therefore SIRCUS is able to explore the lower limits of the instrument responsivity with much improved accuracy.

The two methods of determining the calibration coefficient for the $412 \mathrm{~nm}$ and the $775 \mathrm{~nm}$ channels of the VXR are compared in figure 6. The values of $D_{\mathrm{b}}$ for all measurements of the NPR source from 13 January to 31 August 1999 are plotted for the two radiance levels of the NPR source that were determined on FASCAL. These levels correspond to all four lamps in operation, or only one lamp (designated lamp number 1) in operation. The FASCAL calibration for these settings was performed on 19-21 May 1999. As explained in $\$ 2$, the band-averaged radiance was determined using the $1996 \mathrm{Vis} / \mathrm{SCF}$ relative responsivity data, $r(\lambda)$.

The calibration coefficients derived from the SIRCUS data are also shown in figure 6. At $412 \mathrm{~nm}$, the average $D_{\mathrm{b}}$ from the NPR measurements is $0.041948 \mathrm{~V} \mathrm{~cm}^{2} \mathrm{srnm} \mu \mathrm{W}^{-1}$, and the SIRCUS result is $0.041869 \mathrm{~V} \mathrm{~cm}^{2} \mathrm{srnm}_{\mu} \mathrm{W}^{-1}$, for a difference of $0.19 \%$. At $775 \mathrm{~nm}$, the average $D_{\mathrm{b}}$ is $0.026303 \mathrm{~V} \mathrm{~cm}^{2} \mathrm{srnm} \mu \mathrm{W}^{-1}$, the SIRCUS result is $0.026267 \mathrm{~V} \mathrm{~cm}^{2} \mathrm{srnm} \mu \mathrm{W}^{-1}$, and the difference is $0.14 \%$. This agreement is better than expected given the magnitude of the relative standard uncertainties in $D_{\mathrm{b}}$ and may be fortuitous. Most uncertainty components in $D_{\mathrm{b}}$ are systematic, and cannot be reduced by repeat measurements.

The SIRCUS values for the absolute spectral radiance responsivity can be used in equation (3) to determine $D_{\mathrm{b}}$. This provides a more robust and reasonable comparison of the two methods because the VXR filters could have changed between the Vis/SCF measurements in 1996 and the SIRCUS measurements in 1999. Recalculation of $D_{\mathrm{b}}$ at $412 \mathrm{~nm}$ gives $0.042161 \mathrm{~V} \mathrm{~cm}^{2} \mathrm{sr} \mathrm{nm} \mu \mathrm{W}^{-1}$, which agrees with SIRCUS to within $0.7 \%$. At $775 \mathrm{~nm}, D_{\mathrm{b}}$ changes by less than $0.1 \%$ because the spectral radiance of the NPR changes slowly with wavelength compared to its behaviour at $412 \mathrm{~nm}$, so the calibration coefficient at $775 \mathrm{~nm}$ is less sensitive to the wavelength shift of $R(\lambda)$ with respect to $r(\lambda)$.

\section{Future applications using SIRCUS}

Higher radiance levels, lower wavelength uncertainty, negligible bandpass, and no out-of-band radiation all contribute to reducing the uncertainty in radiance and irradiance responsivity calibrations on SIRCUS. Our current uncertainties in irradiance and radiance responsivity calibrations are $0.1 \%$ and $0.25 \%$, respectively, over the spectral range $406 \mathrm{~nm}-920 \mathrm{~nm}$. With minor improvements in the facility, we expect to be able to reduce our uncertainties for both irradiance and radiance responsivity calibrations to $0.05 \%$ or less (Anderson et al. 1992). SIRCUS could easily be adapted for optical flux linearity studies, and offers an alternative to the NIST beamconjoiner. In addition, the uniformity of the exit aperture of the laserilluminated ISS has been exploited in the study of two-dimensional array detectors.

Reduction of our uncertainties on SIRCUS will impact calibrations of the VXR while extension of the wavelength coverage using secondary working standards will enable calibration of other ultraviolet, visible and infrared transfer radiometers. SIRCUS may be used, for example, to calibrate the SWIR transfer radiometers 

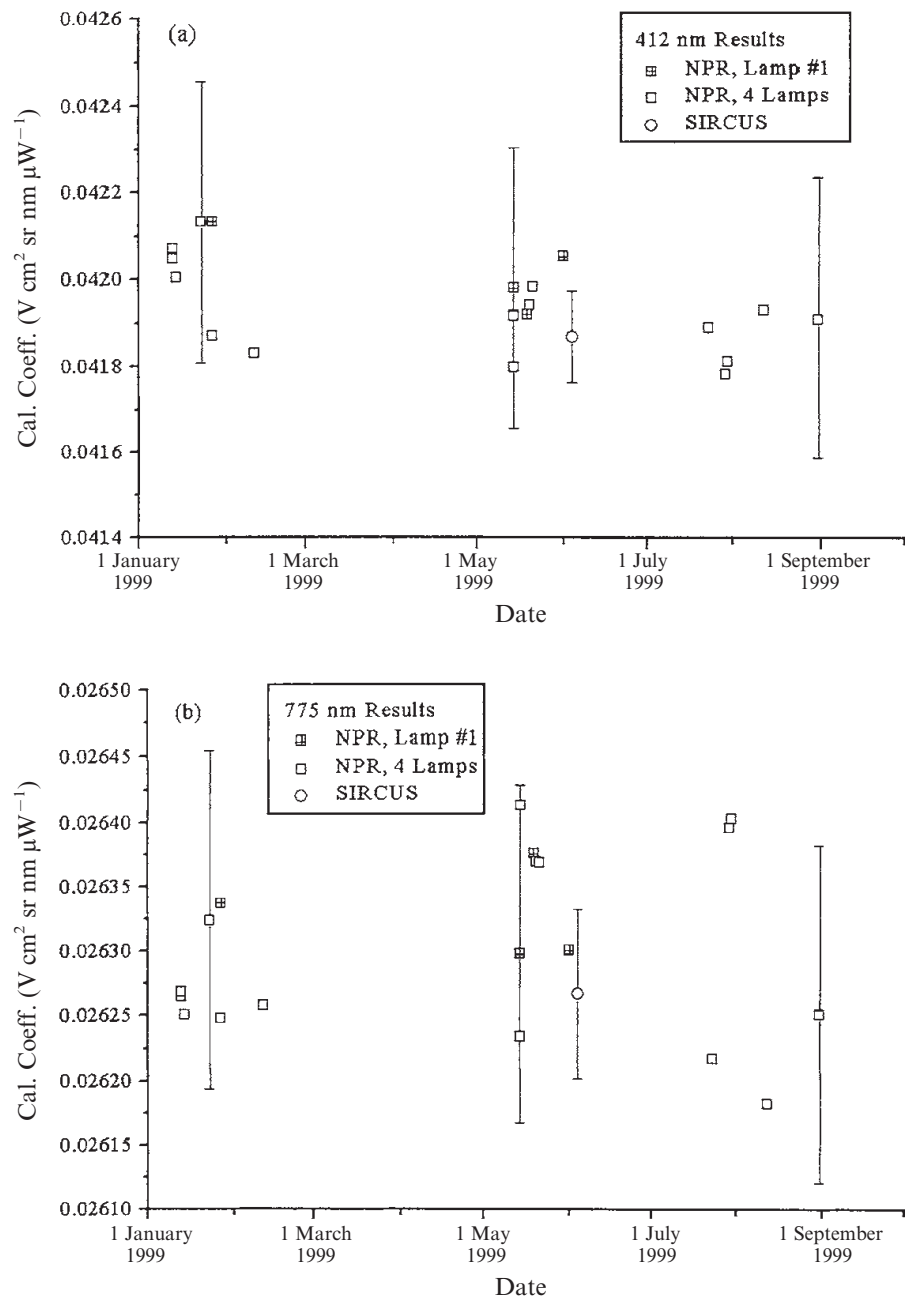

Figure 6. Comparison of the calibration coefficients determined using the NPR ISS and SIRCUS for the (a) $412 \mathrm{~nm}$ and (b) $775 \mathrm{~nm}$ channel of the VXR. Two levels were used with the NPR and they are identified in the legend. The vertical lines illustrate representative relative standard uncertainties.

developed by NIST, the University of Arizona and other institutions. The current variation in sphere radiance measurements in the SWIR is about $4 \%$, which is comparable to the uncertainties in the calibration of the transfer radiometers. With SIRCUS, we expect to be able to calibrate radiometers out to $2.5 \mu \mathrm{m}$ with relative standard uncertainties of $0.5 \%$ or less. Therefore, calibrating the SWIR transfer radiometers on SIRCUS should reduce the variance in radiance measurements with these instruments and provide a better estimate of the accuracy of the scale assignments made by the EOS participants.

Further in the infrared, we anticipate calibrating the Thermal-infrared Transfer Radiometer (TXR) (Rice and Johnson 1998, Rice et al. 2002). The TXR is a vacuumcompatible, liquid nitrogen cooled filter radiometer with two $1 \mu \mathrm{m}$-wide filter 
channels centred at $5 \mu \mathrm{m}$ and $10 \mu \mathrm{m}$, respectively. The TXR is important to the remote sensing community in that it offers a relatively simple and economical method of verifying the radiometric scales that are used for ground calibration of thermalinfrared channels of the many remote sensing instruments in use. As with the VXR, SIRCUS provides an accurate and direct method of calibrating the TXR spectrally against the NIST detector-based radiometric scale. The SIRCUS data will enable a comparison to the relative spectral responsivity determined using the ambient infrared spectral comparator (Migdall et al. 1994, Rice and Johnson 1998). Integration of the SIRCUS data over the TXR channel will provide a calibration coefficient that can be compared to the one derived using an ambient black body source (Rice and Johnson 1998, Rice et al. 2002). The SIRCUS facility will reduce the uncertainties associated with calibration of the TXR at NIST by enabling direct measurements of a number of effects that previously could only be estimated.

In a separate application, SIRCUS is well suited to calibrate instruments used in a number of environmental monitoring and remote sensing applications. For example, a global network of sun photometers, the Aerosol Robotic Network (AERONET) has been established under the auspices of NASA's EOS programme to provide globally distributed near-real-time observations of aerosol optical depths, aerosol size distributions and precipitable water in diverse aerosol regimes (Holben et al. 1998). The sun photometers are filter radiometers with a number of channels with band centre wavelengths ranging from the visible to the near-infrared; the Cimel CE318-2 sun photometer has filters at 440, 670, 870, 936 and $1020 \mathrm{~nm}$, for example. Reduction in uncertainties in the calibration of the sun photometers will aid the validity of data products that result from AERONET.

Finally, SIRCUS is being used to improve the accuracy of NIST's spectroradiometric, photometric and radiance temperature calibration services. The primary impact to the EOS community will be in the reduction of uncertainty in the spectral irradiance values assigned to $1000 \mathrm{~W}$ standard lamps. These standard lamps are currently calibrated from $250-2400 \mathrm{~nm}$ on FASCAL and used by the EOS community as irradiance standards for both irradiance and radiance calibrations (in combination with diffuse reflectance standards). To date, filter radiometers have been calibrated using the Vis/SCF and used to determine the radiance temperature of a hightemperature black body with an uncertainty of $0.5 \mathrm{~K}$ near $2900 \mathrm{~K}$ (Yoon and Gibson 1999). The high-temperature black body will be used to directly determine the spectral irradiance of the standard irradiance lamps. The new method will result in a reduction of the uncertainty in the NIST irradiance scale by a factor of up to five, depending on wavelength. Because the principal source of uncertainty in the calibration of the filter radiometers is the spectral irradiance responsivity, the calibrations using SIRCUS results will reduce the uncertainties even further.

\section{Summary}

The VXR, a portable filter radiometer for radiance measurements with six narrowband channels, has been calibrated using two different methods, both involving measurements of integrating sphere sources. Measurements with the broadband, lamp-illuminated ISS require ancillary measurements on the spectral responsivity, while the laser-illuminated calibration results in a direct determination of the spectral radiance responsivity. We present results for one lamp-illuminated ISS and one laserilluminated ISS for two channels of the VXR; the agreement is within the combined uncertainties. The laser-illuminated ISS method is more accurate. 
The improved accuracy from SIRCUS is the result of many factors. First, systemlevel calibrations are more accurate than combinations of component level measurements, and the high flux levels and appropriate geometric properties of the source make the system-level determinations possible. Secondly, the advancements in tunable lasers allow for thorough studies in the spectral regions of interest. Thirdly, the availability of suitable transfer detectors ensures a short measurement chain to the fundamental measurements on HACR.

The improvement in the calibration accuracy, especially for the spectral region below $500 \mathrm{~nm}$ and above $800 \mathrm{~nm}$, will have a significant impact on the utility of the radiometric intercomparisons performed by NASA's Goddard Space Flight Facility and NIST for EOS. First, SIRCUS will be used to characterize and calibrate the EOS/NIST transfer radiometers in the SWIR and thermal infrared; work with the VXR will also continue. Secondly, SIRCUS may be used with other important EOS radiometers. Thirdly, SIRCUS will be used to improve the accuracy of the NIST spectroradiometric quantities, which will reduce the uncertainties in critical standards such as the $1000 \mathrm{~W}$ quartz-halogen irradiance lamps.

\section{Acknowledgments}

We would like to thank NIST colleagues M. V. Annageri, S. S. Bruce, C. E. Gibson, T. C. Larason and R. D. Saunders for assistance, and acknowledge support by NASA's EOS Project Science Office under contract number S-41365-F and the US Air Force Metrology under contract numbers 97-421 and 98-439.

\section{References}

Anderson, V. E., Fox, N. P., and Nettleton, D. H., 1992, Highly stable, monochromatic and tunable optical radiation source and its application to high accuracy spectrophotometry. Applied Optics, 31, 536-545.

Barnes, R. A., and McClain, C. R., 1999, The calibration of SeaWiFS after two years on orbit. Proceedings, International Society of Optical Engineering (SPIE), 3870, $214-227$.

Brown, S. W., and Johnson, B. C., 1999, A portable integrating sphere source for radiometric calibrations from the visible to the short-wave infrared. The Earth Observer, 11(3), $14-19$.

Brown, S. W., and Johnson, B. C., 2002, Development of a portable integrating sphere source for the Earth Observing System's calibration validation programme. International Journal of Remote Sensing, 00, 00-00.

Brown, S. W., Johnson, B. C., and Yoon, H. W., 1998, Description of a portable spectroradiometer to validate EOS radiance scales in the shortwave infrared. The Earth Observer, 10(3), 43-48.

Brown, S. W., Eppeldauer, G. P., and LyKke, K. R., 2000, NIST facility for spectral irradiance and radiance responsivity calibrations with uniform sources. Metrologia, 37, 579-582.

Butler, J. J., and BARNes, R. A., 1998, Calibration strategy for the Earth Observing System (EOS)-AM1 Platform. IEEE Transactions on Geoscience and Remote Sensing, 36, $1056-1061$

Butler, J. J., Johnson, B. C., Brown, S. W., Yoon, H. W., Barnes, R. A., Markham, B. L., Biggar, S. F., Zalewski, E. F., Spyak, P. R., Cooper, J. W., and Sakuma, F., 1999, Radiometric measurement comparisons using transfer radiometers in support of the calibration of NASA's Earth Observing System (EOS) Sensors. Proceedings, International Society of Optical Engineering (SPIE), 3870, 180-192.

Clark, D. K., Gordon, H. R., Voss, K. J., Ge, Y., Broenkow, W., and Trees, C., 1997, Validation of atmospheric correction over the oceans. Journal of Geophysical Research, 102, 17209-17217.

Cromer, C. L., and Johnson, B. C., 1996, Appendix B-report on the calibration of MOBY. In The Third SeaWiFS Intercalibration Round-Robin Experiment (SIRREX-3), 
19-30 September 1994. NASA Technical Memorandum 104566, vol. 34 (NASA Goddard Space Flight Center: Greenbelt, MD), pp. 71-75.

Early, E. A., and Johnson, B. C., 1997, Calibration and characterization of the GSFC sphere. In Case Studies for SeaWiFS Calibration and Validation, Part 4. NASA Technical Memorandum 104566, vol. 41 (NASA Goddard Space Flight Center: Greenbelt, MD), pp. 3-17.

EARly, E. A., and Thompson, A., 1996, Irradiance of horizontal quartz-halogen standard lamps. Journal of Research of the National Institute of Standards and Technology, 101, 141-153.

Eppeldauer, G. P., Brown, S. W., Larason, T. C., Racz, M., and Lykke, K. R., 2000, Realization of a spectral radiance responsivity scale with a laser-illuminated source and Si radiance meters. Metrologia, 37, 531-534.

Fowler, J. B., Durvasula, R. S., and PARR, A. C., 1998, High-accuracy aperture-area measurement facilities at the National Institute of Standards and Technology. Metrologia, $35,497-500$.

Gentile, T. R., Houston, J. M., and Cromer, C. L., 1996a, Realization of a scale of absolute spectral response using the National Institute of Standards and Technology highaccuracy cryogenic radiometer. Applied Optics, 35, 4392-4402.

Gentile, T. R., Houston, J. M., Hardis, J. E., Cromer, C. L., and Parr, A. C., 1996b, National Institute of Standards and Technology high accuracy cryogenic radiometer. Applied Optics, 35, 1056-1068.

Guenther, B., Barnes, W., Knight, E., Barker, J., Harnden, J., Weber, R., Roberto, M., GodDEn, G., MontGomery, H., and Abel, P., 1996, MODIS calibration: A brief review of the strategy for the at-launch calibration approach. Journal of Atmospheric and Oceanic Technology, 13, 274-285.

Herring, D., 1997, Marine Optical Buoy (MOBY) evolves, while Marine Optical Characterization Experiment (MOCE) continues in support of SeaWiFS, MODIS, and OCTS. The Earth Observer, 9(5), 15-20.

Holben, B. N., Eck, T. F., Slutsker, ?. ?., Tanré, T. D., Bius, J. P., Setzer, A., Vermote, E., Reagan, J. A., Kaufman, Y. J., Nakajima, T., Lavenu, F., Jankowiak, I., and SMIRNOv, A., 1998, AERONET-A federated instrument network and data archive for aerosol characterization. Remote Sensing of Environment, 66, 1-16.

Johnson, B. C., Sakuma, F., Butler, J. J., Biggar, S. F., Cooper, J. W., Hiramatsu, M., and SuZUKI, K., 1997, Radiometric measurement comparison using the Ocean Color Temperature Scanner (OCTS) visible and near infrared integrating sphere. Journal of Research of the National Institute of Standards and Technology, 102, 627-646.

Johnson, B. C., Fowler, J. B., and Cromer, C. L., 1998, The SeaWiFS transfer radiometer (SXR). NASA/TM-1998-206892, vol. 1, NASA Goddard Space Flight Center, Greenbelt, MD, USA.

Johnson, B. C., Early, E. A., Eplee, R. E. JR., Barnes, R. A., and Caffrey, R. T., 1999, The 1997 prelaunch radiometric calibration of SeaWiFS. NASA/TM-1999-206892, vol. 4 , NASA Goddard Space Flight Center, Greenbelt, MD, USA.

Larason, T. C., Bruce, S. S., and Parr, A. C., 1998, Spectroradiometric detector measurements. Special Publication 250-41, National Institute of Standards and Technology, Washington, DC, USA.

Lykke, K. R., Shaw, P.-S., Hanssen, L. M., and Eppeldauer, G. P., 1998, Development of a monochromatic, uniform source facility for calibration of radiance and irradiance detectors from $0.2 \mu \mathrm{m}$ to $18 \mu \mathrm{m}$. Metrologia, 35, 479-484.

Mielenz, K. D., Saunders, R. D. JR., and Shumaker, J. B., 1990, Spectroradiometric determination of the freezing temperature of gold. Journal of Research of the National Institute of Standards and Technology, 95, 49-67.

Migdall, A. L., Eppeldauer, G. P., and Cromer, C. L., 1994, IR detector spectral responsivity calibration facility at NIST. Proceedings, International Society of Optical Engineering (SPIE), 2227, 46-53.

Rice, J. P., and Johnson, B. C., 1998, The NIST EOS thermal-infrared transfer radiometer. Metrologia, 35, 505-509.

Rice, J. P., Bender, S. C., Atkins, W. H., and Lovas, F. J., 2002, Deployment test of the NIST EOS Thermal-infrared Transfer Radiometer. International Journal of Remote Sensing, 00, 00-00. 
Sakuma, F., Johnson, B. C., Biggar, S. F., Butler, J. J., Cooper, J. W., Hiramatsu, M., and SuzuKI, K., 1996, EOS AM-1 preflight radiometric measurement comparison using the Advanced Spaceborne Thermal Emission and Reflection Radiometer (ASTER) visible/near-infrared integrating sphere. Proceedings, International Society of Optical Engineering (SPIE), 2820, 184-196.

WALKER, J. H., and ThOMPSON, A., 1994, Improved automated current control for standard lamps. Journal of Research of the National Institute of Standards and Technology, 99, 255-261.

Walker, J. H., Saunders, R. D., and Hattenburg, A. T., 1987, Spectral radiance calibrations. Special Publication SP 250-1, National Bureau of Standards, Washington, DC, USA.

Yoon, H. W., and GiBson, C. E., 1999, Determination of radiance temperatures using detectors calibrated for absolute spectral power response. In TEMPMEKO '99, The Seventh International Symposium on Temperature and Thermal Measurements, vol. 2 (Delft, The Netherlands: IMEKO/NMi Van Swinden Laboratorium), pp. 737-742.

Yoon, H. W., Johnson, B. C., Kelch, D., Biggar, S., and Spyak, P. R., 1998, A $400 \mathrm{~nm}$ to $2500 \mathrm{~nm}$ absolute spectral radiance comparison using filter radiometers. Metrologia, $35,563-568$. 


\title{
Spectral Irradiance and Radiance responsivity Calibrations using Uniform Sources (SIRCUS) facility at NIST
}

\author{
Steven W. Brown, George P. Eppeldauer, Joseph P. Rice, Jun Zhang, Keith R. Lykke \\ National Institute of Standards and Technology \\ Gaithersburg, MD 20899-8442
}

\begin{abstract}
Detectors have historically been calibrated for spectral power responsivity at the National Institute of Standards and Technology (NIST) using a lamp-monochromator system to tune the wavelength of the excitation source. Silicon detectors can be calibrated in the visible spectral region with uncertainties at the $0.1 \%$ level. However, uncertainties increase dramatically when measuring an instrument's spectral irradiance or radiance responsivity. In addition, the uncertainties are even larger in the UV and IR ranges. We will discuss a new laser-based facility for Spectral Irradiance and Radiance responsivity Calibrations using Uniform Sources (SIRCUS) that was developed to calibrate instruments directly in irradiance or radiance mode with uncertainties approaching those available for spectral power responsivity calibrations. In this facility, high-power, tunable lasers are introduced into an integrating sphere using optical fibers, producing uniform, quasi-Lambertian, high radiant flux sources. Reference standard irradiance detectors, calibrated directly against national primary standards for spectral power responsivity, are used to determine the irradiance at a reference plane. Knowing the measurement geometry, the source radiance can be readily determined as well. The radiometric properties of the SIRCUS source coupled with state-of-the-art transfer standard radiometers whose responsivities are directly traceable to primary national radiometric scales, result in typical combined standard uncertainties in irradiance and radiance responsivity calibrations less than $0.1 \%$ in the visible (larger in the UV and IR). Details of the facility are presented and examples of unique calibrations possible in the facility are given, including system-level responsivity calibrations in support of the National Aeronautics and Space Administration's (NASA's) remote sensing activities.
\end{abstract}

Keywords: calibration, detectors, lasers, ocean color, remote sensing

\section{INTRODUCTION}

NASA's Earth Observing System (EOS) is an international, multi-instrument, multi-platform satellite remote sensing program designed to produce long time series data on global climate change. EOS constitutes the major component of NASA's Mission to Planet Earth and data products from the EOS program will be used as a guide for the implementation of international environmental policies [1]. The utility of these remotely sensed data products in helping to establish and understand driving mechanisms behind global environmental changes occurring on decadal time scales will depend on the accuracy of the calibration of the full ensemble of EOS instruments on the multiple platforms flown over the duration of the EOS mission. State-of-the-art sensor uncertainties are necessary to obtain the required uncertainty in relevant data products. Consequently, detailed sensor characterization and accurate radiometric calibration (both pre-launch and on-orbit) as well as long-term sensor degradation measurements are integral to the success of the EOS program. The EOS program office established a Calibration Validation (Cal/Val) program to validate radiometric uncertainties in instrument builder sources used to calibrate satellite sensors and to validate prelaunch and on-orbit sensor radiometric uncertainties [2].

Primary national radiometric scales are maintained and disseminated by NIST. Artifacts, both sources and detectors, calibrated at NIST directly against primary and secondary standards will have the lowest possible uncertainties. To ensure traceability to primary national (and international) radiometric standards and to ensure radiometric measurements with the lowest achievable uncertainties, the EOS Cal/Val program office has enlisted NIST's assistance in calibration validation activities to support EOS sensors. In this paper, we discuss a new laser-based calibration facility at NIST that helps support the EOS calibration validation program. Unprecedented accuracies in both radiance and irradiance responsivity calibrations are achievable in this facility for Spectral Irradiance and Radiance responsivity Calibrations 
using Uniform Sources, dubbed SIRCUS. While the facility has been in operation for only a few years [3], it has supported a number of EOS programs. We first describe the facility and then go into detail on a few of the systems calibrated using SIRCUS that are related to EOS.

\section{SIRCUS FACILITY}

The SIRCUS facility has been described in detail recently [4], so we briefly discuss the facility here. SIRCUS is shown schematically in Figure 1. In the SIRCUS facility, high-power, tunable lasers are introduced into an integrating sphere producing uniform, quasi-Lambertian, high radiant flux sources. The laser is first directed through an intensity stabilizer that controls the relative optical power in the beam to within $0.01 \%$ of the set point. A portion of the laser beam is sent into a wavemeter that measures the wavelength of the radiation to within $0.001 \mathrm{~nm}$. A beamsplitter sends another portion of the laser beam into a Fabry-Perot interferometer to measure the bandwidth and mode stability of the laser. Finally, the laser radiation is introduced into an integrating sphere, often using an optical fiber [5]. Occasionally, a collimator coupled to the sphere is used as a calibration source. Speckle in the image from the source, originating from the coherent nature of the laser radiation, is effectively removed by either rastering the beam inside the sphere with a galvanometer-driven mirror or by placing a short length of optical fiber in an ultrasonic bath.

Reference standard irradiance detectors, calibrated directly against national primary standards for spectral power responsivity [6] and equipped with a precision aperture measured on the NIST Aperture Area facility [7], are used to determine the irradiance at a reference plane. The source radiance can be readily determined from the measurement geometry as well. A monitor photodiode is located on the sphere to correct for any radiant flux changes in the sphere output between measurements with the reference instrument and the device under test. The sources are located inside a light-tight box. Two baffles are typically installed between the source and the detectors to minimize effects of stray radiation on the measurement. Instruments are calibrated

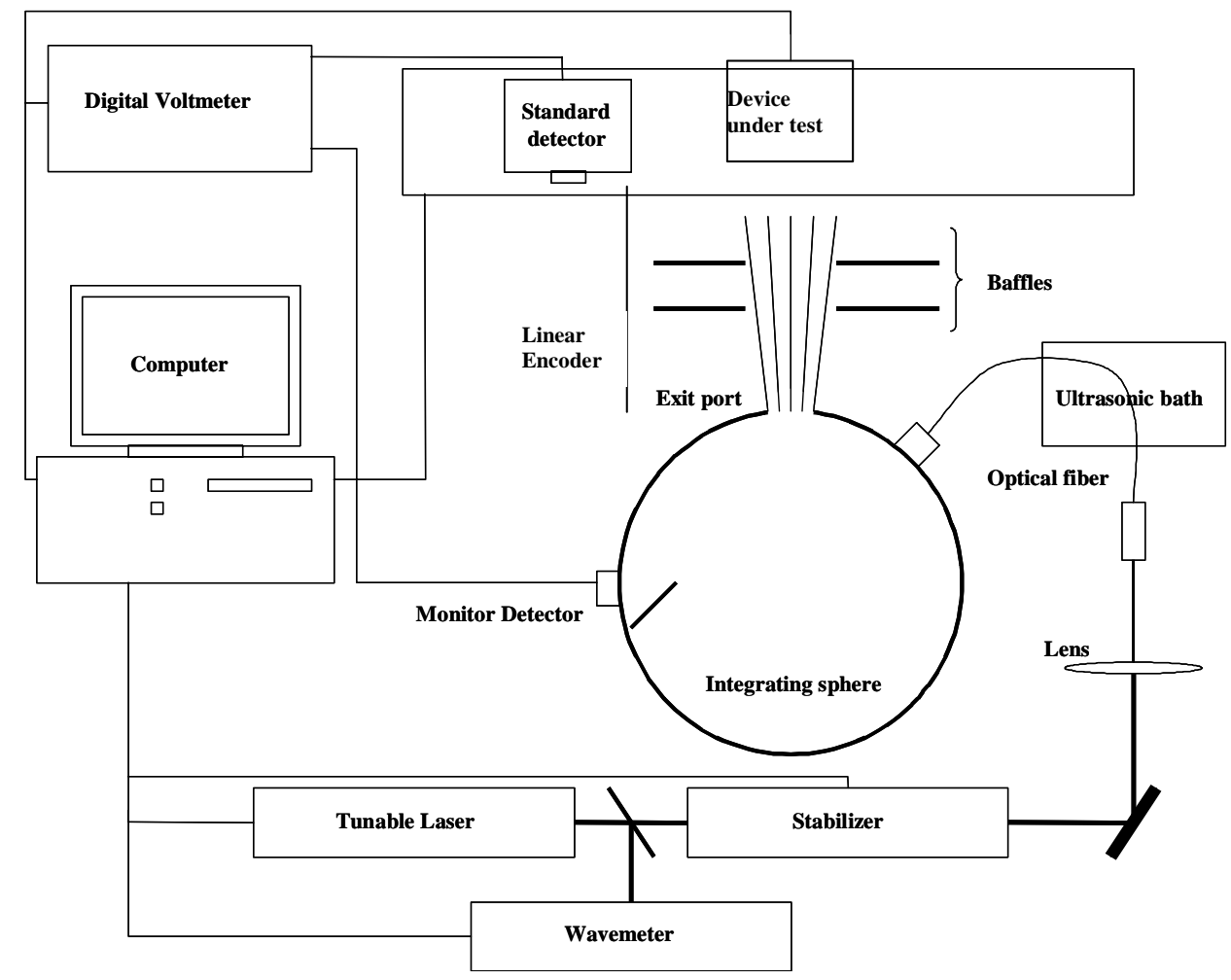

Figure 1: Schematic of SIRCUS facility. 
directly in irradiance or radiance mode with expanded uncertainties $(\mathrm{k}=2)$ of $0.15 \%$, approaching or exceeding those achievable for spectral power responsivity calibrations.

There are two separate SIRCUS facilities; the UV/Vis/NIR SIRCUS and the IR SIRCUS. The UV/Vis/NIR SIRCUS covers the range from $200 \mathrm{~nm}$ to $1.6 \mathrm{um}$ while the IR SIRCUS facility covers the spectral region from $780 \mathrm{~nm}$ to $5 \mathrm{um}$. There is some overlap between the two facilities for scale intercomparisons. The two facilities are very similar; the main distinction is in the laser source used to illuminate the integrating spheres and the reference transfer standards used to determine the irradiance at a reference plane.

\subsection{Sources}

A number of different lasers are used to cover the spectral range from about $210 \mathrm{~nm}$ to $5000 \mathrm{~nm}$. Continuous tunability in the UV/visible/NIR is provided by dye lasers and Ti:Sapphire lasers that are pumped by either an Argon ion laser ( $25 \mathrm{~W}$ all lines visible, $7 \mathrm{~W}$ all lines $\mathrm{UV}$ ) or a frequency-doubled Nd:Vanadate laser (10 W at $532 \mathrm{~nm}$ ). A number of discrete wavelengths are provided by the pump lasers themselves.

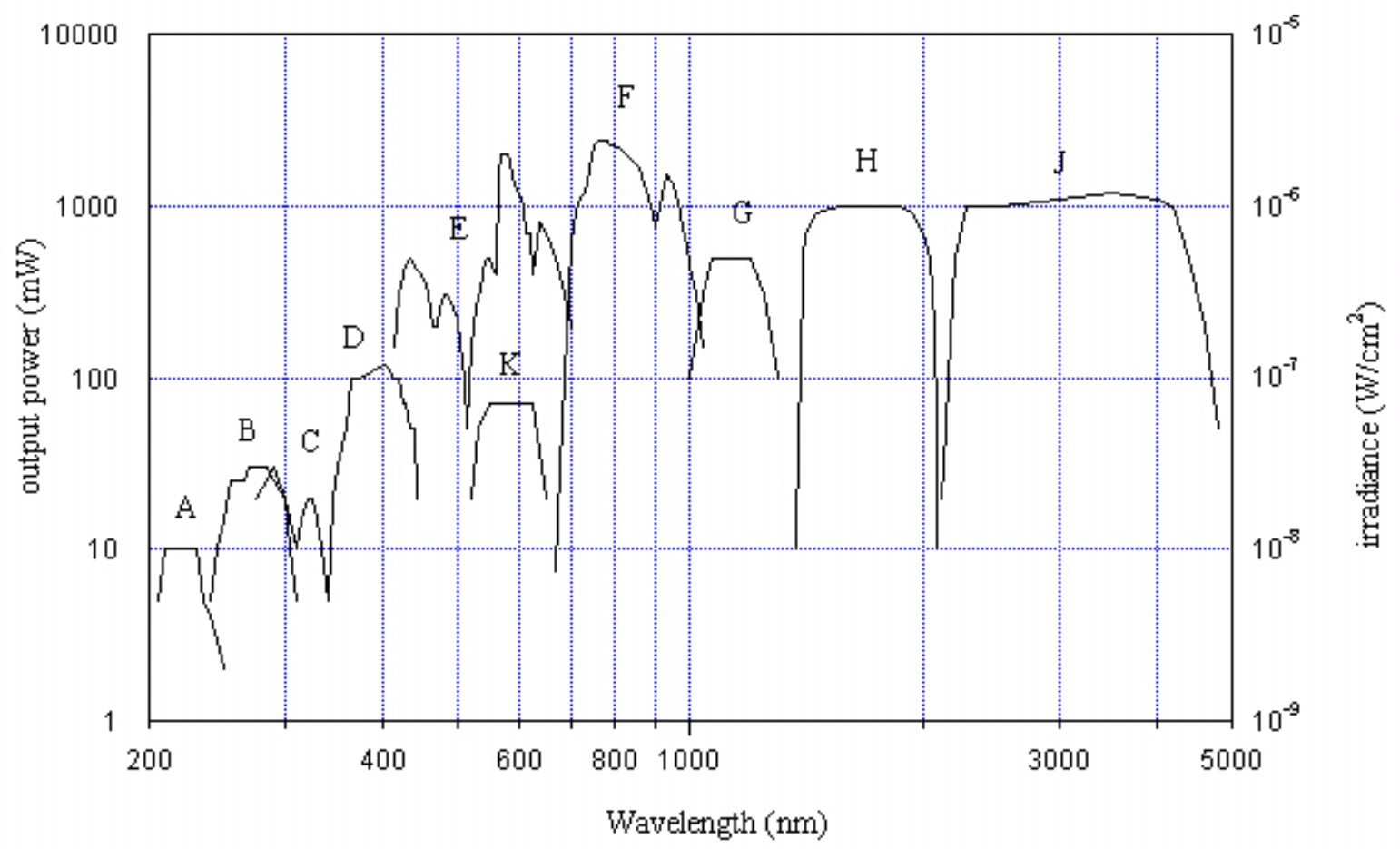

Figure 2: Output powers versus wavelengths for SIRCUS sources.

Continuous tunability in the NIR/IR is provided by optical parametric oscillators (OPO's) pumped by solid state lasers (typically, Nd:YAG, Nd:Vanadate, frequency-doubled Nd:Vanadate). These systems are briefly discussed below and illustrated in Figure 2 [8].

Dye lasers at SIRCUS cover the spectral region from $415 \mathrm{~nm}$ to $700 \mathrm{~nm}$ (E in the figure); dyes used include Stilbene 3 $(415 \mathrm{~nm}-475 \mathrm{~nm})$, Coumarin 480 (470 nm-520 nm), Coumarin 521 (505 nm-565 nm, [9]), Rhodamine 6G (550 nm$620 \mathrm{~nm}$ ), and DCM (610 nm-700 nm). A separate laser is used for each dye so that the full tuning range can be rapidly covered and no dye change or optical reconfiguration is required. Ti:Sapphire lasers cover the spectral range from $680 \mathrm{~nm}$ to $1050 \mathrm{~nm}$ (F in Figure 2) [10]. Frequency doubling of Ti:Sapphire lasers enables tuning from $350 \mathrm{~nm}$ to 500 $\mathrm{nm}$ ( $\mathrm{D}$ in figure). Two different types of frequency doubling are available: The first approach uses an external power 
build-up cavity that requires a frequency-stabilized, single-mode laser to pump it. The second is a NIST-built intracavity-doubled Ti:sapphire laser that requires no frequency stabilization. Over $300 \mathrm{~mW}$ of optical power is available from the dye lasers and the Ti:Sapphire laser (with optical powers of $1 \mathrm{~W}$ or greater over much of the spectral range). The power available from the $\mathrm{cw}$, frequency-doubled radiation is typically in the range from $100 \mathrm{~mW}$ to 200 $\mathrm{mW}$. Computer-controlled actuators rotate the angle of the birefringent tuner in the dye and Ti:sapphire lasers for automated wavelength tuning.

The IR systems developed at NIST include a cw periodically poled Lithium Niobate (PPLN) OPO pumped by a Nd:YAG laser, a mode-locked (220 MHz, 7 ps) PPLN OPO pumped by a Nd:Vanadate laser, and a mode-locked LBO OPO pumped by the $2^{\text {nd }}$ harmonic of the Nd:Vanadate laser. The first two systems tune from approximately1.4 $\mu \mathrm{m}$ to $5 \mu \mathrm{m}$ ( $\mathrm{H}$ and J), while the LBO OPO tunes from approximately $700 \mathrm{~nm}$ to $2000 \mathrm{~nm}$ (not shown in the figure). The power level is in the $100 \mathrm{~mW}$ to $1 \mathrm{~W}$ range.

Mode-locked systems have been shown in the past to work for radiometric situations [11]. A commercial mode-locked system that should allow continuous tunability from about $200 \mathrm{~nm}$ to $3 \mu \mathrm{m}$ (part of the range is shown as $\mathrm{G}$ and F) was recently installed in the facility. One useful feature we hope to exploit is that the bandwidth of these mode-locked systems is considerable broader than the cw systems discussed above. This may help to alleviate some of the problems associated with interference fringes (see below). These mode-locked systems are easy to frequency triple (B in figure) and quadruple ( $\mathrm{A}$ in figure).

Different integrating spheres are used, depending on the radiometric calibration and the wavelength of calibration. Small diameter integrating spheres - typically diameters of $2 \mathrm{~cm}$ to $5 \mathrm{~cm}$ - equipped with precision apertures with diameters ranging from $3 \mathrm{~mm}$ to $8 \mathrm{~mm}$ are typically used for irradiance responsivity calibrations. Larger diameter spheres $-30 \mathrm{~cm}$ diameter - with $5 \mathrm{~cm}$ to $10 \mathrm{~cm}$ diameter exit ports are used for radiance measurements. The spheres are made of either sintered polytetrafluoroethylene-based coating (Spectralon ${ }^{\mathrm{TM}}$,Labsphere, Inc.) that has high diffuse reflectance from about $250 \mathrm{~nm}$ to $2.5 \mu \mathrm{m}$ or infragold for calibrations from $1 \mu \mathrm{m}$ to $20 \mu \mathrm{m}$. Typical irradiance levels at $1 \mathrm{~m}$ using $2.54 \mathrm{~cm}$ diameter integrating sphere with a $5 \mathrm{~mm}$ diameter aperture range from approximately $1 \mu \mathrm{W} / \mathrm{cm}^{2}$ to $10 \mu \mathrm{W} / \mathrm{cm}^{2}$. The irradiance levels can be continuously decreased using the laser intensity stabilizer to check for linearity. The irradiance can be increased by moving closer to the sphere (falls off as $1 / \mathrm{r}^{2}$ ) or by collimating the output of the sphere using either an off-axis parabolic mirror or a lens. Figure 2 shows typical irradiances for different wavelengths. Radiance levels between $1 \mathrm{~mW} / \mathrm{cm}^{2} / \mathrm{sr}$ and $5 \mathrm{~mW} / \mathrm{cm}^{2} / \mathrm{sr}$ are standard for a $30 \mathrm{~cm}$ diameter sphere with a $7.5 \mathrm{~cm}$ diameter output port. Note that the exit apertures are normally calibrated at the NIST facility for aperture area measurement [7]. A monitor detector is mounted on the sphere wall to monitor and correct for fluctuations in the source radiant flux during a calibration.

\subsection{Detectors}

The uncertainty in the spectral irradiance responsivity of reference standard detectors is the largest component in the calibration uncertainty budget on SIRCUS. Ultimately, the quality and characteristics of the reference standard detectors determine the uncertainty achievable on SIRCUS. Silicon tunnel-trap detectors, configured to operate in both radiant power and irradiance measurement modes, are the highest-level transfer standards used in the UV/Vis/NIR SIRCUS facility. They cover the spectral range from $350 \mathrm{~nm}$ to $1050 \mathrm{~nm}$. Nitrided Si reflection traps have been developed to cover the $200 \mathrm{~nm}$ to $400 \mathrm{~nm}$ spectral range. These detectors are calibrated against a cryogenic radiometer [12]. InGaAs detectors, calibrated against an electrical substitution Si bolometer (ESB) [13], cover the range from $1 \mu \mathrm{m}$ to $2.5 \mu \mathrm{m}$ while InSb detectors cover the $2 \mu \mathrm{m}$ to $5 \mu \mathrm{m}$ range. HgCdTe detectors are being developed to cover the spectral range from $3 \mu \mathrm{m}$ to $20 \mu \mathrm{m}$.

\subsection{Calibration method}

The entire data collection sequence is automated. Initially, an electronic shutter that blocks the laser radiation before it enters the optical fiber is closed and a background signal is acquired for both the reference standard trap detector and the sphere monitor. Then the shutter opens and the signals from the trap and the monitor on the sphere are recorded. 
The signals are initially amplified using a current to voltage amplifier and then fed into a digital voltmeter set to average over some number of power line cycles (the signal is averaged for a few seconds at most). Typically 3 'shutter closed' signals and 9 'shutter open' signals are averaged. The mean reference-to-monitor ratio and the standard deviation of the ratio are recorded. After this, the stage moves to the Device Under Test (DUT) position, and the data acquisition sequence is repeated. This gives the DUT mean signal-to-monitor ratio and the standard deviation of the ratio. The standard deviations of the ratios are monitored; trap detector measurement standard deviations larger than $0.01 \%$ are an indication of laser power instabilities.

Under routine calibration conditions, the intensity-stabilized laser wavelength is read by the wavemeter and transferred to the computer during each measurement. Along with mean ratios, the mean and standard deviation of the wavelength are recorded. This enables ready identification of laser wavelength instabilities during a scan. Occasionally, for faster data acquisition, the wavelength is only recorded at the beginning of the acquisition sequence. Following the data acquisition sequence, the wavelength is changed and the sequence is repeated.

\section{EXAMPLES OF SYSTEM-LEVEL CALIBRATIONS}

In the following, we present a few examples of SIRCUS calibrations that relate to the EOS or space-based radiometers. One of the unique features of SIRCUS is that the calibration can be done at system level. The three examples we discuss are the Marine Optical BuoY (MOBY), the SCRIPPS-NIST Advanced Radiometer (NISTAR), and Sun Photometers.

\subsection{MOBY}

Ocean color satellites such as the Sea-Viewing Wide Field-of-View Sensor (SeaWiFS) and the Moderate Resolution Imaging Spectrometer (MODIS) require vicarious calibration for accurate and continuous measurements of the waterleaving radiance. The primary reference instrument for most ocean color satellites is the MOBY, a radiometric buoy stationed in the waters off Lanai, Hawaii $[14,15]$.

Detector array-based spectral imaging systems, such as spectrographs, enable the simultaneous acquisition of an entire spectrum over some finite spectral region of interest; they can acquire a spectral image in a matter of seconds. The ability to rapidly acquire a full spectrum has led to the use of array-based systems in a variety of ocean color applications where the source may not be stable over minutes. Spectrographs commonly consist of an entrance port, a dispersing element (such as a grating) to spatially resolve the spectral components of the incident radiation, and mirrors to image the entrance port (often a slit) onto a detector reference plane. Because of the dispersing element, the spatial image of the entrance port falls on different regions of the detector array, depending on its wavelength; broadband sources form an image across the entire array. The spectral coverage of a spectrograph is determined by the size of its detector array, the dispersion properties of its grating, and its optical layout.

Two CCD-spectrographs, one for the blue spectral region (360 nm to $640 \mathrm{~nm}$ ), referred to as the BSG, and another for the red (560 nm to $940 \mathrm{~nm}$ ), the RSG, form the basis of the Marine Optical System (MOS), the sensor used in MOBY [14]. The same dual-spectrograph design is used for the MOS Profiler, a ship-board-deployable version of the MOBY sensor used in the development of bio-optical algorithms. The response of the two MOS spectrographs to monochromatic laser excitation is shown in Fig. 3. The spectra are similar for both spectrographs. There are four components to the image: a strong sharp peak corresponding to the image of the spectrograph entrance slit on the CCD; a broad, peaked structure around the slit image; a non-zero constant component; and an additional peak. The first three components are similar to specular, haze, and diffuse components of reflectance, respectively. They remain approximately invariant as the excitation wavelength is changed and the image moves across the CCD array. From physical examination of the spectrographs, the fourth component (the additional peak) arises from a spurious reflection off a mirror coupled with higher-order diffraction from the grating. As the image moves across the array, the reflection peak changes size, shape and position with respect to the primary peak. The specular component corresponds to the 

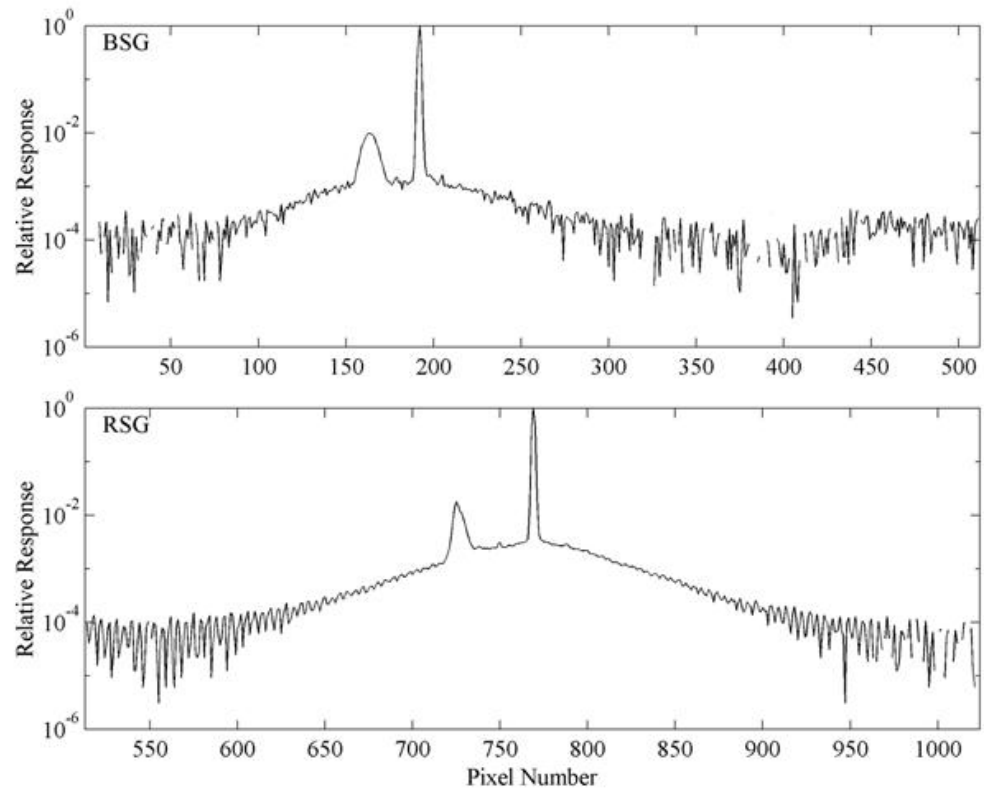

Figure 3: MOS response to monochromatic excitation at (a) $550 \mathrm{~nm}$ (for the blue spectrograph) and (b) $700 \mathrm{~nm}$ (for the red spectrograph).

properly imaged radiation; the other components arise from light scattered in the spectrograph, principally from the grating. The 'specular' component in the spectrograph image is analogous to the 'in-band' component of a filter radiometer's responsivity, while the sum of the other three components is analogous to the 'out-of-band' component.This unwanted background radiation, while small, is fairly typical for single grating spectrographs and can give rise to unforeseen errors, often much larger than anticipated, when the spectral distribution of a source being measured differs significantly from that of the calibration source. Such a situation is routinely encountered in ocean color measurements, where instruments are calibrated against incandescent sources having peak radiances in the near infrared and subsequently measure the optical properties of the ocean, where the up-welled radiance peaks in the blue to green spectral region. Consequently, to fully understand the radiometric performance of a spectrograph used for ocean color measurements, it is important to characterize the stray light in the system.

Knowing the relationship between the excitation wavelength and the position of the image on the array enables us to determine the fraction of incident light at some wavelength that is scattered onto a particular element. For example, for the wavelength $\lambda_{\text {ex }}$ in Fig. 3(top), $0.01 \%$ of the light imaged on element 190 (BSG) is scattered onto element 300. Assuming each element in the detector array has the same average spectral responsivity, the signal from element 300 to radiation at wavelength $\lambda_{\text {ex }}$ is $0.01 \%$ of the signal from element 190 .

A single data set, shown in Fig. 3, does not give us the spectral resolution required to determine a pixel's in-band relative spectral response. For this information, it is necessary to tune the monochromatic excitation source in fine steps, a situation SIRCUS is well equipped to address. Figure 4 shows the relative spectral response of adjacent pixels of the MOS RSG detector array as the laser excitation wavelength on SIRCUS is tuned over the range from $587 \mathrm{~nm}$ to $593 \mathrm{~nm}$ in $0.1 \mathrm{~nm}$ steps. At each excitation wavelength, the responsivity of each pixel is separately determined. By tuning the excitation wavelength over a broad spectral range, a single pixel's absolute spectral responsivity (and slit scatter function [16]) can be determined. 


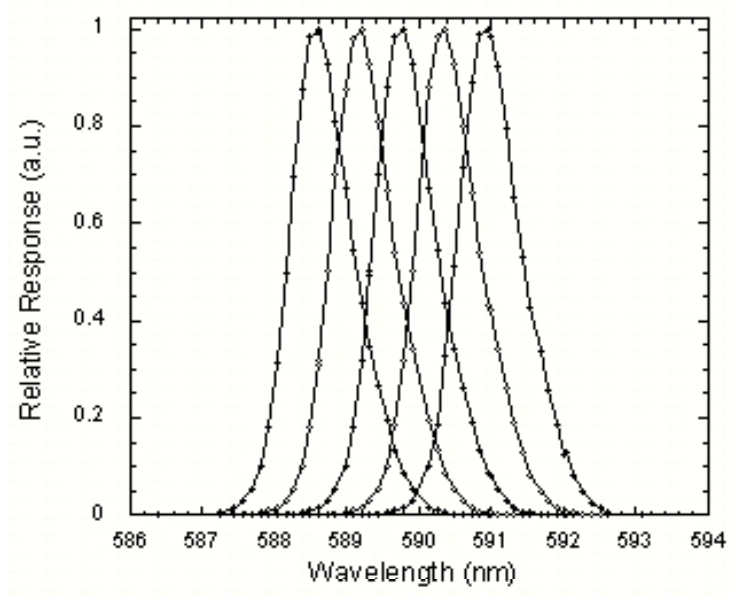

Figure 4: Spectral responsivity of pixels 428 to 432 (from left to right).

As discussed in detail by Brown et al. [17], MOBY's system response has been corrected for stray light. MOBY is used for the vicarious calibration of a number of ocean color satellites and any changes in MOBY-derived water-leaving radiances are directly reflected in satellite sensor calibration coefficients for the relevant channels. Consider the impact on MODIS as a representative example of the impact of the stray light correction of MOBY. MODIS bands 8 through $12(412 \mathrm{~nm}$ to $551 \mathrm{~nm})$ are vicariously calibrated against MOBY [18]. The stray-light-correction factors for MOBY (the correction factors are simply the ratio of the stray-light-corrected to the uncorrected measurements) in terms of $L_{\mathrm{WN}}(\lambda)$ at the MODIS band-averaged wavelengths over 5 years of deployments are given in Fig. 5 . Each left-to-right grouping in the figure is a separate buoy deployment. Note that two different MOS spectrograph systems are used:

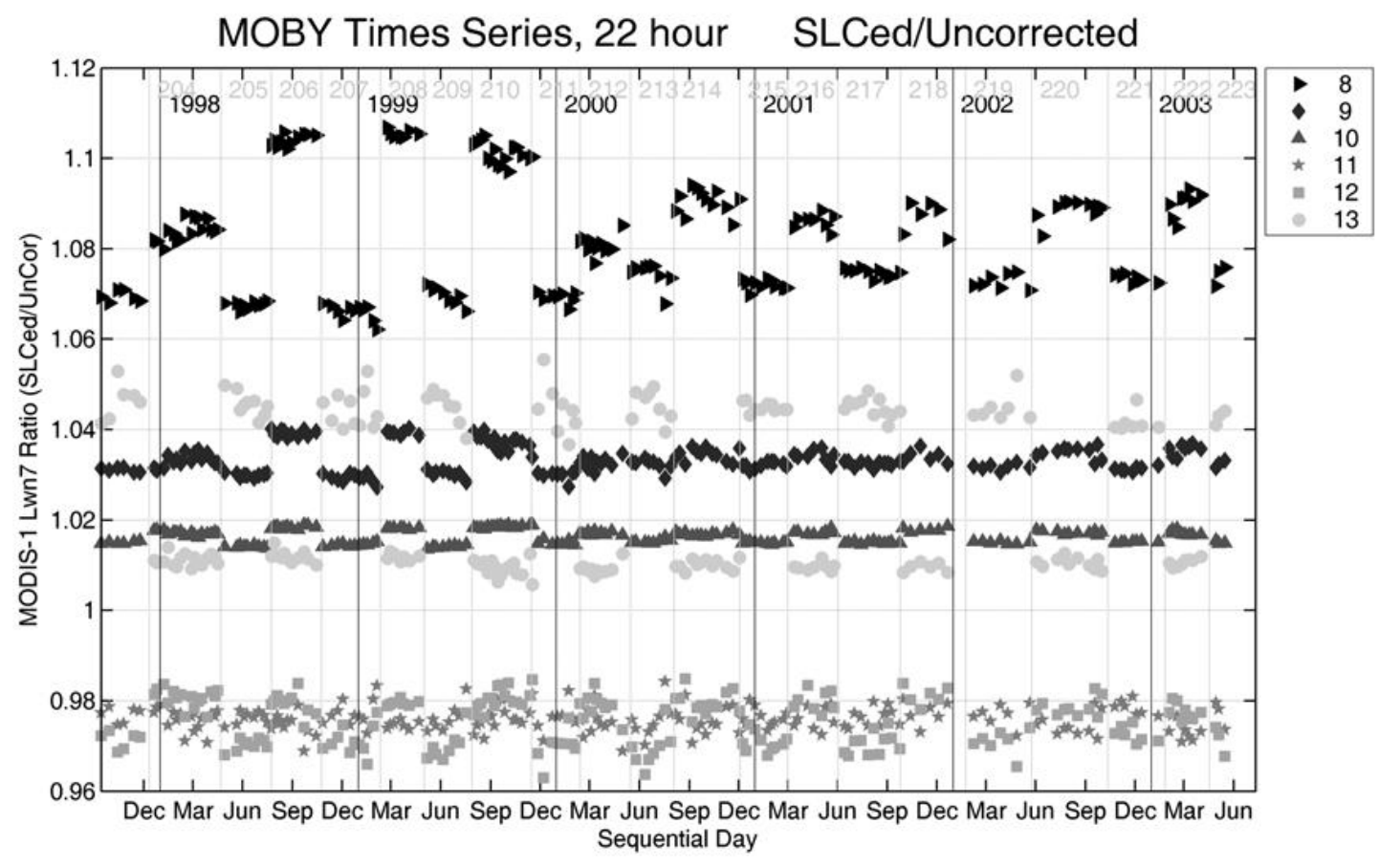

Figure 5: MOBY $L_{\mathrm{WN}}$ stray light correction factors for MODIS bands. 
one for even numbered buoy deployments and another one for odd numbered buoy deployments. The correction factors range from $+8 \%$ for Band 8 to $-2.5 \%$ for Bands 11 and 12. There are slight differences in the stray light corrections for the two different MOS systems. There was an increase in the magnitude of the stray light correction factor for Band 8 for 3 Buoy deployments around 1999. During these deployments, a different optical fiber was used. With the exception of these buoys, the correction factors have been stable over the entire deployment sequence, implying that the MOBY imaging and the MOS slit-scatter functions, along with the ocean color, have remained stable over this time frame. The observed radiometric stability of the MOBY systems $[14,17,18]$ enables us to correct previous deployments for stray light with confidence.

\subsection{NISTAR}

Scripps-NISTAR is a space-qualified radiometer package composed of three electrical substitution cavity radiometers and one photodiode radiometer [19]. It was designed and built to orbit the Earth's Lagrange-1 (L1) point as part of NASA's proposed Deep Space Climate Observatory (DISCOVR) mission and measure the energy emitted and reflected by the Earth with an accuracy of $0.1 \%$. The Scripps-NISTAR package contains 1$)$ a total $(<0.2 \mu \mathrm{m}$ to $>100 \mu \mathrm{m})$ channel (Band A) to measure total radiant power in the UV, visible, and infrared wavelengths; 2 ) a solar ( 0.2 to $4 \mu \mathrm{m}$ ) channel (Band B) to measure reflected solar radiance in the UV, visible and near infrared wavelengths; 3) a near infrared ( 0.7 to $4 \mathrm{~m}$ ) channel (Band C) to measure reflected IR solar radiance; and 4 ) a photodiode (0.3 to $1 \mu \mathrm{m})$ channel to be used as an on-board calibration reference for the Scripps-NISTAR instrument.

SIRCUS was used to make absolute irradiance responsivity measurements of the three cavity radiometers and the $\mathrm{Si}$ photodiode. During pre-flight calibration of NISTAR at the SIRCUS facility, the instrument was in a thermal-vacuum chamber to simulate the space environment. Through windows in the chamber, it viewed the output of a laserilluminated integrating sphere, simulating the geometry of the view of Earth from L1. Shown in Figure 6 is the normalized irradiance responsivity using $532 \mathrm{~nm}$ radiation emitted from a $2.54 \mathrm{~cm}$ diameter integrating sphere located approximately $2 \mathrm{~m}$ from the cavities. The sphere was illuminated by $\sim 10 \mathrm{~W}$ of fiber optically coupled light from a frequency-doubled, diode-pumbed Nd: Vanadate laser.

Shown in Figure 7 is the transmittance of three different Band $C$ filters obtained with collimated light from a Ti:Sapphire laser. For these measurements, the monochromatic, uniform output from a $2.54 \mathrm{~cm}$ diameter integrating sphere was collimated with an off-axis parabolic mirror. Also shown in the figure, for comparison, is the measurement of the transmittance of the filters with a monochromator. The SIRCUS results are a little different (probably because of inter-reflections) from the "piece parts" approach and will help to put them on an absolute scale.

SIRCUS has also helped to calibrate another instrument on the DSCOVR mission, the Earth Polychromatic Imaging Camera (EPIC) [20].

\subsection{Sun photometers}

Sun photometers and sky radiometers are used for atmospheric characterization. Sun photometers determine the atmospheric optical depth while radiance determined from sky radiometers constrains the aerosol models input into radiative transfer codes that calculate the atmospheric contribution to the at-sensor signal. There are several networks of sun photometers distributed globally to measure aerosol concentrations and distributions, e.g. the Aerosol Robotic Network (AERONET) [21]. The Sensor Intercomparison and Merger for Biological and Interdisciplinary Oceanic Studies (SIMBIOS) Project Office at NASA's Goddard Space Flight Center (GSFC) uses in situ atmospheric data to validate MODIS and other ocean color satellite aerosol optical products, to evaluate aerosol models used for atmospheric correction, and to develop vicarious sensor calibration methodologies [22]. 


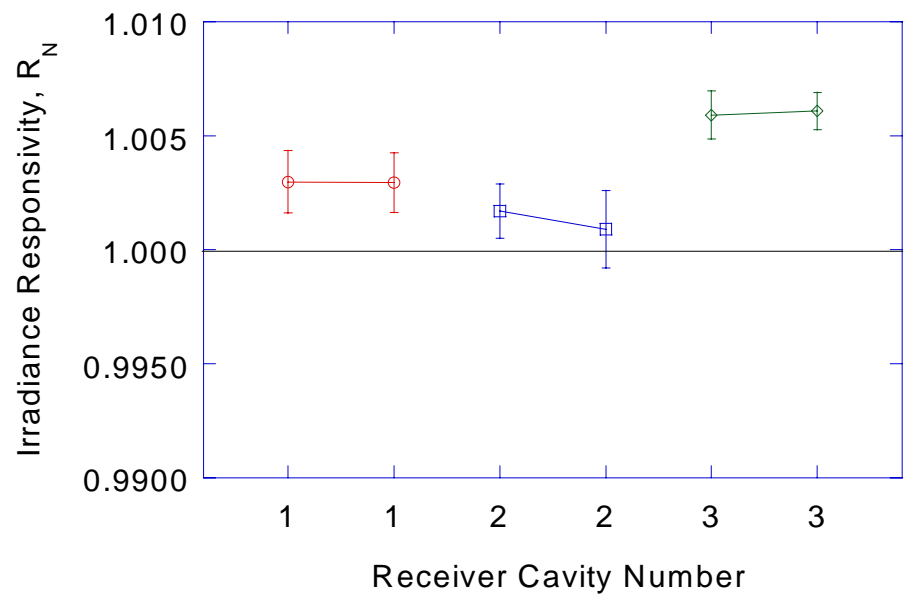

Figure 6: Normalized irradiance responsivity of three receiver cavities calibrated on SIRCUS. For each cavity, the measurements were performed two times in order to assess repeatability within a two week period.

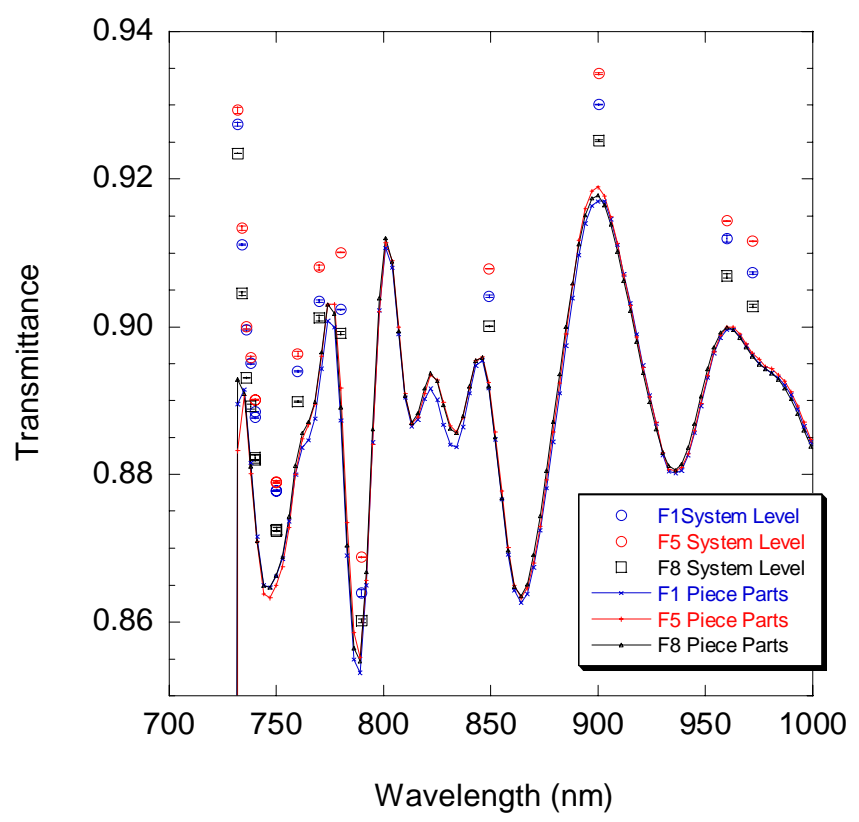

Figure 7: Absolute transmittance for 3 Band C filters measured using either monochromatic measurements forming a piece parts approach (lines) or using the system-level approach enabled by SIRCUS (symbols).

Instruments are calibrated for irradiance responsivity against reference sun photometers using the cross-calibration technique at NASA's GSFC [23]. Reference sun photometers that are part of the AERONET project are calibrated using the Langley-Bouger technique at the Mauna Loa Observatory on regular intervals. The cross-calibration technique consists of near simultaneous solar observations at GSFC with the uncalibrated instrument and a calibrated reference sun photometer. The method assumes that the ratio of the output voltages for the same channel (e.g., same spectral 
responsivity) for the reference and uncalibrated radiometers and a particular air mass is proportional to the ratio of the output voltage at zero air mass. If the spectral responsivities differ, a correction is made for spectral differences related to Rayleigh, ozone, and aerosol attenuation. The uncertainty in the calibration at GSFC is approximately $2 \%$.

Instruments are calibrated for radiance responsivity at NASA's GSFC using a NIST-traceable lamp -illuminated integrating sphere source known as the 'Hardy' source. A reference spectroradiometer equipped with an integrating sphere irradiance collector, the OL746/ISIC, is calibrated for irradiance responsivity against a NIST standard irradiance lamp. The irradiance calibration is then transferred to the Hardy sphere using the OL746/ISIC. Knowing the irradiance responsivity of the OL746/ISIC, the distance between the sphere exit port and the entrance aperture to the 746/ISIC, and the sphere exit port area, it is straightforward to calculate the spectral radiance of the "Hardy" sphere. The uncertainty in the radiance responsivity calibration is estimated to be approximately $5 \%(k=2)$.

As a first assessment of the uncertainties assigned to the radiance and irradiance responsivity calibrations of sun photometers, two multi-channel filter radiometers used in the SIMBIOS program were calibrated for irradiance and radiance responsivity on SIRCUS and the results compared with standard calibrations [24]. The Satellite Validation for Marine Biology and Aerosol Determination (SimbadA) radiometers are eleven-channel filter radiometers with bandpasses of approximately $10 \mathrm{~nm}$ and center wavelengths at $350 \mathrm{~nm}, 380 \mathrm{~nm}, 410 \mathrm{~nm}, 443 \mathrm{~nm}, 490 \mathrm{~nm}, 510 \mathrm{~nm}, 565$ $\mathrm{nm}, 620 \mathrm{~nm}, 670 \mathrm{~nm}, 750 \mathrm{~nm}$ and $870 \mathrm{~nm}$, respectively. For comparison to the GSFC sun photometer cross-calibration results, the SIRCUS-derived irradiance responsivities $s(\lambda)$ were used to predict a top-of-the-atmosphere (TOA) signal Vo(SIRCUS):

$$
V_{o}(\operatorname{SIRCUS})=\int s(\lambda) E(\lambda) d \lambda
$$

where $s(\lambda)$ is the spectral responsivity of one of the radiometer channels and $E(\lambda)$ is an exo -atmospheric solar irradiance spectrum. Souaidia et al. [24] used the exo -atmospheric solar irradiance spectra developed by others. To perform the integration, the $s(\lambda)$ and the $E(\lambda)$ were interpolated to a uniform wavelength interval of $0.25 \mathrm{~nm}$ and integrated.

The dominant source of uncertainty in the SIRCUS-based predicted TOA signal was the solar irradiance spectrum used. Changes of $1 \%$ or so were observed, depending on the exo-atmospheric solar irradiance spectrum chosen. Given a cross-calibration uncertainty of $\sim 2 \%$, we are able to validate the cross-calibration with a combined expanded uncertainty $(k=2)$ of $\sim 4 \%$. Predicted results for the $750 \mathrm{~nm}$ channel and the $490 \mathrm{~nm}$ channel agreed with the crosscalibration to within $2 \%$; the agreement for the $440 \mathrm{~nm}$ channel was approximately $5 \%$. The agreement for the $490 \mathrm{~nm}$ and $750 \mathrm{~nm}$ channels is within the combined uncertainties, but the $440 \mathrm{~nm}$ results are not and warrant further investigation [24].

In order to compare the two sets of radiance measurements, radiance responsivities, $s(\lambda)$, were interpolated to a uniform wavelength interval, as were the sphere spectral radiance values, $L(\lambda)$, for the lamp-illuminated integrating sphere source. The measured signal was then compared to the predicted signal using equation 1, but replacing $E(\lambda)$ with $L(\lambda)$ and using the instrument's radiance responsivity instead of it's irradiance responsivity. We obtained differences in the calibrations between $2 \%$ and $4 \%$, depending on wavelength. The radiance responsivity results were consistent with results of an intercomparison performed at NASA’s Goddard Space Flight Center in 2001 [25, 26].

\section{SUMMARY}

SIRCUS is a laser-based facility recently developed at NIST to provide system-level characterization and calibration of a vast assortment of radiometers, including EOS instruments, with state-of-the-art uncertainties. We continue to improve the radiometric calibration capabilities of SIRCUS by decreasing the uncertainties and increasing the spectral coverage. In many situations, such as with MOBY, it is impossible to bring the instrument to NIST for calibration. Recognizing the need and taking advantage of the miniaturization of lasers, a "traveling SIRCUS" has been developed to perform in-situ calibrations of EOS and related sensors. Currently, the spectral coverage available with this traveling 
SIRCUS is from $360 \mathrm{~nm}$ to $1050 \mathrm{~nm}$; the spectral coverage is being expanded to include the short-wave infrared out to $2.5 \mu \mathrm{m}$.

\section{ACKNOWLEDGMENTS}

We wish to thank John Grangaard for his support of this facility and acknowledge the continued support of the Air Force component of the Calibration Coordination Group of the armed services. We also thank Steven Lorentz, Carol Johnson and Edward Early from NIST for significant contributions to this work.

\section{REFERENCES}

${ }^{*}$ Certain commercial equipment, instruments, or materials are identified in this paper to foster understanding. Such identification does not imply recommendation or endorsement by the National Institute of Standards and Technology, nor dies it imply that the materials or equipment identified are the best available for the purpose.

1. http://eospso.gsfc.nasa.gov/.

2. Butler, J.J. and R.A. Barnes, Calibration strategy for the earth observing system (EOS)-AM1 platform. IEEE Transactions on Geoscience and Remote Sensing, 1998. 36(4): p. 1056-1061.

3. Brown, S.W., G.P. Eppeldauer, and K.R. Lykke, NIST Facility for spectral irradiance and radiance responsivity calibrations with uniform sources. Metrologia, 2000. 37: p. 579-582.

4. Brown, S.W., G. Eppeldauer, and K.R. Lykke, Facility for spectral irradiance and radiance responsivity calibrations using uniform sources (SIRCUS). Applied Optics, 2004. to be submitted.

5. Anderson, V.E., N.P. Fox, and D.H. Nettleton, Highly stable, monochromatic and tunable optical radiation source and its application to high accuracy spectrophotometry. Applied Optics, 1992. 31(4): p. 536-545.

6. Gentile, T.R., J.M. Houston, and C.L. Cromer, Realization of a scale of absolute spectral response using the NIST high-accuracy cryogenic radiometer. Applied Optics, 1996. 35(22): p. 4392-4403.

7. Fowler, J. and M. Litorja, Geometric area measurements of circular apertures for radiometry at NIST. Metrologia, 2003. 40: p. S9-S12.

8. $\quad$ sircus wavelengths powers.

9. Jusinski, L.E. and C.A. Taatjes, Efficient and stable operation of an Ar+-pumped continuous-wave ring laser from 505-560nm using a coumarin dye. Review of Scientific Instruments, 2001. 72: p. 2837-2838.

10. Moulton, P.F., Spectroscopic and laser characteristics of Ti:Al2O3. J. Opt. Soc. Am. B, 1986. 3: p. 125-133.

11. Gentile, T.R. and C.L. Cromer, Mode-locked lasers for high-accuracy radiometry. Metrologia, 1996. 32: p. 585-587.

12. Gentile, T.R., et al., National Institute of Standards and Technology high-accuracy cryogenic radiometer. Applied Optics, 1996. 35(7): p. 1056-1068.

13. Rice, J.P., An electrically substituted bolometer as a transfer-standard detector. Metrologia, 2000. 37: p. 433436.

14. Clark, D.K., et al. An overview of the radiometric calibration of MOBY. in Earth Observing Systems VI. 2002: SPIE.

15. Clark, D.K., et al., MOBY, a radiometric buoy for performance monitoring and vicarious calibration of satellite ocean color sensors: measurement and data analysis protocols, in Ocean Optics Protocols for Satellite Ocean Color Sensor Validation, Revision 3, Volume 2, J.L. Mueller and G.S. Fargion, Editors. 2002, NASA Goddard Space Flight Center: Greenbelt, MD. p. 138-170.

16. Kostkowski, H.J., Reliable Spectroradiometry. 1997.

17. Brown, S.W., et al., Stray-light correction algorithm for spectrographs. Metrologia, 2003. 40: p. S81-S84.

18. Barnes, R.A., et al. The calibration of SeaWiFS on orbit. in SPIE. 2000: SPIE.

19. Rice, J.P., S.R. Lorentz, and T.M. Jung. The next generation of active cavity radiometers for space-based remote sensing, in 10th Conference on Atmospheric Radiation. 1999. Madison, WI: American Meteorological Society.

20. Early, E.A., et al. Radiometric Calibration of the Scripps Earth Polychromatic Imaging Camera. in Earth Observing Systems VI; SPIE. 2001. 
21. Holben, B.N. et al., Aeronet-a federated instrument network and data archive for aerosol characterization. Remote Sens. Environ., 1998. 66: p. 1-16.

22. Fargion, G.S., R.A. Barnes, and C.R. McClain, In situ aerosol optical thickness collected by the SIMBIOS program (1997-2000): Protocols and data QC and analysis. 2001, NASA/TM-2001-209982: Greenbelt, MD.

23. Pietras, C., et al., Calibration of sun photometers and sky radiance sensors, in In situ aerosol optical thickness collected by the SIMBIOS program (1997-2000): protocols, and data QC and analysis, R.B. G. S. Fargion, and C. McClain, Editor. 2001, NASA's Goddard Space Flight Center: Greenbelt, MD.

24. Souaidia, N., et al., Comparison of laser-based and conventional calibrations of sun photometers. SPIE proceedings, 2003.

25. Butler, J.J., B.C. Johnson, and R.A. Barnes, Radiometric measurement comparisons at NASA's Goddard Space Flight Center: Part I. The GSFC sphere sources. The Earth Observer, 2002. 14(3): p. 3-8.

26. Butler, J.J., B.C. Johnson, and R.A. Barnes, Radiometric measurement comparisons at NASA's Goddard Space Flight Center: Part II. Irradiance lamp comparisons and the NIST sphere source. The Earth Observer, 2002. 14(4): p. 25-29. 


\title{
Absolute Radiometric Calibration of Digital Imaging Systems
}

\author{
Steven W. Brown, Thomas C. Larason, Catherine Habauzit, George P. Eppeldauer, \\ Yoshihiro Ohno, and Keith R. Lykke \\ National Institute of Standards and Technology, Gaithersburg, MD 20899
}

\begin{abstract}
We have developed a tunable laser-based facility for the absolute radiometric calibration of digital imaging systems such as CCD cameras, spectrographs, and microscopes. Several types of silicon-based digital imaging systems have been calibrated in this new facility, including a commercially available camera equipped with a removable photopic filter, a custom-designed digital microscope, and a CCD spectrograph. We present results of the CCD camera calibration in detail and discuss relevant aspects of the microscope and spectrograph calibrations. During the radiometric calibration, the pixel-to-pixel uniformity, linearity, and absolute spectral responsivity of each system were determined over the visible spectral range (400 $\mathrm{nm}$ to $800 \mathrm{~nm}$ ). Each of these aspects of the CCD camera calibration will be presented, along with a discussion of the measurement uncertainties.
\end{abstract}

Keywords: calibration, CCD, digital camera, radiometry

\section{INTRODUCTION}

Improvements in the operation and performance of Digital Imaging Systems (DIS's) have led to an explosion in the use of these systems in a variety of scientific, medical, and industrial imaging applications. In many of these new applications, it is important that the spectral characteristics of the imaging system as well as its spatial (geometric) properties be well characterized and its photometric, radiometric, or colorimetric performance known. This has resulted in increased demand for characterization and calibration methodologies traceable to common standards.

By knowing the spectral responsivity of a DIS, one can accurately predict its performance under differing operating conditions. Currently, these systems can be calibrated with relative combined standard uncertainties of approximately $5 \%$ to $10 \%$. In many cases, DIS's are calibrated against broad-band incandescent sources — such as Illuminant A — and subsequently measure sources with very different spectral power distributions. In this case, the measurement uncertainties are not well known in general, and can be much larger than expected.

\section{DESCRIPTION OF THE SIRCUS FACILITY}

We have developed a facility at the National Institute of Standards and Technology (NIST) for Spectral Irradiance and Radiance Responsivity Calibrations using Uniform Sources (SIRCUS) to calibrate DIS's. In this facility - shown in Fig. 1 - lasers are directed into an integrating sphere to produce a monochromatic, Lambertian source for irradiance and radiance responsivity calibrations. This integrating sphere source (ISS) has been used to calibrate the spectral radiance and irradiance responsivity of a variety of detectors and imaging systems [1]. The two critical cornerstones of the facility are broadly tunable continuous-wave (cw) laser sources and reference standard detectors that are well-characterized and calibrated with low uncertainties to determine the radiance of the ISS with low uncertainty.

A variety of lasers are used to provide continuous coverage over the spectral range from $370 \mathrm{~nm}$ to $1000 \mathrm{~nm}$. A Ti:sapphire laser provides tunable coverage from $690 \mathrm{~nm}$ to $1000 \mathrm{~nm}$, frequency-doubling enables tunability from $370 \mathrm{~nm}$ to $450 \mathrm{~nm}$. Discrete lasers were used for the spectral range from $450 \mathrm{~nm}$ to $550 \mathrm{~nm}$ and dye lasers are used to cover the spectral region from $550 \mathrm{~nm}$ to $700 \mathrm{~nm}$. Currently, dye lasers are used to cover the spectral range from $415 \mathrm{~nm}$ to $700 \mathrm{~nm}$.

Reference standard detectors with spectral power responsivities directly traceable to the NIST primary standard equipped with precision apertures were used to determine the sphere radiance [2]. These trap detectors are calibrated for spectral power responsivity directly against a cryogenic radiometer over the spectral range from $405 \mathrm{~nm}$ to $920 \mathrm{~nm}$ [3]. From the power responsivity and the aperture area, the irradiance responsivity of the trap detector is determined. By measuring the distance between the sphere exit port and the trap detector entrance aperture and the area of the ISS exit port, the radiance of 


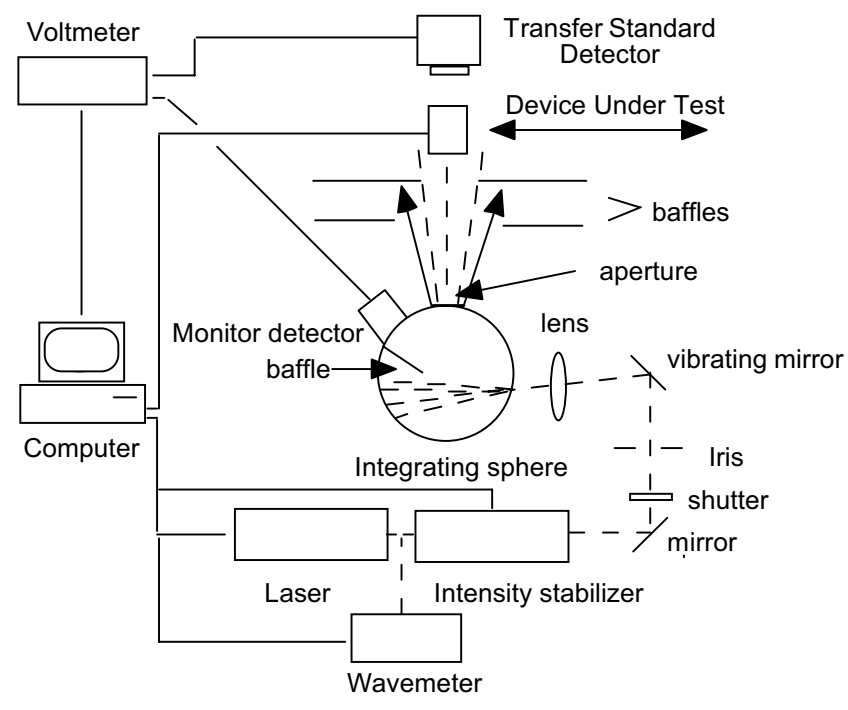

Fig. 1. Schematic diagram of the SIRCUS facility.

the ISS was determined with a relative combined standard uncertainty of $0.1 \%[4,5]$. A silicon monitor detector mounted on the wall of the ISS was used to correct for small power fluctuations in the laser between measurements with the reference standard detector and the DIS.

\section{CALIBRATION RESULTS}

In general, the absolute spectral responsivity (ASR) of an instrument, $R(\lambda)$, relates the signal measured by a detector, $S$, to the spectral radiance of the source being measured, $L(\lambda)$ :

$$
S=\int R(\lambda) L(\lambda) d \lambda .
$$

The integration is over the full spectral bandwidth of the source. A DIS can be considered to be comprised of $i$ by $j$ independent detectors, with an individual measurement equation for each detector, or pixel, in the array. Each pixel in the array will also image a different area of the ISS, and in general:

$$
S_{i, j}=\int R_{i, j}(\lambda) L_{i, j}(\lambda) d \lambda
$$

with $i$ defining the column position and $j$ equal to the corresponding row in the array. $L_{i, j}(\lambda)$ refers to the radiance of the spatial region of the sphere exit port imaged onto pixel $i, j$. Note that for uniform sources, we can eliminate the spatial dependence on radiance: $L_{i, j}(\lambda) \sim L(\lambda)$.

Because we are using narrow-band laser sources (with a bandwidth of approximately $10 \mathrm{MHz}$, or $0.00001 \mathrm{~nm}), L(\lambda)$ can be approximated by a delta function, $L(\lambda)=L\left(\lambda_{o}\right) \delta\left(\lambda-\lambda_{o}\right)$, and Eq. 2 can be rewritten as,

$$
S_{i, j}=R_{i, j}\left(\lambda_{o}\right) L\left(\lambda_{o}\right)
$$

Knowing the source radiance $L\left(\lambda_{o}\right)$ and measuring the CCD signal $S\left(\lambda_{o}\right)$, we obtain a direct measurement of the desired quantity $R_{i, j}\left(\lambda_{o}\right)$ :

$$
R_{i, j}\left(\lambda_{o}\right)=S_{i, j} / L\left(\lambda_{o}\right)
$$

By tuning the wavelength of the calibration source, we directly obtain the absolute spectral responsivity of each element in the DIS.

To complete the radiometric calibration of an instrument, other factors need to be considered as well, including the linearity of the response and the exposure time correction.

\subsection{Camera Calibration}

We calibrated an 8-bit silicon-based CCD camera with 640 × 480 pixels equipped with a 35 mm focal length lens and a removable photopic filter. The instrument field-of-view under normal operating conditions is $13.5^{\circ}$. It comes equipped with 8 user-selectable shutter speeds, enabling measurements of luminance levels as low as $0.34 \mathrm{~cd} / \mathrm{m}^{2}$. It has a short-term repeatability of $0.4 \%$, and is calibrated by the manufacturer against Illuminant A with a stated relative combined standard uncertainty $(k=1)$ of $4 \%$. The camera pixel-to-pixel response uniformity, linearity, and absolute responsivity — both with and without the photopic $\mathrm{V}(\lambda)$ filter on the front of the camera - were determined.

We used a $20 \mathrm{~cm}$ diameter Spectralon-coated integrating sphere with a $5.34 \mathrm{~cm}$ diameter exit port to calibrate the CCD camera. Typical power levels of radiation introduced into the ISS range from a few $\mathrm{mW}$ to a few hundred $\mathrm{mW}$, depending on the laser used, giving a source radiance in the range from $1 \times 10^{-7} \mathrm{~W} / \mathrm{mm}^{2} / \mathrm{sr}$ to $1 \times 10^{-4} \mathrm{~W} / \mathrm{mm}^{2} / \mathrm{sr}$. Prior to determining the responsivity of the $\mathrm{CCD}$ camera, the monitor detector was calibrated against the reference standard detector over the wavelength range of interest. The calibration related the monitor detector signal to the ISS radiance. The CCD camera was 


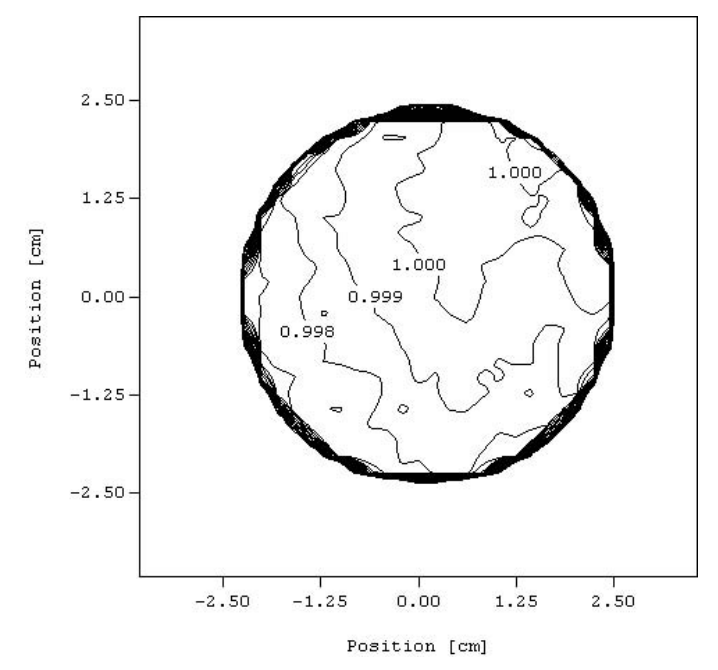

Fig. 2. ISS exit port radiance uniformity with $532 \mathrm{~nm}$ excitation. then aligned in front of the ISS and its radiance responsivity determined. During the camera calibration, the sphere radiance was determined at a particular wavelength by measuring the monitor detector signal.

We measured the uniformity of the ISS at $488 \mathrm{~nm}$, $532 \mathrm{~nm}$, and $633 \mathrm{~nm}$ using a temperature-stabilized Si radiometer with a narrow field of view mounted on a computer controlled two-axis (XY) translation stage. The stage was stepped in $2 \mathrm{~mm}$ increments over a $6 \mathrm{~cm}$ by $6 \mathrm{~cm}$ square area (an area larger than the area of the sphere exit port). The radiometer signal, normalized by the sphere monitor photodiode signal, was recorded at each position. Results are shown in Fig. 2 for the relative radiance over the ISS exit port at $532 \mathrm{~nm}$; results for $633 \mathrm{~nm}$ and $488 \mathrm{~nm}$ were similar. As shown in the figure, the radiance from upper right quadrant of the sphere is slightly larger than the radiance from the rest of the sphere. We therefore allow $L_{i, j}(\lambda) \approx L(\lambda)$, with an added relative standard uncertainty of $0.25 \%$.

We then positioned the camera in front of the ISS exit port. Using a target grid centered on the ISS exit port, we positioned the camera to image the central $4 \mathrm{~cm}$ by $3 \mathrm{~cm}$ region of the sphere exit port. Prior to determining the absolute responsivity of the $\mathrm{CCD}$, it is necessary to measure and correct for any non-linearity in the response of the camera. We therefore measured the response of the CCD array, averaged over the central 50 by 50 pixels, as the sphere radiance was changed. The measurements involved changing the intensity of the laser light entering the ISS (by adjusting the throughput of a laser power controller) and comparing the radiance of the sphere measured by the CCD with the known ISS radiance determined by the calibrated monitor photodiode. The 8-bit camera has a maximum digital number (DN) of 255, corresponding to a $90 \%$ of the CCD full-well capacity; data were taken with the same CCD exposure time $(2 \mathrm{~ms})$ over a radiance range giving count levels varying from approximately $10 \mathrm{DN}$ to $240 \mathrm{DN}$. The resultant data are plotted in Fig. 3 as the ratio of the CCD signal to the sphere radiance versus the $\mathrm{CCD}$ signal $(\mathrm{DN})$. If the $\mathrm{CCD}$ response were linear, the ratio of the $\mathrm{CCD}$ signal to the ISS radiance would remain constant as a function of CCD count level. However, the ratio increased sharply as the CCD camera signal increased from $10 \mathrm{DN}$ to $100 \mathrm{DN}$. Above $100 \mathrm{DN}$, the ratio continued to rise slightly, increasing from a value of approximately 60 at $100 \mathrm{DN}$ to a value of approximately 63 at $230 \mathrm{DN}$. In determining the absolute radiance responsivity of the $\mathrm{CCD}$, we normalized individual data sets to the high-count level, with the individual scaling factors determined by the results shown in Fig. 3.

Note that we only calibrated the linearity of the CCD using a shutter setting corresponding to an exposure time of $2 \mathrm{~ms}$. It is difficult to distinguish between a non-linearity in the CCD response, and a variability in the camera readout offset value. For example, we

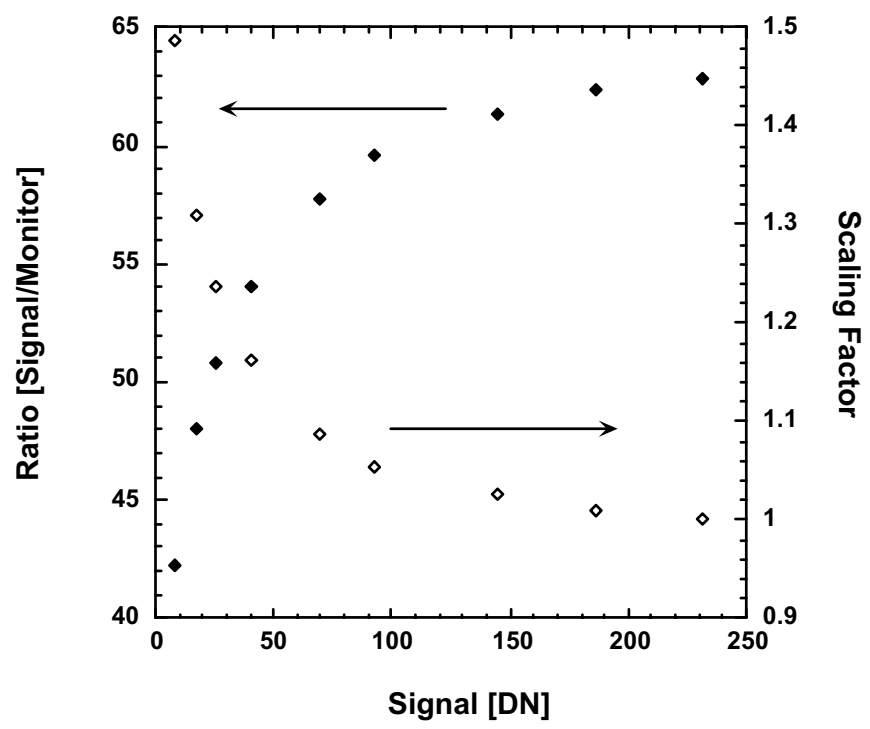

Fig. 3. Non-linear response of the CCD camera and the resultant scaling factors used in the absolute responsivity calibration. 
changing the offset level by 7 DN. Applying this simple correction to the data in Fig. 3, the response of the CCD camera was linear to within $1 \%$ over the range from $25 \mathrm{DN}$ to $230 \mathrm{DN}$.

The CCD camera was then positioned in front of the ISS, and its spectral responsivity, in DN per second per unit radiance, was determined. During the calibration on SIRCUS, the laser was tuned to the wavelength of interest. The CCD camera measured the radiance from the ISS exit port and the radiance responsivity (DN/s/ $\left./ \mathrm{W} / \mathrm{cm}^{2} / \mathrm{sr}\right)$ ) was calculated by dividing the CCD output signal, corrected for the exposure time, by the sphere radiance. During the calibration, images were acquired with several different exposure times. The CCD responsivity was measured for different exposure times at several wavelengths during the calibration. The CCD responsivity agreed to within $1 \%$ for the different exposure times.

In Fig. 4, the pixel-to-pixel responsivity of the camera is shown for $580 \mathrm{~nm}$ excitation. Without the photopic filter in place, the total response variation over the central $90 \%$ of the array was approximately $5 \%$, as shown in Fig. 4(a). With the photopic filter installed, the non-uniformity in the CCD responsivity increased to approximately $10 \%$, as shown in Fig. 4(b). All measurements were performed under the same conditions. The data were not corrected for the non-uniformity of the ISS radiance because it was negligible compared with the non-uniformity of the pixel-to-pixel responsivity of the CCD.

To determine the ASR, we averaged the response of the central 50 by 50 pixels over the wavelength range from $400 \mathrm{~nm}$ to $800 \mathrm{~nm}$. At the time of the calibration, we did not have continuously tunable lasers for the spectral range from $450 \mathrm{~nm}$ to $550 \mathrm{~nm}$. To fill in this spectral region, we calculated the relative responsivity using the output from a lamp-monochromator source ( $4 \mathrm{~nm}$ bandwidth) [6], and determined the absolute responsivity by comparison with results obtained on SIRCUS. For this part of the calibration, relay optics were used to create a parallel $2.5 \mathrm{~cm}$ diameter beam. The radiation, under-filling the CCD field-of-view, was imaged onto a small region in the center of the CCD array. During the calibration, the radiant power in the beam was measured at each wavelength using reference detectors and an image subsequently acquired by the CCD. A 35 by 35 pixel area on the CCD was selected to give an averaged response. The data were subsequently corrected for the nonlinear response of the CCD and normalized to the radiant power in the beam, giving a relative responsivity of the CCD. To determine the absolute responsivity of the camera, the data were normalized to the SIRCUS results near $580 \mathrm{~nm}$. Results are shown in Fig. 5 for the camera (a) with and (b) without the photopic filter attached.

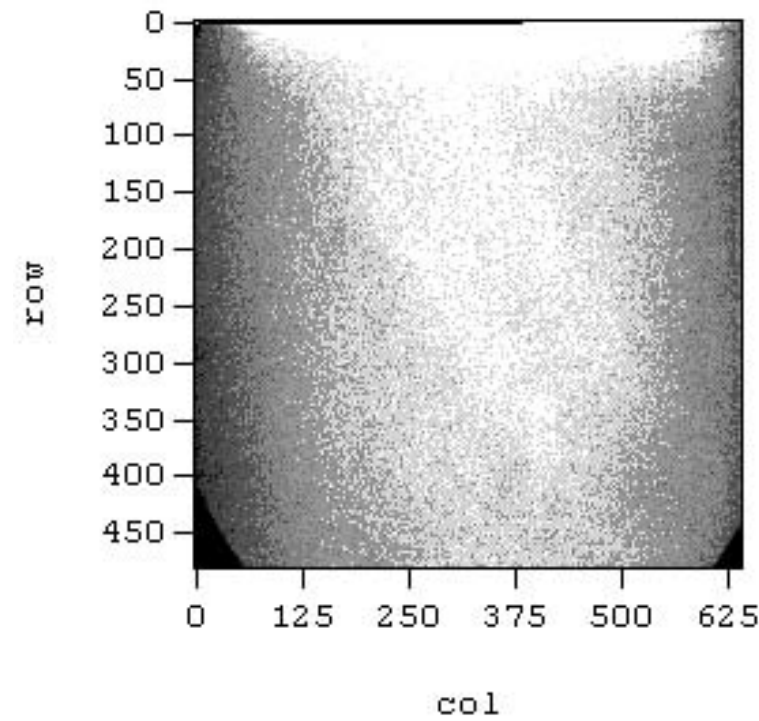

(a)

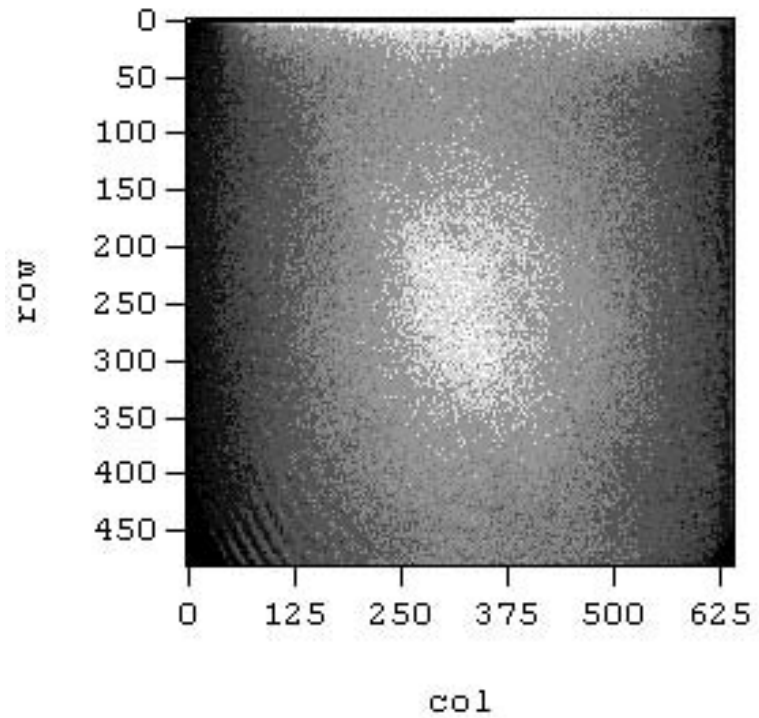

(b)

Fig. 4. Pixel-to-pixel uniformity (a) without and (b) with the photopic filter installed for $580 \mathrm{~nm}$ excitation. The total variation is $5 \%$ in (a) and $10 \%$ in (b). 


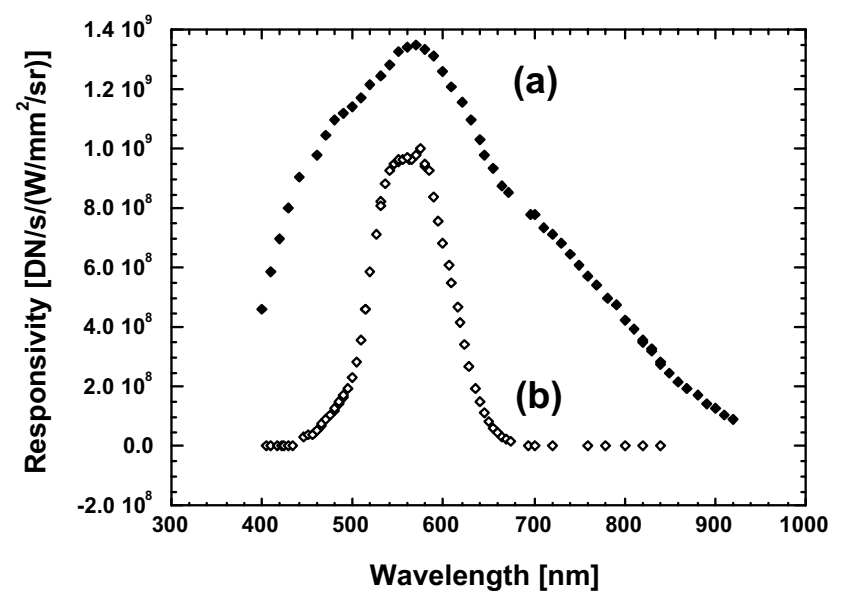

Fig. 5. CCD camera ASR (a) without and (b) with the photopic filter installed.

\subsection{Uncertainty of camera radiance responsivity}

Each component in the calibration chain contributes to the overall uncertainty in the radiance responsivity calibration. We estimated the contributions of all known components to the combined standard uncertainty in the CCD radiance responsivity calibration on SIRCUS. These contributions are listed in Table 1. We estimate the relative combined standard uncertainty in the sphere radiance to be $0.1 \%$; the uncertainty in the non-linear correction to the responsivity to be $1 \%$; the uncertainty in the exposure time correction to be $0.5 \%$; and the reproducibility, including short-term repeatability, alignment and focus, to be approximately $2 \%$. After taking the root-sum-square of the individual components, the relative combined standard uncertainty in the CCD radiance responsivity calibration is $2.3 \%$ (coverage factor $k=1$ ). For imaging applications, there will be an additional uncertainty of approximately $1 \%$ from the non-uniformity of the CCD.

Table 1. Relative combined standard uncertainty of the CCD radiance responsivity calibration on SIRCUS.

\begin{tabular}{|l|l|}
\hline \multicolumn{1}{|c|}{ Component } & Uncertainty [\%] \\
\hline $\begin{array}{l}\text { Sphere Radiance } \\
\text { (Monitor detector calibration) }\end{array}$ & 0.1 \\
\hline Sphere Radiance Non-uniformity & 0.25 \\
\hline CCD Non-linearity & 1 \\
\hline Exposure time correction & 0.5 \\
\hline Reproducibility & 2 \\
\hline \hline Relative Combined Std. Uncertainty & 2.3 \\
\hline
\end{tabular}

The radiant power distribution in the beam from the monochromator was highly non-uniform, leading to increased uncertainty in the calibration of the $\mathrm{CCD}$, both because of the increased uncertainty in the reproducibility of the measurements, and also because of the increased uncertainty in the non-linearity correction of the averaged response. There was an additional uncertainty in the normalization of the response to the measurements on SIRCUS. Consequently, measurements using the lamp-monochromator had a relative combined standard uncertainty of approximately $4 \%$.

For measurements of photometric quantities, a source spectral radiance distribution is weighted by the $\mathrm{V}(\lambda)$ function [7]. The photometric uncertainty is different than the above discussed radiometric responsivity measurement uncertainty and depends on how closely the response of the camera with the photopic filter approximates the $V(\lambda)$ function. For poor matches, the photometric uncertainty can be large and will depend on the source spectral distribution. There will be an additional uncertainty for imaging applications from the larger non-uniformity in the pixel-to-pixel response. 


\subsection{Microscope Calibration}

The microscope consists of an objective lens and a 1024 by 1024 pixel cooled CCD array. It is used to determine x-ray phosphor efficiencies from scintillator crystals [8]. Imaging a scintillation region on a crystal is extremely advantageous because the radiation from scintillators is Lambertian and viewing the source distribution enables the determination of radiometric quantities with lower uncertainties. We measured the linearity, exposure time correction, pixel to pixel uniformity and the absolute spectral radiance responsivity of the microscope on SIRCUS. For these measurements, we used a $5 \mathrm{~cm}$ diameter ISS with a $2 \mathrm{~mm}$ aperture. The microscope, equipped with a 10X objective, was positioned in front of the ISS. Each pixel imaged the same region of the ISS exit port, resulting in minimal additional uncertainty in the calibration from any potential non-uniformity in the sphere exitance.

The CCD array had a 12-bit A/D converter, with $4095 \mathrm{DN}$ corresponding to $90 \%$ of the CCD full-well capacity. We first determined the exposure time and the non-linearity corrections in the response of the microscope; results are shown in Figs. 6 and 7, respectively. The exposure time correction was negligible for exposure times greater that $500 \mathrm{~ms}$. For shorter exposure times, a correction - as large as $100 \%$ for $10 \mathrm{~ms}$ exposure times — had to be applied. In many of these systems, there is a delay in opening and closing the shutter, leading to an exposure time that is slightly different than the nominal value. For this instrument, adding $10 \mathrm{~ms}$ to the nominal exposure time reduced the correction to the $5 \%$ level for the shortest exposure times. The system response was linear for count rates larger than $100 \mathrm{DN}$. Offset variations and uncertainty in the determination of the background signal could contribute to the non-linear behavior at the smaller count rates. During the absolute radiometric calibration, the count rates were kept larger than $1000 \mathrm{DN}$ and the exposure time was kept constant at $100 \mathrm{~ms}$. This is easy to accomplish using a laser-based ISS; we merely adjust the power into the ISS at a given wavelength to achieve these goals.

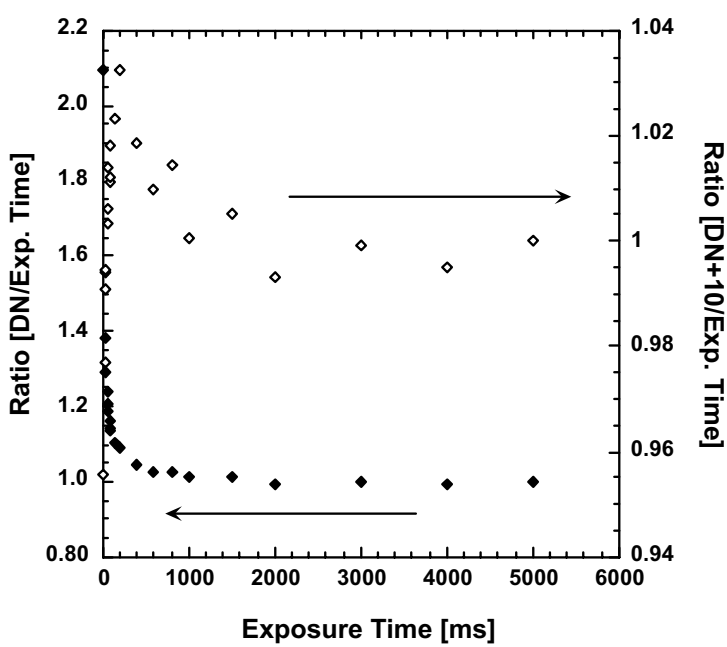

Fig. 6. Digital microscope exposure time correction.

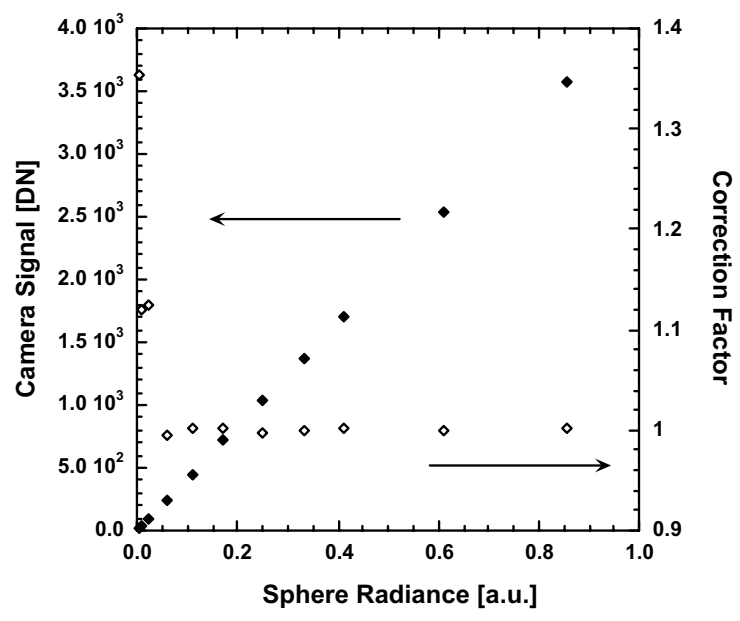

Fig. 7. Microscope response linearity.

The microscope response was uniform to with $0.2 \%$ over the central $90 \%$ of the CCD array; the ASR, averaged over the central 25 by 25 pixels, is shown in Fig. 8. Because of the smaller non-linear correction, the better pixel to pixel uniformity and the 12 bit digitization, we estimate the relative combined standard uncertainty in the microscope ASR measurement to be approximately $1 \%$.

\subsection{Spectrograph Calibration}

Spectrographs are typically calibrated for spectral radiance responsivity against broad-band sources such as lamp-illuminated integrating sphere sources (ISSs) and plaques. Stray light, wavelength errors, and lack of knowledge of the single pixel relative responsivity all give rise to increased uncertainties in the calibration and subsequent operation of these instruments. Many of these sources of calibration error can be greatly reduced or eliminated using tunable, monochromatic laser sources in place of conventional broad-band sources. 


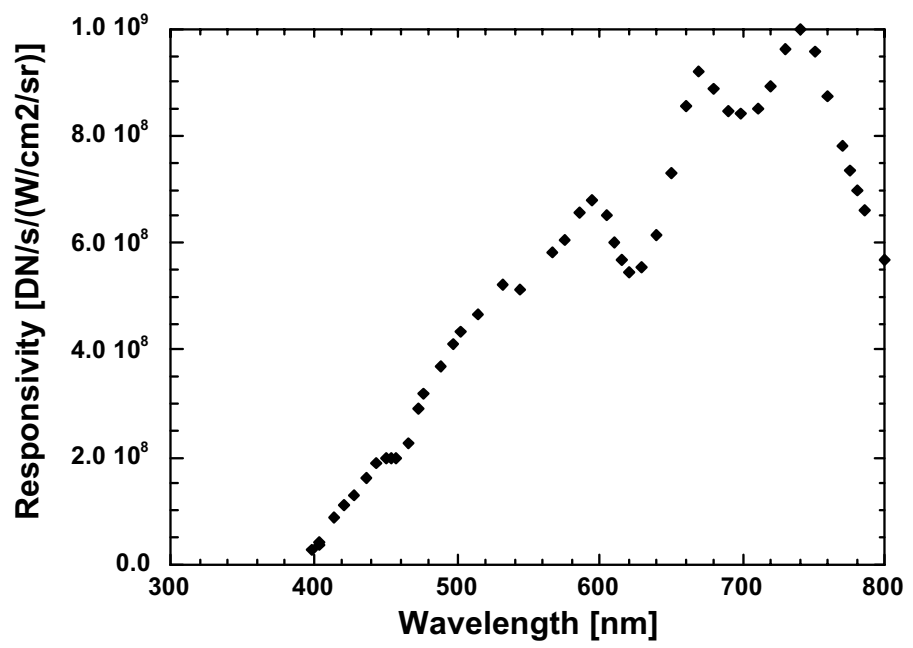

Fig. 8. ASR of digital microscope.

A dual-spectrograph radiometer was calibrated on SIRCUS for radiance responsivity over the spectral range from $400 \mathrm{~nm}$ to $850 \mathrm{~nm}$. Details of the calibration are described elsewhere [4, 5]. In this instrument, radiation enters the spectrograph through an entrance slit, is dispersed using a grating, and imaged onto the CCD array using a mirror, as shown in Fig. 9 . For excitation using monochromatic radiation, the image formed is a line reflecting the image of the entrance slit

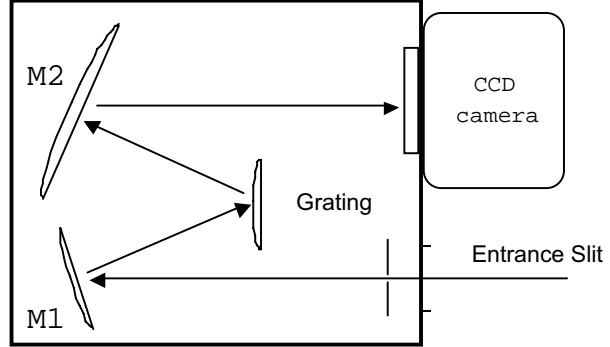

Fig. 9. Spectrograph optical layout. on the CCD (Fig. 10). Note that the image is spread out over several pixels in the horizontal (dispersion) direction, is not centered vertically on the CCD array, and shows a pronounced curvature (Fig. 11). By tuning the excitation wavelength, the image moves across the array and each pixel's ASR can be determined. In this work, we measured the responsivity of the averaged central 100 pixels in each column. Eq. 4 was modified to:

$$
R_{i}\left(\lambda_{o}\right)=S_{i} / L\left(\lambda_{o}\right),
$$

where $i$ now refers to the particular column in the array. In Fig. 12, we show the absolute spectral responsivity, $R_{i}(\lambda)$, for columns $243-247$ over the wavelength range from $735 \mathrm{~nm}$ to $750 \mathrm{~nm}$. We measured a $0.8 \mathrm{~nm}$ separation between the maximum responsivity of adjacent pixels and nominal single pixel bandwidth of $2 \mathrm{~nm}$. The curvature in the spectrograph image, Fig. 11 gave rise to the pronounced shoulder observed at shorter wavelengths in the ASR. This shoulder in the single pixel ASR was attributed to the CCD not being located at the spectrograph image plane. We estimate the relative combined standard uncertainty in the calibration to be approximately $3 \%$. Note that the determination of each pixel's ASR is difficult to obtain using any technique other than the tunable-laser-based ISS approach developed on SIRCUS.

\section{SUMMARY}

We have developed a flexible, detector-based calibration facility using tunable laser sources and high accuracy reference standard detectors for the irradiance and radiance responsivity calibration of digital imaging systems. We have calibrated a number of instruments on this facility, and presented results for a digital camera, a digital microscope and a digital spectrograph. This approach has several advantages over conventional calibration techniques using broad-band sources, including uniform, monochromatic irradiance and radiance sources; lower wavelength uncertainty; no stray light contribution 


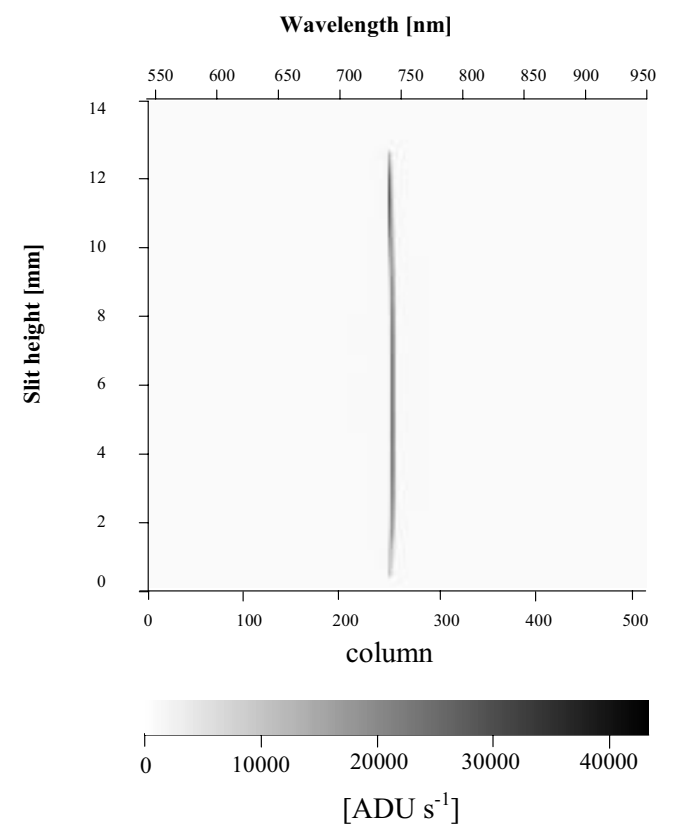

Fig. 10. Spectrograph image of ISS with $745 \mathrm{~nm}$ excitation.

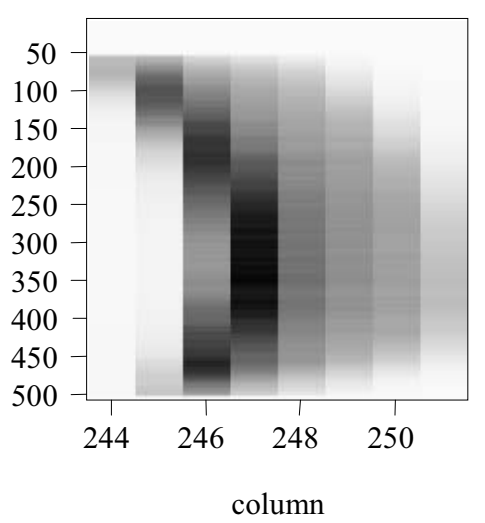

$\begin{array}{lllll}0 & 10000 & 20000 & 30000 & 40000\end{array}$

Fig. 11. Expanded view of spectrograph image of ISS with $745 \mathrm{~nm}$ excitation.

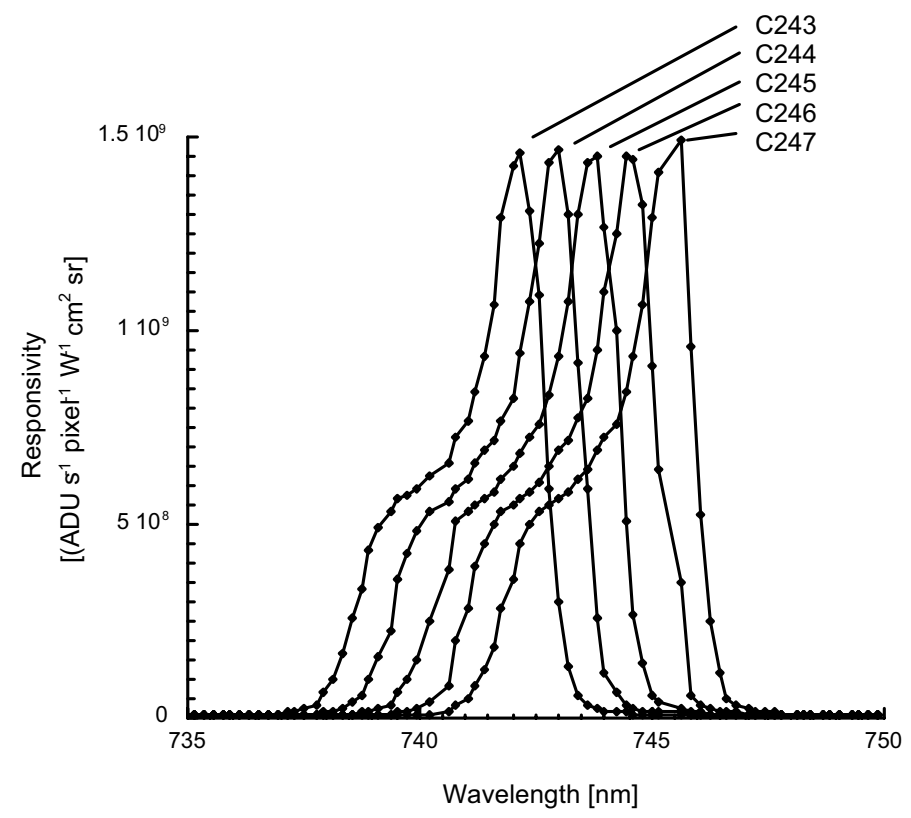

Fig. 12. Absolute spectral responsivity of columns 243 to 247 . 
to the signal; and the ability to determine each pixel's responsivity. By using this approach, uncertainties in the radiometric calibration of DIS's can be reduced by a factor of 2 or greater over conventional calibration techniques.

\section{ACKNOWLEDGMENTS}

We would like to thank Carol Johnson for useful discussions and acknowledge support for this work by the U. S. Air Force Metrology under contracts 97-421 and 98-439.

\section{REFERENCES}

1 S. W. Brown, G. P. Eppeldauer, and K. R. Lykke, "NIST facility for spectral irradiance and radiance responsivity calibrations with uniform sources," Metrologia 37, 579-582 (2000).

2 G. P. Eppeldauer and D.C. Lynch, "Opto-mechanical and electronic design of a tunnel-trap Si-radiometer," J. Res. Natl. Inst. Stand. Technol. (in press).

3 T. R. Gentile, J. M. Houston, J. E. Hardis, C. L. Cromer, and A. C. Parr, "National Institute of Standards and Technology high accuracy cryogenic radiometer," Appl. Opt, $\underline{35}$, 1056-1068 (1996).

4 C. Habauzit, et al., "Radiometric characterization and absolute calibration of the Marine Optical System (MOS) bench unit," J. Atmos. and Ocean. Technol., to be submitted.

5 S. W. Brown, C. Habauzit, B. C. Johnson, and K. R. Lykke, "Tunable-laser-based calibration of a digital spectrograph," Appl. Opt., to be submitted.

6 T. C. Larason, S. S. Bruce, and A. C. Parr, Spectroradiometric Detector Measurements, Natl. Inst. Stand. Technol. Spec. Publ. 250-41, U. S. Government Printing Office, Washington, DC, 1998.

7 Yoshihiro Ohno, Photometric Calibrations, Natl. Inst. Stand. Technol. Spec. Publ. 250-37, U. S. Government Printing Office, Washington, DC, 1997.

8 Steven Grantham, NIST, personal communication. 


\title{
Uniform calibration of night vision goggles and test sets
}

\author{
George P. Eppeldauer \\ Optical Technology Division \\ National Institute of Standards and Technology \\ Gaithersburg, Maryland 20899-8441
}

\begin{abstract}
There are orders of magnitude differences between the $\sim 0.1 \%(k=2)$ uncertainty of NIST reference detector calibrations and the uncertainty of night vision (NV) goggle measurements. NIST developed a night vision radiometer calibration facility and NV radiometer transfer standards. The transfer standards, that propagate the radiance responsivity scale to the military primary standards laboratories, are calibrated against a NIST reference radiometer. The reference radiometer has been calibrated on the NIST Spectral Comparator Facility (SCF) for spectral power and irradiance responsivities. Spectral considerations are discussed to lower the uncertainties of the radiance responsivity scale transfer to the test sets and then to the goggles. Since direct determination of the final uncertainties in goggle calibrations and measurements is difficult, models have been made to estimate the most important uncertainty components based on individual spectral measurements of the source distributions and radiometer spectral responsivities. It is also shown, that because of source spectral mismatch problems, the goggle measurement uncertainty at applications can be much higher than at calibration. A suggestion is being made to mimic the no-moon (stars only) night sky radiation distribution using several LEDs in the test-sets to decrease the large spectral mismatch errors. A broad-band correction factor has been developed to further decrease calibration uncertainty when the goggles to be used have different spectral responsivities than the standard. Geometrical considerations to optimize the radiance measurement angle and the out-of-target blocking are also discussed to decrease the uncertainty in the radiance responsivity transfer.
\end{abstract}

Keywords: calibration, night vision goggle, radiance, radiometer, scale transfer, spectral responsivity, test-set

\section{INTRODUCTION}

The night vision (NV) goggles are the key sensors for human beings for night operations. The goggles have several technical parameters that can be used to characterize them. The most important parameters are spatial resolution, fieldof-view, image distortion, transfer-gain (output luminance / input radiance), and spectral responsivity. The spectral responsivity is one of the most critical parameter for flying safety. The MIL-STD-3009 [1], written in 2001, shows the assumed night vision imaging system (NVIS) spectral response characteristics that shall be used to define NVIS compatibility criteria with aircraft lighting. The standard extends the NVIS radiance requirements from the previous defining document [2]. Though a full framework would be necessary for NVIS radiance measurement requirements, the new (2001) document does not address calibration aspects of the new NVIS technology.

Because of the lack of standardized goggle calibrations, individually applied maintenance actions are performed, such as pass/fail tests to determine whether the goggle will perform as expected when it is used (or as it has performed in the past). At present, there are no standard criteria that state the goggle gain must be higher than some specified value. The users are tasked to locally establish a value based on past performance of the goggles that will satisfy their needs. There are some initial recommendations for each goggle type, but it is still the responsibility of the users to establish their own test methods. Also, in the past two decades, different source distributions have been applied in different test-sets. The earlier used tungsten lamps were replaced with LEDs. Some manufacturers use an $815 \mathrm{~nm}$ (peak) light emitting diode (LED) irradiating source in the test-sets when determining the transfer gain of the goggles. The transfer gain is the luminance at the eye-piece of the goggle divided by the radiance viewed by the goggle. The luminance is a photometric quantity that can be measured easily. However, the radiance viewed by the goggle has a spectral distribution and it is measured by the goggle which has a spectral responsivity different from the human visual system (the CIE standard photometric observer). Accordingly, the radiance viewed by the goggle needs a (broad-band) radiometric measurement

Electro-Optical and Infrared Systems: Technology and Applications IV, edited by David A. Huckridge, Reinhard R. Ebert, Proc. of SPIE Vol. 6737, 67370M, (2007) · 0277-786X/07/\$18 · doi: 10.1117/12.737814 
where photometric units cannot be used. The spectral distribution of the viewed radiance (in the test-sets) has not been standardized. The manufacturers claim that the calibration of the goggles using either a tungsten (close to Planckian) distribution or a narrow-band source distribution is satisfactory because the responsivity of the goggles is constant versus wavelength. They do not examine the errors when the broad-band night sky radiation is measured (at application) as compared to the narrow-band distribution of the LED (at calibration). According to the relative spectral responsivity characteristics of Classes A, B, and C in the MIL-STD-3009, the responsivity curves look flat (constant versus wavelength) because of the logarithmic responsivity scale used for illustration. However, as shown in Fig. 1, the spectral responsivity of different generation Gen-3 goggles can change significantly [3]. As an example, the Class-A standard and realized filter transmittance (cut-on) curves are also shown that will limit the goggle responsivities at the short wavelength side. A model is shown below to show the errors that the $815 \mathrm{~nm}$ LED source application and the constant goggle responsivity assumption can produce in night sky radiation measurements. The night sky irradiance distributions for full moon and no moon (only stars) [4] and the normalized spectral power distribution of a test set LED are shown in Fig. 2a on a logarithmic scale and in Fig. 2b on a linear scale (the full moon distribution is not shown here) to illustrate the difference in the wavelength coverage for the spectral range where the goggles are sensitive.

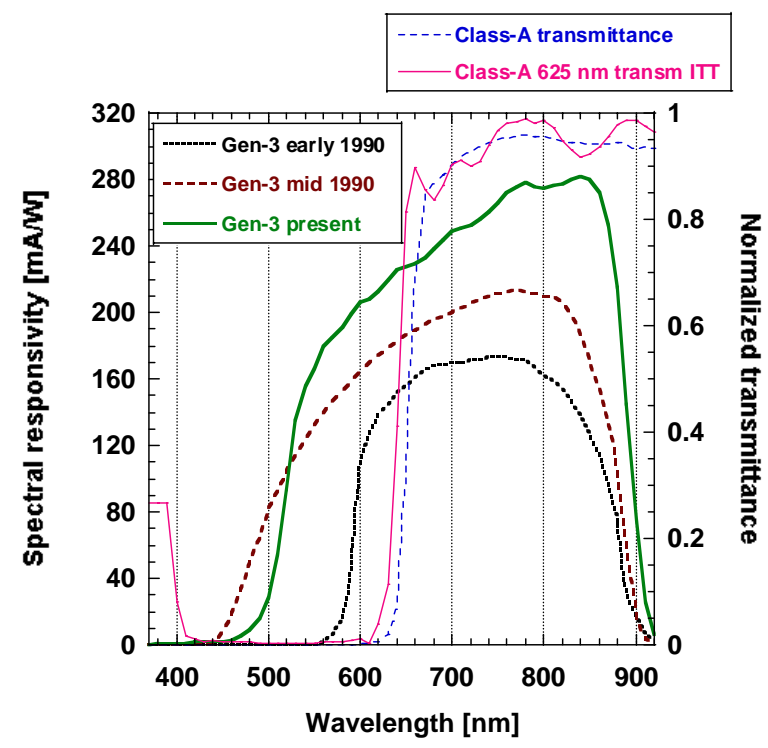

Fig. 1. Spectral responsivity curves of different generation Gen-3 goggles and the transmittance curves of the standardized Class-A and realized ITT [5] minus-blue filter.

A recently published work examined the impact of goggle spectral responsivity changes on NVIS compatibility to aircraft interior lighting [6]. The goggle responsivity changes are caused by the changes introduced in image tube parameters and objective lens coatings. In the referenced publication, the authors believed it is necessary to develop and offer a more complete framework for NVIS related radiometric calculations. They started to explore the cockpit lighting compatibility issues associated with differing night-vision goggle spectral responsivity distributions. In addition to the relative spectral responsivity curves of the Class A and B goggles standardized in the MIL-STD-3009, they calculated the Class C relative spectral responsivity curve, using the transmission of the ("leaky green") objective lens (from MILSTD-3009), and the spectral responsitivity of an ITT image intensifier ("hot”) tube responsive from $450 \mathrm{~nm}$ to $950 \mathrm{~nm}$.

In spectrally broad-band measurements, such as goggle calibrations, the detector spectral responsivities are to be standardized to perform uniform integrated responsivity (INR) measurements. Similarly to the standardized V( $\lambda$ ) function in photometry, the Class A, B, and C spectral responsivity curves can be used as reference goggle spectral 
distributions. The goggle signals can be calculated as the product of the goggle spectral responsivity and the source spectral distribution seen by the goggle. If the realized (manufactured) detector spectral responsivity is different than the

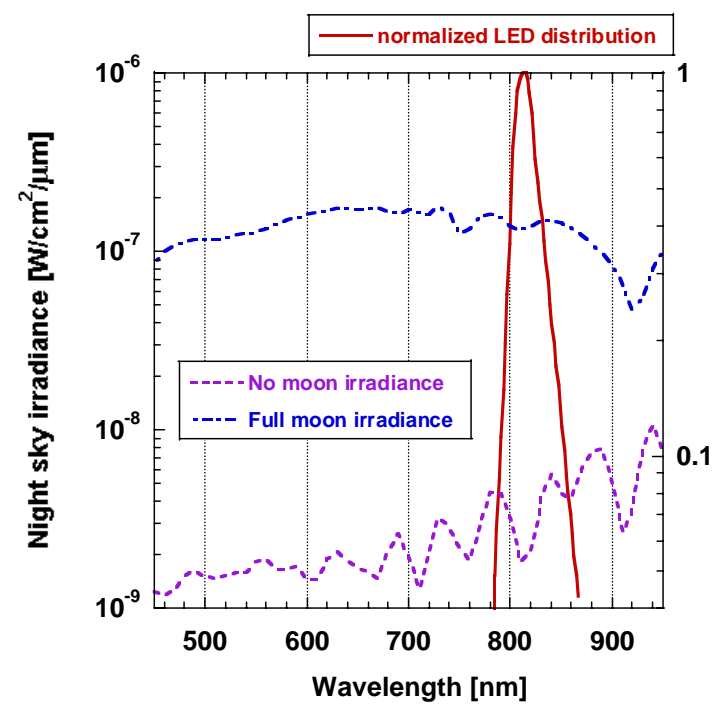

Fig. 2a. Spectral distribution curves of night sky irradiance and a test-set LED on logarithmic scales.

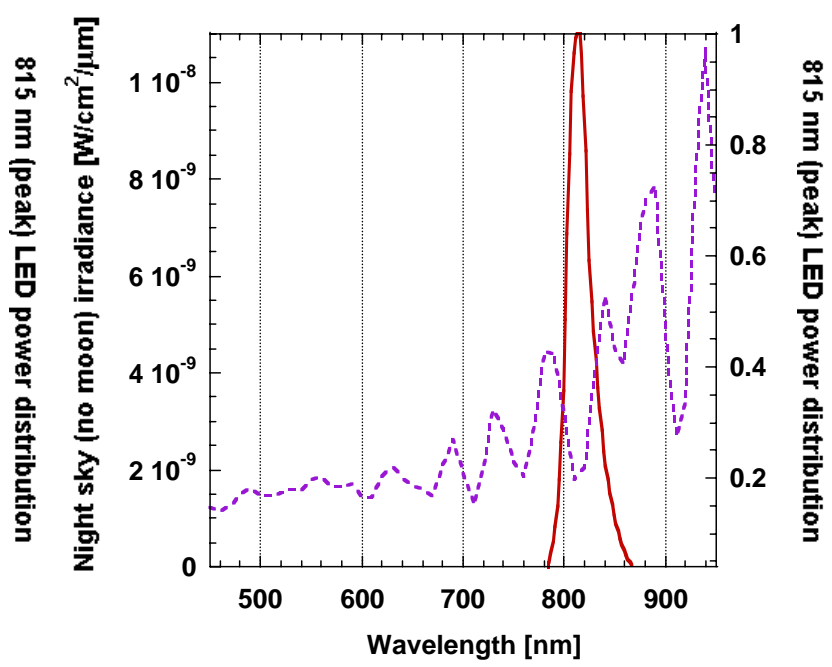

Fig. 2b. Spectral distribution curves of night sky (no moon) irradiance and a test-set LED on linear scales.

standardized responsivity, large systematic measurement errors can be obtained if the source distribution to be measured is very different than the distribution of the source used at calibration.

To avoid these large errors, in addition to the standardization of the detector spectral responsivity, the spectral power distribution of the source at calibration can be standardized (similarly to the CIE Illuminant A, D65, and other distribution functions) as well. In NV goggle calibrations, frequently an LED light source is used in the test-set. Since, the LED spectral power distribution is very different from the night sky distributions (during goggle applications), large goggle measurement errors can be obtained. The measurement uncertainties can be decreased if the source spectral distribution in the test-sets is standardized (at calibration) and the standardized spectral distribution is similar to the night sky radiation (at application). This standard source distribution function does not have to be equal to the different night sky radiation curves. It can be similar to those to obtain low calibration uncertainties. Similar considerations have been made in detector-based photometric and colorimetric calibrations [7]. This idea can be realized using several LEDs $[8,9]$ in a test-set instead of using one LED (that usually peaks around $815 \mathrm{~nm}$ ). Also, a broad-band correction factor (similar to the color correction factor in photometry [7]) that can be easily applied for goggle spectral responsivities that are different than the standard curve, can be developed to decrease goggle calibration uncertainty.

Applying the above discussed INR measurement considerations, the goggle transfer-gain calibrations can be made in a uniform way with low uncertainties. The suggested goggle calibration system is based on the standardized goggle responsivity functions in the MIL-STD-3009 and it is traceable to the SI units through the NIST detector responsivity scales. The suggested uniform calibration system could significantly improve the goggle measurement uncertainties.

\section{THE NIST REFERENCE DETECTORS AND RESPONSIVITY SCALES}

Participation of NIST in the night vision goggle calibration program started in the late 1980s after NIST developed the high sensitivity silicon radiometers [10]. The noise equivalent photocurrent of these radiometers was $10^{-16} \mathrm{~A}$, similar to 
the photomultiplier tubes the Army used for goggle calibrations before acquiring the NIST radiometer standards. A few years later NIST developed a night vision detector (radiometer) calibration facility (NVRCF) funded by the Navy. The NIST responsivity scale is derived from electrical-substitution cryogenic radiometers. Silicon trap-detector transfer standards are calibrated against these primary standards in radiant power measurement mode. The trap detectors are equipped with precision apertures to convert the power responsivity into irradiance responsivity. The trap detectors have relative combined expanded uncertainties of less than $0.1 \%(k=2)$ [11]. The trap detectors propagate the reference responsivity scale to two other NIST responsivity calibration facilities. The first one, the Spectral Irradiance and Radiance Responsivity Calibration using Uniform Sources (SIRCUS) facility [11] is an extended version of the NVRCF and it can be used for general purpose detector irradiance and radiance responsivity calibrations. The other facility is the Spectral Comparator Facility (SCF) [12] which was used to calibrate the NIST night vision reference radiometer for both power and irradiance responsivity [13]. Several NV radiometer transfer standards were also developed at NIST for the Army, Navy, and the Air Force, to propagate the NIST radiance responsivity scale to the military primary standards laboratories. The radiance responsivity of these radiometers is typically determined at the NVRCF. The NIST luminance scale is transferred separately to the three services [14].

\subsection{The NIST Reference Radiometer}

The NIST reference radiometer operates in irradiance measurement mode. The cross-section of the radiometer front is shown in Fig. 3. In front of the radiant power measuring silicon photodiode, there is a diffuser and a thin aperture attached to the front of the diffuser. The aperture determines the reference plane of irradiance measurements. The diffuser dominates the directional responsivity of the radiometer. The input geometry was optimized experimentally to obtain an angular responsivity close to the theoretical cosine function [13]. The field-of-view (FOV) limiter rejects optical radiation outside the radiometer FOV. The detector is temperature stabilized with a thermoelectric (TE) cooler at

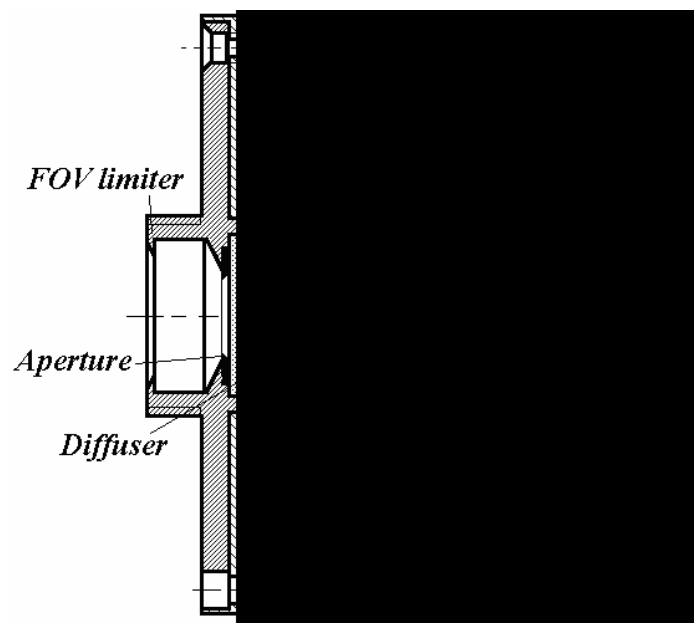

Fig. 3. The cross-section of the NIST night vision reference radiometer

$25^{\circ} \mathrm{C}$. The angular responsivity was measured using a small incandescent lamp at a separation of about $3 \mathrm{~m}$ and the radiometer was rotated around the center of the aperture. The measurement results are shown on Fig. 4 . The solid line is the cosine function and the open circles are the measured data points. The results show, that the irradiance meter has an angular responsivity very close to the cosine function in a FOV of $11.6^{\circ}$ (full angle). 


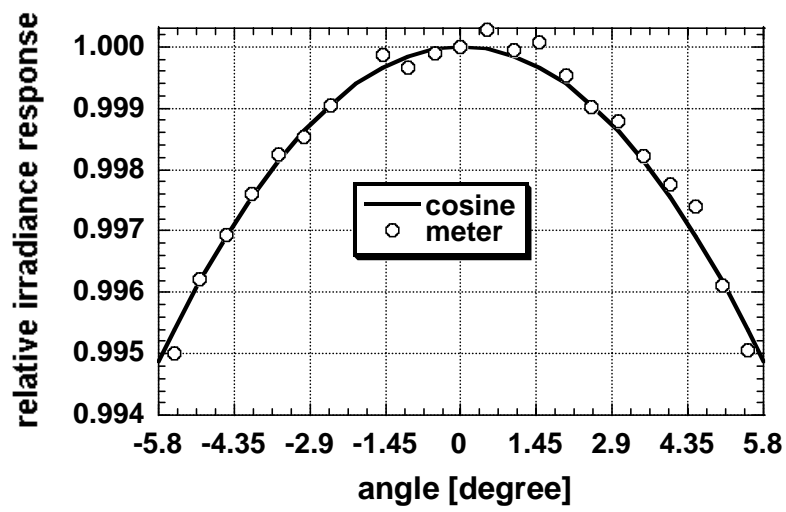

Fig. 4. Relative angular responsivity of the irradiance measuring NIST radiometer standard

\subsection{The NV radiometer transfer standard}

The NV radiometer transfer standards, that propagate the NIST responsivity scale to the military primary standard laboratories, were developed at NIST to perform uniform spectral responsivity transfer and high sensitivity. The cross section of the radiometers are shown in Figs. 5 and 6. The first generation radiometer is shown in Fig. 5. Figure 6 shows the cross section of the second generation radiometer with a $90^{\circ}$ rotation relative to the view for the photodiode holding block in Fig. 5. In both radiometers, the silicon photodiode is mounted in a temperature stabilized copper holder. The copper holder has a threaded hole where the photodiode can be mounted at a given position relative to the input optics using retainer rings and washers. Three nylon screws pull the copper holder against a copper heat sink with a TE cooler/heater in between. The heat sink is attached to the Aluminum housing. The photocurrent meter is mounted in a separate housing (not shown in these figures) which is attached to the bottom of the front part of the measuring head using a four-pin connector. The leads of the TE cooler and a thermistor (not shown) are soldered to the side connector. The input optics attached to the front of the radiometer is different in the two figures. The widely used PVS-7 input optics is shown in Fig. 6 and a simplified input optics (with a radiance measurement angle of $\alpha$ ) is shown in Fig. 5. The design considerations and the angular radiance responsivity measurements are discussed below in Section 3.2. The silicon photodiode has high power responsivity in the spectral response range of the NV goggles. The high photodiode shunt resistance produces low amplification for the amplifier noise in the photocurrent meter, resulting in high sensitivity for the NV radiometer.

The picture of the first generation radiometer transfer standard with the PVS-7 input optics is shown in Fig. 7.

\subsection{The NV radiometer calibration facility (NVRCF)}

The NV radiometer transfer standards are calibrated for radiance responsivity at the NVRCF. The scheme and the picture of the inside arrangement of the NVRCF are shown in Figs. 8 and 9. The standard for the calibration is the NIST reference radiometer. The uniform source for the calibration transfer is a $20 \mathrm{~cm}$ diameter integrating sphere illuminated by a light source. The light source can be either a diode laser (as shown in Fig. 8) or a light emitting diode (LED) attached to the entrance port of the sphere. For a diode laser, both the temperature and the current are controlled, for an LED, only the current is regulated. A silicon monitor detector compensates for intensity changes of the sources. 


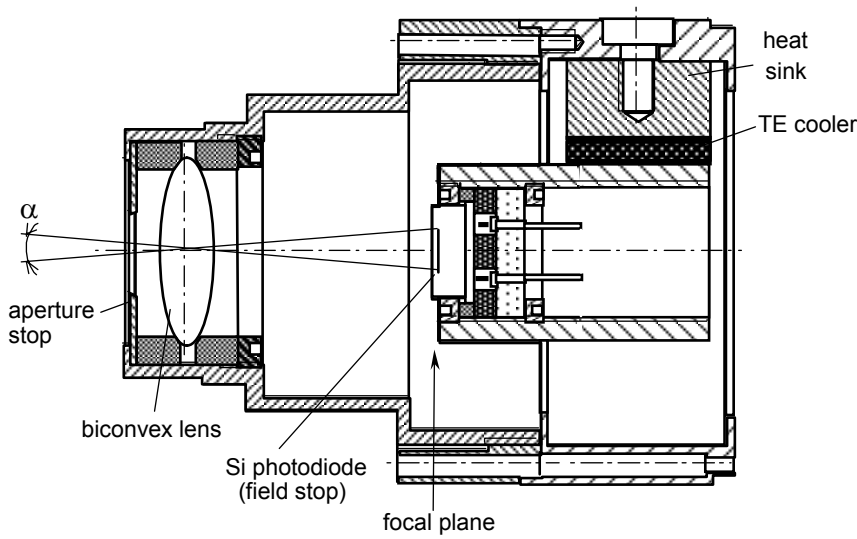

Fig. 5. Cross section of the front head of the NV radiometer transfer standard using a biconvex lens input optics.

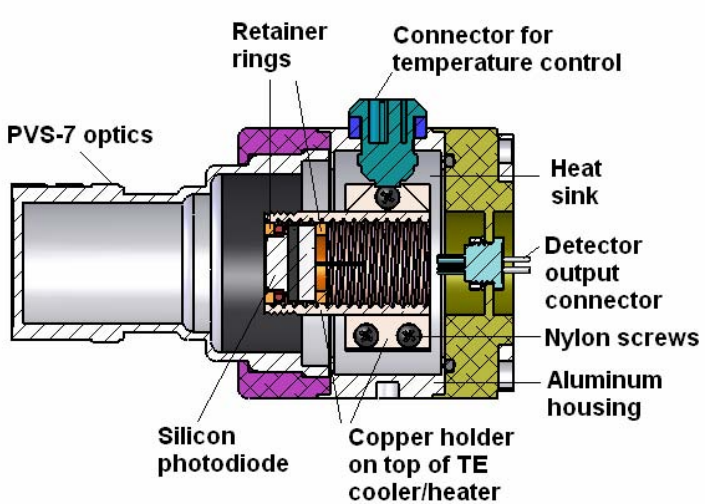

Fig. 6. Cross section of the front head of the new generation radiometer transfer standard with the PVS-7 input optics.

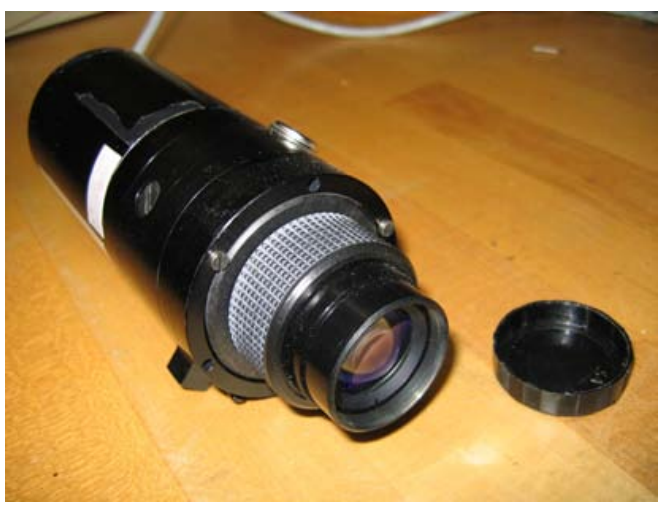

Fig. 7. Picture of the NV radiometer transfer standard with the PVS-7 input optics and the attached amplifier unit.

The radiance, $L$, of the integrating sphere source is determined from a measurement made with the NIST reference radiometer. The flux transfer calculation is based on the geometry of the flux transfer unit. The reference radiometer is calibrated for spectral radiant power responsivity $s_{\mathrm{s}}(\lambda)$ [A/W] traceable to the NIST spectral responsivity scale [12]. The radiant power responsivity for a given light source is calculated from $s_{\mathrm{s}}(\lambda)$ and the spectral distribution of the source. The radiant power responsivity of the reference radiometer for the LED is:

$$
s_{\mathrm{s}, \mathrm{LED}}=\frac{\int_{\lambda} S(\lambda) s_{\mathrm{s}}(\lambda) \mathrm{d} \lambda}{\int_{\lambda} S(\lambda) \mathrm{d} \lambda}
$$

where $S(\lambda)$ is the relative spectral power distribution of the LED. 


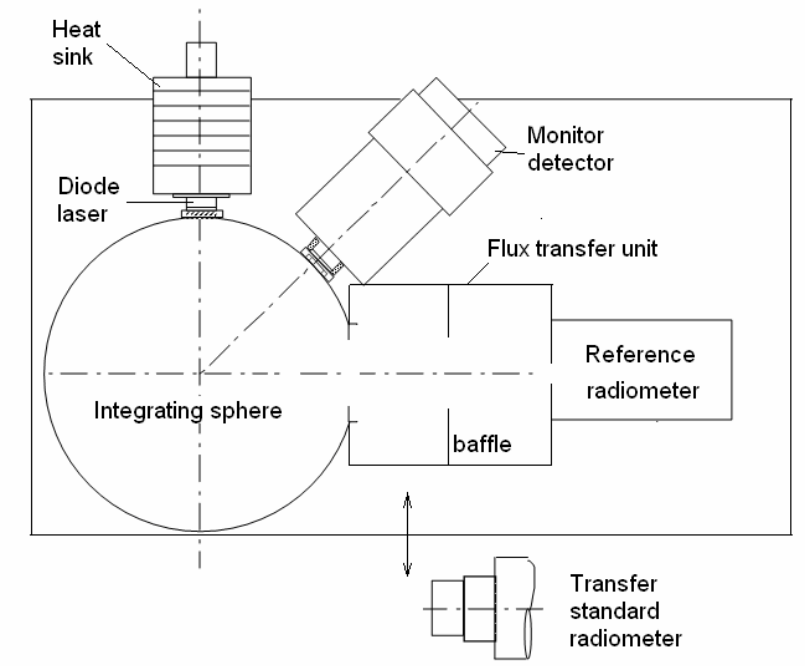

Fig. 8. Scheme of the NV transfer standard radiometer calibration at the NVRCF.

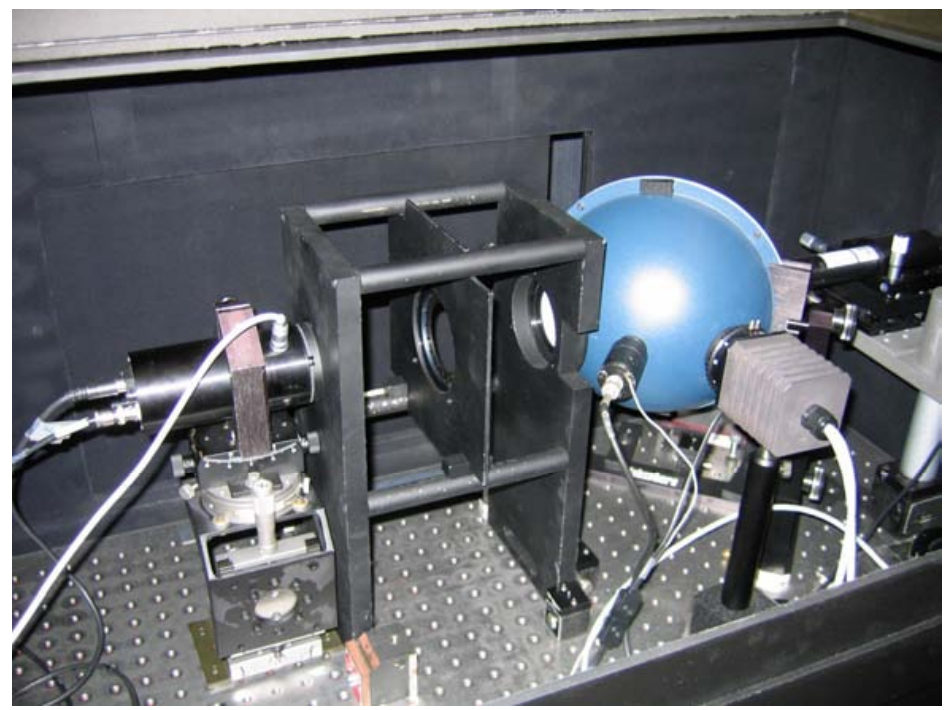

Fig. 9. Picture of the NIST Night Vision Radiometer Calibration Facility.

The cosine-corrected reference radiometer has a sufficiently large acceptance angle for the flux transfer. The irradiance responsivity of the reference radiometer is obtained from spatial responsivity integrals determined by a raster scanning procedure. The effective area of the radiometer aperture is given by the ratio of its total irradiance responsivity to the radiant power responsivity in the center of the aperture.

The radiance $L$ of the sphere exit port is determined from the radiant power $P$ from the source incident on the detector, and the geometric extent $G$ : 


$$
L=\frac{P}{G}
$$

where

$$
G=\frac{\pi^{2}}{2}\left[\left(d^{2}+r_{S}^{2}+r_{D}^{2}\right)-\left\{\left(d^{2}+r_{S}^{2}+r_{D}^{2}\right)^{2}-4 r_{S}^{2} r_{D}^{2}\right\}^{1 / 2}\right]
$$

The $r_{S}$ is the radius of the source (sphere) exit port aperture $(\approx 27 \mathrm{~mm}), r_{D}$ is the radius determined from the effective area of the radiometer aperture $(\approx 4 \mathrm{~mm})$, and $d$ is the distance between the source aperture and the radiometer aperture $(\approx 22.6 \mathrm{~cm})$. In our case, the units are $\mathrm{W}$ for $P, \mathrm{~W} \mathrm{~cm}^{-2} \mathrm{sr}^{-1}$ for $L$, and $\mathrm{cm}^{2} \mathrm{sr}$ for $G$.

As the second step, the NV radiometer transfer standard, with the attached PVS-7 input optics, is calibrated for the exit port radiance. The transfer standard radiometer (as shown in Fig. 8) is placed and aimed at the center of the sphere exit port. The separation between the sphere aperture and the front of the PVS-7 optics is only $50 \mathrm{~mm}$ because of the poor out-of-target rejection of the PVS-7 equipped radiometer (see below in Fig. 13). At this separation, the sphere radiation overfills the radiance measurement angle of the transfer standard and the surrounding angular region where the device has undue sensitivity. The radiance responsivity of the transfer standard is determined as the ratio of its output current to the radiance of the sphere exit port calibrated against the reference radiometer.

The improvement of the out-of-target rejection using a simplified (biconvex) input optics (discussed in Section 3.2) decreased the $2.4 \%(k=2)$ uncertainty component of the distance dependent radiance responsivity of the PVS-7 equipped radiometer to less than $1 \%(k=2)$. The combined relative expanded uncertainty of the radiance responsivity calibrations using the PVS-7 input optics is $3.0 \%(k=2)$.

\section{SCALE TRANSFER}

The military calibration laboratories further transfer the radiance responsivity from the NV radiometer transfer standard to their field NV radiometers. The field radiometers are similar to the construction of the above discussed NV radiometer transfer standard. The radiance responsivity transfer for the field radiometers is made using integrating sphere sources illuminated possibly with the same LED type used in the test sets. The field NV radiometers are used to calibrate the radiance of the test-sets. The goggles are calibrated against the test-set radiance. The spectral distribution of the test-set radiance is dominated by the LED spectral power distribution which is much narrower than both the night sky radiation and the goggle spectral responsivity range.

In order to decrease calibration and measurement uncertainties it is necessary to analyze the spectral and geometrical issues during the radiance scale transfer from the NIST NVRCF to the military primary standard laboratories. The results and conclusions of the analysis below can be applied to the scale transfer from the military primary standard laboratories to the field test-sets. When the radiance scale transfer to the test-sets is performed according to the considerations discussed below smaller than $10 \%(k=2)$ test-set radiance calibration uncertainties can be achieved. Otherwise, the uncertainties can be significantly higher. The additional uncertainties of the radiance scale propagation to goggle calibrations and goggle field measurements are also discussed below.

\subsection{Spectral considerations}

Spectral problems during the scale propagation from the NIST reference detector to test-sets, and then from the test-sets to the night vision goggles, can significantly increase the calibration uncertainties. However, when the spectral characteristics are different at the goggle calibration compared to the follow-on goggle applications, the goggle performance can be very different. To avoid these differences, the spectral issues during the radiance responsivity scale transfer and the following goggle calibrations and applications are to be analyzed. 


\subsubsection{Scale transfer from NIST to field test sets}

During the scale transfer, broad-band detectors (radiometers) measure broad-band sources. Photometric measurements, where the spectral responsivity of the photometer is standardized [7], are a good example to make uniform broad-band measurements. In the above discussed radiometric scale transfer, a similar standardization can be performed if the same silicon detector type is used in all night vision transfer standard radiometers.

The responsivity transfer errors were modeled between LED measurements and laser calibrations using two different types of silicon photodiodes in the transfer standard radiometer. Two (available) LED source distributions were selected with similar peak wavelengths (692 nm and $697 \mathrm{~nm}$ ) and different spectral coverage. Two lasers were used as the third type of source distribution and tuned to the two LED peak wavelengths. Figure 10 shows the Si photodiode spectral responsivities and the two LED spectral power distributions and one laser tuned to the peak of the $697 \mathrm{~nm}$ LED.

Equation 1 can be used to calculate the INR of a detector for an LED source. For the laser lines, instead of the INR, the laser responsivity of the detector at the laser wavelength is used.

Four INRs were calculated first (using Eq. 1) for two Hamamatsu (1227 and 1337) silicon photodiodes and the two LEDs. Then, each INR was compared to the laser responsivity by calculating the percent error between the INR and the laser (reference) responsivity. These examples are shown in Table 1 to illustrate the responsivity error propagations caused by using a different source (LED) at application (or scale derivation) than at calibration (laser). The results show that the errors are smaller with the 1337 detector than with the 1227 . The responsivity of the 1337 detector is linear versus wavelength and it is similar to the unity quantum efficiency line. The 1227 detector has a lowered responsivity in the red and it is very different from a straight line. Also, the responsivity propagation errors are smaller when a narrow band LED (like the $692 \mathrm{~nm}$ peak LED) is measured compared to the errors with the $697 \mathrm{~nm}$ (peak) LED which has a much wider spectral distribution. Utilizing the above considerations, the responsivity errors can be kept small in the responsivity scale propagation even if a laser is used at calibration and an LED is applied at scale derivations or field applications. This conclusion will not apply if more LEDs with different spectral distributions are used in the test-sets.

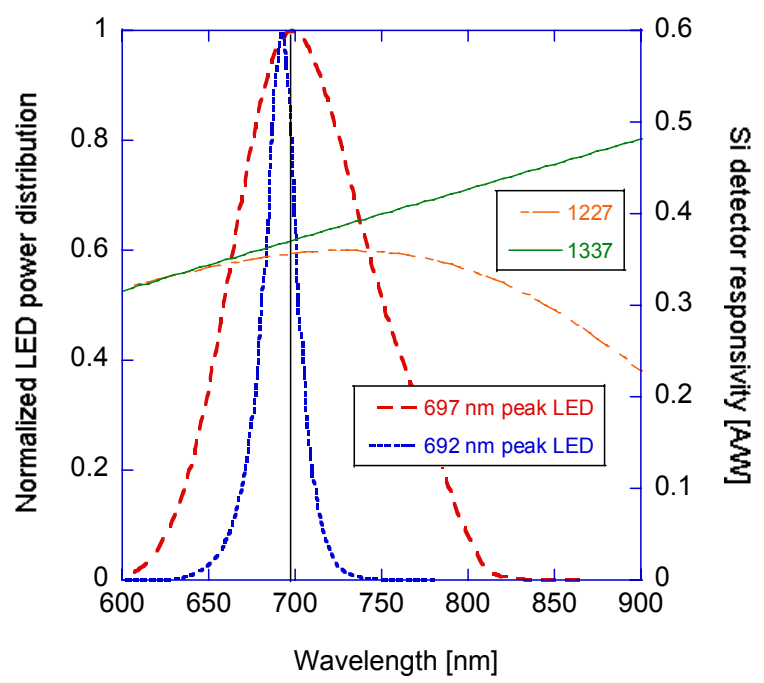

Fig. 10. Spectral responsivity of two different Si detectors, and spectral power distributions of two LEDs with similar peak wavelengths. A laser line is also shown tuned to the $697 \mathrm{~nm}$ (peak) LED.

Proc. of SPIE Vol. 6737 67370M-9 
Table 1.

Percent errors between LED integrated responsivity and laser responsivity measurements for two different LEDs and two different silicon photodiodes.

\begin{tabular}{|c|c|c|c|}
\hline INR (697 nm LED \& 1227) & INR (692 nm LED \& 1227) & INR (697 nm LED \& 1337) & INR(692 nm LED \& 1337) \\
\hline 697 nm laser resp. of 1227 & --o2 nm laser resp. of 1227 & 697 nm laser resp. of 1337 & 692 nm laser resp. of 1337 \\
\hline $4.6 \%$ & $0.6 \%$ & $2.8 \%$ & $1 \%$ \\
\hline
\end{tabular}

\subsubsection{Scale transfer from test-sets to goggles}

It is not enough to determine the radiance responsivity scale propagation uncertainties from the NIST NVRCF to the test-sets. When the radiance scale is propagated from the test-sets to the goggles, the spectral bandwidth of the test-set source distribution and the deviation of that from the source distributions at the goggle applications will have a major influence to the uncertainties of goggle calibrations and applications. The transfer-gain is one of the most important goggle characteristics that should be determined. It is the ratio of the output luminance (at the eye piece) to the input radiance viewed by the goggle. The luminance is usually transferred from the NIST photometric scale [14]. The luminance at the output depends on the goggle signal which depends not only on the test-set radiance but also on the radiance spectral distribution. In order to obtain the same (or similar) goggle transfer gains at both calibration and application(s), the test-set source distribution should be similar to the source distributions at night vision applications.

Third generation (Gen-3) goggles are being used for military applications because of the high resolution and gain produced by improved image intensifier tubes and the Gallium Arsenide coated photocathode that enhanced the sensitivity (responsivity). These goggles can measure very low-level radiation from the visible to about $900 \mathrm{~nm}$. Filters with a cut-on edge of about $600 \mathrm{~nm}$ and $630 \mathrm{~nm}$ are deposited on the input optics of Class-A and B goggles. The Class$\mathrm{C}$ goggles have a cut-on edge of about $660 \mathrm{~nm}$, that allows the use of red cockpit lights without causing large goggle signals or saturation, and also a decreased narrow-band transmittance at about $550 \mathrm{~nm}$ (called leaky green) that allows the goggle user to see a green display (monitor) through the goggle.

The radiance scale is transferred to the goggles from test-sets. A frequently used test-set light-source that irradiates the reticle-plane, is an LED that peaks around $815 \mathrm{~nm}$. There are other test sets using a different LED type that peaks at about $690 \mathrm{~nm}$. In both cases, the spectral power distribution of the LED covers only part of the wavelength range where the goggle is responsive. Also, in applications, where the goggles measure reflections under night sky irradiance, the spectral power distribution is much broader than that of a test-set LED. According to the present calibration considerations, if one LED distribution is used (as a reference distribution) for all Gen-3 goggle calibrations, then the calibration results (the transfer gain of the goggle) will be uniform. The assumption here is that the individual goggle responsivities will not change significantly within the wavelength interval of the LED (of the test-set). Another problem is that the spectral distribution of the source to be measured by the goggle at applications is ignored. As an example, the night sky radiation is very different from the spectral power distribution of an LED. The night sky radiation has a very broad distribution that will cover the overall responsivity range of the goggle resulting in a much larger signal than the goggle signal for a given LED distribution (for normalized distributions). The larger goggle signal at application does not mean that the produced systematic error is in the good direction (that the transfer gain of the goggle will be much higher in field applications than at calibration) because in a correct goggle calibration, the source distribution should be similar to the distribution at applications. The goggle transfer-gain at calibration should be very similar to that at applications. A modeling is described below to estimate the errors for an LED source at calibration and the broad-band night sky radiation at application.

The model shows the errors what an $815 \mathrm{~nm}$ LED source application (during calibration in the test sets) and the "constant goggle responsivity versus wavelength" assumption can produce in night sky irradiated measurements (applications). In the calculations, the night sky irradiance distributions for full-moon and no-moon (only stars) are used as source distributions during goggle applications. The normalized spectral power distribution of an $815 \mathrm{~nm}$ (peak) LED 
with the two different night sky distributions (already shown in Figs. 2a and 2b) are used. In this model, the wavelength coverage for the calibration-source and application-source distributions is very different in the shown spectral range where the goggles are responsive. The different spectral resposivities of Gen-3 night vision goggles from the early 1990, the mid 1990, and present, have also been shown in Fig. 1. Figure 1 also shows that the Class-A (standard and realized) filter transmittance (cut-on) curves that limit the goggle responsivities at the short wavelength end. All three goggles in Fig. 1 used in the modeling are used as Class-A goggles. Their responsivities are calculated as the product of the cut-on filter transmittance (the ITT filter curve) and the real spectral responsivity of the three Gen-3 goggles as shown in Fig. 1. The goggle responsivities are normalized at their peaks. Also, the spectral distributions of the night sky radiation in Figs. 2a and $2 \mathrm{~b}$ are normalized at their peaks to make the spectral products. First, the signal, which is the product of the goggle spectral responsivity and the source spectral distribution, was calculated for the (same) "Gen-3 present" responsivity and the three source distributions (the $815 \mathrm{~nm}$ LED, the full-moon irradiance, and the no-moon (stars only) irradiance). Then, the three different signals were compared by making two signal ratios to the reference signal (using the LED). The ratios in Table 2 show how large the goggle signal errors can be between calibration and (two) applications (using the "Gen-3 present" goggle). Following that, the "Gen-3 mid 1990" and then the "Gen-3 early 1990" goggle responsivities were substituted for the spectral products in the numerators (for both the full-moon and nomoon source distributions) and the denominator of the ratios was the spectral product of the "Gen-3 present" goggle and the $815 \mathrm{~nm}$ LED (used at calibration in the model).

Table 2.

Calculated ratios (systematic measurement errors) of the "Gen-3 present” goggle signals (when measuring two different night sky radiation distributions) to its reference signal (when measuring the $815 \mathrm{~nm}$ LED).

\begin{tabular}{|c|c|}
\hline INR (Gen-3 present \& full-moon) & INR (Gen-3 present \& no-moon) \\
\hline INR (Gen-3 present \& 815 nm LED) & INR (Gen-3 present \& 815 nm LED) \\
\hline 6.00 & 1.41 \\
\hline
\end{tabular}

The calculation results show that a factor of six (equal to $500 \%$ ) error can be obtained with the "Gen-3 present" goggle when it is calibrated using an $815 \mathrm{~nm}$ LED and then applied to measure full-moon night sky radiation. When it measures no-moon (stars only) radiation distribution, the error (ratio) decreases to 1.41 which means a $41 \%$ measurement error. This error is much smaller because of the very different distribution of the two night sky radiation curves as shown in Fig. 2a. The linear Y scale of Fig. 2b better illustrates that the no-moon radiation is much lower at the short wavelength range of the goggle than the full-moon radiation.

In the second part of this model, the spectral responsivities of the two other Gen-3 goggles were substituted for the "Gen-3 present” goggle to model the errors caused by different goggle spectral responsivities. The INRs were calculated for both full moon and no-moon source distributions and were referenced again to the INR of "Gen-3 present" and the $815 \mathrm{~nm}$ LED (as in Table 2). The ratios, that show the measurement errors using these other two goggles instead of the "Gen-3 present” goggle (with the assumption that only the "Gen-3 present” goggle was calibrated using the $815 \mathrm{~nm}$ LED source), are shown in Tables 3 and 4.

Table 3.

Calculated ratios (systematic measurement errors) of the "Gen-3 mid 1990" goggle signals (when measuring two different night sky source distributions) to the reference signal (the "Gen-3 present” measures the 815 nm LED).

\begin{tabular}{|c|c|}
\hline INR (Gen-3 mid 1990 \& full-moon) & INR (Gen-3 mid 1990 \& no-moon) \\
\hline INR (Gen-3 present \& 815 nm LED) & INR (Gen-3 present \& 815 nm LED) \\
\hline \multirow{2}{*}{5.88} & 1.26 \\
\hline
\end{tabular}

Proc. of SPIE Vol. 6737 67370M-11 
Table 4.

Calculated ratios (systematic measurement errors) of the "Gen-3 early 1990" goggle signals (when measuring two different night sky source distributions) to the reference signal (the "Gen-3 present” measures the 815 nm LED).

\begin{tabular}{|c|c|}
\hline INR (Gen-3 early 1990 \& full-moon) & INR (Gen-3 early 1990 \& no-moon) \\
INR (Gen-3 present \& 815 nm LED) & INR (Gen-3 present \& 815 nm LED) \\
\hline 6.54 & 1.21 \\
\hline
\end{tabular}

The ratios, in Tables 3 and 4, show that substituting the "Gen-3 present" goggle (used for calibration) with two other Gen-3 goggles (of different spectral responsivities), the goggle measurement errors can change up to $20 \%$ for no-moon and $54 \%$ for full-moon distributions (related to the 6.00 and 1.41 ratios in Table 2 where the errors are caused by the different source distributions).

All the errors calculated in Tables 2 to 4 are caused by spectral mismatch errors. In Table 2, the spectral mismatch of the applied night sky source distributions is large relative to the spectral distribution of the $815 \mathrm{~nm}$ LED (used at calibration). In Tables 3 and 4, the spectral mismatch is between the "Gen-3 early 1990" and "Gen-3 mid 1990” goggles relative to the "Gen-3 present” goggle (that represents the standard goggle spectral responsivity in this model).

The above measurement errors can be lowered if the spectral mismatch is less. For Table 2, the errors can be drastically decreased if several LEDs are used in the test sets instead of the $815 \mathrm{~nm}$ LED. Since the full-moon and no-moon night sky distributions are very different, and any distribution can happen between these two extreme distributions, the best results can be obtained if the LED distributions in the test sets (using several LEDs) will mimic (roughly) the no-moon distribution. The no-moon distribution is the best choice for reference source distribution because it has the lowest irradiance where large measurement uncertainties are not allowed (to perform safe goggle operations). The goggle measurement uncertainties can be higher at full moon (or between no-moon and full-moon) radiation where the signals are much higher and the measurement uncertainty is less of a problem.

For Tables 3 and 4, the measurement errors can be decreased if goggles are used with spectral responsivities similar to the standards Class A, B, or C. In field applications, the individual measurements of goggle relative spectral responsivities cannot be expected. If possible, goggles should be used with known typical relative spectral responsivities that are similar to each other (e.g. from the same fabrication batch). In this case, a broad-band correction factor can be used to further decrease the errors. This correction factor can be determined as shown below.

The goggle (NVIS) radiance is:

$$
R=\int_{\lambda} G_{s}(\lambda) N(\lambda) d \lambda
$$

where $G_{s}(\lambda)$ is the normalized standard spectral radiance responsivity of the goggle, $N(\lambda)$ is the spectral radiance of the source measured, and $\lambda$ is the wavelength. The signal of the goggle is:

$$
S=\int_{\lambda} G(\lambda) N(\lambda) d \lambda
$$

where $G(\lambda)$ is the (realized/fabricated) absolute spectral radiance responsivity of the goggle that measures the $N(\lambda)$ spectral radiance. The absolute radiance responsivity can be written as 


$$
G(\lambda)=G(\text { peak }) \cdot G_{n}(\lambda)
$$

where $G\left(\right.$ peak) is the absolute radiance responsivity of the goggle at the peak and $G_{n}(\lambda)$ is the normalized relative responsivity of the realized/fabricated goggle. The goggle broadband responsivity is the ratio of Eq. 5 to Eq. 4 :

$$
s=\frac{S}{R}=\frac{G(\text { peak }) \int_{\lambda} G_{n}(\lambda) N(\lambda) d \lambda}{\int_{\lambda} G_{s}(\lambda) N(\lambda) d \lambda}
$$

The goggle calibration factor is the reciprocal of $s$ and the broad-band (spectral mismatch) correction factor is:

$$
C=\frac{G(\text { peak })}{s}=\frac{\int_{\lambda} G_{s}(\lambda) N(\lambda) d \lambda}{\int_{\lambda} G_{n}(\lambda) N(\lambda) d \lambda}
$$

In practice, a broad-band correction factor can be calculated from the typical normalized spectral responsivity curve of the goggle relative to the normalized spectral responsivity curve of the standard (such as Class-A). The source can be the no-moon (stars only) night sky distribution. If the realized/fabricated goggle spectral responsivity is equal to the standardized spectral responsivity of a goggle type (from Class A, B, or C), the correction factor is unity. In case, the relative spectral responsivity of the goggle is known (e.g. measured), the correction factor will compensate for the spectral mismatch errors. The correction factor also can be used when goggles that belong to two different classes (such as Class A or B) are used to measure similar source distributions and uniform measurement results are needed.

While the errors calculated above have not been measured directly, the individual characteristics and functions, such as source distributions and spectral responsivities used in the calculations, were measured. The described modeling was the best approach to estimate the main goggle calibration and measurement uncertainties.

\subsection{Geometrical considerations}

In order to lower uncertainty in both the radiance responsivity calibration of the transfer standard radiometers (described in Section 2.3) and in the following radiance responsivity scale propagations, the traditionally used PVS-7 input optics was tested. This input optics which is a multi-element objective lens, was originally used for goggles where spatial distortions (aberrations) could lower the image quality. The PVS-7 input optics is also used at the front of the transfer standard radiometer where a good out-of-target rejection (radiation attenuation outside of the radiance measurement angle) would be more important than high image quality. An analysis has been made to improve the out-of-target blocking of the PVS-7 equipped radiometer using a simplified input optics of improved angular responsivity. The PVS7 input optics is still needed for the goggles.

The radiance measurement angle depends on the front geometry of the radiometer. Figure 11 shows the radiance measurement scheme of the biconvex lens equipped radiometer. The solid angle $\Omega_{0}$ of the $\mathrm{S}$ source as viewed (subtended) by the radiance measuring radiometer is usually not measured. Instead, the radiometer should have a well defined radiance measurement angle $\alpha$. A radiance meter is usually a modified irradiance meter. At the output of the input optics, either the Si photodiode itself or an aperture can be used as a field stop (L). In the shown example, there is an aperture of diameter $D_{1}$ at the front of the Si photodiode. The diameter of the objective lens is $D_{2}$ and $f$ is the focal length of the biconvex lens. There is an aperture stop A with a diameter of $\mathrm{D}_{3}$ in front of the lens to limit the viewing field. This way, the input optics has a constant $\mathrm{f} \#$, equal to $\mathrm{f} / \mathrm{D}_{3}$. The objective (biconvex) lens can be moved along the optical axis, relative to $\mathrm{L}$ to form an image of the $\left(\mathrm{D}_{4}\right.$ diameter) measured area of source $\mathrm{S}$ in the plane of $\mathrm{L}$. $\mathrm{S}$ must be larger than $\mathrm{D}_{4}$ to overfill L. According to the scheme on Fig. 11, the radiance measurement half-angle is:

Proc. of SPIE Vol. 6737 67370M-13 


$$
\alpha / 2=\tan ^{-1}\left(D_{1} / 2 i\right)
$$

where $i$ is the image distance. If the object distance $o$ between $\mathrm{S}$ and the center of the objective lens is much larger than $i$, then $i=f=$ constant, otherwise $i$ is different than $f$. In the radiometer shown in Fig. 5, no aperture was applied, and the photodiode was in the focal plane of the biconvex lens, that is the $i=f$ condition has been achieved and $\alpha$ is constant.

The angular responsivities of the same transfer standard radiometer head, when equipped with the two different input optics, are shown in Fig. 12 and 13. Using the biconvex lens, instead of the PVS-7 input optics, the out-of-target rejection could be improved by at least an order of magnitude at $+/-12^{\circ}$ and larger angles. The improvements are better illustrated on the logarithmic scale of Fig. 13. The linear scale on Fig. 12 shows that the field-of-view from the FWHM values is $15^{\circ}$ for the PVS-7 and about $10^{\circ}$ for the biconvex-lens input optics.

Using the simplified input optics with the more efficient out-of-target blocking, the distance dependence of the radiance responsivity (between the exit port of the integrating sphere and the input optics of the transfer standard radiometer) could be decreased from a $3.2 \%$ change to less than $1 \%$ during the (radiance responsivity) scale transfer.

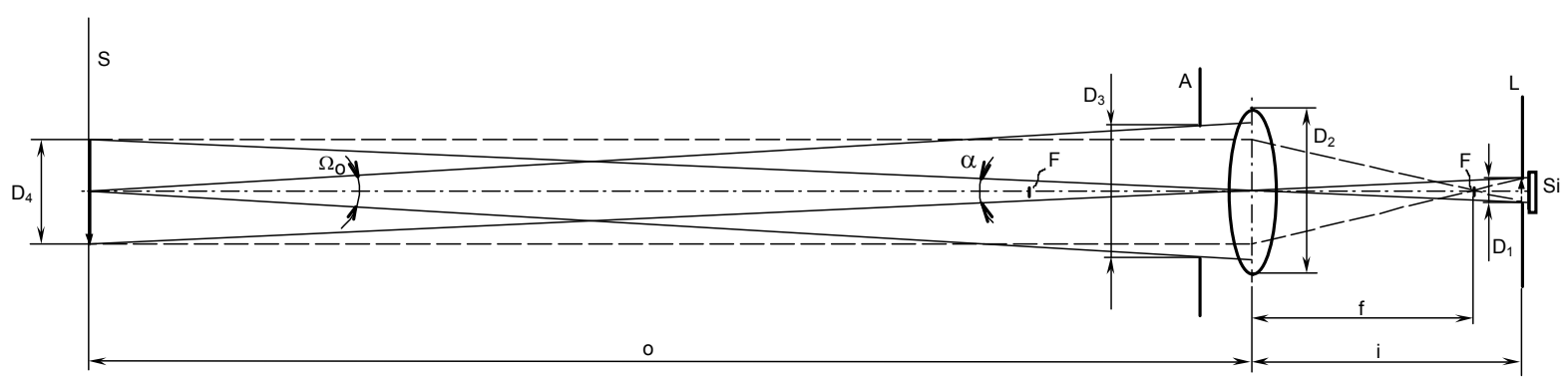

Fig. 11. Radiance measurement scheme of the transfer standard radiometer when using a biconvex objective lens.

For the night vision goggles, $\mathrm{D}_{1}$ is equal to the diameter of the photocathode (since no aperture is used in front of the goggle) which is significantly larger than the diameter of a silicon photodiode used (also without any aperture) in the transfer standard radiometer. Since $\alpha$ depends on $\mathrm{D}_{1}$, different size target spots can be obtained for the goggles and the transfer standard radiometers if the same PVS-7 input optics is used. The uncertainty of the scale transfer (from the transfer radiometer to the goggles) can be smaller if the input optics of the transfer standard radiometer (such as the biconvex lens) is designed such that the target spots of both the radiometer and the goggles are of similar sizes. This requirement can be important if the test-set radiance is spatially non-uniform.

Since both the goggles and the transfer standard radiometers have large radiance measurement angles, the radiance source applied in the radiance responsivity transfer should overfill the acceptance angle of both the radiometers and the goggles. Integrating sphere sources can be used as transfer sources, however, the standard remains the transfer standard radiometer. Application of collimators should be avoided. Also, careful mechanical and optical alignments are necessary in each step of the calibration transfer to keep the calibration uncertainties low. 


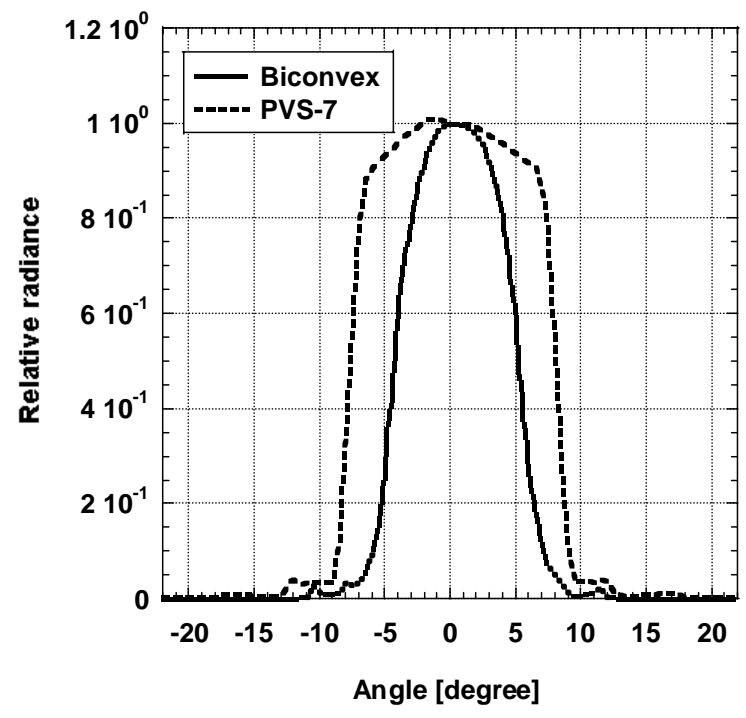

Fig. 12. Normalized angular responsivity of the NV radiometer in a linear scale when equipped either with the biconvex lens or the PVS-7 input optics.

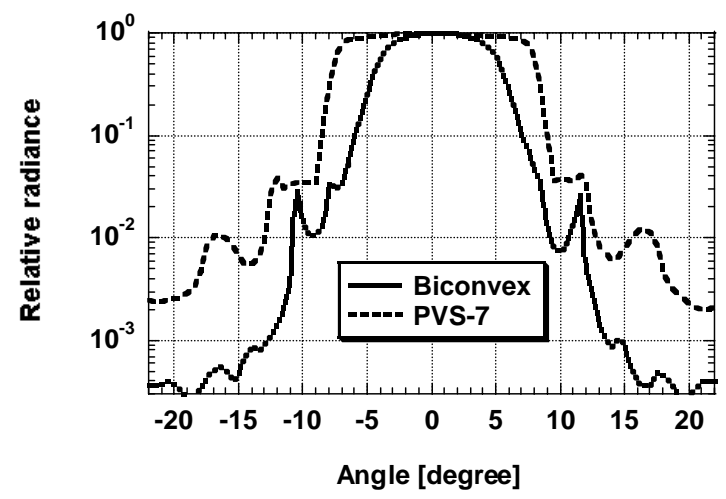

Fig. 13. Normalized angular responsivity of the NV radiometer in a logarithmic scale when equipped either with the PVS-7 input optics or a biconvex lens.

\section{CONCLUSIONS}

The NIST NVRCF has been developed and used on a regular basis to transfer the NIST reference radiance responsivity scale to the military primary standard laboratories. Spectral considerations have been discussed to obtain low uncertainties in the responsivity scale transfer to the test-sets and then to the goggles. A model showed that lasers can be used at radiance responsivity calibrations and scale transfers if the right detector spectral responsivity and test-set source distribution are used. However, use of only one LED type in the test-sets produces a significant spectral mismatch between calibration and applications. At applications, the goggles measure reflections of night sky radiation which has a much broader spectral distribution than that of the test-set LED. As a result of this spectral mismatch, a goggle will have different transfer-gains at calibration and applications. The goggle views a narrow-band LED distribution at calibration and measures a very different broad distribution at applications (night sky radiation). These errors have been modeled. The calculation results showed that $500 \%$ error can be obtained with a Gen-3 goggle when it is calibrated using an $815 \mathrm{~nm}$ LED and then applied to measure full-moon night sky radiation. When the same goggle measures no-moon (stars only) radiation distribution, the measurement error decreases to $41 \%$. In the second part of this model, the spectral responsivities of two Gen-3 goggles were substituted for a third Gen-3 goggle (the reference goggle in this model) to calculate the errors caused by the different goggle spectral responsivities. The calculations showed that the goggle measurement errors can change up to $20 \%$ for no-moon and $54 \%$ for full-moon night sky distributions when different goggles of the same type (uncalibrated in the model) are substituted for the reference goggle. The reason for these errors is the spectral mismatch between the two "test" goggles and the reference goggle.

Suggestions are made to decrease the spectral mismatch between calibration and applications for both the source distributions and the goggle spectral responsivities. In order to decrease goggle measurement errors, it is suggested to use several LEDs with different distributions in the test sets instead of the $815 \mathrm{~nm}$ LED. Since the full-moon and nomoon night sky distributions are very different, and all application distributions are between these two extreme distributions, the best results can be obtained if the LED distributions in the test sets will mimic (roughly) the no-moon distribution. This distribution has the lowest irradiance where large measurement uncertainties cannot be allowed to 
perform safe goggle operations. The goggle measurement uncertainties can be allowed to be higher at other night sky radiations where the signals are much higher and increased measurement uncertainty is less of a problem. A broad-band correction factor was introduced for goggle calibrations (to further decrease measurement uncertainties) for applications where the spectral responsivity of the applied (test) goggle is different than that of the standard.

Geometrical considerations have also been discussed to decrease uncertainties in the radiance responsivity transfer. The design of a simplified radiance measuring input optics was discussed with improved out-of-target rejection. The results of angular responsivity measurements illustrate the optimum radiance measurement angles and the improved radiance measuring characteristics of the NIST developed transfer standard radiometers.

\section{ACKNOWLEDGEMENTS}

The author thanks the U.S. Navy for funding the development of the NV Radiometer Calibration Facility, C. Randy Lustenberger for making the drawing of the NV radiometer front-head, Irena Fryc for participating in the model spreadsheet calculations, and Joe Velasquez for his fruitful discussions on goggle and test-set calibrations.

\section{REFERENCES}

1. MIL-STD-3009, Department of Defense Interface standard for Lighting, Aircraft, Night Vision Imaging System (NVIS) Compatible, 2 February, 2001.

2. MIL-L-85762A, Military specification for Lighting, Aircraft, Interior, Night Vision Imaging System (NVIS) Compatible, 26 August, 1988.

3. Bender, E.J., Personal communication, U.S. Army Night Vision and Electronic Sensors Directorate, Imaging Technology Branch, Science and Technology Division, Fort Belvoir, VA 22060-5806, 2007.

4. Littleton, R., Personal communication, Night Vision and Electronic Sensors Directorate, Fort Belvoir, VA 22060-5806, 2007.

5. Disclaimer, The mention of certain commercial products in this paper is for information purposes only and does not constitute an endorsement of the product by the author or his institution.

6. Task, H.L. and Marasco, P.L., The impact of changing night-vision goggle spectral response on night-vision imaging system lighting compatibility. SPIE proceedings, 2004. 5442.

7. $\quad$ Eppeldauer, G.P., et al., Improved accuracy photometric and tristimulus-color scales based on spectral irradiance responsivity. Proc. of 25th Session of the CIE, 2003. 1: p. D2-30 to D2-33.

8. U.S.patent 20050194516, , Programmable LED spectral light source. 2007.

9. Brown, S.W., Santana, C., and Eppeldauer, G.P., Development of a Tunable LED-based Colorimetric Source. J. Res. NIST, 2002. 107(4): p. 363-371.

10. Eppeldauer, G. and Hardis, J.E., Fourteen-decade photocurrent measurements with large-area silicon photodiodes at room temperature. Applied Optics, 1991. 30: p. 3091-3099.

11. Brown, S.W., Eppeldauer, G.P., and Lykke, K.R., Facility for Spectral Irradiance and Radiance Responsivity Calibrations using Uniform Sources (SIRCUS), Applied Optics, 2006. 32: p. 8218-8237.

12. Larason, T.C., Bruce, S.S., and Parr, A.C., Spectroradiometric detector measurements. NIST Special Publication, 1998. 250-41.

13. Eppeldauer, G., Racz, M., and Larason, T., Optical Characterization of Diffuser-input Standard Irradiance Meters. SPIE proceedings, 1998. 3573: p. 220-224.

14. Ohno, Y., NIST measurement services: Photometric calibrations. NIST Spec. Publ., 1997. 250-37.

Proc. of SPIE Vol. 6737 67370M-16 


\title{
Traceability of photocurrent measurements to electrical standards
}

\author{
George P. Eppeldauer \\ Optical Technology Division \\ National Institute of Standards and Technology \\ Gaithersburg, MD 20899
}

\begin{abstract}
In detector output-signal measurements, where the measurement uncertainty critically impacts the total uncertainty of a detector-based radiometric scale realization, traceability of the photocurrent measurement to SI traceable electrical standards is needed. A reference photocurrent-to-voltage conversion scale has been developed and described here. The new standard has internal reference resistors for all signal-gain selections to decrease noise pickup of traditionally used external reference resistors. The internal resistors were calibrated by the NIST Quantum Electrical Metrology Division against standard resistors. Using the substitution method, the reference photocurrent-to-voltage conversion scale was transferred from the converter standard to other converters up to a maximum signal-gain of $10^{10} \mathrm{~V} / \mathrm{A}$ with an expanded uncertainty of $0.013 \%(k=2)$. The DC conversion scale was extended to AC mode signal-gain calibrations where the photocurrent measurement uncertainty is $0.05 \%(k=2)$.
\end{abstract}

\section{Introduction}

The $0.01 \%(k=2)$ uncertainty of optical power measurements with cryogenic radiometers and the $0.06 \%(k=2)$ uncertainty of irradiance responsivity measurements at the NIST Spectral Irradiance and Radiance Responsivity Calibration with Uniform Sources (SIRCIUS) facility [1] needed the development of a technique to measure photodiode output signals with an expanded uncertainty close to $0.01 \%(k=2)$. Similar uncertainties are needed in those detector output signal measurements where the signal measurement uncertainty critically impacts the total uncertainty of a detector-based calibration. For photodiodes, that are the most accurate transfer and working standards, the output shortcircuit currents are to be measured to perform low signal-measurement uncertainty [2].

Standardized techniques are not available to calibrate photocurrent meters with the required $0.01 \%(k=2)$ uncertainty especially at low (close to $1 \mathrm{pA})$ photocurrents. Traditionally, electrometers (picoammeters) or current-to-voltage converters are used to measure small photocurrents. Research papers report low current-measurement uncertainties. However, these publications usually discuss only electrical current measurements and the calibration of the current meters. Typically, they do not discuss photodiode related low-current measurements which is a special area of low-current measurements where the electrical characteristics of the photodiodes (such as the shunt resistance) can influence the measurement uncertainties.

An intrinsically stable cryogenic current comparator (CCC) was reported recently for accurate measurement of small electrical currents [3]. Uncertainties of $0.002 \%(k=1)$ 
were reported for $300 \mathrm{pA}$ and $0.005 \%(k=1)$ for $100 \mathrm{pA}$ current measurements. A low DC current source, also based on the charging capacitor method using a linear voltage ramp, was reported for the calibration of picoammeters [4]. At $10 \mathrm{pA}$, an uncertainty of $0.003 \%(k=1)$ was reported. The uncertainty increased to $6 \%(k=1)$ at $1 \mathrm{fA}$. A measurement setup to calibrate picoammeters between $100 \mathrm{pA}$ and $100 \mathrm{nA}$ has also been reported where the measurement uncertainty was $0.042 \%(k=2)$ at $100 \mathrm{pA}$ [5].

In photodiode output-signal measurements, the signal measuring circuit operates in a linear mode if the photocurrent of the photodiode is measured. The current is converted into a voltage for data recording and evaluation. For low photocurrent measurements, high load-resistors are needed to obtain a high enough voltage that can be measured with regular digital voltmeters. Since high load-resistors (comparable to the shunt resistance of the measured detector) can produce non-linearity in the signal conversion, operational amplifier based current meters (producing lower load-resistances) are used for low photocurrent measurements. The conversion is made with a large precision resistor in the feedback loop of the operational amplifier [2]. The low-uncertainty signal-conversion is needed up to a feedback resistance of $10 \mathrm{G} \Omega$.

A photocurrent-to-voltage converter calibration method was published [6] where the expanded uncertainties were $0.38 \%(k=2)$ at $1 \mathrm{pA}$ and $0.046 \%(k=2)$ at $10 \mathrm{pA}$. A high constant-voltage was converted into small currents with high-resistance resistor standards and the test-converter measured the small currents. The calibration uncertainty was dominated by two dominant uncertainty components: the voltage-dependence and the stability of the $10 \mathrm{G} \Omega$ to $100 \mathrm{~T} \Omega$ resistor standards and the $1 / \mathrm{f}$ noise from the converter.

In our earlier work, precision resistors were used as reference resistors to calibrate an operational amplifier based current-to-voltage converter. In the first application, the reference resistors were serially connected to the converter input to transfer the converter from current mode into voltage measurement mode. In this case, the unknown feedback resistance of the operational amplifier is determined from the measured voltageamplification which depends on the serial reference-resistance and the feedback resistance. Here, both the input and the output voltages can be measured with low uncertainty. In the other application, the reference resistors are used to measure the current from a stable current source. With both methods, because of the use of external reference resistors, the converter output noise is large especially at high signal-gain selections. Usually, the signal dynamic range and the achievable uncertainty of the converter signal-gain calibrations are limited at high feedback resistances where the noise pickup can be significant and the noise-voltage amplification is high.

In order to obtain improved signal conversion uncertainties, a reference current-tovoltage converter has been developed using internal (electrically shielded by the metal box of the converter) reference feedback resistors. With this design, the noise pickup can be decreased. Circuit design details (such as isolation resistance issues, printed circuit board design, shielding etc) are not discussed here. These questions have been discussed earlier [2]. This work focuses on a signal-conversion scale realization and its propagation to applications. The reference current-to-voltage converter can be used first for the 
current-to-voltage conversion-scale realization and then, in a second step, it can propagate the signal-conversion scale to a test current-to-voltage converter. The signalconversion scale is an electrical scale where the current-to-voltage gains and the corresponding conversion uncertainties are determined. The reference signal conversion scale (implemented by the converter standard) can be transferred to different radiometric and photometric calibrations where it works like a sub-scale. The uncertainty of the subscale must be low enough to avoid dominating the combined uncertainty of the related radiometric or photometric scale realizations. Propagation of the reference sub-scale to field applications with low current-to-voltage conversion uncertainty is also an important requirement.

\section{The current-to-voltage converter standard}

A current-to-voltage converter standard with internal reference resistors has been developed to improve noise performance and uncertainty at high signal-gain calibrations of test converters.

Commercially available precision resistors with excellent electrical and thermal characteristics have been acquired and then calibrated against NIST resistor standards. The manufacturer reported resistances of the purchased resistors (Caddock, TF Series) have $0.01 \%$ tolerances to their nominal decade values between $10^{4} \Omega$ and $5 \times 10^{7} \Omega$ [4]. Two, $5 \times 10^{7} \Omega$ resistors were connected serially to obtain a $10^{8} \Omega$ feedback resistance. The temperature coefficient of resistance for these resistors is $10 \mathrm{ppm} /{ }^{0} \mathrm{C}$ or less. The characteristics of the highest feedback resistor, $10^{9} \Omega$, used in the current-tovoltage converter standard (Ohmite, Mini-MOX) were worse than the lower feedback resistors. The tolerance for the decade nominal value was $0.5 \%$ and the temperature coefficient of resistance was $100 \mathrm{ppm} /{ }^{\circ} \mathrm{C}$.

The traditional circuit diagram used for the current-to-voltage converter standard is shown in Fig. 1. This converter has a BNC IN connector to measure the input current and a BNC OUT to measure the output voltage. One resistor from a set of feedback resistors can be selected with a rotary switch to switch signal-gain (current sensitivity). The selected resistor with its parallel capacitance is switched into the feedback loop of the operational amplifier OA. An electrometer-grade OA is to be used because it has very low bias current, low offset and drift, high open-loop gain and common-mode rejection. The feedback resistors (discussed above) are R1 $=10^{4} \Omega, \mathrm{R} 2=10^{5} \Omega, \mathrm{R} 3=10^{6} \Omega, \mathrm{R} 4=10^{7}$ $\Omega$, and $\mathrm{R} 5=10^{8} \Omega$. Instead of using the manufacturer reported resistance tolerances, the resistors were calibrated against resistor standards at the NIST Quantum Electrical Metrology Division. The highest feedback resistor was R6 $=10^{9} \Omega$. This resistor does not have parallel connected external capacitance to obtain a $3 \mathrm{~dB}$ roll-off frequency of about $100 \mathrm{~Hz}$. The other parallel capacitors C1 to C5 were used for frequency compensations to optimize the fundamental gains and the minimum upper $3 \mathrm{~dB}$ roll-off frequency was again $100 \mathrm{~Hz}$ [2]. The parallel feedback capacitors are C1=C2=6.8 nF, C3=1500 pF, $\mathrm{C} 4=150 \mathrm{pF}$, and C5=15 pF. The converter housing was electrically shielded. The common of the circuit is combined with the shielding wire (connected to the ground-pin 
of the $120 \mathrm{VAC}$ power line connector) at the $0 \mathrm{~V}$ pin of the $+/-15 \mathrm{~V}$ power supply. The printed circuit board of the converter is illustrated with a dashed line on Fig. 1.

In order to utilize the advantage of group policy, two sets of feedback resistors were calibrated for the $10^{4} \mathrm{~V} / \mathrm{A}$ to $10^{8} \mathrm{~V} / \mathrm{A}$ signal-gain selections. The obtained resistance corrections for the manufacturer reported nominal values and the expanded relative measurement uncertainties are shown in Table 1. The results show that the differences between the manufacturer reported nominal resistance values and the NIST Quantum Electrical Metrology Division measured resistances are always smaller than $0.012 \%$ and the highest relative expanded uncertainty of the NIST resistance calibrations was also $0.012 \%(k=2)$.

After the resistance calibrations, all feedback resistors were inserted into the current-tovoltage converter standard. Since the $1 \mathrm{G} \Omega$ feedback resistor has worse electrical characteristics than the others and we wanted to keep the measurement uncertainty low, it was not calibrated against any external resistor standard. Instead, this signal-gain was calibrated against the neighboring signal-gain as it will be discussed below.

\section{DC signal-gain calibrations using the current-to-voltage converter standard}

Improved conversion uncertainty can be achieved with test current-to-voltage converters if they are calibrated against the above discussed current-to-voltage converter standard. In this case, the noise pickup problem can be eliminated and the low uncertainty signal-gain calibration can be extended to the $10^{10} \mathrm{~V} / \mathrm{A}$ gain of a test current-to-voltage converter.

The $10^{9} \mathrm{~V} / \mathrm{A}$ signal-gain (using the $1 \mathrm{G} \Omega$ feedback resistor) was calibrated against the $10^{8} \mathrm{~V} / \mathrm{A}$ gain by measuring the output voltage ratio with the two gains when applying the same (5 nA) constant current to the input of the converter standard.

Table 1.

Evaluation of the NIST Quantum Electrical Metrology Division calibrations for two sets of feedback resistors

\begin{tabular}{|c|c|c|c|}
\hline $\begin{array}{c}\text { Feedback resistor } \\
\text { Serial Number }\end{array}$ & $\begin{array}{c}\text { Nominal Value } \\
{[\Omega]}\end{array}$ & $\begin{array}{c}\text { Correction } \\
{[\mu \Omega / \Omega]}\end{array}$ & $\begin{array}{c}\text { Relative expanded } \\
\text { uncertainty [\%] }\end{array}$ \\
\hline R1 & $10^{4}$ & 42.8 & 0.0001 \\
\hline R2 & $10^{5}$ & 14.7 & 0.0001 \\
\hline R3 & $10^{6}$ & 12.0 & 0.0002 \\
\hline R4 & $10^{7}$ & 116.5 & 0.0014 \\
\hline R5 & $10^{8}$ & 88 & 0.0116 \\
\hline R1' & $10^{4}$ & 2.3 & 0.0001 \\
\hline R2' & $10^{5}$ & -2.8 & 0.0001 \\
\hline R3' & $10^{6}$ & 76.2 & 0.0002 \\
\hline R4' & $10^{7}$ & 124.1 & 0.0014 \\
\hline R5' & $10^{8}$ & 173 & 0.0116 \\
\hline
\end{tabular}




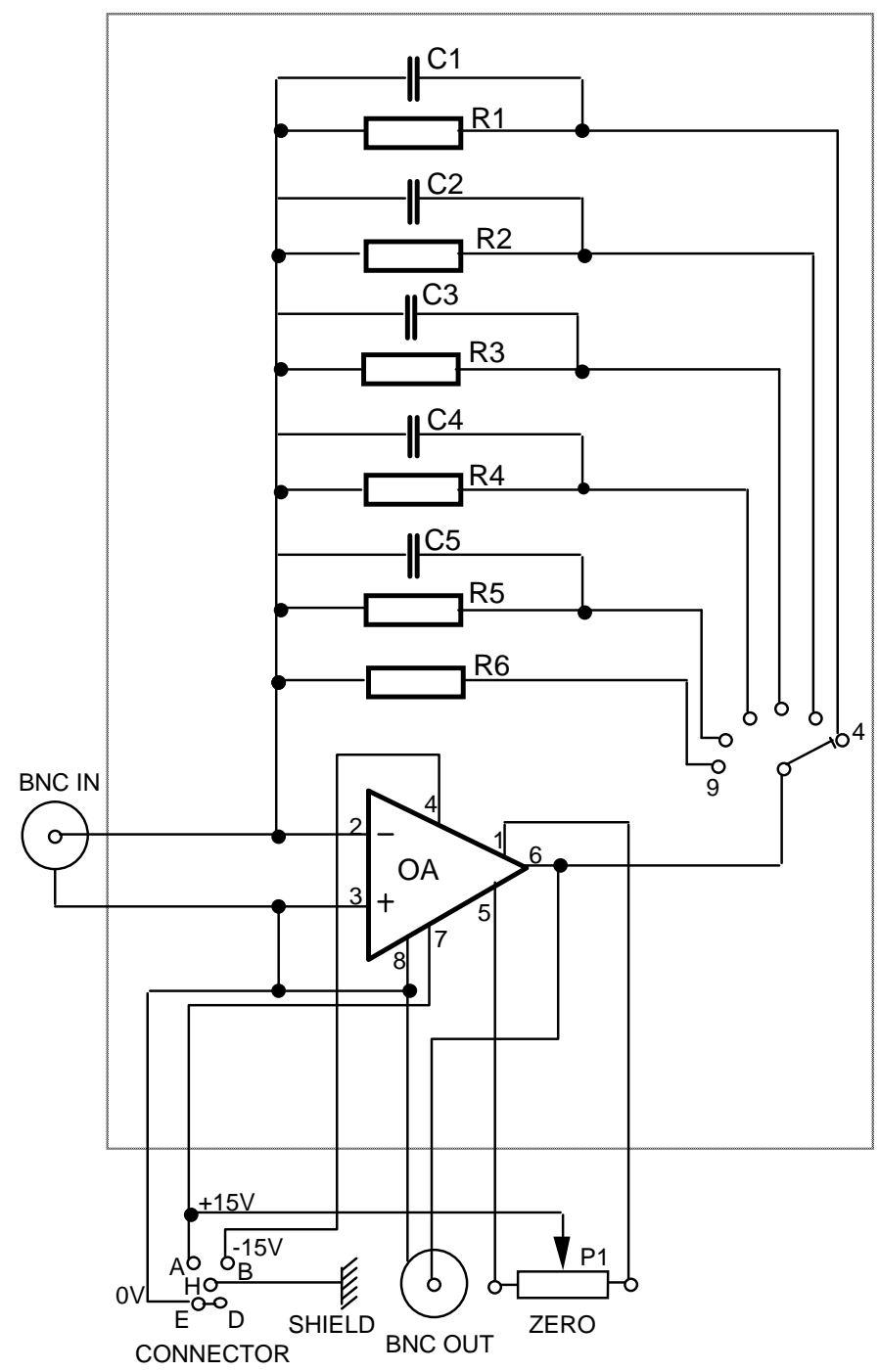

Fig. 1. Circuit diagram of the current-to-voltage converter standard

The current from the variable output current source is measured with the current-tovoltage converter standard. Figure 2 shows the simplified scheme of the substitution type current-to-voltage converter calibration.

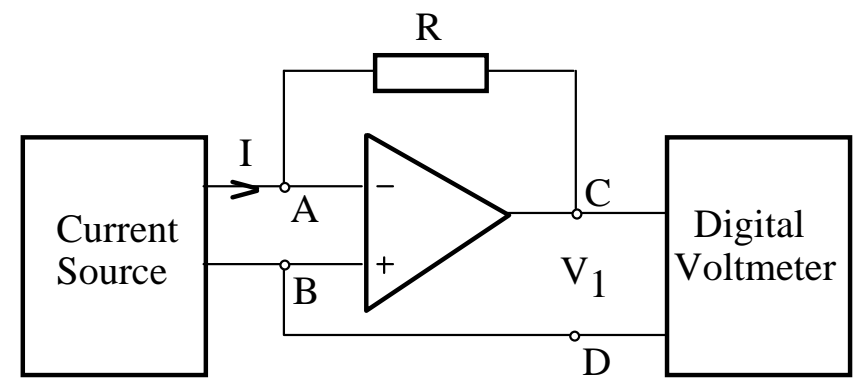

Fig. 2. Current determination with the current-to-voltage converter standard. 
The current I from the electronic current source is introduced into the current-to-voltage converter standard. The output voltage $V_{1}$ of the converter standard can be measured with a digital voltmeter. The value of the input current can be calculated after two output voltage measurements:

$$
I=\frac{V_{1}(I)-V_{1}(I=0)}{R}
$$

where $V_{1}(I)$ is the converter output voltage for input current $I, V_{1}(I=0)$ is the converter output voltage for $I=0$, and $R$ is the resistance of the feedback reference resistor in the converter. The voltage subtraction cancels the output offset voltage of the converter standard.

After the determination of $I$, test current-to-voltage converters can be substituted for the converter standard at points A, B, C, and D. Points B and D are the "common" of the circuits that also give the electrical shielding for the connecting (input and output) cables.

The output voltage of a test current-to-voltage converter is $V_{2}$. The signal-gain of the test current-to-voltage converter is:

$$
G_{S}=\frac{V_{2}(I)-V_{2}(I=0)}{I}
$$

where $V_{2}(I)$ is the test converter output voltage for input current $I, V_{2}(I=0)$ is the test converter output voltage for $I=0$, and $I$ is the current as determined by Eq. 1 . The output offset voltage of the test converter is canceled out by the voltage subtraction in Eq. 2. In order to obtain high signal-to-noise ratios, $G_{S}=R$ is the best choice. However, when $G_{S}=$ $10^{10} \mathrm{~V} / \mathrm{A}$ in the test converter, the maximum built-in feedback resistance of $R=10^{9} \mathrm{~V} / \mathrm{A}$ (corresponds to the highest feedback resistor of $1 \mathrm{G} \Omega$ ) is used in the converter standard. In this case, the test current from the current source must be lowered to $0.5 \mathrm{nA}$ where the output resistance of the current source (Keithley Model 263) is 1 G $\Omega$. This sourceresistance increase is needed to keep the output noise (and the signal-gain uncertainty of the test-converter) low.

\section{Loop-gain related DC signal-conversion uncertainty}

When a small load-resistor converts a large current into a voltage, the conversion is done according to Ohm's law and the conversion uncertainty can be low. In case of small photocurrent measurements, when an operational amplifier is used with high feedback resistors, the conversion uncertainty can be high. This complication is the reason why national metrology institutes (NMIs) do not offer calibration services for low current measurements. 
The signal-gain of a photocurrent-to-voltage converter in DC (low-frequency measurement) mode is:

$$
G_{S}=R \frac{1}{1+G_{L}^{-1}} .
$$

In an ideal case,

$$
R=\frac{V}{I}
$$

where $V$ is the output voltage and $I$ is the input current of the current-to-voltage converter, $R$ is the feedback resistance of the operational amplifier and $G_{L}$ is the loop gain of the analog control loop of the converter. A realized current-to-voltage conversion can be close to the ideal case if the conversion uncertainties are kept low. Otherwise, the loop-gain can produce a significant component in the uncertainty of the current-tovoltage conversion. The DC loop gain can be written as

$$
G_{L}=A_{0} \beta_{0}
$$

where $\beta_{0}$ is the DC feedback attenuation and $A_{0}$ is the DC open-loop gain of the operational amplifier used in the converter. When $R_{\mathrm{S}}$ photodiode-shunt-resistance is connected to the input of the converter, the DC feedback attenuation will be

$$
\beta_{0}=\frac{R_{S}}{R_{S}+R}
$$

For a Si-tunnel-trap detector, if the photodiodes are selected for equal shunt resistance, the typical resultant shunt resistance is between $30 \mathrm{M} \Omega$ and $50 \mathrm{M} \Omega$ [2]. With $R_{S}=50 \mathrm{M} \Omega$ shunt resistance and $R=1 \mathrm{G} \Omega$ maximum feedback resistance (R6 in Fig. 1), $\beta=0.048$ that produces a loop gain of about $G_{L}=5 \times 10^{4}$. This loop gain will result in a current-tovoltage conversion uncertainty of $0.002 \%$. The calculation also shows that the shunt resistance for a $0.01 \%$ conversion-uncertainty cannot be smaller than $10 \mathrm{M} \Omega$ when using gain $10^{9} \mathrm{~V} / \mathrm{A}$. The output resistance of the current source in Fig. 1 at $5 \mathrm{nA}$ test-current was $100 \mathrm{M} \Omega$ resulting in a conversion uncertainty of $0.001 \%$ using gain $10^{9} \mathrm{~V} / \mathrm{A}$ at calibration.

\section{Uncertainty of DC current measurement using the converter standard}

The uncertainty components of current measurement $I$ using the current-to-voltage converter standard at gain $10^{8} \mathrm{~V} / \mathrm{A}$ are shown in Table 2 . The test-current from the current source was $5 \mathrm{nA}$ and the output resistance of the current source was $100 \mathrm{M} \Omega$. The gain-ratio (step-down) method was used to calibrate the $10^{9} \mathrm{~V} / \mathrm{A}$ (highest) gain of the converter standard against the calibrated $10^{8} \mathrm{~V} / \mathrm{A}$ gain. The highest feedback resistor calibrated by the NIST Quantum Electrical Metrology Division was $10^{8} \Omega$. 
Table 2. Uncertainty of input current measurement at gain $10^{8} \mathrm{~V} / \mathrm{A}$ of the current-to-voltage converter standard

\begin{tabular}{|l|c|c|}
\hline \multicolumn{1}{|c|}{ Uncertainty components } & Type & $\begin{array}{c}\text { Relative uncertainty } \\
{[\%]}\end{array}$ \\
\hline Feedback resistance & $\mathrm{B}$ & $5.8 \times 10^{-3}$ \\
\hline Short-term instability of input current $I$ & $\mathrm{~A}$ & $1 \times 10^{-3}$ \\
\hline$V_{1}$ voltage measurement & $\mathrm{B}$ & $2 \times 10^{-4}$ \\
\hline Output noise and drift & $\mathrm{A}$ & $1 \times 10^{-4}$ \\
\hline Loop gain & $\mathrm{A}$ & $2 \times 10^{-4}$ \\
\hline Combined standard uncertainty $(k=1)$ of $I$ measurement & & $5.89 \times 10^{-3}$ \\
\hline Expanded total uncertainty $(k=2)$ of $I$ measurement & & 0.012 \\
\hline
\end{tabular}

During the gain calibration transfer, two uncertainty components will change. Because of the increased closed-loop voltage gain [2], the output noise and drift produced uncertainty component will increase to $1 \times 10^{-3} \%(k=1)$. The increased feedback attenuation will increase the loop-gain produced uncertainty component to $1 \times 10^{-3} \%$ $(k=1)$. The combined standard uncertainty of the current measurement at $10^{9} \mathrm{~V} / \mathrm{A}$ will be $6.06 \times 10^{-3} \%(k=1)$, and the rounded expanded total uncertainty will remain $0.012 \%$ $(k=2)$. The results show that the uncertainty of the current measurements at both the $10^{8} \mathrm{~V} / \mathrm{A}$ and $10^{9} \mathrm{~V} / \mathrm{A}$ gains is dominated by the uncertainty of the feedback resistance measurement (of the $10^{8} \Omega$ in Table 1 ).

The final uncertainty determination of the current measurements with the current-tovoltage converter standard has been made experimentally, by measuring the neighboring gain ratios (using the step-down method). At each neighboring gain ratio of $(n+1) / n$, where $n$ is the gain number ( 4 to 7 in this test), the input-current was kept at a constant value. The reference gain-resistance ratios in Table 1 were divided by the measured output-voltage ratios for all gains. The four gain ratios from $10^{8} / 10^{7}$ to $10^{5} / 10^{4}$ were determined five times (from five different current determinations) and all the four average ratios were smaller than 1.00010. The conclusion after these gain ratio (reference to measured) comparisons is that the current measurement uncertainty of the converter standard is $0.012 \%(k=2)$ for gains $10^{8} \mathrm{~V} / \mathrm{A}$ and $10^{9} \mathrm{~V} / \mathrm{A}$, and $0.01 \%(k=2)$ for the lower gains.

\section{Uncertainty of a test converter DC signal-gain calibration}

In order to calibrate a test current-to-voltage converter with the $10^{10} \mathrm{~V} / \mathrm{A}$ highest gain, the test-current from the current source must be lowered to $0.5 \mathrm{nA}$ and the $10^{9} \mathrm{~V} / \mathrm{A}$ gain of the converter standard must be used to determine the current. At the $0.5 \mathrm{nA}$ input current, the output resistance of the current source is $1 \mathrm{G} \Omega$, one order of magnitude higher than at the $5 \mathrm{nA}$ selection (used above). This source-resistance increase decreases the output noise and drift and also the loop-gain produced uncertainty components of the current determination but the expanded total uncertainty will remain $0.012 \%(k=2)$. However, the $1 \mathrm{G} \Omega$ source resistance makes it possible to keep the output noise and drift and also 
the loop-gain produced uncertainty components of the test-converter calibration low in spite of the $10^{10} \mathrm{~V} / \mathrm{A}$ (maximum) gain selection of the test converter. The uncertainty budget of the signal-gain determination of a test current-to-voltage converter using $0.5 \mathrm{nA}$ input-current at the $10^{10} \mathrm{~V} / \mathrm{A}$ signal-gain is shown in Table 3.

Table 3. Uncertainty of the signal-gain determination of a test current-to-voltage converter at gain $10^{10} \mathrm{~V} / \mathrm{A}$ using $1 \mathrm{G} \Omega$ current-source-resistance

\begin{tabular}{|l|c|c|}
\hline \multicolumn{1}{|c|}{ Uncertainty components } & Type & $\begin{array}{c}\text { Relative uncertainty } \\
{[\%]}\end{array}$ \\
\hline$I$ measurement & $\mathrm{B}$ & 0.006 \\
\hline Short-term instability of input current $I$ & $\mathrm{~A}$ & $2 \times 10^{-3}$ \\
\hline$V_{2}$ voltage measurement & $\mathrm{B}$ & $2 \times 10^{-4}$ \\
\hline Output noise and drift & $\mathrm{A}$ & $1 \times 10^{-3}$ \\
\hline Loop gain & $\mathrm{A}$ & $1 \times 10^{-3}$ \\
\hline Combined standard uncertainty $(k=1)$ of $G_{S}$ measurement & & $6.48 \times 10^{-3}$ \\
\hline Expanded total uncertainty $(k=2)$ of $G_{S}$ measurement & & 0.013 \\
\hline
\end{tabular}

Using the above introduced DC signal-gain calibration method and procedure, even at the highest signal-gain selection of a test converter $\left(G_{S}=10^{10} \mathrm{~V} / \mathrm{A}\right)$, an expanded total gainuncertainty of $0.013 \%(k=2)$ can be achieved. During the calibration part of this method and procedure, photodiodes have not been used.

If the above discussed calibration/measurement uncertainties are to be repeated in real photocurrent measurements (applications), where different photodiodes are connected to the input of a current-to-voltage converter (test or standard), the shunt resistance of the connected photodiode must be known. E.g., the shunt resistance must be $100 \mathrm{M} \Omega$ or higher when the $10^{9} \mathrm{~V} / \mathrm{A}$ signal-gain of the converter-standard is used (as discussed in Section 7). Similarly, the shunt resistance must be higher than $1 \mathrm{G} \Omega$ when the $10^{10} \mathrm{~V} / \mathrm{A}$ (maximum) signal-gain of a test converter is selected. Otherwise, the output noise and drift uncertainty component in Tables 2 and 3 will increase resulting in an increase in the combined uncertainties as well.

The shunt resistance of the applied photodiode will determine the smallest photocurrent that can be measured with the above reported uncertainties (obtained using the electronic current source). E.g., when the noise equivalent current (NEC) is $1 \times 10^{-15} \mathrm{~A}(k=2)$ [2], the minimum signal-current should be at least four orders of magnitude higher (10 pA) to obtain a maximum $0.01 \%(k=2)$ uncertainty contribution (to the $0.012 \%$ and $0.013 \%$ expanded total uncertainties of Tables 2 and 3) from the output signal-to-noise ratio of the converter. This would result in a $10 \mathrm{pA}$ photocurrent measurement expanded total uncertainty of $0.016 \%$.

Using AC mode current-to-voltage conversion (discussed below), the NEC can be decreased to $8 \times 10^{-17} \mathrm{~A}$ [2]. However, the inherent uncertainty of AC signal-gain calibrations is somewhat higher than that of the above discussed DC calibrations. 


\section{Extension of the DC current-to-voltage conversion to AC}

The signal-gain versus frequency characteristics of a current-to-voltage converter at different gain selections are different because the $3 \mathrm{~dB}$ upper roll-off points are usually at different frequencies. In practice, fine tuning of the feedback capacitances to obtain the same $3 \mathrm{~dB}$ roll-off frequencies is either a very difficult or an impossible task.

In order to describe the signal-gain of a photocurrent-to-voltage converter in AC measurement mode, Eq. 3 (that describes the DC signal-gain only) must be extended with the frequency dependent components of the loop-gain and the current-to-voltage conversion [2]:

$$
G_{S}(\omega)=R \frac{1}{1+j \omega R C} \frac{1}{1+\left[G_{L}(\omega)\right]^{-1}}
$$

In order to achieve low photocurrent-to-voltage conversion uncertainty in AC mode, both the current-to-voltage conversion and the loop-gain are to be optimized at the signal (chopping) frequency.

When the above described DC photocurrent-to-voltage conversion scale is extended to AC, even if the signal frequency is kept constant, additional AC mode measurements are needed. The above determined DC signal-gains are the reference values of a DC and AC mode current-to-voltage converter for signal frequencies close to $0 \mathrm{~Hz}$. In order to propagate the DC signal-gain scale to higher signal frequencies, the signal-gain versus frequency curves are to be known for all signal-gain selections of the converter. When these curves are known, the above discussed current-to-voltage converter standard can be used in AC mode as well.

As an example, Fig. 3 shows the measured signal-gain versus frequency curve of the current-to-voltage converter standard (discussed above) for one signal-gain selection. The photocurrent from a Si tunnel-trap detector-standard was converted into voltage by the converter standard, using signal-gain $10^{5} \mathrm{~V} / \mathrm{A}$, and the voltage was measured by a lock-in amplifier. During the measurement, the detector-standard was illuminated by the chopped radiation of a tungsten halogen lamp. The frequency of the chopper was tuned from $4 \mathrm{~Hz}$ to $180 \mathrm{~Hz}$ and the lock-in amplifier was synchronized to the chopping (signal) frequency. Each data point is an average of 20 measurements. The data points are shown with an open circle on the left-Y logarithmic scale. This AC signal-gain curve is used as a relative curve and it will be converted into an absolute curve using a DC (0 Hz) absolute tie point (as discussed above). The standard deviation of the mean of the 20 measurements is shown with open squares on the linear right-Y axis of the graph. The frequency (X-axis) scale is also a logarithmic scale to obtain the popular Bode-plot for the signal-gain function. As shown in the graph, a one-time-constant RC roll-off curve-fit was applied to the measured (response) data points: 


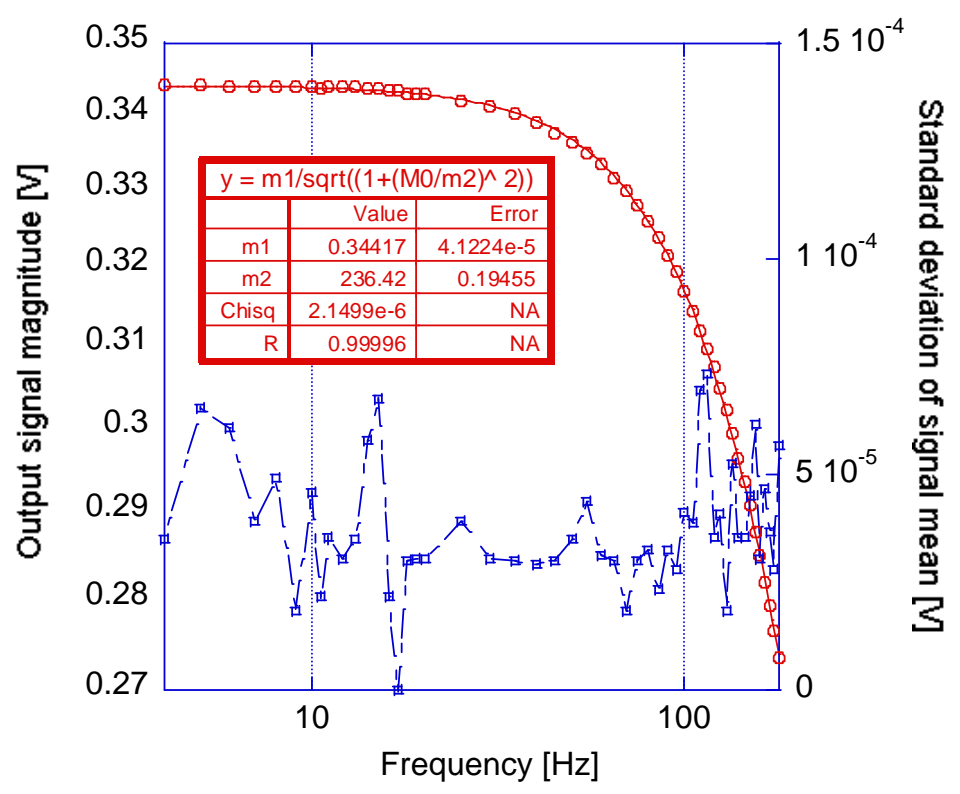

Fig. 3. Frequency dependent response of the converter standard at signal-gain $10^{5} \mathrm{~V} / \mathrm{A}$.

$$
y=\frac{m_{1}}{\sqrt{1+\left(\frac{M_{0}}{m_{2}}\right)^{2}}}
$$

where $m_{1}$ is the DC response, $M_{0}$ is the frequency, and the upper roll-off frequency is

$$
m_{2}=\frac{1}{2 \pi R C}=236.4 \mathrm{~Hz}
$$

The fit verifies that the upper roll-off of a Si-photodiode current meter is exclusively determined by the time constant of the feedback RC components [2].

The integrating time-constant at this $10^{5}$ V/A signal-gain (see Section 4) is $R_{2} C_{2}=6.8 \mathrm{x}$ $10^{-4}$ s gives a $3 \mathrm{~dB}$ upper roll-off frequency of $234.2 \mathrm{~Hz}$. The $3 \mathrm{~dB}$ roll-off frequency obtained from the curve fit is slightly higher because the actual $\mathrm{C}_{2}$ is different from the $6.8 \mathrm{nF}$ nominal value of the (externally connected) feedback capacitor. From the fit parameters and the standard deviation of the data points, an AC measurement uncertainty of $0.04 \%(k=2)$ can be estimated.

Similar signal-gain versus frequency measurements were made for the other signal-gain selections as well. The one-time-constant curve-fit worked well for all signal-gain selections. The results are summarized in Table 4. 
Table 4.

Results of the $3 \mathrm{~dB}$ upper roll-off frequency measurements from one-time-constant curve fits at the different signal-gain selections of the converter standard.

\begin{tabular}{|c|c|c|c|}
\hline $\begin{array}{c}\text { Signal gain } \\
{[\mathrm{V} / \mathrm{A}]}\end{array}$ & $\begin{array}{c}3 \mathrm{~dB} \text { roll-off } \\
\text { nominal frequency } \\
{[\mathrm{Hz}]}\end{array}$ & $\begin{array}{c}3 \text { dB roll-off } \\
\text { measured frequency } \\
{[\mathrm{Hz}]}\end{array}$ & $\begin{array}{c}\text { Standard deviation } \\
\text { of the signal mean } \\
{[\%](k=1)}\end{array}$ \\
\hline $10^{4}$ & 2341.7 & 3131.5 & 0.014 \\
\hline $10^{5}$ & 234.2 & 236.4 & 0.015 \\
\hline $10^{6}$ & 106.2 & 102.7 & 0.02 \\
\hline $10^{7}$ & 106.2 & 105.3 & 0.025 \\
\hline $10^{8}$ & 93.7 & 91.5 & 0.025 \\
\hline
\end{tabular}

At signal-gain $10^{8} \mathrm{~V} / \mathrm{A}$, the nominal frequency was calculated with a $2 \mathrm{pF}$ estimated stray capacitance parallel connected to the $\mathrm{C} 5=15 \mathrm{pF}$ feedback capacitor. The standard deviation of the measured data points are shown for all signal-gain selections in the last column. The above described $0.04 \%(k=2)$ relative expanded uncertainty of the signalgain $10^{5} \mathrm{~V} / \mathrm{A}$ also applies to the signal-gains of $10^{4} \mathrm{~V} / \mathrm{A}$ and $10^{6} \mathrm{~V} / \mathrm{A}$. The uncertainty (shown by the standard deviations) is increased to $0.05 \%(k=2)$ for the signal gains of $10^{7} \mathrm{~V} / \mathrm{A}$ and $10^{8} \mathrm{~V} / \mathrm{A}$.

The results indicate that the signal-gain versus frequency measurements may not be needed if the $\mathrm{C}$ feedback capacitors are measured (before mounting them into the circuit) and the parallel stray capacitances are known. In this case, the RC time constants and then the relative roll-off functions can be calculated. The uncertainty may increase at the higher gain selections where the value of the parallel stray capacitance is comparable to the value of the externally connected feedback capacitor. The parallel stray capacitance that can be performed with a regular printed-circuit-board (PCB) design is about $2 \mathrm{pF}$. This capacitance can be decreased by an order of magnitude if three layers are implemented on the PCB. In this case, the inverting-input and the output pin of the operational amplifier are to be soldered to two different layers (the top and bottom) that are separated with the shielded (third) copper layer to minimize the stray capacitance between the input and the output (where the feedback resistors are connected).

Even if the relative roll-off functions can be calculated, measurement of the frequency dependent signal-gains may be necessary to avoid gain peaking. Optimization of the signal- and the loop-gains needs partial frequency compensations for the different gain selections [2]. Frequency compensation can be applied to eliminate gain peaking.

For an AC measuring test current-to-voltage converter, the reference values (tie points) of the signal-gain versus frequency functions are the DC signal-gains. Usually, the signalgain at a given signal frequency is not transferred directly from the reference current-tovoltage converter to the test AC-mode converter. Only the DC signal-gains are transferred from the reference converter to the AC-mode test converter. In detector responsivity measurements, using the here discussed method, the DC responsivity of the 
detector standard can be extended to the AC measuring current-to-voltage converter attached to the detector. Usually, NIST reports the DC responsivity of the issued detector-standard without the signal-gain of the current-to-voltage converter standard used to calibrate this detector. In this case, the signal-gain versus frequency curves of the AC measuring test converter (used with the NIST issued detector standard) should be known to extend the detector DC responsivity with the AC signal-gain of the attached test-converter at the signal (chopping) frequency. This method can produce similar AC signal-gain uncertainties for both the reference and the test current-to-voltage converter.

Since the DC and AC signal-gain calibration uncertainties are random and uncorrelated, the root-mean-square-sum of the DC and AC signal-gain uncertainties will produce the combined uncertainty of an AC-operated test current-to-voltage converter.

\section{Conclusions}

In order to satisfy the less than $0.02 \%(k=2)$ expanded total uncertainty requirement of NIST reference radiometric calibrations, a new calibration method and procedure has been developed for calibrating the signal-gains of current-to-voltage converters that operate in a wide photocurrent range. A reference current-to-voltage converter has been developed where all the feedback resistors are inside of an electrically shielded housing to avoid noise pickup. The feedback resistors from $10 \mathrm{k} \Omega$ to $100 \mathrm{M} \Omega$ have been calibrated at the NIST Quantum Electrical Metrology Division against resistor standards. The $0.0116 \%$ expanded uncertainty $(k=2)$ of the $10^{8} \Omega$ feedback resistor of the converter standard made it possible to realize a photocurrent measurement scale with $0.012 \%(k=2)$ uncertainty for the $10^{8} \mathrm{~V} / \mathrm{A}$ and $10^{9} \mathrm{~V} / \mathrm{A}$ gains, and $0.01 \%(k=2)$ uncertainties for the lower gains. These are about a factor of three improved uncertainties over the uncertainties of our traditional (external reference-resistor based) calibrations at the $10^{9} \mathrm{~V} / \mathrm{A}$ signal-gain. During the scale realization, the stable output current of a DC current source of high output-resistance was measured with the current-to-voltage converter standard. The output offset voltage of the converter was compensated at both the scale realization and the following scale transfer. Secondary (and field) current-tovoltage converters up to $10 \mathrm{G} \Omega$ feedback resistors could be calibrated against the reference current-to-voltage converter using the (converter) substitution method. The uncertainty obtained at the $10 \mathrm{G} \Omega$ (maximum) feedback resistor of a test converter, was an order of magnitude lower than with the traditional method. Following the electronic signal-gain calibrations of the current-to-voltage converters, considerations have been made for the minimum shunt resistances of the photodiodes that can be connected to the input of the calibrated converters. As a result of high photodiode shunt-resistance selections, a 10 pA photocurrent can be measured with $0.016 \%(k=2)$ uncertainty. By determining the gain versus frequency characteristics of the converter standard for all signal-gain selections, the method/procedure (based on the converter standard) was extended to AC mode calibrations where the signal-gain uncertainty was $0.05 \%(k=2)$.

It is planned to compare the current-to-voltage converter standard with reference lowcurrent sources at $10 \mathrm{pA}$ and $100 \mathrm{pA}$. 


\section{References}

[1] S. W. Brown, G. P. Eppeldauer, and K. R. Lykke, Facility for Spectral Irradiance and Radiance Responsivity Calibrations using Uniform Sources, Applied Optics, Vol. 45, No. 32, p. 8218-8237, 2006.

[2] G. P. Eppeldauer, NIST Technical Note 1438, Optical Radiation Measurement with Selected Detectors and Matched Electronic Circuits Between $200 \mathrm{~nm}$ and $20 \mu \mathrm{m}$, U.S. Government Printing Office, Washington, DC, 20402, 2001.

[3] G. Rietveld, P. de la Court, and H. E. van den Brom, Intrinsically stable CCC for accurate measurement of small currents, Conference on Precision Electromagnetic Measurements (CPEM), Conference Digest, p. 156-157, 8-13 June 2008, Broomfield, Colorado.

[4] W-S Kim, M-S Kim, K-T Kim, Y. chong, H-J Kim, M. Kim, and H. N. Tauscher, A high precision calibration setup for low-current meters in the range of $10 \mathrm{pA}$ to $1 \mathrm{fA}$, Conference on Precision Electromagnetic Measurements (CPEM), Conference Digest, p. 358-359, 8-13 June 2008, Broomfield, Colorado.

[5] P. P. Capra, F. Galliana, and M. Astrua, A measurement setup to calibrate picoammeters in DC current in the range $100 \mathrm{pA}-100 \mathrm{nA}$, Conference on Precision Electromagnetic Measurements (CPEM), Conference Digest, p. 578-579, 8-13 June 2008, Broomfield, Colorado.

[6] P. Sipila, R. Rajala, P. Karha, A. Manninen, and E. Ikonen, Calibration of currentto-voltage converters for radiometric applications at picoampere level, Proc. NEWRAD, p. 223-224, 17-19 October 2005, Ed. J. Grobner, Physikalisch-Meteoroligisches Observatorium Davos, Weltstrahlungszentrum, Switzerland.

[7] Certain commercial equipment, instruments, or materials are identified in this paper to foster understanding. Such identification does not imply recommendation or endorsement by the National Institute of Standards and Technology, nor does it imply that the material or equipment are necessarily the best available for the purpose. 